\title{
Hello (more-than-human) World. \\ Prototyping an Internet of Things for water with the project wildthings.io
}

\author{
Birgit Bachler
}

\author{
A thesis \\ submitted to the Victoria University of Wellington \\ in fulfilment of the requirements for the degree of \\ Doctor of Philosophy
}

Victoria University of Wellington 


\section{Abstract}

Departing from the concept of an Internet of Things as a means to give voice to non-human 'things', this thesis examines the development of experimental prototypes for grassroots, community-run digital networks and DIY electronic devices as artistic interventions, as an exploration of how design communities can learn from the more-than-human world when building networked media. Since its emergence in the early 1990s, the 'Internet of Things' (IoT)-which imagines a global network infrastructure, in which physical 'things' are connected to the Internet-has mainly developed around human, market- and technologydriven concerns, where 'smart' devices are employed to make daily lives easier. While the current vision of an IoT is largely dominated by human intentions, this design research reconsiders an IoT which amplifies the voices of non-human 'things', and proposes avenues to shift focus from a human-centred to a more-than-human-centred design paradigm.

With a focus on wai/water, this design research engaged with local stream ecologies in Pōneke/Wellington that have largely disappeared from the cityscape, having been piped underground due to urban development. Data collected during fieldwork combines a variety of knowledges through interviews, observations, and immersions, as well as audio and video field recordings and sensor data, such as GPS, humidity, conductivity, and temperature. This intersection of knowledges inspired and informed the creation of design artefacts to learn how the more-than-human world can inform and inspire the development of networked media.

Creative outputs were openly documented in an online repository and concluded in the network installation "Papawai Transmissions", which imagined novel ways of understanding and (re-) connecting with disconnected streams, their communities, and their ecosystems in urban Aotearoa/New Zealand. The research narrative of this thesis is presented in a combination of written word and audio-visual materials to make audible the many voices recorded during the lab- and fieldwork. 
The research revealed how methods of slowness, seamfulness and openness-despite standing in contrast with the domination of quick development cycles in industry-provide opportunities for unheard voices to contribute towards more sustainable ways of designing networks within a more-than-human context.

\section{Keywords}

- more-than-human

- design

- design research

- seamful design

- Internet of Things (IoT)

- networks

- streams

- iterative prototyping

- Pōneke/Wellington

- wai / water

- open

- seamfulness

- slow 


\section{Acknowledgements}

Firstly, I would like to thank all participants, the humans and the freshwater streams, who generously contributed their time and knowledges to this research project.

My sincerest gratitude goes to my primary supervisor Dr Anne Galloway, for your continuous encouragement, your guidance and wisdom, and your thoughtful advice throughout my research journey, and for always being the supervisor I needed at any stage of this project. Your dedication to and leadership in doing better design within a more-thanhuman world has had a profound influence on me and this project, and helped me grow as a researcher.

To Prof Sally Jane Norman thanks for being an inspiring secondary supervisor, for keeping me on my toes with thoughtful and generous feedback and for allowing this work to blossom. I also want to say thanks to Dr Dugal McKinnon for being part of my supervisory team during the early stages of this project.

Thanks to all School of Design staff who made me feel so welcome on the other side of the planet. A special thanks goes to the school administration team, Eli Feth, Mailyn Webb, Bronwyn Knight, Kim Victoria, Gisela Broom, Robyn Isaacs and the workshop technicians, especially Garry Sammons, who provided invaluable support for this project.

Thanks to all my colleagues across academic, technical, administrative and professional staff at Massey's Te Rewa O Puanga/School of Music and Creative Media Production for supporting me and my research in so many ways. Here I would especially like to thank research coordinator and dear friend Dr Catherine Hoad, for engaging in and providing critical feedback on my work, and updating me on vital wisdoms from reality TV shows I missed out on because I was writing. 
To my family, Mum, Dad, Gerhard, Nicole, Eva, Martin, Doris, Michi, and my nieces Julia, Sophie and Diana. Danke, dass ihr mich immer in all meinen Vorhaben unterstützt habtaus der Ferne, aber mir nie fern. Ohne euch hätte ich das nie geschafft.

Thanks to all fellow postgraduate students from WIG001, especially Dr Nabil Allaf, Dr Mailin Lemke and Dr Gillian McCarthy-I will always value your kindness and friendship throughout our studies and your helpful advice on navigating this dauting territory that is a $\mathrm{PhD}$. To the wonderful humans of the more-than-human-lab, thanks for sharing a part of this journey with me. I also want to thank Dr Catherine Caudwell for providing an amazing example of doing a PhD in Design, and encouraging me that I can do it too.

I am grateful to the examiners of this dissertation, Prof Karen O'Rourke, Prof Paul Coulton, and Dr Leon Gurevitch, for taking the time to engage with my research. Here I would also like to thank all the kind people who invited me to present or publish my research and who gave valuable feedback on my work in progress.

A huge thanks to Meredith Robertshawe for the diligent proofreading work.

Ngā mihi nui to my Te Reo teachers Adrian Tangaroa Wagner, Te Ataahia Castorina and Krissi Smith. Your generosity and knowledge supported my learnings here in Aotearoa immensely.

A big round of thanks goes to all my awesome friends, new and old, far and close, who stood by me during my studies, and reminded me that life is not only work. I could not have done this without you, thanks for all the beautiful memories that kept me going. Thanks to Minki the cat for watching me work and keeping my lap warm during late nights.

To Frank. Thanks for always being in my corner and cheering me on. I will never forget your patience and loving support during turbulent times, and I am looking forward to spending many days filled with LOLs, art and music with you. 


\section{Table of Contents}

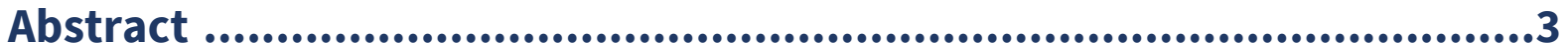

Acknowledgements .....................................................................5

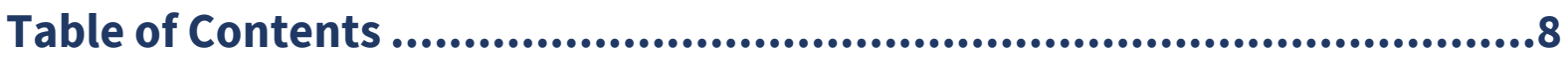

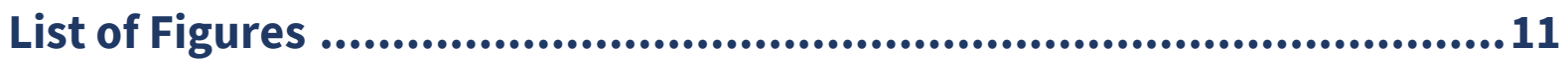

List of Tables....................................................................................20

Chapter 1: Introduction ............................................................21

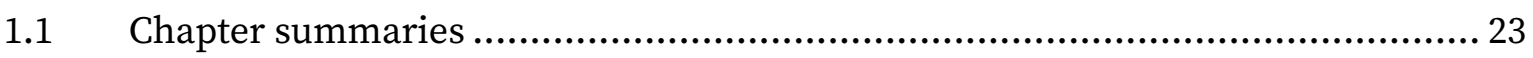

Chapter 2: Charting new waters: An Internet of Things .......................26

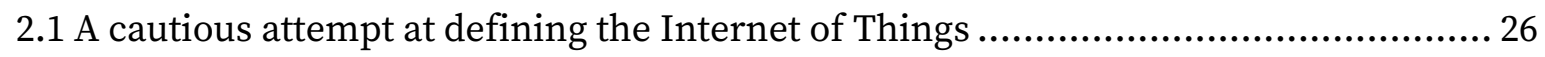

2.2 Mainstreams: A commercial IoT timeline ................................................ 31

2.2.1 Global perspectives: The 1990s and the first Things on the Internet ................. 31

2.2.2 The 2000s and a world of opportunities and standards................................. 36

2.2.3 The 2010 s and the maturing of IoT solutions .......................................... 44

\section{Chapter 3: Encountering turbulences: Troubling loT design in}

Aotearoa/New Zealand with more-than-human concerns ...................50

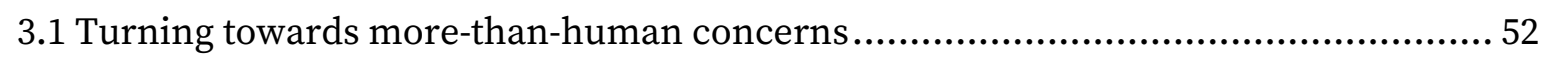

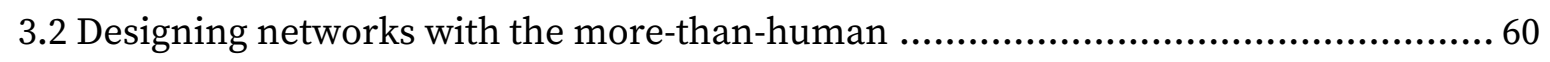

3.3 Getting to know Wai/Water networks in Aotearoa/New Zealand ..........................67 67

3.4 A more-than-human Internet of Things with water ....................................... 71

Chapter 4: Methodology...............................................................73

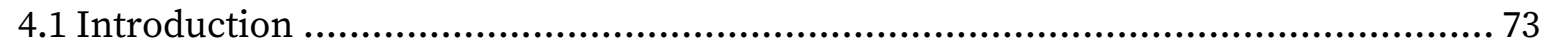

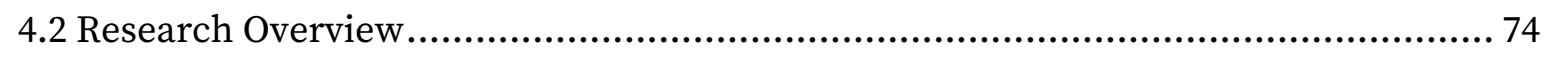

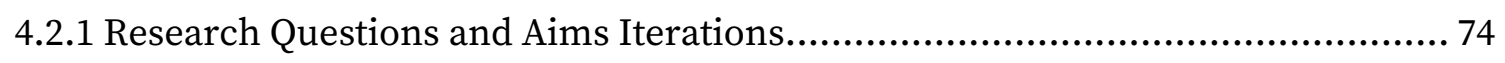

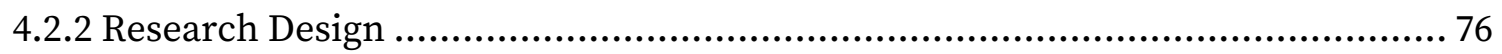

4.3 Limitations/Role of the Researcher ........................................................ 79

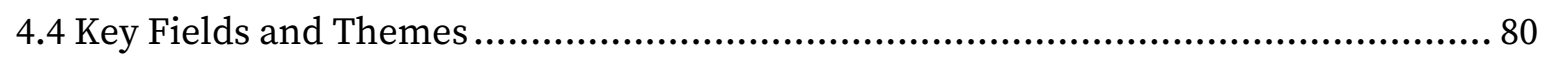




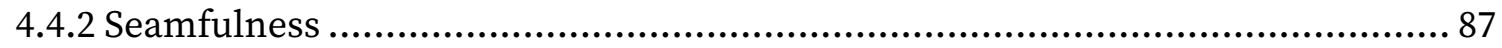

4.4.3 Openness - Opening the design process to more-than-human voices ................ 91

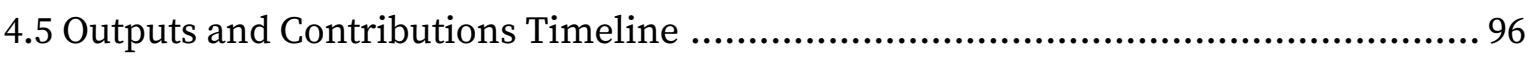

Chapter 5: Field Explorations .............................................................97

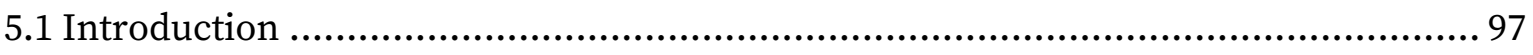

5.2 Exploring Papawai Stream by foot [Blog post 22 October 2016) ............................ 99

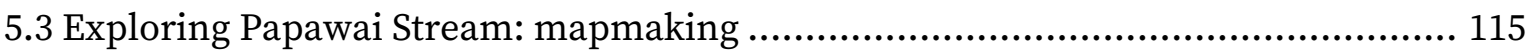

5.4 Walking with: Walkshops in Moturoa Stream, Papawai Stream \& Korokoro Stream 125

5.5 Water on the Internet? Or Worlds that are not supposed to meet.......................... 129

5.6 Participating in the SHMAK workshop........................................................ 131

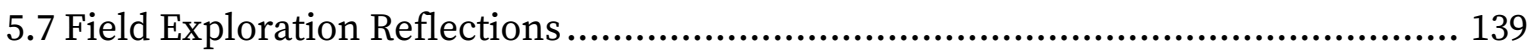

Chapter 6: Prototyping Log .............................................................. 144

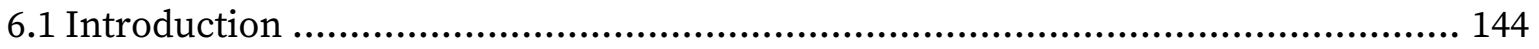

6.2 Building a low-cost hydrophone .............................................................. 146

6.3 Hello World. Building a DIY Internet of Things. [Lab Notebook, 19 December 2016-26

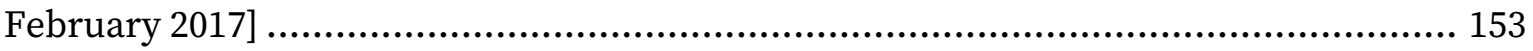

6.4 Wi-Fi in a bottle: recycling materials for waterproofing electronics.................... 166

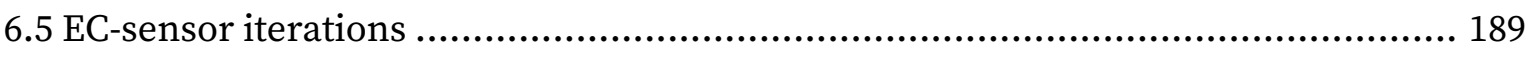

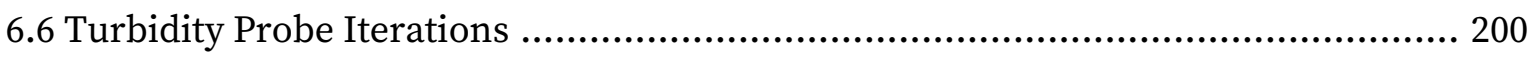

6.7 Giving voice to the stream: Output node iterations ........................................ 243

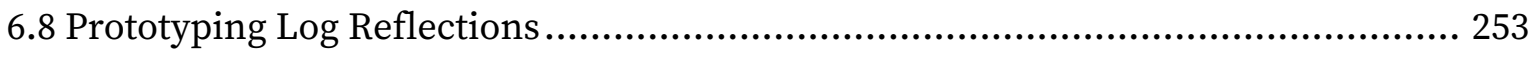

Chapter 7: Field Lab...................................................................... 259

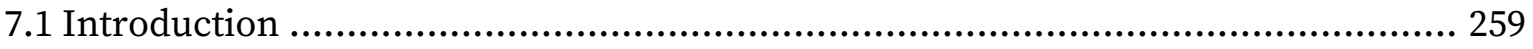

7.2 Moturoa Transmissions - prototyping quick iterations for Brooklyn's Central Park 260

7.3 Testing DIY EC sensors in Papawai Stream [Lab Notebook, 16 December 2018] ...... 271

7.4 Papawai Transmissions - Field Evaluations ................................................. 282

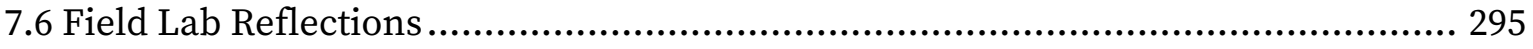


References................................................................................. 309

Appendix A................................................................................... 325

Human Ethics Application 0000023322 : Participatory design walkshops for a more-than-

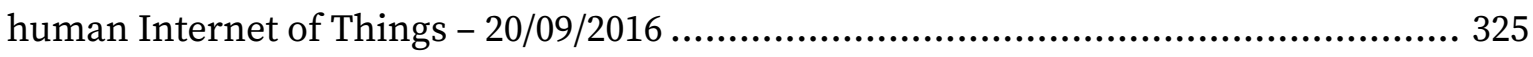

Appendix B .............................................................................. 336

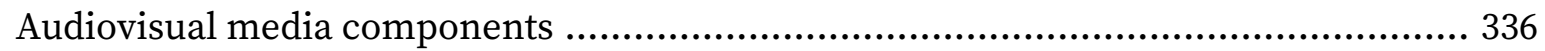




\section{List of Figures}

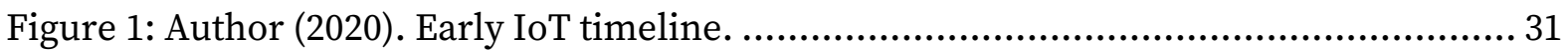

Figure 2: Know Your Meme (n.d.) The Internet Coke Machine.................................... 32

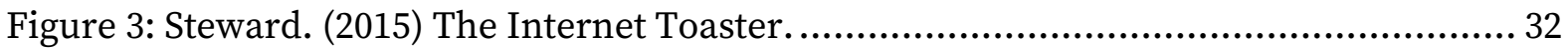

Figure 4: Boulton. (2014). The Trojan Room Coffee Pot............................................ 32

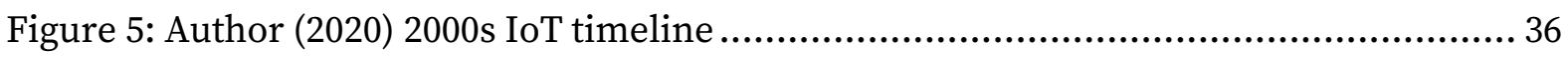

Figure 6: Haque (2011). Pachube Screenshot....................................................... 41

Figure 7: Author (2020). The 2010s and the maturing of IoT solutions........................... 44

Figure 8. Author. (2019). Iterative design timeline............................................ 78

Figure 9: Author. (2016). Noticing the vegetation upon departure upstream. .................. 99

Figure 10:Author. (2016). Google Maps satellite imagery screenshot of Prince of Wales Park

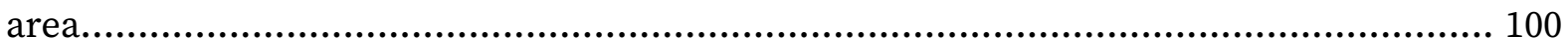

Figure 11. Author. (2016). The stream on the side of the sports field is surrounded by ground vegetation. 100

Figure 12. Author. (2016). Following the pathway that leads towards the stream crossing. 101 Figure 13. Author. (2016). Observing the streambed just under the bridge. ................... 102 Figure 14. Author. (2016). Rocks in the stream bed are accompanied by red bricks. ........ 103 Figure 15. Author. (2016). A fallen-over Pine tree crosses the walking path and the stream.

Figure 16. Author. (2016). Looking downstream with the stream on the left and the path on the right. 104

Figure 17. Author. (2016). The streambed is dry and muddy..... 105

Figure 18. Author. (2016). A bottle sticks out from the dried-out streambed. 106

Figure 19. Author. (2016). The course of the waterflow can be read from the flow of the land and vegetation.

Figure 20. Author. (2016). Further atop the streambed gets steeper and reveals more bricks and old glass rubbish.

Figure 21. Author. (2016). The drain that appears to connect through a pipe to Papawai Stream. 
Figure 22. Author. (2016). The official paved walkway downhill connecting Brooklyn and

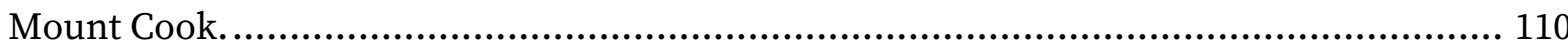

Figure 23. Author. (2016). View from the walkway towards Newtown........................... 110 Figure 24. Author. (2016). Stream culvert close to the Prince of Wales Park sports field... 111 Figure 25. Author. (2016). Streambed erosion by the Park caused the unrooting of a tree. 111 Figure 26. Author. (2016). Streambed erosion created a deep narrow canyon by the Park. 112 Figure 27. Author. (2016). Screenshot of GPS trail recorded during walk with Open GPS Tracker. 113

Figure 28. Author. (2016). Screenshot of QGIS with the following map layers: OSM Map tiles NZ property titles .shp - teal (downloaded from LINZ) NZ street address (2) .shp green (downloaded, LINZ) Streams 1936 .gdb - blue (acquired via WCC). 117 Figure 29. Author. (2016). Screenshot of the stream addresses layered on top of Open Street Map. 118 Figure 30. Author. (2016). Outlines of properties in Wellington crossing territory of original streams. 119

Figure 31. Author. (2016). Historical Papawai Stream and surrounding Wellington Stormwater Network today. 120

Figure 32. Author. (2016). Map of Wellington's stormwater network (teal) and the original streams (blue).

Figure 33. Author. (2016). Screenshot of a prototype of an Open Street Map geo-tagged with photos taken during an exploration of a less accessible part of the stream. 123

Figure 34. Author. (2016). Screenshot of a successful capture of the route walked around Papawai Stream viewed in Google Earth. 128

Figure 35. Author. (2016). SHMAK kit equipment used in the workshop. 132 Figure 36: Author. (2016). A kōkopu is being shown to the participants in a white bucket. 133 Figure 37: Author. (2016). The electric conductivity meter and the data sheet for recording the measurements. 134

Figure 38: Author. (2016). Assessing turbidity with the black disk method. .................... 135

Figure 39: Author. (2016). Assessing Nitrate with a colorimetric test........................... 136 
Figure 40: Author. (2016). Collecting and identifying benthic macroinvertebrates..... 137

Figure 41: Author. (2016). Demonstration of the E.coli test.

Figure 42. Author. (2016). Overview of materials used to build the first hydrophone prototype.

Figure 43. Author. (2016). Detail of the electret microphone in the film canister............ 149

Figure 44. Author. (2016). Detail of the assembled and connected preamp.

Figure 45. Author. (2016). Hydrophone without casing connected to headphones beside Papawai Stream.

Figure 46. Author. (2016). Testing the DIY hydrophone with a repurposed branch used as "fishing pole"

Figure 47: Author. (2017). Screenshot of Hello world output from testing the HelloMesh.ino with two modules. 155

Figure 48. Author. (2017). Testing to WeMos D1 nodes. 159

Figure 49. Author. (2017). Raspberry Pi with multiple terminal windows open for testing of MQTT network switching LEDs of two connected nodes on and off via pub/sub. 160 Figure 50. Author. (2017). Screenshot of the hacked forum.WeMos.cc website showing one legit user post in between spam. 164

Figure 51. Author. (2017). Draft of the star network setup with the hub at the centre connecting possible nodes together.

Figure 52: Author. (2017). Example of a plastic bottle found during a location visit at Moturoa Stream. 170

Figure 53. Author. (2017). Early prototype using a solar charged power bank with a WeMos D1 mini on a small breadboard in a glass jar. 170

Figure 54: Countdown Ltd NZ. (2019) Product image of the water bottle. 171

Figure 55. Author. (2017). Drying empty water bottles on the deck in the sun. 172 Figure 56. Author. (201). First proof-of-concept for using a water bottle as project enclosure.

Figure 57. Author. (2017). Sketchbook showing early drafts for bottle inlays with laser-cut cardboard concepts. 
Figure 58. Author. (2017). Prototyping lab table showing sketchbook with early drafts for EC sensor probe with laser-cut cardboard concepts and components.

Figure 59. Author. (2017). First concept for enclosure assembly with bent metal rod for

suspension.

Figure 60. Author. (2017). WeMos D1 mini incl. relay shield attached to acrylic inlay

prototype with header pins.

Figure 61. Author. (2017). WeMos D1 mini incl. relay shield attached to acrylic inlay prototype with header pins.

Figure 62. Author. (2017). Overview of the iterations showing variations of inlay designs and the final bottle assembly

Figure 63. Author. (2017). Laser cutting one of the acrylic inlays for keeping battery and microcontroller in place.

Figure 64: Author. (2017). The finished acrylic insert holding both battery and

microcontroller in place.

Figure 65: Author. (2017). Final prototypical rendition of the acrylic inlay inside the bottle

enclosure.

Figure 66. Author. (2017). Final prototypical rendition of the bottle enclosure including hanging mechanism.

Figure 67: Author (2017). Workbench in the electronics workshop while assembling

Moturoa_Transmissions.

Figure 68: Author (2020). Temperature probe with screw top cable gland.

Figure 69. Author (2017). Moturoa Transmissions node disassembled for battery charging.

Figure 70: Author (2017). Close-up of the Wi-Fi hub node with a steel blue ladybug exploring the outside of the enclosure.

Figure 71. Author. (2019). Examples of documented guises of glass jars in the project: first transported to the lab as lunch container, then thoroughly cleaned, then used as test enclosure for new nodes or as vessels for storing stream samples. 
Figure 72. Author. (2019). The simple inlays for the bottle enclosures made from recycled cardboard.

Figure 73: Author (2017). Iterations of networked prototypes showing the Wi-Fi hub and MQTT broker (1), the design of the Moturoa Transmissions water temperature sensor (2), and the Papawai Transmission EC-sensor(3) and EC-visualisation (4) probes................. 188 Figure 74. Author. (2017). Attaching two nichrome wires to the audio cable. 189 Figure 75. Author. (2017). Hardware setup for testing the EC probe with a WeMos D1 mini in a breadboard. 190

Figure 76. Author. (2017). Test setup of conductivity meter. 191 Figure 77. Author. (2017). The final assembled design of the first unsuccessful EC sensor probe.

Figure 78. Author. (2017). Testing a repurposed power plug as second EC probe. 193 Figure 79. Author. (2017). Comparing readings of the DIY probe with readings from the TDS meter.

Figure 80. Author. (2018). Comparing the readings of two EC probe prototypes from the same stream sample.

Figure 81. Author. (2018). The terminal output shows the EC probes publishing the measured values under the topics moturoa/ec and moturoa/ecrua. While the test recording was done, the DHT11 sensor was also active in the lab, publishing air temperature (moturoa/atemp) and air humidity (moturoa/ahumid).

Figure 82. Author. (2018). Two bottles for taking water test samples directly from Papawai Stream.

Figure 83. Author. (2018). Testing two EC probes with the same water sample in the lab. 198 Figure 84. Author. (2018). The basic concept illustrated with the two key components: An LDR facing a white LED. 201

Figure 85. Author. (2018). Preparing the hose. 202

Figure 86. Author. (2018). WeMos D1 breadboard setup. 203

Figure 87. Author. (2018). Image of the inside of the garden house with the LDR and LED. 
Figure 88. Author. (2018). Breadboard Setup including garden hose. 205

Figure 89. donblair. (2015). Turbidity sensor design. 205

Figure 90. Author. (2018). First attempt of using hot glue to attach the sensor and LED to the hose. 206

Figure 91. Author. (2018). Arduino... Tools... Board showing list of ESP8266 boards. 208

Figure 92. Author. (2018). Testing the LDR connected to a WeMos D1 with the AnalogIn Arduino example. 209

Figure 93. Author. (2018). I drilled 5mm hole into the hose to fit the LDR neatly. 210 Figure 94. Author. (2018). The prongs of the LED are soldered to strands of the CAT-8 cable.

Figure 95. Author. (2018). Testing the values read by the sensor with the Arduino serial monitor.

Figure 96. Author. (2018). Testing the LED at full brightness encapsulated in the hose. ... 212 Figure 97. Author. (2018). Failed assembly. The sealant has not dried enough overnight to keep the components in place.

Figure 98. Author. (2018). LED and LDR sealed with colour coded heat shrink. 215

Figure 99. Author. (2018). Testing the LED and LDR before assembly in the hose. 216 Figure 100. Author. (2018). Using a vice to keep components in place during assembly... 217 Figure 101. Author. (2018). Hotgluing the components together turns out to be a tricky task.

Figure 102. Author. (2018). The first layers of hot glue applied already reveal some possible weaknesses of the design. 219

Figure 103. Author. (2018). Detail of the sensor probe with dried hot glue. 220 Figure 104. Author. (2018). Test setup of turbidity probe and battery-powered WeMos D1 mini connected to Wi-Fi network. 221

Figure 105. Author. (2018). Cleaning a used bottle casing for repurpose as a new output node

Figure 106. Author. (2018). Draft for turbidity visualisation with six LEDs. 223

Figure 107. Author. (2018). Measuring out the placement of the copper tape. 224 
Figure 108. Author. (2018). Attaching the copper tape to the bottle casing. 225

Figure 109. Author. (2018). Soldering the LEDs carefully to not melt the plastic too much.226

Figure 110. Author. (2018). Testing the solder connection of the LED. 227

Figure 111. Author. (2018). Testing all six LEDs in planned jar enclosure...................... 228

Figure 112. Author. (2018). WeMos D1 mini connected to control the six LEDs. .............. 229

Figure 113. Author. (2018). Copper rods of various diameters. .................................. 230

Figure 114. Author. (2018). Tests of soldering cables to the rods................................ 231

Figure 115. Author. (2018). Shaped copper rod with copper wire before being soldered. . 232

Figure 116. Author. (2018). Testing a group of three LEDs connected to the copper

prototype.

Figure 117. Author. (2018). Detail of assembling grouping of LEDs to copper wire before soldering 234

Figure 118. Author. (2018). Detail of copper wire prototype in progress. 235

Figure 119. Author. (2018). Prototype with two groupings of three LEDs attached. 236

Figure 120. Author. (2018). Final prototype design with three groupings of three LEDs. .. 237

Figure 121. Author. (2018). Detail of assembled prototype with test code. 238

Figure 122. Author. (2018). Terminal output of turbidity data measurements. 239

Figure 123. Author. (2018). Turbidity sensor node being tested in a bowl of tap water. .... 240

Figure 124. Author. (2018). The turbidity sensor node submerged in a bowl of cold tea. .. 241

Figure 125. Author. (2018). While the prototype is configurable in space, the visual data readout is not clear. 242

Figure 126: Author. (2017). Early testing of an output node featuring an OLED shield...... 243

Figure 127. Author. (2017). Early node design featuring a piezo disk for sound output..... 244

Figure 128: Author. (2017). Moturoa status node in lab without bottle casing. 245

Figure 129. Author. (2017). Moturoa status node showing a blue light, indicating data is being transmitted on the MQTT network. 247

Figure 130. Author. (2017). Moturoa status node showing a blue light, indicating data is being transmitted on the MQTT network 248

Figure 131: Author. (2019). Final assembled circuit for the first bespoke LED node. 249 
Figure 132. Author. (2018). The components that make up the audio node: The Adafruit Feather Huzzah microcontroller with the Music Maker FeatherWing shield powered by a battery via USB.

Figure 133. Author (2018). The audio node being tested with a pair of headphones, placed next to the visual nodes for temperature and electric conductivity.

Figure 134. Author. (2020). Screenshot of the GitHub repository iRGBit/wildthings.io. .... 255

Figure 135. Author. (2017). Moturoa Transmissions nodes (water temperature sensor, air temperature/humidity sensor, rain sensor, status node and EC sensor) gathered by the install location for setup.

Figure 136. Author. (2016). Moturoa Transmissions base station during install.

Figure 137. Author. (2017). Installation at Moturoa Stream showing two sensor nodes and one and Wi-Fi Access Point/MQTT Broker.

Figure 138. Author. (2017). Adjusting the cables of the temperature sensor node as part of the install. 265

Figure 139: Author. (2017). Moturoa_Temp node suspended from a tree with sensor cables secured to the branch. 266

Figure 140: Author (2017). Moturoa humid node suspended in a tree as part of the Upstream Brooklyn Art Trail.

Figure 141: Author. (2017). Test installing Moturoa Transmissions nodes at Papawai Stream.

Figure 142: Author. (2017). Contact sheet of iterations of chart design. 269

Figure 143: Author. (2017). Moturoa Transmissions chart, Saturday 4 March 2017. 270

Figure 144. Author. (2017). Moturoa Transmission data visualisation, Sunday 5 March 2017.

Figure 145. Author. (2018). Two wooden crates containing the Wi-Fi Access point and two EC-sensor probes to be tested in jars. The electronics are temporarily secured in place with bubble wrap. Additional materials in the crates are spare enclosures, cables, and batteries. 
Figure 146. Author. (2018). The pathway to access the installation test site besides Papawai

Stream. 273

Figure 147. Author. (2018). Two repurposed extension cables measuring electric conductivity in Papawai Stream. 275

Figure 148. Author. (2018). The temperature node is added to the two EC-probes in the stream.

Figure 149. Author. (2018). The test setup beside the stream................................... 280

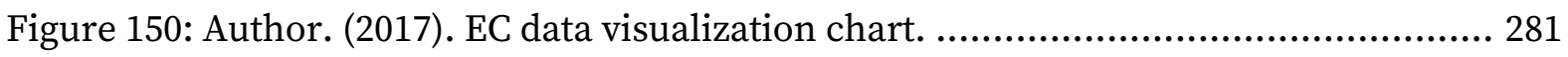

Figure 151. Author. (2019). Papawai Site install beside the sports field......................... 284

Figure 152. Author. (2019). Temperature and EC-sensor nodes in the stream................. 285

Figure 153. Author. (2019). EC sensor node in a glass jar........................................ 285

Figure 154. Author. (2019). Temperature LED probe in a bottle repurposed from the

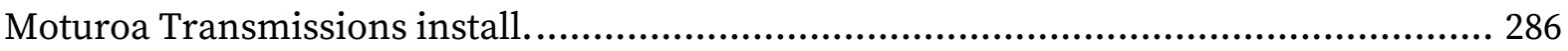

Figure 155. Author. (2019). Overview of the install site by the playing field. ................... 286

Figure 156. Author. (2019). Two versions of LED probes used for the test install............. 288

Figure 157. Author. (2019). The Wi-Fi hub bottle rests in the crate with a drinking water

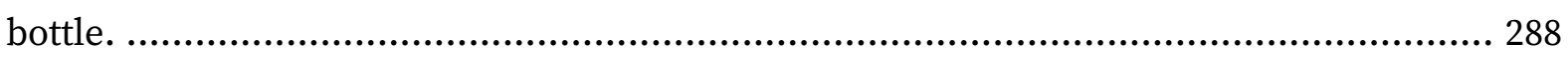

Figure 158. Author (2019). During the install the two sensor probes are suspended from a

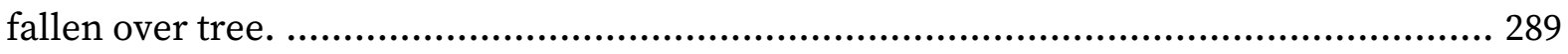

Figure 159. Author. (2019). Sunday install LOG01.CSV data plotted with sensordata_visualisation.pde. 292

Figure 160. Author. (2019). Monday install LOG02.CSV data as plotted through sensordata_visualisation.pde. 293

Figure 161: Author. (2020). Screenshot of fieldexplorations.mp4 .............................. 336

Figure 162: Author. (2020). Screenshot of prototypinglog.mp4 ................................ 337

Figure 163. Author. (2020). Screenshot of fieldlab.mp4 ........................................ 338 


\section{List of Tables}

Table 1: Author. (2020). Places where I have disseminated and discussed my research...... 96

Table 2: Author. (2016). Materials List for building a DIY hydrophone......................... 148 


\section{Chapter 1: Introduction}

The concept of an 'Internet of Things' was first mentioned in 1999 at the Auto-ID Centre at Massachusetts Institute of Technology, connecting products to the Internet as a means to improve the efficiency of supply chain management. While this scenario has been largely dominated by human-centered interests such as market explorations and business opportunities, IoT developments at the fringes of the commercial developer spectrum offer alternative visions of an IoT. Often based in creative research, these experimental visions are established through collaborative co-creation between human and non-humans and illustrate how different types of creative experiments across academia, industry and the arts can manifest deep and complex links, as well as indicate some clear points of rupture and divergence in IoT research. The opportunities to move away from a human-centred vision into more-than-human research territory promises new avenues of giving voice to often under-acknowledged life-forms, such as the more-than-human world of water, and centring them in an IoT research project. By centring the interests of the 'things' themselves, design research is able to explore new avenues of work that might stand as a response to urgencies of the Anthropocene, such as the global climate crisis, the impact of which is slowly being felt in small, localised environments. By attempting to develop an IoT from the bottom-up, and locating the research in a suburban freshwater stream that has been piped underground and subsequently neglected before being restored by a local community group, this research proposes an IoT that responds to localised challenges that affect neighbourhoods, and local more-than-human communities alike.

The motivation to do this research stems from my professional creative practice as a media artist, designer and researcher, and my love for the more-than-human world that has always been grounded in connections with local freshwaters. Having grown up amongst the Eastern Central Alps in a small city in Austria, I always felt a strong connection with the River Mur. I fondly remember watching it flow, downstream and imagining the masses of water merging with larger European rivers before arriving in the Black Sea at some stage. Knowing this river mostly through its connections with its springs, I was in awe when I moved to Rotterdam and spent many hours beside the majestic Maas River, watching it 
patiently flow towards the North Sea for the last few kilometres. Arriving in Pōneke/Wellington and not knowing the local river felt unusual to me. The body of water mainly associated with this city is the Wellington Harbour, the Te-Whanganui-a-Tara, Great Harbour of Tara. At first sight, the city appeared to be devoid of the streams feeding into the harbour.

After hearing stories of eels swimming under Taranaki Street (see for example Manning, 2009, Te Punga Somerville, 2015) I learned about the history of the disconnection between the city and its multiple freshwater streams, buried underground in pipes as a result of colonisation and urban development. While discovering some of the preserved or restored freshwater streams in my new neighbourhood, I wondered if I could respond to this disconnect, offering creative ways of reconnecting those lost water features with the city through network technology. As a media designer, I have always been interested in networks. In my previous work, I have investigated or curated unusual, playful, experimental connections between network nodes: across the more-than-human spectrum, networking citizens and windows, urban features and social network profiles, loyalty card data and anonymous voicemail boxes.

Coming from Europe and being familiar with mostly Eurocentric philosophical perspectives, I initially considered New Materialism as an avenue to understand working with more-than-human worlds, and as opposed to my previous research projects, centring the 'things', the streams themselves, in the research. However, as my research evolved, I began to understand how European scholarship has only recently engaged with ideas of decentring the human in philosophy. In Aotearoa/New Zealand, a Te Ao Māori worldview offers many more established insights into working with the more-than-human world, having never separated humans from their mountains, land, and water.

In this way, this thesis discusses the complexities of working within "worlds that are not supposed to meet”, (see 5.5) attempting to weave together lost narratives of local freshwaters through the use of network technology. By providing narratives from my 
research progress across field explorations, prototyping activities, and field lab work, I discuss how, through embracing methods of slowness, seamfulness and openness, the design work developed network prototypes as part of the research project wildthings.io. The work seeks to respond to the question of how we, as a design community, can learn from the more-than-human world when building networked media. Through the development of experimental prototypes for grassroots, community-run digital networks, and DIY electronic devices as artistic interventions, the project gives way for further investigation how slow development cycles of networked technology can open new avenues for understanding designing within and for the more-than-human world.

\subsection{Chapter summaries}

The first two chapters following the introduction present a literature review of the Internet of Things, followed by attempts to enrich and extend the conversation around an IoT through more-than-human concerns. The fourth chapter illustrates methodological concerns and introduces the three key themes that guided the design research trajectory. The subsequent three chapters present the design research in the form of narratives, concluding each chapter with a reflection. The final chapter presents the conclusions of the research project.

\section{Chapter 2}

This chapter presents a timeline of developments around the Internet of Things and how the idea of networking 'things', has developed, from its beginnings in the since the late 90s, through to the 2010s, to ground this research in the historical conversations around networking objects and understanding the opportunities inherent in expanding the research trajectory further into the territory of more-than-human worlds.

\section{Chapter 3}

In this chapter, I explore how the Internet of Things, as it informs design research, has been critiqued, reimagined, and re-negotiated through the (re-)centring of the more-thanhuman world. By considering the implications for IoT design in Aotearoa/New Zealand, and 
the intersections it traverses with more-than-human concerns, this chapter further maps the theoretical groundwork that informs this thesis project, and introduces and contextualises the methodology

\section{Chapter 4}

In this chapter, I outline the methodology that ties the research project and all activities together. I first introduce the research questions and research aims and provide an overview of my research design across field explorations, prototyping development, and field labs. I subsequently discuss how my research process has been continuously shaped and re-shaped by iterations of practical design research, underpinned by theoretical inquiries into more-than-human thinking. The chapter concludes with a presentation of key fields and themes that recurred throughout my research journey and inspired the research designs throughout various stages.

\section{Chapter 5}

In this chapter, I present my fieldwork activities from the period of 2016 to 2019. Here I focus on field explorations, which involved discovering and connecting to the streams by spending time with them. These activities include explorations in the form of hour-long walks which took on diverse forms: alone or in conversation with invited participants, recorded and unrecorded, paying attention to how we understand the more-than-human world of water and the role it could play in my design research.

\section{Chapter 6}

With this chapter I seek to give the reader an impression of the prototyping activities that underpinned the design research of wildthings.io, between 2016 and 2019, often happening concurrently with the activities listed in the preceding chapter, Field Explorations. In this chapter I focus on the hard- and software development and prototyping in electronics lab and the 3D workshop. The narratives documented in this section shine light at design developments, the small moments of success and failure, that often go unnoticed when only attending to the final outputs of a design process. This chapter thus illustrates how the 
deliberate transparency of my design research process mobilises the key themes of the work into actual documentation, in the form of writing, photos and video recordings.

\section{Chapter 7}

In this chapter, I focus on research activities that sit at the edges or the seams-somewhere in between my field exploration and prototyping work. This chapter focuses on the moments where development from the electronics lab and explorations from earlier fieldwork meet, in the form of installing, testing, and exhibiting my IoT prototypes at stream sites in Pōneke/Wellington.

\section{Chapter 8}

In this concluding chapter I discuss how this research presents a humble departure into a more-than-human research trajectory through creative IoT development. This chapter offers reflections on central contributions, limitations, and future opportunities of this research project. 


\section{Chapter 2: Charting new waters: An Internet of Things}

This chapter presents a timeline of developments around the 'Internet of Things' and how the idea of networking 'things', has developed since the late 1990s, grounding my research in a field that is characterised by conversations across academia, the tech industry, and the arts.

In the first section, I briefly discuss definitions of the term 'Internet of Things' (IoT) and highlight current projections of its market potential alongside the visions and market ambitions of the current 'Internet Big Five' - Amazon, Apple, Facebook, Google and Microsoft. In the second section, I give an overview of how this vision has evolved since the first mention of the term in the late 1990s, outlining the discussion of the IoT throughout the 1990s, 2000s and 2010s.

\subsection{A cautious attempt at defining the Internet of Things}

In this section, I highlight the key works defining and discussing the term 'Internet of Things' (IoT) since it was coined in 1999 at Massachusetts Institute of Technology. This literature survey encompasses a range of views on the IoT, stemming from a divergent range of stakeholders: technologists, developers, marketers, business journalists, makers, and academics from a range of disciplines, including computer science, telecommunications, design, arts, and the social sciences.

The current technical advancement of the IoT is brought forward by the increasing availability of low-cost and energy-efficient hardware and developments in wireless sensor network technologies. Yet, there is still no stable definition for the IoT. In general terms, the Internet of Things is described as a network of interconnected "things", which are uniquely addressable in the network and have the ability to sense and actuate, and to transmit and receive data online. Departing from the idea of a global infrastructure for RFID tags, the vision of the IoT is described as a "future where pretty much everything is online" (Dodson, 2003, para. 10) and people are able to be ever more efficient and productive. 
In 2009 A. Furness, CTO of the EU-funded project CASAGRAS (Coordination and Support Action for Global RFID-related Activities and Standardization), drafted the following definition of the IoT:

\begin{abstract}
A global network infrastructure, linking physical and virtual objects through the exploitation of data capture and communication capabilities. This infrastructure includes existing and evolving Internet and network developments. It will offer specific object-identification, sensor and connection capability as the basis for the development of independent cooperative services and applications. These will be characterized by a high degree of autonomous data capture, event transfer, network connectivity and interoperability (as cited in Patel et. al, 2011, p.1).
\end{abstract}

The IoT is also sometimes referred to as Web 4.0 (Pemberton, 2006; see also The Hammersmith Group, 2009; Khoo, 2010), following Web 2.0, the Social Web and Web 3.0, the Semantic Web. This is, however, not a clear definition, as there are others (Floridi, 2009) who see the IoT as part of Web 6.0, the "Web Onlife" (p.33).

According to a report from CISCO and DHL, "humans will more than triple the number of "things" connected to the Internet, growing them from 15 billion today to 50 billion by 2020" (Macaulay et al., 2015, p.26). One of the earliest ambitious number projections of "50 billion devices by 2020" stems from a press release from tech company Ericsson in 2010 (Ericsson Media Relations Team, 2010), followed by the same prediction by Cisco one year later (Evans, 2011, p.3). Intel Corporation (n.d.) even saw the number of connected IoT devices in 2010 rise up to 200 billion. In the meantime, these projections for 2020 have been narrowed to an estimate of 20-30 billion connected devices (Nordrum, 2016).

From the beginnings of this research project in 2015, to the final data collection stages in late 2018, the Internet of Things resided at the "Peak of Inflated Expectations" of Gartner's Hype Cycle of Emerging Technologies and was expected to reach the "Plateau of Productivity" in 5 to 10 years' time (Rivera, 2015; Panetta, 2018). This graph, which is often cited in popular media (see for example Brewster, 2014 or Deschamps-Sosino, 2015), commercial research reports (e.g. Hewlett Packard Enterprise, 2015), and academic 
publications (see for example Gubbi et al., 2013; McNely \& Rivers, 2014 or Li, et al., 2018), shows IoT-related technologies such as mesh-sensor-networks and RFID entering and leaving the graph during the past ten years. Thus, Deschamps-Sosino (2015, para. 11) concludes that it does not matter where the term sits on the chart, its long-term presence "in multiple guises" shows its success and growth. In its latest 2019 version, the term 'Internet of Things' has correspondingly disappeared from the Gartner graph, but is mentioned as part of trends within sensing and mobility (Panetta, 2019), and can again be found at the peak of inflated expectations in the guise of $5 \mathrm{G}$ technology (see also Li et al, 2018).

However, complementary critiques note that eventually the 'Internet of Things' is just a "code term that powerful stakeholders have settled on for their own purposes" (Sterling, 2004, p.1). Following this claim, a look at the IoT endeavours of the 'Internet Big Five' Amazon, Apple, Facebook, Google and Microsoft - can outline the mainstream market as follows:

- Amazon is entering the Internet of Things market by developing the managed cloud platform “AWS IoT" for connecting billions of devices "easily and securely with cloud applications and other devices" (Amazon Web Services, Inc., n.d.).

With AWS IoT, your applications can keep track of and communicate with all your devices, all the time, even when they aren't connected. (Amazon Web Services, Inc., n.d.)

Alexa, Amazon's virtual assistant AI (Artificial Intelligence) is also advertised in combination with AWS, offering to "enrichen the customer experience" by controlling their devices through the voice commands offered by Alexa (Amazon Web Services, Inc., n.d.-b).

- Apple has introduced a domestic solution called "HomeKit" (Apple Inc., 2014). Under the slogan "Your home at your command", Apple advertises its Home app as the central hub to control accessories such as lights, switches, speakers, air conditioning sensors and other compatible appliances from one Apple device such as an iPhone or an iPad. 
- Facebook's first steps into IoT started in 2013 when acquiring the company Parse. Parse was originally aimed at facilitating web and mobile application development. Since 2015, the company has offered a developer platform for embedded and IoTspecific devices (Parse, n.d.). Parse was shut down in 2017 (Isaac \& Hardy, 2016), but continued to operate as the open-source Parse Server (Parse-community/parse-server, 2016/2020). Facebook's latest involvement with IoT technology is Portal (Facebook, n.d.) marketed as "smart video calling" hardware and software compatible with Amazon's virtual assistant Alexa.

- Google's first widely known IoT project was its 2014 acquisition of the smart home company Nest Lab. The "Nest Learning Thermostat" \& "Smoke + CO Alarm" and “Nest Cam” (Nest Labs, Inc., n.d.) are the products offered by Nest Labs. Similar to Apple's HomeKit, these products focus on home security and compatibility with a range of other smart products and domestic appliances (Nest Labs, Inc., n.d.). Google currently operates Google Cloud IoT, offering a platform consisting of "scalable, fully-managed cloud services; an integrated software stack for edge/onpremises computing with machine learning capabilities for all your IoT needs" (“Google Cloud IoT - Fully managed IoT services,” n.d.).

- Microsoft (n.d.) focuses on real-time business solutions and presents the "Microsoft Azure Internet of Things Suite". The company presents the Internet of Things as a "practical and applicable technology trend that can generate return on investment (ROI) and drive efficiencies and insights for organizations that know how to use it" (Edson, 2015).

All five companies direct their IoT development towards mainstream markets, focussing on business and smart home related solutions. Currently, the IoT market is highly competitive, and other businesses are still trying to find their niche. However, compatibility with other solutions (see Nest Labs, Inc., n.d. or Apple, n.d.) are valuable features of commercial IoT ecosystems. 
The following overview provides highlights of three decades of discourse on the Internet of Things. It reflects on the emergent and fluid nature of the IoT, and also demonstrates how the ambiguity of the term and uncertainty of underlying concepts allows a diverse range of stakeholders to drive their own agendas. Things themselves, however, are inevitably a part of the term, and play a crucial role in development and design of the IoT concept. In all cases, things are given a 'voice' and capacity to act in the physical world. They become nonhuman actors in, and users of, an ecosystem of digital networks that was previously only inhabited by human agendas. 


\subsection{Mainstreams: A commercial loT timeline}

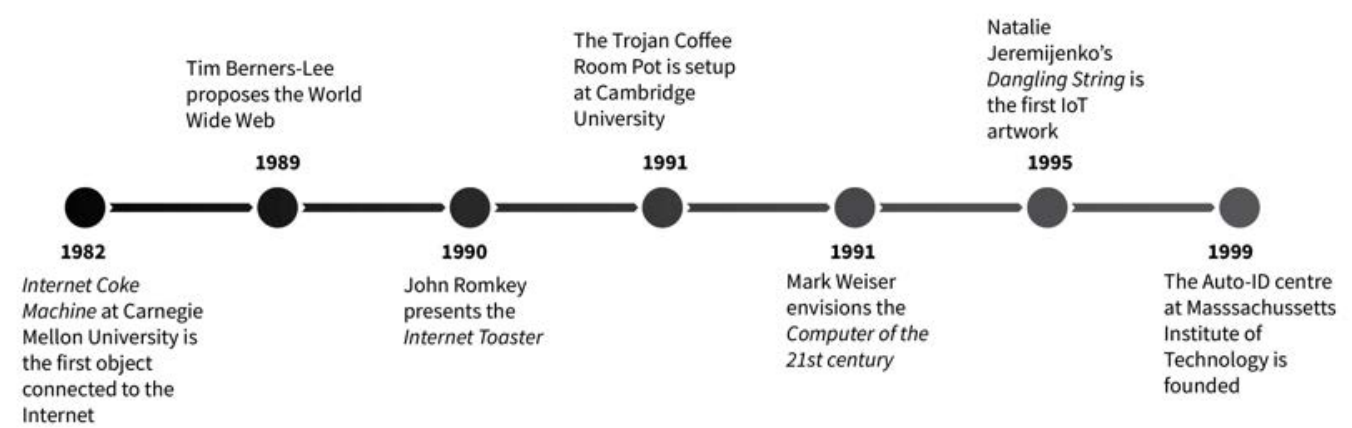

Figure 1: Author (2020). Early IoT timeline.

\subsubsection{Global perspectives: The 1990s and the first Things on the Internet}

The term 'Internet of Things' originated in 1999 at the Auto-ID Centre at Massachusetts Institute of Technology. Kevin Ashton (2009), co-founder and executive of the Auto-ID Centre, presented the idea of improving the efficiency of Procter and Gamble's supply chain management by connecting products via RFID technology to the Internet:

\section{Adding radio-frequency identification and other sensors to everyday objects will create an Internet of Things, and lay the foundations of a new age of machine perception (as cited in Santucci, 2009, p.2).}

However, the idea of connecting objects to the Internet is not entirely new, and Internetbased technologies such as sensor networks have existed since the 1990s (see for example Perera et al., 2014). The first 'everyday' object connected to the Internet was a Coke machine at the Carnegie Mellon University Computer Science Department (Figure 2). The system, developed in 1982, monitored the out-of-product lights on the machine's push buttons and published the status of the machine online, including notification if a column was empty and needed to be refilled, and which column contained the coldest can (Everhart, 1990). Thus, besides inventory control and quantity tracking, the machine also attempted to indicate product quality. 


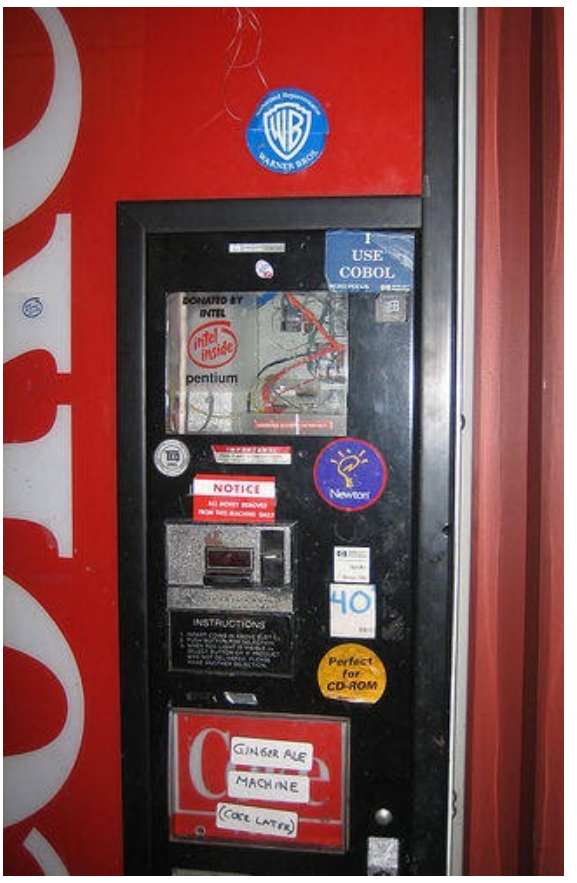

Figure 2: Know Your Meme (n.d.) The Internet Coke Machine.

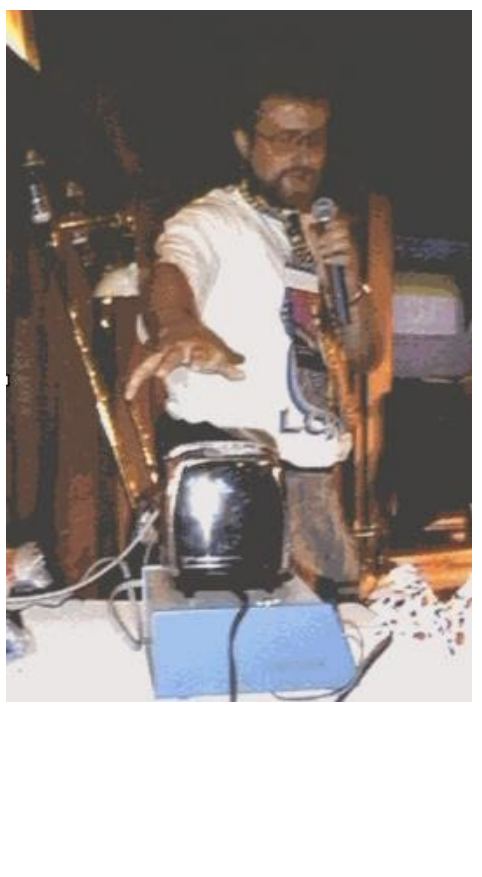

Figure 3: Steward. (2015) The Internet Toaster.

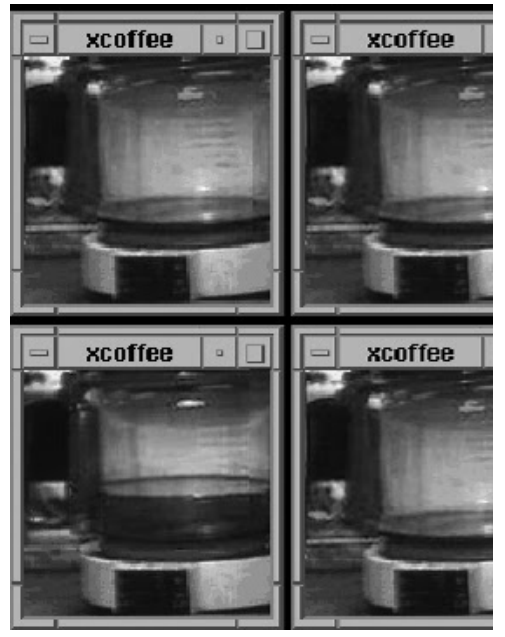

Figure 4: Boulton. (2014). The Trojan Room Coffee Pot.

In the early 1990s, developer John Romkey connected a toaster to the Internet at the Interop Internet Networking show in Las Vegas (Figure 3). The toaster could be controlled via TCP/IP and SNMP (Simple Network Management Protocol). One year later, the project was further modified by adding a robotic arm that was able to load the appliance with slices of bread. Subsequently, more experimental prototypes of networked things were presented at the show such as the talking Internet Weather Bear, outputting local and remote weather data via voice synthesis (Malamund, 2000; Stewart, n.d.; Savetz, 1994; Dern, 1992). Another early computer lab project is the Trojan Room Coffee Pot (Figure 4), which included a video frame-grabber locally streaming the live image of a coffee pot locally to researchers in the building. Later, the image feed was made available on the World Wide Web, and was allegedly one of the most popular sites on the web at the time (Stafford-Fraser, 2001). 
Playful explorations of how everyday objects could be connected to the Internet were substantially on the rise in commercial as well as artistic contexts in this period. One of the earliest artworks often associated with the Internet of Things is Dangling String or Live Wire by Natalie Jeremijenko (1995):

Created by artist Natalie Jeremijenko, the "Dangling String" is an 8 foot piece of plastic spaghetti that hangs from a small electric motor mounted in the ceiling. The motor is electrically connected to a nearby Ethernet cable, so that each bit of information that goes past causes a tiny twitch of the motor. A very busy network causes a madly whirling string with a characteristic noise; a quiet network causes only a small twitch every few seconds. Placed in an unused corner of a hallway, the long string is visible and audible from many offices without being obtrusive. It is fun and useful. The Dangling String meets a key challenge in technology design for the next decade: how to create calm technology (Weiser \& Brown, 1996).

The movement of the string is directly linked to the amount of network traffic that goes through the connected Ethernet cable. The string is kept "alive" through the presence of network traffic. The way this work renders abstract network data concrete and visualises data flows in a material form was a novel approach for the time (Weiser \& Brown, 1996).

In Mark Weiser's (1991) envisioning of the computer of the 21st century, he describes an environment in which networked computers of various sizes and forms vanish into the background. In his vision, machines reside in the human world and pose no barrier to physical interaction, unlike the then-popular desktop computer.

Machines that fit the human environment, instead of forcing humans to enter theirs, will make using a computer as refreshing as taking a walk in the woods.

(Weiser, 1990) 
In later talks (1994; 1995) Weiser addresses the misleading concept of seamlessness and argues for "seamful systems", with "beautiful seams". He also rejects the idea of an interface as a boundary between people and machines and argues that the basic unit of design should involve social people, in their environment, plus their device (Weiser, 1995, p.21). Later, Chalmers (2003) similarly argues for 'seamfulness' in design, and describes it as

...taking account of the finite and physical nature of digital media. Seamful design involves deliberately revealing seams to users, and taking advantage of features usually considered as negative or problematic (Chalmers, 2003, p.1).

Chalmers points out that the revealing of the seams in the infrastructure of ubiquitous computing can be an opportunity for user understanding and empowerment. Seams could also offer a trajectory towards the creation of more dynamic systems, which can adjust to interaction patterns originally not envisioned by the designer.

In pursuit of Mark Weiser's ubiquitous computing vision, Schilit and Theimer (1994) describe an active map service, capable of publishing information of networked things called "located-objects" and their changes over time. Located-objects can include anything with an associated physical location, ranging from workstations and printers to a single person. In this vein, Lamming and Flynn (1997) predicted the 21st century would be defined by what they call "intimate computing". Their Forget-Me-Not project proposes a wearable device that offers computer-based support for human memory. What they describe as a "prosthetic episodic memory device" (p.2) appears similar to an early conception of today's smart phone or smart watch. Interestingly, Lamming and Flynn saw privacy and security as major concerns for users of their Forget-Me-Not (p.8). Similarly, Gershenfeld (1999, p.200) saw the need for unobtrusive computing, as opposed to ubiquitous computing: smart materials and gesture recognition, giving the still inert personal computers the ability to hear, see, move, and respond. Such responsiveness thus forms a key feature of this mode of computing, where there is a leap between passive hearing, seeing, and moving, and the active engagement of these faculties as responses. 
Towards the end of the 1990s, research towards augmenting physical objects through RFID technology foresaw the development of invisible interfaces for the workspace of the future (see for example Harrison et al., 1999). These futuristic interfaces promised a combination of the intuitiveness of the physical world with the advantages of computation. They saw more interesting scenarios possible when wireless network connections are available; however, the mentioned examples stay within the workplace paradigm.

One notable project that aimed to study how these technologies can be designed for home use is the "Aware Home". In their living laboratory, equipped with tracking and sensing technology, Kidd et al. (1999) followed a technology-centred and a social, human-centred research agenda. They also questioned how useful the frameworks designed for office applications aimed at enhancing productivity, efficiency, and profit, could be in a home context (Kidd et al., 1999).

In the early 1990s, the first IoT prototypes were developed in computer science departments, connecting common electric appliances to the Internet as proofs-of-concept. In the mid-90s, Mark Weiser's vision of ubiquitous computing set new ambitions for the field, thinking beyond the spatial restrictions of the then-dominant desktop computer. The commercial development of RFID technology at the end of the 1990s created hope for new possibilities of networked products, resulting in the coining of the term 'Internet of Things'. 


\subsubsection{The 2000 s and a world of opportunities and standards}

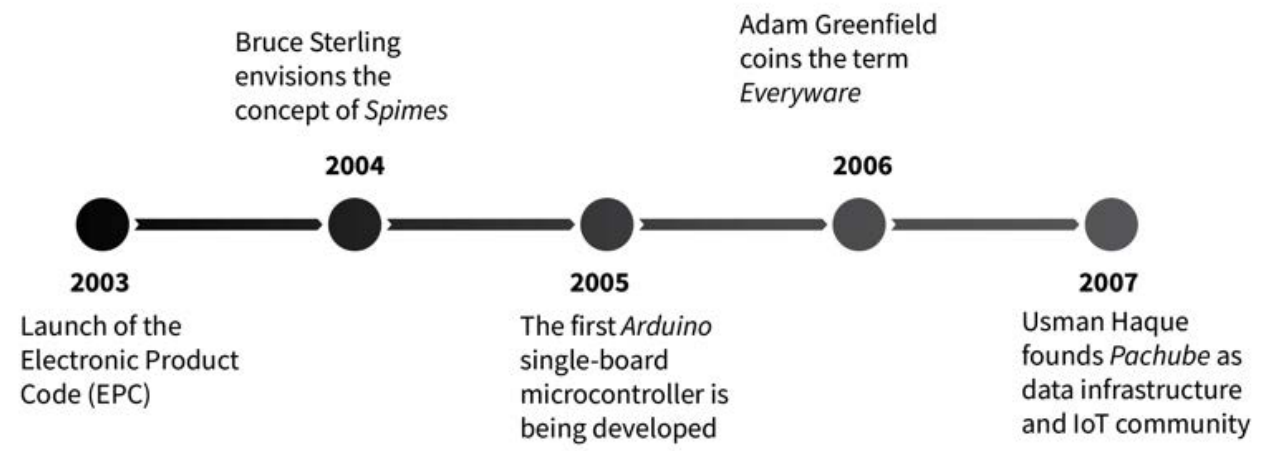

Figure 5: Author (2020) 2000s IoT timeline

The Internet of Things was a new concept that emerged at the end of the 1990s, and the idea of networking and tracking products through RFID gained momentum in the 2000s.

At the Auto-ID Centre, the "networked physical world" was regarded as the next generation of computing, where technologies such as the Electronic Product Code (EPC), the Bluetooth standard or the Physical Markup Language (PML) would significantly change humancomputer and human-object interfaces in warehousing, logistics, industrial and utility systems (Sarma et al., 2000). Schoenberg (2002) describes sensors in retail contexts as "the ultimate in inventory management" (para.2). He lists three of the EPC's advantages over the barcode: less need for manual labour (scanning), fewer inventory glitches, and a decrease in shoplifting (para.4).

In this period, in business publications (see for example Ferguson, 2002), the market potential of the IoT was recognised to have the capacity to not only reshape companies' supply chains, but entire industries. Object-to-object communication, it was assumed, would enable a form of "silent commerce" that would require no human interaction (para.3) and provide new levels of customer insights, allowing monitoring of an item even after purchase (Adams et al., 2001, p.2), and predicting the emergence of an "object internet” (p.1). Accordingly, research groups were established to explore new opportunities of the IoT. Notably here was the EU funded Disappearing Computer Initiative (2003) which 
sought to "see how information technology can be diffused into everyday objects" through the commission of 17 research projects across European institutions.

In the early 2000s, notable research projects around the 'smart home' similarly gained traction. MIT's Project Oxygen (2004) explored Pervasive Computing, with the intention of centring research around human needs, as opposed to machine requirements. The Philips research laboratory HomeLab investigated Ambient Intelligence for the future home (De Ruyter, 2003) by testing new prototypes in a laboratory built as a functional two-storey house.

Kindberg, Barton, \& et al. (2000) considered the World Wide Web (WWW) as a suitable network for building an Internet of Things. People, places, and things could have an online presence through an assigned Uniform Resource Locator (URL) making them accessible via Hypertext Transfer Protocol (HTTP), just like web pages. Waldo (2002, p.9) at Sun Systems noted that the Internet was becoming the communication fabric between devices and services, and humans were becoming a minority online. He proposed Jini as a networking technology capable of meeting the demands of an ever larger and more heterogeneous network for devices (p.17).

However, in the wake of the launch of the Electronic Product Code, media (see for example Dodson, 2003) raised the concern that through a broad introduction of tagged retail items, customers would be the ones being traced instead of products. Alongside privacy concerns, the emerging technology was also troubled by the lack of a network standard and growing competition in the development of wireless standards, like the competitive ZigBee and WiMax protocol (Weisman, 2004).

Computer scientists (Dickerson et al., 2008) anticipated large data streams created by the sensor nodes of the Internet of Things and developed abstractions that allowed these streams to be "first-class citizens on the Web": every data stream should have a URL that 
could be manipulated through the HTTP protocol and would be hyperlinked to other objects on the Web (p.360).

Further looking into feasible network designs, Gershenfeld and Vasseur (2004) drew an analogy with the early Internet and its battle against then competitor Bitnet, a centralised system for linking computers through mainframes. Eventually, the decentralised Internet grew more popular. Similarly, the IoT also faced a battle between a centralised version, where 'dumb' devices are wired to a proprietary controller with an Internet connection, and a decentralized version in which 'smart' devices have their own IP addresses. This latter scenario "enables innovation at the edges" (p.66). Besides encouraging innovation, they considered scalability, robustness, and security as key guiding principles of an IoT development, based on the success of the early Internet (p.67).

By this point, the techno-scientific developments of the Internet of Things also had an influence on the media arts, resulting in the field of locative media:

\section{Locative media emerged over the last half decade as a response to the decorporealized, screen-based experience of net art, claiming the world beyond either gallery or computer screen as territory (Tuters \& Varnelis, 2006, p.357).}

Tuters and Varnelis (2006) predicted that the Internet of Things could engender a world in which objects become sentient, and liberate themselves from "human bondage", envisioning a "machinic third nature" (p.363). Sterling (2004) similarly described speculative objects tagged with their own histories and the capacity to record their location in time and space with the term "spimes". In his keynote address at SIGGRAPH 2004, he envisioned spimes to have searchable identities and histories. They would be precisely located in space and time, continuously recorded, tracked, inventoried, and always associated with a story. Sterling further elaborates on spimes as "sustainable, enhanceable, uniquely identifiable, and made of substances that can and will be folded back into the production of future spimes" (2005, p.11). 
In 2006 Greenfield used the term "Everyware" for describing the leap of computing away from the desktop paradigm into everyday life, and saw technologies such as RFID and 2D barcodes as a "low-impact way to 'import' physical objects into the datasphere, to endow them with a digital shadow" (p.210). Furthermore, Kitchin and Dodge (2011) noted that

\section{... everyware in its contemporary deployment is highly partial in nature, uneven, and unequal in distribution, density, penetration, sophistication, and form. Access is dependent upon economic resources, knowledge to use technologies, location, and whether appropriate infrastructure is available, and devices and networks being interoperable (Kitchin \& Dodge, 2011, p.216).}

Dennis (2006) described the emergence of an Internet of Things as one centre of the new modulations of social, continuous control as described by Deleuze (1992, p.5). Dennis argued that "these ubiquitous and mobile technologies shred 'taken-for-granted' categories of late modernity, such as the once-conventional distinctions between public and private" (Dennis, 2006, para.4). The first release of the open hardware prototyping environment Arduino in 2005 also played a key role in disseminating IoT-related technology among the 'maker' scene, a term originating from the US-based Make Magazine, founded in 2005 by Dale Dougherty (2012). The Arduino platform served as a catalyst for 'maker' projects, providing an affordable platform for building networked projects.

In his Manifesto for Networked Objects artist and technologist Bleecker (2006) dismissed the idea of an Internet of Things as solely a technical framework for "a television talking to a lamp" (p.17), and saw its capacity for creating "more habitable worlds" (p.19) in which our engagement in a more lively way with things, engaging in trans-species dialogues. Perhaps because he was writing his manifesto at the height of blogging culture, Bleecker assumed that social impact on the Internet was facilitated through blogging. He created the term "blogject" for describing things interacting and participating in the "meaning-making" apparatus of the social web (2006, pp.2-3). Bleecker called agency the most provocative aspect of the blogject feature set, and he saw that agency happening within the ecology of networked publics: 
The Blogject capacity for producing effects is far more powerful because it has always been pervasively, ubiquitously, everywhere tethered to the far reaching, speedy, robust network of social exchange and discourse that humanity has every constructed. In the Internet of Things, that kind of agency happens within the ecology of networked publics - streams, feeds, trackbacks, permalinks, Wiki inscriptions and blog posts (Bleeker, 2006, pp.8-9).

Similarly, Stanford-Clark's (2009) term “tweetjects” referred to objects that exhibit a kind of agency within the ecology of networked publics by participating in social exchange on the microblogging platform Twitter. Stanford-Clark combined home automation with the social web: he installed sensors in his house with the capacity to publish updates via the Twitter handle @andy_house, publicly sharing the status of his electricity and water meters, lights and fountains.

The question of agency is again visited by Kranenburg (2007), who discussed the possible impacts and caveats of an IoT's “Ambient Intelligence” which, besides the opportunity to improve supply-chain management, has the potential to reshape entire cities. He argues for an open and inclusive process of IoT development, using public consultation to avoid the development of an ultimate tool for surveillance. Interestingly, he also described artists as the ones who have "always exploited the conditions for technological change, applications and services” (p.16), and predicted that the poetic process of making meaning and creating experiences would no longer happen at the level of design, but would instead be found within IT architecture, its standards, and its protocols (p.16).

Encouraging and facilitating the collaborative sharing of sensor and actuator data, Pachube (Figure 6) was an open web platform for connecting IoT devices, active between March 2007-July 2011 where developers could connect their virtual and physical IoT devices and share them publicly (Haque, n.d.). 


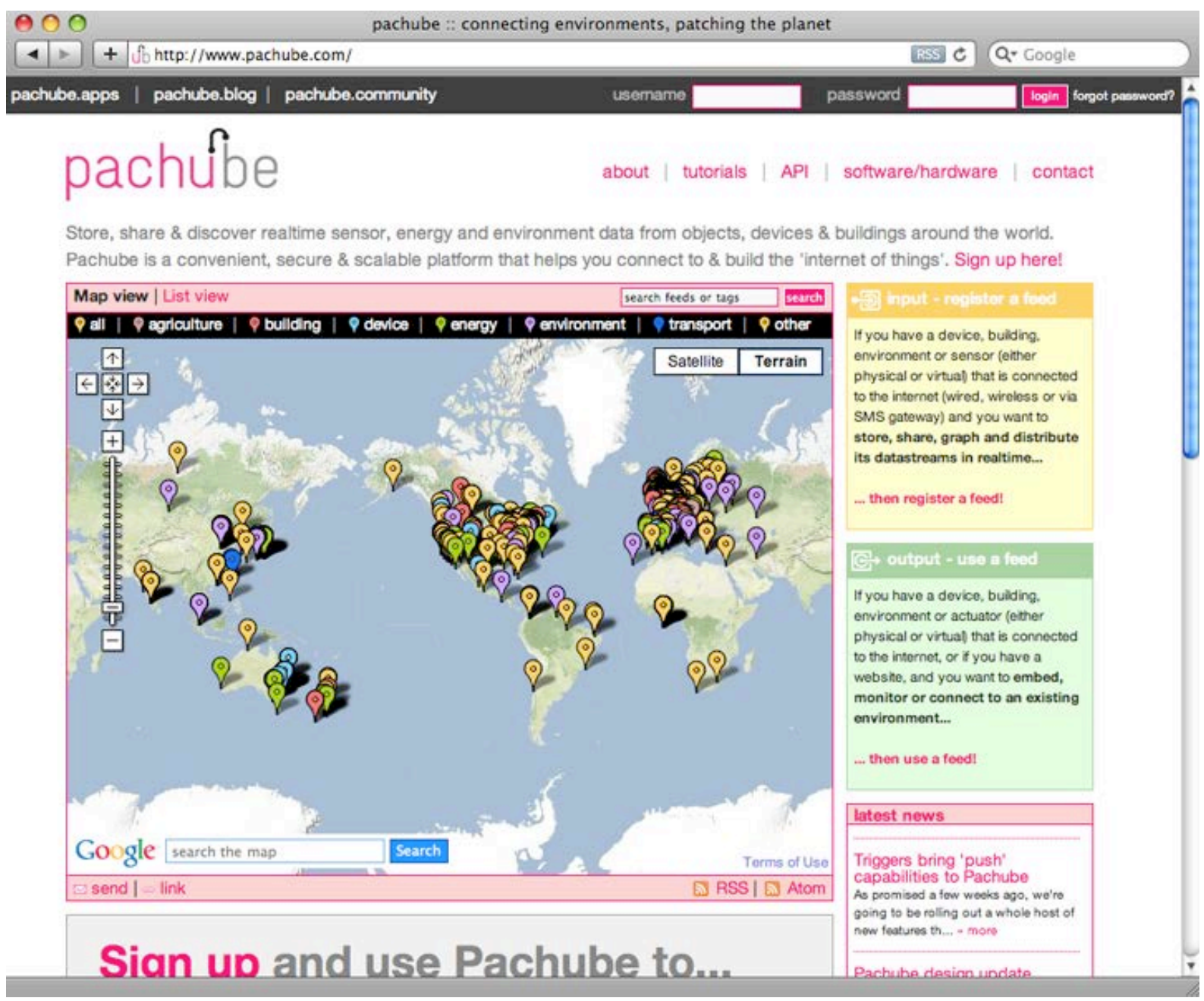

Figure 6: Haque (2011). Pachube Screenshot.

The project was first acquired and rebranded as Cosm in 2011 (Butcher, 2011), and is now run under the name Xively, which is currently part of the Google Cloud Platform family and offers an enterprise IoT platform and application solutions (Xively, 2015). Code examples and tutorials in books (for example Pfister, 2011) for DIY IoT applications still feature Pachube as a means to stream sensor data to the web and need to be updated. This exemplifies how the acquisition of a centralised IoT platform can disrupt an IoT developer ecosystem.

The death of businesses within an apparently lively market can be observed among many initially popular IoT middleware solutions: The development of the once popular opensource IoT application and API ThingSpeak was abandoned in 2015 (ioBridge, 2010/2020), succeeded by a more advanced commercial, hosted DotCom version (ThingSpeak, n.d.). The open-source IoT API Nimbits was archived in 2018 (Sautner, 2011/2019). Of the two 
projects the developer mentions to follow up on, Node-RED is still active (Node-RED, 2013/2020), while Stringify has been shut down (Summerson, 2019). The open-source project for visualising IoT sensor data, Emoncms, still operates as a hosted and selfhostable (Emoncms/emoncms, 2012/2020).

The 2000s also saw a rise in creative projects that incorporated ideas of an Internet of Things into their designs. For example, the Java Toaster (2001), a Brunel University London product design graduation project, used bread as a display device for meteorological information sourced from the Internet (Ward, 2001). No Place Like Home (Wilcox, 2012) is a pair of GPS shoes; Wi-Fi Dowsing Rod (Thompson, 2007) is a divining rod indicating the strength of wireless signals. In a more disruptive manner, Constraint City (2007) translated the strength of nearby closed Wi-Fi networks into a painful bodily experience to a human user, by tightening a chest strap embedded in a jacket (Savicic, n.d.). The interaction was described as "pushing the urban networks intangible reality onto the individual's body" (Canonico, 2007).

The Immaterials series were an output of the research project Touch (2005-2010), from the Interaction Design department at the Oslo School of Architecture and Design. The makers described their work as 'discursive design' that makes the invisible structures of wireless technology visible "as both celebration and critique, drawing attention to the spectacle of otherwise invisible structures and opening them up for reflection" (Arnall, 2014). Ghost in the Field (2009) was an attempt at visualising the spatiality of RFID radio waves with light. Later work Light painting Wi-Fi (2011), made use of the same concepts to visualise Wi-Fi signals in urban spaces, Satellite Lamps (2014) linked the strength of GPS signals to the brightness of a lamp. These works show how a range of actuators can be employed for data representation in a network, simplifying interfaces by removing the common display and adding the possibilities for material, tangible interactions with networks.

The 2000s were characterised by the search for technological frameworks for building an IoT that was capable of connecting things on a global scale. Developments of the IoT were 
closely linked to the advancement of the WWW away from the workplace and into people's private lives. The release of the Arduino platform included non-professionals in the development of networked objects, and critics advanced the discussion around the impacts of the IoT on people's lives. 


\subsubsection{The $2010 \mathrm{~s}$ and the maturing of IoT solutions}

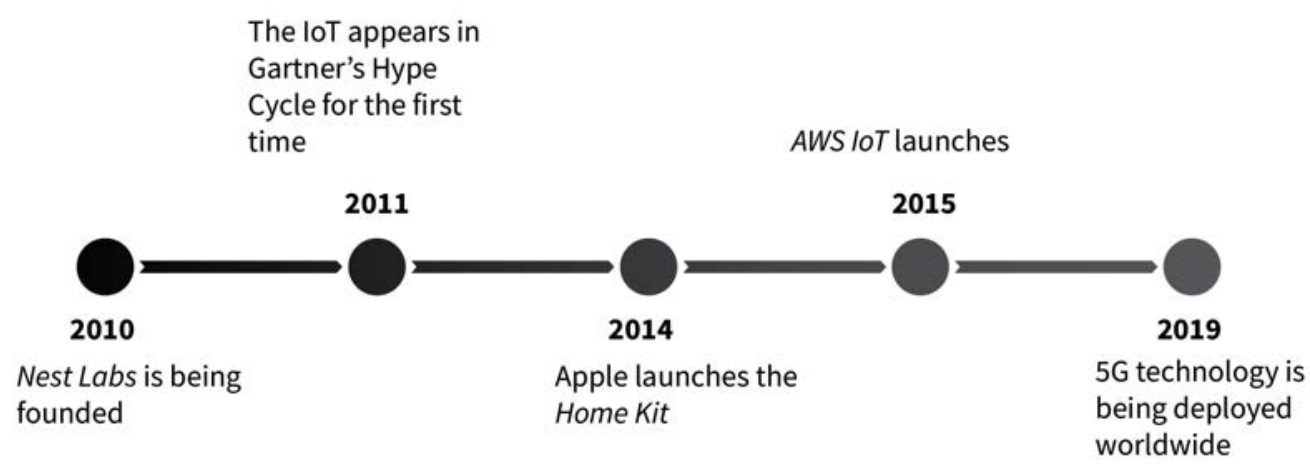

Figure 7: Author (2020). The 2010s and the maturing of IoT solutions.

The computer has escaped the box, and ordinary objects in space are carriers of digital signals (Easterling, 2012, p.1).

The search for possible standards, protocols, and frameworks for realizing an IoT has continued throughout the 2010s, and philosophical considerations of the implications of agentic and social capacities of objects have grown denser throughout a variety of disciplines.

Kranz et al. (2010b) investigated new opportunities for Human Computer Interaction (HCI) within the Internet of Things and described a set of guidelines for "embedded interactions" to let users communicate and interact with the IoT. They also recommended the use of specialised components over all-in-one devices for specific tasks. The guidelines suggested careful consideration of the trade-off between clearly visible and seamlessly integrated controls in a product design. Furthermore, they suggested that an object's original behaviour should not change, but the added value through its IoT functionality should still be perceived by the user (p.52).

In 2010, Atzori, Iera \& Morabito published a survey highlighting that industries and disciplines such as telecommunications, informatics, electronics, and the social sciences have different visions of the Internet of Things. They claimed that the different visions 
depended largely on whether one approaches the paradigm from an "Internet-oriented" or a "Things-oriented" perspective. Additionally, they introduced a third "Semantic-oriented" perspective, which encompasses the challenge of uniquely addressing a large number of heterogeneous objects, and the representation and storing of exchanged information. In an attempt to widen the scope of objects connected through the IoT, Leder et al. (2010) developed a website as a platform to attach stories and memories to old artefacts that evoke a feeling of connectedness and togetherness.

Valhouli and Constantine (2010) described networked objects that blur the line between bits and atoms as mashups between physical objects and "informational shadows" (see also Greenfield, 2006, p.210). Similarly, Shingleton (2011) saw the phenomenon of the IoT as an anticipated technical and cultural shift where links between concrete objects and abstract data produce a hybrid of physical and electronic spaces. He asserted that if objects are treated like code, the

\section{messages they encode will emerge from the pattern of social relations being expressed, allowing the Internet of Things to provide the meta-data that enables data to self-organize, assembled out of an unthinkable number of associations created by agents both human and non-human (Shingleton, 2011).}

Speed and Shingleton (2012) also discussed object agency, placing it within a speculative "third generation IoT" which involves - borrowing from the UNIX operating system file permission categories - the reading, writing and acting on objects. In their design fiction, the acting on a connected object is performed through artist-written instructions, which demonstrate a form of non-human agency through the voice of a human.

By contrast, Easterling (2012) explained that objects possess agency already before a digital/media layer is added. She criticised the prevailing belief that agency is mainly attributed to active forms such as electric current and moving cars, but not to the infrastructure; the static arrangement of an electrical grid or a concrete highway system. Writing about urban contexts, she described the Internet of Things as a "world embedded with so many digital devices that the space between them consists not of dark circuitry but 
rather the space of the city itself. The computer has escaped the box, and ordinary objects in space are carriers of digital signals" (p.1). However, Parks and Starosielski (2015) criticised the ongoing privatization of these infrastructure spaces and utilities, claiming it as a result of the absence of public involvement due to a lack of technological literacy (p.6).

Arnall (2013) proposes considering material and mediational approaches to technology, arguing that invisibility, seamlessness and materiality are central issues in the design of contemporary interfaces. In his research he aimed to increase the legibility of 'background' infrastructures like RFID, Wi-Fi and GPS

... in order to gain material understanding for our own design practice, but also for new agency, new forms of control, discussion and debate across disciplinary boundaries (Arnall, 2013, p.157).

Lloyd (2013) also criticised the cultural conversation about the IoT as tending toward a highly functionalist, utilitarian set of ideas, and proposed scenarios in which objects contain their own narratives and perspectives, becoming storytellers in the form of portals, subjects or oracles. McGuirk (2015) addressed the domestic revolution facilitated by the advent of the 'smart home', seeing new forms of labour, privacy and monopolisation of control arise. Analogue to its inhabitants, who produce immaterial labour through participation on social networks, the smart home-connected, always on and producing data-is also capable of fabricating subjectivities and can thus be considered to become an extension of our immaterial labour. Here, ideas around a quantified self can be regarded as a counterpart to this fabrication of subjectivities, where narratives and statuses are created based on monitored interactions with objects through corporeal, physiological, or neurological parameters.

Business experts continue propagating the IoT's marketing potential for venture capitalist investors and entrepreneurs. Kellmereit and Obodovski (2013) see better collaboration between the industry and technology sector, and the building of communities around the IoT to help advance consumer awareness as necessary driving forces for an accelerated adoption of the IoT. The rise of smart products in the form of a "third wave of IT", (Porter \& Heppelmann, 2014) provided an opportunity for businesses for rapid innovation and 
economic growth, as businesses develop products that will be "far more efficient, effective, safe, reliable, and more fully utilized, while conserving scarce natural resources such as energy, water, and raw materials" (Porter \& Heppelmann, 2014).

However, concerns around security vulnerabilities of current IoT devices on the market have been raised, and the need for better encryption of IoT data is widely discussed (see for example Hewlett Packard Enterprise, 2015). The 'spamming refrigerator' or the 'hacked car' are often mentioned scenarios in popular media (see for example Brewster, 2014; Starr, 2014; Greenberg, 2015), but according to Symantec, malware for IoT devices is "still in its infancies" (Thomas, 2014).

The increasing popularity of smart products resulted in the emergence of humorous websites and social media accounts dedicated to criticising the unnecessary addition of complexities and security vulnerabilities when connecting daily objects or products to the Internet. The Tumblr page weputachipinit features a collection of IoT products under the theme "It was just a dumb thing. Then we put a chip in it. Now it's a smart thing." The site welcomes submissions of content that features an object with a chip in it, of questionable utility.

Another example, the Twitter account @internetofshit, was created mid-2015 and has 401.7K followers as of December 2019. The account's profile bio states “whatever, put a chip in it” (Internet of Shit, n.d.). The account and the associated hashtag \#internetofshit comprise a collection of posts and retweets commenting on absurdities around IoT products:

when i[sic] started this account five years ago, i[sic] worried i[sic] would run out of material too quickly, but there really is an unlimited supply of shit (@internetofshit, 2019).

Jenkin's (2015) critique of current IoT technologies addressed the reliance on "centralized servers, well-defined APIs, black-boxed electronics for the end user [that are] built to be used in condoned specific ways" (p.449). In his dissertation, Jenkins (2018) proposed the term 'object ecology' to describe "how objects hold memberships inside multiple networks - information, electronic, legal, cultural, material and more” (p.2). Rather than regarding 
the IoT as a single-household product, the Co-Housing IoT extends the notion of object ecology from one home to neighbouring homes, and designs this IoT with shared responsibilities in mind.

Approaching IoT design through a creative narrative, Addicted Products (2012) by Rebaudengo and Haque illustrates the Internet-connected toaster as a tragic character. The project aims to playfully employ the implications of agency of products in everyday life. Brad the toaster is connected to other toasters in the network and is therefore able to compare his performance with them. Brad also has the ability to request being moved to another place if he does not get enough attention from his human host (Rebaudengo, 2012). The series Radiotropism (Brain, 2015) encompasses experimental Wi-Fi routers that "misbehave in unexpected ways". The routers have a deliberately inconsistent signal by being tethered to the lunar cycle, the lighting of a candle, or a yet-to-be-watered house plant. With these interventions, Brain aims to "include the agenda of other non-human organisms and natural systems" when defining success or failure in a system in the form of the presence of a Wi-Fi signal (Brain, 2015). These networked objects expect a specific form of engagement with their human users, and have the capacity to actively intervene and behave unexpectedly.

More artistic IoT themes can be found in the programme of the German media art festival and conference "Transmediale", which acts as a catalyst for contemporary media arts discourse. Curators address the question of whether

tech developers, hackers and artists can respond in both critical and constructive ways to the challenges of the quantification of every-thing in ways that reach beyond the banal visions of the self-ordering fridge and the city as a digitally assisted playground (transmediale, 2015a).

Sterling addresses the Internet of Things in his opening talk at the Transmediale 'afterglow' and insists that the IoT and its real-world consequences are imminent (transmediale, 2015a), calling for "domestic Internet Art designed for the home, IoT art” (transmediale, 2015b). Sterling presents Casa Jasmina, a two-year open source IoT pilot project. Casa Jasmina is an apartment located in Torino, Italy, combining lab, gallery space, and bed \& 
breakfast. The project offers opportunities for individual artists, designers, and hackers as well as companies to test projects in a real-world test bed (Arduino, 2015).

The programme for the 2016 edition of the Transmediale festival also suggests that bottomup options in the form of tweaking, disrupting, and appropriating existing infrastructures offer viable alternatives to the "prevailing vision of the Internet of Things as a top-down mega-infrastructure of interconnected every-things" (transmediale, 2016). This bottom-up alternative of the IoT envisions the human users as makers and developers of the IoT. In order to also include non-human users as active developers of the IoT, the next section explores projects that playfully engage with the perspectives and agency of the connected things and their environments.

This historical overview of the discussion of the IoT illustrates how the increasing availability of networking technology, in combination with the pursuit of new markets and business opportunities, have served as main catalysts for the development of the IoT. It also notes the risks that critics have put forward, such as the loss of privacy, and issues of agency and control from the user perspective.

The following chapter complexifies and extends the narrative of the IoT by introducing more-than-human thinking into the development of networked things, specifically when the 'things' in question are members of more-than-human communities, and more specifically still when they are the freshwaters of urban Pōneke/Wellington. 


\section{Chapter 3: Encountering turbulences: Troubling loT design in Aotearoa/New Zealand with more-than-human concerns}

In this chapter, I explore how the Internet of Things as it informs design research, has been critiqued, reimagined, and renegotiated through the (re-)centring of the more-than-human world. By considering the implications for IoT design in Aotearoa, and the intersections it traverses with more-than-human concerns, this chapter further maps the theoretical groundwork that informs this thesis project, and introduces and contextualises the methodology discussed in Chapter 4. The 'turbulences' I speak to in the title are the key tensions that have underpinned the conceptual and critical development of this work, and the wider material context in which IoT design exists. Through attending to critiques of human/non-human binaries, examining the embracing of the potentialities of the morethan-human world in network design as it is situated in Aotearoa/New Zealand, and redressing the marginalisation and erasure of Māoritanga (Māori culture, traditions, and ways of life) in academic discourses, this chapter presents a series of encounters that have shaped the trajectory of wildthings.io.

The chapter traverses the following central discussions. In the first section, I examine contemporary Western academic discourses that challenge traditional divides between humans and non-humans and attend to the distributed knowledges of a more-than-human world. Particularly, I focus on slowly unfolding concepts that have been established within Te Ao Māori (a Māori world view) and Mātauranga Māori (the Māori way of engaging with the world) long before European academia started to pay attention to such concerns. In the second section, I describe how more-than-human theory has influenced creative practice within arts and design and its specific relevance for IoT design, looking at the field of Human Computer Interaction. I examine HCI as a site of development which has shown increasing interest in the decentring of the human in response to environmental and Anthropocene-related concerns. I build on these concerns with sustainability and technology in the third section, analysing how the idea of networks and more-than-human worlds specifically relate to water in Aotearoa/New Zealand. In reflecting on my own relationship to wai/water, I critically consider the situatedness of this research, and its 
localised implications therein. In the fourth section, I summarise these key theoretical ideas, and provide an overview of how these connections helped establish the methodology around slowness, seamfulness and openness, and how they correspond to IoT design with more-than-human sensibilities grounded in Aotearoa/New Zealand. 


\subsection{Turning towards more-than-human concerns}

In recent decades, Western academia has observed an increasing interest in the nonhuman, in concerns for the more-than-human world, and the decentring of the human. In this section I provide a brief overview of the recent emergence of more-than-human thinking within academic discourse as a response to the tensions emergent within IoT projects (discussed in the previous chapter) that speak to a broader paradigm where the category of 'human' itself increasingly comes into question.

A decentring of the human, and a corresponding shifting of attention towards concerns for the non-human can be found in a wide variety of recent and current western philosophical lines of thought (Grusin 2015, p.vii), among them Object-Oriented Ontologies (Harman, 2018), New Materialism (Dolphijn \& Tuin, 2012), and Speculative Realism (Shaviro, 2014). This relatively recent widespread interest in challenging the seemingly traditional divides between humans and non-humans in academia has contributed to a growing push for methods that can work with the distributed knowledges, experiences and values of a morethan-human worlds.

One such response to a critical reimagining of the divides between 'human' and 'nonhuman' has been that of the 'post-human'. The concept of the post-human dates back to the $19^{\text {th }}$ century (Blavatsky, 1888/1997), p.684) but gained notable recent popularity through the writings of Hayles (1999) and Braidotti (2013). Coming from literature studies with a concern for cybernetics and informatics, Hayles predicted an increased urgency of questions concerning the post-human for the new millennium. In her terms, the prefix 'post' describes both the connotation of "superseding the human and coming after it" (p.283). Hayles concludes her book with:

\section{Although some current versions of the posthuman point toward the antihuman and the apocalyptic, we can craft others that will be conducive to the long-range survival of humans and of the other life-forms, biological and artificial, with whom we share the planet and ourselves (Hayles, 1999, p.291).}


In contrast, Braidotti's post-humanism (2013) is characterised by its anti-humanist and feminist tenets. Braidotti sees a need for a renewal of the ideal conception of the human in the Vitruvian man (p.13) and describes post-anthropocentric conceptions of subjectivity as a needed renewal of the Humanities (p.55).

New Materialism emerged around the same time as the popularity of the 'posthuman' during the early 2000s, and describes a heterogeneous philosophical movement, spanning the humanities, natural and techno-sciences, with roots in feminism and Marxism. New Materialism is characterised by a return to matter and a concern for material objects. The dualist-dominated thought of humanism is turned into a monist perspective with a special attention to matter (Dolphijn \& Tuin, 2012, p.85). The reconfiguration of persistent dichotomies such as nature/culture, body/thought, concrete/abstract is a central interest in the New Materialist agenda (Parikka, 2010). Shifting these dualist structures allows for the conceptualisation of the flux of nature and culture, matter and mind (Dolphijn \& Tuin, 2012, p.48).

Plumwood (2001) discusses the complexities and traps inherent to language and dualistic thinking, highlighting how "progressive naturalism requires that we disentangle the liberatory roles of the concept of nature from the anti-liberatory ones" (p.6). She highlights how "conservative naturalism seeks to naturalise oppression" (p.6) often "functioning in colonizing frameworks to naturalise inferior treatment" (p.6), a claim which requires attention when researching in Aotearoa/New Zealand. In terms of doing design research particularly for and with the more-than-human world, Plumwood (2001) proposes nature as 'a biospheric other' which should not be a background part of our field of action or subjectivity, but a positive presence and ethical subject to which we can owe a debt of gratitude, generosity, and recognition as prior and enabling presences or ancestors (pp.1617). New Materialism, as a philosophical movement, tries to redress humanist thinking in continental and anglophile philosophy with an interest in the non-human, exploring questions of sentience and interspecies communication (Boyd, 2015, p.6). Approaching the ‘nonhuman turn', Grusin (2015) describes the nonhuman as “understood variously in terms 
of animals, affectivity bodies, organic and geophysical systems, materiality, technologies” (p.vii).

The rhetoric of the 'non-human' itself is nonetheless situated in a complex discursive terrain. The term "more-than-human world", originally coined by Abram (1996) as a way of referring to earthly nature, has been rapidly adopted and further discussed in academia. The field of human geography, for example, adopted the signature 'more-than-human' instead of referring to the post-human, since it helps to distinguish itself from the posthuman as an apocalyptic hyperbolic future critiqued by other human geographers (see Castree \& Nash, 2004, p.1342; Greenhough, 2014, p.95 on Fukuyama, 2002).

For the field of human geography, more-than-human literature emerges from the research of Sarah Whatmore (2002) and has roots in Science and Technology Studies (STS) and anthropology.

The term 'more-than-human' has been defined in the Dictionary of Human Geography as follows:

1. A term used critically to remind human geographers that the non-human world not only exists but has causal powers and capacities of its own.

2. A term used positively to highlight the absolute dependence of humans on a vast and complex array of non-human entities, only some of which are subject to human control.

In both cases the more-than-human accents a relational worldview in which parts cannot be dissociated readily (Rogers et al., 2013).

Noorani \& Brigstocke (2018) define the ‘more-than-human' as follows:

\footnotetext{
More-than-human

Describes how human societies are always composed of varied relations between humans and non-human forces and agencies such as objects, animals, microbes, and technologies. It challenges the idea that humans are separable from their worlds, or society is separable from nature. More-than-human can refer to realms or entities beyond the human, or to larger ensembles that include the human (Noorani \& Brigstocke, 2018, p.39).
} 
The 'more-than' signature has also been used in a critical encounter with things in an IoT, where Gabrys (2016, p.11) describes how "things are always more-than-things as immediately encountered", the 'more-than' is widely used to grasp the wider implications of things as "plural, processual, relational, incomplete, and even as provocations that open into practice” (Gabrys, 2016, p.11; referring to James, 1996; Grosz, 2005; Mackenzie, 2010). In light of such plurality and incompleteness, the 'more-than' signature stresses that it is what "exceeds the human rather than what comes after the human" and that it "conjures a different kind of historicity", arguing that "one never arrives at a time/place when the human was not a work in progress" (Whatmore, 2004, p.1361). The challenge of more-thanhuman research lies in its experimental imperative and the implicated need to take risks (p.1362), an approach adopted from science studies (Greenhough, 2014, p.104). Whatmore (2004, p.1362) draws on Paulson (2001, p.112), who argues that learning new types of encounter with non-humans (see also Greenhough, 2014, p.103) can give rise to new modes of relations with humans, and I suggest, reimagining of matter itself.

Both more-than-human research in the social sciences and New Materialist theory in the humanities are connected to strands of post-human thinking but aim to describe distinct traditions of thought. Both New Materialists and more-than-human geographers engage with the concept of active and vibrant matter (see Parikka, 2010; Bennett 2010).

Materiality is always something more than 'mere' matter: an excess, force, vitality, relationality, or difference that renders matter active, self-creative, productive, unproductive (Coole \& Frost, 2010, p.9).

Matter, and material assemblages, of which embodied humans are an integral part, are affective-affected, agential, and morphogenetic. Bennett (2010) describes assemblages as "ad hoc groupings, as living, throbbing confederations" or "open-ended collectives", that are not governed by any central head (pp.23-24). Materialist assemblages (Bennett, 2010, p. 23; Braidotti, 2013, p.159; DeLanda, 2006, p.12) and more-than-human entanglements (Barad, 2007) share commonalities that are worth further exploration to critically examine the complexity and fluidity of the IoT and all its parts: silicone chips, network protocols, start-up incubators, smart fridges, microcontrollers, radio compliance standards, underwater cables, self-watering pot plants, and so on. 
More-than-human and New Materialist research then proposes a heterogeneous conception of social life by acknowledging the significance of the non-human, and the fluidity between Structuralist scientific binaries such as subject/object, and nature/culture/society. Such binaries have hence been subject to rigorous criticism from researchers engaged with the dynamism of the more-than-human. For Bennett, 'humanness' is always already situated both within and intertwined with 'non-humanness': "Humans are always in composition with nonhumanity, never outside a sticky web of connections or an ecology of matter" (2004, p.365). Plumwood (2001) foregrounds the vital importance of such situatedness, and hence critically deconstructs these stark dichotomies, stating:

As part of escaping dualist construction, we should reject certainly the idea that culture is self-enclosed and nature purely other (which seems often to be part of what people who talk about dissolving boundaries have in mind). But rejection of these polarizing constructions of nature does not require dispensing with nature, prioritizing culture over nature, or absorbing the one pole of nature into the other pole of culture. Such reductionist measures are not helpful reinventions of the concept of nature - rather they move with the mainstream of the Western tradition of anthropocentrism and backgrounding. They support rather than disrupt the modern sense, especially fostered in urban life, that we humans are completely immersed in a self-enclosed sphere of our own we can call "culture" (Plumwood, 2001, p.26).

Further attempts to move beyond binaries when doing research with participants that inhabit different worlds, for managing both diversity and cooperation, can be found in the notion of the 'boundary object' Star \& Griesemer, 1989; Bowker \& Star, 1999):

\section{Boundary objects are those objects that both inhabit several communities of practice and satisfy the informational requirements of each of them. Boundary objects are thus both plastic enough to adapt to local needs and constraints of the several parties employing them, yet robust enough to maintain a common identity across sites (Bowker \& Star, 1999, p.297).}

Here 'boundary objects' emerge as both sutures that hold multiple boundaries together; yet they themselves are fluid and readily adaptable; and further, foreground the tensions in applying Western concepts of time across worlds. Following Plumwood's (2001) call for challenging Western accounts of time and agency, Bastian (2009) then argues that 
a critical component of opening up who or what can be understood as possessing agency involves challenging the conception of time as linear, externalised and absolute, particularly in as much as it has guided Western conceptions of process, change and invention (Bastian, 2009, p.99).

Bastian's call to challenge linear conceptions of time reaffirms the necessity of imagining temporality beyond Western, colonial constructions. Nevertheless, notions of time from an indigenous vantage point have often struggled to be amplified in academic contexts; such imaginings of time through an academic lens have often reified the imagining of "an 'other' time, whose logic and historical expression are incommensurable with the normative temporality of a clock and calendar associated with Western modernity" (Ganguly, 2004, p.162).

Examining where a more-than-human IoT sits within the varying intersections of New Materialism and the decolonisation of design practice further reveals the potentiality of this research to deconstruct and renegotiate anthropocentric thinking. New Materialism is often mentioned alongside posthumanist and multispecies thinking and practice, as they

... share goals through art and theory to dissemble anthropocentric modes of performance and to provoke, through the evocative potential of art, changes in material-cultural practices (Boyd, 2015, p.6).

Such work may echo a wider paradigmatic shift in which the shortcomings of anthropocentrism are increasingly experienced as lived, material realities. Greenhough (2014) claims that natural disasters and an increased spread of zoonotic diseases are urging Western societies to shift their focus away from the human towards the non-human (p.94). The major human impact on earth and atmosphere at a global scale has resulted in the proposal of naming the current geological epoch the "Anthropocene" (Crutzen \& Stoermer, 2000). Haraway (2015) demands that it is "our job, to make the Anthropocene as short/thin as possible and to cultivate with each other in every way imaginable epochs to come that can replenish refuge" (p.160). 
Notably, these Anthropocene-related urgencies, among them the looming climate crisis, have been voiced by indigenous peoples long before western discourse has acknowledged them. Rosiek et al. (2020) point out how the "pervasive context of settler colonialism cannot help but influence both the practice of new materialist scholarship and its reception" (p.332) and identify how indigenous scholarship shows "strengths in precisely some of the places new materialist social science is facing challenges" (p.331).

From the vantage point of the colonized, a position from which I write, and choose to privilege, the term 'research' is inextricably linked to European imperialism and colonialism. The word itself 'research', is probably one of the dirtiest words in the indigenous world's vocabulary (Smith, 2012, p.1).

This sentence stands central at the opening to the work of Linda Tuhiwai Smith's Decolonizing Methodologies (1998; 2012) and "perhaps the most quoted sentence in the book" (2012, p.xi). Aotearoa/New Zealand academia increasingly attempts to integrate Māori perspectives into previously Western-centred design curricula (see for example Victoria University of Wellington, 2019a;2019b). However, current academic frameworks do not easily accommodate non-Western ways of thinking and researching, as they, for example, privilege text-based forms of publication, and often more competitive than collaborative. A good overview on these critiques can for example be found in Kaupapa Māori research (Walker et al., 2006).

"[D]ecolonizing design" is not a question of improving the status quo but a
question of learning to differentiate between designs that facilitate the
productivist drive towards devaluing and appropriating human and non-
human natures, and designs that facilitate a process of delinking and
redirection into other modes of being/becoming (Schultz et al., 2018, p. 82).

Members of the Decolonising Design group have observed the "currency of the term ‘decolonizing' being reduced to a hollow gesture” (Schultz et al., 2018, p.84), following the same route as sustainability, that was co-opted for neoliberalist means in design. 
While the academic conversations around more-than-human thinking can help push a field like IoT design away from centring on solely human concerns, and guide the research towards a more holistic worldview, in which the things themselves receive greater attention, we shouldn't overlook the longstanding existence of comparable philosophies in indigenous thinking. When working in Aotearoa/New Zealand, we must pay attention to how the care for the more-than-human worlds stands central in Te Ao Māori and Mātauranga Māori. 


\subsection{Designing networks with the more-than-human}

In this section I discuss how more-than-human theory is enmeshed in creative practice in arts and design and its relevance specifically for IoT design, looking at developments within the field of Human Computer Interaction (HCI). After discussing select relevant examples of IoT designs that push the boundaries between human/non-human interactions, I examine HCI as a site of development which has shown increasing interest in the decentring of the human, evolving in what can be called a more-than-human computer interaction.

In a commercial scenario, an Internet of Things appears an almost rigid network structure for enhancing remote corporate control over consumer goods, and possibly even consumers themselves. However, from a developer's perspective, this framework is yet to be built, currently lacking standards and a consistent, secure infrastructure. Accordingly, artists and designers are able to re-define this still-malleable concept using tactics of subversion, intervention and (re-)appropriation of network technologies and infrastructures.

One of the first networked art projects connecting online users with plants was TeleGarden (1995). The art installation allowed web users to view and interact with a remote garden filled with living plants. Members could plant, water, and monitor the progress of seedlings by controlling an industrial robot arm. The creators described the application of a robot arm as "most absurd", as they consider a garden to be very "human, immediate and tactile" (Goldberg \& Santarromana, 2008). Their project creates a tension between the 'natural' living organic environment and the 'unnatural' robotic arm interacting with it through remote, human commands.

A decade later, the project Botanicalls (2006) encourages "a happy relationship between plants and people" through the use of a hardware kit for plants (Faludi, 2013). Equipped with sensors, plants have the capability to call and send text messages to their owners when requiring assistance (Faludi, 2013). Dialogue (2008) by Haapoja explores audio-based forms 
of human-plant interaction through facilitating a dialogue between the breathing human and the non-human photosynthesis process, established through sound (Haapoja, 2008).

While TeleGarden and Botanicalls make use of microcontrollers and network technology to facilitate and mediate the conventional notion of humans taking care of a house plant, other artworks such as Spore 1.1 (2005) or Natural Fuse (2009) conceptualise the idea of an abstracted, non-immediate ecosystem in which a set of protocols define the fate of a plant. Spore 1.1 cuts out the need for a human caretaker and creates a "self-sustaining ecosystem for a rubber tree plant purchased at The Home Depot” (Easterly \& Kenyon, n.d.), linking the health of the plant directly to the stock market performance of its vendor. In Natural Fuse (2009) pot plants are connected in a city-wide network comprising a self-regulated ecosystem based on the electric and carbon-absorbing capacities of plants. Human interaction within the network is limited to the turning of a knob. The three states 'Off', 'Selfish' and 'Selfless', to power a lamp, require compromises between the human need for electricity and the health of a plant, joined up together in one circuit (Haque, 2009a; Haque, 2009b).

Another environmentally engaged project emerges in Trash Track (2009), by the MIT Senseable City Lab. The project extended the notion of supply-chain-management, which Cooper and Ellram (1993) defined as the "distribution from supplier to user" (p.13), by tracking a product's journey after it had been disposed of. Equipping non-human things with sensing capability revealed previously invisible infrastructures: the journeys of various items of trash (MIT Senseable City Lab, 2009). Artists have also responded to privacy concerns, as sensing capabilities of electronic gadgets could allow unwanted tracking of citizens (see for example Albrecht, 2002 or CASPIAN, 2003). Artist-led workshops, such as the New Brave World RFID Radio Frequency Identification Workshop (iMal, 2008) promoted the creative appropriation of RFID technology in creative practice.

The tracking of non-human lives has been used as an unconventional approach to mapping the experience of urban spaces. One example, the Brooklyn Pigeon Project (2004), was an experiment in capturing “unconventional portraits” (Lasch, 2004) of Brooklyn, NY by 
equipping trained pigeons with video and audio recording backpacks. Correspondingly, Urban Eyes (2006) was described as a "critical design concept combining RFID technology, aerial photography and pigeons to create an explorative experience for urban spaces” (Kirsch \& Ängeslevä, 2008). Beatriz da Costa's Pigeon Blog (2006) was described by Bleecker (2006) as "an early protozoa [sic] on the Blogject species evolutionary chain" (p.4). Pigeons equipped with miniature sensing devices collected air pollution data that is rendered live over Google Maps (Da Costa, 2006).

Natalie Jeremijenko's Amphibious Architecture (2007) expanded the idea of tracking nonhuman animals by allowing forms of inter-species communication between humans and fish through interactive buoys installed along the East and Bronx Rivers in New York. An array of LEDs served as a visualisation of a feedback loop between human generated textmessages, the presence of fish, and the quality of the water (Environmental Health Clinic, 2009). In Usman Haque’s installation Sky Ear (2004) LED lights and mobile phones are combined as interfaces for experiencing natural phenomena. A dozen mobile phones with EMF (electromagnetic field) sensors were released into the night sky with glowing helium balloons. People could call the phones floating up to 100 metres above them and listen to the sounds of the sky, while the lights visualised patterns of signals from above (Haque, 2007).

The range of projects discussed above use networked technologies to tap into the experiences of non-human animals and plants, connecting a human audience with environmental phenomena through experimental use of sensors and actuators. The need to (re-)connect humans with their environment, or allow humans to see a more-than-human perspective on human impact is increasingly coming into focus in the context of the Anthropocene: Bridging concerns of the Anthropocene with the opportunities of networked objects that have the capacity to shift human perception, the Internet of Things has been increasingly discussed in the light of philosophical movements that focus on the nonhuman (Lindley et al., 2017). STS scholars and philosophers turned their focus towards the 'things' in the IoT themselves, which were "considered as exerting influence in the 
construction and maintenance of action and experience” (DiSalvo \& Lukens, 2011, p.421). Non-humans here include animals, the built environment, objects of all kinds, including software, hardware, protocols, and networks. "From the perspective of environmental ethics", as DiSalvo and Lukens argue, "it is important to attend to nonhumans in order to avoid decision-making, planning, and design that serve human interests at the expense of other species” (2011, p.427). Ciortea et al (2014) proposed socio-technical networks (STNs) as an ontology that enables non-human agents as participants in a network: "An STN encodes the different relationships among people and smart things, such as friendship, ownership, provenance or co-location.”

Correspondingly, Coulton \& Lindley (2017) proposed a more-than-human centred design for the IoT after surveying a range of projects that "illustrate a consensus forming around the idea that non-anthropocentric theories have increasing relevance for contemporary design" (p.470). However, they claim that "although rendered from disparate philosophical foundations the direct impact of these various theories on practical design work is, relatively speaking, negligible" (p.470). They continue, "while a relationship with theory is often important to these design research projects, the precise nature of that relationship often appears not to be of direct consequence to the outcomes" (p.470). They also point out that network anxieties resulting from pervasive socio-technical ecologies, like the IoT, have encouraged researchers to consider non-anthropocentric theories to inform their inquiries (p.471).

Stead et al. (2019) expanded the discourse around the unsustainability of contemporary IoT design practice further by developing Sterling's $(2004 ; 2005)$ concept of spimes, presenting an IoT design manifesto in which, for example, planned obsolescence (Stead, 2017) and the short life spans of IoT gadgets are tackled by envisioning a spime object's lifecycles through phases of deactivation, disassembly and extraction of recyclable resources before reentering the design stage (Stead et al., 2019, p.2137). Stead et al. describe the conceptual underpinning of the spime object accordingly: 
Transparency, coupled with a focus on product disassembly, and recyclable parts and componentry, would be some of the principal aspects of a spime object's design specifications (Stead et al., 2019, p.2136).

Knutsen discussed the complexities designers face when developing "hybrid products, comprising of atoms and bits” (2014, p.129) and regards seamlessness, besides immateriality and smartness, as unhelpful tropes.

Obscuring the seams, edges and grains of the foundational building blocks what we now take for granted as the 'Internet', leads to a lack of understanding and agency both for designers and the larger public sphere" (Knutsen, 2014, p.139).

These projects emphasise the richness to be born from the entanglements of New Materialism, post-humanist, and multispecies thinking and practice where, as previously mentioned, such work can re-negotiate and re-map art and theory to "dissemble anthropocentric modes of performance and to provoke, through the evocative potential of art, changes in material-cultural practices" (Boyd, 2015, p.6). As one strand of Western theory that engages Anthropocene-related urgencies, New Materialism might offer insights into the development of theories and methods for a more-than-human IoT. Despite its critiques (Sullivan 2012; Rekret, 2016), the theories find fertile ground in media theory (Parikka, 2012), communication design (McNely \& Rivers, 2014), music (Fure, 2016), and creative arts research (Barrett \& Bolt, 2014). In the latter, a 'material turn' is experienced in moving beyond discursive constructions towards more artistic mappings of complex relationships between nature, culture, body, language and knowledge (see Barrett \& Bolt, 2013; 2014). More-than-human geographers are also collaborating with artists (see for example Yusoff, 2015) and searching for "experimental practices that amplify other sensory, bodily and affective registers" (Whatmore, 2006, p.606) to supplement the humanist repertoire of methods. Similarly, Escobar (2018) introduces design literature to nondesign readers and, conversely, up-to-date social theory approaches to design experts with little background in the social sciences and the humanities.

Whatmore's call for the "redistribution of expertise beyond engaging with other disciplines to engage knowledge practices and vernaculars beyond the academy in experimental research" (p.607) then aligns well with a design agenda that extends beyond academia into 
the more-than-human world, creating new knowledge on the IoT in collaboration with more-than-human communities.

Human Computer Interaction (HCI) is one such field which has engaged in these expanding knowledge practices. HCI has shown increasing interest in the decentring of the human, particularly as a "response to concerns about environmental sustainability, technology obsolescence, and consumerism” (Liu et al., 2019).

As spatial (and temporal, and beyond?) relationships between computers, nodes and networks become relevant, the field of more-than-human geography offers insights into working within an evolving field on the interface of the nature/culture dichotomy. Morethan-human geography expands the scope of ethnographic practice, not just attending to humans and animals, but also to things, materials, and forces. The figurative escape of the computer also brings to mind the 1974 novel by Ted Nelson, Computer Lib/Dream Machines, cited as "the most important book in the history of new media" (Wardrip-Fruin, p.301) which was published just before the personal computer revolution that laid foundations for the field of Human-Computer Interaction. To acknowledge and meaningfully engage with this sticky web of connections as part of an Internet of Things research and design practice, we need to be actively engaged with broadening the field of Human-Computer Interaction towards more-than-Human-Computer Interaction.

In doing so, however, we need to listen to warnings from the decolonising design community, on how "technological revolutions have played in the industrialisation and eradication of plural memory and imagination” (Schultz, 2018, p. 81) and regarding "technological colonisation of imagination as a 'defuturing ontological elimination design event'. An event that is defuturing Indigenous futures and therefore defuturing (eliminating) options for sustainable futures for all humans and lifeworlds upon which humans depend" (Schultz, 2018, p. 81). Especially when researching and designing with and for whenua/lands in Aotearoa/New Zealand, the more-than-human interests should have a strong voice in our design decisions. 
In summary, the earlier creative projects discussed demonstrate a variety of approaches for designing interfaces and networks introducing more-than-human perspectives and agency into relationships with nature and natural phenomena. The tensions some of the works provoke speak to a broader project in which the category of 'human' itself increasingly comes into question, which I discuss in the following chapter, aiming to locate the research more specifically in Aotearoa/New Zealand, further developing the complexities entailed in building networks within a more-than-human context, focussing on wai/water. 


\subsection{Getting to know Wai/Water networks in Aotearoa/New Zealand}

Ki te taha o tōku pāpā

Ko Ateria te whenua

Ko Dachstein te maunga

Ko Altausseer See te roto

Ko Traun te awa

Ko Bachler tōku whanau

Ko Birgit tōku ingoa

I want to start this section with a short excerpt of my pepeha, introducing myself in te reo Māori through my more-than-human connections to the world. I chose to do this to highlight how Māori whakapapa (genealogy) acknowledges someone's connections to the more-than-human world by introducing features of land and water, before naming their human ancestors. In this specific pepeha, I refer to the rohe/region of my paternal lineage, which I have never lived in myself, but connect to through my ancestry. I also chose to present the lineage of my father here, because the family history of my father's side is better known, and also, because my connection to water, and my curiosity to learn more about how the quality of local waters connects to the quality of life, was sparked by him. During my teenage years, his work in the Knittelfeld City Council was focussed on environmental issues which I remember not the least from assisting him in writing the Umweltbericht (Stadtgemeinde Knittelfeld, 2004). I want to acknowledge his impact on my research journey through his ambition to stay curious and keep looking for answers outside the most obvious places.

From this very personal account, upon arriving in Aotearoa/New Zealand I found the state of water in Pōneke/Wellington, and the wider country strikingly different from how it is presented in the public image, as spearheaded by tourism-centred campaigns advertising New Zealand for example as ' $100 \%$ Pure' (Tourism New Zealand, n.d.). Voicing the growing concerns about the health and wellbeing of Aotearoa/New Zealand's more-than-human world, Joy (2015) describes a freshwater crisis resulting from human impact on the lands, 
fuelled by policy that prioritises short-term economic growth over long-term environmental sustainability (p.17).

However, these concerns are not an issue specifically localised in Aotearoa/New Zealand, as highlighted by the worldwide surge of climate change protests, led by young people all over the world in 2019 (RNZ, 2019). Turning more specifically to concerns about water, projects on a global scale such as the 17 United Nations Sustainable Development Goals specifically list "Goal 6: Clean Water and Sanitation” and "Goal 14: Life below Water" as objectives that directly link to water quality, with other goals, such as "Goal 11: Sustainable Cities and Communities" indirectly speaking to the quality of life in relationship to environmental health (United Nations, n.d.).

The significance and value of water is nevertheless not a recent concern for Aotearoa/New Zealand. Māori have been vocal about the concerns for the natural world, including water, for a long time (see for example Douglas, 1984) but it is only recently, that Māori understandings have slowly found their way into policy (see for example Tipa \& Teirney, 2006).

Water is fundamentally important for its life-giving essence and spiritual values. Māori philosophy takes a holistic, ki uta ki tai (mountains to the sea) approach to looking after water resources (Tipa \& Teirney, 2006, p.1).

In their cultural impact report, Gilmore and Mellish (2004, p.8) advised the Wellington City Council that the waters of the Waitangi Stream system are viewed by the local tribes as a taonga (treasure) and as mauri (life force).

Water is a taonga - a treasure, essential for life. Water is a resource, a vital input for agricultural production and manufacturing. Rain, rivers and lakes are sources of inspiration and creativity. Water permeates ecosystems, jurisdictions, and communities, linking complex and emergent social, cultural, technological and economic systems (Russell et al., 2011, p.iii). 
However, as Swanson et al. (2017) raise, in the age of the Anthropocene, the conversation of creativity has shifted towards the arts of living on a damaged planet:

\section{The seductive simplifications of industrial production threaten to render us blind to monstrosity in all its forms by covering over both lively and destructive connections. They bury once-vibrant rivers under urban concrete and obscure increasing inequalities beneath discourses of freedom and personal responsibility (Swanson et al., 2017).}

The impact of European settlement on Te-Whanganui-A-Tara's water is beautifully described by Alice Te Punga Somerville, referencing Manning (2009) when describing how the former Waitangi Lagoon was mightily disrupted by two events:

the major 1855 Wellington earthquake, and the laying of pipes during the nineteenth and twentieth century projects of public works which means the Waitangi stream now exists almost entirely underground through human-made pipes. Much of Wellington city was erected on land that was partially reclaimed and partly drained (Te Punga Somerville, 2015).

Local New Zealand artists have increasingly engaged with concerns centring wai/water, specifically in the Wellington region, with initiatives like the Common Ground: Hutt Public Art Festival (2017) focussed on exploring our relationship with water through art, ranging from walks and screenings (Mechen et al., 2017), to participatory artworks (Priest, 2017), to revitalisations of neglected stream sites (Parker et al., 2017). Parker (2014) also created a sound installation in the Wellington CBD, reconnecting pedestrians aurally back to Kumutoto Stream, nowadays piped underground. The employment of IoT technology in local arts often aided the connecting of non-human data with the human world, such as in the project The Park Speaks (Clothier et al, 2011) where data gathered by environmental sensors placed in a botanical garden connect to media in a gallery. Similarly, in educational works such as Breathe (Smith et al., 2017), underwater temperature sensors in a freshwater stream connect to an exhibit that informs how water oxygen levels relate to the ability for fish to breathe. In line with the IoT art projects are other local community-engaging projects such as Riversense (n.d.) and Community Based Water Monitoring (Valois, 2019), or commercial water sensing solutions such as RiverWatch (n.d.), and Aotearoa/New Zealand researchers studying how indigenous knowledge can benefit the health of local rivers (see for example Parsons et al., 2019; Fisher \& Parsons, 2020). 
In sum, the engagement of creative communities and makers with issues concerning the more-than-human lifeworld of water sheds light on the lack of connection people have with their local waterways, and offers artistic engagements and participatory activities to reconnect with lost understanding of water ways through art. 


\subsection{A more-than-human Internet of Things with water}

In this chapter, I navigated the complexities of conceptualising a more-than-human Internet of Things with water through discussing contemporary academic discourses concerning more-than-human thinking, including concepts of Te Ao Māori and Mātauranga Māori that are slowly being picked up by Western scholars. The interweaving of these concepts has also gained traction in the field of HCI, where questions around sustainability or technological obsolescence are increasingly gaining attention from scholars and developers. Moving those concerns back to Aotearoa/New Zealand-specifically focussing on local freshwater-adds another layer of complexity, particularly when learning about how colonisation and urbanisation have disrupted natural connection to freshwater. Such an endeavour invites a questioning of if and how technology is able to remediate this disconnection, or if this just adds another layer of colonial baggage onto an already troubled more-than-human world.

With these considerations in mind, I had to both unlearn and disrupt certain designerly habits that have been part of my practice before embarking on this research journey. This involved developing the confidence to sometimes let go of external pressure and deadlines, allowing time to tune into the voices of the stream and unravelling all their more-thanhuman connections, instead of spending a finite amount of time with them and then attempting to map and represent them accurately. What started as a research project that aimed to centre a small stream in my neighbourhood which had been adopted and restored by a local gardening group slowly revealed larger narratives. Small conversations around local freshwater neglect and pollution moved into larger concerns about restoration and revealed freshwater issues in Aotearoa/New Zealand at larger scale. The disconnect between people and their local freshwater streams through urbanisation is garnering more attention, with artists thematising these issues through a variety of media, pushing for new ways for bringing the forgotten more-than-human world of local freshwater back into people's memories. 
Combining ideas of more-than-human thinking and recent developments in HCI and IoT development, I discuss how methods of slowness, seamfulness and openness served as guidance for my research centring Papawai Stream and the more-than-human world enmeshed in this small, fairly unknown, freshwater feature of the Wellington town belt. 


\section{Chapter 4: Methodology}

\subsection{Introduction}

This research departs from the concept of an Internet of Things (IoT) as a means to give voice to non-human 'things' and tentatively proposes avenues to shift focus from humancentred to a more-than-human-centred design paradigm. In Chapter 2, I traced the IoT back to its first mention in 1999 and examined how the discourse around IoT development has been shaped by ambitious visions of a network capable of disrupting internet ecologies worldwide. Today, however, the complexity of IoT technology still does not afford one single, stable technological framework for building a global, profit-driven top-down IoT. On the other end of the developer spectrum, the rising availability of low-cost IoT technology has allowed artists and designers to develop bottom-up alternatives to the prevailing market-driven human-centred scenario. The review also revealed that, in times of Anthropocene-related urgencies, design methods-classically characterised by humancentredness-are increasingly troubled by more-than-human concerns. With a focus on wai/water, this project imagined novel ways of understanding and (re-)connecting with disconnected streams, their communities and their ecosystems in Pōneke/Wellington Aotearoa/New Zealand. The research narrative is presented in a combination of written word and audio-visual materials to make the many voices recorded during the lab- and fieldwork heard. The thesis proposes ways of acknowledging and engaging with local knowledges to inform art and design research. With this chapter, I describe my process of finding productive avenues of engaging with the more-than-human ecologies of wai/water in Aotearoa/New Zealand for designing an Internet of Things.

I first introduce the research questions and aims that emerged from my literature review as part of my thesis proposal (4.2.1). Then I provide an overview of my research design across lab- and fieldwork and discuss how my research process has been continuously shaped and re-shaped by iterations of practical design research underpinned by theoretical inquiries into more-than-human thinking (4.2.2). The chapter concludes with a presentation of key fields and themes that recurred throughout my research journey and inspired the research designs throughout various stages (4.3). 


\subsection{Research Overview}

\subsubsection{Research Questions and Aims Iterations}

In my initial research proposal for this thesis project, I drafted two research questions that were set out to guide the design of my project:

I. (How) can New Materialist theory produce a useful theoretical framework that can be applied to and more-than-human IoT design?

II. Can a more-than-human IoT be realised through participatory design and if so, how?

These research questions informed the following two aims:

\section{Theorise a more-than-human IoT.}

II. Explore and evaluate the theorised more-than-human IoT through research design.

While these questions and aims created a solid basis for inquiry from the outset of my research journey, the focus of inquiry naturally shifted once I had confirmed Papawai Stream as my first research site and more-than-human participant in my study.

As the first research question shows, I initially considered focussing on a Euro-centric school of thought to guide the theoretical grounding for my inquiry. Considering my own background as a European, New Materialism and related philosophies such as ObjectOriented Ontology offered tools to solidify my argument that more-than-human concerns are considered 'valid' within contemporary academic discourse. However, once I engaged more with Papawai Stream and consequently connected with other streams in the region, among them Waitangi, Moturoa and Korokoro Stream and rivers of Te Ika-a-Māui/the North Island, most notably Whanganui River, I found that I had cast my net too narrowly. Once I got acquainted with the rich and robust body of knowledge on engaging with the more-than-human world in Te Ao Māori/the Māori world, I did not want to exclusively focus my research on a Eurocentric way of thinking and making.

Ka pu te ruha ka hao te rangatahi - As an old net withers another is remade. The second research question proposes participatory design as an avenue to involve the more-than-human world into the research process as participant. 
This required unpacking what participation means in a more-than-human context, and if and how traditional human-centred participatory design methods can provide new perspectives on designing with and for water and connected ecosystems. Proposing morethan-human participation within the time frame of this thesis project appears overambitious in hindsight. Instead, I feel more comfortable claiming to have spent time with, paid attention to and amplified the voices of the more-than-human world throughout my design research across the field- and lab work I conducted and the design outputs I produced.

With these considerations in mind, my research questions have gone through a few iterations before landing at the following version that voices the nature of my inquiry more accurately:

How can artists and designers learn from the more-than-human world when building networked media? 


\subsubsection{Research Design}

This research views the global development of an 'Internet of Things' (IoT) through a morethan-human lens of urban streams and waters in Pōneke/Wellington Aotearoa/New Zealand, to gain understanding of the intersection between networked technologies, and in a more-than-human world. Through an iterative creative research practice underpinned by theoretical inquiries into more-than-human concerns, I attempt to understand how specifically IoT technology that is currently globally advertised, can be developed for and together with local urban freshwaters. The overarching approach of this research can be described as qualitative, using methods of observation, walking interviews, immersions, audiovisual narratives, and a creative reflective/diffractive practice.

During my fieldwork, in which I spent time with urban freshwater streams and connected communities, methods inspired by participatory design and sensory ethnographic practices aided my understanding of paying attention to the more-than-human voices of the streams and their life worlds. Following an iterative design approach, the fieldwork stimulated the production of design artefacts which act as artistic outputs as well as means for further data collection. Field notes contained sensory observations, transcripts of conversations and monologues, geolocation data from walking interviews, geo-tagged photos, audio and video recordings of interviews, and field observations. Additionally, the IoT network prototypes collected sensor data such as conductivity, temperature, and turbidity. Prototyping work was predominantly concerned with the development of the IoT prototypes and took place across different computers and microcontrollers, Linux terminals, and various software development environments. Hardware prototyping was mainly situated in the 3D workshop and electronics lab, using sketch books, design software, soldering irons, basic hand tools and power tools and laser cutters. My lab development also produced tangential research outputs, such as maps created with the geo-tagged field data and experimental graphs generated from the sensor data. 
The fluid character of this research made it impossible to make a strict separation between fieldwork and prototyping work: as software development and debugging also took place in the form of field lab (Chapter 7) right in the dirt beside the stream and methods inspired from fieldwork (Chapter 5) were employed during prototyping sessions in the lab (Chapter 6). Besides being characterised by its iterative character, my work can be described as a creative reflective/diffractive practice: being an active participant in local arts, design and technology-related events, the study offers perspectives on the physical, material, and social fabric of maker culture and bottom-up community engagement from within an Aotearoa/New Zealand context. 
Iterative Design Overview

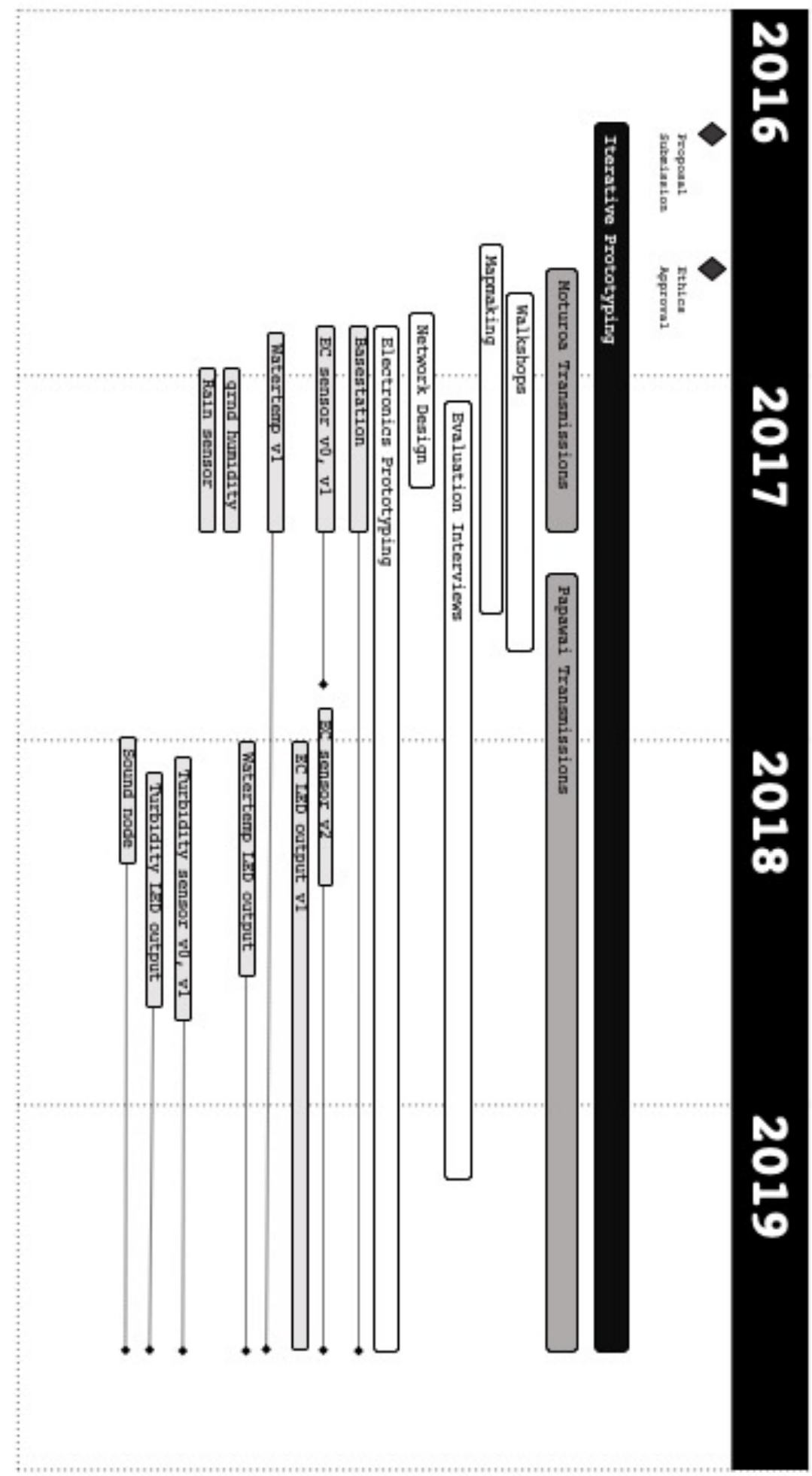

Figure 8. Author. (2019). Iterative design timeline. 


\subsection{Limitations/Role of the Researcher}

This research journey has further been complicated by my own practice as artist, designer and hard- and software developer, which gave me the privilege to approach the topic from multiple perspectives and engage with material on a variety of levels. This simultaneously troubles the understanding of research materials, having oscillated between theory and design practice, writing a thesis, and writing code, audio-visually recording human and non-human voices and using DIY sensors to record stream data. Situated between soldering labs, workshops, office desks, electronics suppliers and the 'field', the surroundings of Pōneke/Wellington streams, this research embraces not only theoretical but also practical messiness.

One important aspect of my work was that I continuously sought to challenge my own knowledge and experiences, and to keep being attentive to new knowledges that would change the initially projected course of my research journey. I also attempted to participate in as many communities and attend related events in the region to keep exploring new and often unexpected angles to approach my research questions.

The biggest challenge appeared to be the often seeming disconnect between the fields I am working in. I came across the term 'Internet of Things' in the commercial sphere as a buzz word in start-up lingo and marketing speech, in government and local authority memos, praised as a tool for innovation as well as a more figurative concept in the arts and literature. Being an artist, designer, and developer myself, I never found myself comfortably situated in any of the disciplines that the term kept appearing in.

This in-between space that I am constantly meandering through in my own practice also manifested itself in the way I did my research. The constant oscillation between creative arts and design methods in the field and the lab back to the drawing table designing circuitry, soldering PCBs, and building wireless networks that would withstand the demands of Pōneke/Wellington weather was an equally exhausting and rewarding exercise. 


\subsection{Key Fields and Themes}

The search for methods involving the decentring of the human, help to establish a theoretical grounding for design research that aims to navigate this complex territory, with the aim to introduce new perspectives to the development of an Internet of Things. My research, as situated in Aotearoa/New Zealand, presented further opportunities for engaging in a methodological approach responding to this call for a diversity of perspectives in design research. As a European born researcher, having only lived in New Zealand for three years at the start of this project, I need to learn about local, situated knowledges (Haraway, 1988) and perspectives. Working within the context of Aotearoa/New Zealand offered rich learning opportunities, where a Māori worldview offers a deep, intricate understanding of 'thing' networks.

From a designer's perspective, new, more malleable, and open frameworks for approaching research problems are emerging, among them post-qualitative research (Lather and St. Pierre, 2013) and non-representational approaches (Vannini, 2015). However, given that they are still in their infancies, these new, cross-cultural traditions face many challenges when trying to weave diverse attributes and non-text focused work into Western academic publishing structures, where they might be described as "messiness" (Law, 2004), or "slowness" (Ulmer, 2017). These factors, in turn, have become core to my research paradigm where, as I outline below, slowness, seamfulness (cf. infra p. 86), and openness have been integral to the development of a networked artwork as part of wildthings.io.

From the outset of the research, I developed tentative parameters to evaluate my research outputs against. While I started with a larger, more detailed and specific set of parameters to work with, as illustrated in the lab notebook entry for Moturoa Transmissions in 4.2, three categories express the character of my research journey across data collection, generative design research and evaluation. At first glance, the three categories of slowness, seamfulness and openness appear as shortcomings or hindrances to developmentespecially from the perspective of the technology industry, where quick development 
cycles, seamless solutions, and 'agile' prototype development towards exit strategies are idealised. Embracing these themes as productive methods, especially in the early stages of the research, proved difficult, as they made it hard to see results and outcomes of the work. Only later, once I felt more confident to share early prototypes publicly, I noticed how they created spaces for spending meaningful time with and paying close attention to, how my work sits within the more-than-human networks.

In this section I introduce these three themes briefly as methodological approaches to my research overall. The narratives (as presented in Chapters 5-7) provide a more in-depth discussion of the themes in specific contexts of the research process. I start this discussion here with $\underline{\text { slowness, }}$ which was a theme that articulated itself in later stages of the project, entangled within the other two key themes of seamfulness and openness. Seamfulness emerged early as a key theme in the research as part of the literature review on the Internet of Things, and also connects to ideas of openness which have been central to my artistic work before embarking on this doctoral journey.

\subsubsection{Slowness}

In this section I discuss the theme of slowness as the first of three key themes permeating this research project. I start by introducing it first as a concept that has recently gained attention in academia, followed by a discussion of slowness as a design tool, specifically when engaging in creative research with land and waters of Aotearoa/New Zealand.

\section{Slowness is a process of unlearning and unsettling what has come before (Springgay \& Truman 2019, p.15).}

Before developing devices, and designing new nodes and connections for an environment, it was necessary to take time to reveal and learn about existing networks. Unlearning and unsettling included acknowledging many of my European-centric perspectives I brought into this project, through my upbringing and previous education and art practice; and tuning into the complexities of the more-than-human world, specifically the freshwaters of suburban Aotearoa/New Zealand. 
The call to slowing down has been voiced by scholars as a response to the escalating pace of academic production (Ulmer, 2017, p.201). Advocating for a 'Slow Ontology', Ulmer describes how, in New Materialist qualitative scholarship, a more-than-human, entangled approach to research involves the writing of environmental landscapes, as well as "writing on/with/through/in aspects of nature" (p.207), calling for more-than-methodologies which “involve material, ecological, and temporal inquiries” (Ulmer, 2017).

An approach to slowness when working with more-than-human ecologies resonates with how Pigott and Lyons (2016) discuss their artistic practice as a

\section{[...] slow attunement and creative 'listening'. This process involved a distillation of a rhizomic mesh of conversations and encounters, embracing place identity, species, technology and communication (Lyons, 216, p.144).}

Embracing slowness also afforded time to pause and understand what it means to be a designer in Aotearoa/New Zealand.

Viewing slowness through a critical, reflective lens, I turn to my own research progress as I map the entanglements of this mode. Such slowness, in wider design discourse, has responded to 'time' and 'temporality' as constructs which mediate not only research processes, but the wider material and political contexts in which such work is situated. Here, to draw on Odom et al. (2018), temporality-the state of existing within time-shapes virtually all aspects of how we experience and construct the world around us. By embracing slowness, I was afforded time to (re-)engage with my own positionality as a researcher in the context of Aotearoa/New Zealand. Here, slowness as a conceptual tool, allowed me to pause and reflect on the ongoing flow of research and knowledge production in this setting, both in terms of indigenous epistemologies, and the more-than-human world which exists beyond the bounds of the University, and indeed anthropocentric constructions of time.

'Slowness', as a design tool, has emerged from a wider research trajectory which emphasises embracing different experiences of time. Here, turning to 'slowness' as a component in the design process can support experiences that include moments of mental rest and solitude (Odom et al., 2018, p.384). As they note, another area of work has 
investigated temporality and slowness as different ways of framing the design of interactive systems themselves, where slowness has also been applied in design efforts to support experiences of anticipation, social connection, and longer-term relations with everyday computational objects (p.384). Moreover, embracing not only different experiences of time, but different perspectives on time can expand the theoretical realm of design methodology in order to acknowledge the ways in which time itself functions as a mode of colonial knowledge (cf. Rifkin, 2017).

If time is then the medium through which an "interactive dialogue between a human and computer begins, unfolds, and resolves" (Odom et al. 2018, p.384), turning our attention towards examining different perspectives of time can equip designers with a means through which to critically (re-)engage with such beginnings and resolutions, and indeed the temporal nuances entrenched within. In looking to extant research in this area, Lindley (2015) and Pschetz (2015) envision time as always already socially entangled and relational, highlighting the need for alternative expressions of temporality in design. Taylor et al. (2017) further addresses the decolonising potential of such approaches, through a crosscultural design project that emphasises time from an Australian Aboriginal community's perspective. Here the designers propose the concept of the 'Situational When', that emphasises an approach to understanding time in interface design "not as a point on a calendar or clock, but as a set of converging circumstances that constitute "the time" for happenings to take place” (2017, p.6461). Attention to the 'Situational When', they argue, "pens up new possibilities for design that put greater emphasis on the social and relational aspects of time, the situational insights embodied in local narratives, and the tangible (e.g. people) and intangible (e.g. energy) circumstances that together make up the 'right' time" (2017, p.6461).

Such research highlights the need for new design methods to embrace the diversity and fluidity of 'time', as well as the complexity in designing in timeframes that may well expand beyond the lifetime of the design team itself (Odom et al., 2018, p.384). Researchers have also proposed different themes, such as 'narrative time' and 'ephemerality' as resources for 
design. Returning to Taylor et al.'s 'Situational when', the process of 'slowing down' to attend to the situation has also been a rich vein of design research. Whatmore and Landstrom, for example, contrasted a conventional participatory ethos of empowering local people with an ethos of empowering the situation, where the aim is to 'force thought' in those affected by it and to 'slow down' the reasoning (in Noorani \& Brigstocke 2018, p.24). The effect of embracing slowness, in this instance, has direct implications for my own research journey:

\section{Through intentionally building stages and spaces for the intermingling of human and non-human agencies, and slowing practices down, hybrid forums of knowledge and expertise can off er innovative practical and political responses (Noorani \& Brigstocke 2018, p.24).}

While quick iterations of design outputs might appear to constitute an effective way of pushing the progress of a design work further, considerations of the impact of my research, such as introducing networked technology into the more-than-human worlds of local freshwaters, slowed my research progress down, from an academic perspective. However, this slowness allowed a richer and deeper engagement with Te Ao Māori concepts, glimpsing a future of being able to contribute to research on the interface between science and indigenous knowledge (as described by Durie, 2004).

As part of the development, slowness-first mistaken as a hindrance to my research progress - then allowed me to take the time needed to listen and connect to the field, and allowed early, seemingly unproductive activities to slowly reappear as productive outcomes in my design interventions. For example, early exploratory walks were primarily aimed at testing prototypes of a DIY hydrophone that never ended up being used as part of a final installation. These walks later evolved into field explorations with a focus on listening. Eventually I used some of the resulting field recordings (see attached example) as aural backdrop during prototyping and evaluation activities in the electronics lab. I was reminded here of the work of Hallnäs and Redström (2018), who argue that a basic principle of slow technology is to amplify the presence of things to make them into something more than just a silent tool for fast access to something else (2001, p.209). 
Given the experiences presented here, I frame my own approach and response to 'slowness' in line with Hallnäs and Redström (2001, p.210), who propose two basic guidelines for slow technology:

focus on slowness of appearance (materialisation, manifestation) and presence - the slow materialisation and design presence of form.

focus on aesthetics of material and use simple basic tools of modern technology - the clear and simple design presence of material (Hallnäs \& Redström, 2001, p.210).

Embracing these principles as part of the field explorations required me to slow down and take the time to acknowledge the rough edges encountered and later created during my fieldwork. By aiming to create complete recordings and documentation of my research, I overlooked the fact that tending to recording equipment would inhibit my ability to employ my own senses while being in the field. Hence, I later conducted several un-documented walks to the sites, in which I tried to pay attention to the stream, instead of focussing on my recording devices. During the first field explorations, the process of walking and conversing meandered between slow movements and immersions.

The lens of slowness also afforded more time to understand what it means to be a designer in Aotearoa/New Zealand. Slowness allowed me to pause and take the time to acknowledge that research and knowledge production have been part on this land and waters before Europeans arrived. Here, I turn to ways Escobar (2018) has written about designing with and for a world of many worlds, as well as literature specifically addressing methodologies for working with and on indigenous lands (Smith, 2012).

Slowness may also be ascribed to a crucial latency phenomenon, where intertwined cultural perspectives must be addressed in all their complexity. Latour (2013) also describes this latency phenomenon also through the need of understanding how human impact on Earth often only materialises in the form of effects much later. 
This is what it means to live in the Anthropocene: "sensitivity" is a term that is applied to all the actors capable of spreading their sensors a little farther and making others feel that the consequences of their actions are going to fall back on them, come to haunt them. When the dictionary defines "sensitive" as "something that detects or reacts rapidly to small changes, signals, or influences," the adjective applies to Gaia as well as to the Anthropos - but only if it is equipped with enough sensors to feel the retroactions (Latour, 2013, p.141).

Taking literally this sensitivity to small changes, signal or influences literally, a sensor network for a small suburban stream could equip small communities to feel the retroactions of climate change on a local scale, understanding the connections of the morethan-human world and how human impact alters the wellbeing of its inhabitants. Slowly unfolding these complexities also not only requires letting go of a 9-5 working rhythm when doing fieldwork, but also tuning into the temporal worlds of the stream and understanding its natural rhythms and flows as part of the inquiry.

If organic rhythms are at the forefront of a Slower inquiry, then our thoughts might follow the rhythms of celestial bodies, land formations, climates and seasons, flora, fauna, and our material selves (Ulmer, 2017, p.207).

The openness to tune into natural rhythms of our worlds is a skill that stands central within Te Ao Māori, where, for example, the Maramataka offers knowledge based on the lunar cycles. Skills, that need to be relearned, require the unlearning of standardised concepts of time. 


\subsubsection{Seamfulness}

In this section I introduce the second key theme of this research, seamfulness. I first introduce seamfulness as originally coined by Weiser (1991) and then discuss how it has developed in later design research, before exploring how the previously discussed themes of slowness are entangled within ideas of seamfulness.

The concept of seamfulness came to my attention after a conversation with Anne Galloway which directed me to her talk (2006; see also 2007) in which seams are discussed as

... intimations of past actions and interventions, of things joined together and things cut apart. The whole that they create is a hybrid, something both new and old. And perhaps most importantly in cultural terms, when we think about them actually being made, they mark liminal space (Galloway, 2006, p.2).

The concept of seamfulness, originating from Weiser's (1991) vision of the computer of the 21st century helps to guide this research across a very dense and complex history of developments, protocols, standards, applications, products that define the contemporary discourse of the IoT today. Seamfulness as a methodology helps preserve access to the many layers of a complex technology and entangled research process without the urge to smooth over the edges.

\section{Machines that fit the human environment, instead of forcing humans to enter theirs, will make using a computer as refreshing as taking a walk in the woods (Weiser, 1991, p.104).}

In later talks, Weiser $(1994 ; 1995)$ addresses the misleading concept of seamlessness and argues for "seamful systems", with "beautiful seams". Weiser also rejected the idea of an interface as a boundary or difference and argued that the unit of design should involve social people, in their environment plus their device (Weiser 1995, p.21). Later, Chalmers and MacColl (2003) argued for seamfulness in design and described it accordingly:

\section{taking account of the finite and physical nature of digital media. Seamful design involves deliberately revealing seams to users, and taking advantage of features usually considered as negative or problematic (Chalmers \& MacColl, 2003, p.1).}

Chalmers et al. (2003) pointed out that the revealing of the seams in the infrastructure of Ubiquitous Computing can be an opportunity for user understanding and empowerment. 
Seams could also be a way towards the creation of more dynamic systems that are able to adjust to interaction patterns originally not envisioned by the designer.

Seamlessness in IoT devices is problematic not only in terms of privacy concerns but also in relation to obfuscating functionality to users, preventing understanding of what networked devices really do, at any given point in time. Seamful design tries to "reveal inevitable seams in ubicomp systems and use them to increase awareness for system infrastructures, their heterogeneous components and otherwise neglected yet useful information within the system", aiming to "smooth over the bumps and cracks" (Broll \& Benford, 2005, p.155). In their literature review of seamful design, Inman and Ribes (2019) describe how this concept has initially sought to counterbalance the "long-standing but unacknowledged design virtue" (Inman \& Ribes, 2019, p.5) of seamlessness. Instead of discussing the two concepts as binary opposites, they consider seamful and seamless design as complementary concepts and describe "beautiful seams" as

\section{a phrase that seems to capture both the spirit of user-friendly, coherent design emphasised by seamlessness and the heterogeneity, contingency, and appropriability of seamful design (Inman \& Ribes, 2019, p.12).}

This appropriability has also been discussed, framed as opportunities of appropriation (Oulasvirta, 2004a; Chalmers, 2004), to "support emergent practices by making the basic design itself flexible enough" (Oulasvirta, 2004a) or let users "take advantage of [seams] and appropriate them for their own uses and interpretations" (Chalmers et al., 2004, p.175). Fuller and Haque (2008) specifically mention non-specialists as a group who "may feel mystified by technological seamlessness" (p.31), whereas the opportunity to "crack something open helps provide a sense of individual responsibility and technical audacity" (p.31).

Despite the relative gap in the literature, there are nonetheless important insights into how the concept of slowness can be found within the context of seamful design. For example, Inman and Ribes (2019) note that "[a]s UbiComp considered space as an opportunity to deploy more and more sensors, connecting the physical space with the virtual, time has been delegated in less conspicuous ways" (p.6) and note that "less consideration has 
focused on the disappearance, concealing, or standardization of time” (p.7). Broll and Benford (2005) note the technical limitations of UbiComp: "Patchy network coverage, fluctuating signal strength, deviations in positioning and the generally limited resources provided by mobile devices are an everyday reality for their users" (p.155). Analogously, when Oulasvirta (2004a) notes examples of seams, differentiating between "obvious technological seams" and "less obvious seams", they argue that seams do not necessarily need to be understood as "spatial", despite the literal meaning of a seam being "a line of stitches joining or separating two pieces of cloth", which points to seams that operate on a temporal, or contextual level.

\section{We must shift attention from technologies that seamlessly blend in with everyday life, towards technologies that move people and enable them to move others (de Lange, 2013).}

With my research activities situated in heterogenous contexts across field explorations (Chapter 5), lab development (Chapter 6) and field lab (Chapter 7) seams as a metaphor aid to "draw our attention to those places where multiple infrastructures are stitched together to achieve fleeting, nonstable, even ephemeral moments of alignment" (Vertesi, 2014, p.277). The narratives provided in Chapters 5, 6 and 7 present how these design research stitches these environments and contexts together, which is illustrated, particularly, by the field lab in Chapter 7, where electronics development and deployment moved into the field, creating new connections between the field and the design work, which in turn, also uncovered new seams. The seam further "directs designers' attention to seeing infrastructure as a resource (and source of errors) for users. It's part of the context" notes Oulasvirta (2004b, p.13). Some of those seams were discovered during early fieldwork, understanding how the development of infrastructure, in the form of the stormwater network has disconnected the original freshwater streams from their source. This infrastructure disconnect would later come back during the design research, when the stream environment itself would provide challenges of prototyping a technological infrastructure as part of the networked install. Through the narratives, I aim to bring the reader closer to context, through using the opportunity of exploiting prototyping and field documentation as a seamful design itself. Seams reveal themselves not only in the design of the prototypes themselves, but also in the documentation provided as part of this thesis, 
including the audiovisual components, which are supplied to bring the research environments closer to the reader of this dissertation.

Embracing slowness as one of the key methods that informed the research framework of wildthings.io allowed me to listen and connect to the field; and allowed early, seemingly unproductive activities, to slowly reappear as productive outcomes in my design interventions. Engaging with slowness, as a method, further revealed an opportunity for critically mapping the ways in which design research and practice have considered questions of temporality; as Lindley (2015) has noted, "unlike concepts such as 'space' and 'place', which have been the subject of careful enquiry and definition, time is considered sporadically and in rather heterogeneous ways" (p.1442). The question of how temporality may be represented, or materialised, within design process thus represents a broader field of inquiry. Time, as Inman and Ribes (2019) argue, is often delegated in inconspicuous ways; "time has become a commodity rather than a contextually important facet for design" (Inman \& Ribes 2019). Less consideration has been focused on the disappearance, concealing, or standardisation of time, and moreover, how such obfuscation of the temporal might be represented within the discourse of seamlessness.

This question of seams, and their ability to serve, as Crabtree and Rodden (2008) have argued, as "mechanisms of interaction" (p.491), formed the second of the key methods of this project. Ultimately, seamlessness and seamfulness speak to longstanding discussions about the revelation and concealment of human and technological operations (Inman \& Ribes 2019). However, a major gap in much design research to date has been examples of seamful design as it relates to temporality. Inman and Ribes' literature review found that while much of the literature characterises 'seamful' and 'seamless' as a way of dealing with heterogeneous data and scenarios-spatially, temporally, and technologically-the majority of the research to date has focused on spatial rather than temporal contexts $(2019$, p.11). These intersections of seamfulness and slowness were thus important to the development of my design outcomes, where the literal seams of my interventions sought to respond to questions of the user agency, but at times, designer or system agency, by facilitating quick 
access to common operations (Chalmers, 2004). The seams also encourage a temporal slowness and considered-ness within these interactions.

The seams also reveal themselves with my positionality as a European-born researcher doing work with wai/water in Aotearoa/New Zealand, where a process of unlearning plays into the work of understanding the limitations of technology when attempting to recognise the rich knowledge inherent in the more-than-human world of local freshwater streams. The need to be open, to learn and to listen when doing this kind of work is another central aspect of this project, which I discuss in the following section.

\subsubsection{Openness - Opening the design process to more-than-human voices}

Openness was one of the first parameters of my research, mostly due to my background in open-source development and my familiarity with open tools. Where embracing seamfulness and slowness within the research process, as articulated above, allowed me to engage with the intersections and encounters of the project's multiple facets, and its situatedness therein; drawing on openness has added further dimension to my understandings. As Gasparotto (2019) notes, notions of openness in design have a plurality of meanings that can be "summarized through the features of open source, collaboration and access" (p.27). In this section I first discuss openness from a collaboration angle, through a participatory design approach, then delve into the complexities of open-source development and questions of access when working across art and science fields and doing research on indigenous lands.

Openness as collaboration, Gasparotto (2019) notes, "evokes the aggregation of a plurality of subjects" (p.21). In this sense, she continues, the open paradigm seeks to emphasise the possibility of people working together, where collaboration can imply participation, cooperation, and sharing. From the outset of the research, I considered Participatory Design (PD) as an avenue to involve not just people, but the more-than-human world into the research process as participants. This required unpacking what participation means in a more-than-human context, and if and how traditional human-centred participatory 
design methods can provide new perspectives on designing with and for water and connected ecosystems.

\section{It takes work, and new ways of thinking, and new kinds and methods of openness, to bring substantively new voices into a conversation (Muller 2009, p.166).}

The opportunities and challenges of adding new voices and perspectives into a design conversation are widely discussed in the field of Participatory Design (see for example Bannon \& Ehn, 2012; Kensing \& Greenbaum, 2012). A more-than-human participatory research agenda, as described by Bastian et al. (2016), supports the inclusion of marginalised voices in the research process, and "makes research accountable to those it affects" (p.5). Akama et al. (2020) describe how "pluriversality requires us to work with multiple worldviews and link them in our personal thinking and design research practices" (p.3) and calling to “trouble PD’s dominant borders and assumed 'Western'-centricity” (p.10).

In finding myself working with local communities and ecosystems in an Aotearoa/New Zealand context, it is vital to acknowledge and incorporate non-Western traditions and modes of thought (see Smith, 2012). Blomberg and Karasti (2012) discuss the opportunity to include ethnographic sensibilities into a PD approach, but warn that:

\section{We should not assume that the tools and techniques of Participatory Design developed for Scandinavian (and other European and North American) audiences will enable multiple voices to define and inform the design when transported to very different traditions (Blomberg \& Karasti 2012, p.107).}

As an artist, designer, coder, and researcher taught within Western academia, most of my tools and technologies stem from a Western background. I must avoid a technological colonisation of Aotearoa's more-than-human worlds through my research and the tools I develop, respecting that "data is a living taonga" (Te Mana Raraunga, 2017, p.1), with relationships between people worlds and the spirits are inherently linked through whakapapa/genealogy. 
In the "perhaps the most quoted sentence in the book" (Smith, 2012, p.xi), one which stands central in the introduction of the work Decolonizing Methodologies, Smith reminds us:

\section{From the vantage point of the colonised, a position from which I write, and choose to privilege, the term 'research' is inextricably linked to European imperialism and colonialism. The word itself 'research', is probably one of the dirtiest words in the indigenous world's vocabulary (Smith, 2012, p.1).}

I want to be humble and acknowledge the privilege of doing research with and for the water of the streams of Aotearoa/New Zealand. Similarly, I need to scrutinise my background in open-source development and keep assessing if and how open sharing of my design research benefits the more-than-human communities it affects. Openness, manifested as a willingness to share my process and give the knowledge back to communities who care for their streams, implied access to open licensing and publishing of hardware, software, writings, and recordings of my design processes. Whilst it is not within the scope of this section to fully unpack the complexities and tensions which can arise from mobilising 'open' culture into spaces grappling with the implications of decolonisation, emergent work in this field reminds us that as researchers, we must always be critically aware that underlying much 'open' discourse' is the assumption of the universality of knowledge systems, often dictated by hegemonic knowledge groups (see for example Adam et al., 2019). I take up this approach to openness in my own work with this caution in mind.

Pākehā engagement with kaupapa Māori that goes beyond cultural sensitivity, and that demands from educational researchers a personal quality not directly teachable, but developed through an openness to being taught by experience, a tolerance for uncertainty, and an understanding of power (Jones, 2012, p.100)

"Open knowledge production and universal criticism, too, are regarded as failing the interests of Indigenous peoples when the critics in power remain ignorant or careless about the philosophical bases(kaupapa) of indigenous knowledges” (Jones, 2012, p.104). 
Proper engagement with kaupapa Māori requires Pākehā individuals to become ordinary, at ease in Māori contexts, open to Māori knowledges, and familiar with te reo Māori. And to achieve this is to be oriented to learning, watching, listening, in relationships of depth and longevity (as well as having a sense of humour, suspended judgment and humility!)-along with a necessary consciousness of the wider relationships of power in which this engagement takes place. This sense of alert ease takes a long time for those who cannot whakapapa into te ao Māori, and even for many who can. It takes years of taking part, working with Mäori friends and colleagues, and being seen (kanohi kitea) in Māori communities, projects and events. Without this, it is difficult for Pākehā researchers, or Māori researchers for that matter, to properly engage, positively or critically, with kaupapa Māori, including with Māori communities (see also Glyrm, 2008) (Jones, 2012, p.107).

In his presentation, Taiuru (2015) provides a "holistic description and comparison of Māori Culture and Society (Te Ao Māori) and Open-Source Culture (Te Ao Tino Rangatiratanga)”, describing how they could benefit each other, and highlights similarities between open source and Māori communities. He highlights the benefits of open-source technologies to Indigenous peoples and warns how proprietary systems and software could take away Intellectual Property of the traditional owners, making users pay for the right to access their own information.

Being mindful that protocols of Indigenous communities often do not align with conventional tech protocols, the open-source project Mukurtu CMS is developed as a digital archive that acts as a "safe keeping place" for Indigenous community content sharing. The makers however recognise that "while the standard open-source model provides an avenue for growing a development community, Mukurtu users do not have the resources, infrastructure, and programming skills to contribute to Mukurtu's development in the same way as other open-source platforms" (Christen et al., 2017). In response, the project is working on a support structure through training, community engagement, community development and development sprints, and supplying infrastructure through academic institutions. 
As Kiem notes, decolonising design

is a question of who controls, profits from, or is protected (or not) by the ways in which intellectual and other forms of re/production and consumption are organised (Schultz et al, 2018, p.83).

This highlights how openness, in the sense of opening sharing project material, requires further engagement when working outside of the open-source community, who are familiar with concepts of software and hardware development. When collecting data of the more-than-human-world, for example, this would involve finding ways of giving back to the communities and embracing reciprocity. However, it is worth noting here, that decolonising is a process, rather than a product, and these steps outlined form humble first steps as part of this journey.

Prioritising open-source technology when developing designs for this research project can aid the keeping of inner workings and politics of used hardware or software more transparent and accessible; and opening the possibility to adapt or expand functionality of a device to suit a more-than-human design scenario. However, even with a dedication to open-source ideology, the objectification of knowledges can never be eliminated, because technology always prescribes some level of representation of data. The challenge of representation of more-than-human perspectives through technologies is finding a suitable way to acknowledge and preserve the individual characteristics of each voice; and making them accessible to each other, and finding ways to provide compatibility to enable connections between perspectives.

This openness, along with slowness and seamfulness, has allowed a richer and deeper engagement with concepts of Te Ao Māori, glimpsing of a future of contributing to research at the interface between science and indigenous knowledge (as described by Durie, 2004). 


\subsection{Outputs and Contributions Timeline}

Places where I have disseminated and discussed my research

\begin{tabular}{|c|c|c|}
\hline Title [type] & Place & Date \\
\hline Moturoa Transmissions [Installation] & $\begin{array}{l}\text { Upstream Brooklyn Art Trail, } \\
\text { Wellington, New Zealand }\end{array}$ & 02-05 March 2017 \\
\hline $\begin{array}{l}\text { Networking Natures } \& \& \text { Coding Cultures [Artist } \\
\text { Talk] }\end{array}$ & $\begin{array}{l}\text { Digital Art Workshop - Building } \\
\text { Blocks, Aftermath Gallery, } \\
\text { Christchurch, New Zealand }\end{array}$ & 22 July 2017 \\
\hline $\begin{array}{l}\text { Materialising a more-than-human Internet of } \\
\text { Things [Artist talk] }\end{array}$ & $\begin{array}{l}\text { Sonic Acts Academy, Dansmakers, } \\
\text { Amsterdam, The Netherlands }\end{array}$ & 24 February 2018 \\
\hline $\begin{array}{l}\text { FOSS+ART. Unleashing Artistic License with FOSS } \\
\text { [Talk] }\end{array}$ & $\begin{array}{l}\text { ITx, New Zealand's Conference of IT. } \\
\text { TSB Bank Arena, Wellington, New } \\
\text { Zealand }\end{array}$ & 11 July 2016 \\
\hline Papawai Transmission [Prototype demo] & $\begin{array}{l}\text { MakerFaire Wellington, Shed 6, TSB } \\
\text { Bank Arena, Wellington, New Zealand }\end{array}$ & 20 October 2019 \\
\hline lab.wildthings.io [Lab Blog] & Online & \\
\hline $\begin{array}{l}\text { https://github.com/iRGBit/wildthings.io [Code } \\
\text { Repository] }\end{array}$ & Online & \\
\hline $\begin{array}{l}\text { Prototyping a more-than-human Internet of } \\
\text { Things [Poster presentation] }\end{array}$ & $\begin{array}{l}\text { Propagate - Faculty of Architecture } \\
\text { and Design Inaugural PhD } \\
\text { Symposium. Victoria University of } \\
\text { Wellington, New Zealand }\end{array}$ & 24 October 2019 \\
\hline $\begin{array}{l}\text { Prototyping a more-than-human Internet of } \\
\text { Things [Presentation] }\end{array}$ & $\begin{array}{l}\text { College of Creative Arts Research } \\
\text { Symposium, Massey University } \\
\text { Wellington, New Zealand }\end{array}$ & 8 February 2019 \\
\hline $\begin{array}{l}\text { Korokoro Stream - sensitive urban design and } \\
\text { restoration [Roundtable Discussion [ }\end{array}$ & $\begin{array}{l}\text { Common Ground Public Arts Festival, } \\
\text { Lower Hutt, New Zealand }\end{array}$ & 27 February 2017 \\
\hline $\begin{array}{l}\text { Internet of Water - methods for building } \\
\text { networks within more-than-human context } \\
\text { [Presentation] }\end{array}$ & $\begin{array}{l}\text { Sustaining the Seas Conference } \\
\text { Sydney. University of Sydney, Sydney, } \\
\text { Australia }\end{array}$ & 11 December 2017 \\
\hline Tributaries, Nodes and Networks [Panel] & $\begin{array}{l}\text { Sustaining the Seas Conference } \\
\text { Sydney. University of Sydney, Sydney, } \\
\text { Australia }\end{array}$ & 11 December 2017 \\
\hline $\begin{array}{l}\text { Materialising a more-than-human Internet of } \\
\text { Things [Artist Talk] }\end{array}$ & $\begin{array}{l}\text { From Cosmos to Genes New } \\
\text { Materialist Training School, Charles } \\
\text { University, Prague, Czech Republic }\end{array}$ & $23-26$ August 2016 \\
\hline $\begin{array}{l}\text { How do the things like your Internet of Things? } \\
\text { [presentation] }\end{array}$ & $\begin{array}{l}\text { UX design meetup, Victoria University } \\
\text { of Wellington, New Zealand }\end{array}$ & 27 June 2017 \\
\hline $\begin{array}{l}\text { Materialising a more-than-human Internet of } \\
\text { Things }\end{array}$ & $\begin{array}{l}\text { Internet of Things meetup, Biz Dojo, } \\
\text { Wellington }\end{array}$ & 7 June 2016 \\
\hline $\begin{array}{l}\text { Greater Wellington Audience Engagement Hui } \\
\text { [Presentation] }\end{array}$ & $\begin{array}{l}\text { Government House, Wellington, New } \\
\text { Zealand }\end{array}$ & 5 July 2016 \\
\hline $\begin{array}{l}\text { Materialising a more-than-human Internet of } \\
\text { Things [Artist talk] }\end{array}$ & $\begin{array}{l}\text { International Symposium on } \\
\text { Electronic Art (ISEA), Universidad de } \\
\text { Caldas, Manizales, Colombia }\end{array}$ & 14 June 2014 \\
\hline $\begin{array}{l}\text { Materialising a More-than-human Internet of } \\
\text { Things [Presentation] }\end{array}$ & $\begin{array}{l}\text { Arduino Day, Victoria University of } \\
\text { Wellington, New Zealand }\end{array}$ & April 22016 \\
\hline $\begin{array}{l}\text { Discussion/Meetup groups I have been part of } \\
\text { loT Meetup, Friends of Papawai Gardening } \\
\text { Group }\end{array}$ & Wellington, New Zealand & \\
\hline $\begin{array}{l}\text { Open, seamful and slow: a more-than-human } \\
\text { Internet of Things [Paper] }\end{array}$ & XCOAX 2020, Graz, Austria - online & 8-10 July 2020 \\
\hline $\begin{array}{l}\text { Backyard Networks - Reconnecting Localised } \\
\text { Experiences of Climate Change [Paper] }\end{array}$ & Mapping the Anthropocene & 26 September 2020 \\
\hline
\end{tabular}

Table 1: Author. (2020). Places where I have disseminated and discussed my research. 


\section{Chapter 5: Field Explorations}

\subsection{Introduction}

In this chapter, I present my fieldwork activities from the period of 2016 to 2019, focussing on field explorations, which involved discovering and connecting to the streams by spending time with them, exploring them through hour-long walks, both alone or in conversation with invited participants, paying attention to how we understand the morethan-human world of water and what role it could play in my design research.

This section presents five key activities within my field explorations. The first, Exploring Papawai Stream by foot, was documented as a blog post on 22 October 2016. The second, Exploring Papawai Stream utilises mapmaking as a means to refind and reconnect 'lost' streams. The third, Walking with, explores the participatory method of 'Walkshops' in Moturoa Stream, Papawai Stream, and Korokoro Stream. The fourth, Water on the Internet? Or Worlds that are not supposed to meet, locates conversations as sites of fluid, active research. The fifth field exploration, Participating in the SHMAK workshop, documents my negotation of my positionality as both researcher and participant within my field work.

The media component of this chapter is a video (fieldexplorations.mp4) recorded during field explorations, showing visuals of Papawai Stream in slow motion, accompanied by a audio field recording taken the same day. The video could be played as an intro to reading this chapter, either as a separate component, tuning into the environment of the stream both visually and aurally, or played in the background while reading this chapter and immersing yourself in the sonic environment of Papawai Stream.

I invite the reader into narratives in which I seek to understand and explore more-thanhuman networks in the field, followed by tapping into these networks through my design research outputs, which are described as part of the prototyping log in Chapter 6, followed by the field lab in Chapter 7. 
My field explorations are reproduced from their 'originary' recordings. My focus throughout the project has been to record in the present tense; in doing so, to borrow from Saka (2008), I seek to narrate what would normally remain fragments of fieldnotes. The narrations here take on different forms; in locating these explorations in different narrative modes and methods, I make visible their slowness, seamfulness, and openness, as they emerge in myriad forms. 


\subsection{Exploring Papawai Stream by foot [Blog post 22 October 2016)}

This blog post describes my first exploration up Papawai Stream, beyond the easily accessible footpath, on the search for where the water originates.

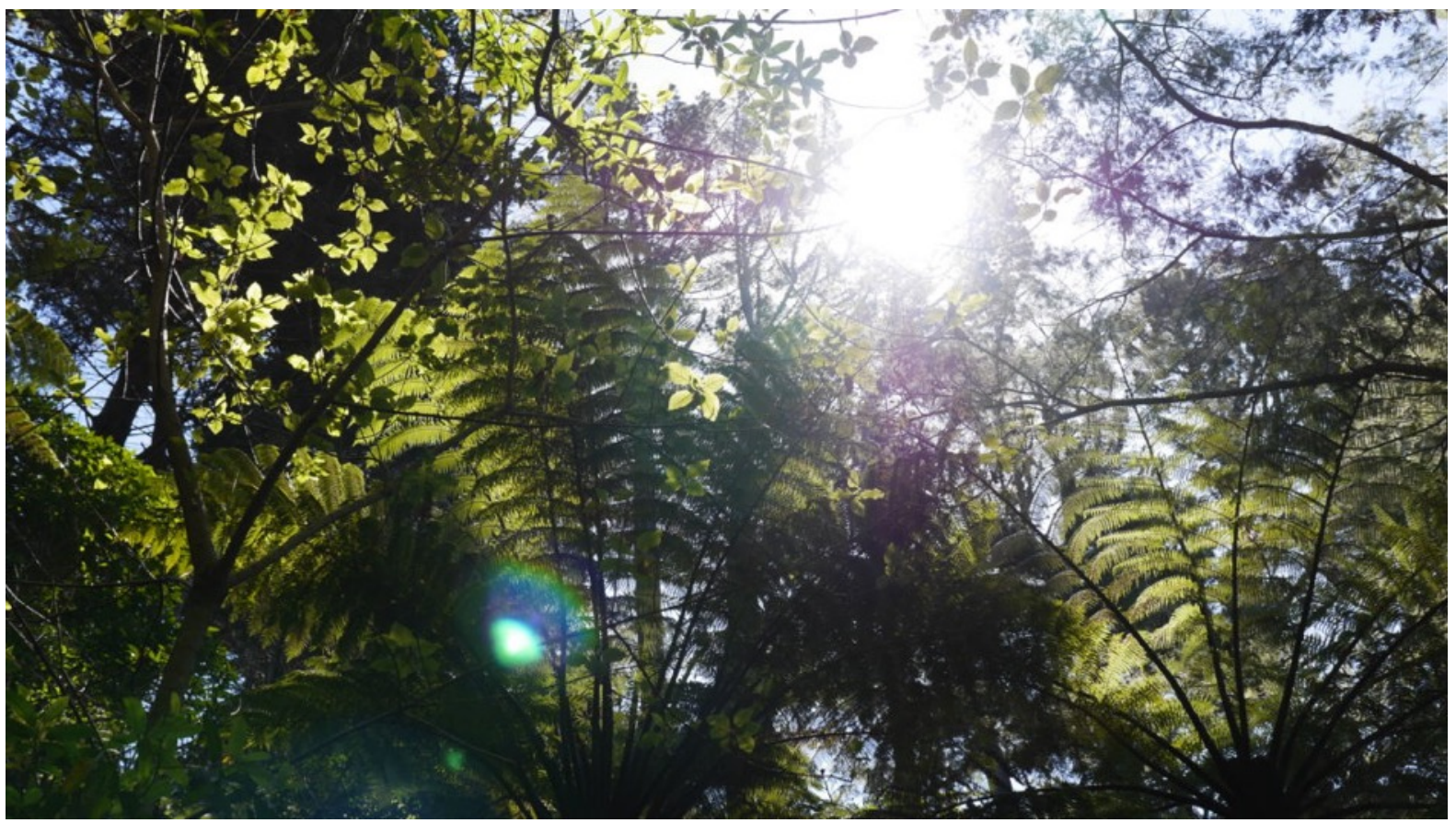

Figure 9: Author. (2016). Noticing the vegetation upon departure upstream.

Unfortunately, the photo camera I took with me on the walk had no built-in GPS, so I cannot accurately pinpoint the high-quality images taken during this first walk to a location in retrospect. I did, however, bring a tablet to record the walk with the software Open GPS Tracker that also allows annotation of waypoints with text, image, and video recordings.

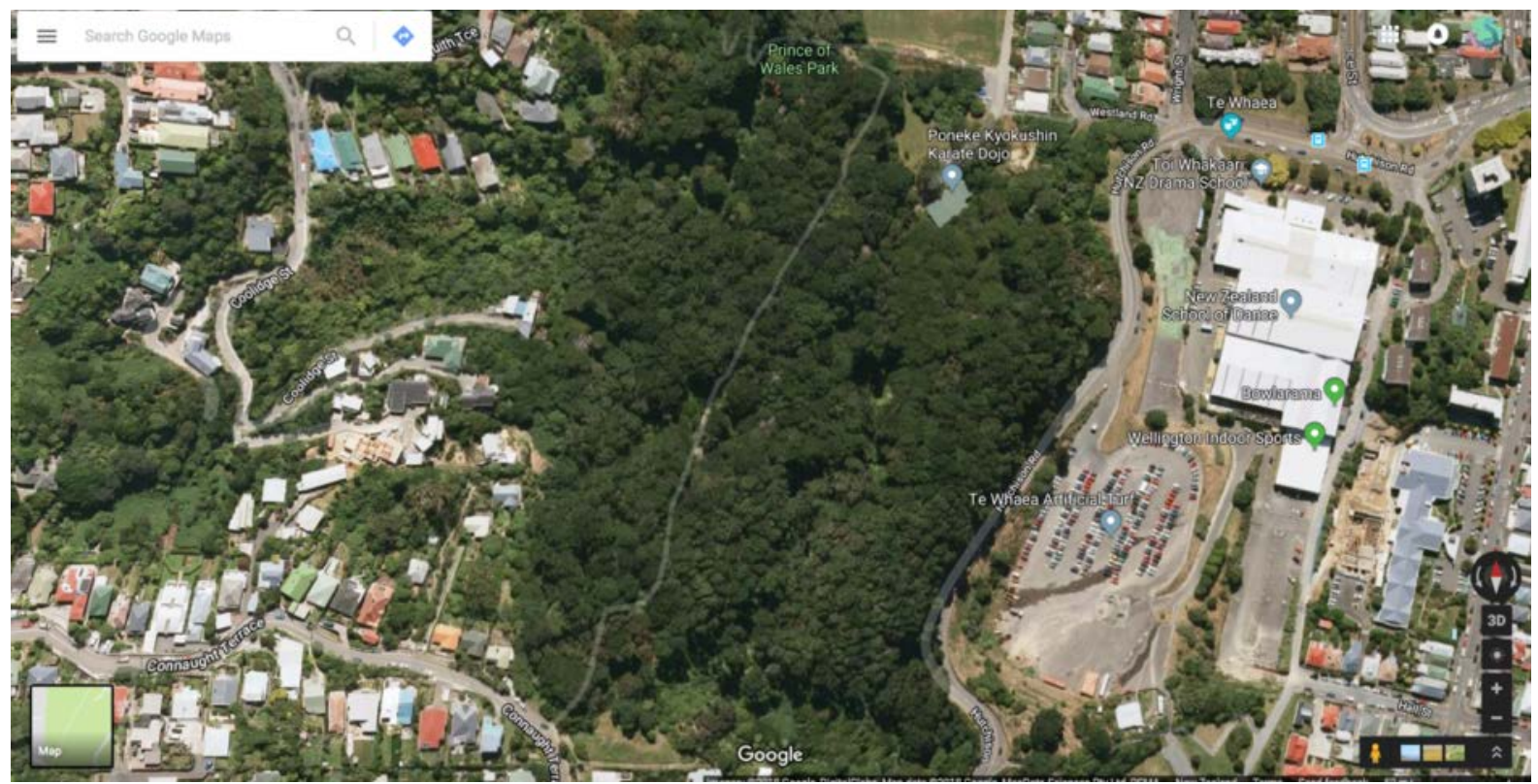


Figure 10:Author. (2016). Google Maps satellite imagery screenshot of Prince of Wales Park area.

It is an early Saturday afternoon, the weather is fair, it is late October, early spring in New Zealand. My flat on Salisbury Avenue is just a short walk away from the stream and I would pass the stream on a daily basis, taking the path through Papawai Reserve, which briefly is accompanied by the stream on my daily walking commutes. I can see the playing fields from the west-facing windows, with the green belt vegetation as a backdrop.

I cross the lower playing field to enter Prince of Wales Park, which is part of the Wellington Green Belt, an outdoor recreational area relatively close to the city centre.

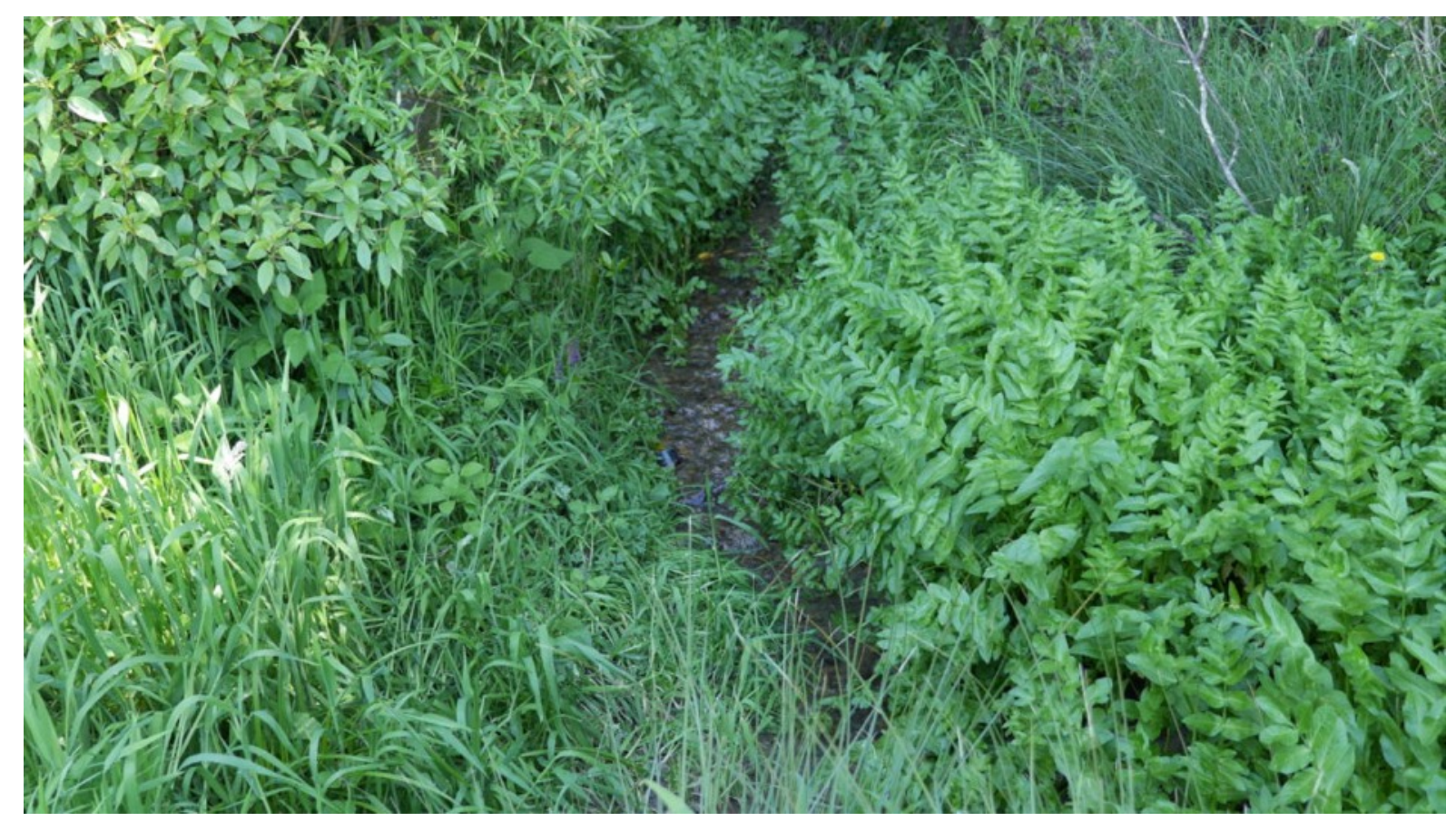

Figure 11. Author. (2016). The stream on the side of the sports field is surrounded by ground vegetation.

On the sides of the field, I can see the stream; surrounded by vegetation, almost hiding, but slowly gurgling away. To follow its course further, I take a walking path uphill, entering the shaded, forested part of Prince of Wales Park. The stream is on the right side of the track, not easily accessible, but partly visible through the vegetation. 


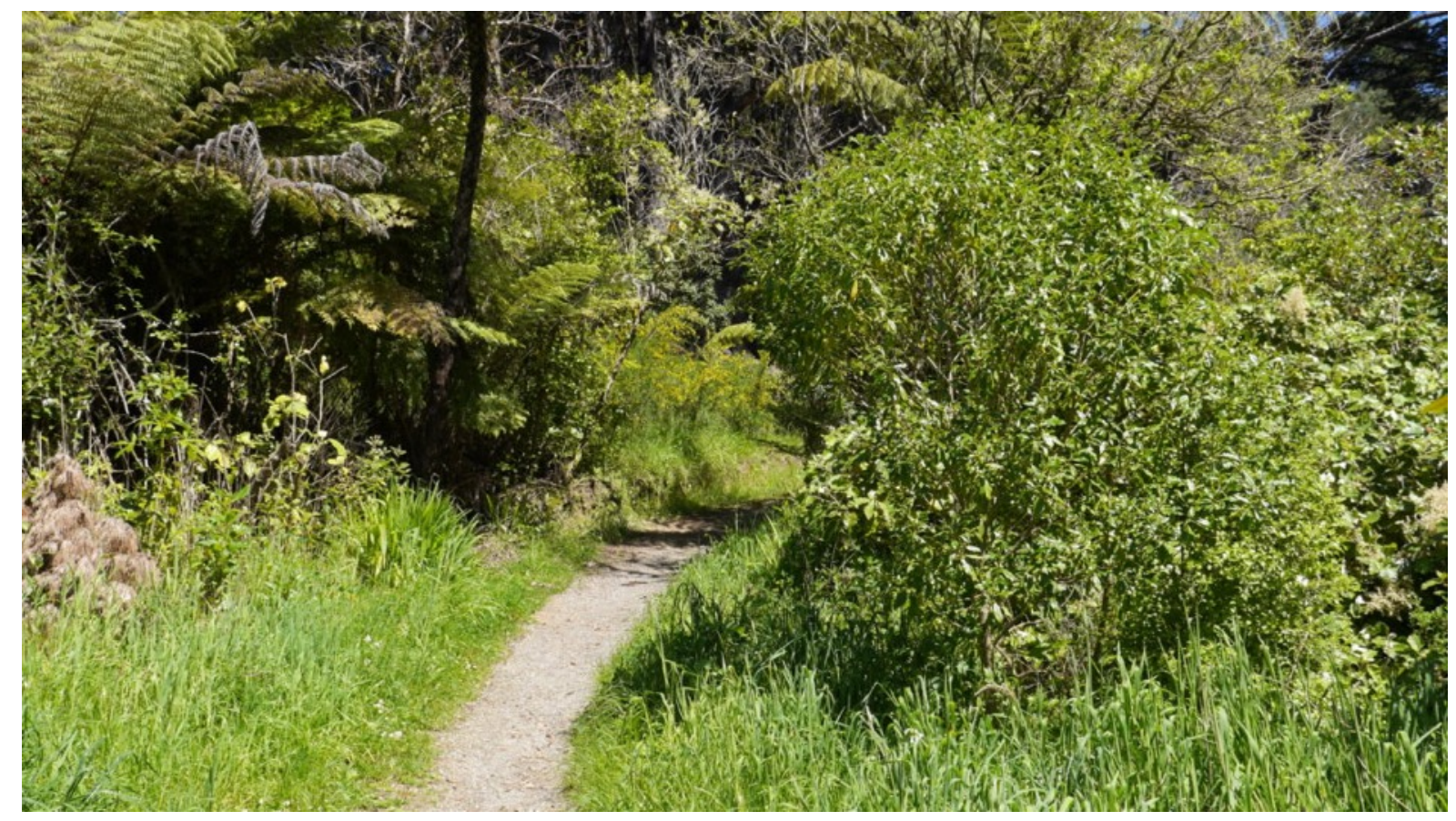

Figure 12. Author. (2016). Following the pathway that leads towards the stream crossing.

I arrive at a bridge that crosses the stream. The stream is accessible from here-I would need to duck to walk underneath the bridge, but I choose to walk back up and use the more accessible way on the other side of the bridge. 


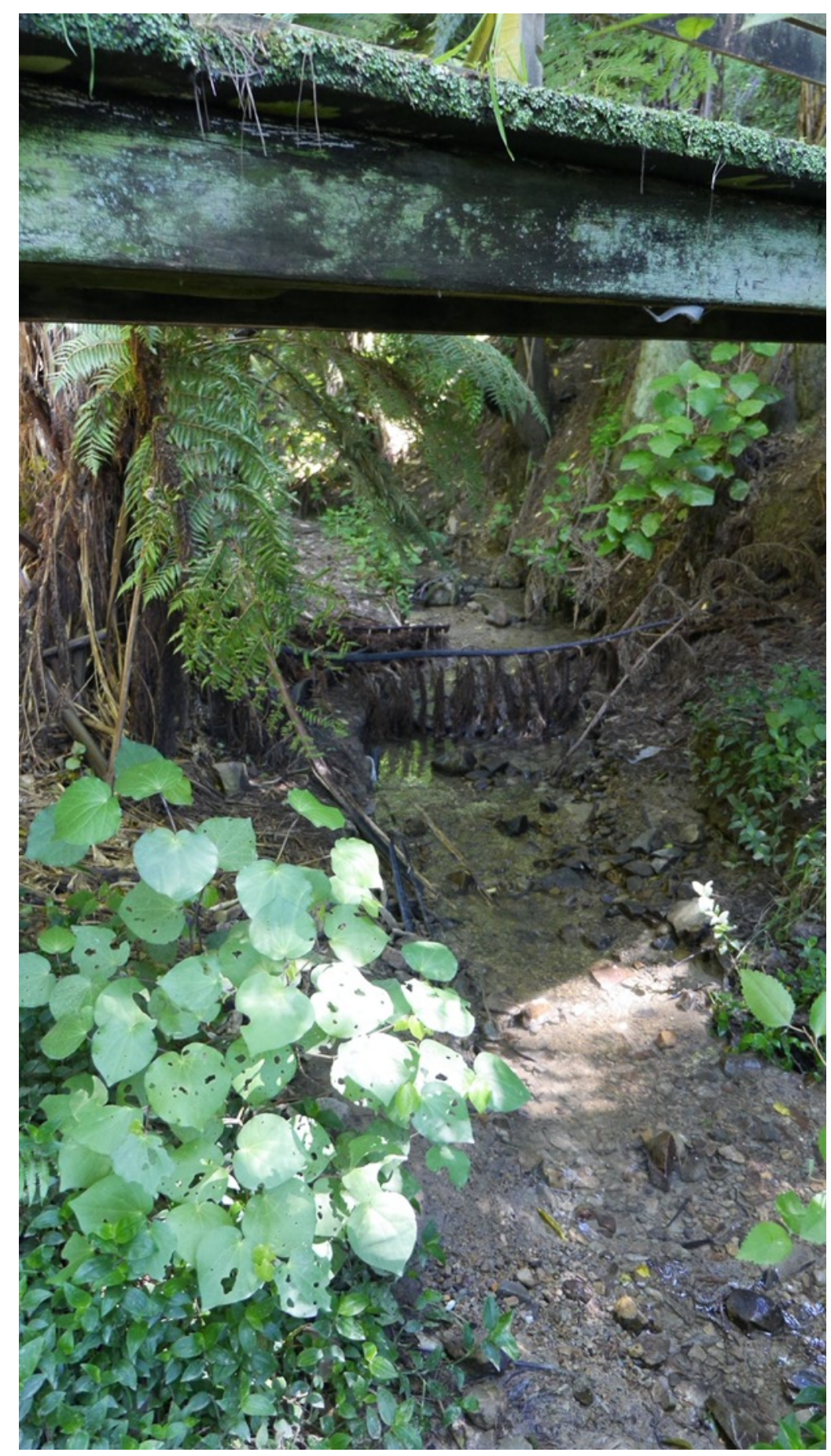

Figure 13. Author. (2016). Observing the streambed just under the bridge.

The bridge would lead me to a paved path uphill, leading up to Dorking Road, Brooklyn. I, however, head south-west, following the stream on an unpaved walkway that at times resembles a small gully. 
The stream bed consists of stone and gravel, but I notice some old red bricks, adding colourful highlights to the otherwise merely brown environment.

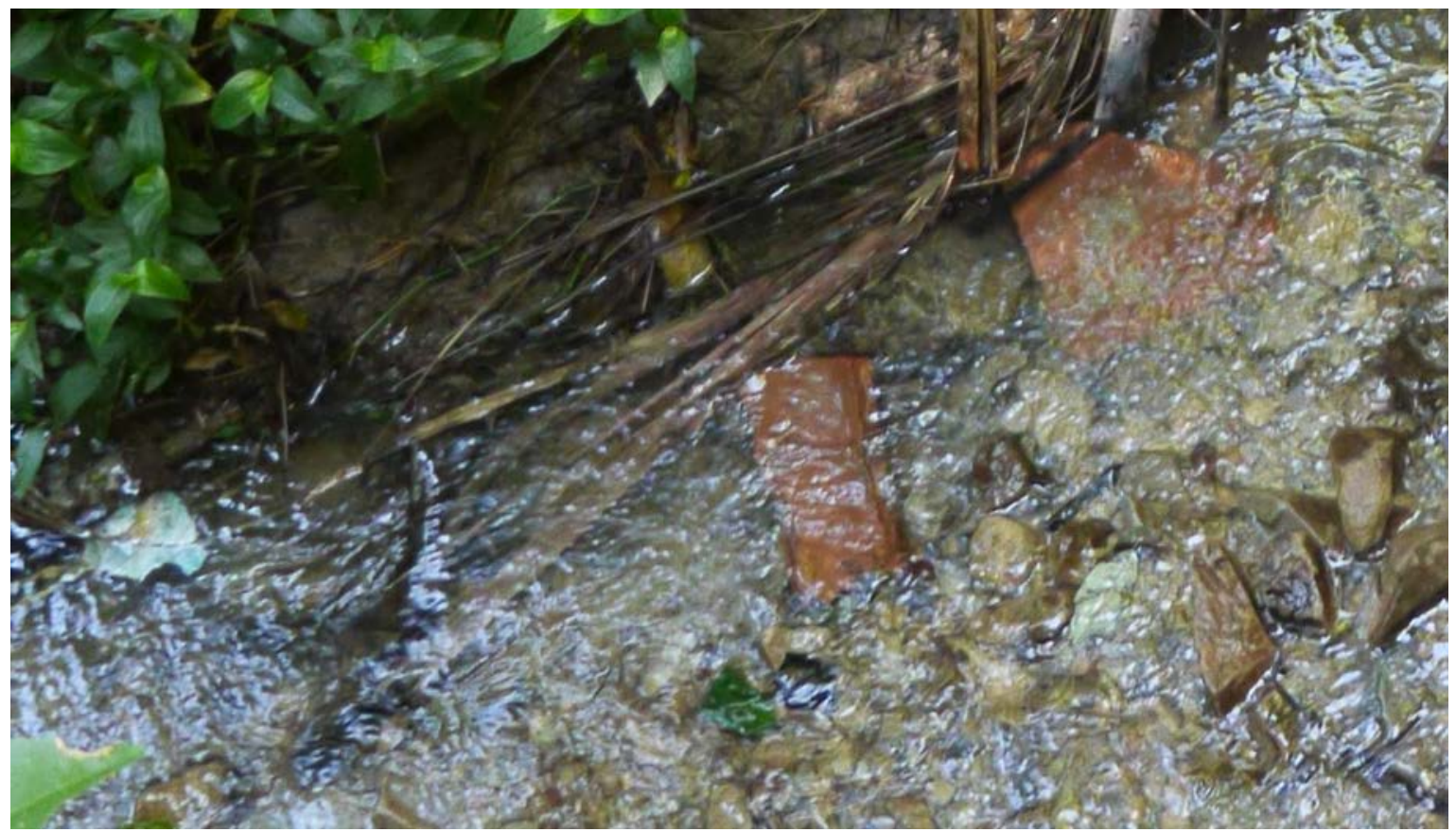

Figure 14. Author. (2016). Rocks in the stream bed are accompanied by red bricks.

Walking further upstream, I hear bird calls and the creaking of trees in the wind. The stream is still visible and audible on the right side of the walking path, flowing a little lower than the walkway. Fallen trees are obstructing the track further up but can be passed to continue the walk.

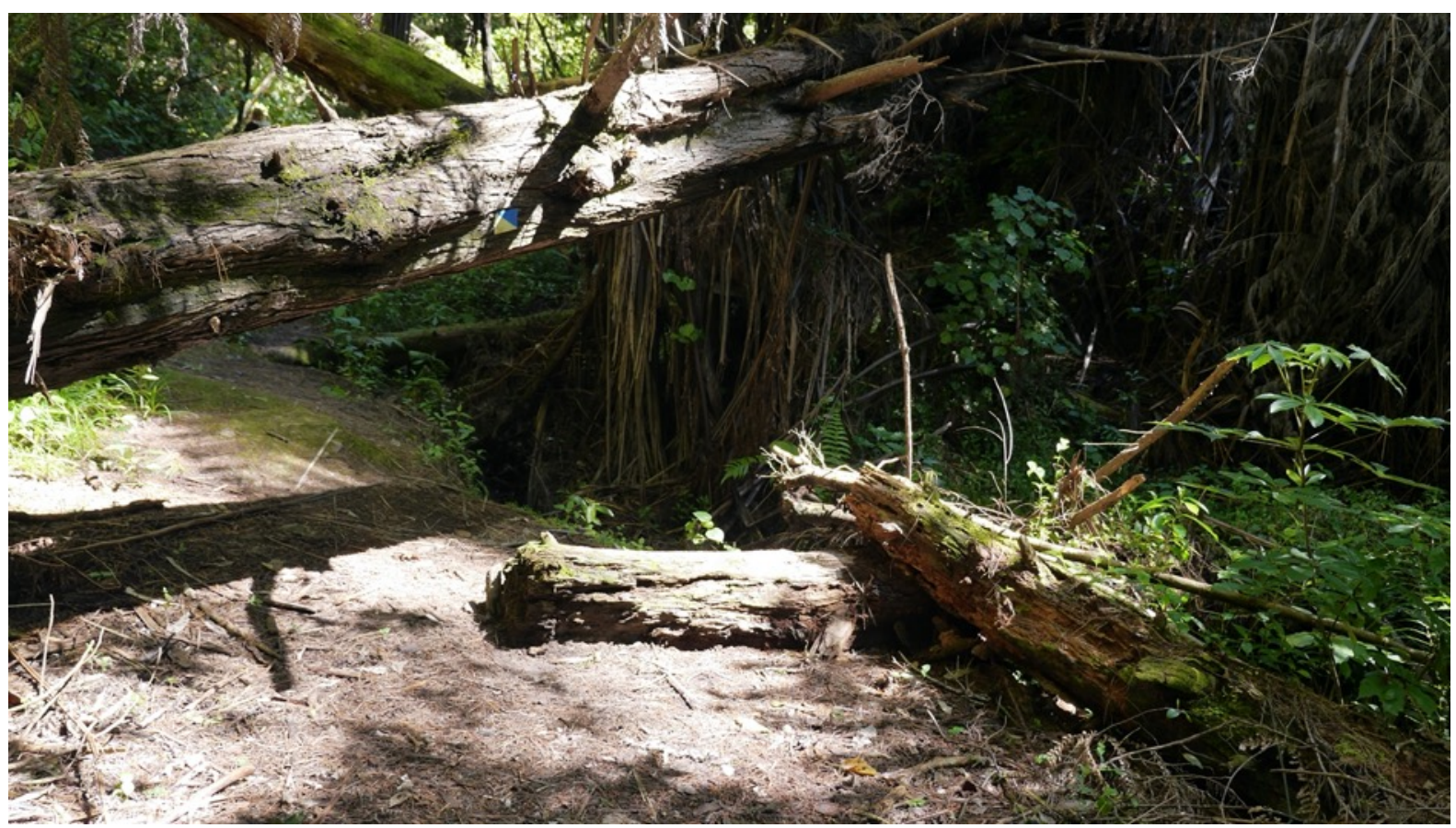

Figure 15. Author. (2016). A fallen-over Pine tree crosses the walking path and the stream. 
The sun is coming through the trees at times and creates nice highlights of the landscape. The path is softly covered with pine needles and lined by green vegetation. I see very tall pine trees and beautiful ponga/tree ferns. The track appears to be getting more rough, narrower and city noises have entirely disappeared from the soundscape. I hear the gurgling of the stream beside me, accompanied by bird song and the sound of the wind brushing through the trees.

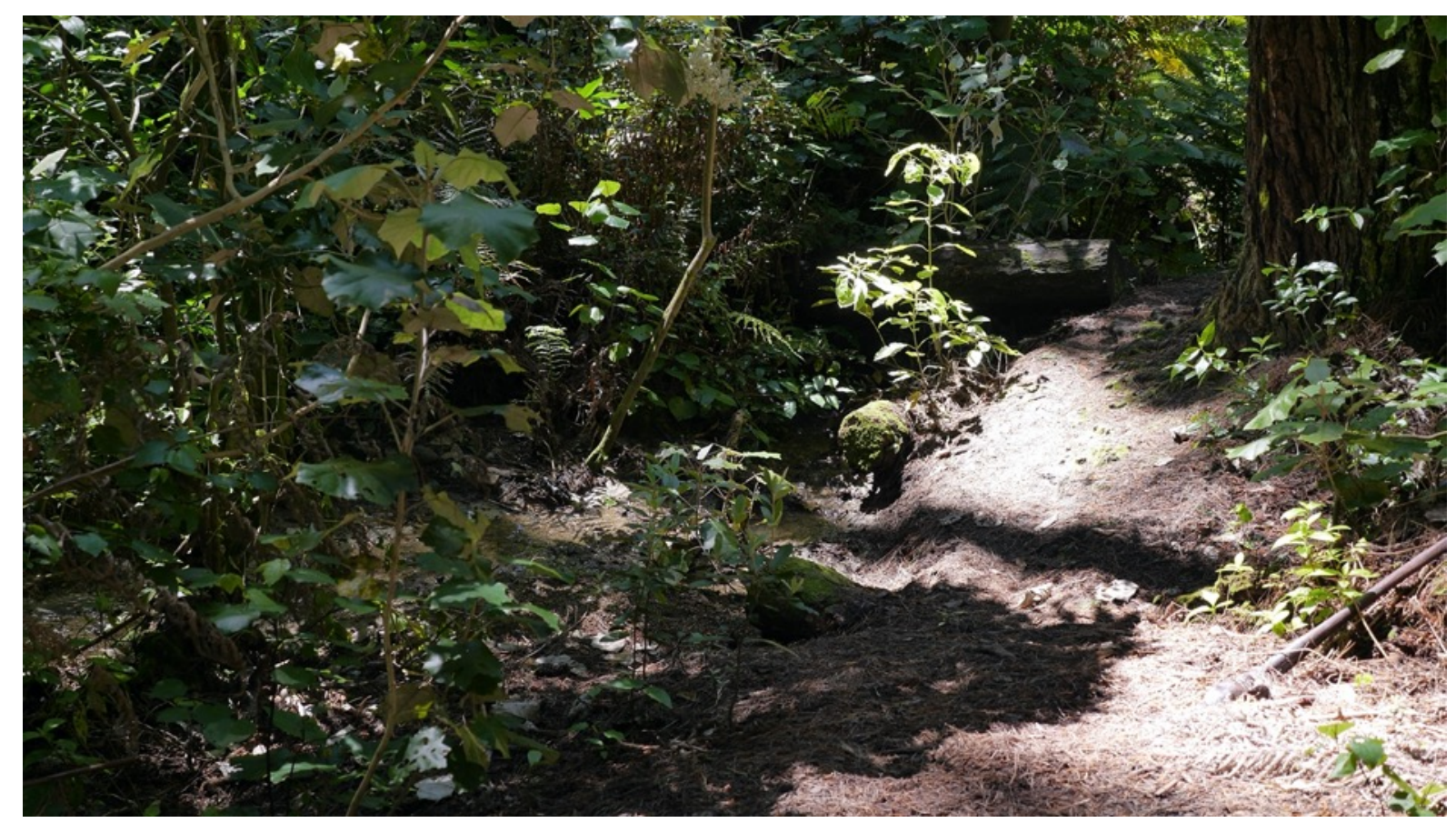

Figure 16. Author. (2016). Looking downstream with the stream on the left and the path on the right.

Walking further uphill, I notice the stream has suddenly disappeared. I head back down a few metres to reach the spot where the flow has died down and blends quietly into a brown boggy patch of forest ground. 


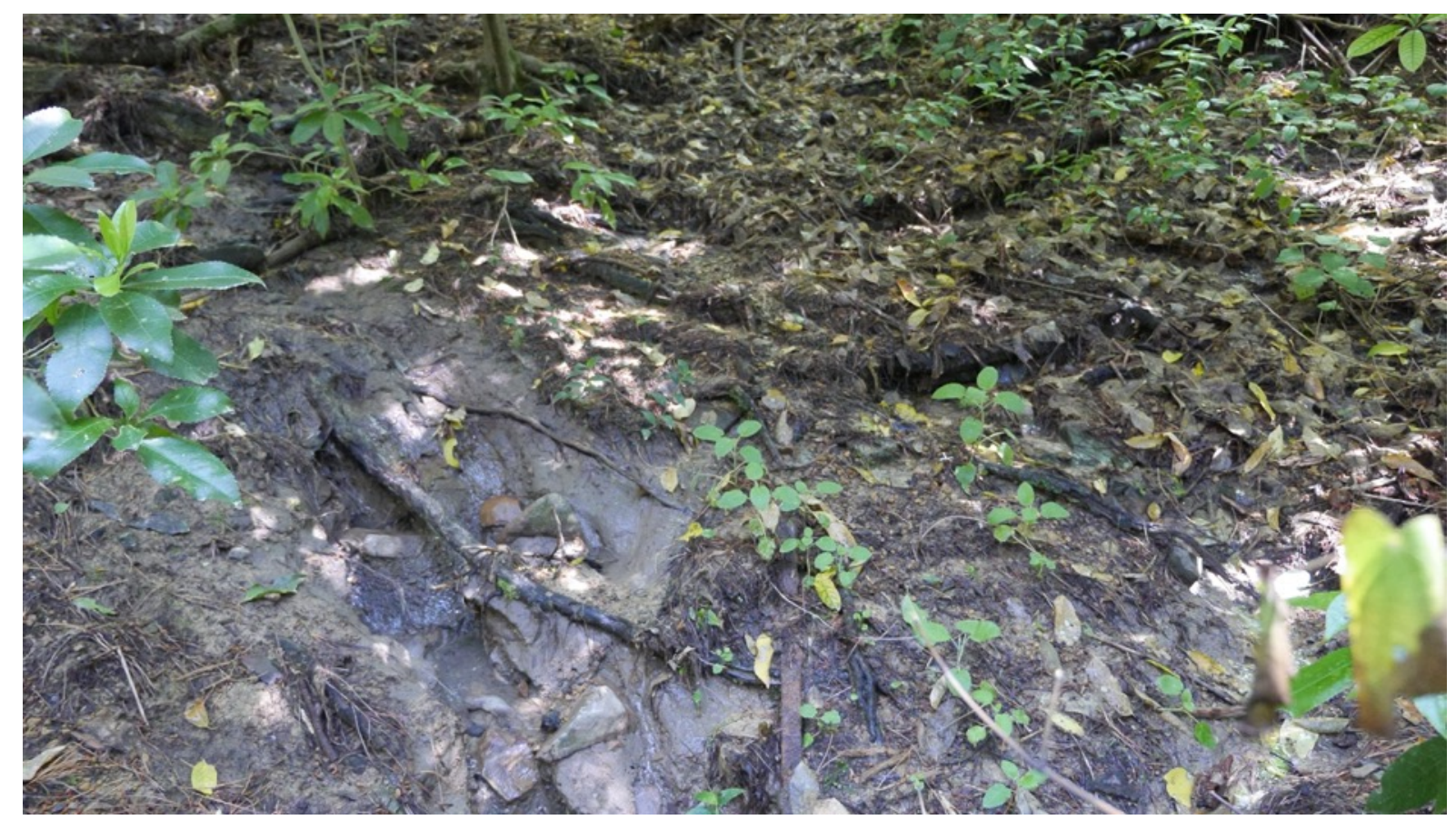

Figure 17. Author. (2016). The streambed is dry and muddy.

By the looks of the area, it seems this part has higher water flows, perhaps during or after heavy rains. The further uphill I walk the more trash I encounter, mainly bottles and plastic packaging often buried halfway in the ground. It appears the garbage is being moved further down as part of a mudslide. 


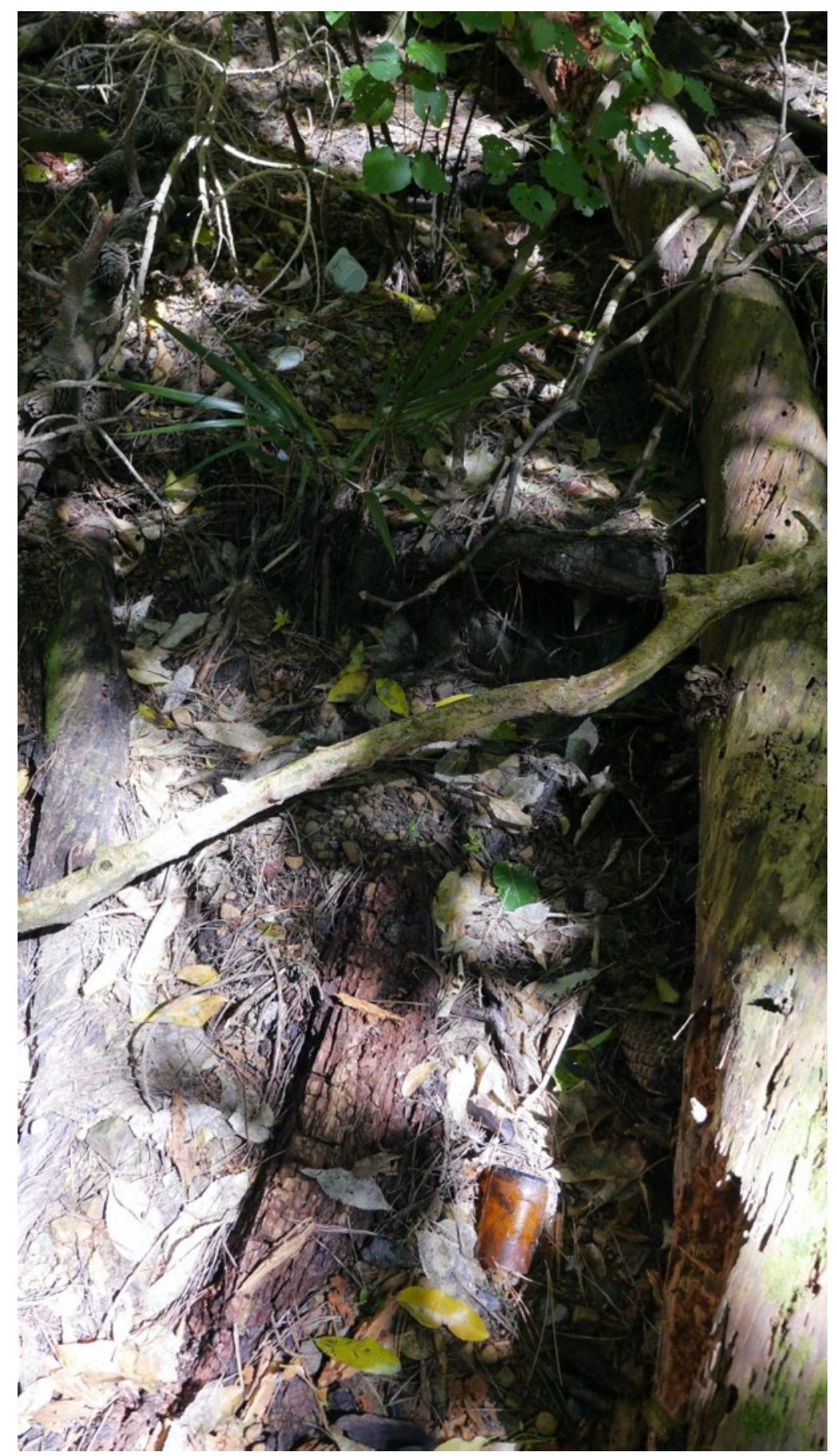

Figure 18. Author. (2016). A bottle sticks out from the dried-out streambed.

The territory is getting steeper, and accessibility starts to become an issue. I need to watch my steps as the ground is very boggy and slippery at times. I hold onto branches of trees for stability. The look of the territory suggests even more strongly that a mudslide has brushed down the area recently, washing down earth and vegetation. 


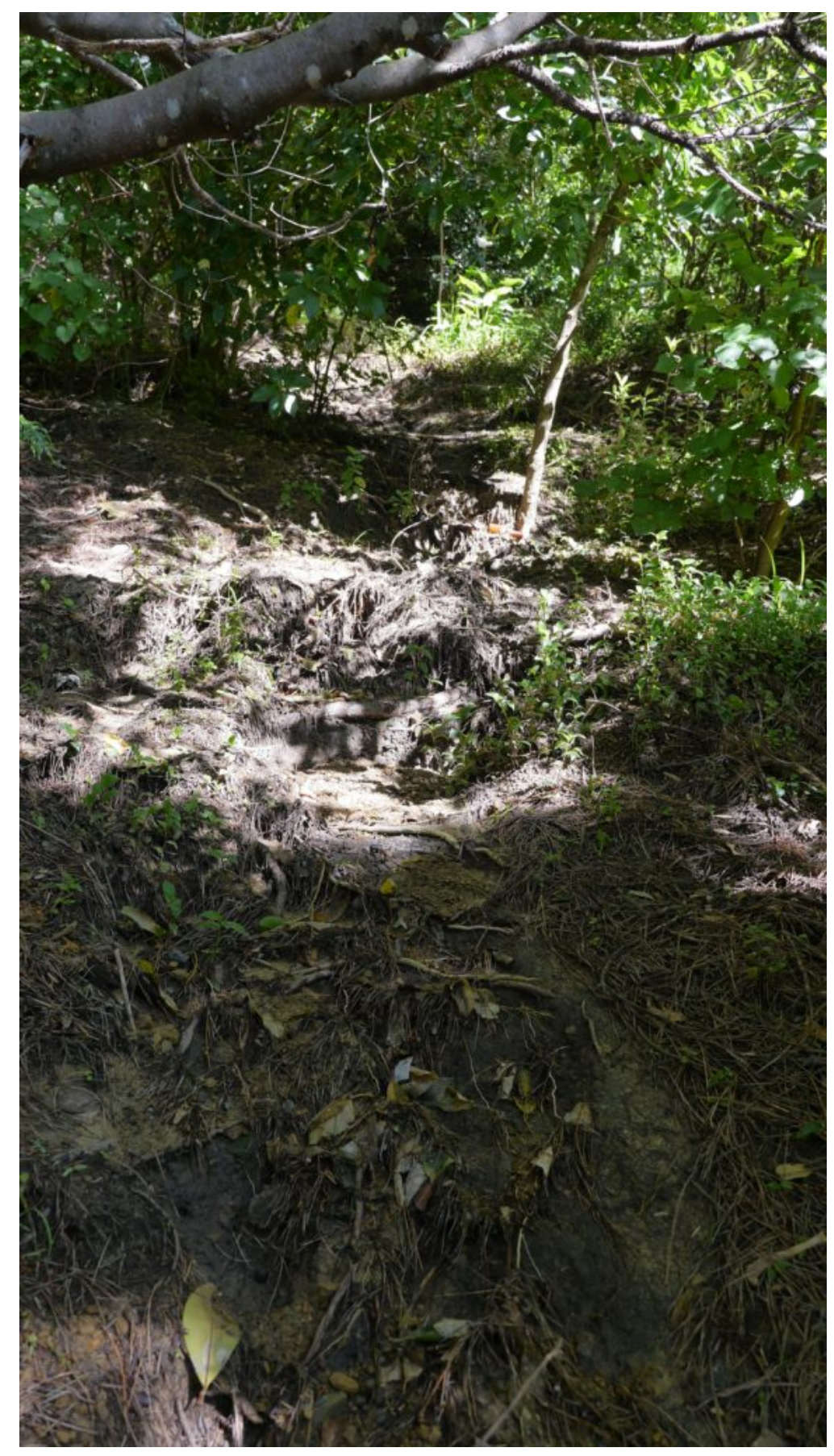

Figure 19. Author. (2016). The course of the waterflow can be read from the flow of the land and vegetation.

I appear to reach the top of the hill as I can see more of the sky again. The boggy ground is littered with brown bottles and red bricks. I need both my hands to hold onto bushes and trees as I carefully crawl uphill so I cannot use my camera to take more photos. 


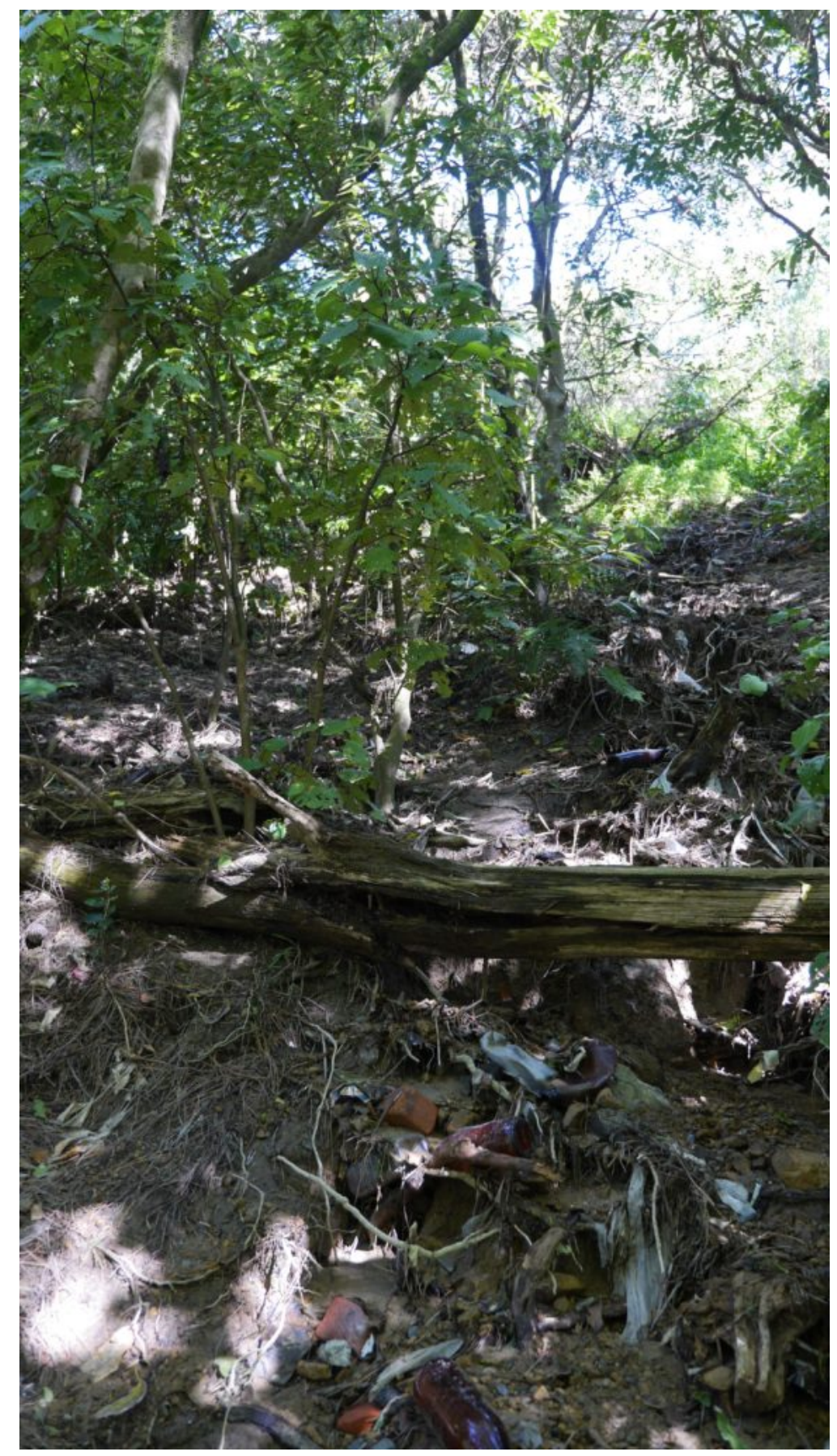

Figure 20. Author. (2016). Further atop the streambed gets steeper and reveals more bricks and old glass rubbish.

I see a pipe and what appears to be an old railroad rail sticking out of the hill. I also see the bottom of a house, and I am not sure if I am on private land. There is no water coming from the pipe. I need to watch my steps, but I manage to reach the top of the hill.

I end up on a street that-as I would later find out-is called Connaught Terrace. I end up coming up and out of the gully just next to the garage of a private property. I look back down into the area I just came out. I mostly see grass and trees. 
I walk back to the street where I find a drain cover that must be part of the network of the pipe leading down to Papawai Stream. It is marked with a red dot. Leaves partly cover the grill. It appears this drain collects rainwater and runoff from Connaught Terrace, which would end up feeding into Papawai Stream.

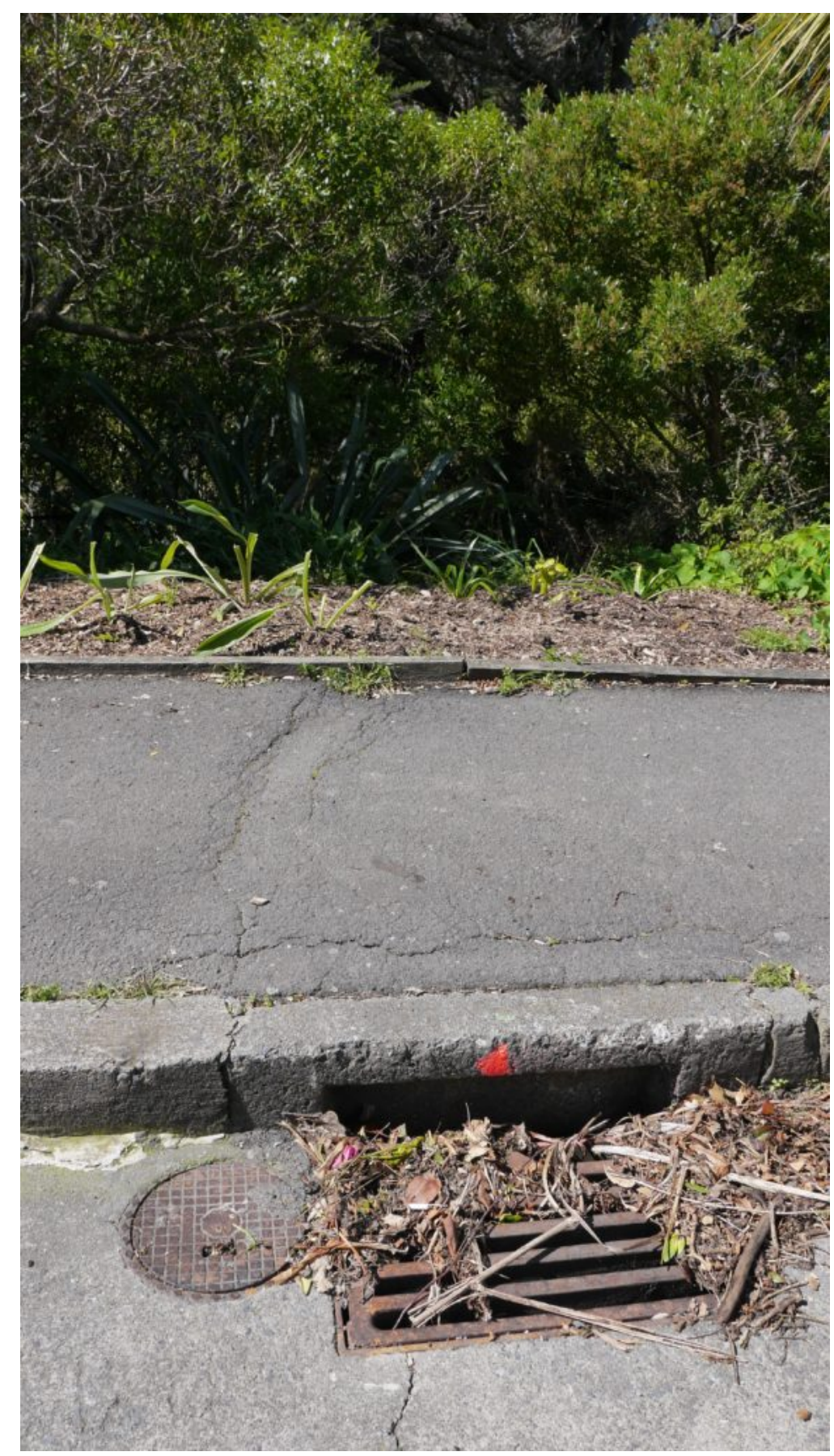

Figure 21. Author. (2016). The drain that appears to connect through a pipe to Papawai Stream.

I notice an official paved path that ends up approximately 10 metres next to where I exit the reserve and decide to take this way back down to Mount Cook. It appears this is the public 
path as marked on Google Maps, connecting the lower playing field of the Prince of Wales Park with Connaught Terrace.

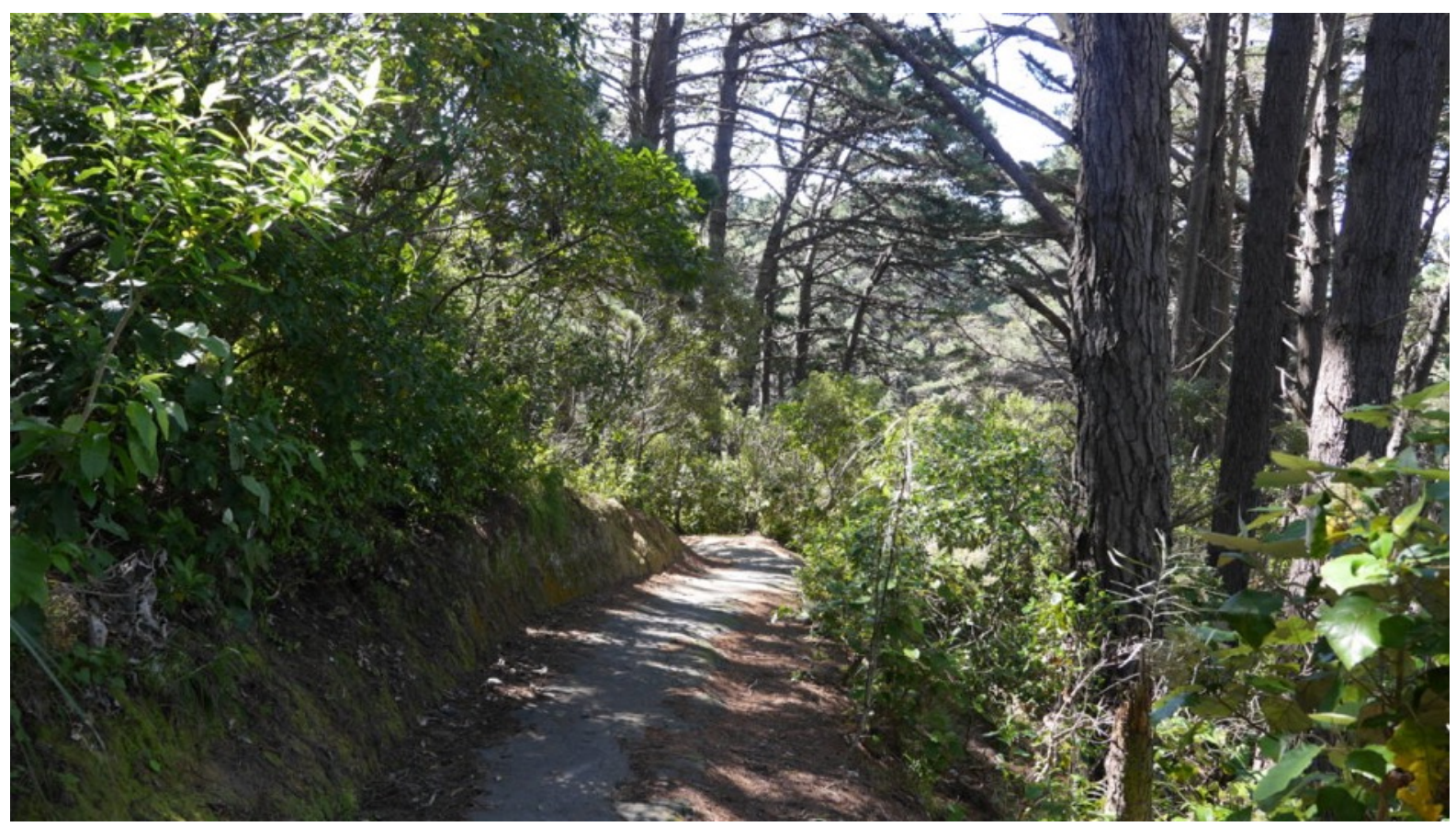

Figure 22. Author. (2016). The official paved walkway downhill connecting Brooklyn and Mount Cook.

The above photo shows the paved walkway downhill, which is very different from the path I chose for my uphill adventure. Papawai Stream must be somewhere in a parallel valley behind the hill on the left side. On the right side, I get a good view through the pine trees to what must be the suburb of Newtown.

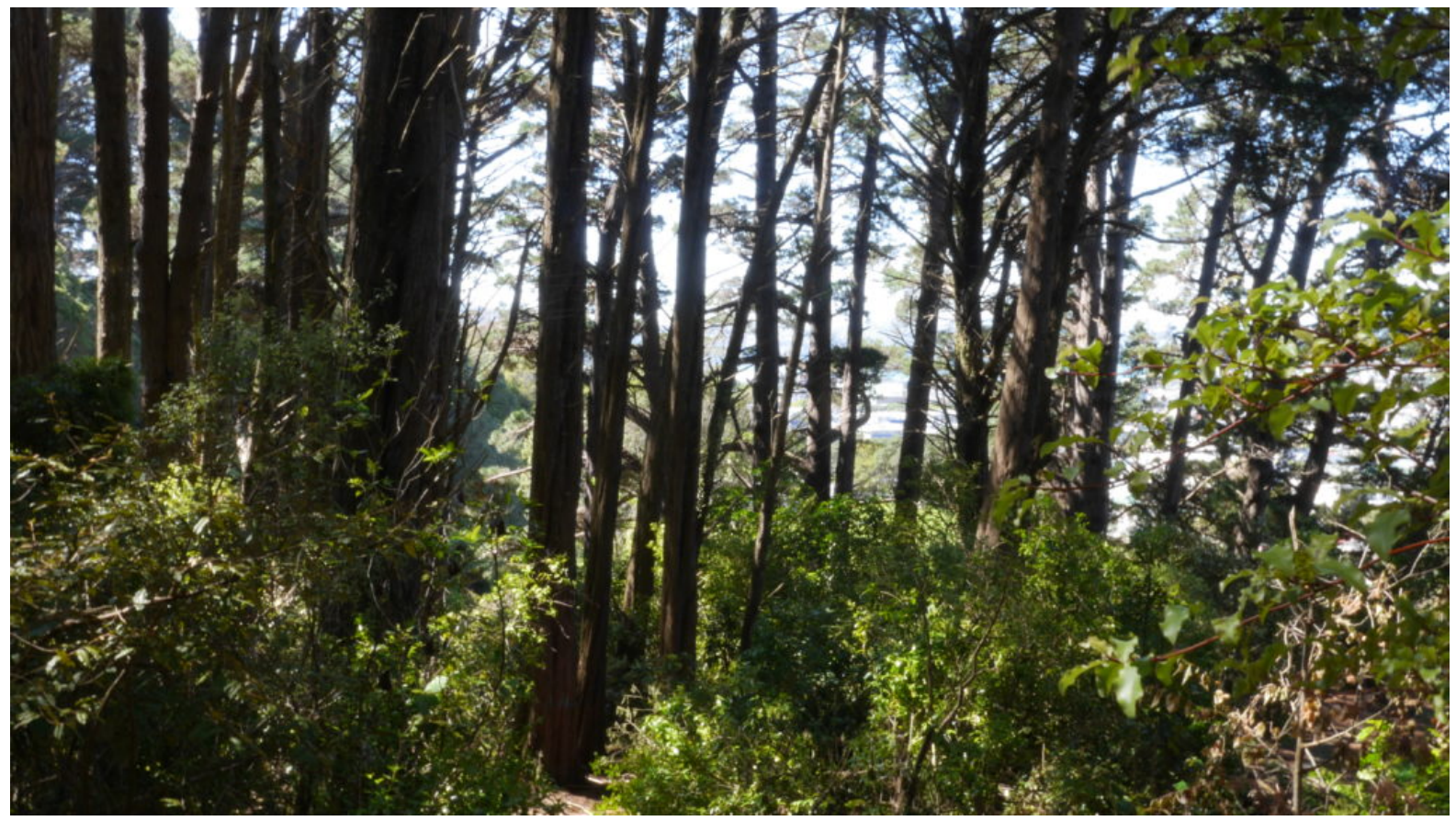

Figure 23. Author. (2016). View from the walkway towards Newtown. 


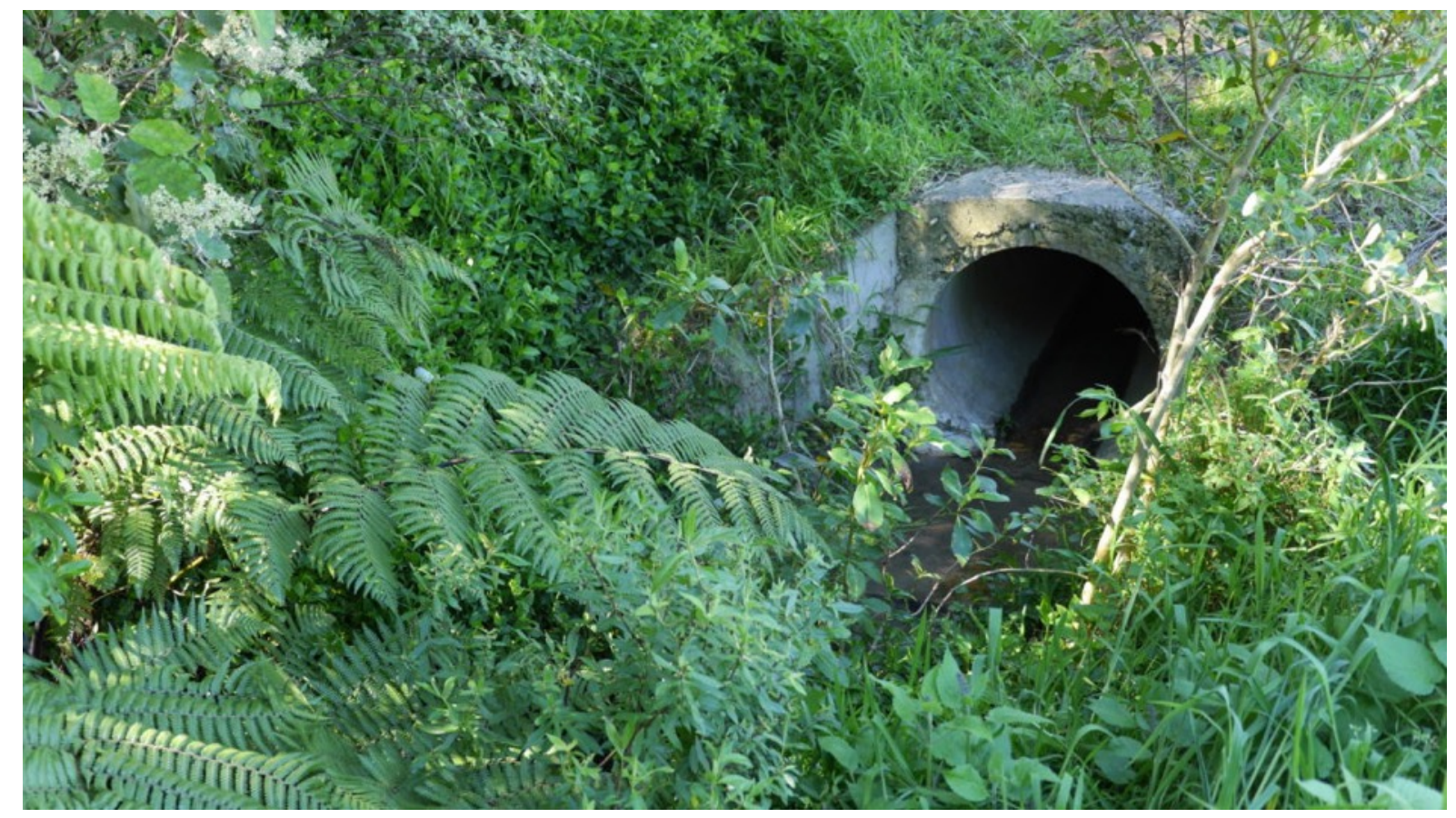

Figure 24. Author. (2016). Stream culvert close to the Prince of Wales Park sports field.

I end up walking back down to the fields, following the stream around the bund and snap another photo of it entering the culvert next to the playing field changing sheds.

Further downstream it appears that there has been some significant event recently, causing washouts and uprooting trees.

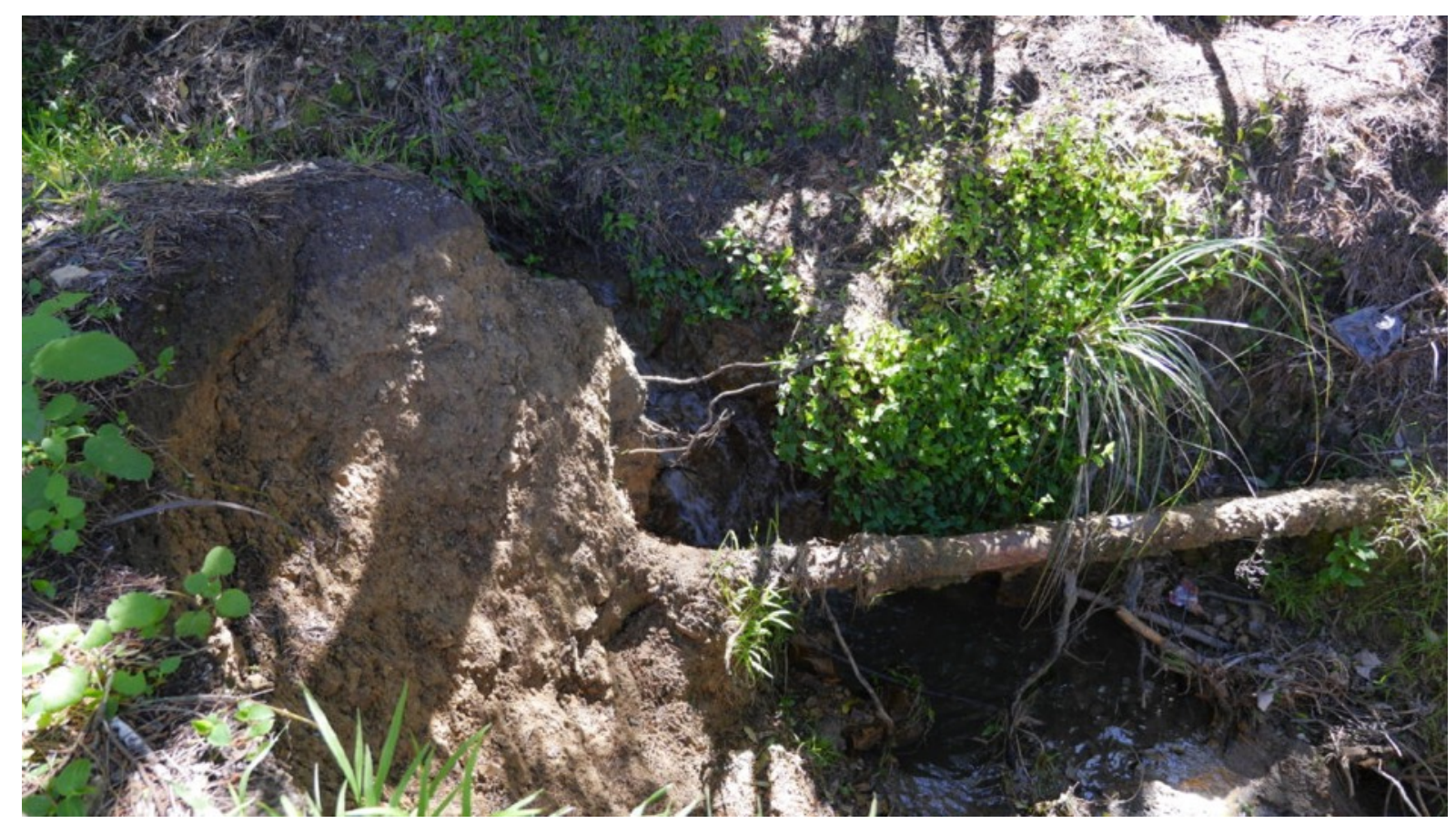

Figure 25. Author. (2016). Streambed erosion by the Park caused the unrooting of a tree. 
The stream is flowing through a canyon that appears to be the result of a washout, possibly after heavy rain. The stream bed is shallow. Vegetation has suffered from slides.

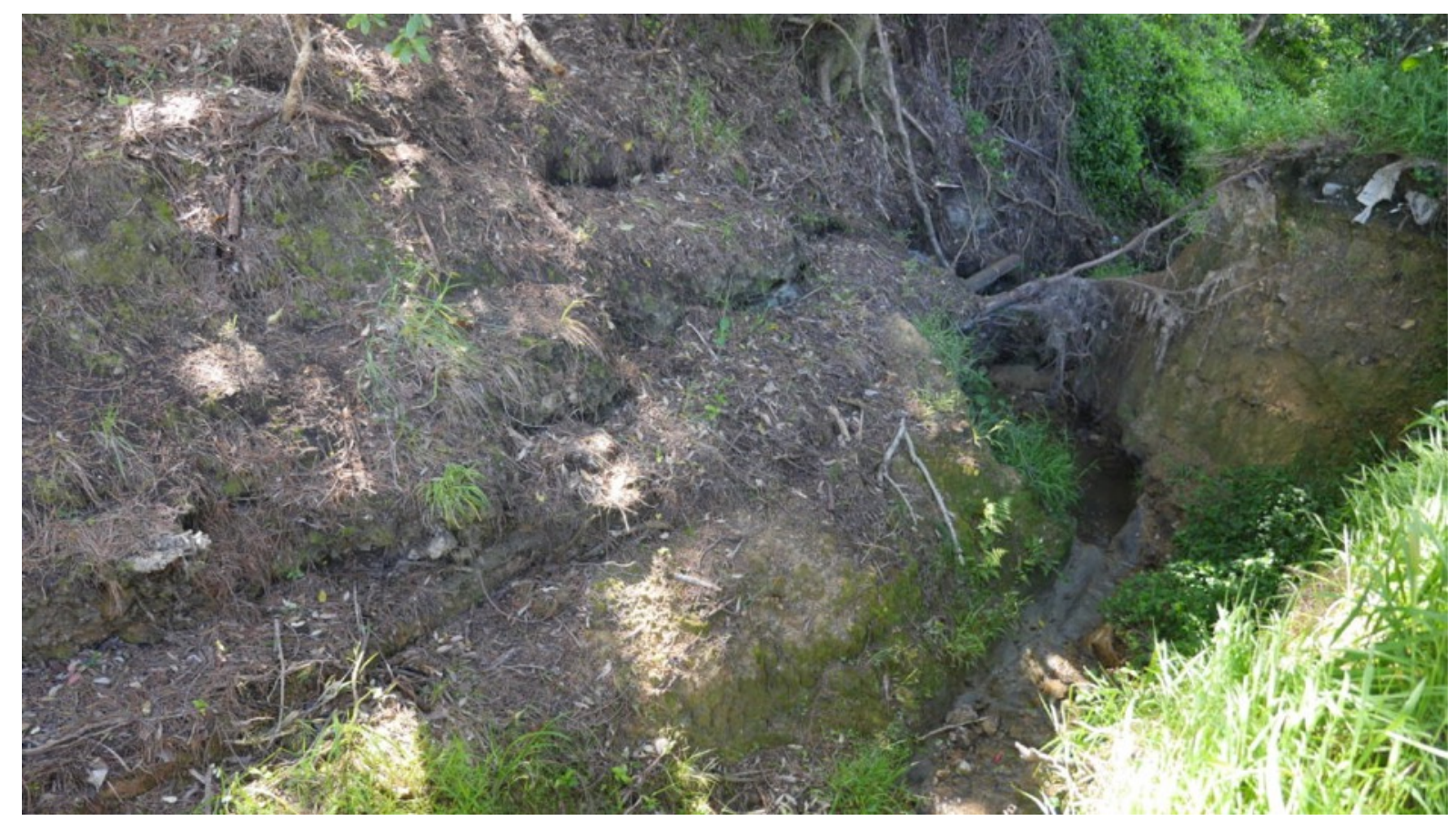

Figure 26. Author. (2016). Streambed erosion created a deep narrow canyon by the Park.

I conclude my walk here having explored some of the more inaccessible parts of this stream. Connaught Terrace seems to be the highest point of the stream, but it is not entirely clear to me where the water is originating from. The area just about where the stream is losing flow has been particularly curious, as it appears that people are frequenting this area despite its inaccessibility. 


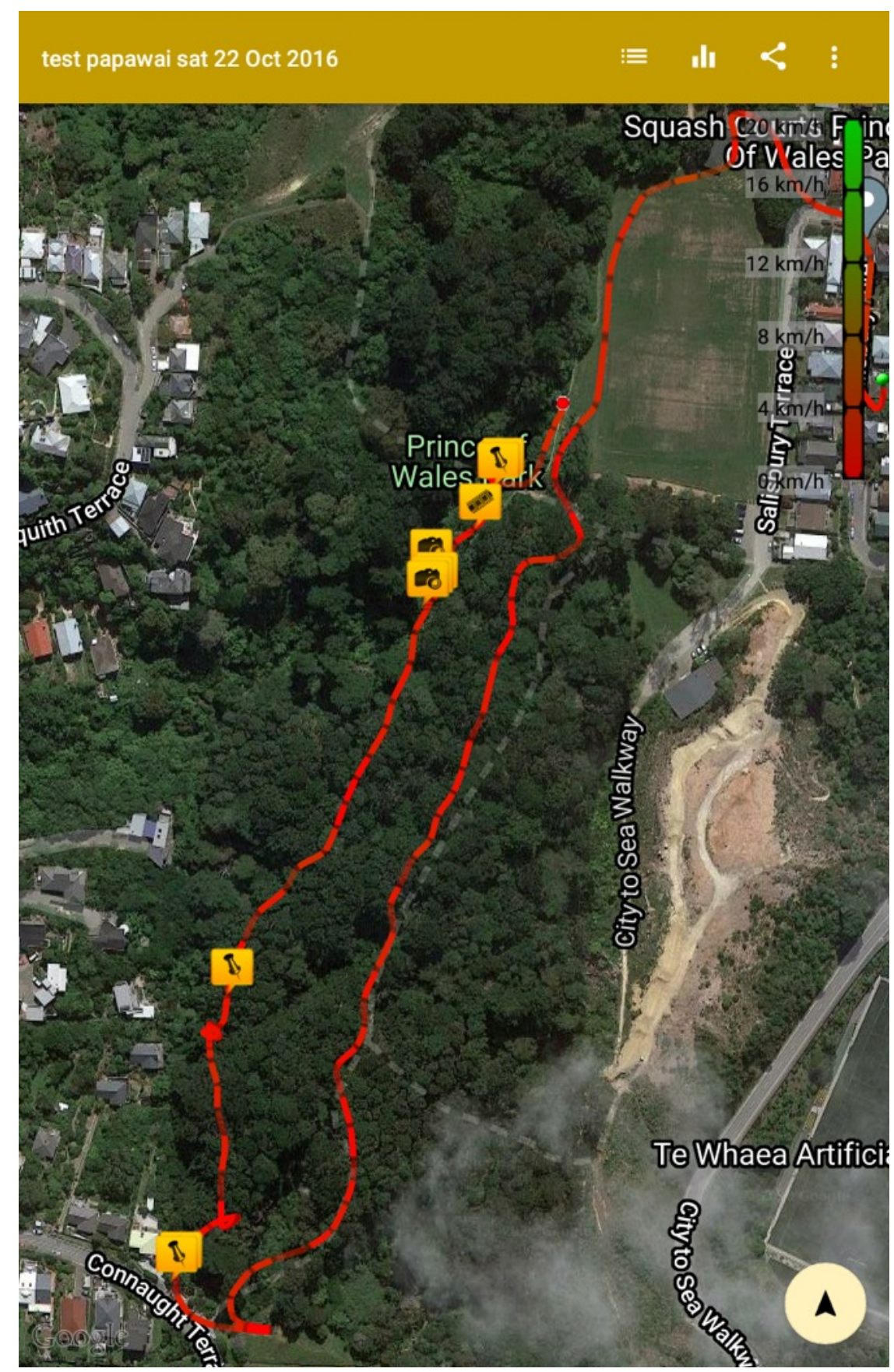

Figure 27. Author. (2016). Screenshot of GPS trail recorded during walk with Open GPS Tracker.

Back at home I look at the data recorded with the tablet and inspect the map I created by walking (see also O'Rourke, 2013). While I could carry my camera with the strap hands-free, around my neck or over my shoulder, the tablet was more unwieldy. While the app records GPS data in the form of a track in the background, I also wanted to add manual annotations, such as written notes, photos, or videos. This required both hands to navigate the interface. The notes ended up being very short descriptions of the area to pinpoint certain waypoints such as "Greggo Troll Bridge" at the departure of the walk or "utterly steep slope" and 
"gutter" reaching the top of the street. The photos and videos are grainy and of low quality in comparison to the photos taken with the photo camera.

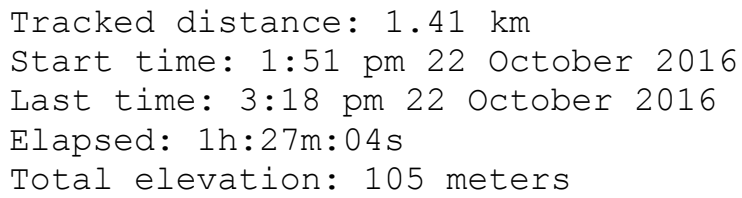

While the GPS might not be accurate, affected by the shape of the land and the thick cover of foliage, the trail (seen in Figure 27 departing from the red dot by Prince of Wales Park) gives an approximation of the accessible part of the stream from the park up to the pipe on Connaught Terrace in Brooklyn. 


\subsection{Exploring Papawai Stream: mapmaking}

After having established Papawai Stream as the site for my research, one of the first fieldwork tasks involved exploring the area surrounding the stream on my own, as there is little documentation of it online. The stream is undocumented on most Wellington Maps (Google, OpenStreetMap, WCC Map). The connected reserve and community garden can only be found online through the sites of the Community Groups (Communityfinder.org.nz, MtCookMobilised.pbworks.com). A web search on the stream first reveals that there is another Papawai Stream located in the Wairarapa. Looking more specifically for "Papawai Stream, Wellington" returns articles related to the restoration group, news about recent flooding (Seaman, 2015) and more recently, information regarding the planned Omāroro/Prince of Wales reservoir. Burnett (2013) published a report on mapping Wellington's unpiped streams and recommended for future mapping projects of Wellington streams the knowledge of community groups should be tapped into generously. (Burnett, 2013, p.1). The fieldwork of Burnett's project involved on-site mapping with handheld GPS devices walking the length of the stream (p.10). The maps created in this project gave a first overview of the forks and tributaries of Papawai Stream.

Online and archival research was conducted to estimate the locations of piped streams and to relate the present-day underground course of the stream to the original course. The Wellington City Council Stormwater Pipe spatial dataset (Wellington City Council, 2015) gave a first indication of how the course of Papawai Stream continues to the harbour once it disappears in the stormwater network at Papawai Reserve. A first visual analysis of the dataset in the spatial analysis software QGIS gave a rough idea of the course of the stream. Most pipes are aligned to streets and urban developments while the pipes departing from the Scruffy Dome have a distinctively organic shape and thwart the prevailing rectangular structure of the network. A later acquired Historic Streams Dataset showing the 1936 course of Wellington streams confirms the initial heuristic assessment of the stormwater dataset.

This section describes an exploration of a dataset that I acquired mid-2017 containing location data of Wellington's streams from the year 1936 via e-mail from Wellington City 
Council. Having access to this historical spatial data prompted an exploration on understanding where those original streams are located in relation to Wellington's geography now. Combining this data with open datasets of current property addresses available online, I generated a new dataset that contains all addresses that are located on historical stream land. This section describes a quick write-up on how I generated the new data from multiple maps. As opposed to explorations of the stream through fieldwork, this process gives me access to a top-down view on the streams, which I thought could be useful for exploring clues to stream locations in more urban settings.

The dataset of the historical Wellington stream data from 1936 is formatted as a GPS database file (.gdb). I open the file using the software QGIS, a free and open-source geographic information system. To be specific, I am using QGIS 16.2.3 Mac OSX. By using spatial tools, I want to know the current addresses where the stream layer crosses a property. 
To achieve this task, I have downloaded both an open property dataset and an address dataset, which I then placed on top of an Open Street Map layer.

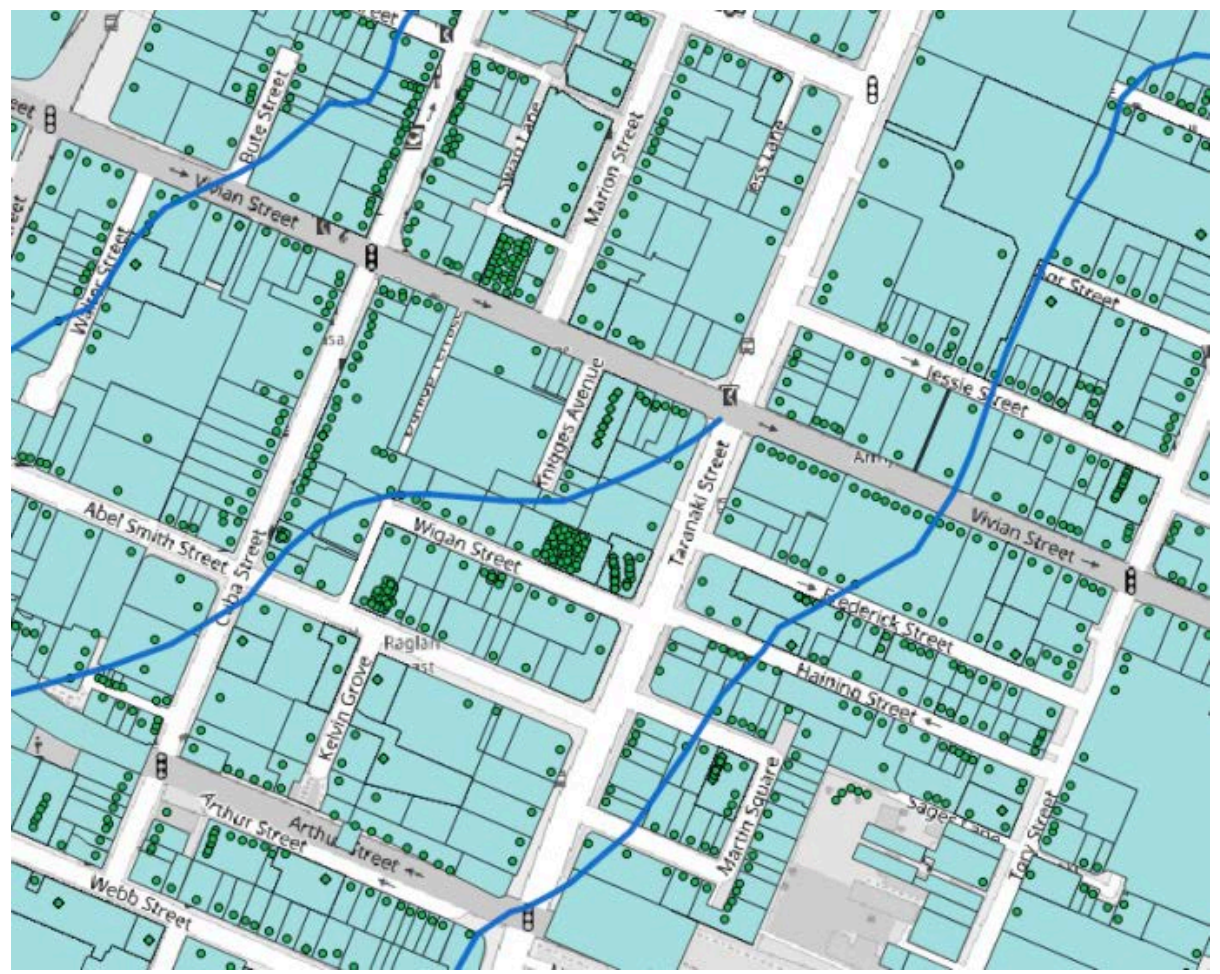

Figure 28. Author. (2016). Screenshot of QGIS with the following map layers:

OSM Map tiles

NZ property titles .shp - teal (downloaded from LINZ)

NZ street address (2) .shp - green (downloaded, LINZ)

Streams $1936 . g d b$ - blue (acquired via WCC).

To create a table that contains all addresses of properties that intersect with one of the streams, I use a two-step process:

First, identify all properties that overlap with a Stream feature.

Then, identify the address(es) of these properties and export them in a somewhat readable form (.csv). For the result to be correct, I need to make sure that the projections of all spatial datasets match.

The following three steps are necessary to create a list of addresses:

1. Spatial Query: Intersect the properties with the streams.

2. Spatial Query: Select the addresses that are within a selected property.

3. Export the final selection as CSV

I select the feature attribute full_add_2 of NZ-street-address -2 , which gives me the format of the address I want in my data output (e.g. 2/11 Arlington Street, Mount Cook, Wellington). 
The exported table contains exactly 2716 addresses in Wellington whose properties are built on a location which was historically crossed by one of the original streams of Wellington, and published in the project repository (irgbit, 2017).

Because the tabular output is only useful for someone who might be interested in further processing the data but might appear hard to digest for a lay user, I experimented with creating a map that contains the addresses as a layer on top of the map.

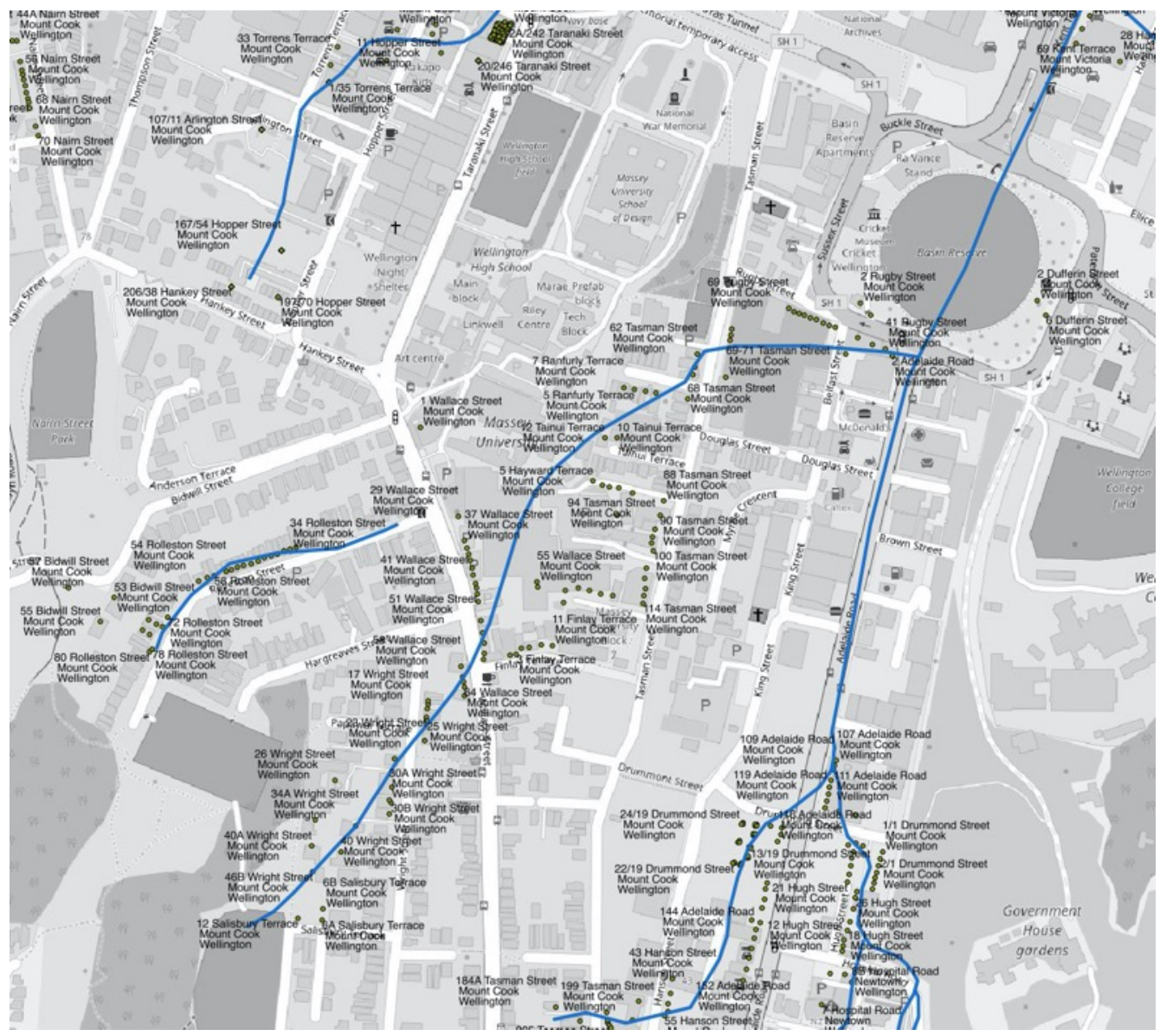

Figure 29. Author. (2016). Screenshot of the stream addresses layered on top of Open Street Map.

This view does make it easier to find properties and specific addresses, but it overall appears quite cluttered, especially as addresses in areas with high property density are hard to read. In an attempt to create a more abstract visualisation of the resulting data, I removed the base map layer and only showed the property outlines that intersect with a stream. 


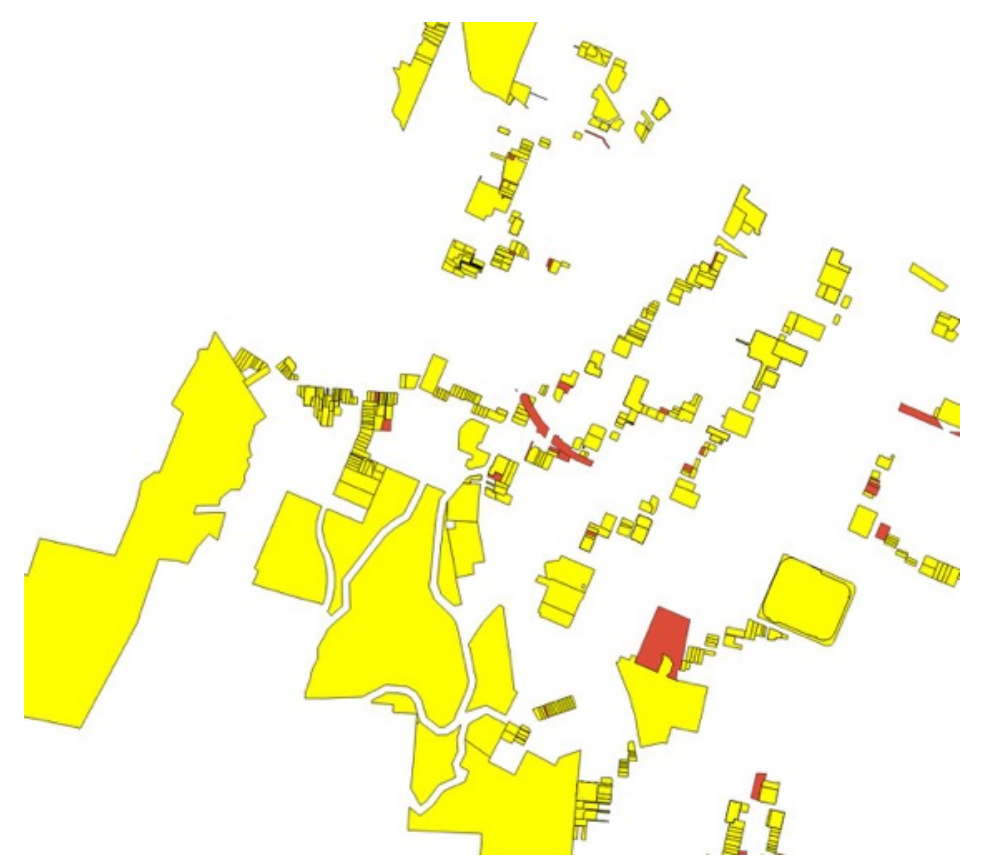

Figure 30. Author. (2016). Outlines of properties in Wellington crossing territory of original streams.

This output (Figure 30) is visually interesting because it might not be recognised as a map at first glance, but it moves too far away from the original goal. It does, however, show, that there are many properties across town that are built on the location of a historical stream and might spark the interest of residents who are interested to learn more about the history of the stream they are living on.

Figure 31 shows an overlay of the Historic Streams Dataset 1936 (blue) with the selection of the Wellington City Council Stormwater Dataset 2015 on an Open Street Map of Wellington City (greyscale). 


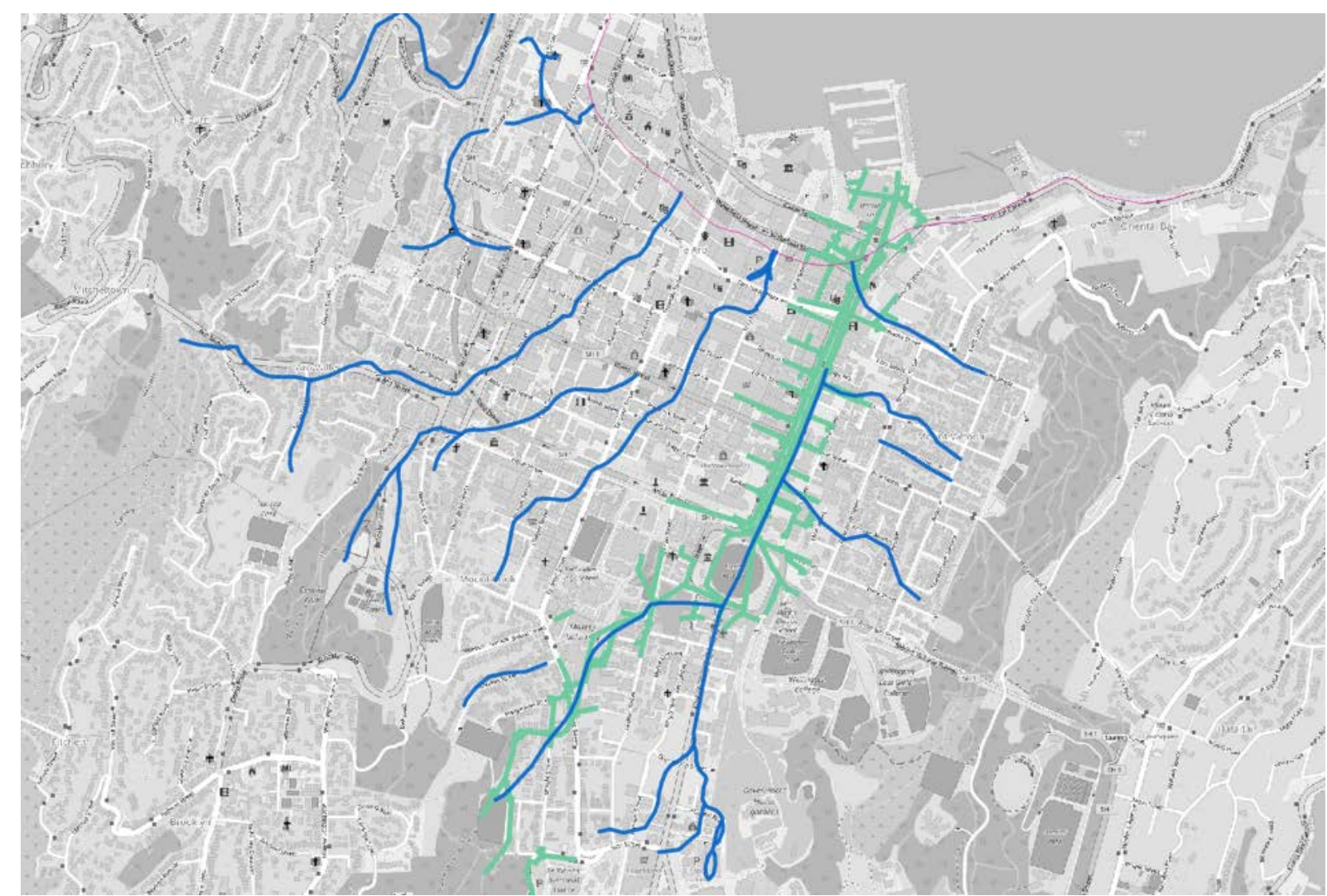

Figure 31. Author. (2016). Historical Papawai Stream and surrounding Wellington Stormwater Network today.

First experiments with recording audiovisual material alongside GPS data guided my research towards literature on the concept of "geo-narratives" (Kwan \& Ding, 2008) or "spatial transcripts" (Jones \& Evans, 2012), in which spoken words and location are “accurately connected” (Jones \& Evans, 2012, p.851). After having decided to do mobile interviews and record additional data from sensors during walks with participants, I wanted to find a way of representing that data accurately on a map. The intention was to have a clear overview of spoken words, measurements made, background noises, observations, weather, stream temperature etc. These hours researching open-source software solutions and attempting to create a geotagged video, a geotagged transcript of audio and installing software packages, libraries, experimenting with abandoned old code repositories, and contacting developers about year-old software, did not yield the expected results. However, in hindsight, I realised how this kind of data representation would only serve as a form of prettified data analysis and force the observations and experiences I collected into one single format. Regarding ethical implications, the data collected during interviews is confidential to my supervisors and myself, so such a representation would have possibly aided the ability to give a unique, innovative overview of the collected data, 
but would have been dictated by the possibilities and limitations of a given software, ethical permissions, and not necessarily aided my pursuit in learning from more-than-human networks.

As a deviation, however, my research into geotagged amalgamations of data was useful for the development of the idea of a "Stream Diary" together with the "Friends of Papawai Stream Community".

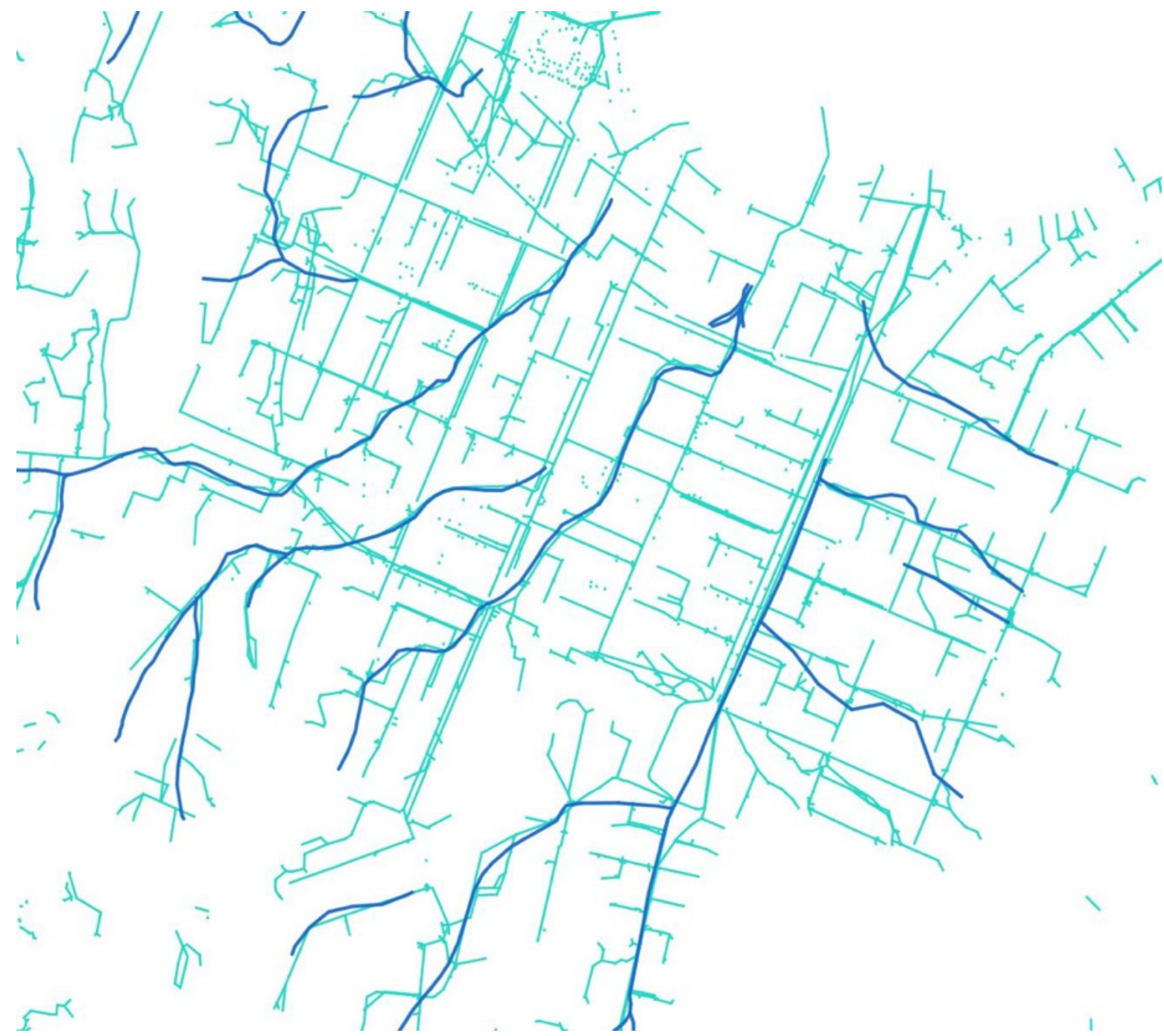

Figure 32. Author. (2016). Map of Wellington's stormwater network (teal) and the original streams (blue). 
To collaboratively map and explore a part of the stream not accessible by official pathways, I joined a small group of core members on one of their surveying walks. After already having done formal walks with the involved community members as participants, I now found myself in the role of participant as well as explorer and advisor. The group members have had a long interest in monitoring the stream themselves and have taken advice from experts such as engineers and ecologists onboard in their decision making. In my role as an artist and design researcher, I could not give the group any scientific advice but offered to discuss options on documenting the stream over time through geotagged images. In a quick exchange during one of the informal walks, I helped a member with the location services settings on their phone to enable geotagging of photos they took along the walk. Initially, the group brought a notebook to the walk to record any observations with pen and paper. It soon turned out that recording data with pen and paper while hiking through steep muddy terrain proved unwieldy. The notes taken during the first walk turned out to be hard to read, and locations of observations could only be described vaguely. Since the members were equipped with smartphones, we discussed possible more convenient methods to record observations, e.g., with a mobile phone or tablet and tagging notes and images with GPS location. After I got access to the geotagged photos taken by one member on their mobile phone during the first walk, I provided the group with a map that featured the location of the walked tributary of the stream as well as the photos that were taken during the hike, placed on location. 


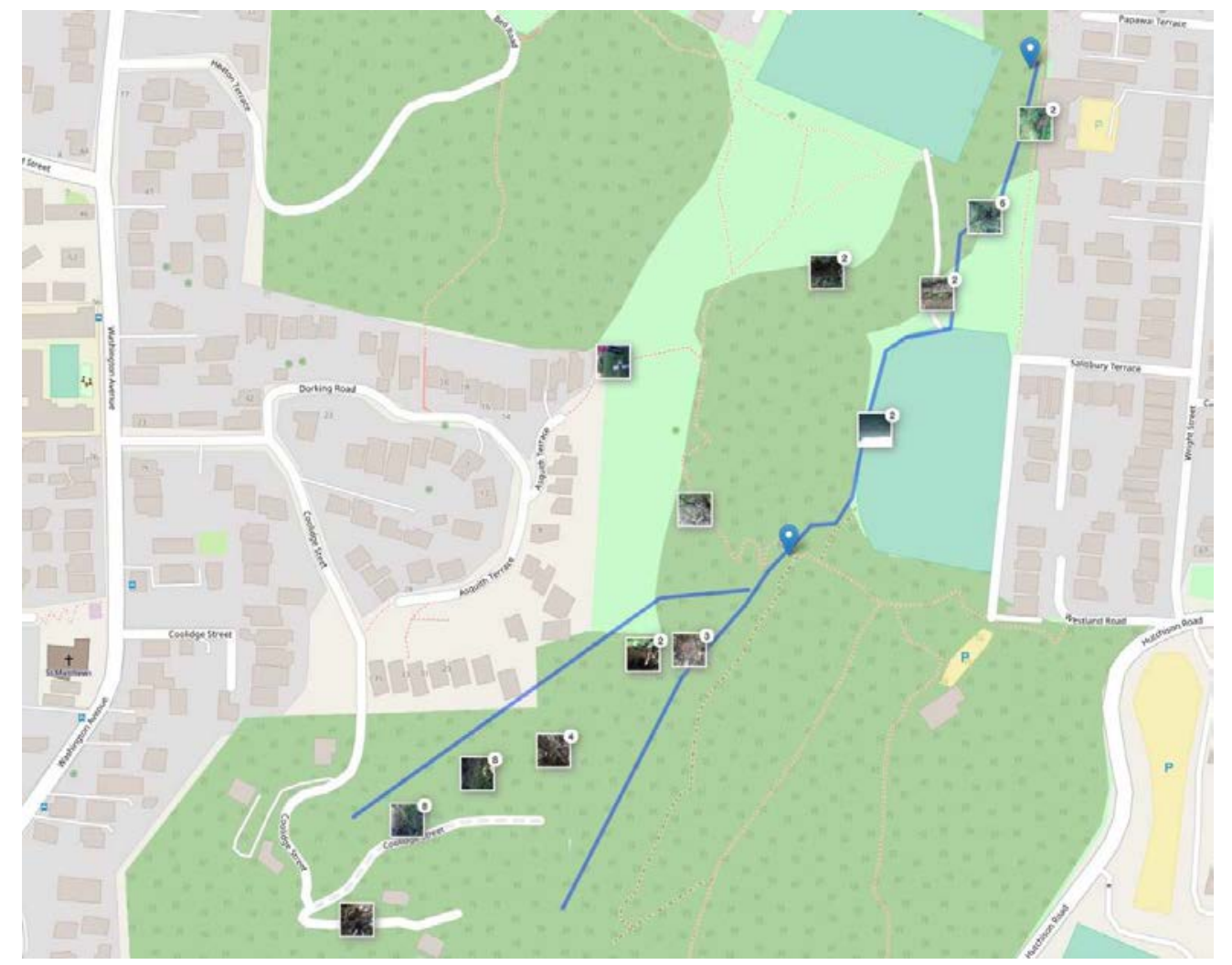

Figure 33. Author. (2016). Screenshot of a prototype of an Open Street Map geo-tagged with photos taken during an exploration of a less accessible part of the stream.

This prototype sparked the group's interest to archive and to map the material they have been collecting about the stream in the past, and I advised them of the possibilities of creating a digital archive of material in the form of a map.

The members informally called the project "The Stream Diary" and I adopted the conceptual and technical development as a design output for this thesis as it aligns well with my research question.

From the perspective of a software design project, the community would be interested in having a map of Papawai Stream and its point of interests. These include man-made structures such as the Scruffy Dome, the Changing Sheds or "Greggo's Bridge" as well as features of the landscape such as the large ponga tree or the stream fork.

The map should show any geotagged photos taken in the reserve. Further desired features included a timeline or filter function to observe and compare the state of points of interests along stream over time. 
In this scenario, humans would act as sensors recording visual, possibly audiovisual data of the stream. To open participation from the wider community spending time in the reserve or the wider town belt, members were open to the idea to install small signposts at locations of interest and invite the public to participate in The Stream Diary by recording an image and submitting it to the archive.

Additional to these signposts, networked nodes of Papawai Transmissions could be installed at points of interest to add sensor data such as electrical conductivity or temperature, which gives an indication of changes of water quality over time. 


\subsection{Walking with: Walkshops in Moturoa Stream, Papawai Stream \& Korokoro Stream}

At the time of the first walkshops I only had partial maps of Papawai Stream and no clear records of its course beyond some of the unpiped sections around the reserve and the council walking tracks. From the outset of the research, I wanted to explore the stream in its entirety, from spring to ocean. Due to the unpiped sections allowing a relatively accessible exploration by foot, I would usually meet participants at the point where the stream disappears underground to flow towards the ocean as part of the city's stormwater network. At Papawai Stream this meeting point would be somewhere between the "Scruffy Dome" at the lower end of Papawai Reserve, or by the picnic lawn at the upper end of Papawai Reserve. Similarly, the meeting point at Moturoa Stream would be by the lower entrance of Central Park, close to where the stream disappears into a culvert.

As I arrive at the agreed meeting place for my first walkshop with a participant (Walk 01), I am nervous not knowing whether I have brought the right amount of technology with me to properly record this walk. The backpack I am carrying is filled with writing and drawing utensils and a clipboard with paper for the planned mapping and ideation activities as well as copies of the information sheet and consent forms, just in case. The action camera is set to video mode and tied to the shoulder strap of the backpack at about chest height. The 7" Samsung Tablet in the outdoor casing in my left hand is meant to record the geodata of our walk so. My right hand is occupied with wielding the dedicated audio recorder. After having briefed the participant (Walk 01) about the rough plan for the workshop, we head towards the stream to a spot they selected for the sensitising activity.

The participant draws a map and talks along while I record our conversation with the audio recorder. After about half an hour the participant (Walk 01) has finished a mostly textual map on one of the A3 sheets. The mapping activity reveals early connections with water through childhood memories of local rivers and more recent engagements through conversation activities, being a longstanding member of the Friends of Papawai Group. 
The early connection with water or through childhood memories was also mentioned by later participants (Walk 02, Walk 03). The engagement with restoration was a common connection across participants during the first exploratory walks (Walk 02-06), mostly due to the fact that participants were contacted via the local restoration communities. The sensitising activity worked well in the first walk but was not as successful in the following walks. In all of the following walkshops, sitting down for a sensitising activity before departing for the walks appeared impractical, for example because participants were eager to go for a walk immediately as they had brought a dog along (Walk 02), or because the walk was prioritised because of time restrictions (Walk $04 \&$ Walk 09) of the participant.

In some cases (Walk 02), it worked out to combine the ecosystem mapping exercise with the ideation session at the end, though it then did not serve as a sensitising session for the entire walkshop but worked out as a conversation starter for the last ideation session.

In the first walk the participant (P01) guides me towards terrain that I was vaguely familiar with due to first tests and solo walks. While we head off, I want to start the GPS recording, but the tablet appears not to be connected to any satellites yet and defaults to a location in Europe. I press record on the action camera strapped to my chest and on my handheld audio recorder.

As I offer the participants to lead the walk, participants engaged with stream restoration are happy to show me forks of the Papawai stream (Walk 01, 02, Walk 11) and Moturoa Stream (Walk 03-06) that I have not encountered before. In one later walk (Walk 09) the participant is not familiar with the terrain, but is invited to lead the walk towards features they are interested in. Focusing on the streams, most participants decide that the walk ends at the point where the stream disappears underground. Having already walked for at least an hour, most participants are happy to conclude the walk at this point and wrap the walkshop up with the ideation session. The walks gave me insights into the features of the stream and the surrounding terrain as well as points of interest that could be suitable for future tests or installs of my networks. 
The ideation session at the end of the walk is designed around questions "How could an Internet of Things give voice to the stream and its environment? What could an Internet of Water look like?”. This is the point of the walkshop in which the conversation directly addresses my design work.

While we sit down to talk through ideation, I offer the participant (Walk 01) some koha in the form of biscuits.

It turns out that water quality or stream health was most frequently mentioned by walkshop participants (Walk 01-06) during the ideation session. More specifically, participants were interested in stream health in relation to care for non-human inhabitants (Walk 01, Walk 02, Walk 03, Walk 05), but also the quality of pūhā/watercress (Walk 01), and the safety for humans to work and play in the stream (Walk 03, Walk 04). Participants also note interest in the presence of fish (Walk 01, Walk 02, Walk 05) in the stream, but also show interest in the wider non-human world such as eels (Walk 01, Walk 05), native birds such as tui or kākā (Walk 02, Walk 06).

With stream health being a common thread across walkshops, and the focus of the installation design directly responding to the walks, Moturoa Transmissions, focusses on measuring stream data in relation to water quality, with dedication to make the installation safe for the more-than-human inhabitants of the stream.

At home I immediately transfer the walk data to my hard disk, starting with the audio recorder. I have recorded five audio files instead of one, because I accidentally stopped recording, when attempting to pause. I note that the cuts in the recording caused by my frequently pausing the device, make it hard to understand the flow of the conversation during transcription. During the first walkshop, I recorded about 2 hours of audio. The geolocation recording appears to have failed. With the interface not being easy to navigate during the walk, it seems I have never properly started tracking, which means I have carried the tablet around without recording any geolocation data. I have about one-hour of 
footage from the action camera. The image quality is reasonable, but quite grainy and shaky from the walking. We mostly see the participant in frame, as I am following them along. Often, they are obscured by my hands holding the audio recorder and the tablet.

In subsequent walkshops, I learn that the audio recording is my key data and I focus on this device, in case other devices inhibit the natural flow of the conversation or walk. I replace the tablet with a GPS watch so I can have that hand free when needing to navigate rough terrain. The continuous GPS data ends up not as essential to my process as envisioned in the beginning of the research, as contextual data to discuss locations with participants (e.g. "where the wastewater pipe crosses the stream" or "at the beginning of the bund") are more useful as location data than a continuous set of GPS markers (see Figure 8) when communicating with participants. Using geolocation data attached to images also makes it easier to create a photo map containing points of interest on a map.

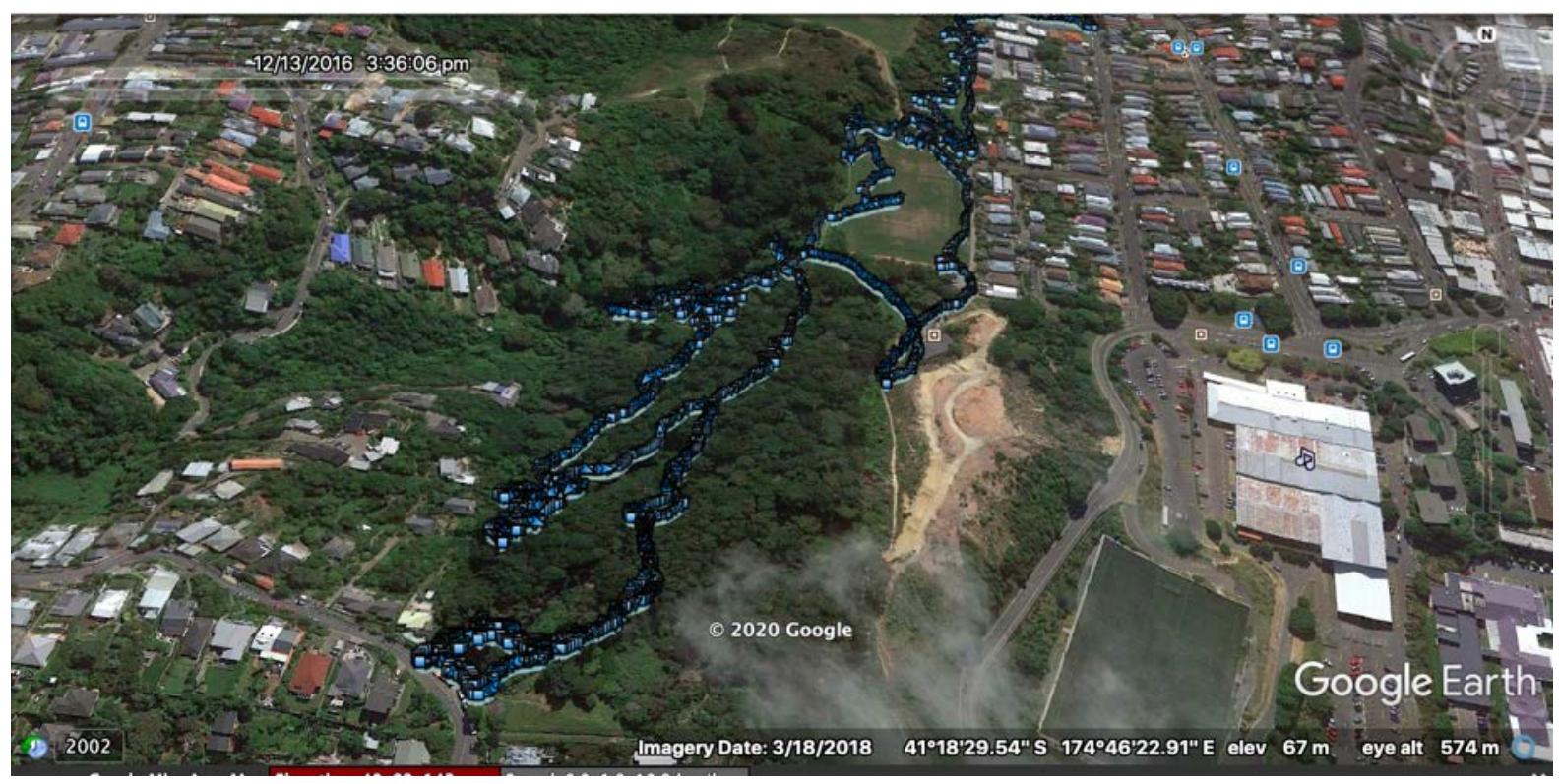

Figure 34. Author. (2016). Screenshot of a successful capture of the route walked around Papawai Stream viewed in Google Earth. 


\subsection{Water on the Internet? Or Worlds that are not supposed to meet}

I like... I think that question is really interesting. How is water... how would a river have a voice on the Internet... how would it have an agency on the Internet...

Water being in the Internet, like I can imagine a river flowing through a server farm and cooling the Internet. I don't know what that means but, I like the idea of a river being diverted into a building to cool the server or something...

I like the idea... I don't know... how can you have the river on the Internet. Like how do you have that energy.

I like the idea of streaming... streaming the river on the Internet. Just the sound... isn't that data? Does it need to be data?

I had an idea about, like if people are genuinely interested in the chemical makeup of the river. I'm not sure whether that's necessary at all those levels... we don't really understand what the safety levels are... but... if you did imagine the Internet... the water having a voice... you could imagine each stream painting a different colour, depending on its chemical makeup. So you could have four streams, painting something together. If it's a voice, it could be a colour, on a screen, that could be the river, in concert with other rivers maybe or other streams, or other pieces of water. So you had different colours, that would be their voice. I just think that the ability to transform data into something meaningful is much harder. It might be better if it just remained in the abstract.

But changing, eventually. So maybe you could see over the course of weeks, how the colours change, so there's a long line painting, you know. I don't know. I'm just thinking how does the water have its own desire to do much more than flow. I don't really think it wants to communicate. I mean personally, I'm not so sure about you. Do you think it wants to communicate?

Or we want to take something from that, a meaning from that. But I'm not sure if the... is it perhaps not about the intention?

Have you seen that movie Cowspiracy? It's basically suggesting that cows have trained us to cut down the Amazon and to get us clear half of America so that they can become a super-breed...

Yeah there is also one about maize...

Yeah there is even one about corn...

or you could say the suburban grass has trained us... So it's more us that's reacting...

What did the water train us to do?

Well I guess... that's what I am saying, I'm wondering whether you... t's trained us to...

It's also on the level of, the theory of corn... in the US people have eaten so much corn syrup, as a sugar, as a sweetener, their genetic makeup has changed to resemble corn. So in that sense if drive that one step further you could say, like okay, your genetic makeup is part corn, the corn might be driving the human, to give free reign to the corn.

So water is $60 \%$ in the human.

Yeah exactly...

Maybe indeed, maybe if water doesn't want to communicate, it also might have some kind of innocence. 
I feel water is not the cow, or the corn, in that way. And we... The health of the stream how scientists measure the health is about whether what it's doing to other people, or other things. Whether it's killing the fish that used to live there. or whether we're getting sick from swimming in it. Not the health of the river itself, but all the other species...

So, for E.coli as a species it would be a whole different idea what a good water would be.

I'm just thinking about like cameras, like iPhone cameras. People photographing the rivers, whether that's kind of a form of, what that is. Those often make their way onto the Internet as well.

That's interesting also with the drive from making microelectronics waterproof, like the Sony phone.

Waterproof. That's a weird thing actually. Making something waterproof.

Especially with electronics.

Yeah, exactly, this sort of... they are worlds that are not supposed to meet, somehow, are they?

Excerpt from transcript of a conversation during a group walk along Korokoro Stream as part of a walk conducted during the Common Grounds Art Festival.

In the interest of respecting the privacy of the participants, I edited out sections which were not pertinent to the immediate focus of the research. 


\title{
5.6 Participating in the SHMAK workshop
}

\#CitSciNZ2018 Freshwater Quality Monitoring Workshop, April 82018

\begin{abstract}
The aim is to identify the opportunities and tools available for communities to monitor freshwater, including fish monitoring, invertebrates, algae, chemical, microbiological and visual water quality analysis and habitat assessment.
\end{abstract}

\begin{abstract}
A further aim is to give participants a taste of the old and new tools available for measuring water quality and for them to get a better understanding of how to monitor to get good results and what to monitor to be able to answer their water quality questions
\end{abstract}

It's Sunday morning, just a couple of minutes before 9.30am and I am with a group of people gathering at the visitor centre of Ōtari-Wilton Bush, waiting for further instructions by the organisers of the \#CitSciNZ2018 Freshwater Quality Monitoring Workshop. I have heard about the SHMAK (Stream Health Monitoring and Assessment Kit) before, in conversations with participants, and when I saw the opportunity to get acquainted with the use of this kit, I was excited and signed myself up straight away.

Hello all,

Thank you for registering for Sunday's water quality monitoring workshop. Please find detailed information for the day along with a map of Ōtari Wilton Bush reserve attached.

A few last points

- Transport is not provided (bus information on attachments)

- BYO food

- Make sure you bring warm clothes

- Enjoy!

While waiting, I find myself nervously assessing other people's clothes and outdoor gear and wonder if my pair of hiking boots and waterproof jacket are a suitable outfit for the day ahead. This is also the moment where I learn what "waders" are. I am relieved to find out that the wearer of said item is one of the organisers. Most of the participants are wearing what I would call hiking or tramping outfits, and I also spot some gumboots.

The purpose of the workshop is to assess some changes that have been made to the original kit and understand how participants use the kit, and whether the instructions are easy enough to follow. For me, this day was a great opportunity to get my hands on one the kits myself and understand how measuring is done with the kit. Besides that, I also got a chance 
to learn how participants would interact with the SHMAK kit and note down any observations that would be useful for the development of my devices.

After a brief intro by the organisers the group slowly makes its way down to the stream, everyone carrying a bit of equipment. I am carrying a large orange cone that I find out later is called a bathyscope. I notice that the kit features some reasonably large equipment, some of them gathered in large plastic tubs, so would probably require a couple of people to be transported safely.

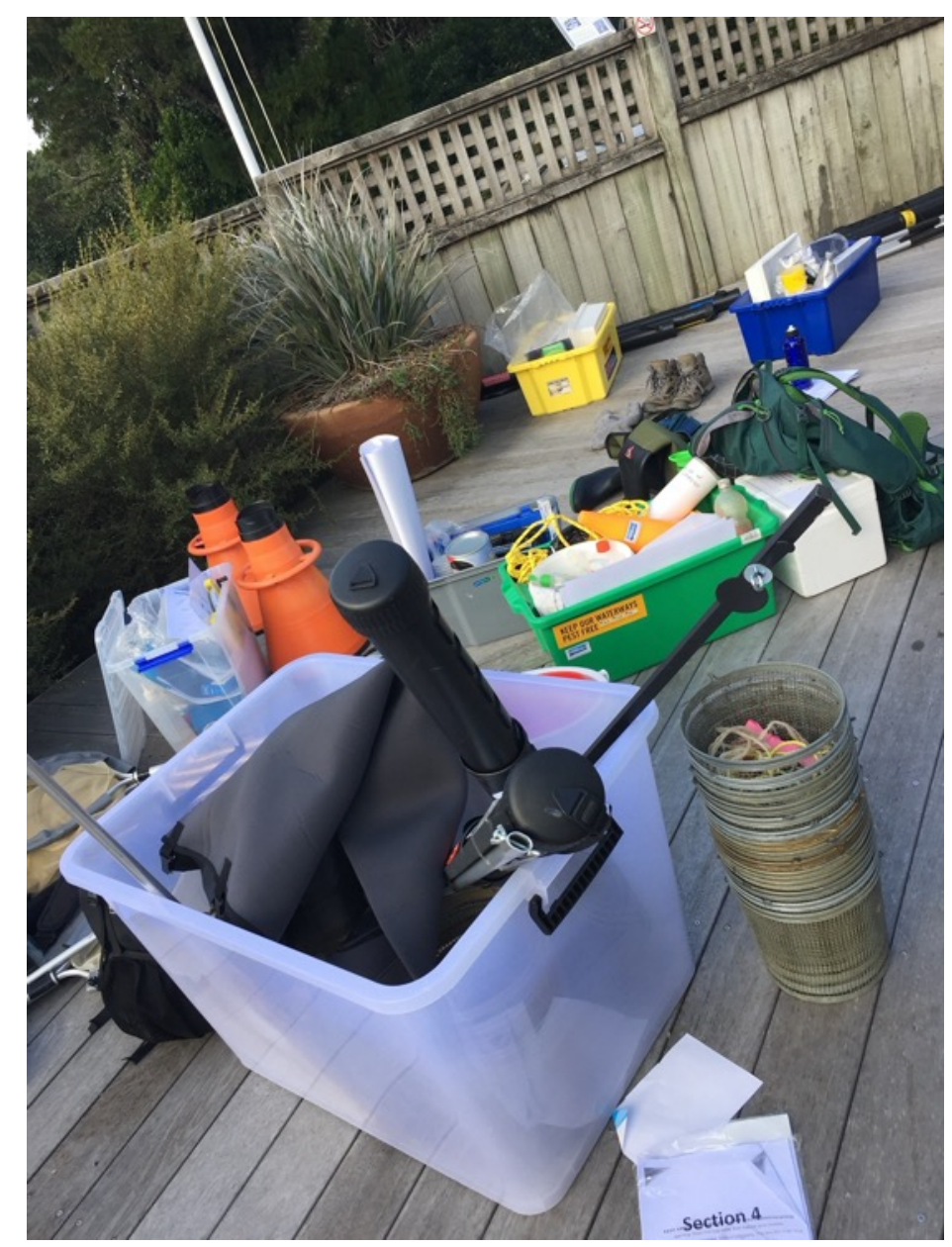

Figure 35. Author. (2016). SHMAK kit equipment used in the workshop.

After a couple of minutes of walking, mostly downhill, we reach the picnic lawn, a grass field nestled within a bend of Kaiwharawhara Stream - our spot for the day. The group of participants gets loosely divided into smaller groups and we follow a designated instructor giving us a the SHMAK data sheet and introducing us to the different kind of measurements we will be taking today. 
One of the instructors catches a kōkopu from the stream and puts it into a white bucket for all workshop participants to see. I walk up to the bucket and observe the little fish wiggling about, and I am reminded of one of the nightly "fish hunts" I participated in, accompanying an ecologist and some community members of Papawai Stream. As other participants approach to have a look at the catch, I recall the excitement that is tied to the realisation that there actually are fish inhabiting the streams, and that these would be the ones migrating through the pipes under the city.

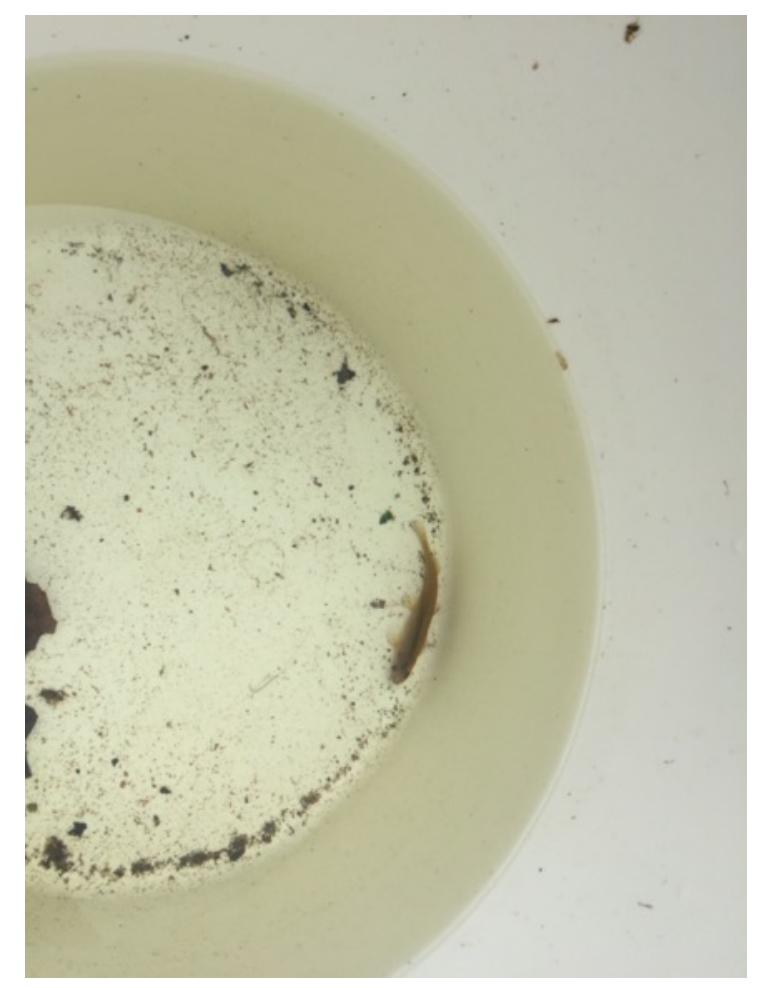

Figure 36: Author. (2016). A kōkopu is being shown to the participants in a white bucket.

The fish is soon after released back into the stream and we observe the workshop performing some of the techniques used with SHMAK kit for us. Some of the measurements do require standing in or wading through the stream (see for example Figure 38) and I immediately regret not having brought along a pair of gumboots myself. I do however enjoy the task of noting down the results and measurements in our log, trying to understand what these numbers or values mean.

I take notice of the ways data is being measured and documented in the workshop, especially electric conductivity, which was also captured during my installation Moturoa Transmissions. With the SHMAK kit, electric conductivity and temperature are measured 
with a handheld probe and the resulting values are noted down into the SHMAK data sheet. A measurement over time is not included in the method.

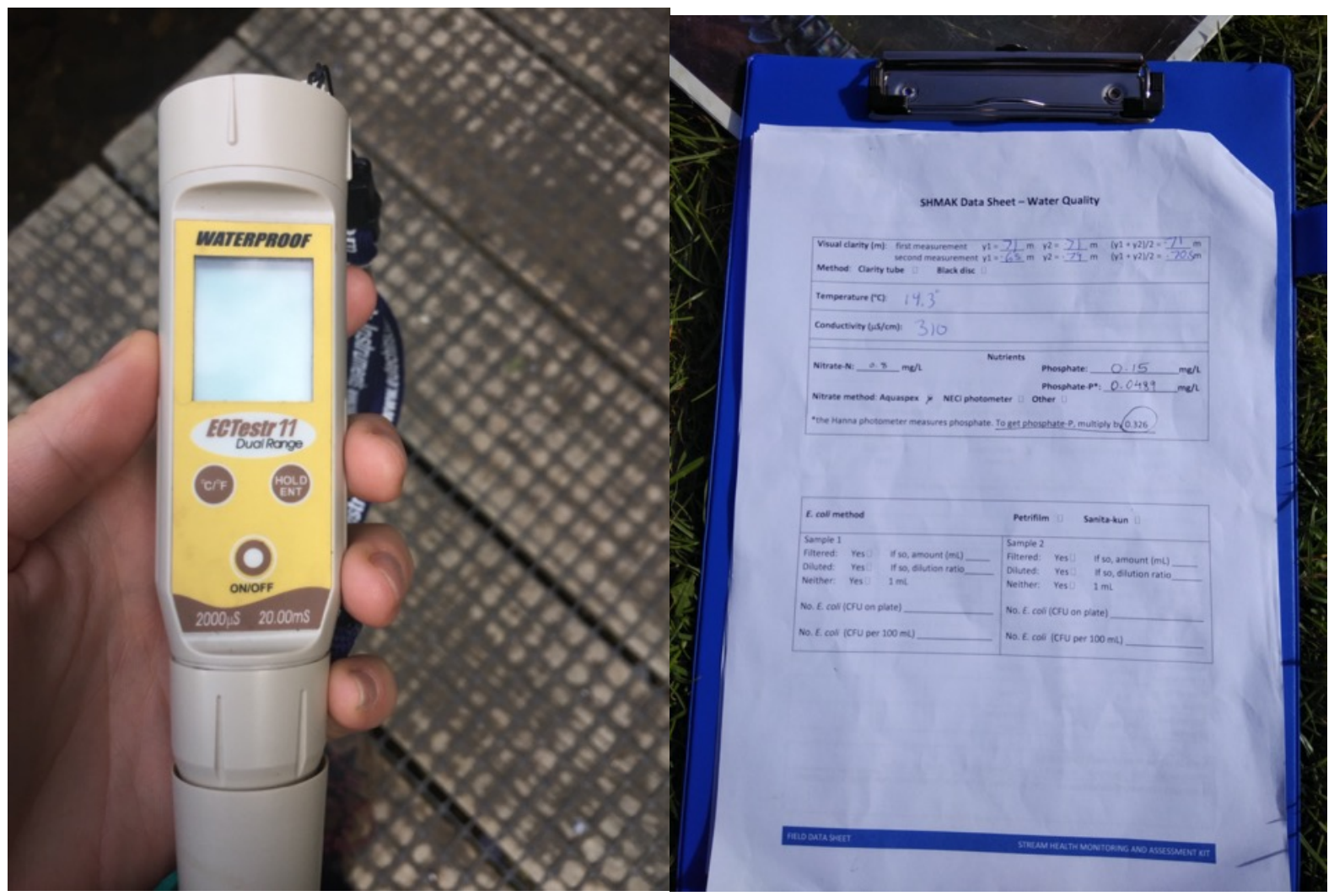

Figure 37: Author. (2016). The electric conductivity meter and the data sheet for recording the measurements.

The measurement of water clarity was demonstrated with two different methods, the clarity tube, and the black disk method - both of which seemed to be quite reliant on the perception of the participant carrying out the assessment. The black disk method (see Figure 38) for example required collaboration between two participants - one looking through a viewer and moving until the disk becomes visible/invisible and noting down the respective distances. The positions are changed over, and all measured values are to be averaged. The use of this probably requires some practice in terms of correct procedure and handling, as values differed a lot from participant to participant, and the averaging out of measurements just appeared as an approximation in the end, which felt disappointing after all the effort. 


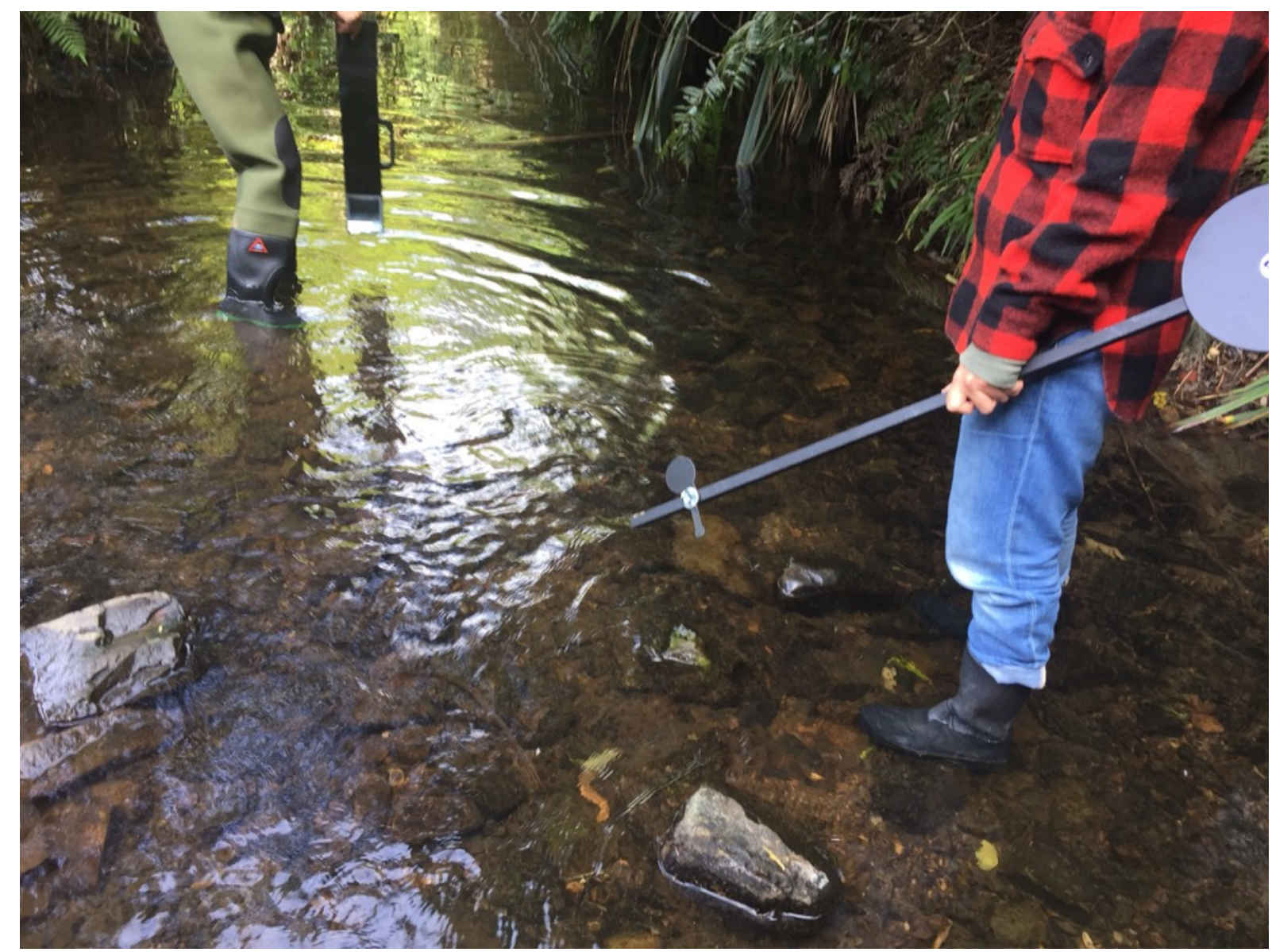

Figure 38: Author. (2016). Assessing turbidity with the black disk method.

Similarly, the nitrate test required some careful handling of substances, including filling stream water samples into two small vials, mixing it with liquid reagent, then adding powdered reagent with a tiny spoon. One of the instructors assisted us with the spoon and advised us to stay out of the wind. After some waiting for the substances to mix, we tried to assess our samples. The assessment required comparing the colour of the reference vial and the sample vial to a colour chart (see Figure 39) and assessing if the colours match. The corresponding concentration is to be filled into the data sheet, in $\mathrm{mg} / \mathrm{L}$. Again, the assessment appeared quite subjective, and our group was hesitant to reach a consensus on which of the colours matched closest. 


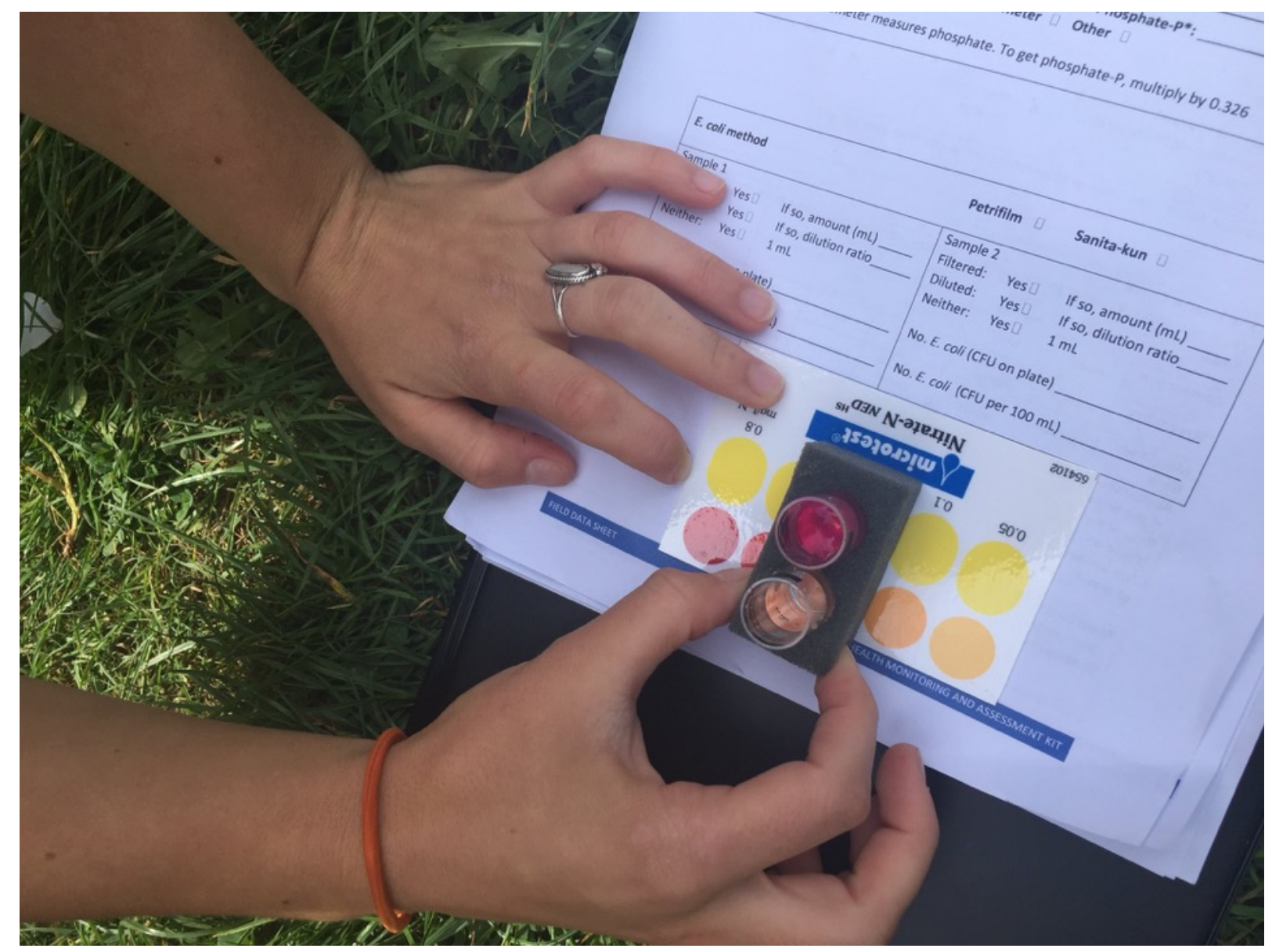

Figure 39: Author. (2016). Assessing Nitrate with a colorimetric test.

Throughout the day the participant groups start to disperse, and walkers and their families mingled with workshop participants. While some of our group tried to identify macroinvertebrates out of a stream sample provided by one of the instructors, children joined and helped us spot them and sorting them into a tray. The act of trying to suck a little bug into a dropper or capturing them with tweezers requires some concentration and tactility. We find many flies, some worms and snails and a very committed small group of us tries to make sense of how to transfer the data into the field sheet, but we struggle to understand the meaning of the provided table and how to calculate a total of our meticulously collected tiny animal collection. 


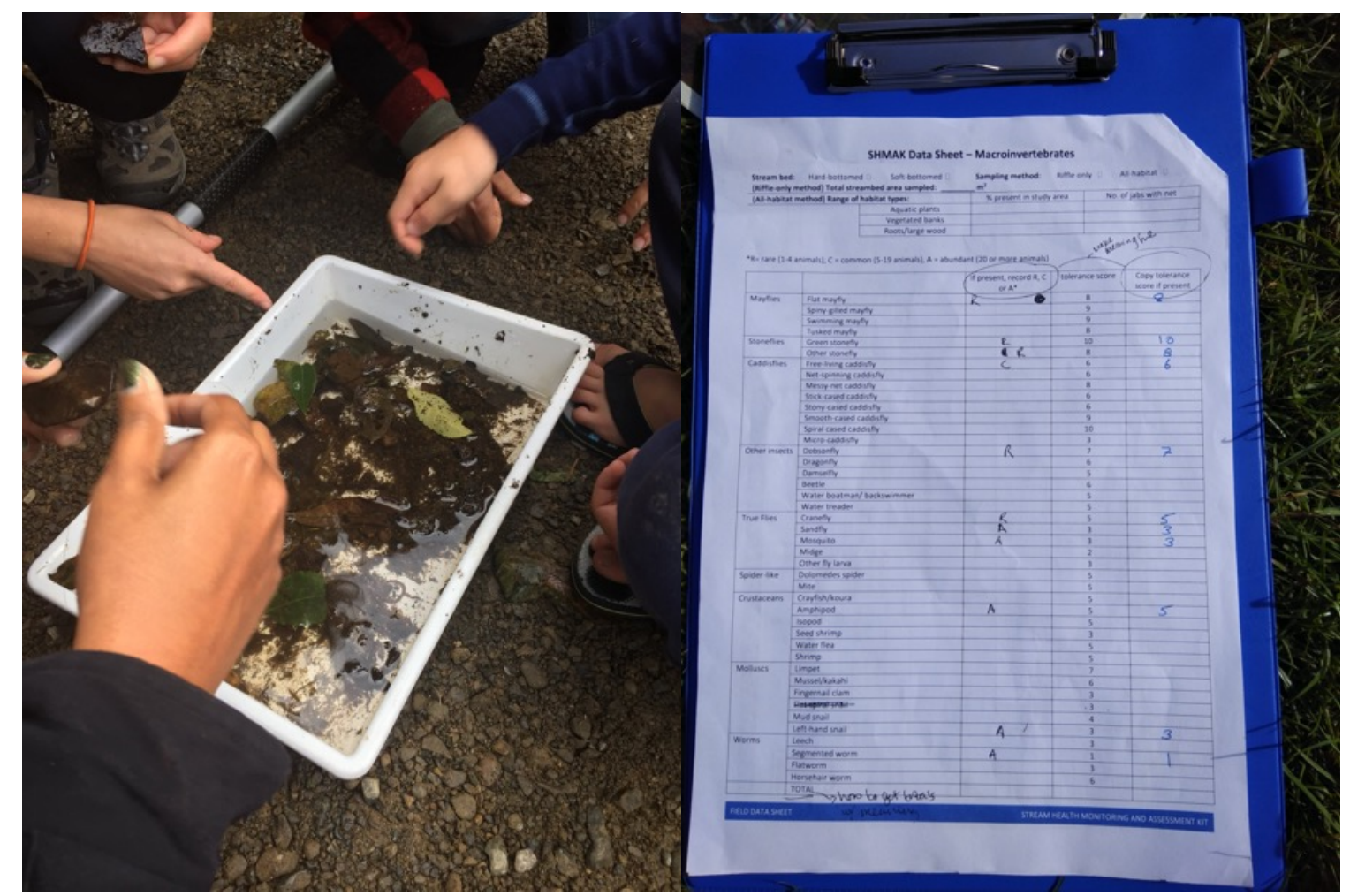

Figure 40: Author. (2016). Collecting and identifying benthic macroinvertebrates.

A test that appeared quite of interest to many participants was the E. coli testing - possibly due to the fairly recent E. coli contamination of Havelock North's water supply (as reported by Price, 2015). The test, however, is carried out offsite, as it requires some incubation time, hence the instructors have brought some already developed samples to the workshop to run participants through the process (see Figure 41).

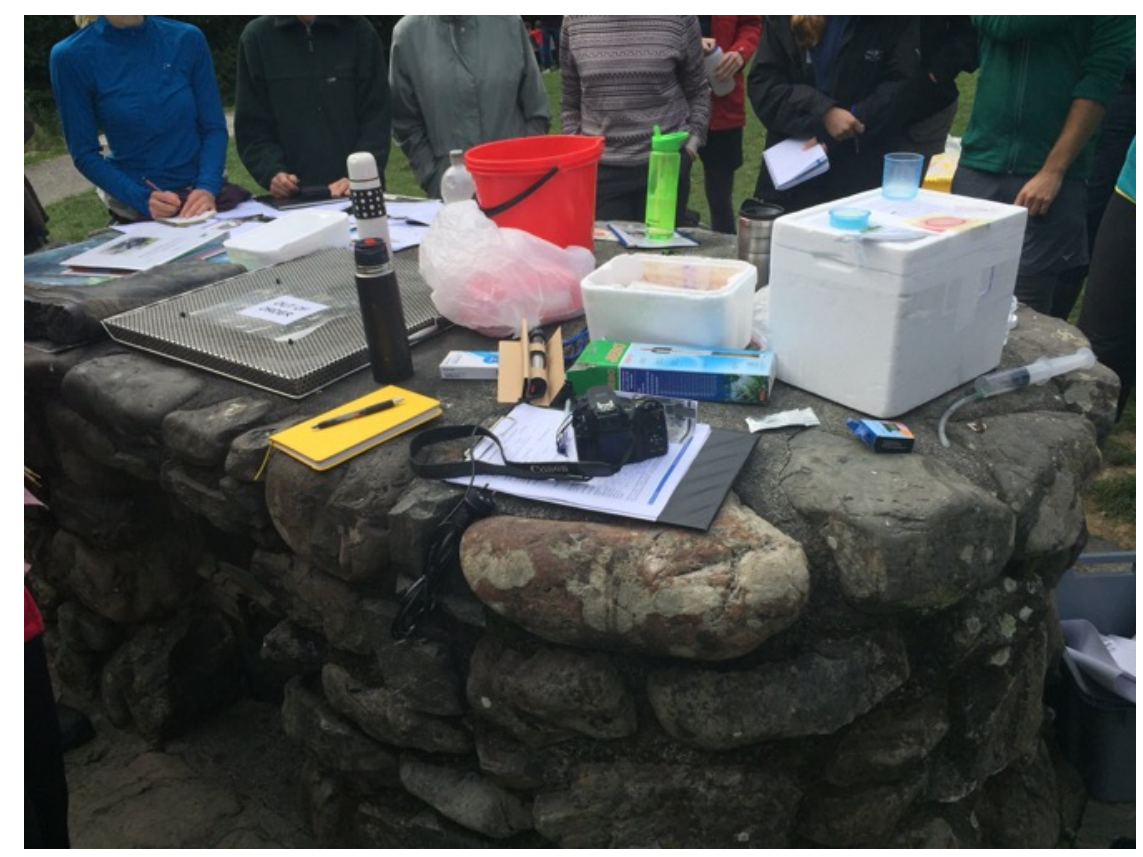

Figure 41: Author. (2016). Demonstration of the E.coli test. 
At the end of the workshop, we are invited to discuss the usability of the kit and the clarity of instructions. My main observation is the lack of design of the field manual and the kit which could help connect the instructions better to the required items of the kit. Our group depended on the support from instructors when it came to identifying the correct parts of the kit and proper usage. For example, colour coding and numbering all parts of the kit associated with one or multiple tests could have helped us collect required items without the help of a supervisor.

The lack of understanding how to properly transfer some of the more complex measurements into the data sheet, and how to make meaning out of them was also discussed. The apparent subjectivity of values, depending on the how (untrained) participants who carry out the measurements see or interpret measurements, resonated a lot with how I see the devices I built to collect data.

How my devices collect data might offer an alternative to by-hand measuring and recording, or they could, perhaps, add another layer of subjectivity when used in combination with manual citizen science methods, as presented in the SHMAK kit. 


\subsection{Field Exploration Reflections}

The selected narratives in this chapter, as recounted above, illustrate different stages of my explorations within the field. I start by presenting my first exploratory walk of Papawai Stream in the form of a blog post (5.2), followed by an account of attempting to draw the course of the stream on a map with GIS software (5.3). The documentation of walks with human participants $(5.4,5.5)$ outline conversations across multiple sites of encounters at Papawai, Moturoa and Korokoro Streams. My own participation in the SHMAK workshop considers questions of subjectivity and value, where these tensions coalesce in my own work (5.6).

The documentation of these stages, in their various forms, highlight my process of learning about the sites I work with through walking, sensing, and connecting. In this final section, the reflection, I discuss how the core research themes of slowness, seamfulness and openness informed the field activities, and also underscored the documentation of and reflection on the field research, and my own positionality as a researcher therein.

\section{Openness}

As part of the explorative work, openness extended into how I situated and negotiated my own participation within this research. While in the early phases of my research, I needed to be open and carefully pay attention to the diverse participants of the research, allow myself to acknowledge the stream and its more-than-human entanglements as voices I need to be listening to. For example, the first narrative presented outlines my first documented exploration of Papawai Stream, narrated along a series of photographs and field notes taken during the walk.

Walking by myself $(5.2)$, and with participants $(5.4,5.5)$ was a helpful method to form connections with the more-than-human world of the stream at a stage of the research where I was unfamiliar with the stream as a site, and with the more-than-human world as a research participant for my design research. As my research question departed from the 
paradigm of an "Internet of Things", an early quote by Weiser's (1991) idea envisioning the computer of the $21^{\text {st }}$ century, accompanied me during my first walks:

Machines that fit the human environment, instead of forcing humans to enter theirs, will make using a computer as refreshing as taking a walk in the woods (Weiser, 1991).

While doing my walks, I imagined what Weiser had in mind-computers that were unobtrusive to the environment I found myself in; boggy, sometimes steep terrain, partly dominated by thick bush and weeds, a trickle of a stream lined by fallen trees, sometimes littered with old rubbish or bricks.

While walking, I imagined building a network that connects to the stream, while paying attention to both the peculiarities I noticed from a design perspective, as well as the places and sections of the stream that the participants connect with and gravitate towards; and what they notice during walking and conversing. These insights informed the prototyping process of the field lab, discussed in the following chapters (6 and 7).

Further, being a participant myself, as in the SHMAK workshop, opened new perspectives on complementary practices as part of citizen science endeavours, particularly in relation to stream sensing.

Methods of openness have contributed new perspectives to the field explorations, through processes of walking with, conversing with and listening to, and participating within the communities around the more-than-human worlds of freshwater streams.

\section{Seamfulness}

The first seams as part of the field explorations emerged from my initial efforts to understand how Papawai Stream connected from the mountain to the sea. While this is a literal attempt of understanding the geography, I learned how in Māori philosophy, a ki uta ki tai (mountains to sea) approach encompasses a holistic way of looking after water resources and their management (see Tipa et al. 2006; Tipa et al. 2016). The difficulty of 
finding the stream made apparent how urbanisation has created a rupture within the precolonial landscape, removing a clear connection between the freshwater stream and its link to the harbour from the cityscape.

My early walks also served as testing territory for recording equipment that would help me gather data, such as photos or audio recordings, or tagging of GPS locations, and taught me to be mindful of equipment use in the field. Seamfulness reveals itself in the practice of the act of walking, documenting the walks and the attempt of piecing various forms of data and media collected together for write-up. As Lee and Ingold (2006) note,

... walking itself can consequently become a practice of understanding, so that the record of the walk, and of the experience it affords, is just as importantand just as a valid source for field material - as the record on 'discourse' that might have accompanied it (Lee \& Ingold, 2006, p.83).

The process of mapmaking, as outlined in 5.3, aided the unearthing of the subterranean canvas of Wellington's forgotten freshwater. The seams between the piped and unpiped freshwater served as places to plug in my design work (as discussed in Chapter 5).

Seamfulness is hence reflected in the field explorations in discovering apparent seams present in the field, including piped/unpiped, water/waterproof, and the research process, such as generating multiple layers of data and media, that underpin the design process as described in Chapters 6 and 7. 


\section{Slowness}

The embracing of seamfulness as part of the field explorations also required me to slow down and take the time to acknowledge the rough edges encountered and later created during my fieldwork (as discussed in Chapter 5).

By aiming to create complete recordings and documentation of my walks tending to recording equipment inhibited my ability to employ my own senses for being in the field, and fully immersing myself in the sensory more-than-human world. Hence, I later conducted several un-documented walks to the sites, in which I tried to pay attention to the stream, instead of focussing on my recording devices. During the first field explorations, the process of walking and conversing meandered between slow movements and immersions, as outlined in 4.5.

The research journey also revealed how early efforts, such as conducting the walkshops or my participation in related events (such as the SHMAK workshop as narrated in 4.6), did not have a clear connection to my work in the developing stages. However, these manifested later on, once my design work found its footing, and could more playfully engage with learning. This is exemplified in the design of LED nodes, as shown in Chapter 5 .

To recapitulate as noted earlier (cf. supra p.83), quick iterations of design outputs would be an effective way of pushing the progress of a design work further, but considerations of the impact of my research-such as introducing networked technology into the more-thanhuman worlds of local freshwaters-slowed my research progress down from an academic perspective. However, this slowness allowed a richer and deeper engagement with concepts from a Te Ao Māori perspective, to glimpse a future of being able to contribute to research on the interface between science and indigenous knowledge (as described by Durie, 2004). 
As part of the field explorations, slowness, first mistaken as a hindrance to my research progress, allowed me to take the time needed to listen and connect to the field, and allowed early, yet seemingly unproductive activities to slowly reappear as productive outcomes in my design interventions (as presented in Chapter 7).

As this chapter has shown, the highlighted fieldwork explorations discussed here exemplify how methods of walking and conversing, as well as participating and observing have been informed by slowness, seamfulness and openness, and laid foundation for prototyping processes and field lab, as demonstrated in the following chapters. 


\section{Chapter 6: Prototyping Log}

\subsection{Introduction}

With this chapter I want to give the reader an impression of the prototyping activities that underpinned the design research of wildthings.io, between 2016 and 2019, often happening concurrently with the activities listed in the preceding chapter, Field Explorations. In this chapter I focus on the hard- and software development and prototyping in electronics lab and the 3D workshop. The narratives documented in this section shine light on design developments, the small moments of success and failure that often go unnoticed when only attending to the final outputs of a design process. This chapter thus illustrates how the deliberate transparency of my design research process mobilises the key themes of the work into actual documentation, in the form of writing, photos and video recordings.

The media content accompanying this chapter is a video (prototypinglog.mp4) documenting the assembly of prototypes in the electronics lab. Because the video contains no sound, the clip could be played as a moving image accompaniment to reading this chapter, perhaps on a secondary screen, particularly when reading sections $6.5,6.6$. or 6.7.

For this section, I highlight six activities that illustrate my prototyping work. The first narrative Building a low-cost hydrophone is presented as a blog post on how to build a lowcost hydrophone to record underwater sounds, and discusses a prototype designed before fieldwork with participants began. Covering lab book entries from roughly two months, Hello World. Building a DIY Internet of Things offers an insight into finding a technologically and conceptually viable way of building networked media to be deployed by the stream. In Wi-Fi in a bottle: Recycling materials for waterproofing electronics, the narrative zooms further out in the prototyping timeline, showing how the designs of low-cost enclosures for the electronic components has developed over the course of the entire design research. The last sections, EC-sensor iterations, turbidity probe iterations and Giving voice to the stream: Output node iterations give a detailed development overview of DIY component design that stood central to the IoT installations. In the last section, Prototyping Log Reflections, I highlight how the narratives compare and contrast within my design journey, and in what 
way the key themes of slowness, seamfulness and openness played into the prototyping process overall. 


\subsection{Building a low-cost hydrophone}

This section describes the very first prototyping effort as part of the research process and manifests the first attempt of providing a transparent step-by-step recount of hands-on development, including written accounts and photos documenting the process on the go. A version of this narrative was posted on my research blog, which remains the most-often visited entry to date.

It is October 2016, and I am at the very beginning of my practical design research, excited as my research proposal has just received ethics approval a few weeks ago.

Before heading into the field with participants I wanted to build some prototypes of devices that could be used as part of the research. These prototypes would be used to capture some layers of the more-than-human world that are inaccessible to our human senses, but also to demonstrate an example of a DIY device that I could see myself developing as part of the larger research project. Inspired by the sound ethnographies of rivers by Aotearoa/New Zealand Annea Lockwood (1989; 2008), my first intuition is to build a hydrophone which would enable me to listen to and record sounds of the stream.

Hydrophones are used in scientific research for acoustic monitoring, such as for tracking marine animals, are relatively expensive and even DIY solutions feature pricy elements (see for example Dawson, 2012). Low-cost DIY solutions often re-use old audio components as headphones or radios (e.g. digifishmusic, 2008) which are made waterproof with silicone or hot glue.

After reviewing a variety of instructions, I decide to combine the parts of two (Decker, 2013 and University of Waikato, 2011) for my first build. Both of them use an electret microphone element as the primary audio component. In the instructions of Decker (2013) the element is submerged in an empty film canister filled with mineral oil. I choose this design for the first version following a suggestion by a member of the Wellington Sonic Arts Facebook group in response to my question:

BB: Hi everyone! I need to build a couple of low-cost hydrophones for a project, currently looking at plethora of builds in the DIYuniverse online but wondering if anyone here has good suggestions/experience? Will use it outdoors to listen to Wellington urban streams (also piped ones). Thanks 
$R R$ : I've read about buying little electret elements then soldering them to a cable and putting it in a film canisters filled with mineral oil then connecting that to a little preamp. I think jaycar had a little electret preamp kit for \$20ish. Not sure if they still sell the mic elements, but you can rip those out of old phone handsets. And film canisters can be picked up from WPS on featherston st for free (Bachler, B. 2016, October 11).

The instructions by the Science Learning Hub (2011) also feature an electret microphone, with the only design difference being their recommendation to cover the microphone with flexible sealant, instead of using the film canister, which is also mentioned as an alternative design by Decker (2013). Most importantly, the Science Learning Hub (2011) instructions contain a list of components that are all available in Aotearoa/New Zealand, with most parts being sourced from Jaycar, a local electronics supplier with a store close-by in Pōneke/Wellington.

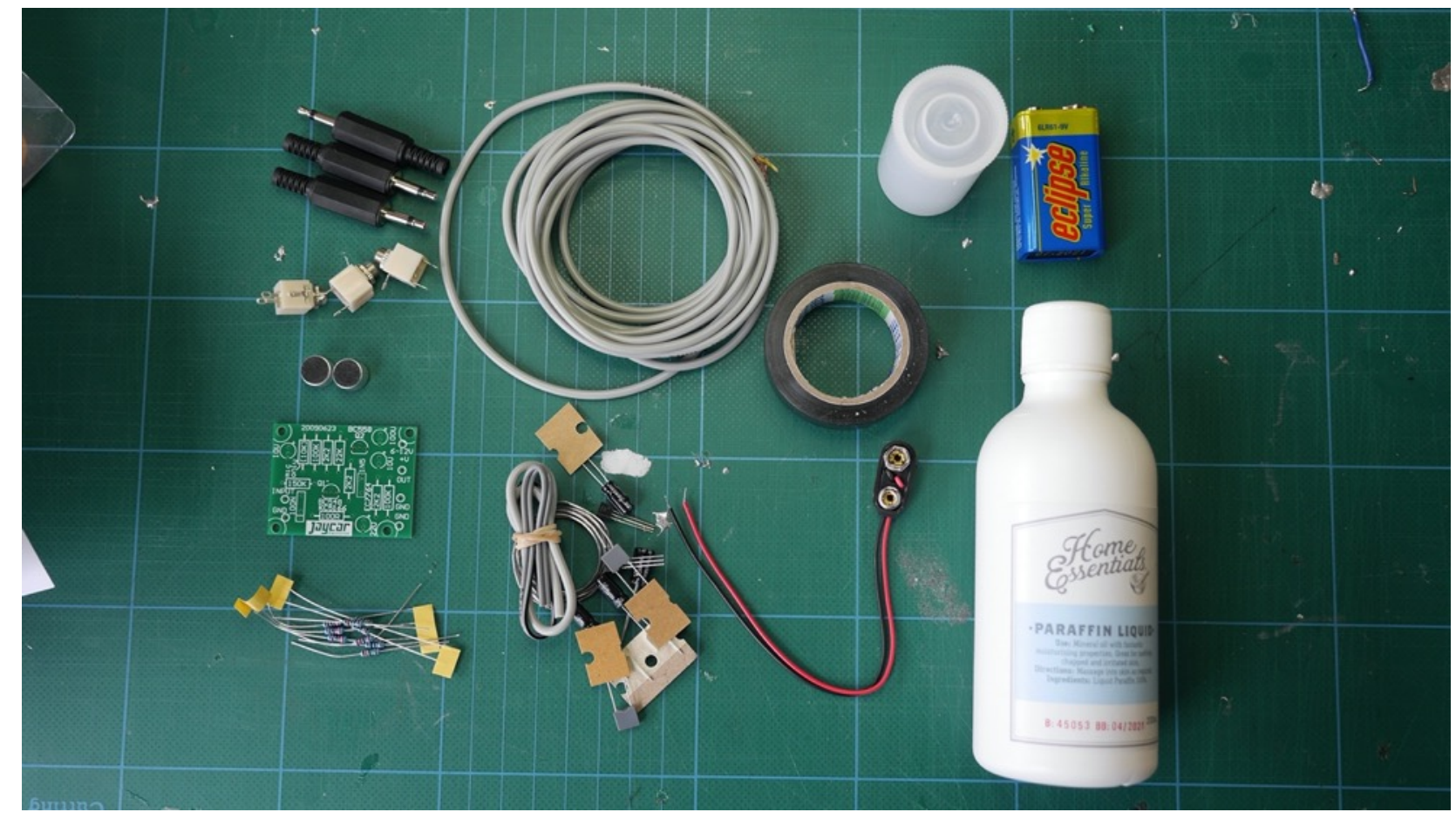

Figure 42. Author. (2016). Overview of materials used to build the first hydrophone prototype. 


\section{Materials List Hydrophone}

\begin{tabular}{|c|c|c|c|}
\hline $\mathrm{D}$ & 1x Pre-Champ Versatile Preamplifier Kit & $\begin{array}{l}\text { https://www.jaycar.co.nz/pre- } \\
\text { champ-versatile-preamplifier- } \\
\text { kit/p/KC5166 }\end{array}$ & $\$ 12.90$ \\
\hline $\mathrm{C}$ & 1x Electret Microphone insert (2 pictured) & $\begin{array}{l}\text { https://www.jaycar.co.nz/standard- } \\
\text { microphone-insert-with- } \\
\text { pins/p/AM4011 or AM4010 }\end{array}$ & $\$ 3.40$ \\
\hline B & 2x 3.5mm enclosed Socket (3 pictured) & $\begin{array}{l}\text { https://www.jaycar.co.nz/3-5mm- } \\
\text { enclosed-socket/p/PS0122 }\end{array}$ & $2 \mathrm{x} \$ 1.45$ \\
\hline A & $3.5 \mathrm{~mm}$ miniature plug - black ( 3 pictured) & $\begin{array}{l}\text { https://www.jaycar.co.nz/3-5mm- } \\
\text { miniature-plug-black/p/PP0114 }\end{array}$ & $\$ 1.10$ \\
\hline $\mathrm{E}$ & Single Screened Audio Cable (3 meters) & $\begin{array}{l}\text { https://www.jaycar.co.nz/single- } \\
\text { screened-audio-cable-sold-per- } \\
\text { metre/p/WB1500 }\end{array}$ & $\$ 0.75 \times 3$ \\
\hline $\mathrm{F}$ & Insulation Tape & $\begin{array}{l}\text { https://www.jaycar.co.nz/pvc- } \\
\text { insulation-tape-black-5m/p/NM2800 }\end{array}$ & $\$ 1.55$ \\
\hline G & 9V Battery Snap & $\begin{array}{l}\text { https://www.jaycar.co.nz/9v-battery- } \\
\text { snap-standard/p/PH9230 }\end{array}$ & $\$ 1.05$ \\
\hline $\mathrm{H}$ & Film canister & Sourced from photography shop & $\$ 0.00$ \\
\hline I & 9V Battery Alkaline & $\begin{array}{l}\text { https://www.jaycar.co.nz/9v-battery- } \\
\text { alkaline-eclipse/p/SB2423 }\end{array}$ & $\$ 5.10$ \\
\hline $\mathrm{J}$ & Paraffin Liquid (mineral oil) & Available at pharmacies & $\begin{array}{l}\text { Approx } \\
\$ 10.00\end{array}$ \\
\hline & Selleys 80g All Clear Multipurpose Sealant & $\begin{array}{l}\text { https://www.bunnings.co.nz/selleys- } \\
\text { 80g-all-clear-multipurpose- } \\
\text { sealant_p0227570 }\end{array}$ & $\$ 13.60$ \\
\hline & Total & & $\$ 53.85$ \\
\hline
\end{tabular}

Table 2: Author. (2016). Materials List for building a DIY hydrophone.

Building the first version of the hydrophone took less than a day and involved researching the best possible option to be built within the timeframe of one day, sketching a schematic alongside a shopping list of necessary parts, planning the quickest route around town to buy all necessary parts and then around two hours of assembly in the soldering lab, plus some extra time for testing, troubleshooting, and documenting along the way. 


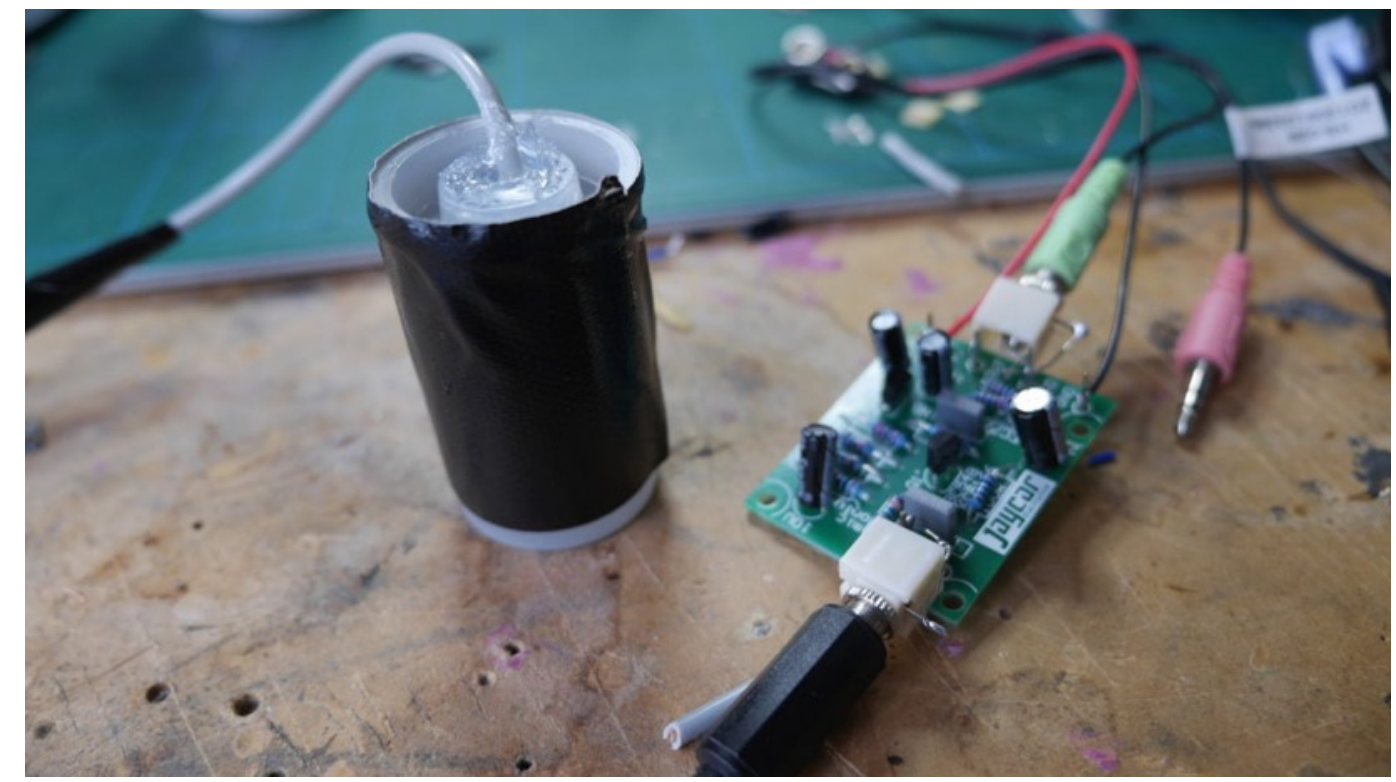

Figure 43. Author. (2016). Detail of the electret microphone in the film canister.

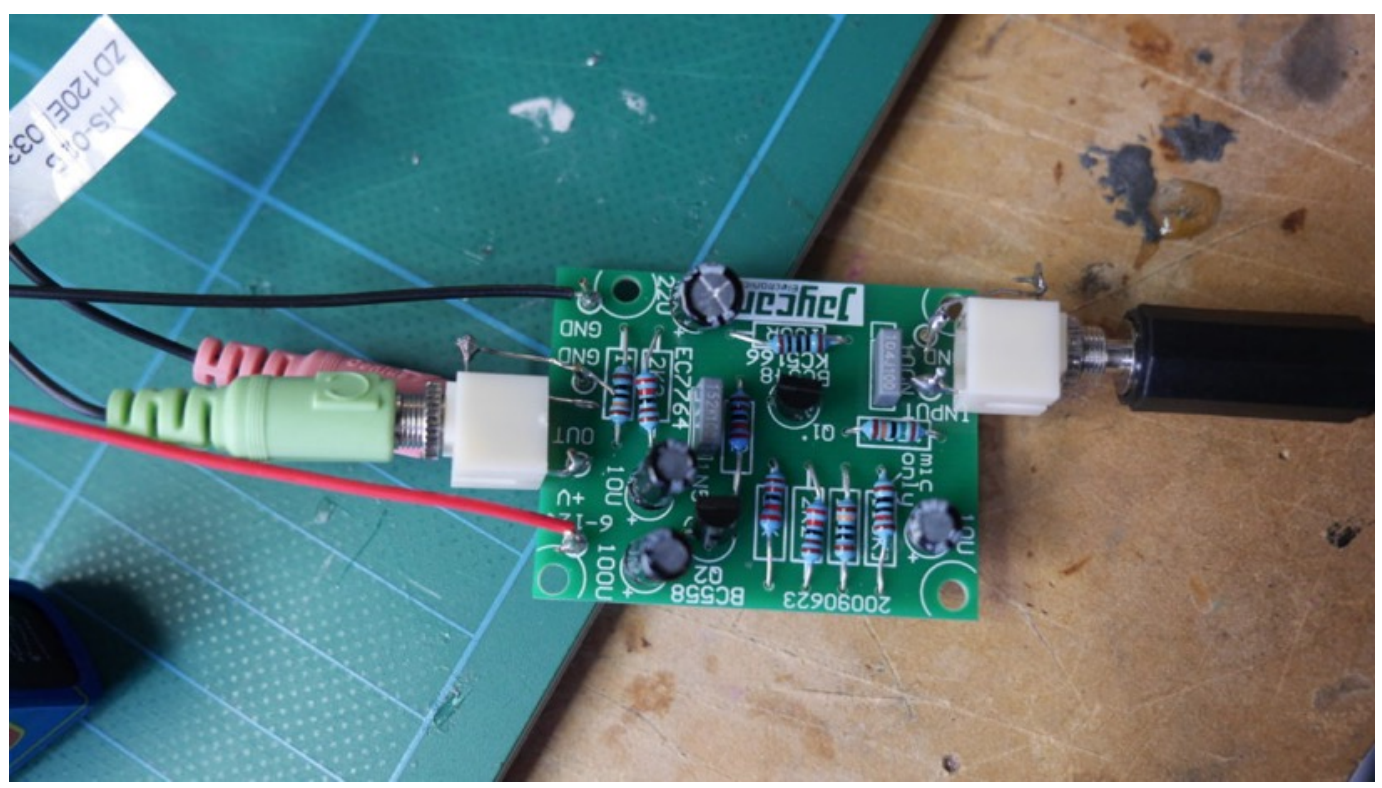

Figure 44. Author. (2016). Detail of the assembled and connected preamp.

I assembled the hydrophone as described in Decker (2013) but instead of an 1/8" plug used a $3.5 \mathrm{~mm}$ mono headphone jack at the end of the 3-metre-long cable. I also soldered $3.5 \mathrm{~mm}$ sockets to the pre-amp for in- and outputs to keep the device as modular as possible, and to use it with different kinds of speakers or an audio recorder.

I let the sealant on the hydrophone dry overnight, but discovered some leaks the next morning, so I reapplied another layer of sealant and waited another day before testing the hydrophone in water. 
After the first indoor tests in water glasses, mainly to make sure no sealant is leaking when the hydrophone is submerged underwater, I take the hydrophone with me on my first exploration of Papawai Stream (which is also narrated in 4.2).

I bring a pair of headphones to plug into the pre-amp and I hope to be able to listen to sounds of the stream that could only be heard from under the water surface. At this stage, the electret has no enclosure, so all contacts and the battery are exposed, but as it is a reasonably dry day, I can test the prototype regardless, resting the preamp on a dry rock while attempting to launch the hydrophone into the stream.

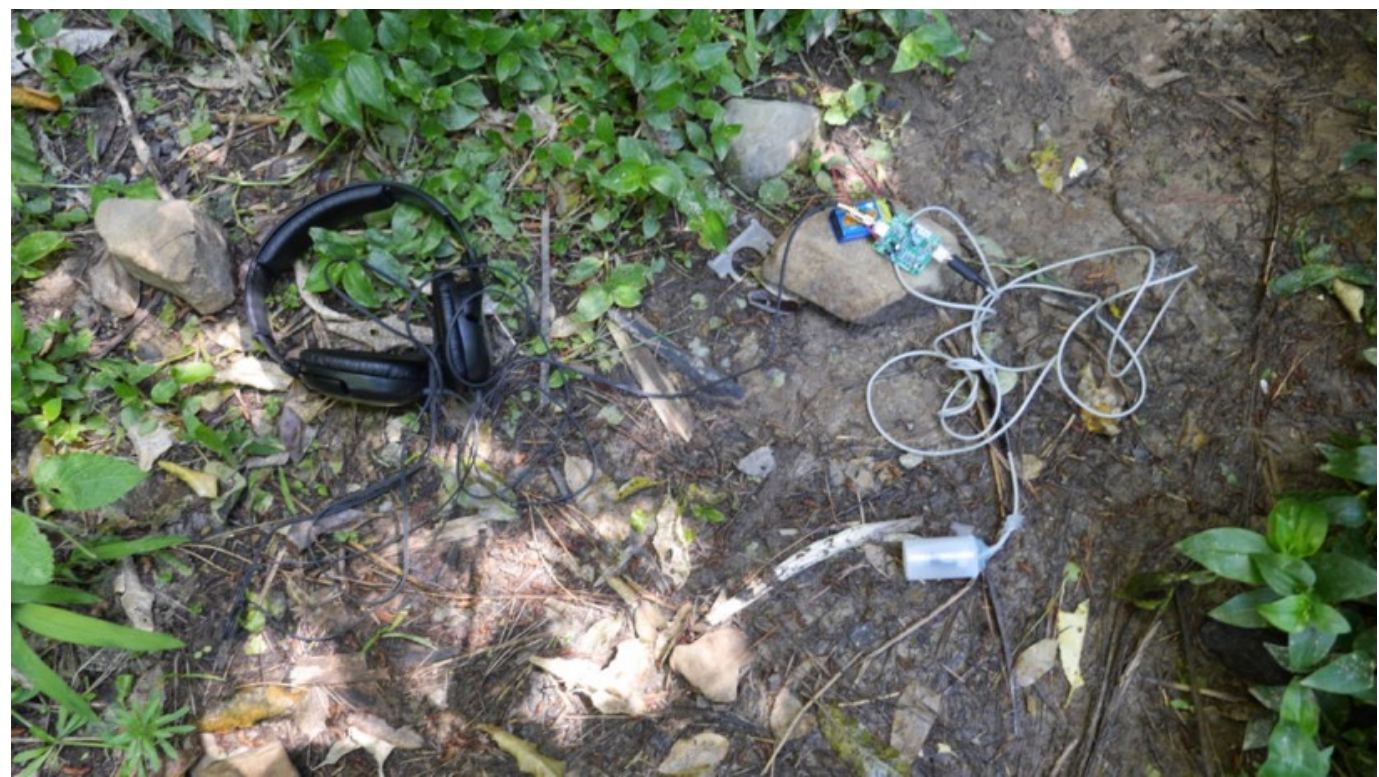

Figure 45. Author. (2016). Hydrophone without casing connected to headphones beside Papawai Stream.

During this first short test I learn a few things that will prove useful during tests and installs of more complex prototypes at a later stage of my research journey:

- When working by the stream with electronic prototypes I will need flat, dry surfaces or containers to safely keep components out of the water

- To launch probes accurately into the stream a makeshift pole like a robust branch will come in handy

- Bring tissues or a small towel to dry components after submersion and a waterproof pouch to store and transport them. Hand sanitiser and/or gloves might be recommended when working with waters of unknown quality. 


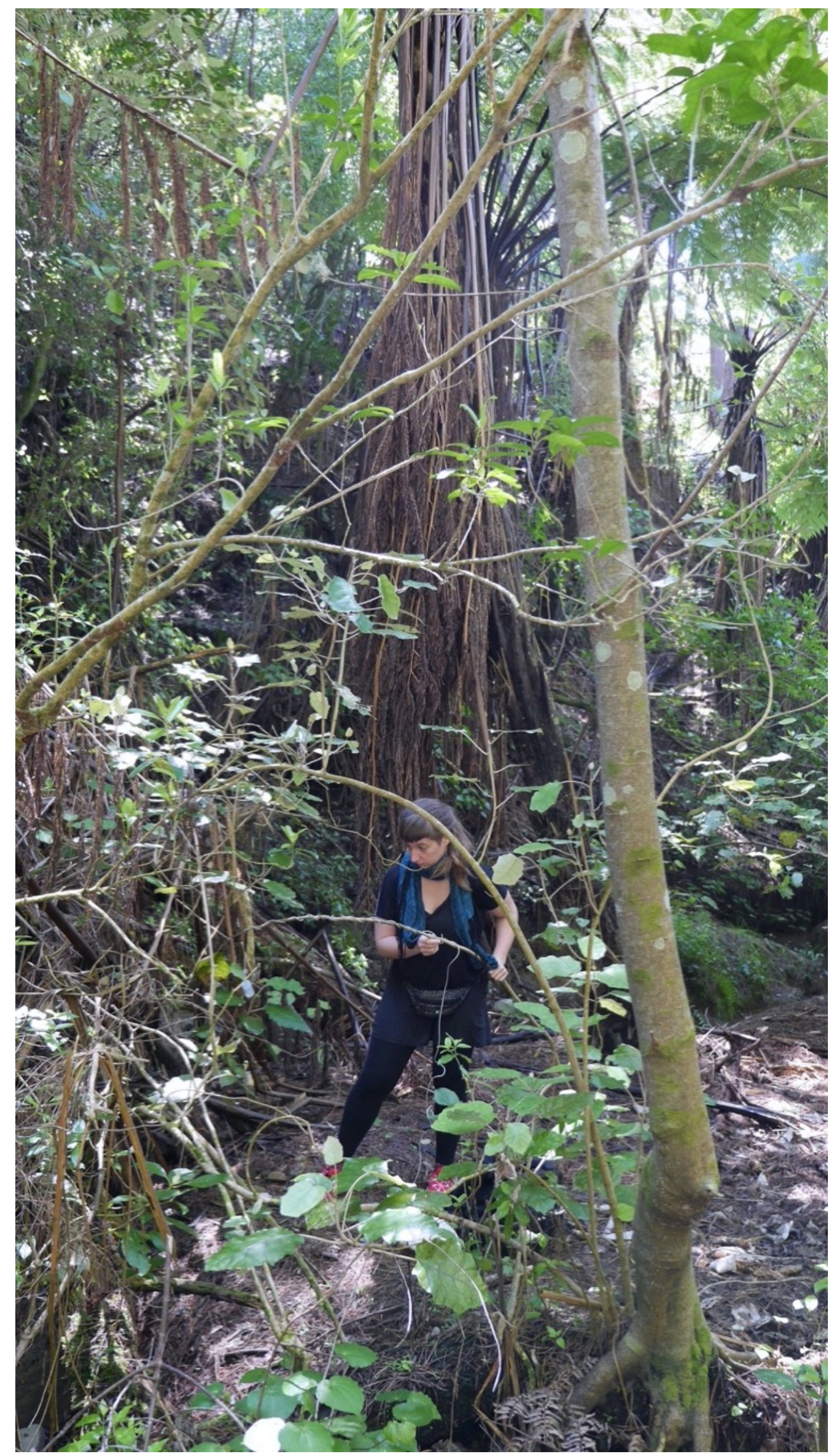

Figure 46. Author. (2016). Testing the DIY hydrophone with a repurposed branch used as "fishing pole". 
The hydrophone itself accompanies me during a few walks, but the way I set it up initiallyjust as a listening device to be immersed into the water-proves unsuccessful, especially when walking with others. For example, on one of the group walks along Korokoro Stream (featured in the form of a transcript excerpt in 4.5) I notice that the sound picked up by the hydrophone is similar to the gurgling and gushing that can be heard beside the stream without the need for an amplifying device. As a result of this, the hydrophone ends up not being further used in walks with participants. The role of tuning into the stream by listening to the sounds of its environment, however, is a central focus during solo explorations. Some of my solo field immersions involved audio recordings-such as wildthings.io (2018) which are played during evaluation sessions in the lab as well as embedded in my public development blog (Bachler, 2016)-to give the stream a presence at points in the research, where I am unable to be at its side, but to remind me for who and with who I aim to be developing my designs for. 


\subsection{Hello World. Building a DIY Internet of Things. [Lab Notebook, 19 December 2016-26 February 2017]}

In this section I present the first steps of developing a working prototype of an IoT network, which lays the technical foundation for the upcoming research. The narrative of this development journey is built from material as documented in my lab notebook, in which I kept track of my daily progress, working code, ideas, frustrations and successes.

Development started with the assessment of possible hardware and software to start prototyping with, aiming at developing a proof-of-concept for IoT technology that would be suitable for a basis to build further hard-and software from. The first experiments of setups I sketched were based on hardware I had available from previous prototyping projects, including some one single-board computers, the Raspberry Pis and microcontrollers, such as the Arduino Uno, as well as some WeMos D1 microcontroller boards, which are based on the ESP8266 microchip. The ESP8266 is suitable for IoT development because of its low cost (less than \$5NZD), and its Wi-Fi capabilities. Similarly, having a background in prototyping with the Arduino development environment, this was my first choice for drafting early code examples for heuristic evaluation.

The entries presented here are selected notes concerning the prototyping process starting with early research into IoT development and finishing with the first success running a MQTT Pub/Sub network, which laid the foundations of all further prototyping efforts towards the final install in 2019. In my original lab notebook, the prototyping entries are accompanied by notes from fieldwork by the water, recaps of correspondence and interviews with participants and annotations of readings which, while they had a direct impact on this prototyping journey, have been edited out to keep the narrative focused on the IoT development journey. 
19 December 2016.

I bookmarked some tutorials involving ESP8266 WeMos boards and Raspberry Pis as MQTT (Mosquitto) brokers. I installed Raspian on my oldest Raspberry Pi (2011) and it does work. ;)

\section{December 2016.}

Perhaps I should stop using the Raspberry Pi v1 for my project as it lacks onboard Wi-Fi - I just installed Mosquitto on v1 and v2 - forgot v3 at home.

Note: The DHT humidity sensor shield I have is a DHT-pro.

The WeMos and the shield seem to work. Now I need to look now how to use it with the Raspberry Pi and make it talk via MQTT.

Perhaps I should focus on figuring out the simplest way of sending messages between my sensors and my raspi but I could also check out something like OpenHAB (n.d.): “A single user interface to all of your systems".

\section{Jan 2017.}

I am downloading Platformio (2017) for Atom on Mac. I have also been checking out the ESP8266 github (Grokhotkov \& Molinari, n.d.).

\section{February 2017.}

I am working through an Adafruit tutorial (Schwartz, 2015) using a Raspberry Pi and the ESP8266 for home automation. The tutorial shows how to connect multiple devices to one Raspberry Pi. That setup features a router which in turn requires power, which I won't easily have in the field, so I decide to look into ad-hoc networks that don't require a Wi-Fi network present to work (see Walkevar \& Agrawai, 2016).

At this stage I decide to start from scratch with the WeMos D1s again. I download Arduino, install the ESP library, then download the WeMos drivers for Mac. After installing some drivers and replacing a faulty USB cable I get the Example code HelloMesh.ino from the esp8266 Arduino examples to work (see Figure 47). 
received: Hello world response \#4 from Mesh_Node12824165.

receireceived: Hello world response \#7 from Mesh_Node12824165.

received: Hello world response \#8 from Mesh_Node12824165.

received: Hello world response \#9 from Mesh_Node12824165.

received: Hello world response \#10 from Mesh_Node12824165.

received: Hello world response \#11 from Mesh_Node12824165.

received: Hello world response \#12 from Mesh_Node12824165.

received: Hello world response \#13 from Mesh_Node12824165.

received: Hello world response \#14 from Mesh_Node12824165.

received: Hello world response \#15 from Mesh_Node12824165.

received: Hello world response \#16 from Mesh_Node12824165.

received: Hello world response \#17 from Mesh_Node12824165.

received: Hello world response \#18 from Mesh_Node12824165.

received: Hello world response \#19 from Mesh_Node12824165.

received: Hello world response \#20 from Mesh_Node12824165.

received: Hello world response \#21 from Mesh_Node12824165.

received: Hello world response \#22 from Mesh_Node12824165.

received: Hello world response \#23 from Mesh_Node12824165.

received: Hello world response \#24 from Mesh_Node12824165.

Figure 47: Author. (2017). Screenshot of Hello world output from testing the HelloMesh.ino with two modules.

Then I test other Arduino examples, such as the microcontroller's function to act as a

Webserver/Access Point. The access Point example works, generates a little webserver with

SSID \& password, accessible under http://192.168.4.1

\section{Feb 2017.}

I am ordering parts from the WeMos shop. The practical issues with developing a network for the stream are technical limits towards:

- Moisture/weather/wind

- Battery life / Power consumption

- Connectivity/Wi-Fi range

Apart from conceptual and aesthetic considerations these three cannot easily be anticipated during lab development and need to be tested on site.

Currently, the aim is to develop a work that can run for min 8 hours a day, charge 16 hours a day.

\section{Feb 2017.}

Argh. It appears that due to the large amount of available sensor devices, the multiple protocols they support, across Wi-Fi or any Bluetooth-derivative - there is no simple, straightforward solution to building custom IoT solutions. 
Eames (2017) has a good tutorial on using a Raspberry Pi 3 in combination with multiple WeMos D1 mini nodes as temperature sensors. The setup is described well but it makes use of an existing network and the Raspberry Pi runs a server itself, which might be too complex of an application. The HTTP response in this example might be useful for alteration of the example.

There are quite a few posts on the WeMos forum of users trying to find ways of connecting multiple microcontrollers via a network, with multiple WeMos connected to a sensor measuring data like temperature, and then sending that data to one central node, which could be a WeMos as well.

Going back to my early bookmarks, I decide to give Pub/Sub and MQTT another go, even though I have zero experience with these protocols.

OK the plan now is:

I use a Raspberry Pi as MQTT broker.

I am not sure if/how the Pi should also be serving as the Access Point.

The Pi could run a Python script that reads and saves all data and publishes the outputs again to the nodes.

I proceed to try using my Raspberry Pi as a local Wi-Fi access point, trying some tutorials with no success (e.g. Martin, 2017), but finally succeed following a step-by-step guide on the Adafruit website (lady ada, 2013).

I have created a Wi-Fi access point with my Raspberry Pi 3. I used an external Wi-Fi dongle with an external antenna to improve connectivity and range of the network.

I followed the tutorial step by step but left out the part for Network Address Translation because at this point, I only want this network to be used locally without any uplink to the World Wide Web. After setting up the daemon, the network automatically starts on boot. The SSID is Moturoa_Transmissions after the name of the artwork. I can see the network on my laptop and on my phone just after less than half a minute after booting the Raspberry Pi. Yay.

Getting closer. 
Now that the Wi-Fi is up, I am trying to get the WeMos with the DHT22 sensor to talk to the Raspberry Pi via MQTT.

The test setup looks like follows: I am following a MQTT tutorial (Lewis, 2016) to connect my Raspberry Pi with my WeMos board.

The Raspberry Pi has multiple terminal windows open (see Figure 49) during testing.

The first one runs MQTT in verbose mode via the command

mosquitto $-\mathrm{v}$

The second one is subscribed test-wise to a debug topic with the line Mosquitto_sub -h 127.0.0.1 -i testsub -t debug

In the third window I test the broker by publishing the test message to the topic debug: Mosquito_pub -h 127.0.0.1 -i testPublish -t debug -m 'Hello World'

Success: I can see the debug messages bouncing around the network. Now I need to connect the WeMos to the network with a basic script. I connect a LED to the board via pin D4. The LED can be switched on and off via Mosquito_pub -h 127.0.0.1 -i testPublish -t ledStatus -m '0'

The full working Arduino code:

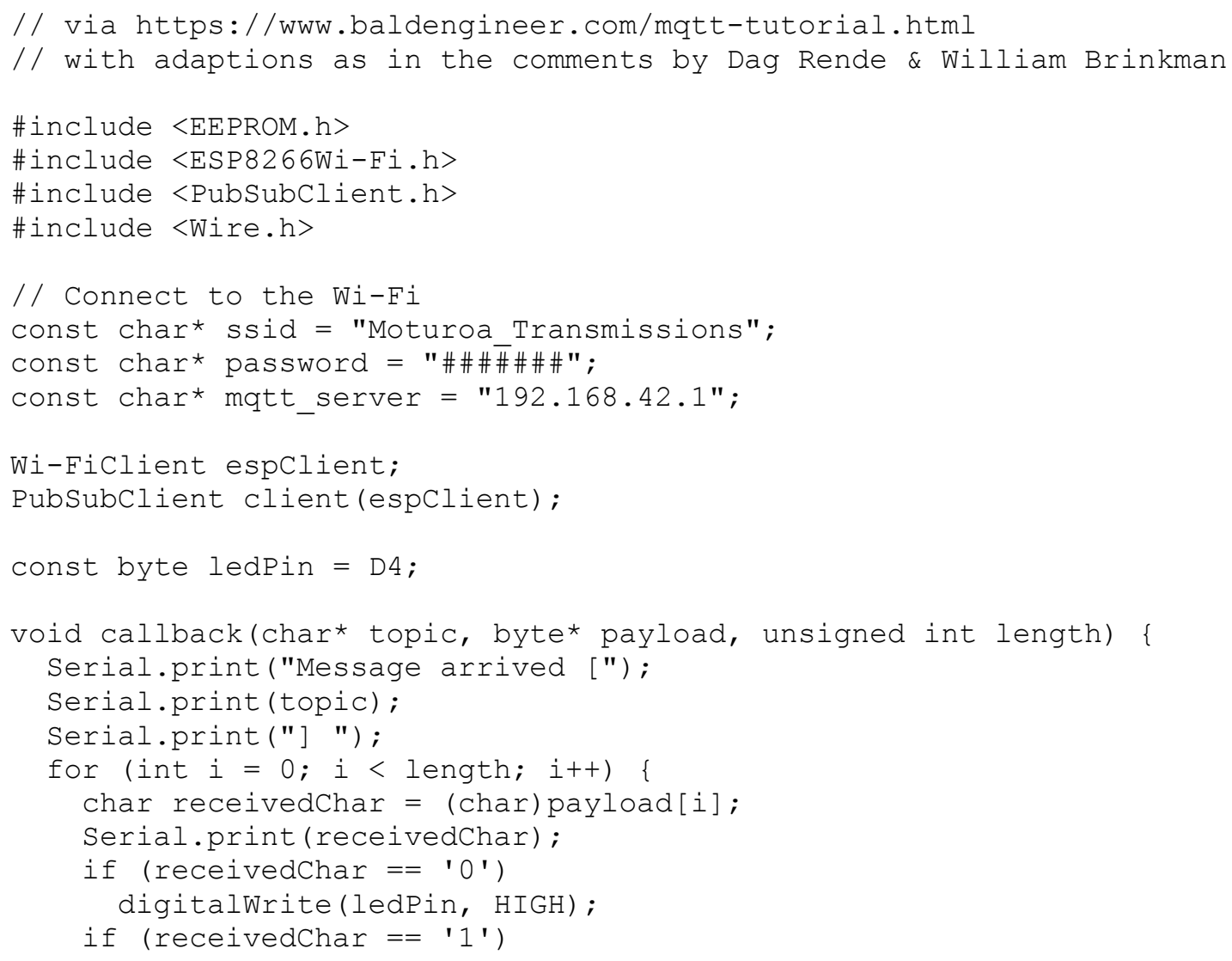




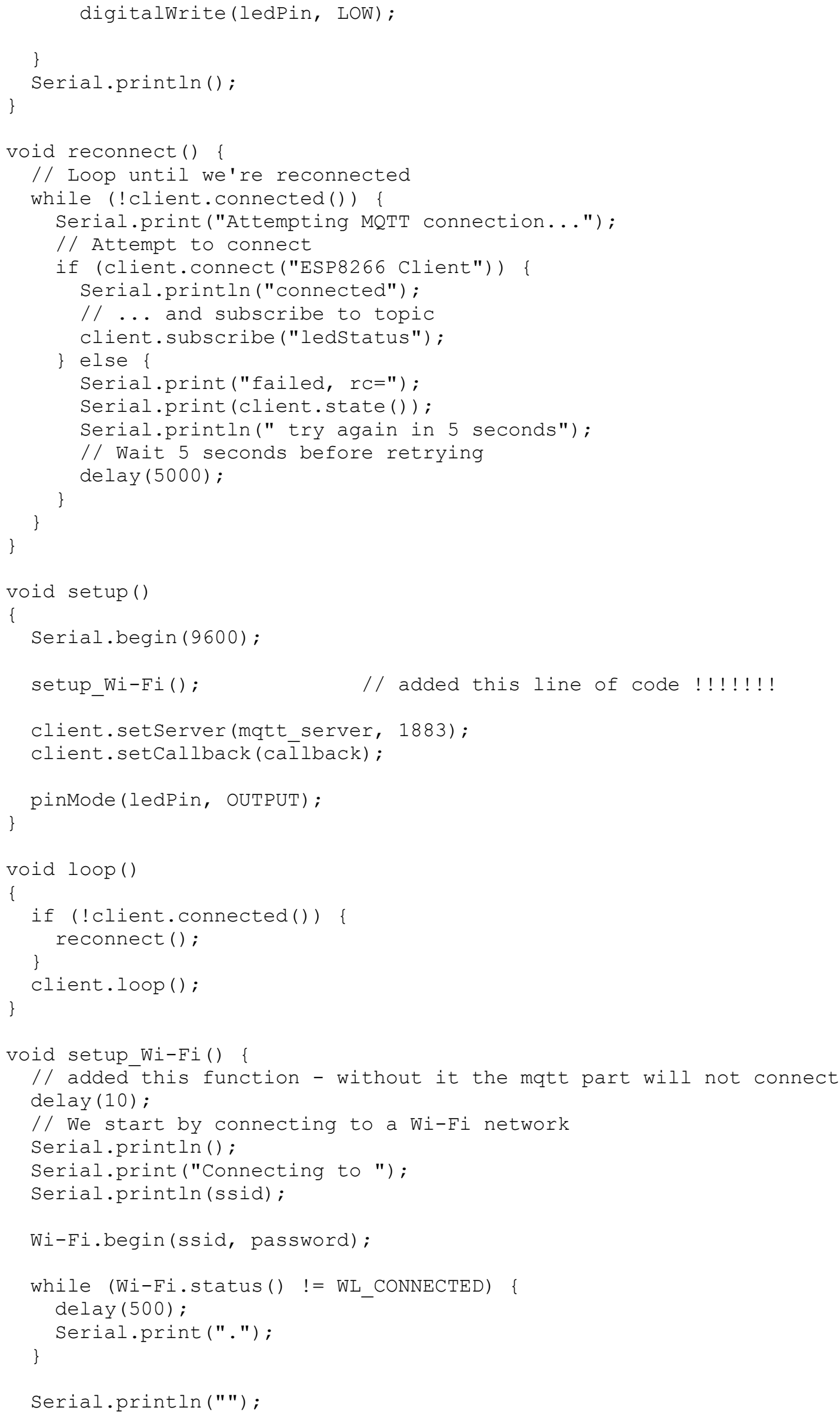




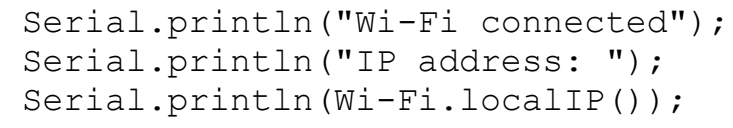

Note: When connecting multiple clients make sure the line

if (client.connect ("ESP8266 Client2")) \{

contains a different name, otherwise the broker will get confused. Node names need to be unique.

Other than that, the publication of LEDs to two nodes simultaneously works well (see

Figure 48). I can follow the network traffic on my multiple terminal windows (Figure 49) and the communication is reliable.

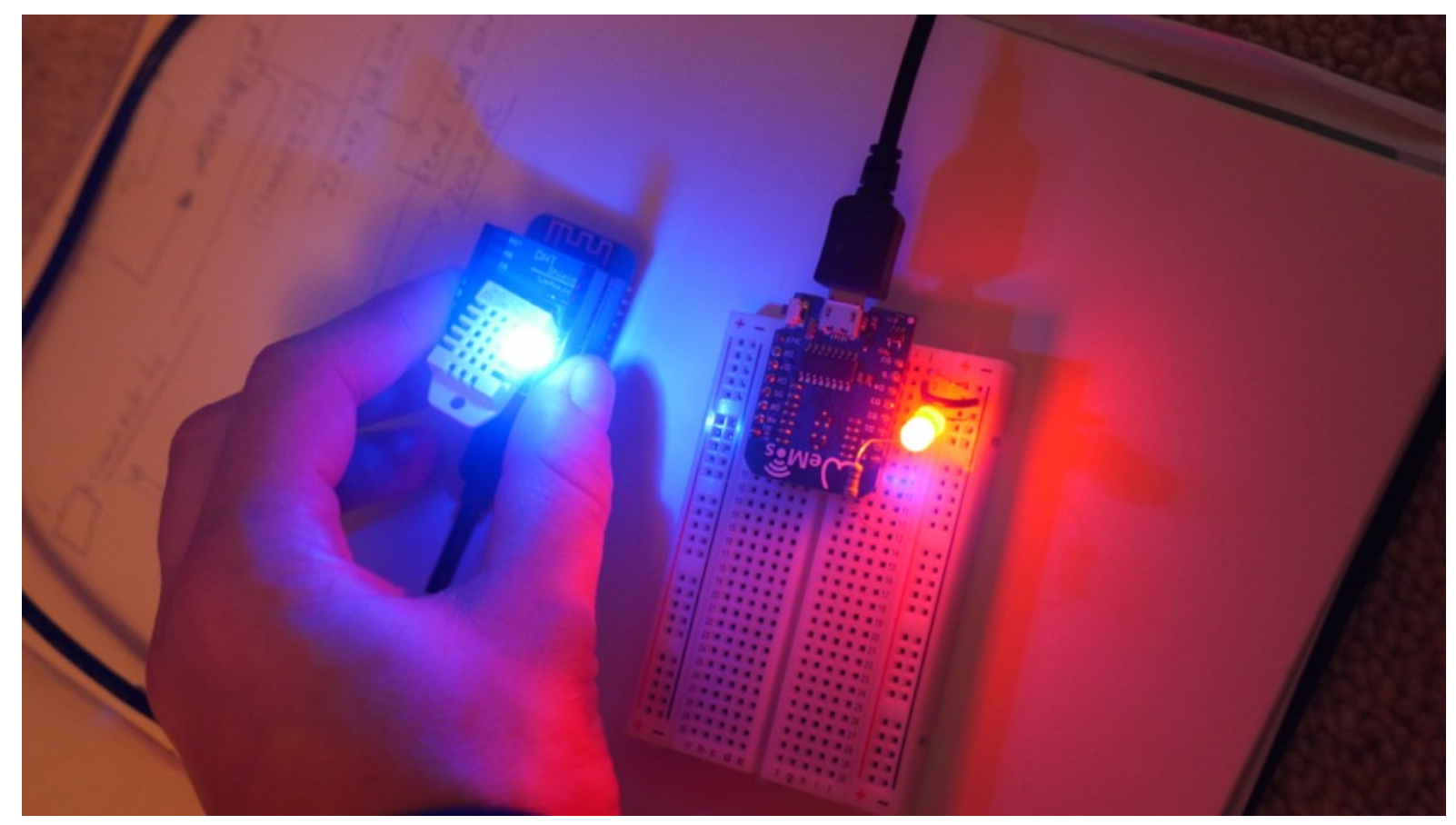

Figure 48. Author. (2017). Testing to WeMos D1 nodes. 


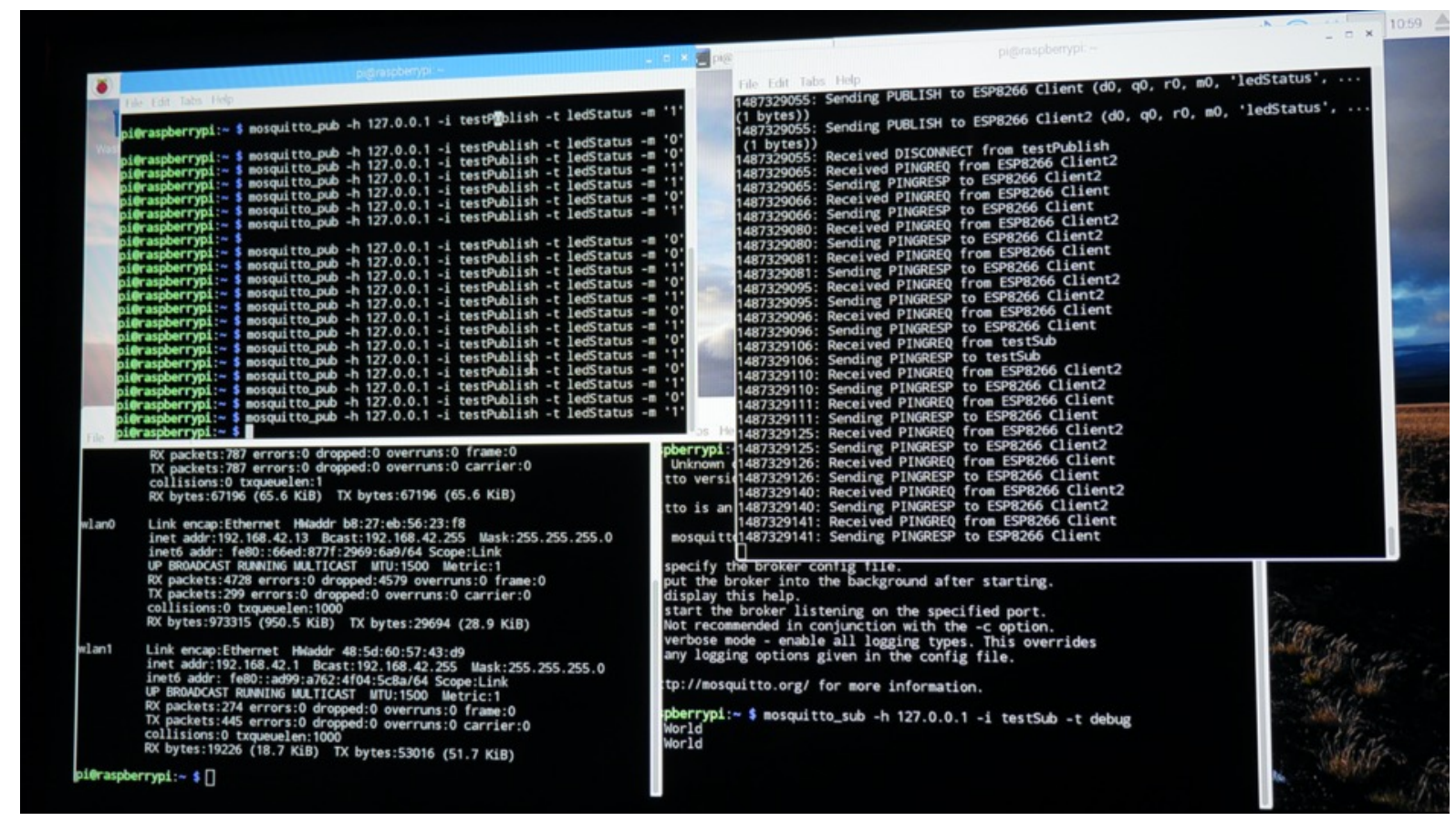

Figure 49. Author. (2017). Raspberry Pi with multiple terminal windows open for testing of MQTT network switching LEDs of two connected nodes on and off via pub/sub.

\section{February 2017}

In the next step I try using my MQTT Pub/Sub network to report back an analog sensor value via MQTT, similar to what I would need when sensing data by the stream. I use the Arduino script that already worked but will use the callback function to report the reading of a light sensor, to test if it works.

For the design work itself this data might be the water conductivity, the temperature of the water, possibly also even the light the stream gets as these are all indicator for the health of the stream.

I connect a test circuit with an Arduino Uno and read the values out via Serial connection to make sure the sensor itself works properly.

After this I connect the circuit to my WeMos D1 mini.

In a blog post Schoutsen (2015) uses an ESP8266 board to report temperature and humidity to a MQTT broker. The node reads the sensor values every five seconds. The function checkBound ( ) compares the new reading with the previous one and only publishes it to the respective topic if it differs by float diff $=1.0$. 
The next step involves cleaning up the code and removing unnecessary debug messages and add a function to reconnect if Wi-Fi is lost, as demonstrated in the Home Automation code of ItKindaWorks (2016).

To sum up, what I have done prototyping-wise:

I have tested the WeMos D1 mini ability to act as an access point.

I connected two WeMos D1 mini together, having one act as the access point and the other one as a node. The communication went via HTTP/Rest and did work generally.

Because of the limited memory and the relatively small antenna of the WeMos D1 I decided to use a Raspberry Pi 3 as the master node for my network.

The Raspberry is reasonably small and can be powered with a power bank.

After I managed to set up the Pi3 with an external antenna as an access point I tested the MQTT protocol for communicating with the D1s. The PubSubClient library (O'Leary, 2016) is available for the Arduino IDE and allows for straightforward communication between the nodes and the Raspberry Pi broker in the Arduino IDE.

I will use the Paho Python client (Eclipse, n.d.; Light, n.d.) on the Raspberry for managing connections.

I plan to use 18650 LiPo cells with $3100 \mathrm{mAh}$. The ESP8266 uses about 80mA when the Wi-Fi is on (see ESP8266 datasheet), $350 \mathrm{~mA}$ peak (see Spiess, 2016).

\section{February 2017}

I had a small incident with melting plastic due to a short-circuited set of 4 AA batteries this made me re-think the choice of using LiPoly batteries and a battery shield and resort to a safer version of a readymade store-bought USB charger that is as small as possible. The Digitech 2600mAh power bank houses an $18650 \mathrm{Li}$ Ion battery and works with USB charging at 1 Ampere. 
It appears that the bank does not charge when the red LED is blinking - the indicator light needs to be on constantly. The manual only describes colours but not static/blinking behaviour.

The 10400mAh power pack intended to power the Raspberry Pi 3 MQTT broker caused the Raspberry to indicate power instability (a little lightning bolt symbol at the top right of the interface). In comparison, the $8500 \mathrm{mAh}$ solar power bank only showed this at startup when the Pi draws the most energy.

Since the Raspberry Pi 2 uses less energy and I am using the external Wi-Fi antenna for the network I changed the microSD into my Pi model 2B and since the OS booted correctly, I only needed to configure wlan1 to wlan 0 and the Mosquitto broker now runs on a less power-hungry Pi. (for power consumption see (FAQs - Raspberry Pi Documentation, n.d.)

OK, I might just keep a list of relevant files on my Pi here, in case I need to change something later:

/etc/network/interfaces

/etc/dhcp/dhcpd.conf

/etc/default/isc-dhcp-server

/etc/hostapd/hostapd.conf

Also, here's my calculation for running the Pi using a 10400mAh battery:

battery time = amp-hours / drawn-current

battery time $=10400 / 700 \Rightarrow 14.8$ hours

I'm also planning to use Python Paho, the MQTT client Python library for subscribing/publishing on my local machines, as most usefully described by Light (n.d.).

\section{February 2020.}

OK, some useful notes, on how to start MQTT broker on startup (Joe_Q, 2016), remotely accessing the Raspberry Pi via SSH (SSH using Linux or Mac OS, n.d.) and starting a service headless - without a monitor connected (matt_symes, 2016). 
The commands are as follows (matt_symes, 2016):

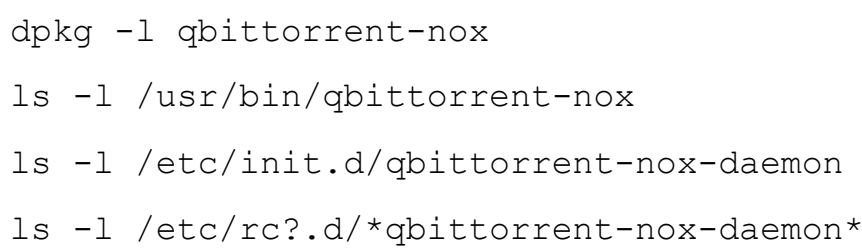

\section{Feb 2017}

Steps I took today on the Raspberry Pi:

1. Make sure there is a user mosquitto

2. I enable ssh in the -config file

3. I set a new password for the Raspberry Pi: \#\#\#\#\#

4. I also tell in raspi-config to wait for network until start

5. I added mosquitto $-\mathrm{d}$ to etc/rc.local before the exit 0 line so it runs at boot. Woo hoo! Getting close.

The test, starting the Raspberry Pi as if in the field with no monitor attached, by itself, hoping the network would launch and the MQTT broker would properly work upon launch: No screen connected to the Raspberry Pi. Cold starting the Pi after being unplugged from power supply.

Connecting to Moturoa_Transmissions with my Macbook.

Running python paho-test-connedct.py $-v$ in

It works.

Hooray! 
On the downside, I discover later today that the WeMos forum, which has been one of my main resource for communicating with other developers using this board, has been hacked (Figure 50).
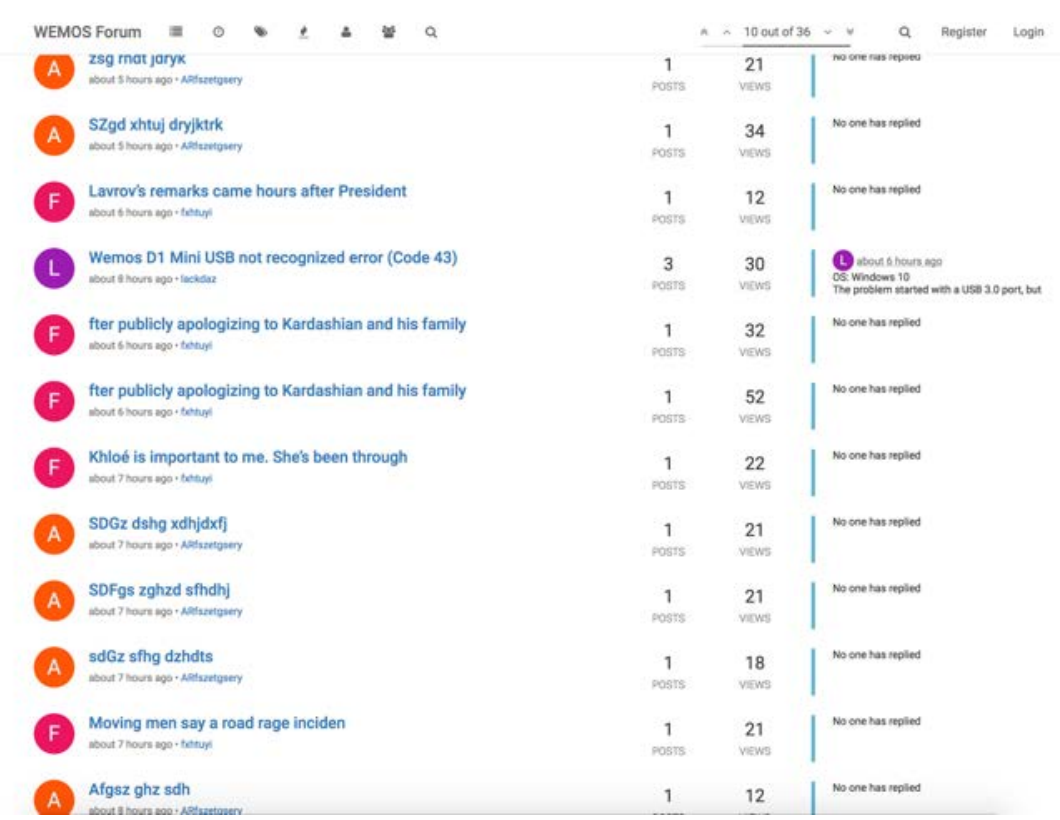

Figure 50. Author. (2017). Screenshot of the hacked forum.WeMos.cc website showing one legit user post in between spam.

I am glad that I copy-pasted some of the essential commands and instructions into my lab notebook or took the time to write up my step-by-step progress instead of just bookmarking the URL of a useful resource. However, many conversations with other users, posts of related questions and helpful answers on this forum are deleted from the web and appear to be no longer retrievable. 


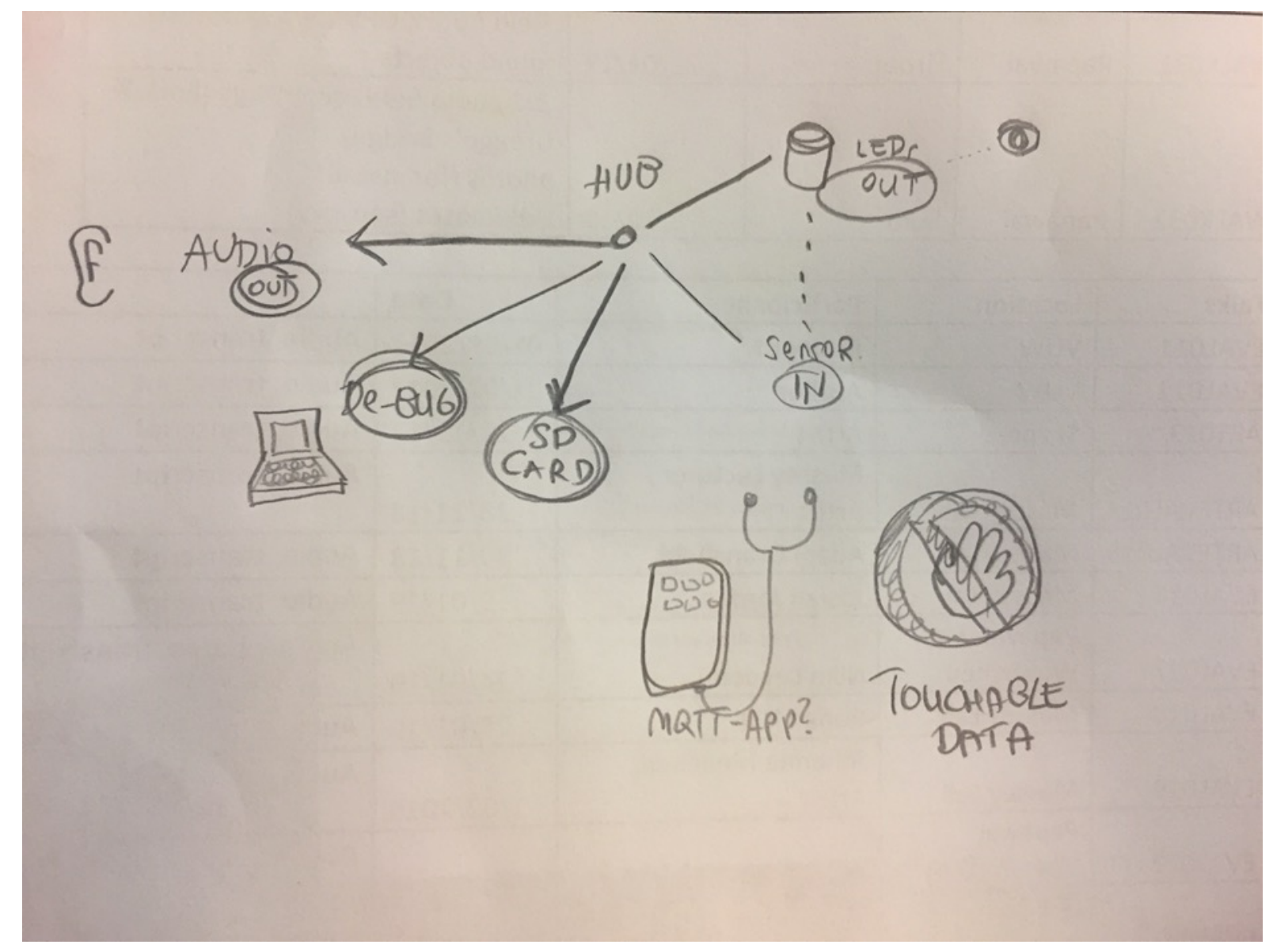

Figure 51. Author. (2017). Draft of the star network setup with the hub at the centre connecting possible nodes together. 


\subsection{Wi-Fi in a bottle: recycling materials for waterproofing electronics}

With this narrative I provide an overview of design developments starting in 2017 concerning the waterproof enclosures I built as part of the design research. I focus on the practical concerns that emerged when attempting to place electronics in an outdoor environment.

One early concern when embarking on the journey of developing an IoT with and for the stream was how to safely place the electronics into the environment, without the morethan-human world being harmed by the electronics, and, conversely, the electronics being safely enclosed and sheltered from the elements.

Designing the right kind of enclosures was a key concern during early prototyping that could only be addressed once I had settled on a hardware solution to move forward with and knew final dimensions of all components needed to complete one node. Once I had decided that a combination of multiple WeMos D1 mini microcontrollers as sensor nodes and one Raspberry Pi single-board computer as IoT hub were the final constellations for the Moturoa Transmissions installation, I had less than two weeks left before the work would be shown at its first public exhibition, the Upstream Art Trail. At this stage, the design parameters for the upcoming development sprint, as scribbled in my notebook, were the following:

- Working, open-source technology based customisable IoT setup

- Stable power supply for all nodes

- Consistent network communication between DIY nodes

- Safe, outdoor-proofed electronics

- Aesthetics and accessibility embracing a seamful design approach

With these parameters in mind the design of enclosures needed to be

- customisable to accommodate a variety of hardware configurations depending on the nature of the node 
- allowing space for a power supply

- not interfering with the Wi-Fi signal

- non-toxic to the environment, waterproof

- aesthetics: transparent, or otherwise allowing visual access to the components inside the nodes

Early research on IoT enclosures did not yield many examples where aesthetics were specifically taken into account. Tutorials would mostly focus on the electronics themselves, usually deployed in a lab for teaching purposes, where enclosures did not play a major role. Documentation of citizen science projects used either off-the-shelf enclosures or recycled enclosures (see for example Public Lab contributors, n.d.) or custom 3D-printed casings (see for example Adafruit, n.d.), which ended up obfuscating the electronic parts. Usually, developers would prefer inconspicuous designs of their deployed electronics to not attract attention out of concerns for theft and vandalism.

Online research yielded some inspirations for designing unconventional project enclosures. For example, a collection by make (Ragan, 2012a) includes materials beyond the usual off-the-shelf plastic and metal enclosures including recycled tin cans, parts from an old telephone, a coconut shell, or using resin to enclose an amplifier circuit (see also Hirst, 2011).

After assessing project enclosures that are predominantly used in electronics projects, particularly outdoor IoT works, it appeared that enclosures were primarily chosen for protecting, often hiding away circuitry, and their design and sculptural quality often play a secondary or no role in the project. Some creative project enclosures, going beyond the standard plastic or laser-cut box, are more commonly found in arts and music projects and include for example the re-use and recycling of cans and jars, parts of old electric devices, the use of natural materials such as wood and coconut or resin to house an electronics project (see for example Hirst, 2011; Ragan, 2012a; 2012b; protoman, 2009). 
Inspired by the combination of transparency and waterproofing qualities I started looking at the possibility of making the WeMos D1 weatherproof with resin (see for example Ragan, 2012b; protoman, 2009). However, this would take at least 48 hours to finish, involving steps of preparing a silicone mould first and then casting the components in resin. The advantage would be that components are fixed in one place and could not disconnect. The disadvantages are that parts would not be accessible anymore, and that the resin might interact with the circuits-however protoman (2009) mentions in a comment on his post that painting the circuit with acrylic lacquer would be an option to prevent chemicals from interacting with circuitry. Looking at the design parameters, customisability would not be impaired by components fixed in one place, and the process would not be easily repeatable. Given that this was an early iteration with little time for thorough testing, I abandoned the idea for pursuing a resin cast enclosure design.

Another early idea for material to be used to house my sensors was wooden pieces often found around the stream area after the deforestation of old pine trees. This idea, however, was abandoned early, before even considering the above design parameters, as the weight of the wood, just from carrying one piece back from the field, foreshadowed that this would be problematic during future installs, with multiple sensors in use.

Once I succeeded in having stable communication between nodes bouncing Hello World messages across my network, I started with the development of the low-cost electronic conductivity sensor, as the central component of this setup. Read more about the design of this sensor in section 6.5. The development of the sensor involved calibrating the readouts and comparing them to the digital TDS reader (see Figure 79). For this process I used distilled water to be used as the test liquid because it has the lowest possible conductivity. This process allowed me to ensure that the sensor is waterproof, as well as able to give consistent readouts when submerged in different kinds of water. The water used was purchased at the supermarket and came in 1-litre plastic bottles. By adding measured amounts of salt, I was trying to test, if and how my sensor reacted to changes of conductivity. 
Once the network was satisfactorily relaying readouts from the electric conductivity sensor node, and I had a good idea of the size and form of the components, I needed to make a decision on the design of the nodes. The design should be both suitable for presenting the piece as an installation at a public exhibition, with aesthetics underpinned by concepts of seamfulness, as well as protect the electronics and circuitry from more-than-human forces such as moisture, wind and heat.

The requirements for my project enclosure were the following:

Aesthetics of the enclosures was a main consideration for presenting the network in the context of a public art trail, with consideration for the following parameters for choosing materials and form of enclosures:

- Seamfulness (see also 4.4.2) Most off-the-shelf enclosures for electronic projects are rectangular boxes to fit components together as tightly and compactly as possible. While seamlessness helps make a project small and portable, the components are hidden from sight and the shape or form does not reveal the functionality of a project to a viewer.

Seamful design involves deliberately revealing seams to users, and taking advantage of features usually considered as negative or problematic (Chalmers, 2003, p.1 referring to Weiser, 1994; 1995).

A seamful IoT design provides access to the inner workings of the components and radically reveals and exploits limitations of technology as part of the design. Seamfulness can open up participation in the form of dialogue with an audience.

- Connection to location/participants: The design interventions are informed by the data collected together with participants during previously held design walkshops.

- Low environmental impact \& low cost: Recycled/recyclable materials should be considered for building enclosures rather than sourcing new materials from scratch, also minimising the cost for sourcing new materials and making the project more affordable to communities. 


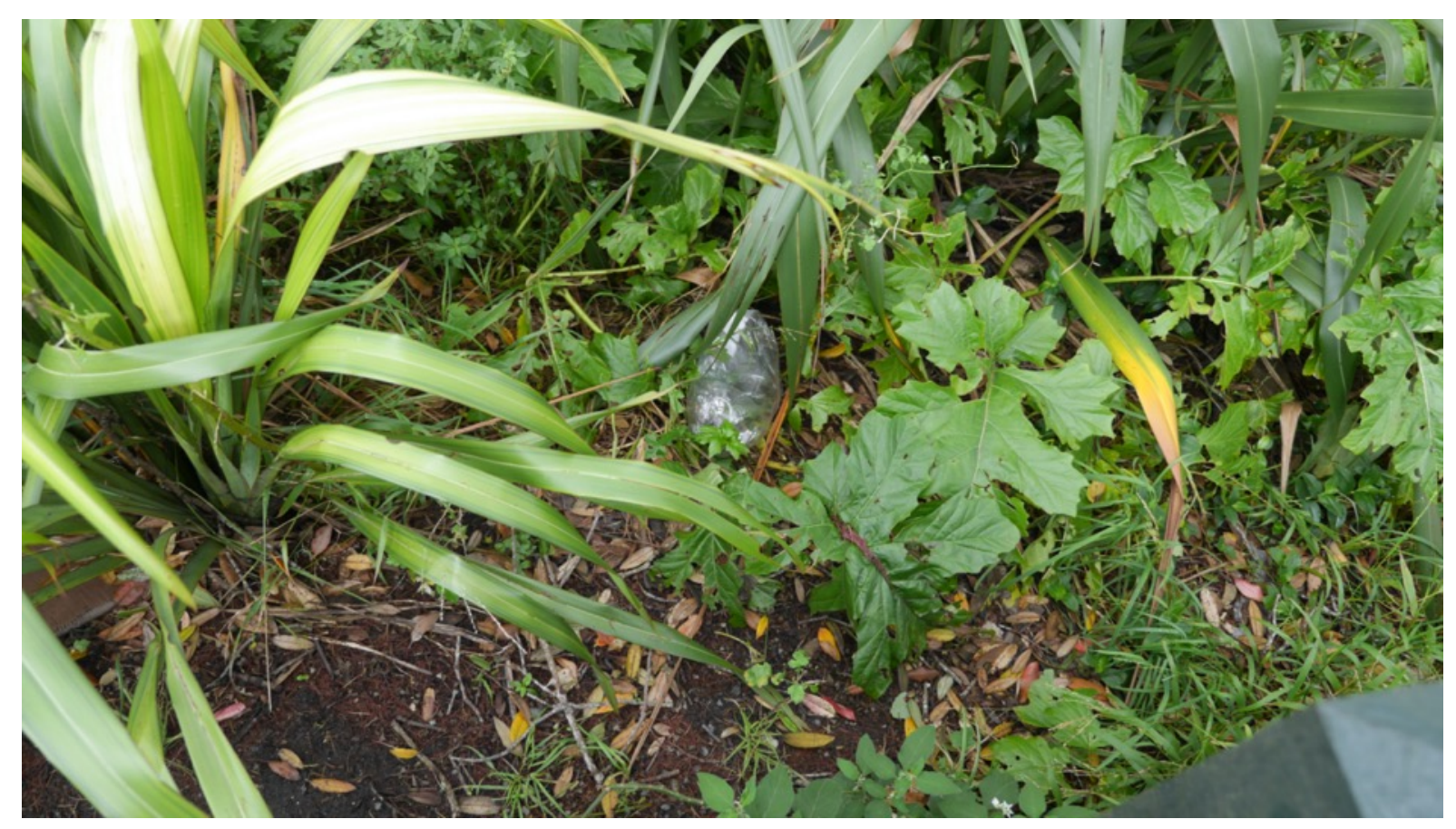

Figure 52: Author. (2017). Example of a plastic bottle found during a location visit at Moturoa Stream.

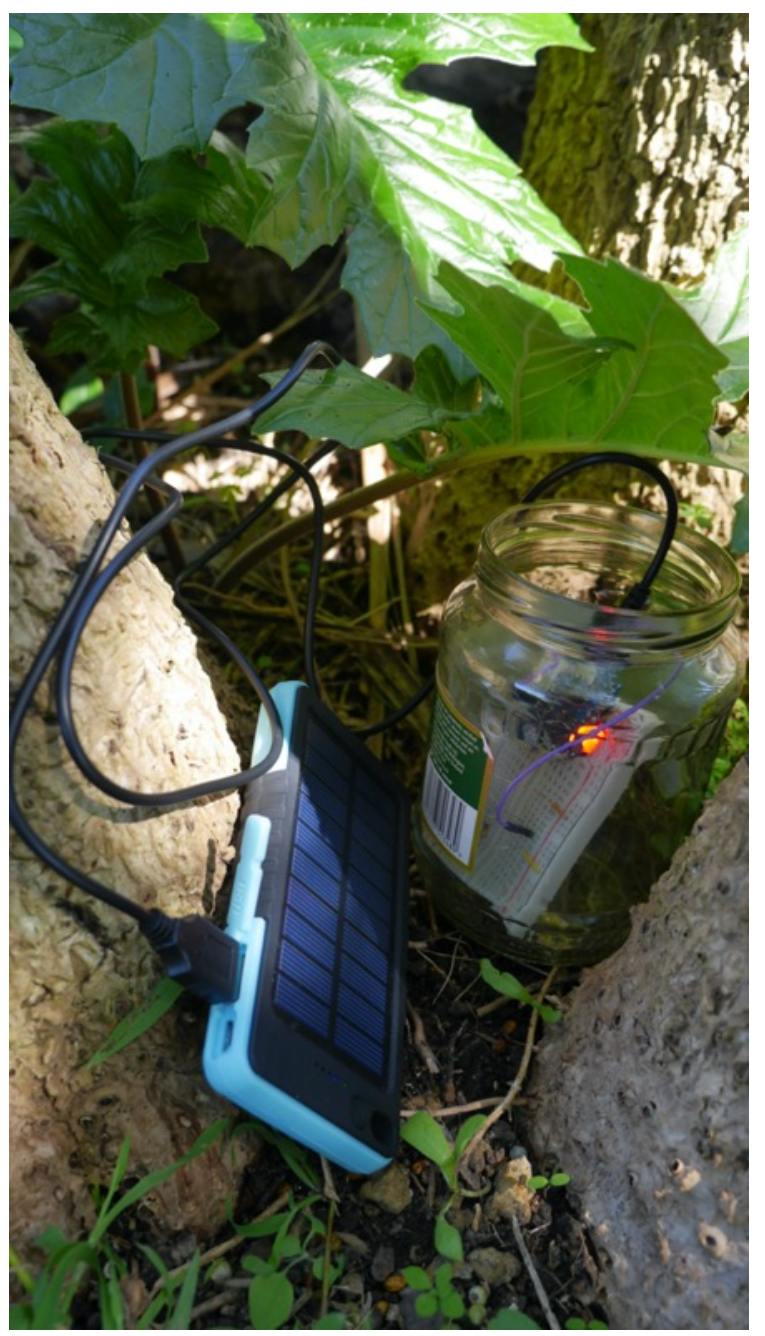

Figure 53. Author. (2017). Early prototype using a solar charged power bank with a WeMos D1 mini on a small breadboard in a glass jar. 
After some tests with off-the shelf enclosures and recycled glass and plastic containers, I decided to use the empty plastic bottles I had left over from testing my conductivity sensor in distilled water as a good resource for housing my components. The transparent casing additionally gives an audience visual access to all the electronics, the microcontrollers and components, and reveals the processes that went into the assembly of the hardware by revealing wires, connections, and solder points that are usually hidden away in consumer electronics.

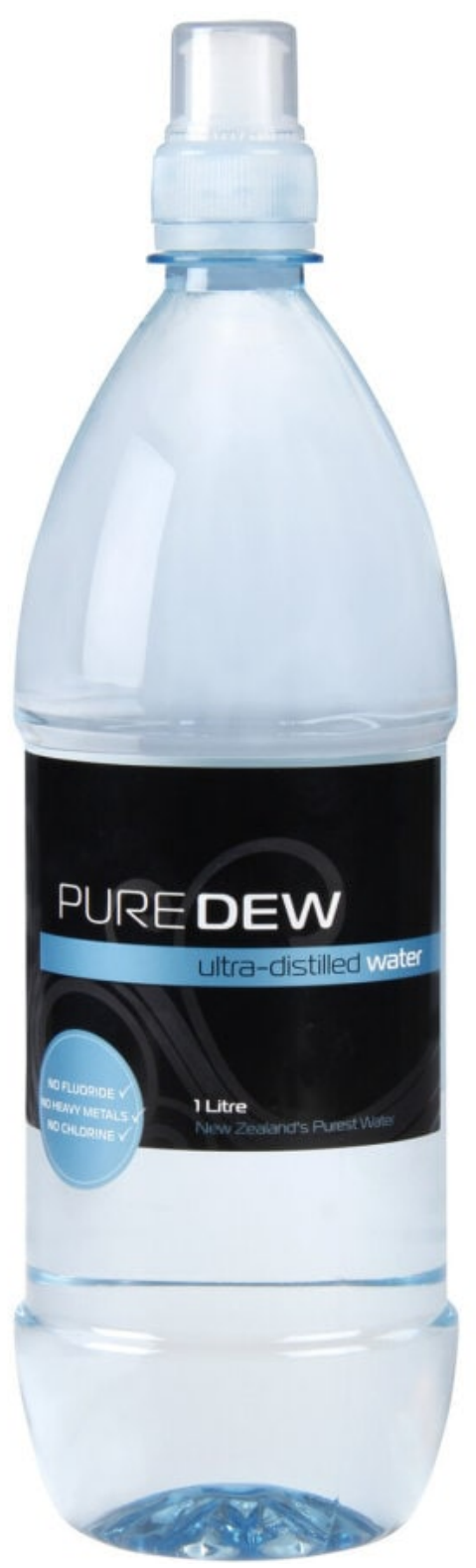

Figure 54: Countdown Ltd NZ. (2019) Product image of the water bottle. 
To re-use the empty bottles as enclosures for housing my electronics prototypes, I place them outside on my clothes airer (see Figure 55). Removing the label turned out to be quite a delicate task, and the remaining glue on the bottle left a quite sticky film, which not only attracted dust and other particles to it, but also lessened the transparency of the material (see Figure 56) which I could only carefully remove with an acetone-based nail polish remover.

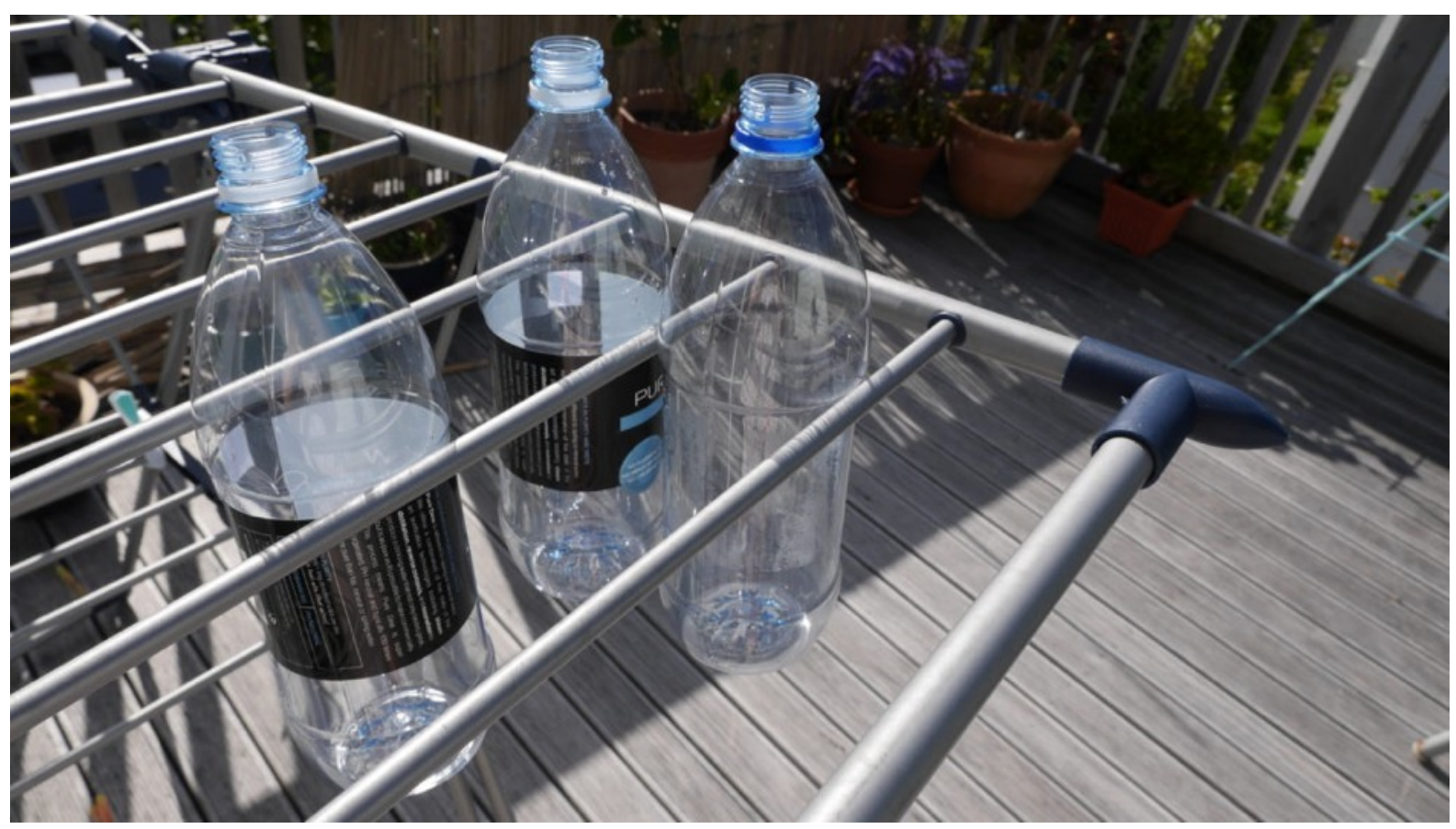

Figure 55. Author. (2017). Drying empty water bottles on the deck in the sun.

The next step was to find a suitable way to fit components into the bottles, which I started by carefully slicing my bottles open and experimenting with different placements of the components inside them. Housing the Raspberry Pi with the external Wi-Fi antenna and the larger battery pack into one of the bottles was the first challenge, as I envisioned the antenna on the outside of the bottle, with the electronics safely housed on the inside. In a first proof-of-concept (Figure 56), I imagined the bottle being suspended sideways, with the antenna sticking out of the opening at a right angle, plugged into one of the four USB ports, which neatly aligns with the bottle opening. While the bottleneck was big enough to fit the antenna through, it was too thin to fit the antenna board. This led me to remove its plastic cover, which did make the board itself more vulnerable with some of the fragile connections exposed, but the look eventually did align with the design idea to leave components uncovered and open. 


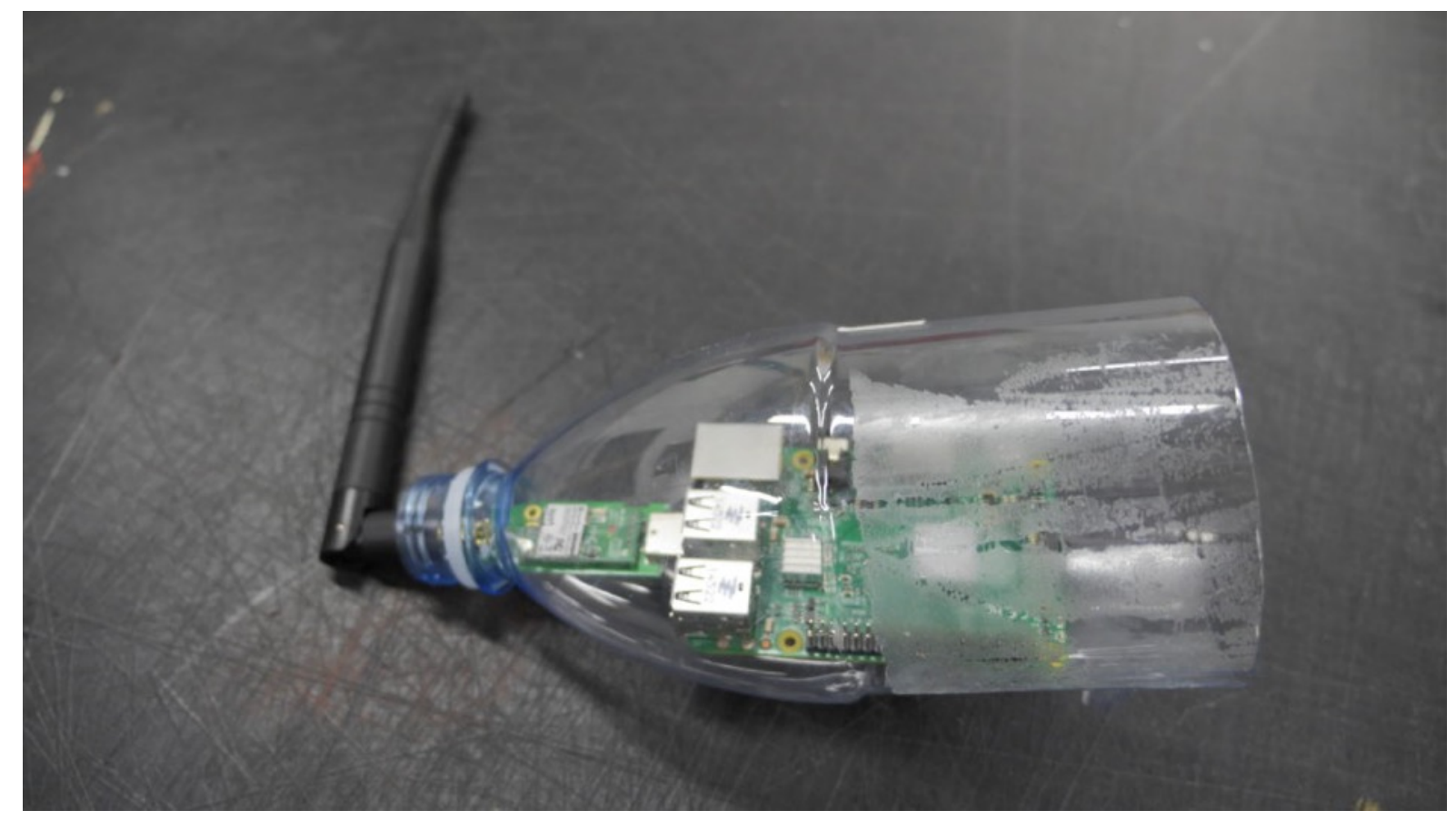

Figure 56. Author. (201). First proof-of-concept for using a water bottle as project enclosure.

Once I had a fair idea that the combination of bottles and electronics would be the way forward, I needed to make sure that components would sit neatly and safely inside the bottles in order to minimise the risk for any of the connections to break or disconnect. With the support of 3D workshop staff, I designed laser-cut cardboard prototypes which would act as inserts inside the bottle to keep components in place without obfuscating them. The first iterations of these inserts assumed that they would act as a flange between the two bottle halves, as well as provide a stable ring around the enclosures which could be used to suspend the bottles during installation. 


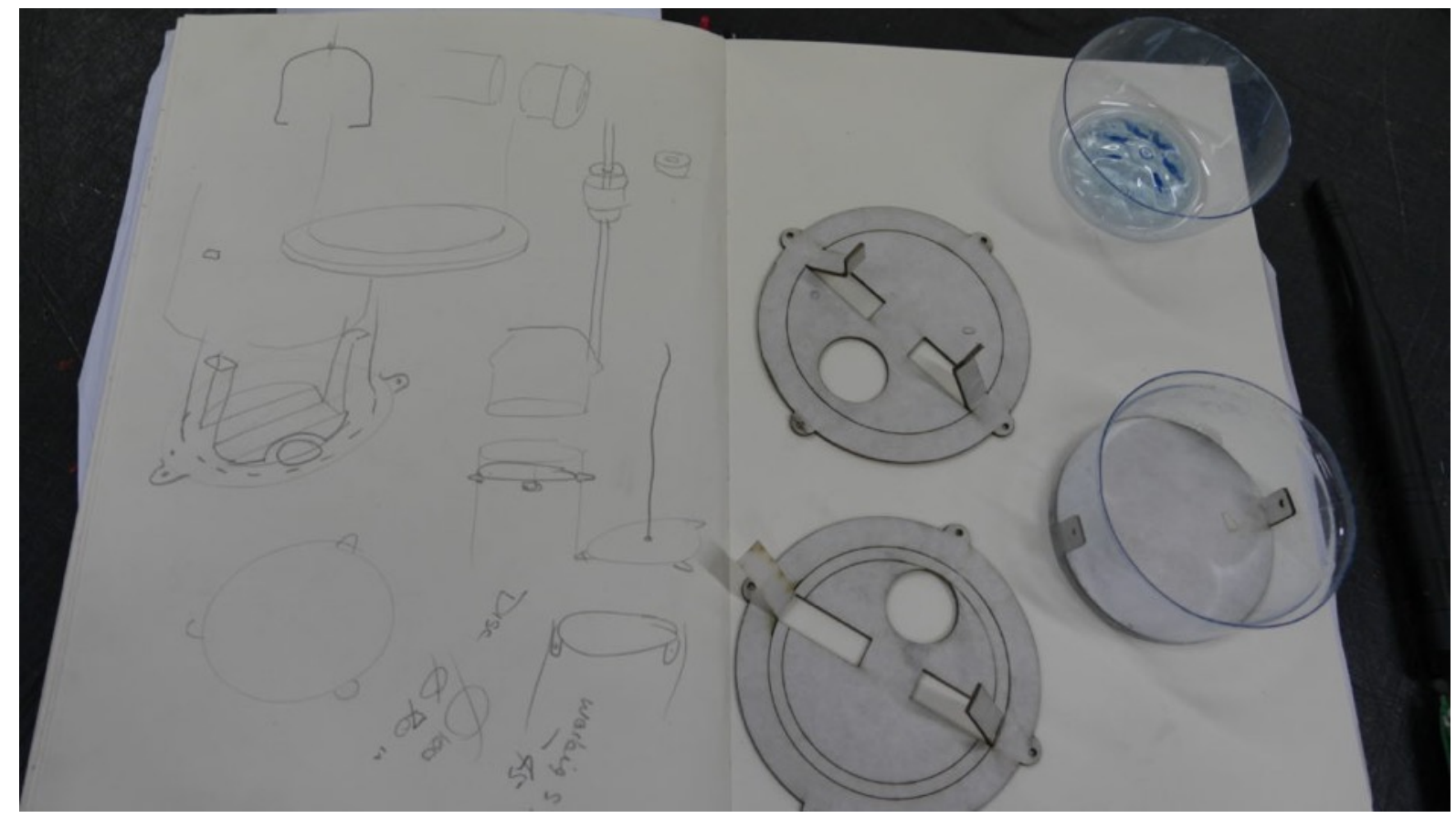

Figure 57. Author. (2017). Sketchbook showing early drafts for bottle inlays with laser-cut cardboard concepts.

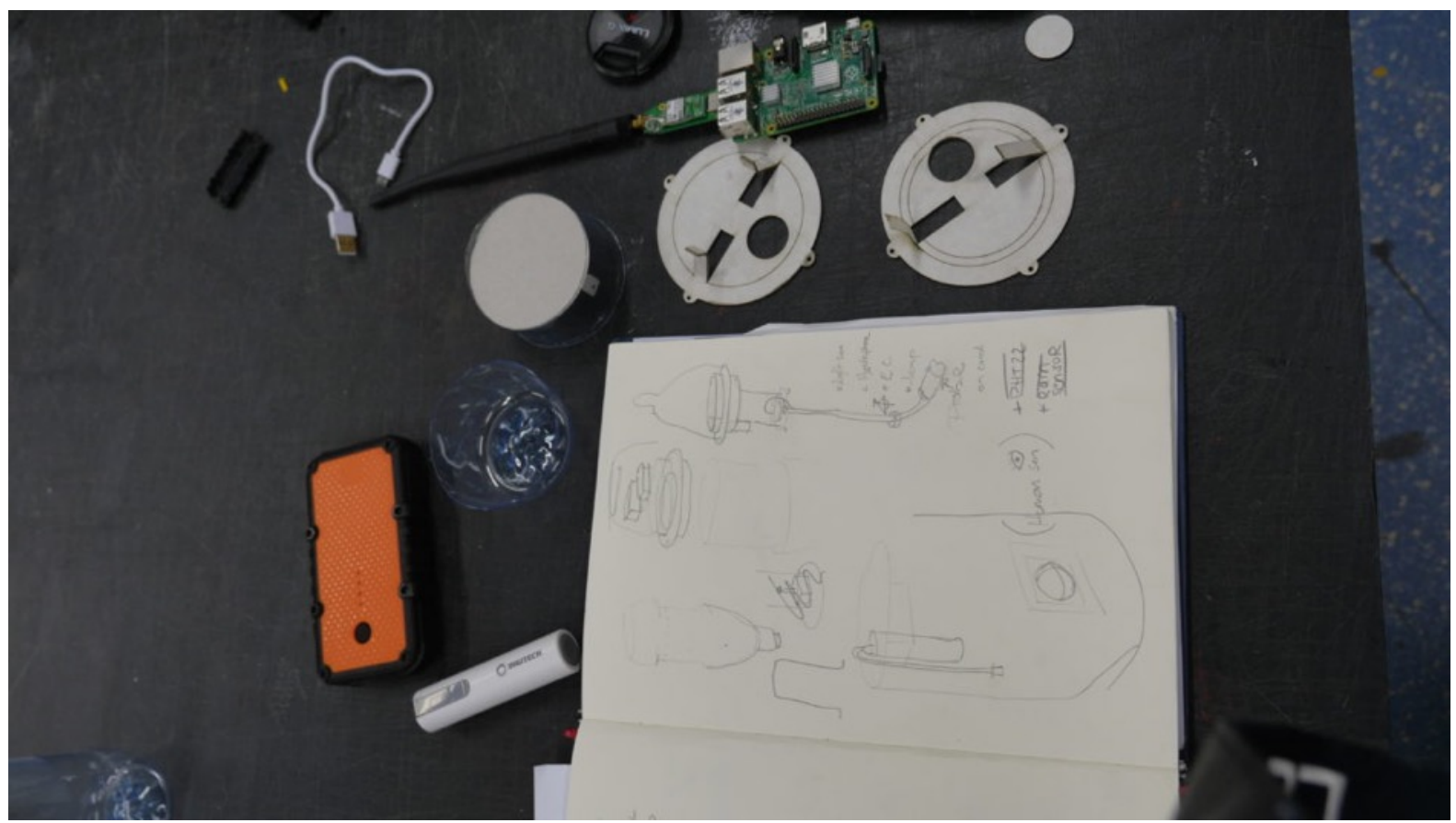

Figure 58. Author. (2017). Prototyping lab table showing sketchbook with early drafts for EC sensor probe with laser-cut cardboard concepts and components.

While the idea of the inserts technically worked for housing the components and providing a surface for a hanging mechanism, the flange would need to be sealed to waterproof the components, which added a layer of complexity to a design that needs to be easily deassembled and re-assembled. After experimenting with a few different sizes of inlays, all ideas for hanging mechanisms were scrapped due to waterproofing issues. Eventually, the 
idea of simply stacking the top of the bottle half into the bottom half to create a reasonably sealed transition provided new ground for design experiments. The first prototype of this idea (Figure 59) featured a bent metal rod that held both bottle halves together, as well as kepeping the cardboard insert in place.

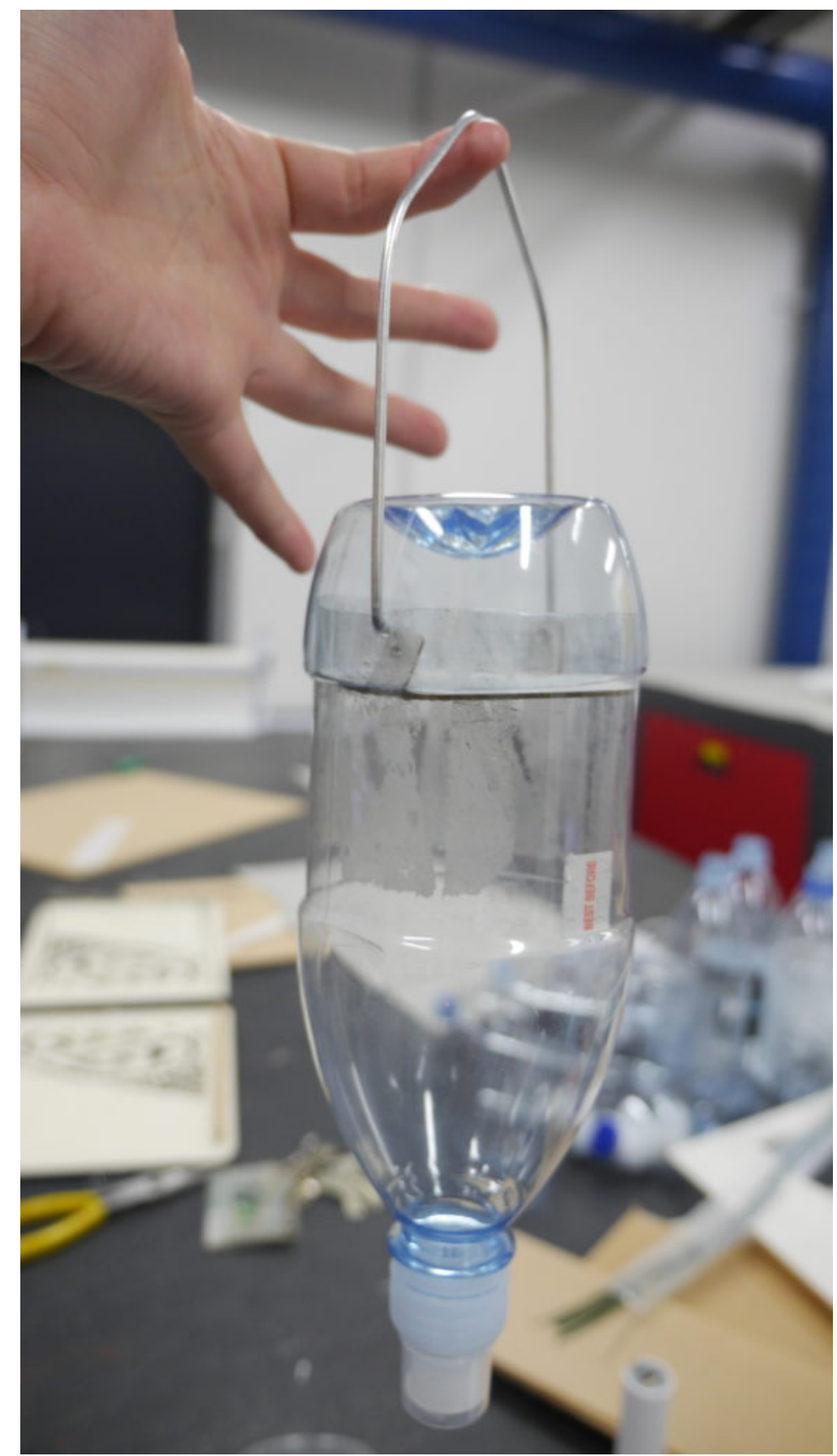

Figure 59. Author. (2017). First concept for enclosure assembly with bent metal rod for suspension.

The idea of suspending the bottle on two points through drilled holes with one bent metal rod appeared satisfactory at this stage, as it provided a simple and quite stable hanging mechanism that was easy to replicate and made use of minimal additional material. At this 
point, the focus turned to iterating on the design of the inlays in correspondence with the hanging mechanism to house the microcontroller and its battery.

Again, starting with designing cardboard prototypes, I developed the idea of a bracket to keep the WeMos D1 mini board in place. The long header pins soldered to the board would be used to attach the board to the angled inlay (Figure 60 \& Figure 61).

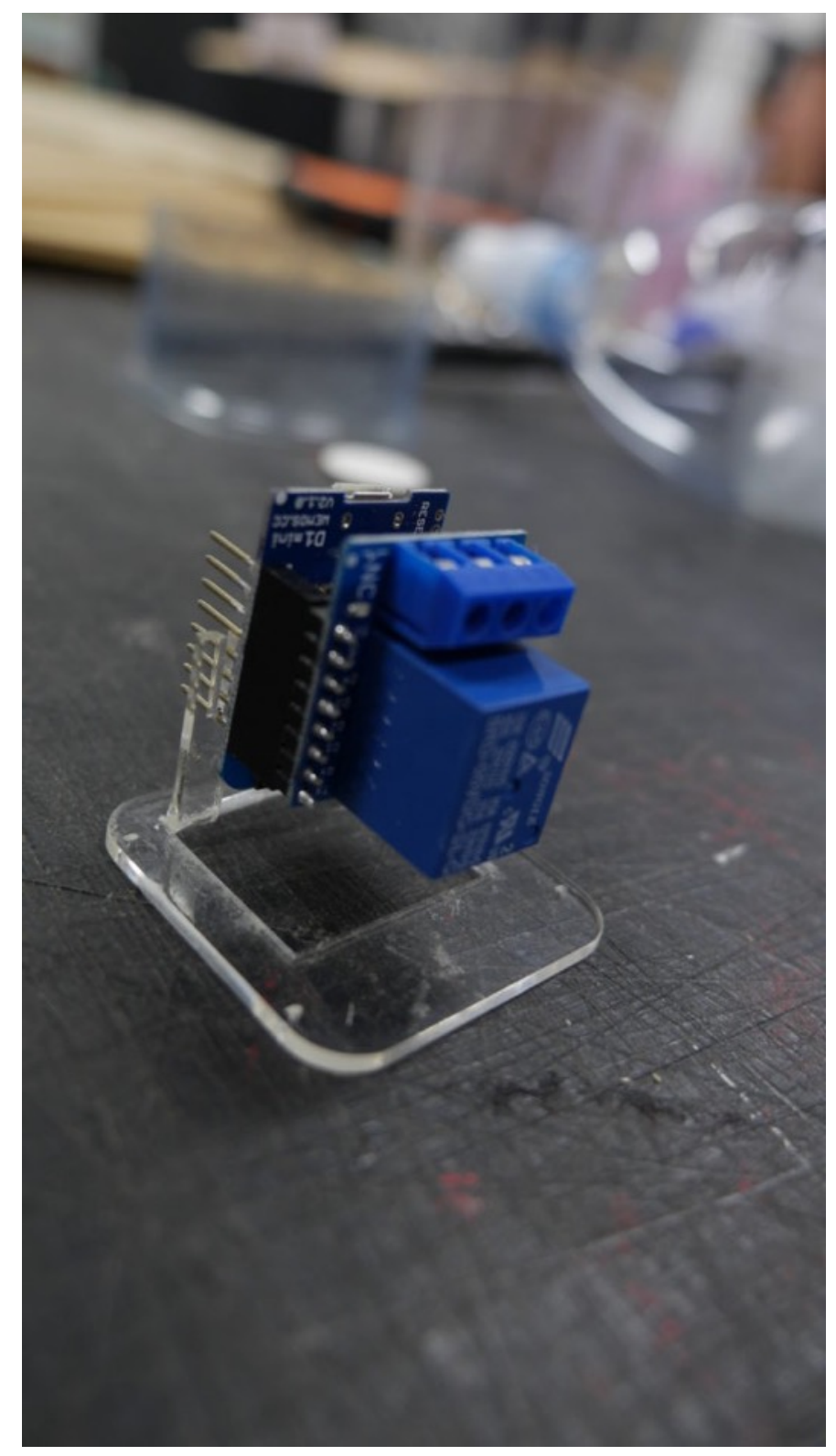

Figure 60. Author. (2017). WeMos D1 mini incl. relay shield attached to acrylic inlay prototype with header pins. 


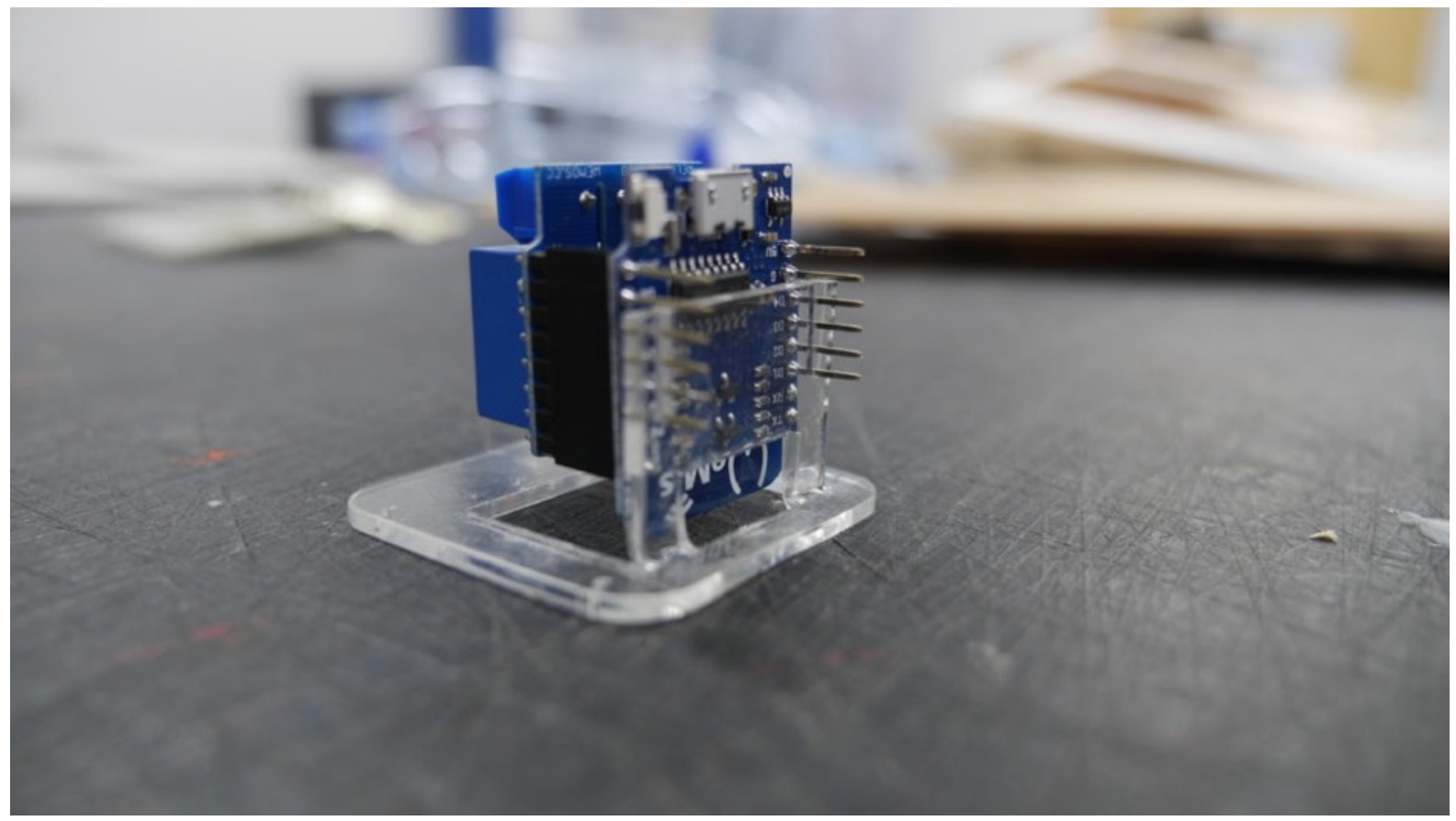

Figure 61. Author. (2017). WeMos D1 mini incl. relay shield attached to acrylic inlay prototype with header pins.

The design of this component should accommodate keeping the microcontroller securely in place as well as allowing enough room for extra shields to be stacked up on the board (see for example the relay shield attached in Figure 60) to keep the sensor design open for future additions and iterations.

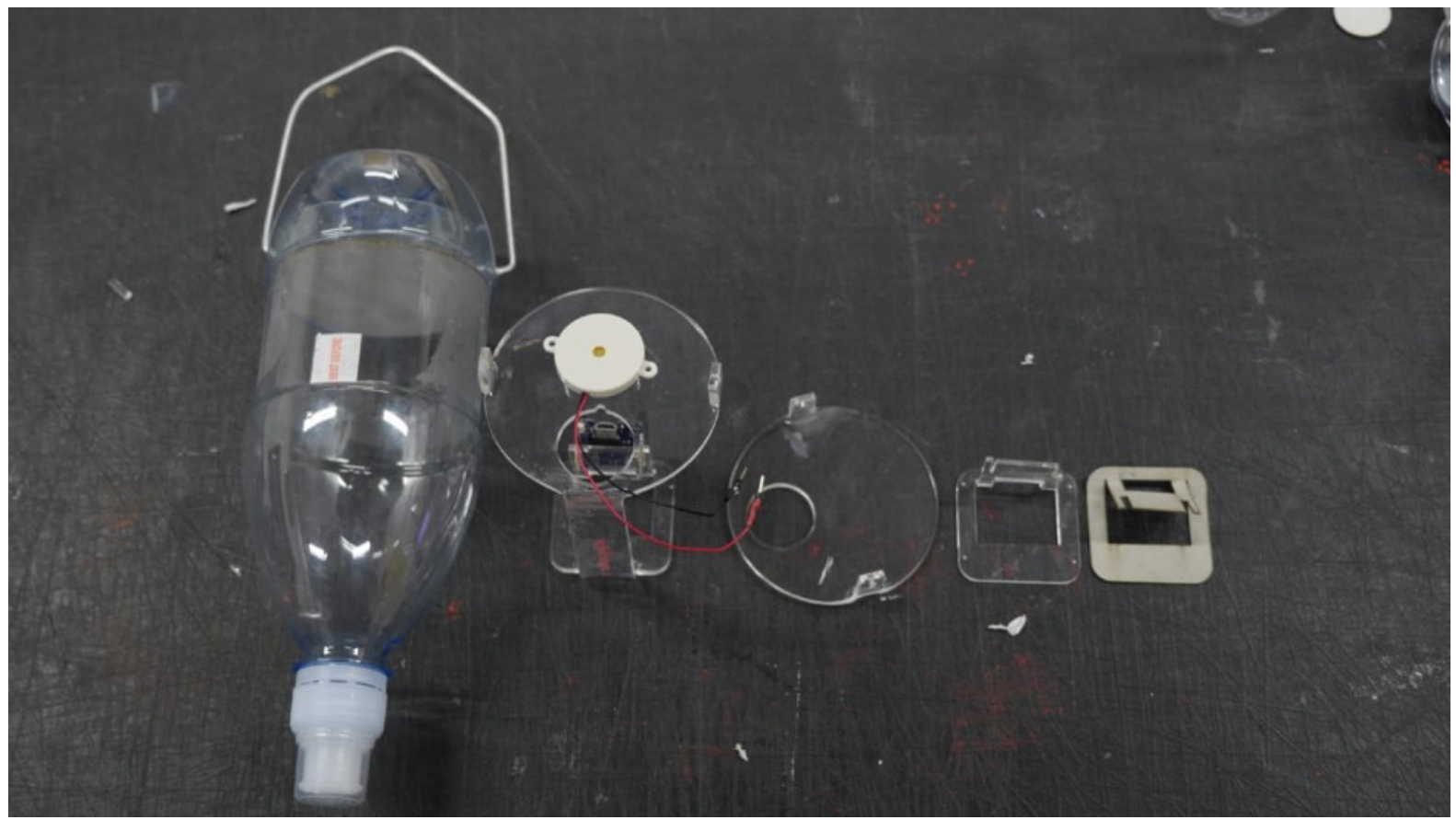

Figure 62. Author. (2017). Overview of the iterations showing variations of inlay designs and the final bottle assembly 
The next iteration of the inlay saw a combination of the new board attachment with the previously designed disk as part of the enclosure assembly. The added bridge between the two parts is designed to give support to the battery and also features the wildthings. io lettering.

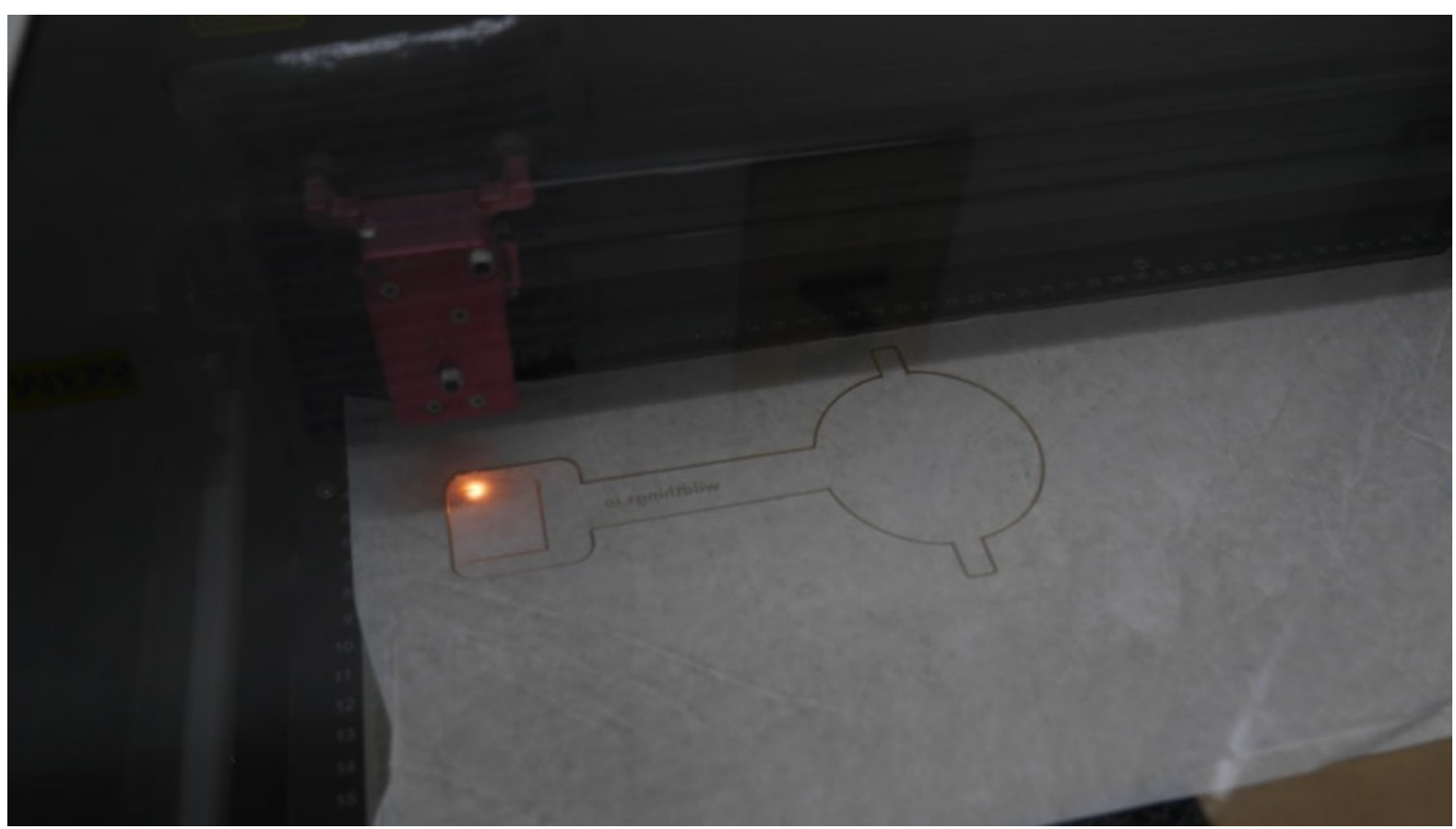

Figure 63. Author. (2017). Laser cutting one of the acrylic inlays for keeping battery and microcontroller in place.

After shaping the inlay with the heat bender and adding a red colour for testing the lettering, the first assembled prototype for the components to be fitted into one of the bottles is completed (Figure 64). 


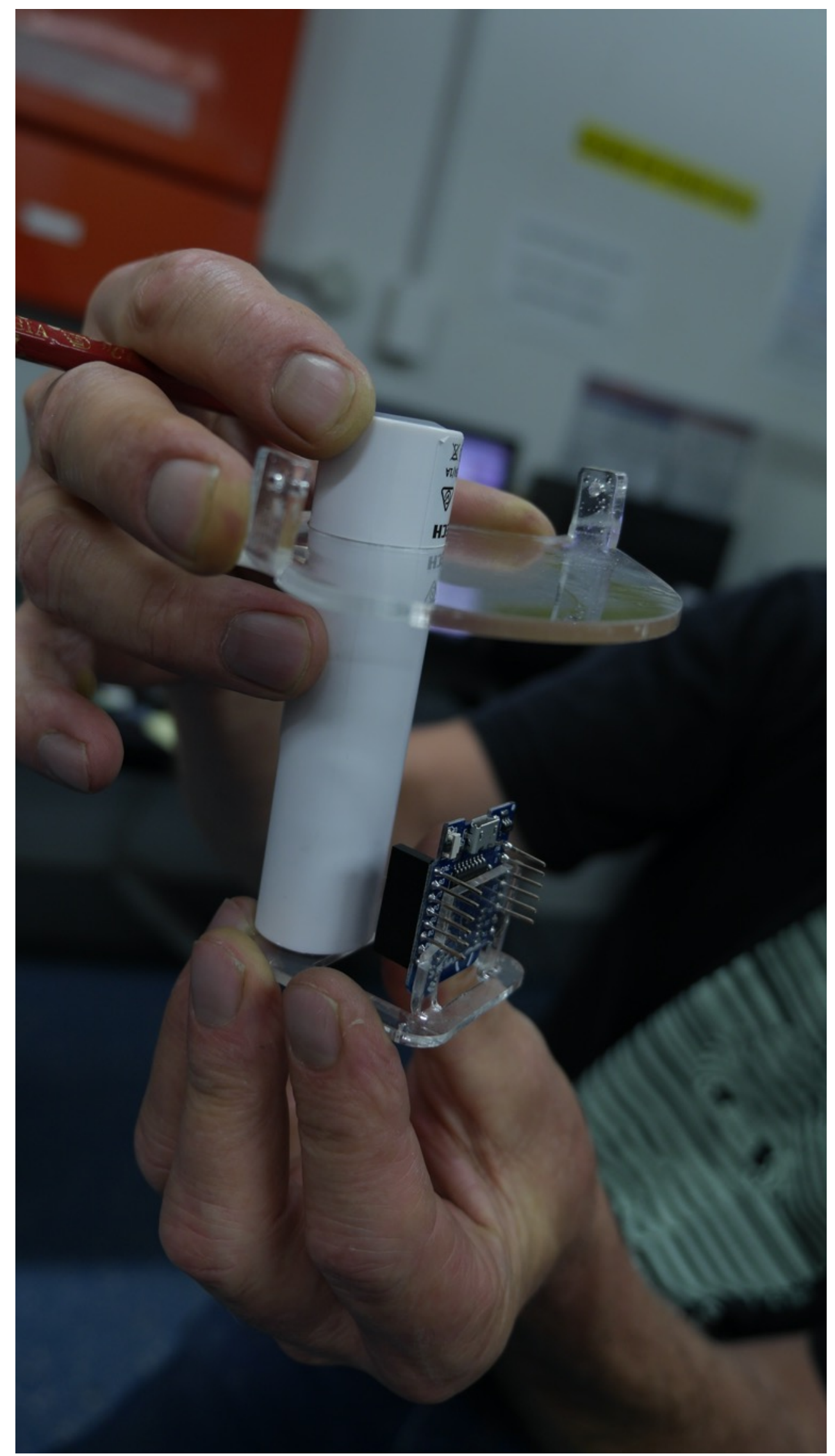

Figure 64: Author. (2017). The finished acrylic insert holding both battery and microcontroller in place. 


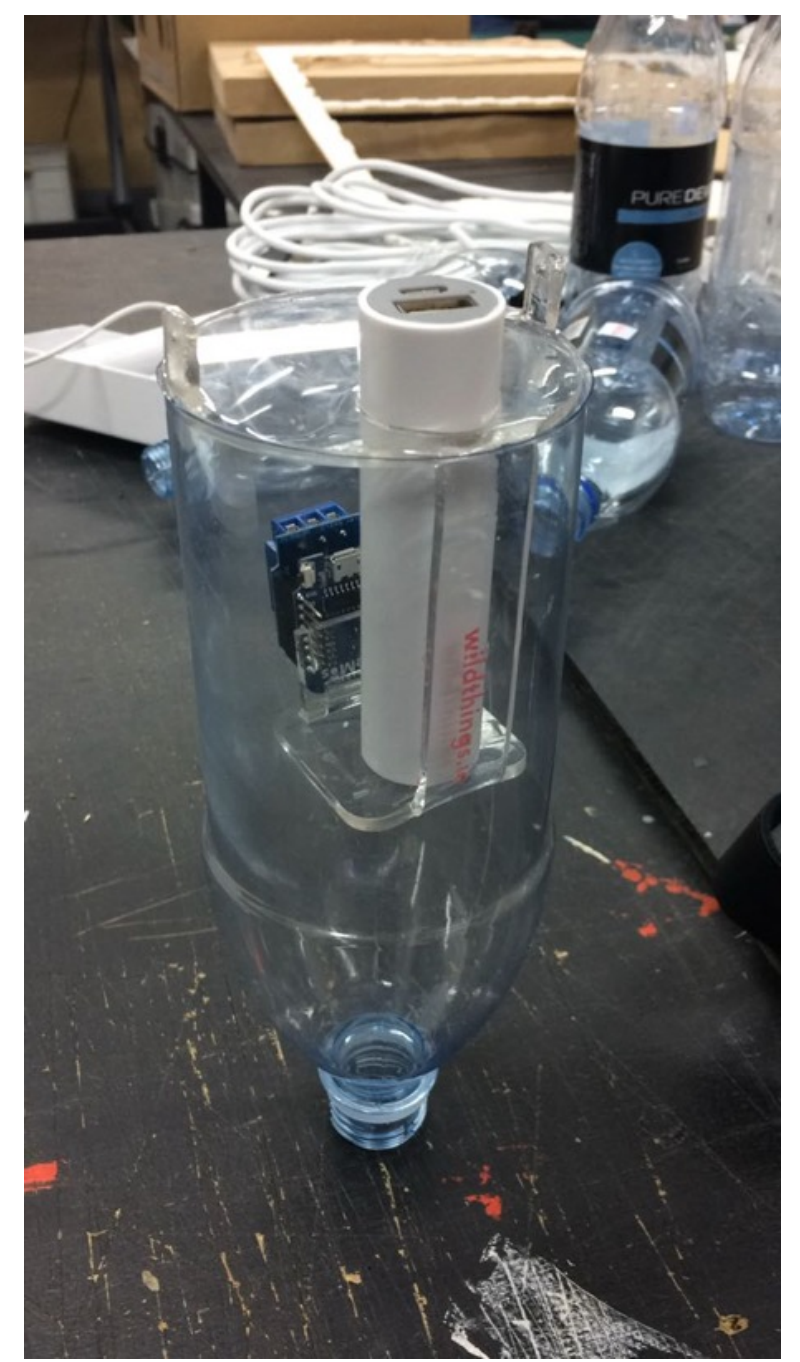

Figure 65: Author. (2017). Final prototypical rendition of the acrylic inlay inside the bottle enclosure.

I posted a photo of the assembled prototype (Figure 66) on the WeMos forum with the following question:

Me:

I re-used the water bottle I did my DIY EC conductivity testing with and am currently a custom inlay for battery and WeMos+shield(s)

The sensor cable will go through the bottom. The top will be covered with an end piece of the bottle with a slightly bigger diameter to shield from rain entering the circuit. I also add a bag of silica to get rid of damp inside.

Response by deshipu:

If this is going to be installed outdoors, you might also make sure that it is insect-proof (and other creepy-crawlies), as well as protected against mold[sic]. That means that you want to cover those USB sockets with something tight, not just protect it from rain from above. (birgit \& deshipu 2017, February 24). 
Tests before the planned three day install during the Upstream Brooklyn Art Trail showed that the overlapping bottle parts were tight enough to keep rainwater out and the added silica bags should help absorbing any excess humidity that may accumulate inside the bottle during the install. The drilled holes in the plastic for the metal rod suspension were more likely to be the weak spots of the design in terms of outdoor resistance, hence waterproof sealant was added after assembly to minimise the risk of water or insects entering the enclosure through this opening.

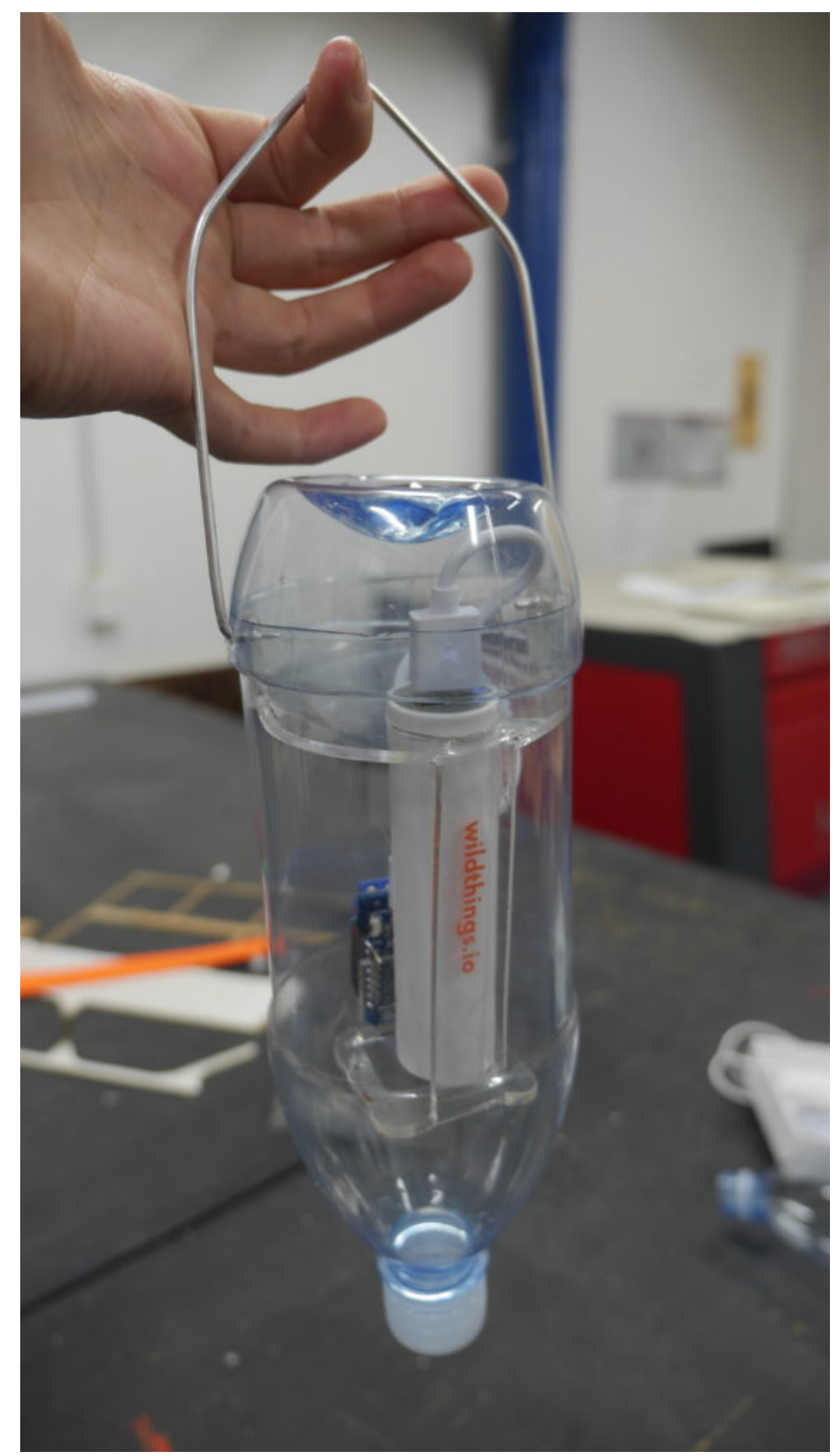

Figure 66. Author. (2017). Final prototypical rendition of the bottle enclosure including hanging mechanism. 


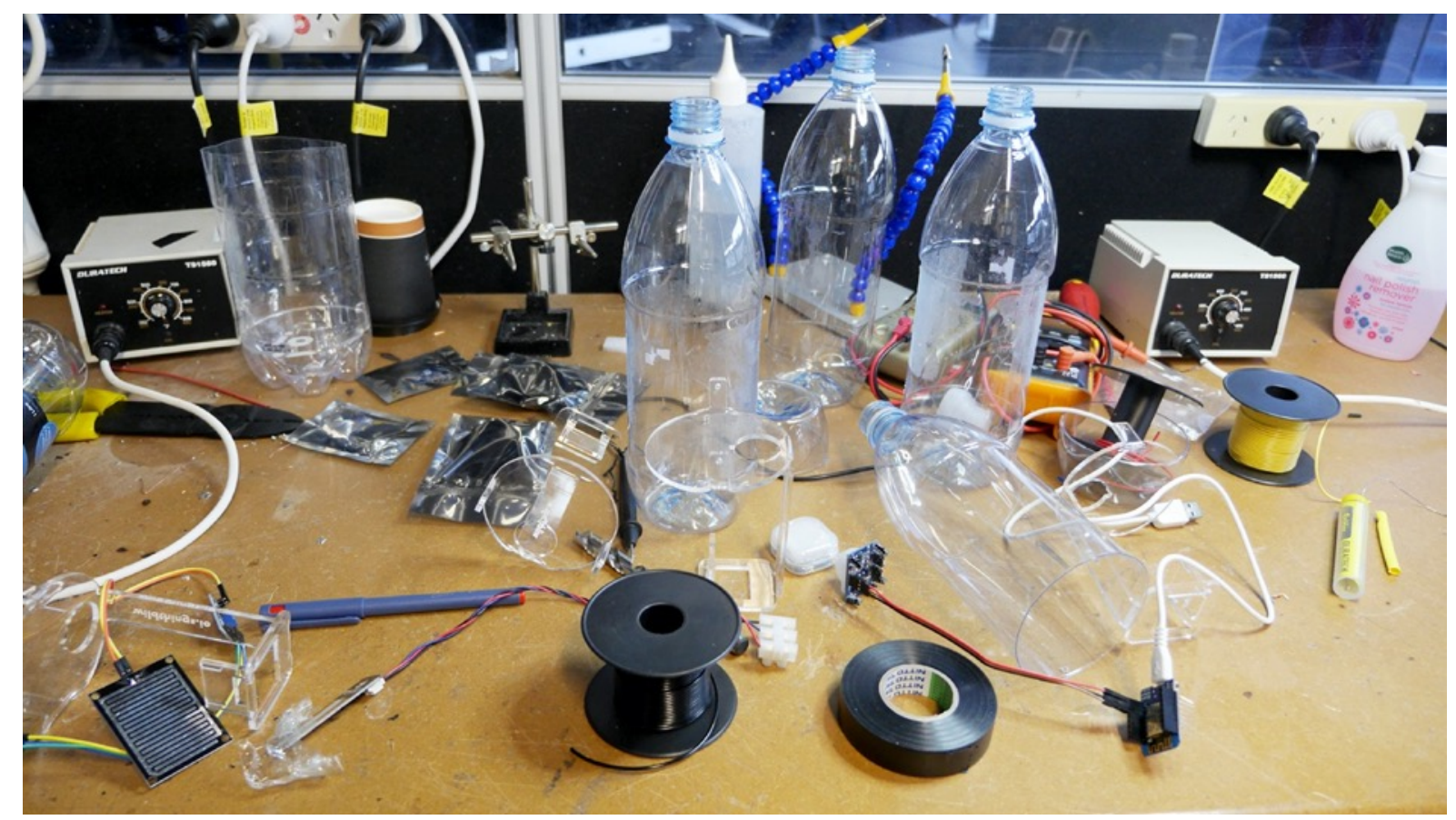

Figure 67: Author (2017). Workbench in the electronics workshop while assembling Moturoa_Transmissions.

Once the plastic bottle with the acrylic insert was confirmed as the basic enclosure for Moturoa Transmissions, considerations on how this design could be modified to accommodate each kind of probe needed to be made. Probes featuring a sensor to be submerged in the water, such as the EC sensor and the water temperature sensor, needed consideration on how the wires could be safely channelled to the outside, without impeding on the waterproof qualities of the enclosure. The bottle opening offered a convenient option to be used as a cable duct. Fitting the screw top with a store-bought waterproof cable gland the provided a secure mount for the wires exiting the bottle. The design imagines the possibility to remove or change the sensor if needed, only requiring some rewiring work on the inside of the bottle and leaving all wires on the outside untouched. For extra safety I added some sealant on the inside of the gland. It was also important to leave enough cable on the inside of the bottle to remove the screw top, if needed (Figure 68).

Another component that made use of the bottle screw top was the external antenna for the Wi-Fi hub. The external antenna connector was attached through a fitted hole drilled in the bottle top, securing the circuit board safely on the inside of the bottle, while the antenna sits on the outside (Figure 70). 


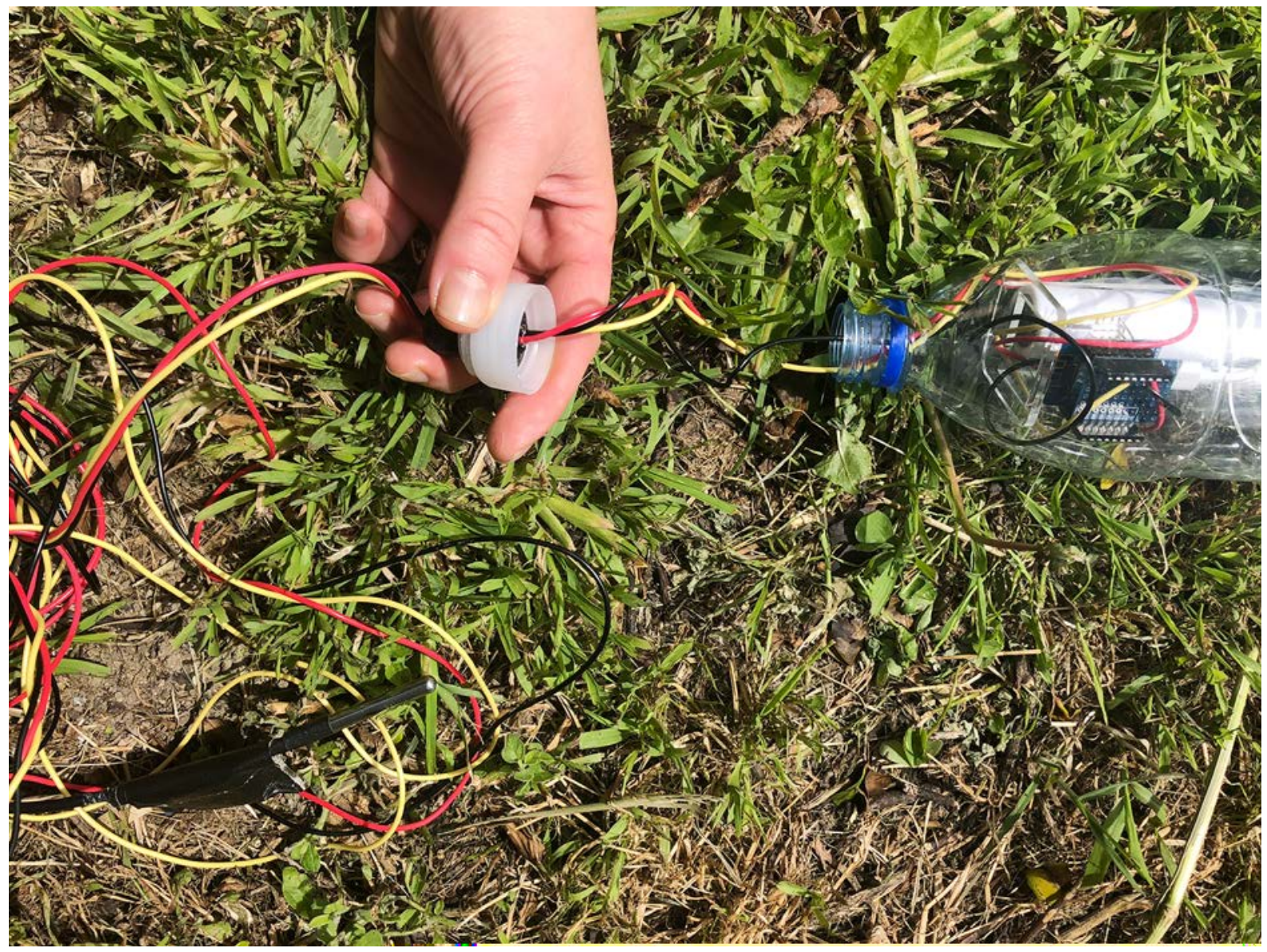

Figure 68: Author (2020). Temperature probe with screw top cable gland.

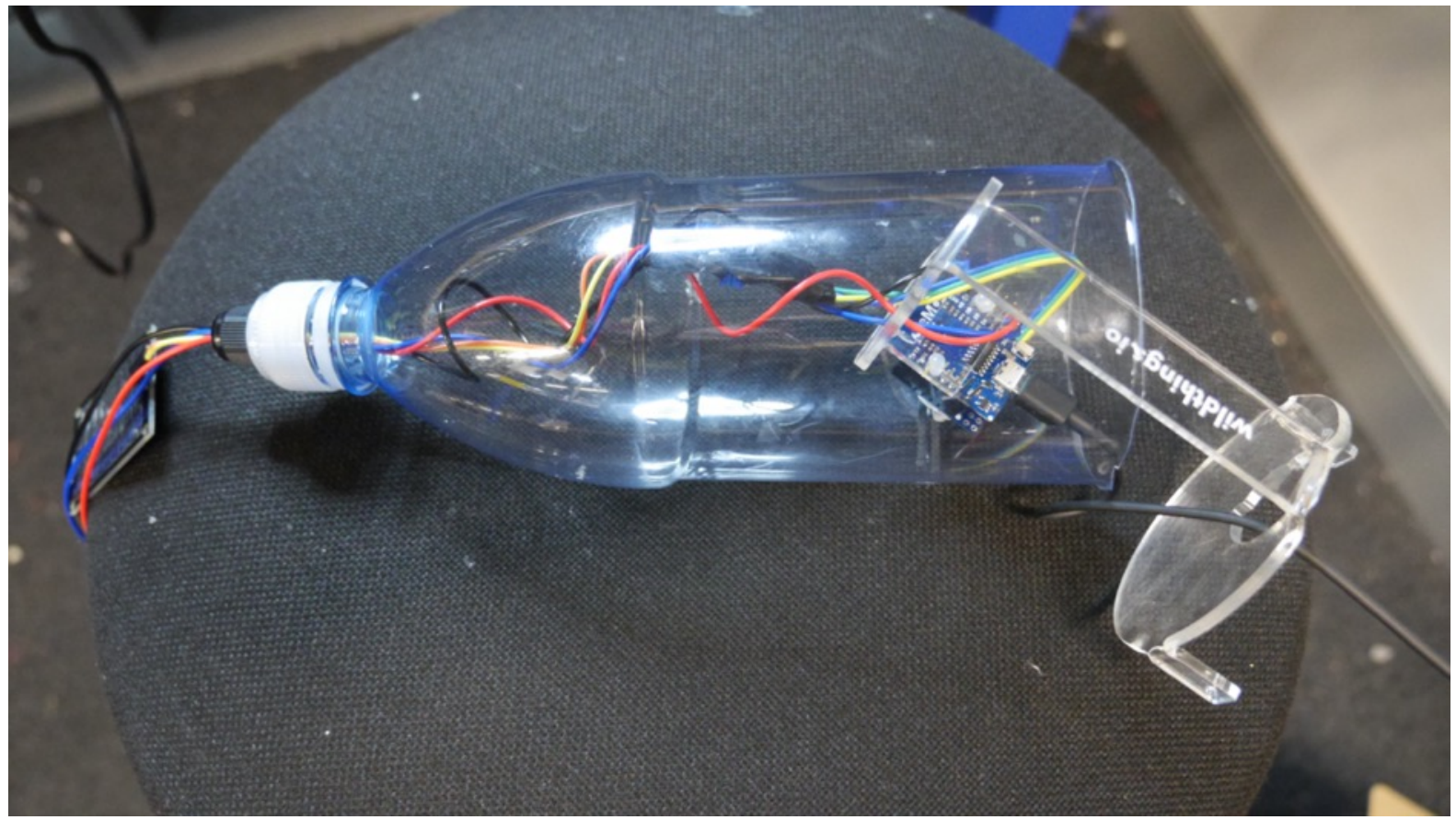

Figure 69. Author (2017). Moturoa Transmissions node disassembled for battery charging. 


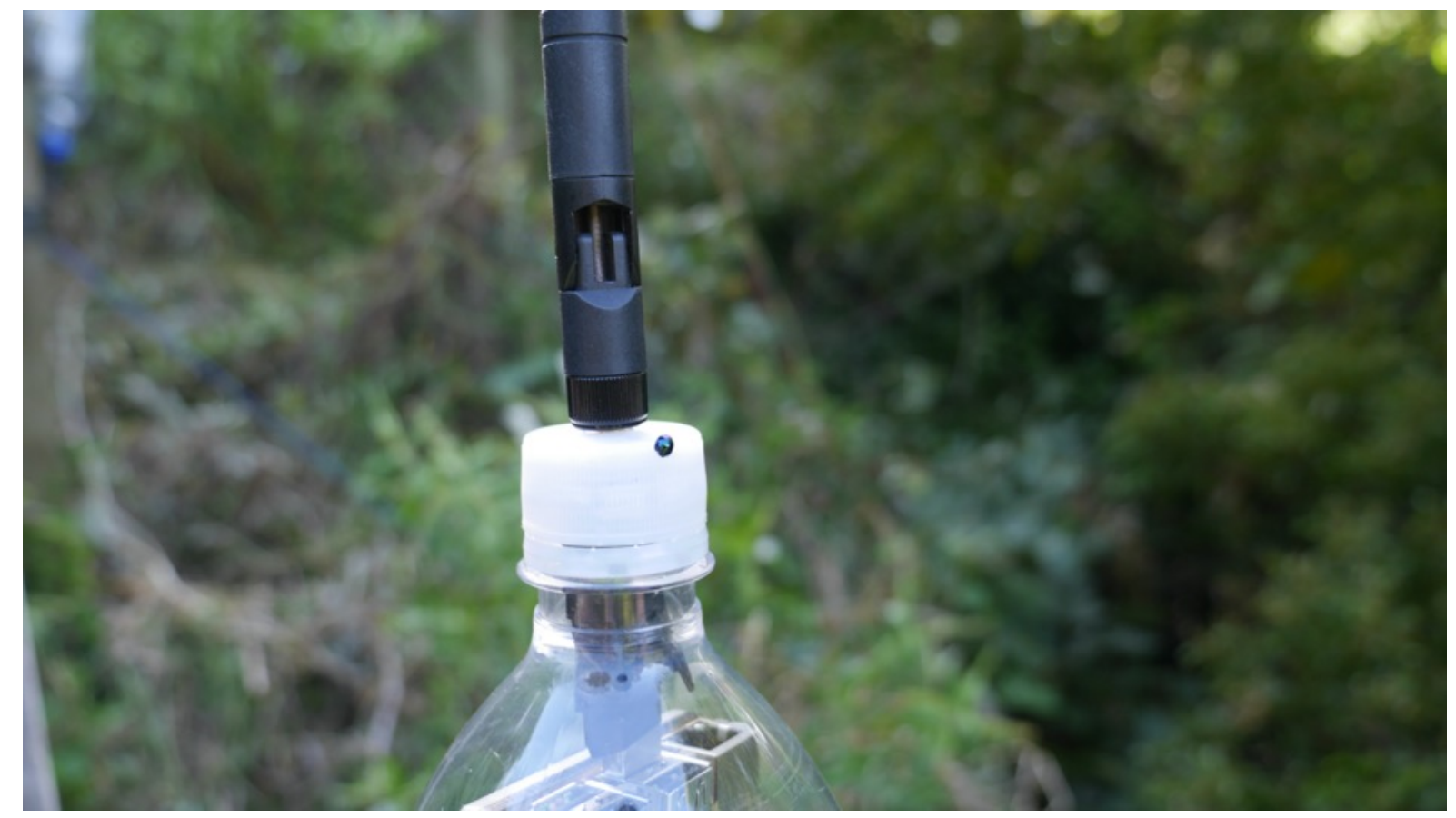

Figure 70: Author (2017). Close-up of the Wi-Fi hub node with a steel blue ladybug exploring the outside of the enclosure.

After the first public install, I assessed how the enclosures made from recycled plastic in combination with laser-cut acrylic fared as a material suitable within a more-than-human design context. The upcycling of the water bottles generally worked well, but did not present a sustainable design concept, as the plastic already showed early wear in the form of small cracks from repeated opening and closing of the containers for charging. With the idea in mind that the nodes could be easily re-built, the access to a laser cutter could be a barrier in the design, so this component would also benefit from simplification.

Accordingly, for further iterations of the enclosure design, modified glass jars replaced outworn bottle enclosures, and the laser-cut acrylic inlays were simplified in the form of paper and cardboard pieces. Glass containers were also encountered as trash during my early stream explorations (see Figure 18), so again this material gives a nod as to how human-made materials are in composition with the non-human world, slowly reappearing on the surface after having been disposed of. 
To give the glass jars the longest and most useful possible life span as part of this project I would often bring them to the electronics lab in the form of a lunch container. Then, after thoroughly cleaning the jars, they would be used as test enclosures for new nodes, or as vessels to store water for testing of submerged sensors in the lab (see Figure 71).

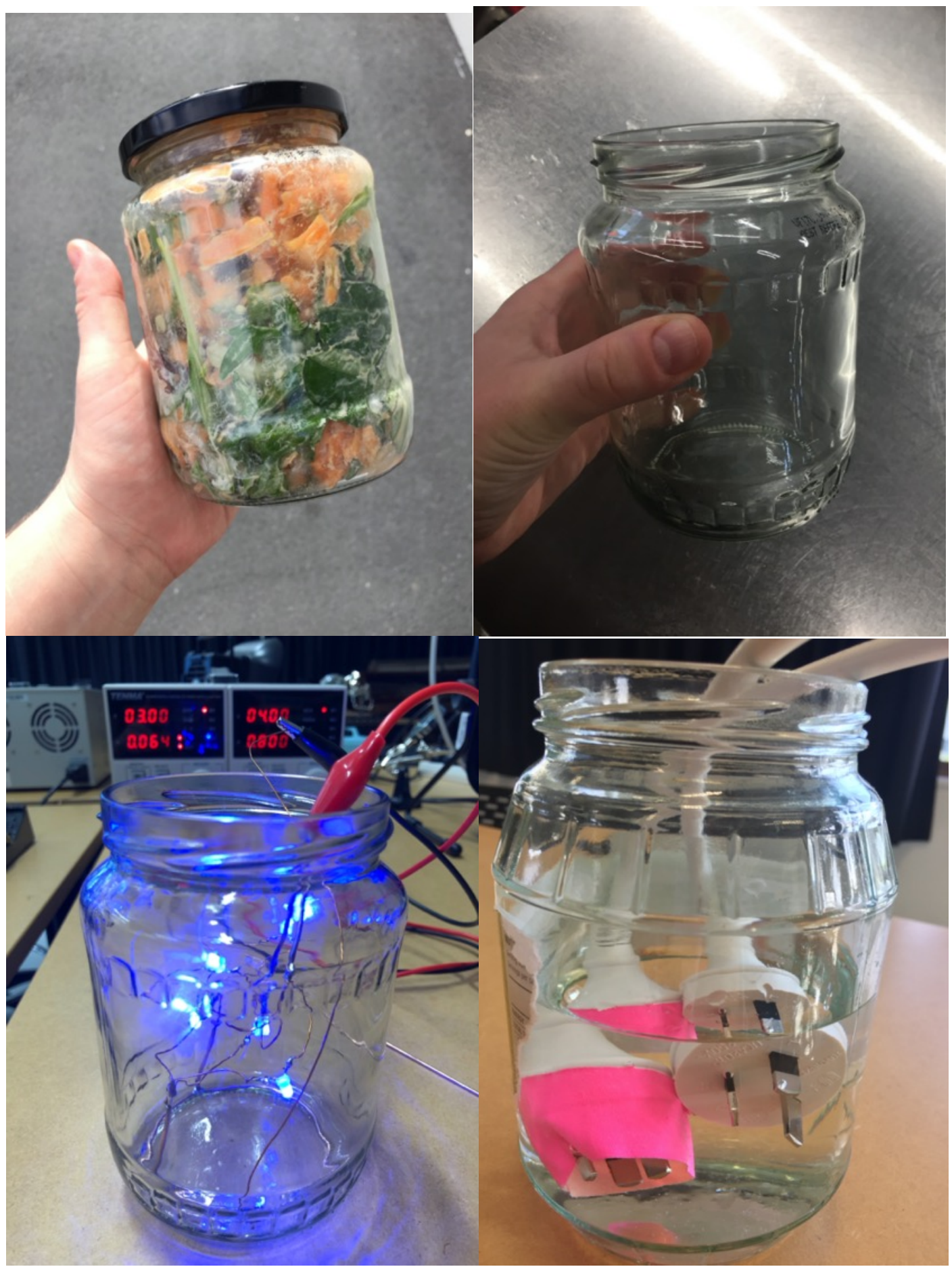

Figure 71. Author. (2019). Examples of documented guises of glass jars in the project: first transported to the lab as lunch container, then thoroughly cleaned, then used as test enclosure for new nodes or as vessels for storing stream samples. 
Selecting suitable jars involved testing whether the basic microcontroller setup and the USB battery would comfortably fit inside when connected, which requires at least $12 \mathrm{~cm}$ height. The inlay was simplified to a basic structure made from cardboard and hot glue. The main function of the inlay was to protect the components from short-circuiting. Because the glass jar itself provides a more stable casing than a plastic bottle, stability was not necessary and both battery and microcontroller were loosely placed inside the jar. This makes it easier for assembly and disassembly or changing components between enclosures. The cardboard design is also easily reproduced and can be recycled once not needed anymore. It does, however, have a less impactful look than the handcrafted transparent acrylic.

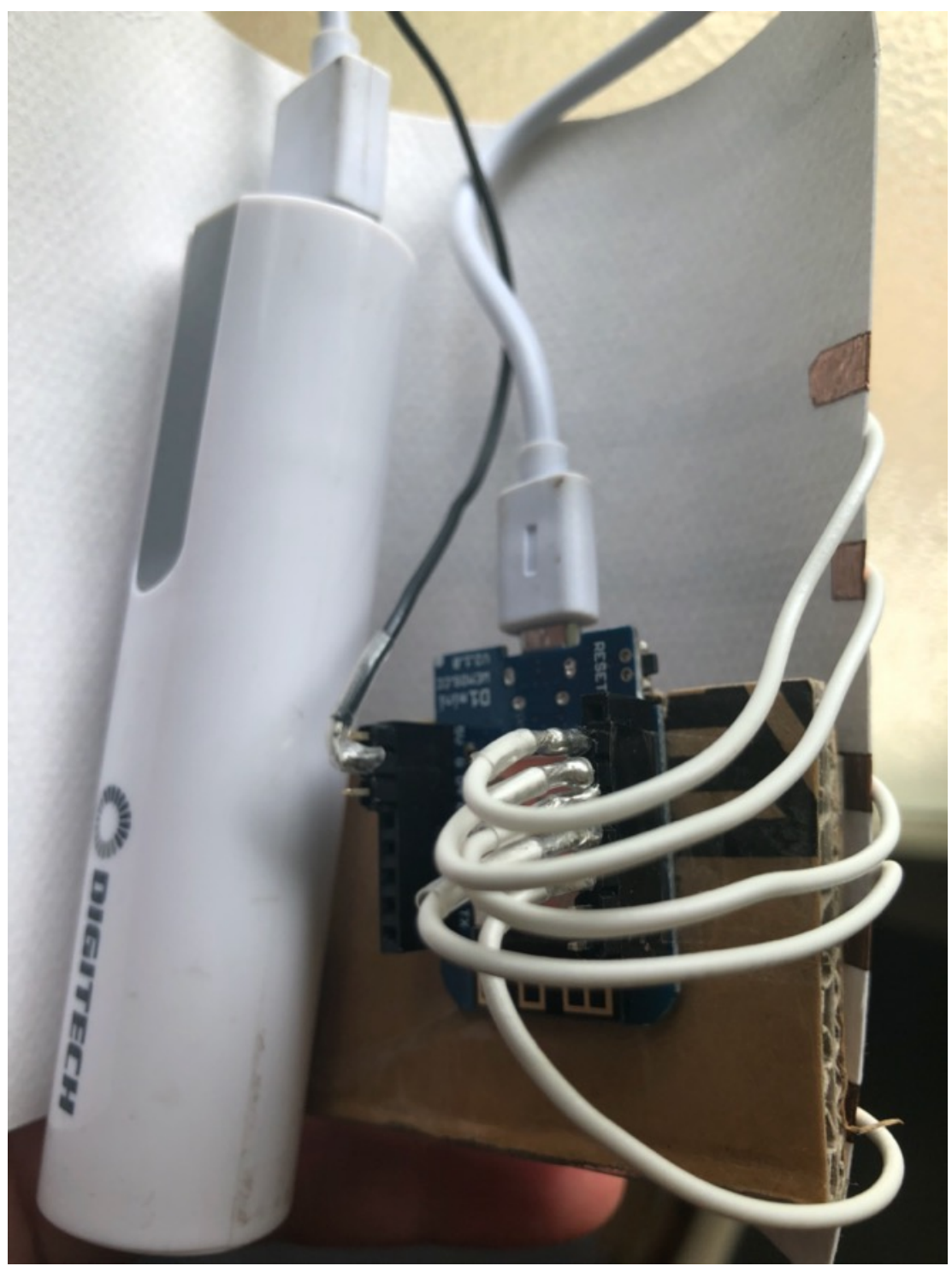

Figure 72. Author. (2019). The simple inlays for the bottle enclosures made from recycled cardboard. 
Overall, the transparent design of both the bottle and the jar versions were well received by the participants during evaluation interviews.

I like the fact that you can see through them. And that it tells you what it's doing. Cause I feel like if I was walking along a stream, and I came across this I would be like "what is that", but I think it's really nice that it's so simple. Well. It looks very complicated to me cause I don't know how circuits and things work. But the fact that it tells you simply what it is and what it's doing seems very un-terrifying. Which is something that I tend to associate IoT with, being a little bit scary. cause being interconnected is very useful in one sense, but it's also 14:24 a little bit terrifying. Because all the information is known by every part of the thing.

I think they would seem a lot more kind of malicious, or if you're up to something if they were all blacked out or something. If they were in some serious looking box. Like this, it looks very friendly. Like made out of recycled things, effectively (Evaluation 12, July 2017).

I think it's pretty cool. Totally, from what you are trying to do, or at least from what I think you're trying to do I think a lot of it makes perfect sense (Evaluation 14, November 2018).

I quite like the containers, because they look like bird feeders, and it is that style, that works, it does not look so valuable (Evaluation 15, November 2018).

I like the lo-fi kind of look (Evaluation 17, January 2019).

As a designer and creator, I can be hard on myself when I feel that re-using materials like this is like cheating, as I am not creating something new. While I came into the project with the idea that the recycled plastic bottles would just be a first step of developing the ideal enclosure for this project, the continuously positive feedback of participants encouraged me to delve deeper into the quest of only using recycled materials and aiming to produce as little as possible waste from this project. Hence, the bottle prototypes that are still usable, such as the temperature sensor from the Moturoa Transmissions installation, or the Wi-Fi hub in the large bottle will still be in use during further iterations of the work. Using a variety of versions of enclosures (Figure 73) during an install also gives an audience an insight into how the project developed and demonstrates how the design offers modularity as part of the concept. Parts from each kind of enclosure are re-usable for another iteration, if needed, and with a simple design, changes can be easily implemented by makers, who would prefer using other materials that are more suitable for their particular project. 

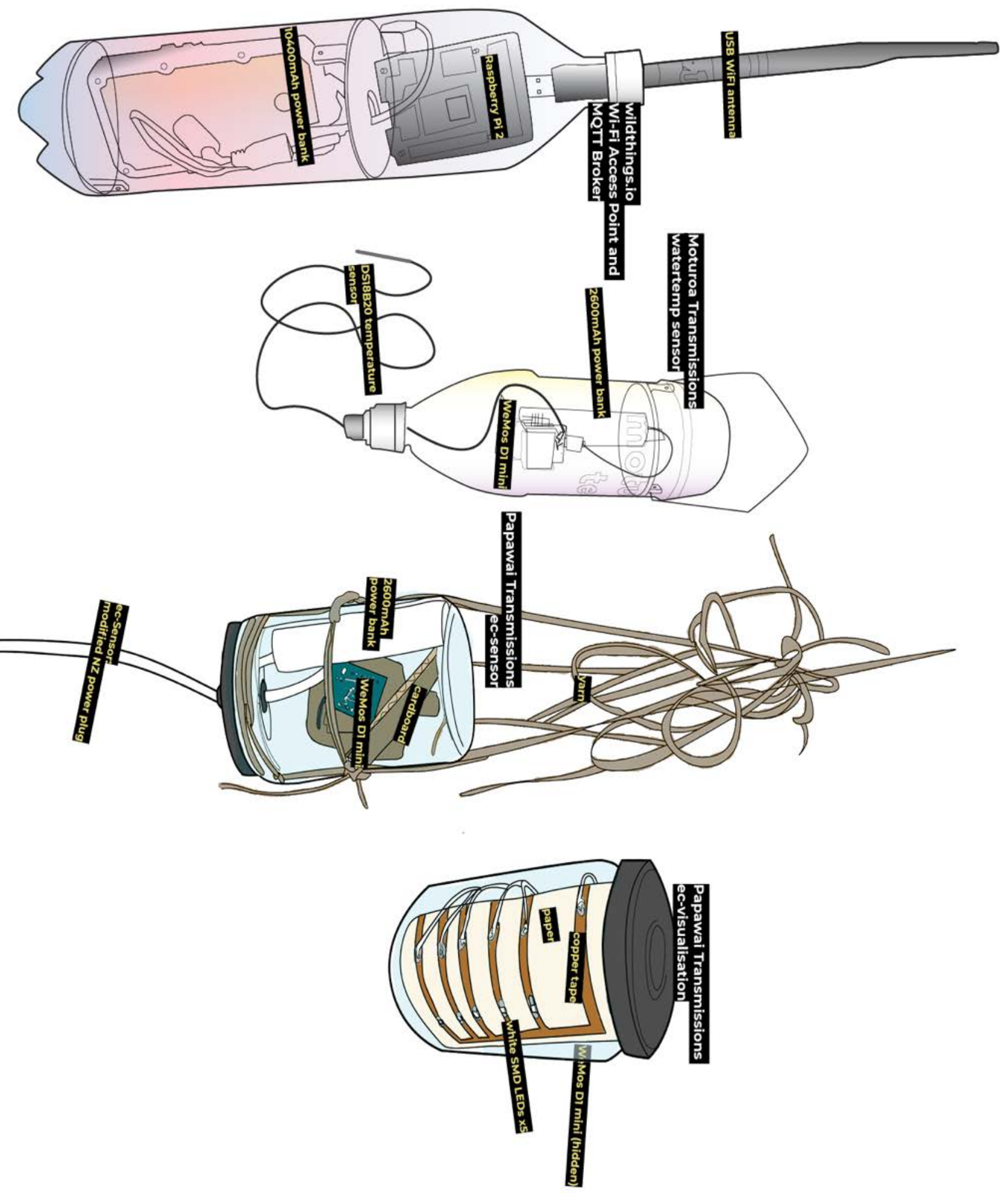

Figure 73: Author (2017). Iterations of networked prototypes showing the Wi-Fi hub and MQTT broker (1), the design of the Moturoa Transmissions water temperature sensor (2), and the Papawai Transmission EC-sensor(3) and EC-

visualisation (4) probes. 


\subsection{EC-sensor iterations}

This narrative provides a closer look at the iterations of the first DIY sensor built as part of this project, which is the EC (Electric Conductivity) sensor, and how the design departed from early, unsuccessful experiments and developed into a stable solution that stands at the heart of the final networked installation Papawai Transmissions.

\section{DIY Electrical Conductivity: Trial and Error}

\section{EC Version 1: Pens, Nichrome and too much tape}

The first EC meter I built was based on the instructions by Practical Maker (2011). The probe involves two Nichrome resistance wires attached to an empty pen tube and covered with electrical tape.

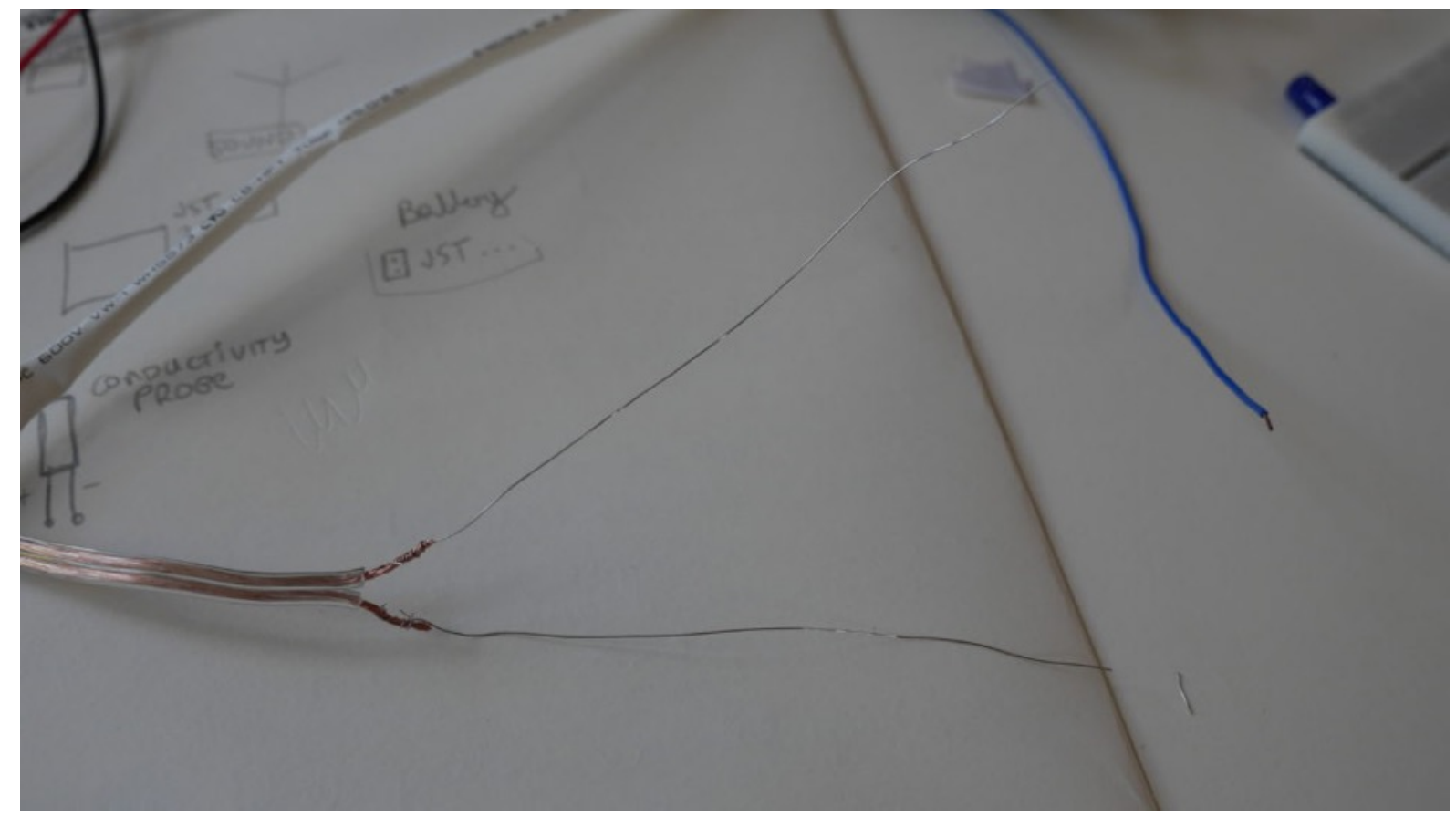

Figure 74. Author. (2017). Attaching two nichrome wires to the audio cable.

The Nichrome wires (Figure 74) are soldered to stranded core copper wire from an audio cable and connect via a $10 \mathrm{k} \Omega$ resistor to the analogue input of a WeMos D1 mini. 


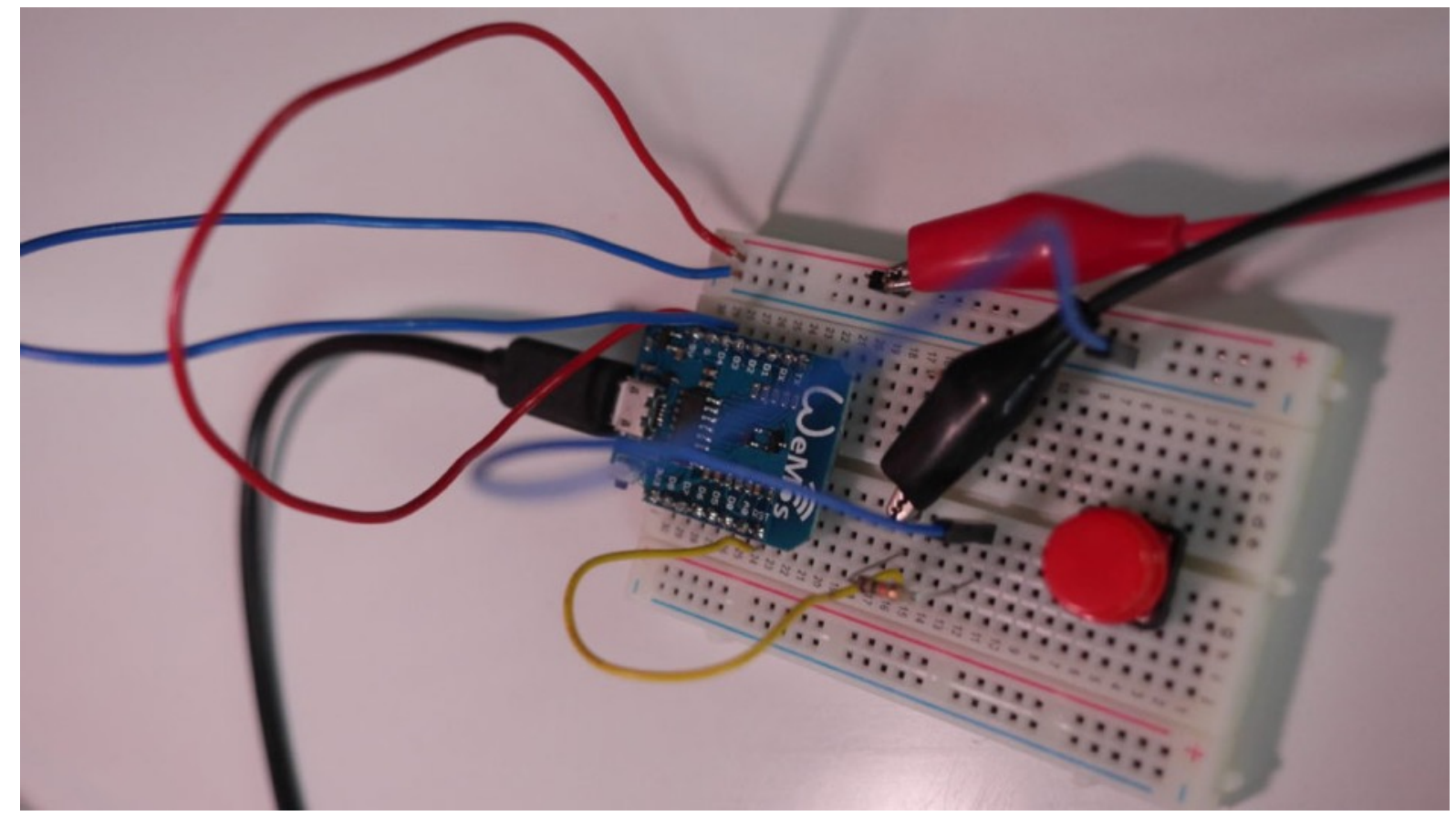

Figure 75. Author. (2017). Hardware setup for testing the EC probe with a WeMos D1 mini in a breadboard.

For testing I would use the WeMos D1 mini in combination with a small breadboard (Figure 75) and jumper wires and header pins connecting to alligator cables to hook up my probe to the micro controller and my computer. 


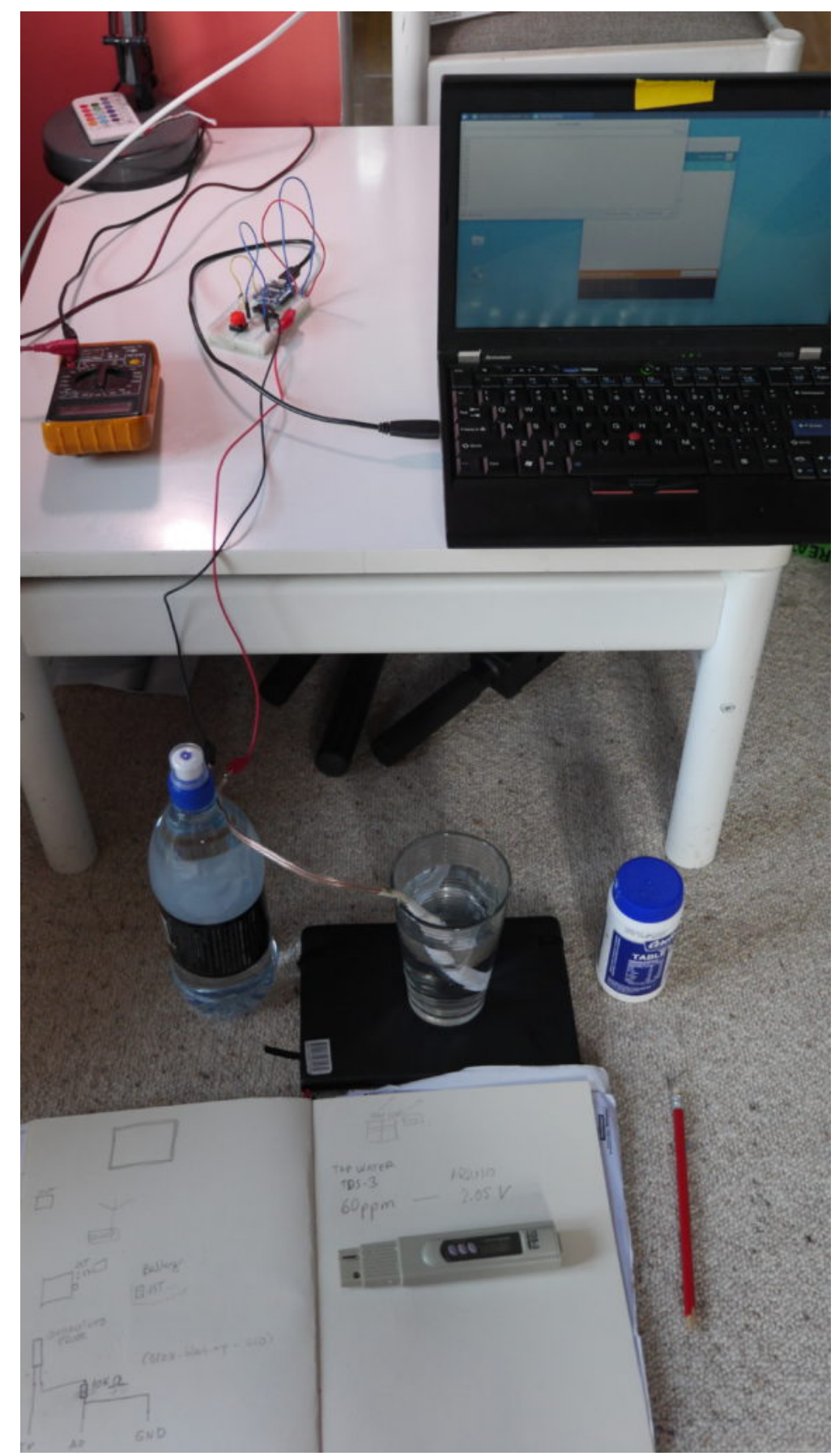

Figure 76. Author. (2017). Test setup of conductivity meter.

For the test setup, I initially submerged the probe in a glass of distilled water. I would read out the voltage via the Arduino Serial Monitor and note the voltage value down in my notebook. I would also take a reading with my TDS-3 meter and note the value down next to the voltage. I would add a measured amount of salt to the water solution and repeat this process.

While the initial voltage readouts looked promising, the material assembly of the probe proved problematic and generated inconsistent voltage readings. 


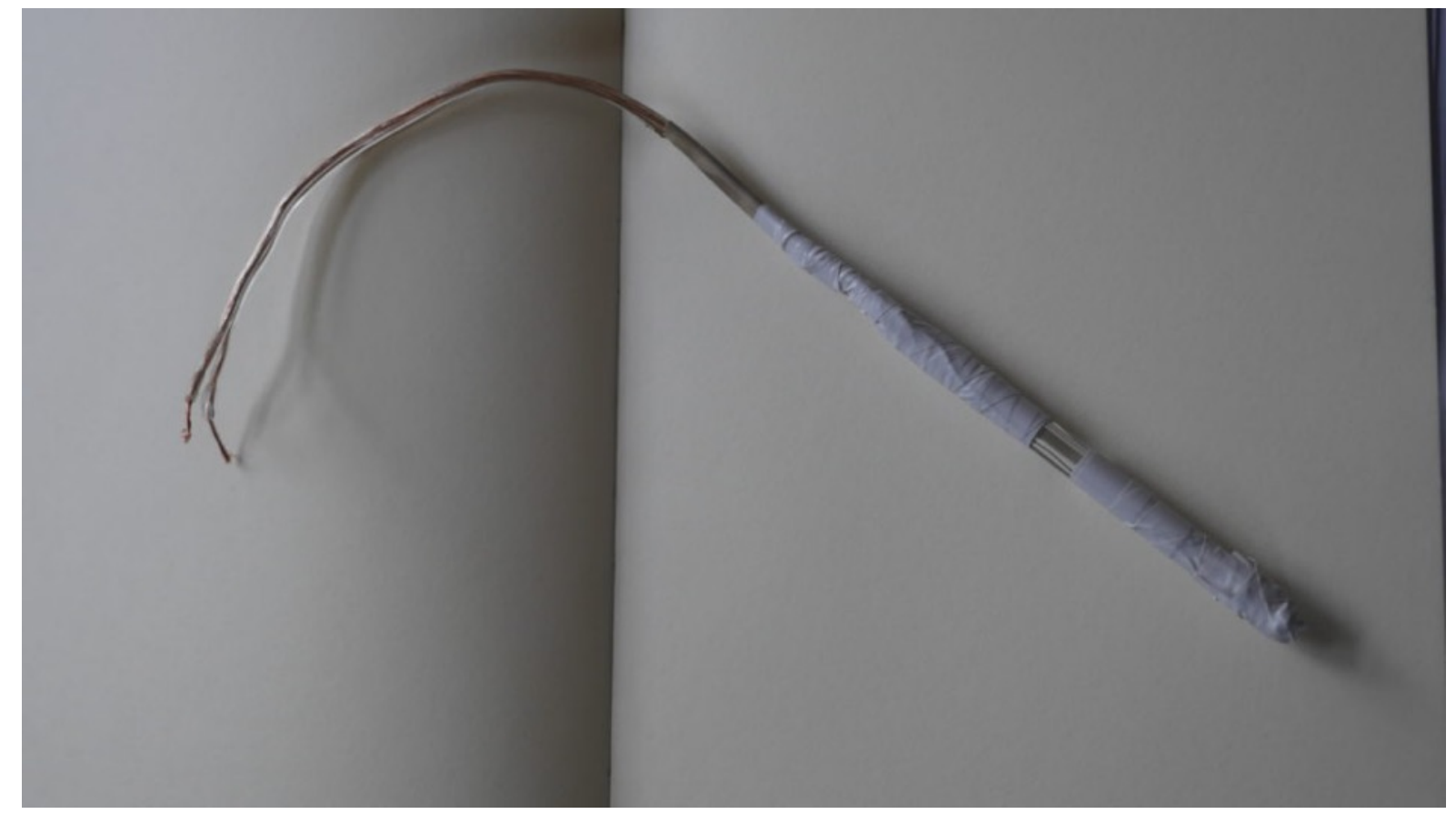

Figure 77. Author. (2017). The final assembled design of the first unsuccessful EC sensor probe.

The electrical tape trapped water in between the multiple layers, which generated inconsistent readouts. The probe would also still show conductivity once I lifted it out of the water solution, as the solution slowly dripped from the parts, and also slowly lifted and moved the electrical tape. To sum it up, this probe design was not only quite complex and flimsy, but also unreliable in terms of readouts. This meant I needed to look for another solution.

\section{EC Version 2: Repurposing a wall plug}

In a very detailed blog post Ratcliffe (2015) described how to build an EC meter for Arduino for $\$ 3$. His solution involved repurposing a Type A Two Prong US plug. I took one old New Zealand plug and connected it to my previous setup. 


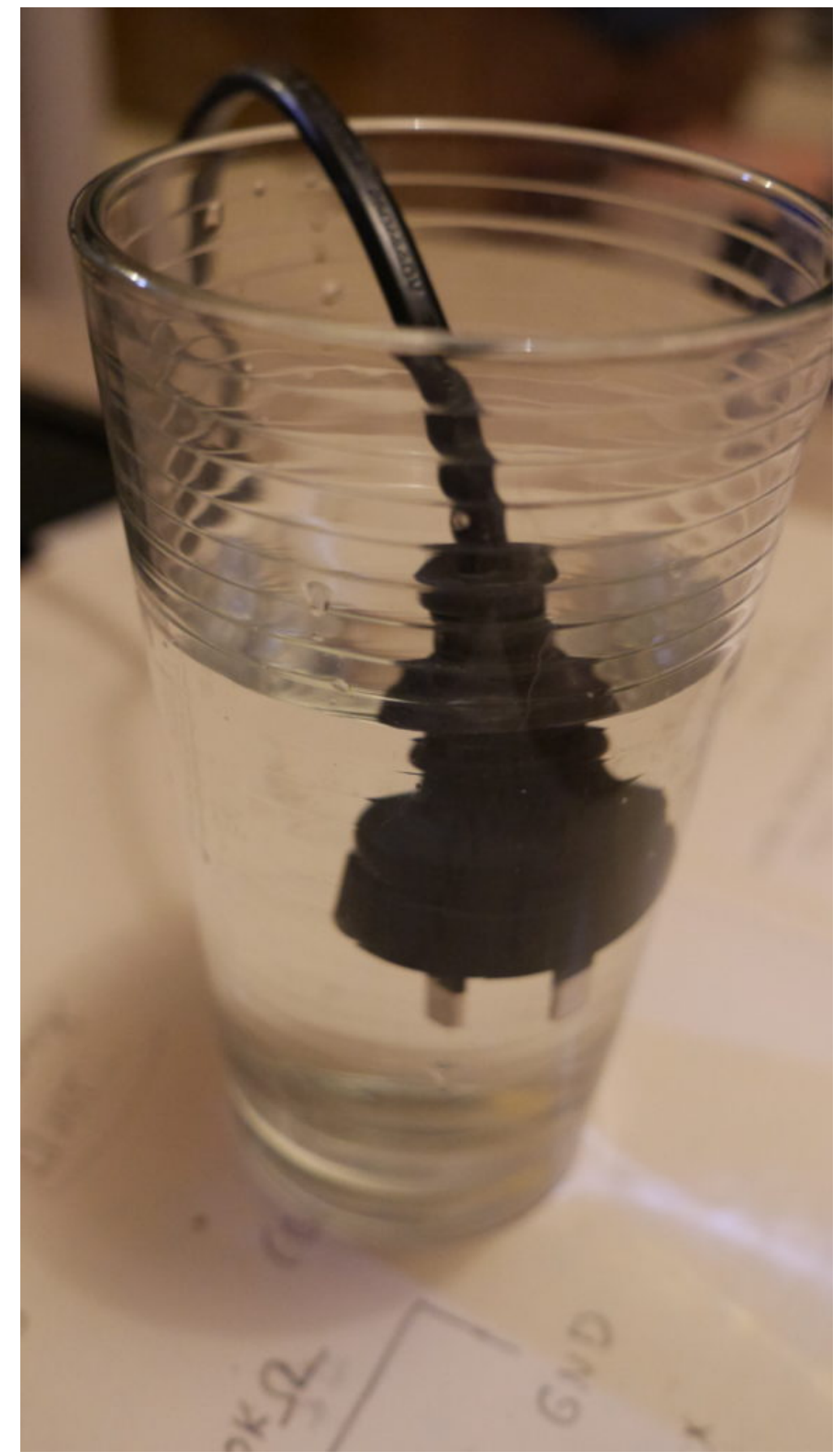

Figure 78. Author. (2017). Testing a repurposed power plug as second EC probe.

The appearance of a power plug suspended in a glass of water looked very strange from the start. However, the initial tests showed that the plug was an easy, hassle-free way of generating reliable voltage readings alongside the TDS meter. 


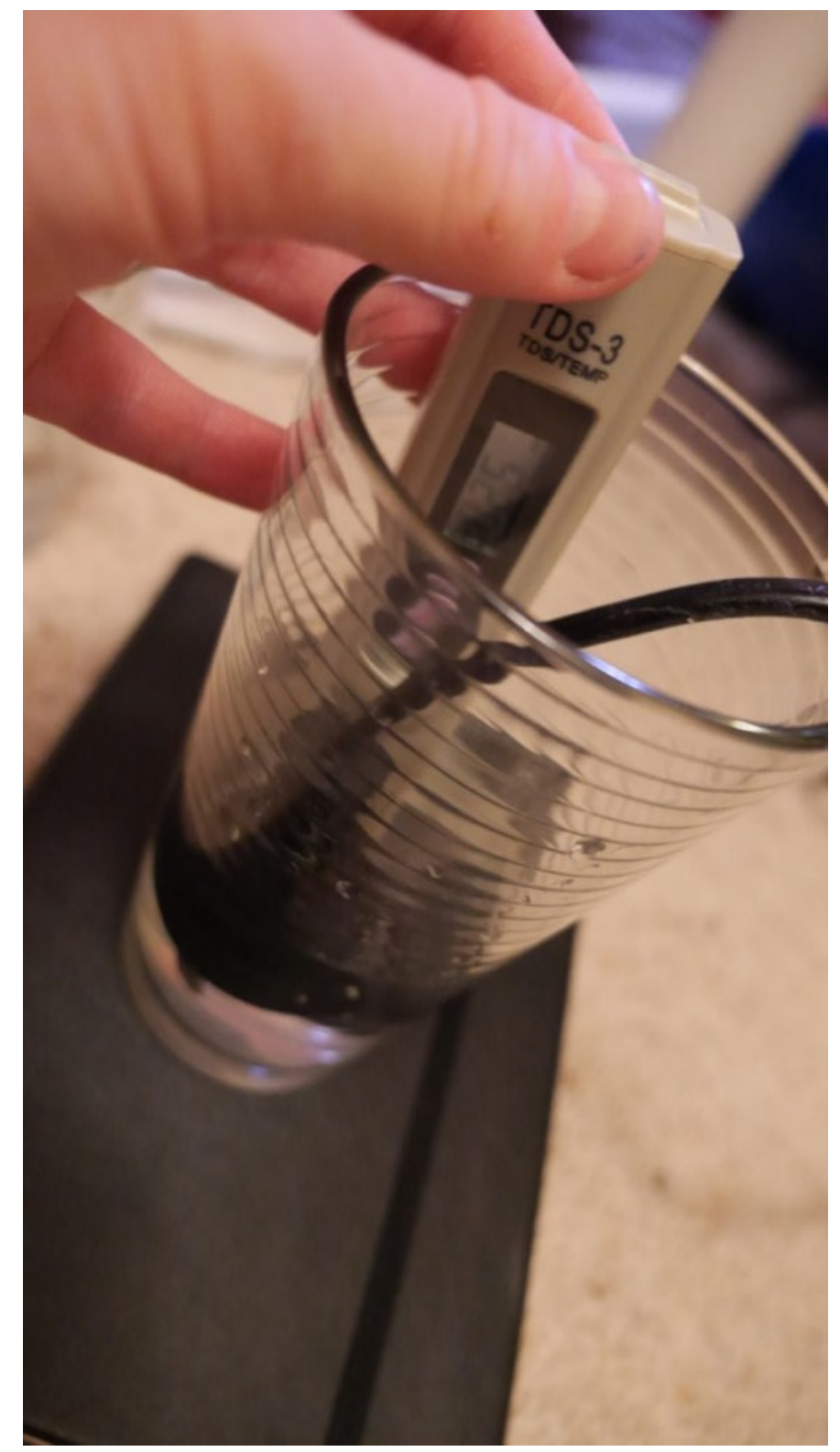

Figure 79. Author. (2017). Comparing readings of the DIY probe with readings from the TDS meter.

Before using more EC probes in the field and gathering data from different parts of the stream I tested them in a controlled environment in the lab.

\section{Experiment 1:}

The two versions tested use the same materials, same length of chord and same $560 \mathrm{Ohm}$ resistor. The first test involved a jar filled with tap water, immersing both sensors in the jar and monitoring the data via mosquitto_sub. 


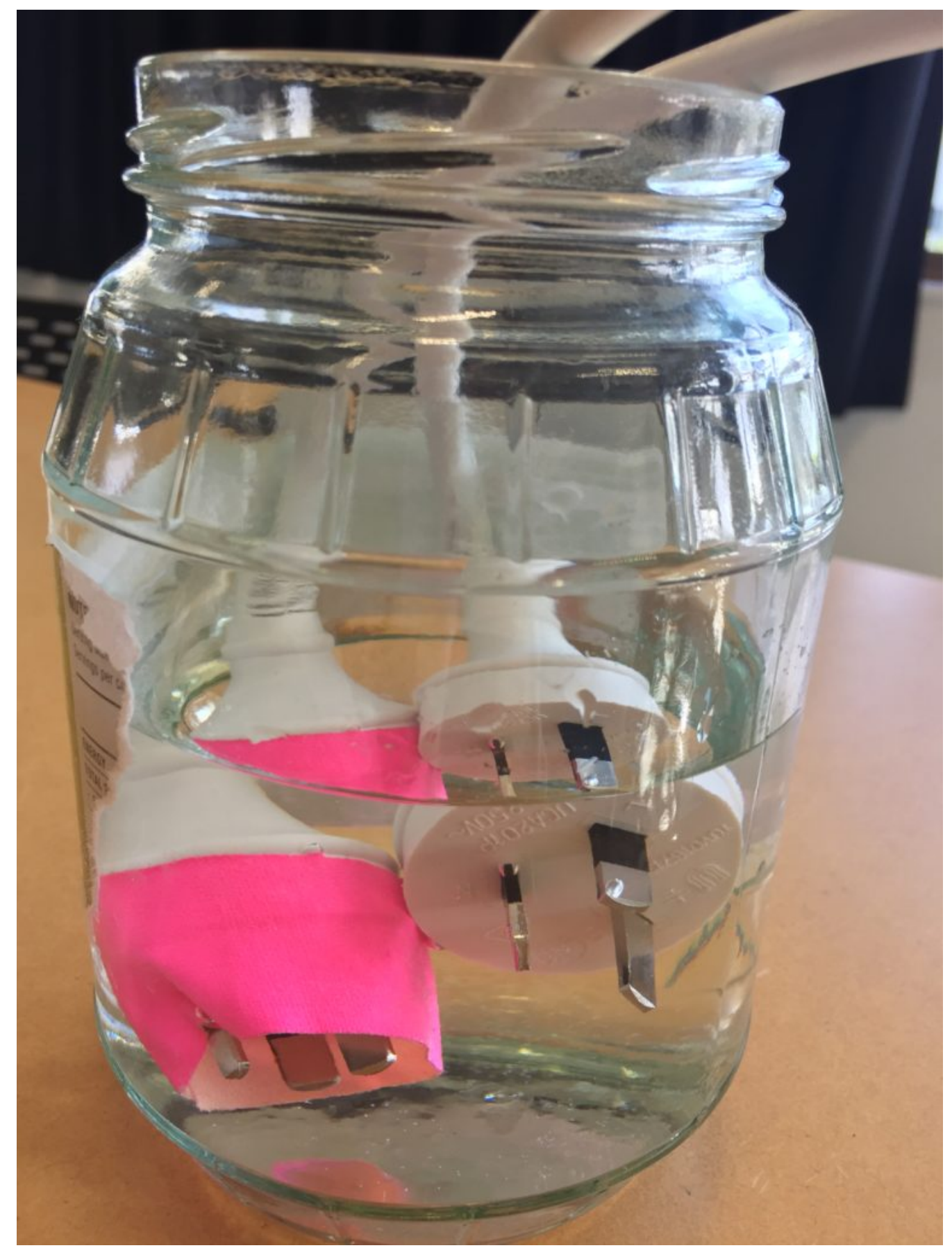

Figure 80. Author. (2018). Comparing the readings of two EC probe prototypes from the same stream sample.

Note: The pink tape wrapped around one probe was an attempt to avoid the probe mistaken with a power plug on the lab table, which posed a severe hazard. The probes are stored away safely when unattended to ensure health and safety. This version of the probe uses the Live (L) and neutral $(N)$ prong of the plug. To improve the safety of the probe the live $(L)$ prong should be removed and Neutral $(N)$ and Ground $(\stackrel{\perp}{\equiv})$ should be used for measuring the electric conductivity. 


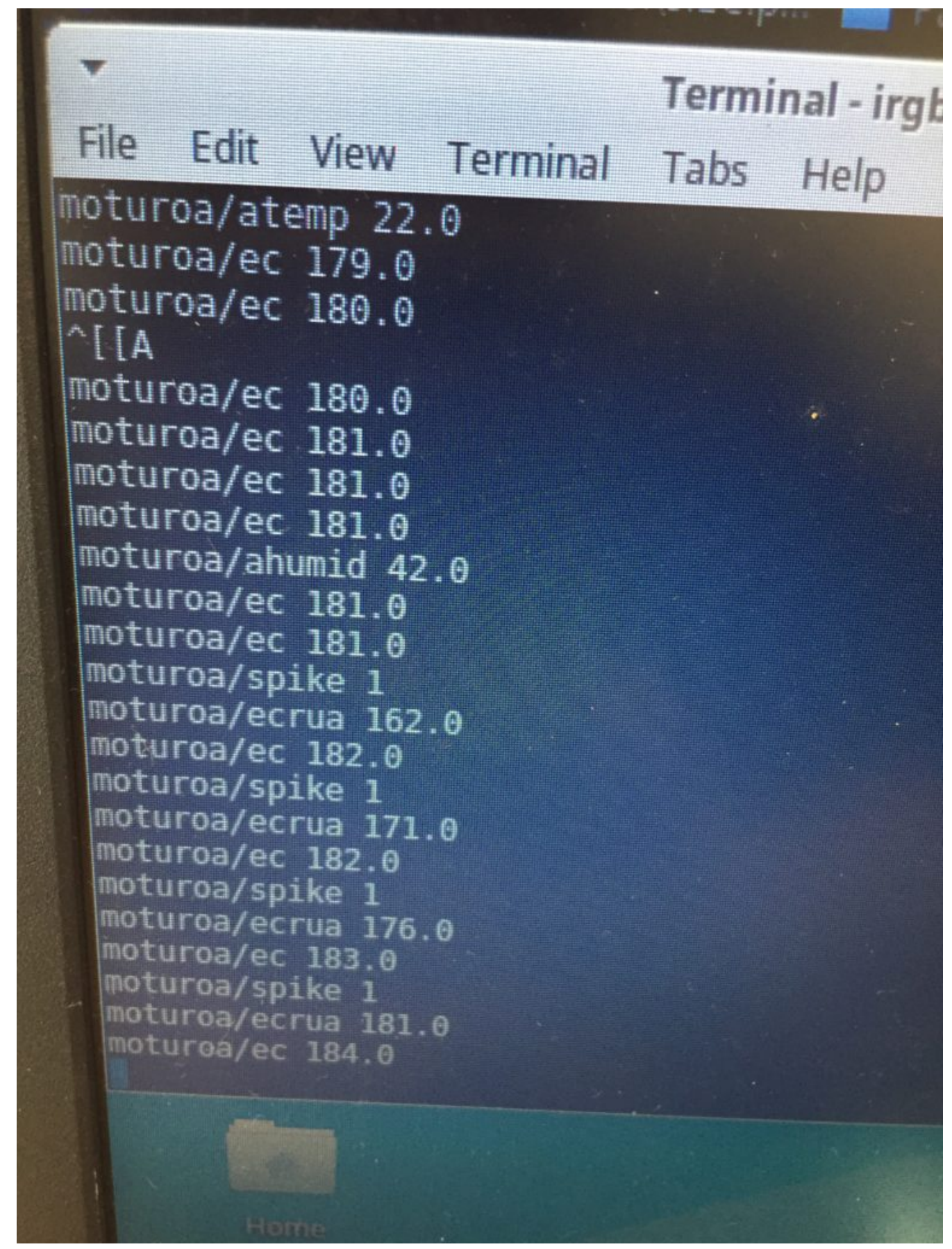

Figure 81. Author. (2018). The terminal output shows the EC probes publishing the measured values under the topics moturoa/ec and moturoa/ecrua. While the test recording was done, the DHT11 sensor was also active in the lab, publishing air temperature (moturoa/atemp) and air humidity (moturoa/ahumid).

This first test showed a deviation of around 10 between both probes, behaving relatively consistently. The next test would require measurements in the stream to see whether the probes return coherent readings from flowing water. 


\section{Experiment 2:}

Due to bad weather and high winds, it was too dangerous to conduct testing in the field.

However, to get a better idea of the consistency between the two probes I went to an easily accessible part of the stream outside of the forested area and collected two samples of stream water.

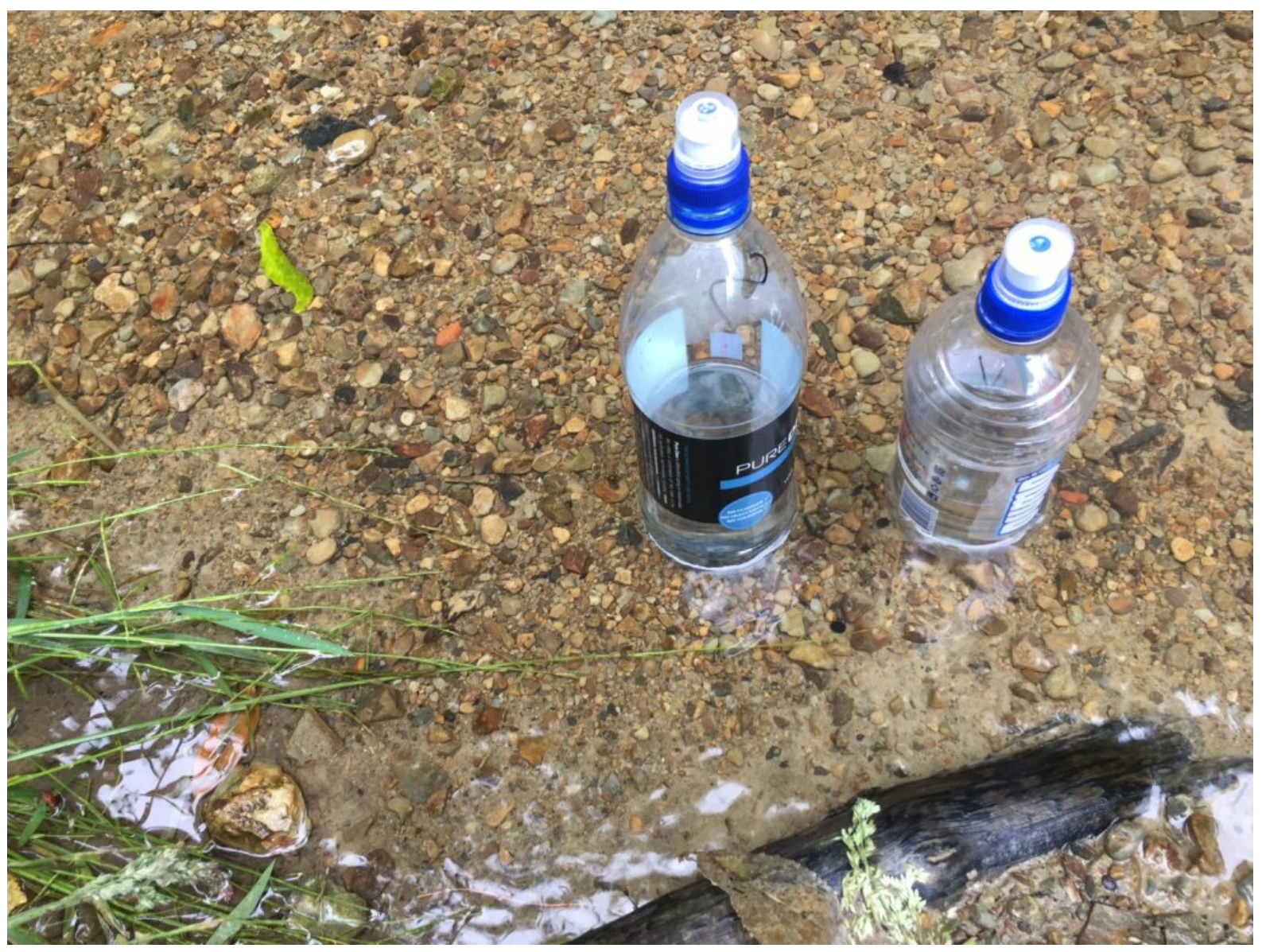

Figure 82. Author. (2018). Two bottles for taking water test samples directly from Papawai Stream.

Back in the lab I pour the first sample into a clean jar that is big enough to contain all three sensors. I prepare a paper sheet for keeping experiment notes, starting with date, time, and location of sample taken, last weather and readings from the TDS meter at the beginning and end of the test.

1. I boot the Raspberry Pi (the Pi acts as Wi-Fi Access POint hosting the Moturoa_Transmissions network and acts as the MQTT-broker). 
2. Connect laptop to Moturoa_Transmissions and start log with timestamp

mosquitto sub -v -h 192.168.42.1 -p 1883 -t '\#' | xargs -d\$' \n' -L1 sh -c 'date "+D $\circ$ T $\$$ "' > data.log

3. Immerse probes into water sample and activate by connecting the WeMos D1 micro controllers to power supplies (USB batteries).

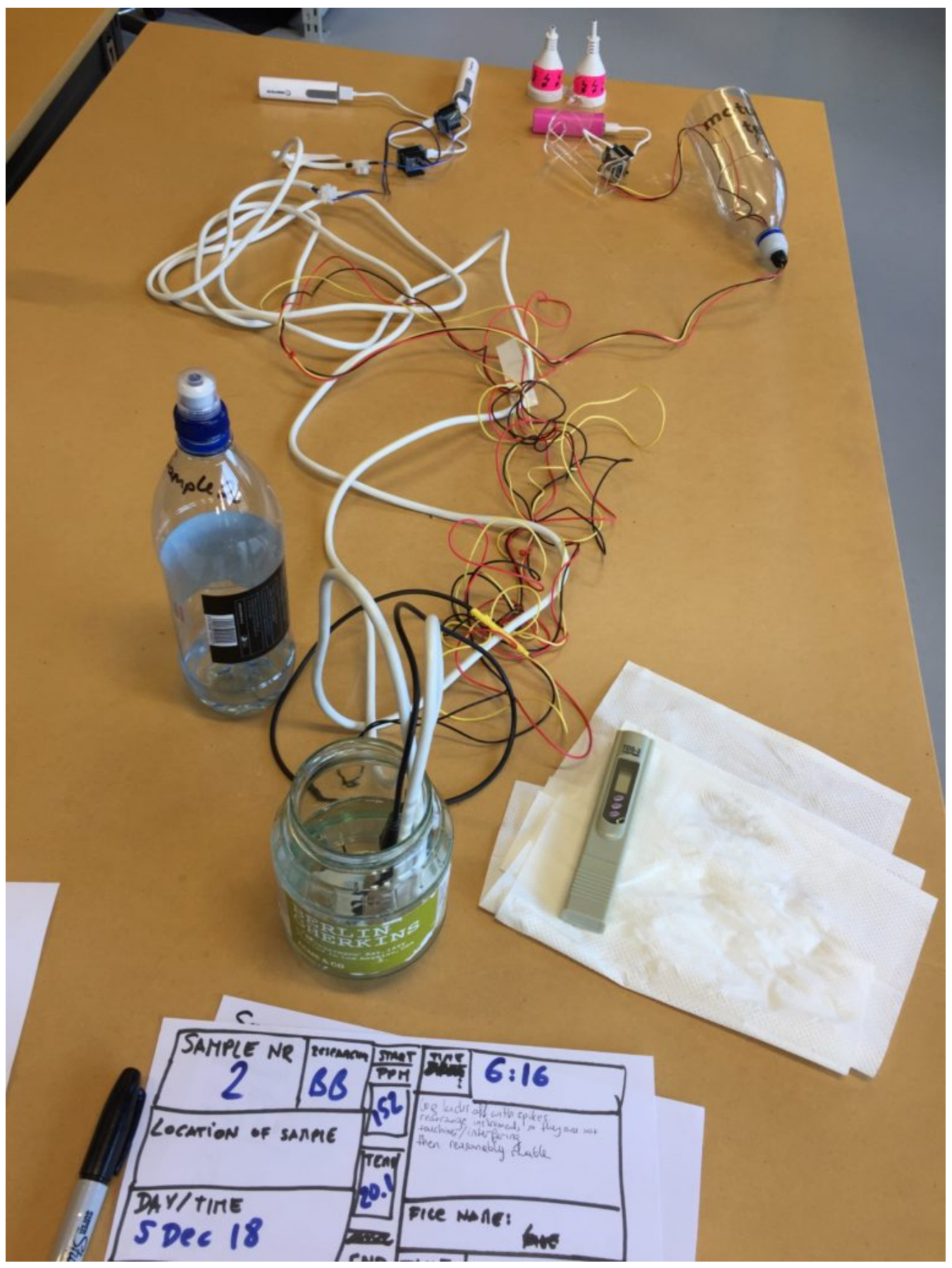

Figure 83. Author. (2018). Testing two EC probes with the same water sample in the lab.

The raw data of both test results can be found on the development repository. In the end, however, I decided to use only one of the two probes in the final install, acknowledging that 
a proper calibration of the sensors is outside of the scope of the thesis. The sensor can, in combination with readouts from the temperature probe, give a good insight into sudden changes of the stream water, when taking samples over a long period of time (see for example visualisations in Sections 7.3 and 7.4), which could give some early indication of issues with the stream, that could be followed up by taking measurements with scientific instruments. 


\subsection{Turbidity Probe Iterations}

In this narrative I present the documentation of the iterative development of the turbidity sensor node, that was the last addition to Papawai Transmissions.

\section{Turbidity Sensor Iteration I - prototyping a DIY probe}

When asked what kind of sensor they would like to see added to the current set of sensors (electric conductivity and temperature), participants indicated the interest to know more about the clarity of the stream water.

The SHMAK kit (see for example NIWA, 2008; see also Section 5.6) also features the measurement of water clarity either with the Clarity Tube or the Black Disk method. The training guide states that " $[\mathrm{w}]$ hat you are looking for in your clarity results is any change over time. If there is a change to more turbid, then you then need to look for reasons" (NIWA, 2008).

Hence a probe constantly measuring the clarity of the water appears to be a useful addition to the sensor family.

\section{Research on other DIY turbidity sensors:}

donblair (2015) provides a good overview of turbidity, "how it's usually assessed, and various approaches one might take to measuring it.” The Open Water Project Github repository features comprehensive documentation of their turbidity sensor design. Kelley et al. (2014) present the build of an affordable open-source turbidimeter featuring a combination of off-the-shelf electronic components and 3D-printed hardware. The simplest DIY design involves only two components, a light source aimed at a light-sensitive photocell (see for example Marchetto's design as described in donblair (2015). With all components (an LED, an LDR and various resistors) at hand in the electronics lab, the next step involves considering different enclosures and ways of waterproofing the components. An option suitable for Papawai Stream needs to work in relatively shallow waters and should not obstruct the natural course of the stream. Hence, a tube with a diameter of around $1-3 \mathrm{~cm}$, akin to the usually shallow depth of Papawai Stream would be a good first iteration of a 
prototype. The tube should be dark to minimise ambient light impacting the sensor readings.

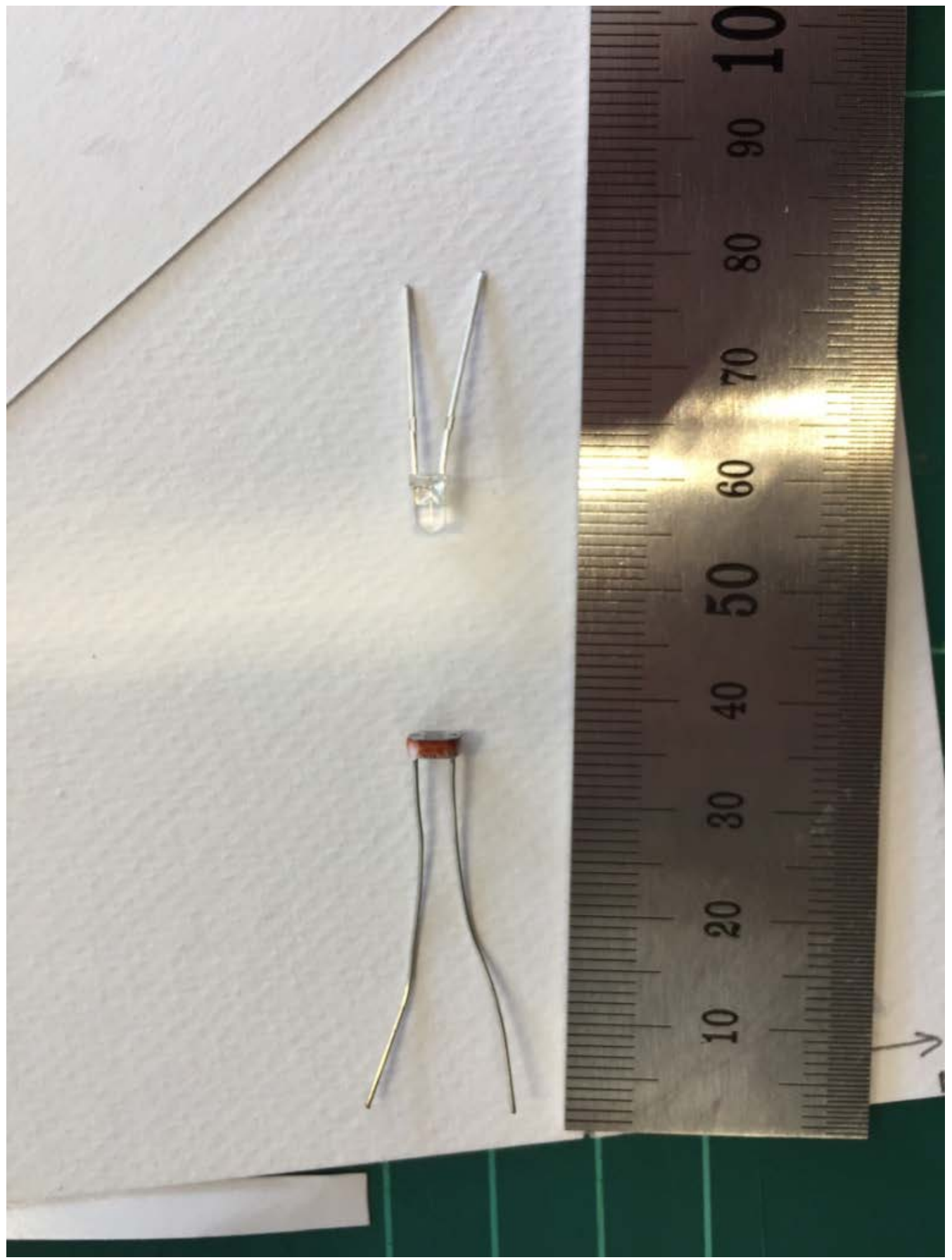

Figure 84. Author. (2018). The basic concept illustrated with the two key components: An LDR facing a white LED. 


\section{Turbidity Sensor Iteration I: Garden Hose enclosure}

A relatively cheap and easy to recycle material that is suitable to be used in water is a garden hose. For Prototype 1, I use a piece of garden hose of about $10 \mathrm{~cm}$.

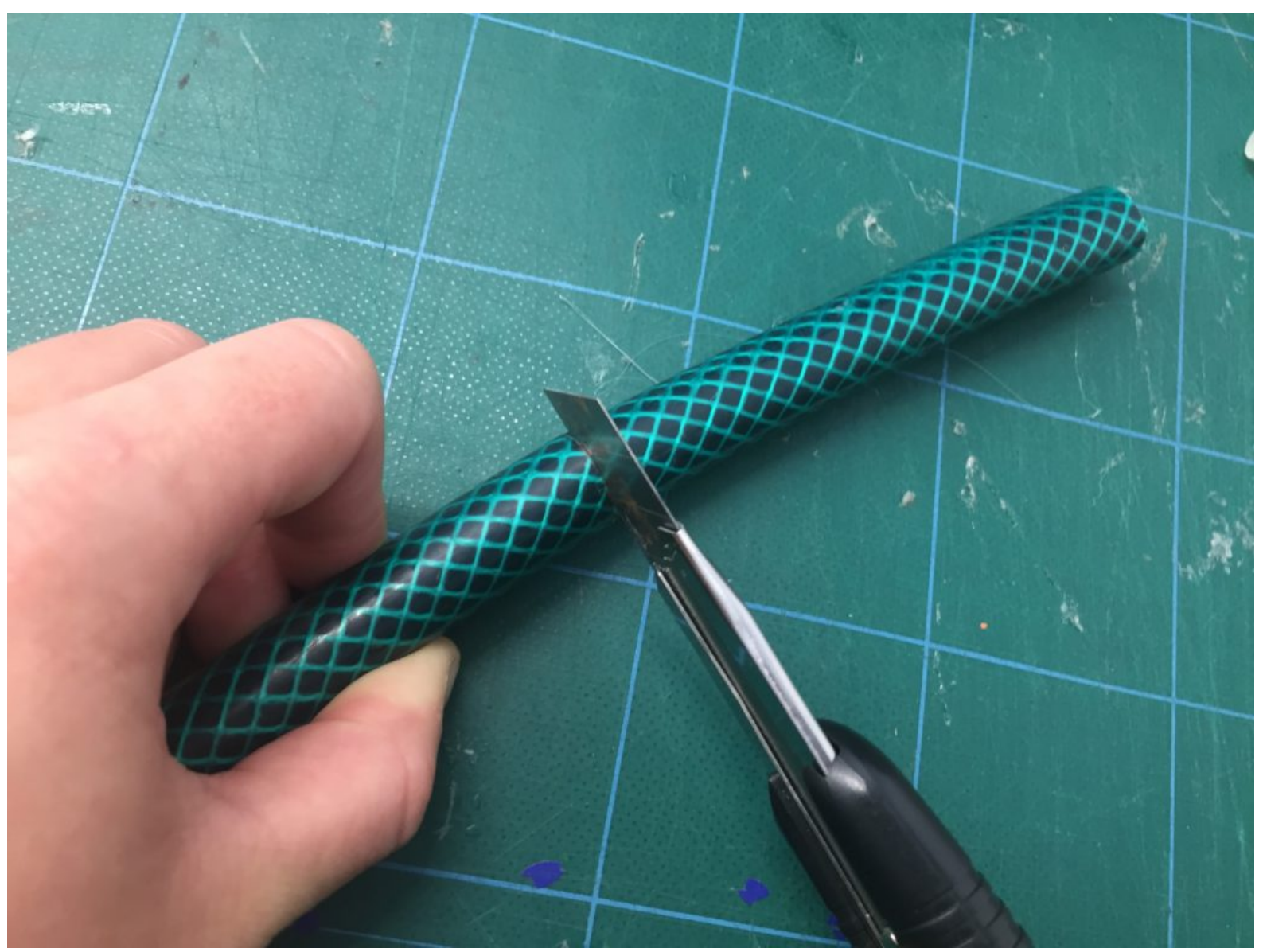

Figure 85. Author. (2018). Preparing the hose.

First, I connected the LDR sensor and the LED on a breadboard to test the sensor readings via Serial. The Arduino code used for this version of the prototype can be found here. 


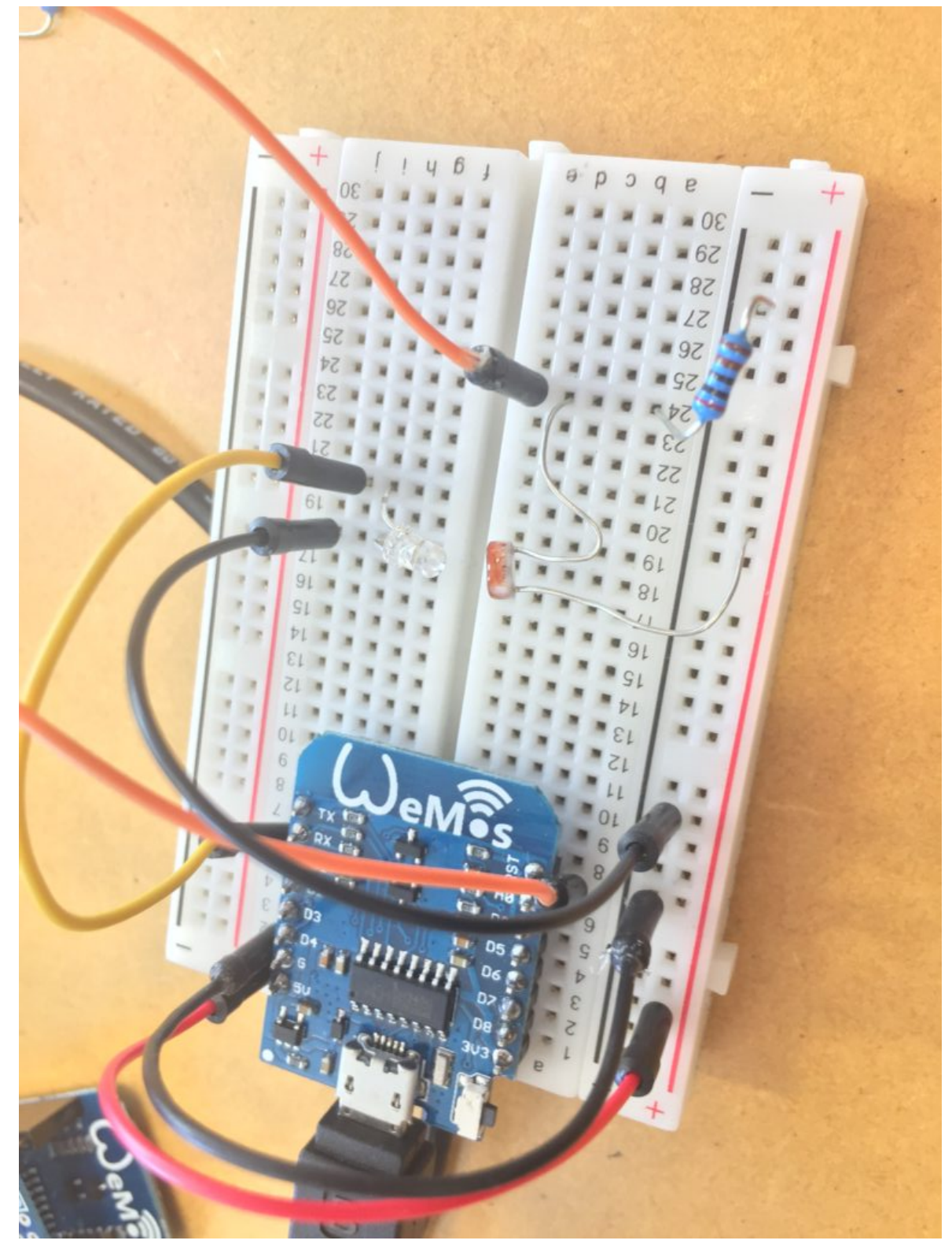

Figure 86. Author. (2018). WeMos D1 breadboard setup.

It turned out that a $2 \mathrm{~K}$ resistor for the LDR and a relatively low brightness value for the white LED shows a consistent change in the tube.

analogWrite(ledPin, 64);

These values are good enough for general testing of the design and will likely need to be adjusted to the conditions in the field. 


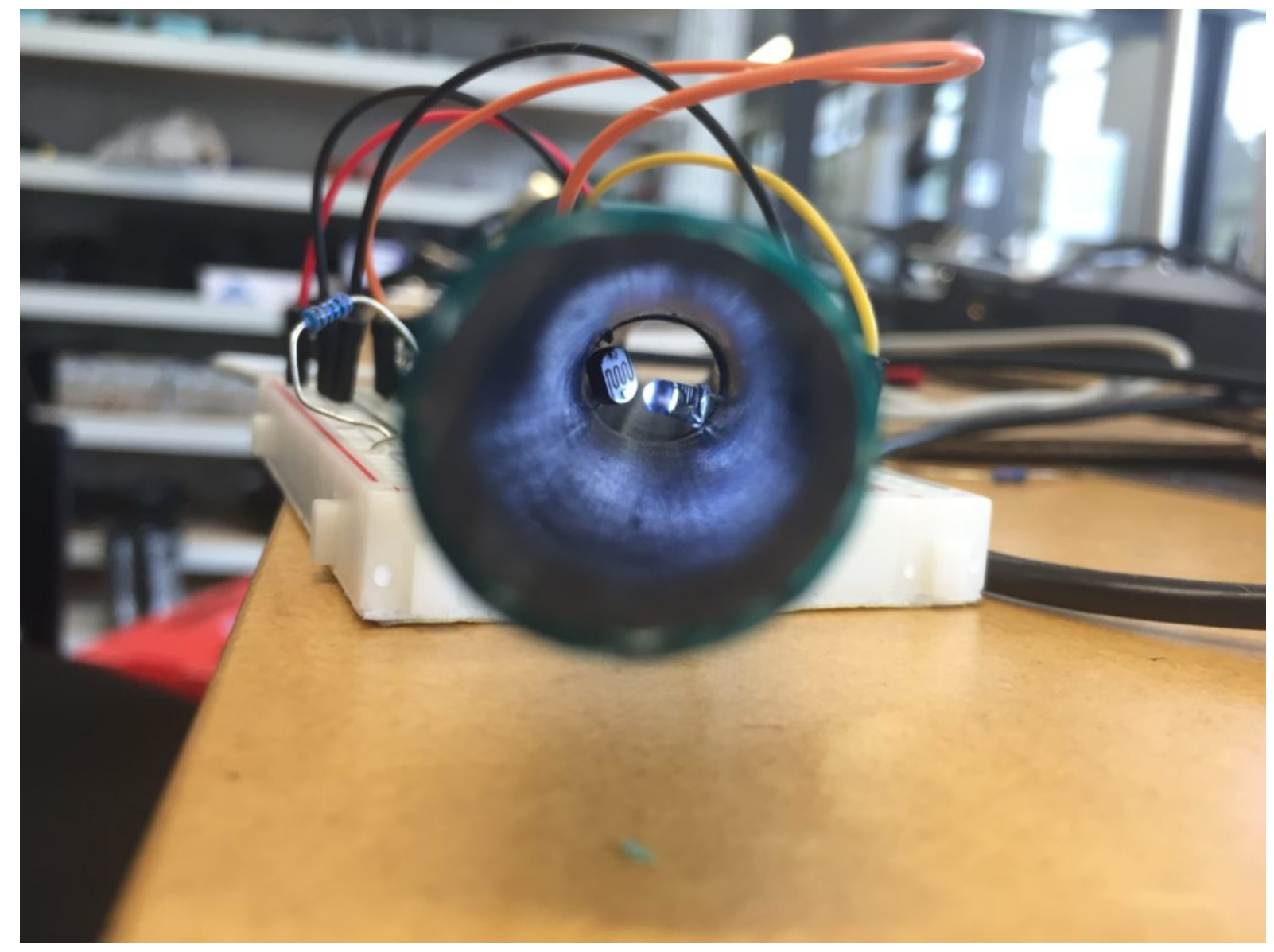

Figure 87. Author. (2018). Image of the inside of the garden house with the LDR and LED.

I cut the hose in half to position the components inside and used transparent Sellotape to attach the two halves back together. This design generally worked but required some work making sure that the electronics do not short circuit. 


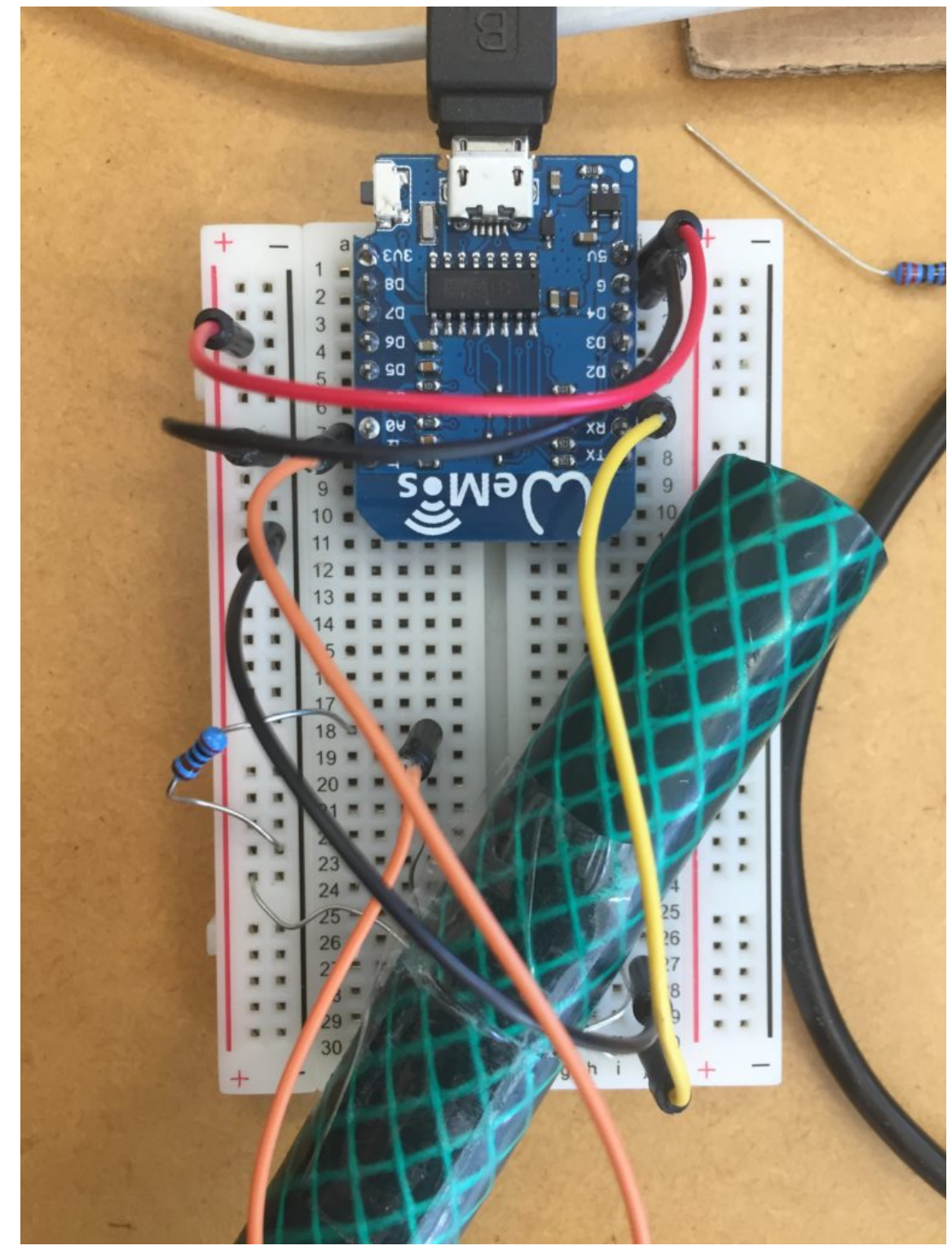

Figure 88. Author. (2018). Breadboard Setup including garden hose.

The next challenge is to waterproof the probe. For this first iteration, I chose hot glue to seal the exposed wires of the components, similar to Marchetto's design (as cited in donblair, 2015, see Figure 89).

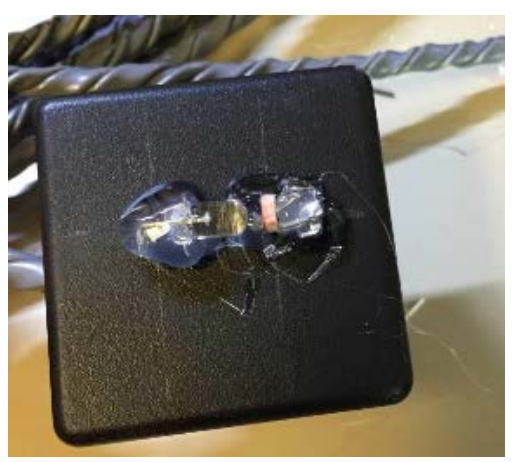

Figure 89. donblair. (2015). Turbidity sensor design. 
The four wires sticking out of the submerged part of the sensor need careful waterproofing. While hot glue generally works, it runs the risk of breaking once set. A flexible waterproof sealant would be safer.

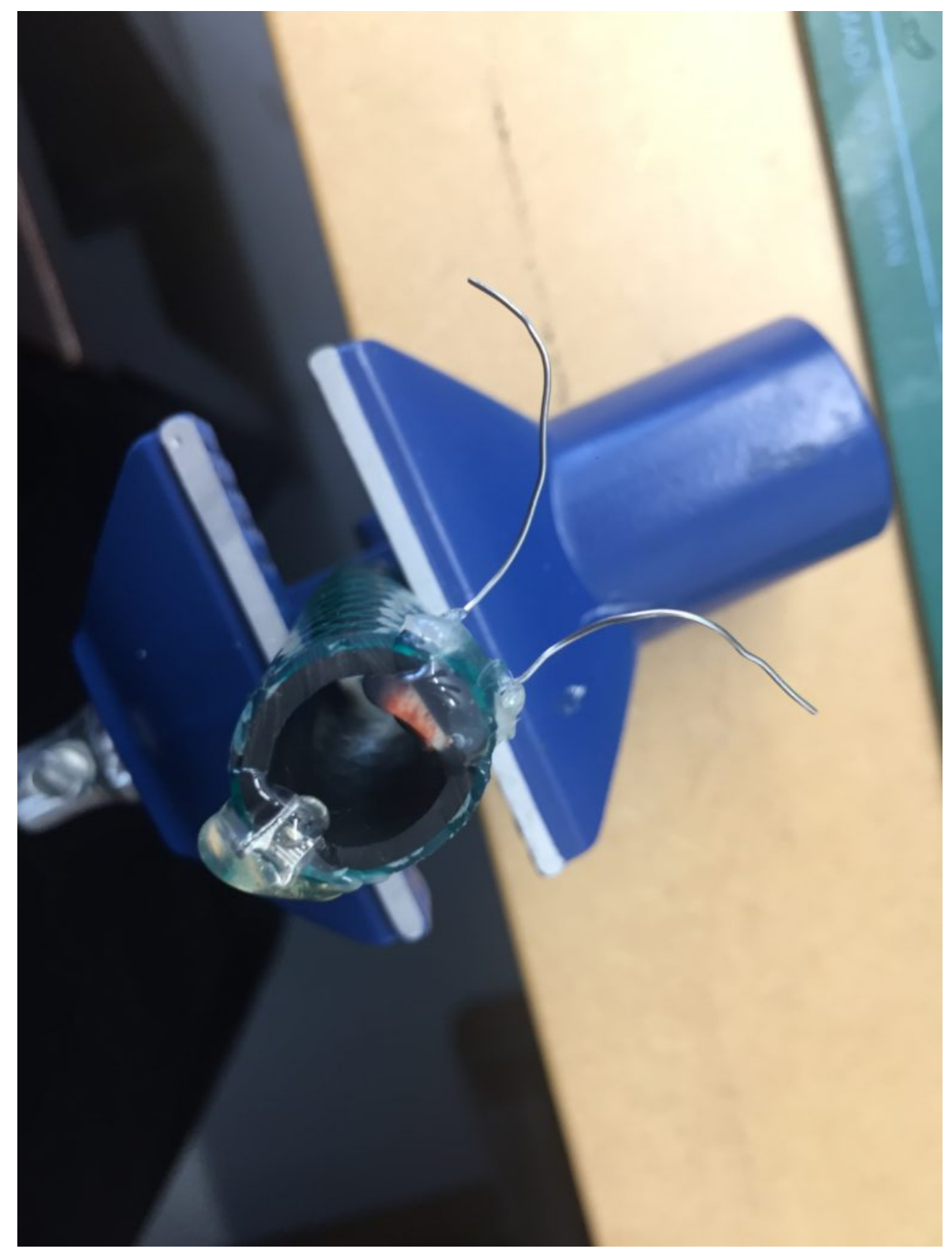

Figure 90. Author. (2018). First attempt of using hot glue to attach the sensor and LED to the hose.

I will redo the design with proper cables connected to the sensors for more safety and the ability to test the sensor submerged in water. 


\section{Component List:}

\section{Hardware}

- Computer with USB interface

- WeMos D1 mini (now known as LOLIN)

- $1 \mathrm{LDR}$

- $12 \mathrm{k}$ Resistor

- $13 \mathrm{~mm}$ white LED

\section{Software}

- $\quad$ Arduino

- $\quad$ CH340 drivers for your operating system

- Add ESP8266 to your Board Manager 
Arduino NG or older

Arduino Robot Control

Arduino Robot Motor

Arduino Gemma

Adafruit Circuit Playground

Arduino Yún Mini

Arduino Industrial 101

Linino One

Arduino Uno WiFi

ATtiny Microcontrollers

ATtiny25/45/85

ATtiny $24 / 44 / 84$

ESP8266 Boards (2.5.0-beta3)

Generic ESP8266 Module

Generic ESP8285 Module

ESPDuino (ESP-13 Module)

Adafruit Feather HUZZAH ESP8266

Invent One

XinaBox CW01

ESPresso Lite 1.0

ESPresso Lite 2.0

Phoenix 1.0

Phoenix 2.0

NodeMCU 0.9 (ESP-12 Module)

NodeMCU 1.0 (ESP-12E Module)

Olimex MOD-WIFI-ESP8266(-DEV)

SparkFun ESP8266 Thing

SparkFun ESP8266 Thing Dev

SweetPea ESP-210

$\checkmark$ LOLIN(WEMOS) D1 R2 \& mini

LOLIN(WEMOS) D1 mini Pro

LOLIN(WEMOS) D1 mini Lite

WeMos D1 R1

ESPino (ESP-12 Module)

ThaiEasyElec's ESPino

Wifinfo

Arduino

4D Systems gen4 loD Range

Digistump Oak

WiFiduino

Amperka WiFi Slot

Seeed Wio Link

ESPectro Core

Figure 91. Author. (2018). Arduino... Tools... Board showing list of ESP8266 boards.

\section{Turbidity Sensor Iteration II - Improved design with material issues}

With this revised prototype I aimed to create a better, more stable design by inserting the LED and the photocell through holes into the hose while improving the sealing of electrical components from the beginning. I also wanted to use a cable with the actual length for use in the field and chose an approximately $2 \mathrm{~m}$ long stranded core CAT- 5 cable.

First, I soldered the photocell to the green pair of cables and tested it with the code from yesterday. 


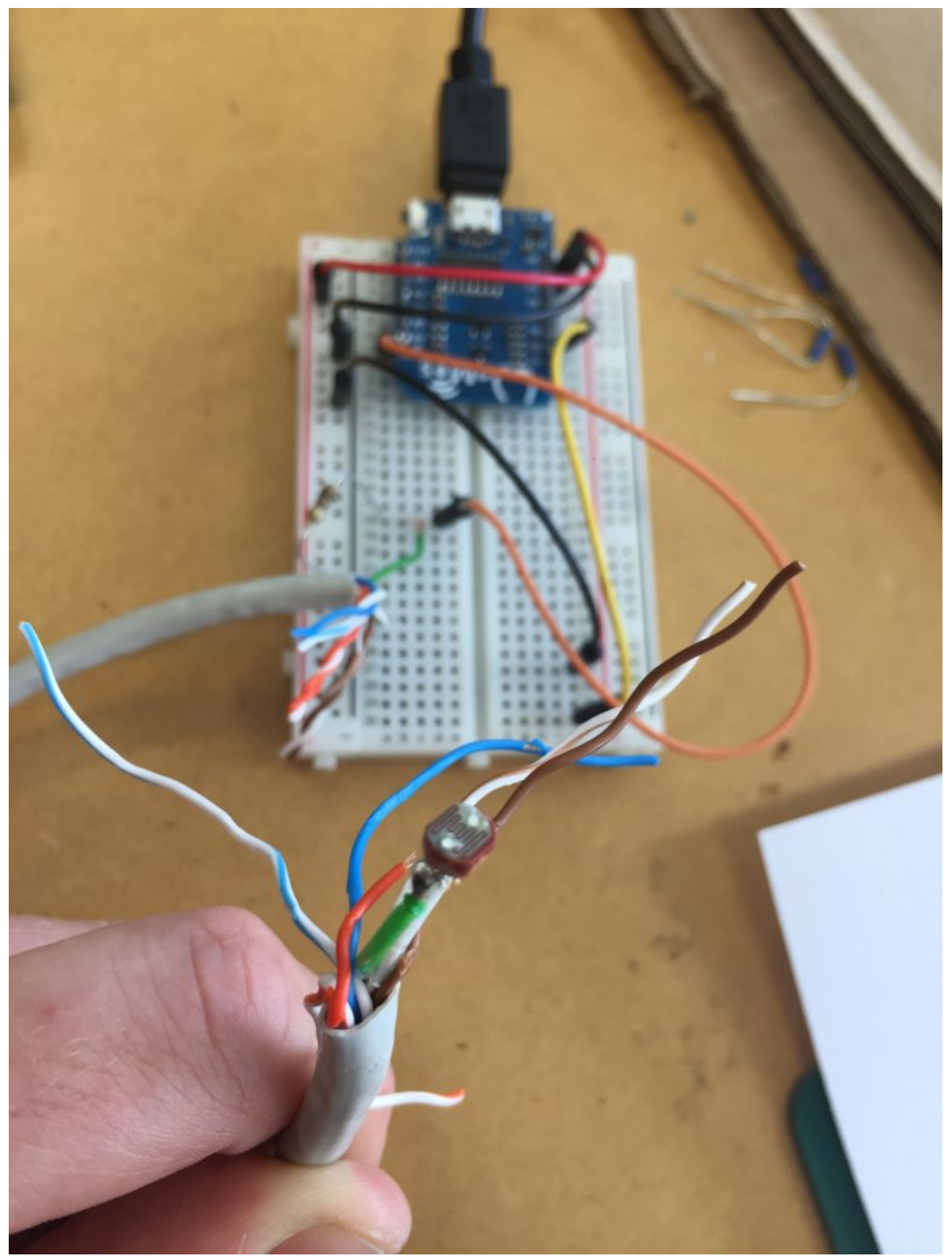

Figure 92. Author. (2018). Testing the LDR connected to a WeMos D1 with the AnalogIn Arduino example. 


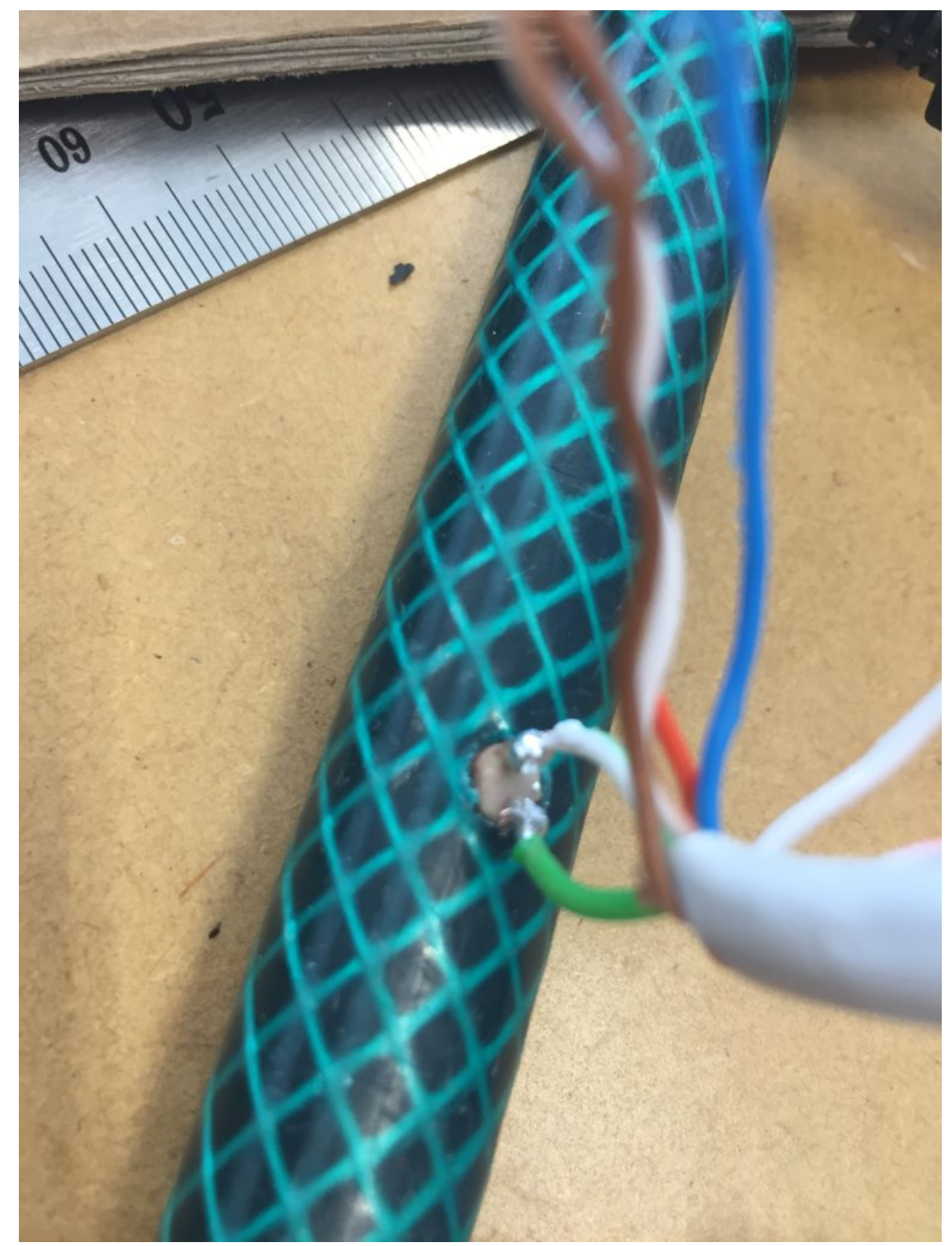

Figure 93. Author. (2018). I drilled $5 \mathrm{~mm}$ hole into the hose to fit the LDR neatly.

The $3 \mathrm{~mm}$ LED requires a $3 \mathrm{~mm}$ hole. I drilled the $3 \mathrm{~mm}$ through the $5 \mathrm{~mm}$ hole to make sure the holes are nicely aligned. 


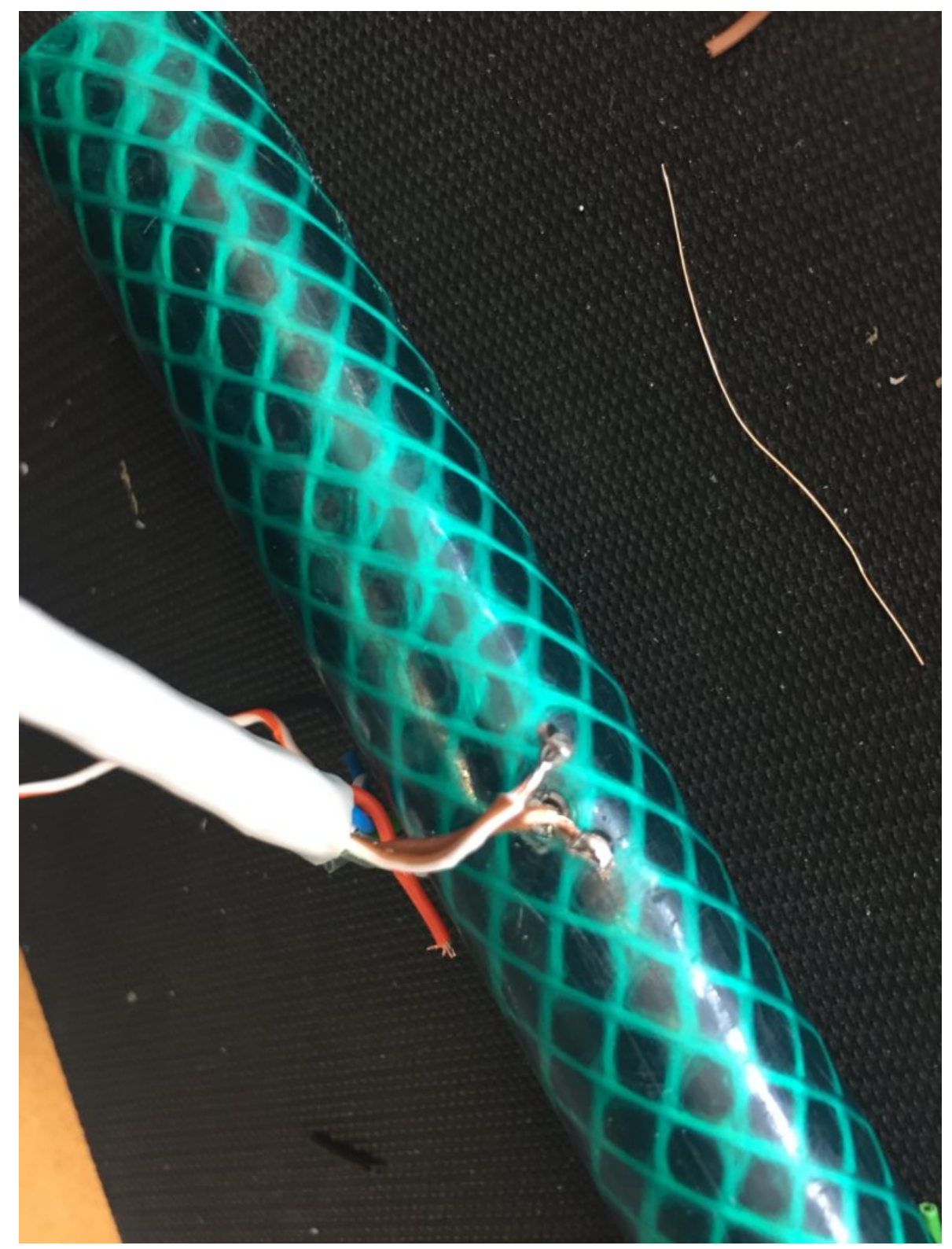

Figure 94. Author. (2018). The prongs of the LED are soldered to strands of the CAT-8 cable.

Reconnecting the wires with the breadboard from the previous prototype, I ended up swapping the resistor to a $10 \mathrm{k}$ one which gave me more consistent readings when the LED was on half brightness. 


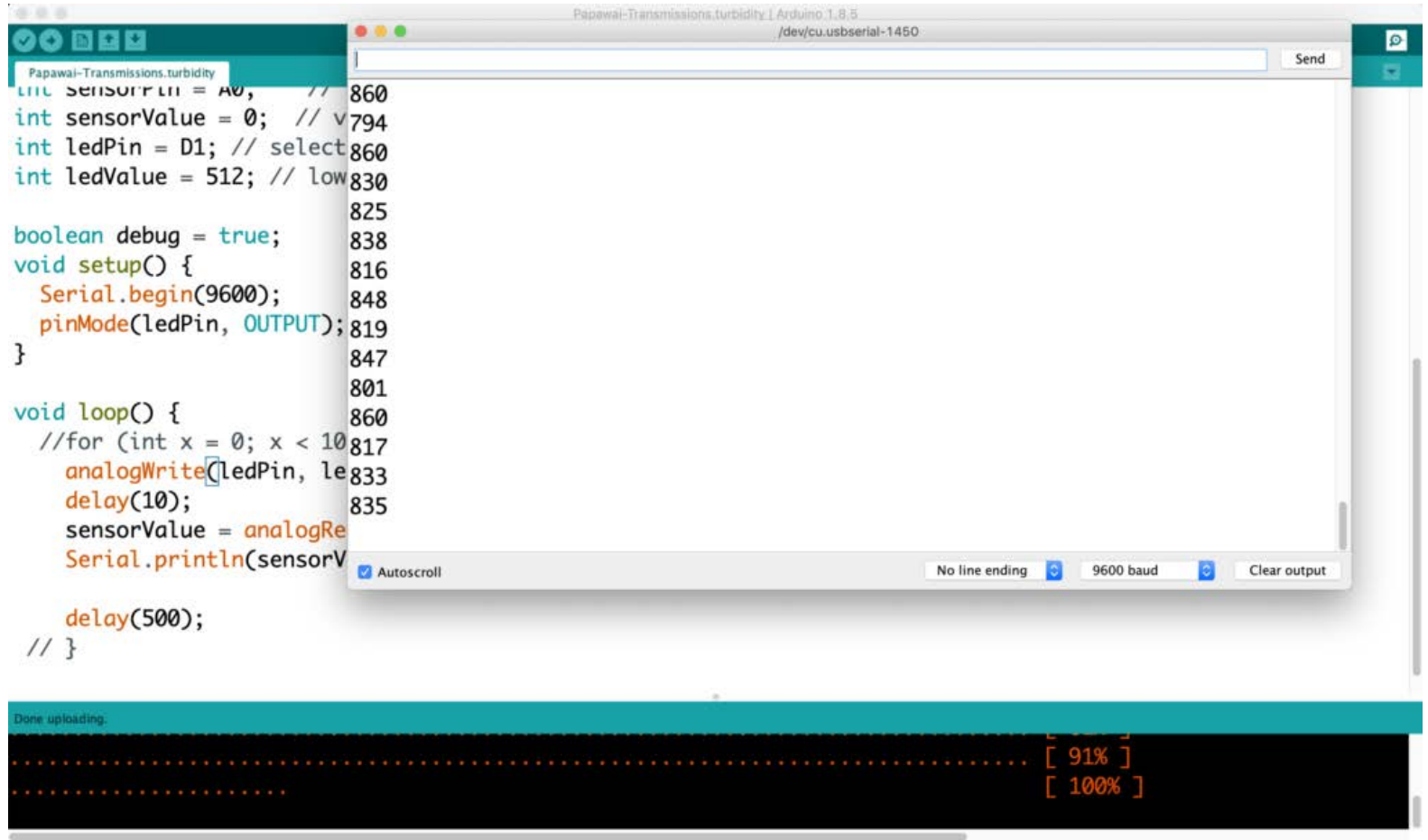

Figure 95. Author. (2018). Testing the values read by the sensor with the Arduino serial monitor.

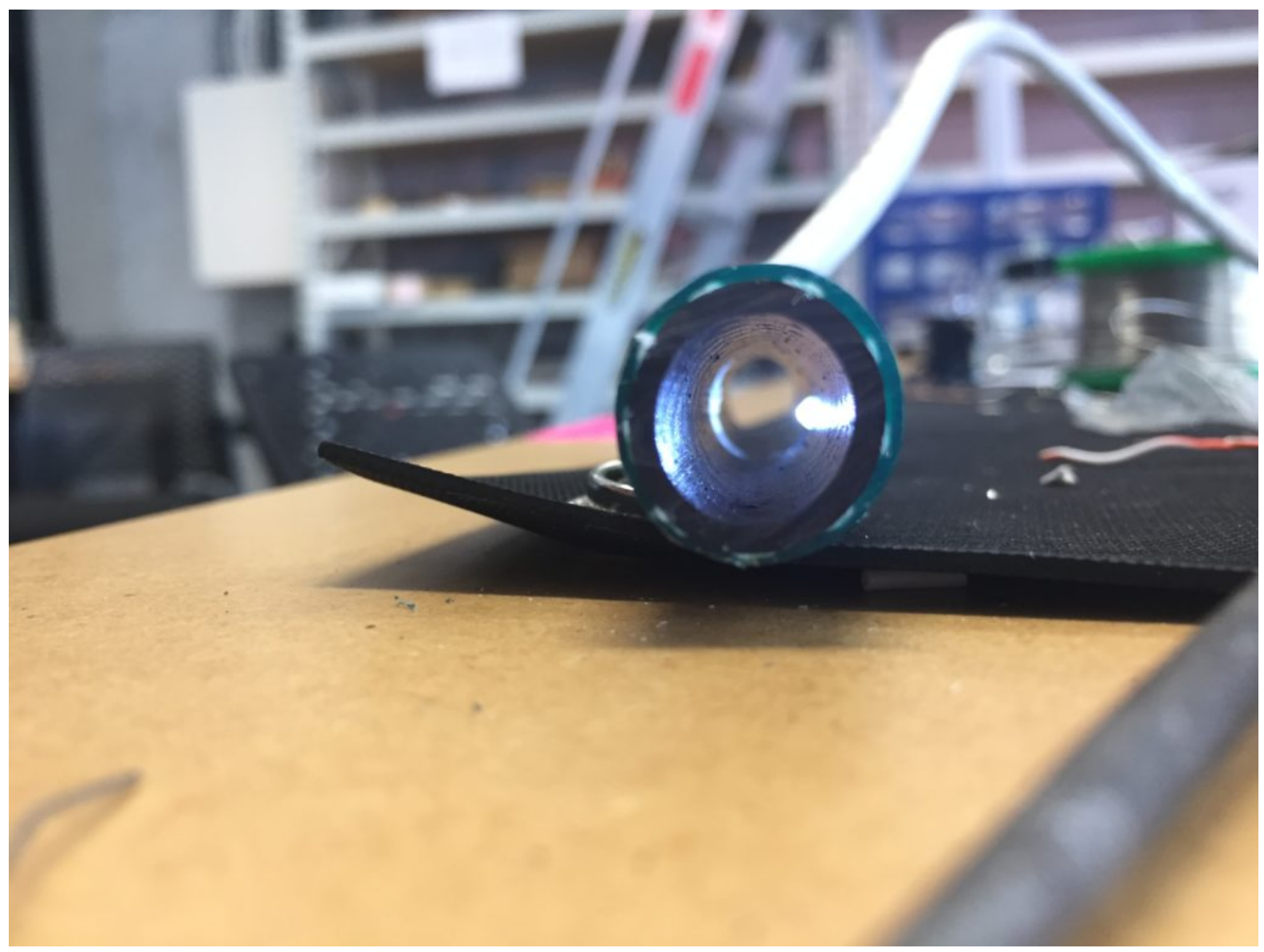

Figure 96. Author. (2018). Testing the LED at full brightness encapsulated in the hose. 
For the sealing, I purchased the All Clear sealant that I have previously used for waterproofing my hydrophone. As opposed to hot glue, this material stays flexible when dried out and doesn't run the risk of getting brittle.

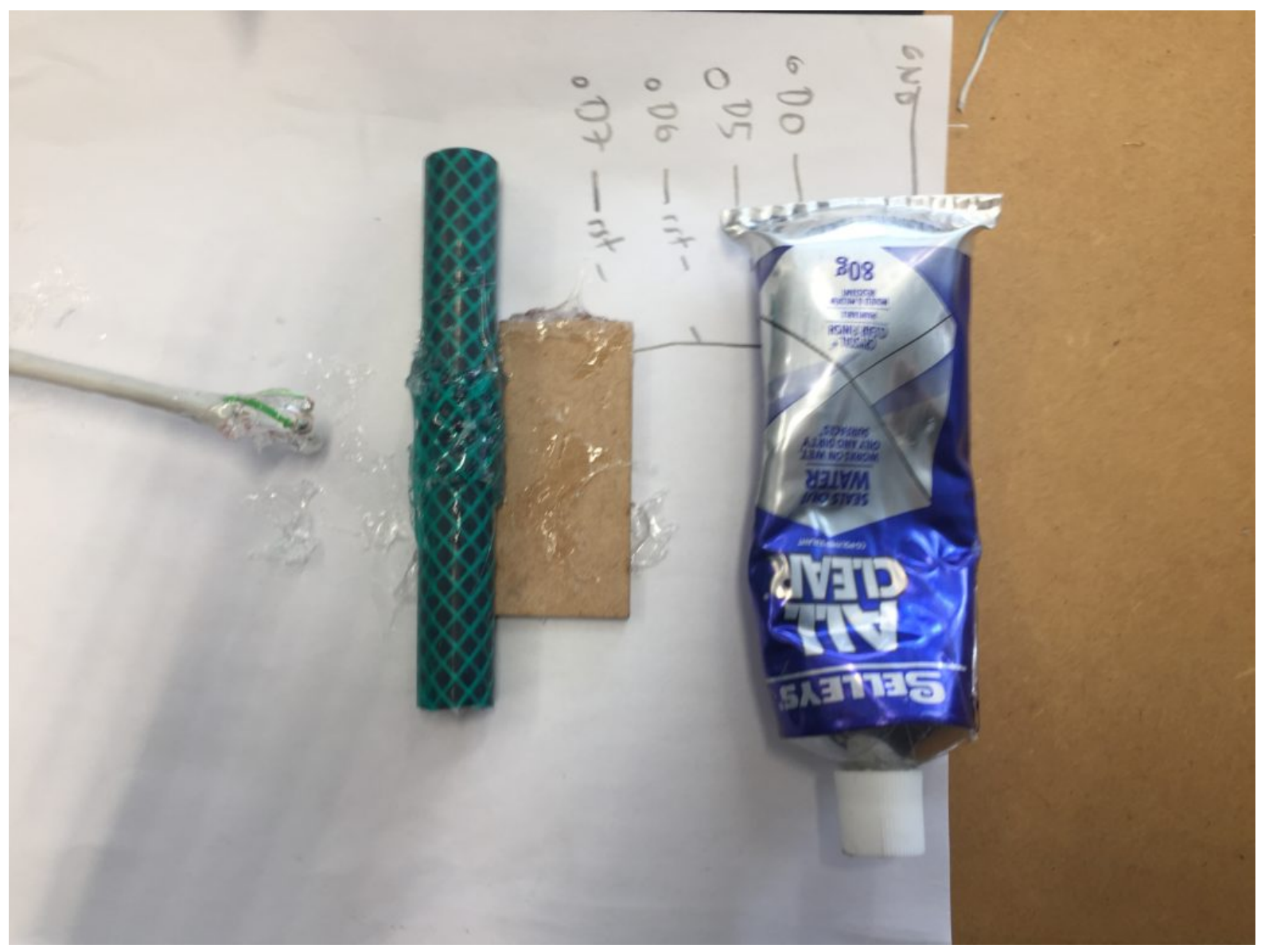

Figure 97. Author. (2018). Failed assembly. The sealant has not dried enough overnight to keep the components in place.

Unfortunately, one of the LED solder points was not well done, and I only discovered it after having added the sealant. With the cable disconnected, this prototype is unusable in the field, but the process of building it helped me understand possible avenues for improvement:

- The design with the components stuck through tight-fitting holes is cleaner but needs to be revised with waterproofing in mind.

- Solder points need to be stress tested and - if necessary, re-done - before adding sealant.

- Cables need to be fixed into place before adding sealant. Sealing might need to be re-done in the 3D workshop with proper ventilation and safety gear as this might require the use of turpentine. 
- Sealant might need to be changed as it might not be ideal in combination with cables/electronics.

\section{Component List:}

Hardware:

- Computer with USB interface

- WeMos D1 mini (know known as LOLIN

- $1 \mathrm{LDR}$

- $110 \mathrm{k}$ Resistor

- $13 \mathrm{~mm}$ white LED

- Drill with $3 \mathrm{~mm}$ and $5 \mathrm{~mm}$ bits

- All Clear Sealant

\section{Software}

- $\quad \underline{\text { Arduino }}$

- CH340 drivers for your operating system

- Add ESP8266 to your Board Manager. The next iteration of the turbidity sensor requires more thorough waterproofing from the beginning. Prototype I started as a simple proof-of-concept of the component combination (LED, LDR in a garden hose enclosure) and had no consideration of waterproofing. After that, the focus of Prototype II lay on improving the initial design's lack of water-proofing and adding long cables so it can be eventually tested out in the field. 


\section{Step 1: Components, cables and heat shrinking tube}

For this turbidity sensor design, I used an approximately two-metre-long stranded core CAT-5 cable to connect my white LED and my LDR to my WeMos D1 board.

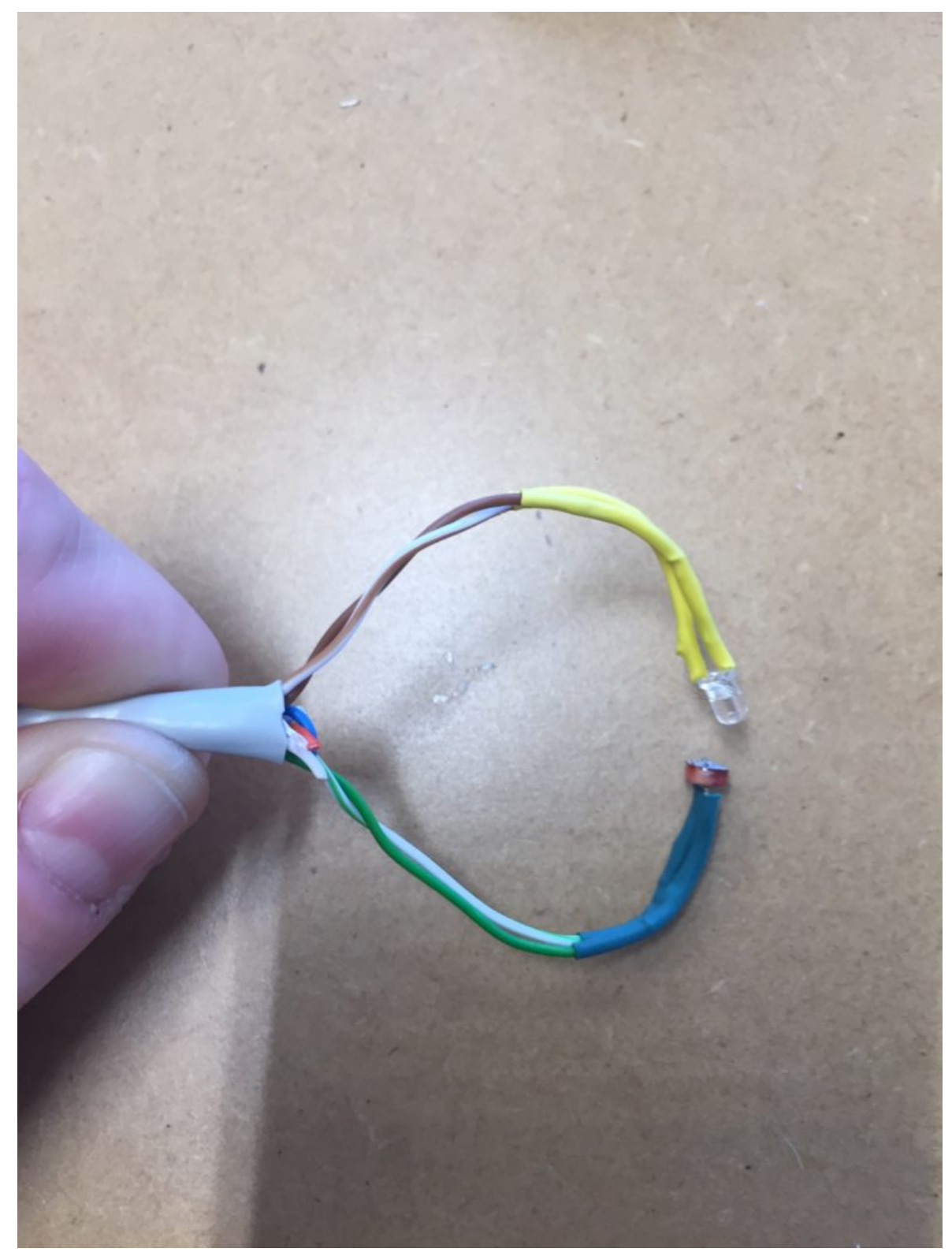

Figure 98. Author. (2018). LED and LDR sealed with colour coded heat shrink.

After assembly, I immediately sealed the components that eventually get submerged into the water with heat-shrink tubing (yellow for the LED, green for the LDR). 


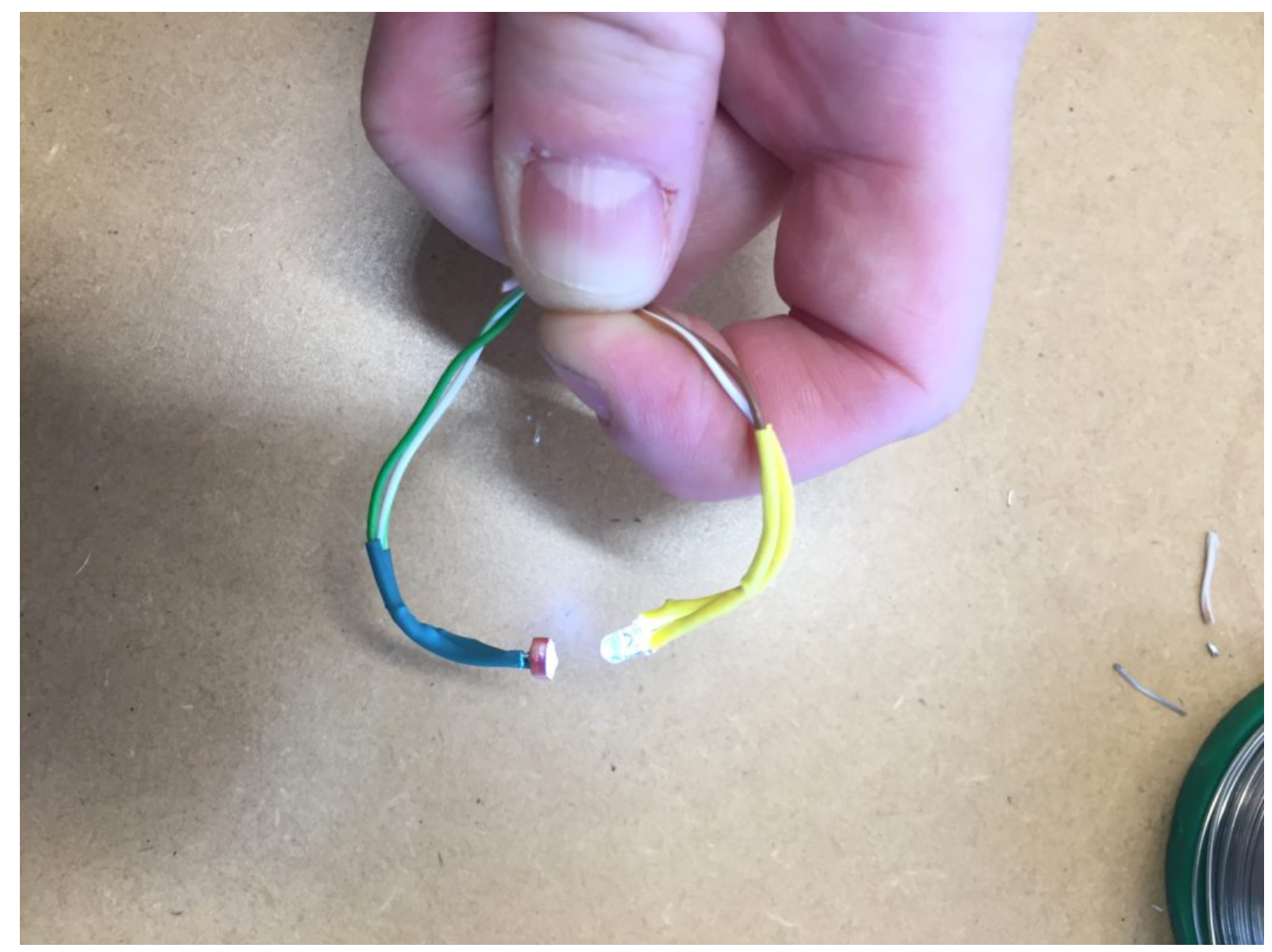

Figure 99. Author. (2018). Testing the LED and LDR before assembly in the hose.

My first attempt at running the test code with the components through the two-meter-long wire went well. I tested the incoming values roughly by concealing the LDR with my finger.

\section{Step 2: Housing the components in the "test tube."}

Similar to the previous prototype, I cut a new $10 \mathrm{~cm}$ piece off the garden hose and drilled two opposing holes of the size of the components in the centre. To attach the LDR and the LDR I used hot glue only this time. The reasoning behind this is that hot glue has a much shorter drying/hardening time than the All Clear sealant. This allows an efficient applying of layer after layer within a relatively short period. The sealant ideally requires overnight drying which means assembly would span several days instead of hours. 


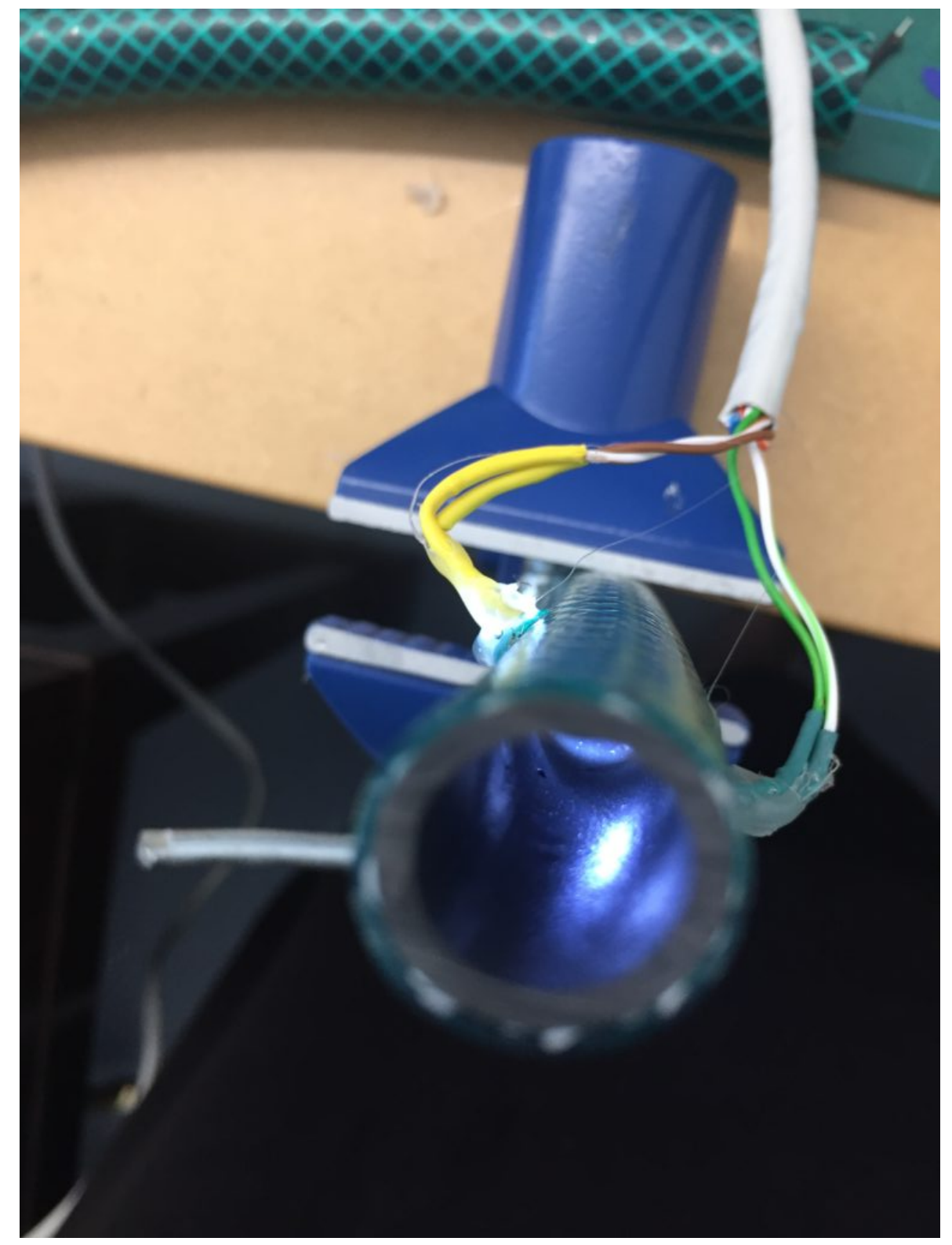

Figure 100. Author. (2018). Using a vice to keep components in place during assembly. 


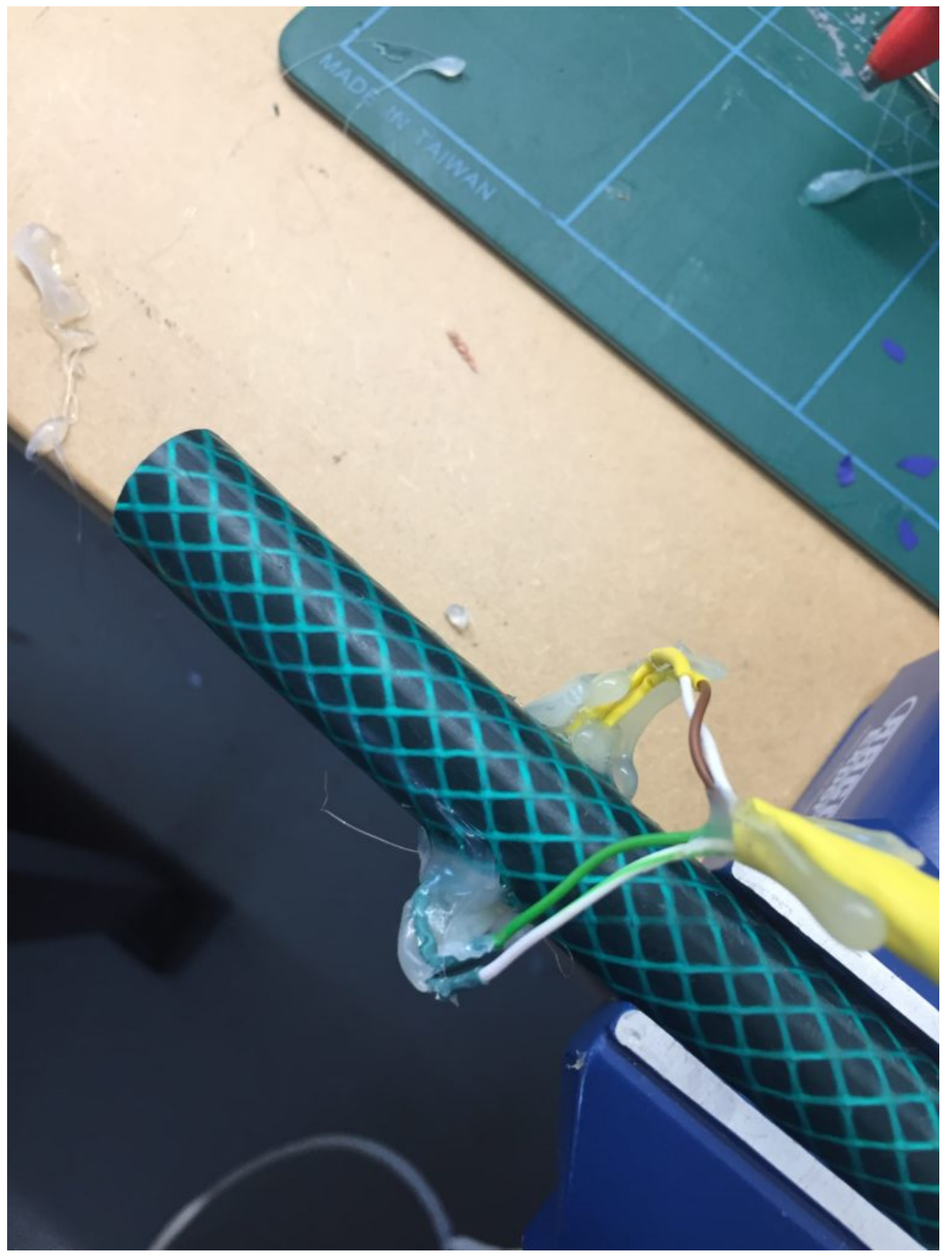

Figure 101. Author. (2018). Hotgluing the components together turns out to be a tricky task.

The work with hot glue is messy and requires diligence. Therefore, the purpose of the first layer is to attach the components to the hose and make sure they are facing each other correctly. 


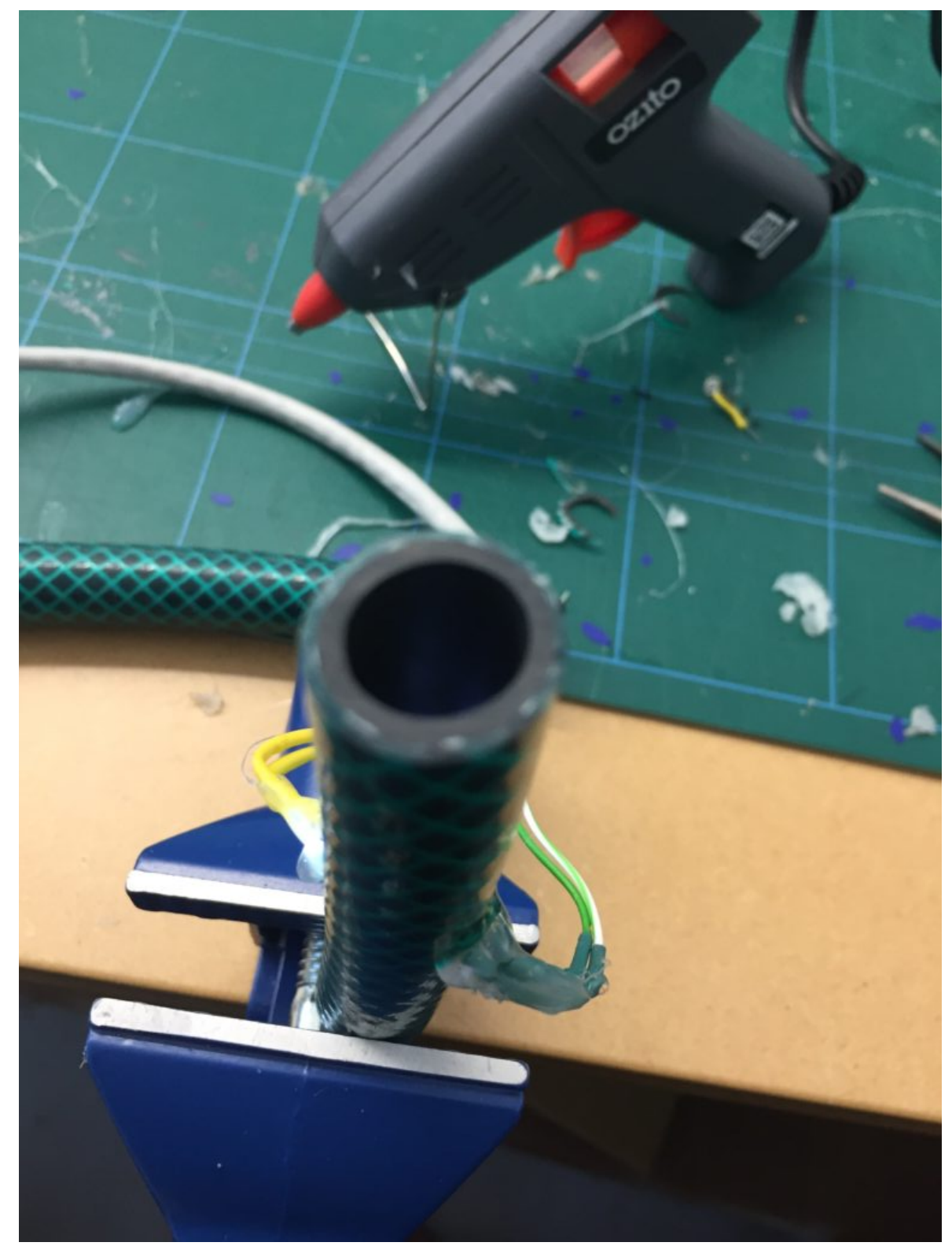

Figure 102. Author. (2018). The first layers of hot glue applied already reveal some possible weaknesses of the design.

After the first layer has been applied, I test that the components have not been damaged in the process of hot gluing. The incoming values look good so far, but I am not entirely happy with the design. It is quite hard to apply hot glue around the components evenly. I already spot some small grooves in the glue that could cause some leaks later on. 


\section{Step3: Dear hot glue, please protect my components}

While applying the next layer of hot glue, I have already made peace with the fact that this probe is going to look very odd. Basically, I am looking at a small dark-green piece of garden hose attached to a long cable with semi-transparent blobs of glue. I also notice that the wire close to the components appears to be under strain caused by the angle the components are attached to the hose. I should have immediately bent the connectors at a right angle to avoid this oddly shaped glue blob altogether.

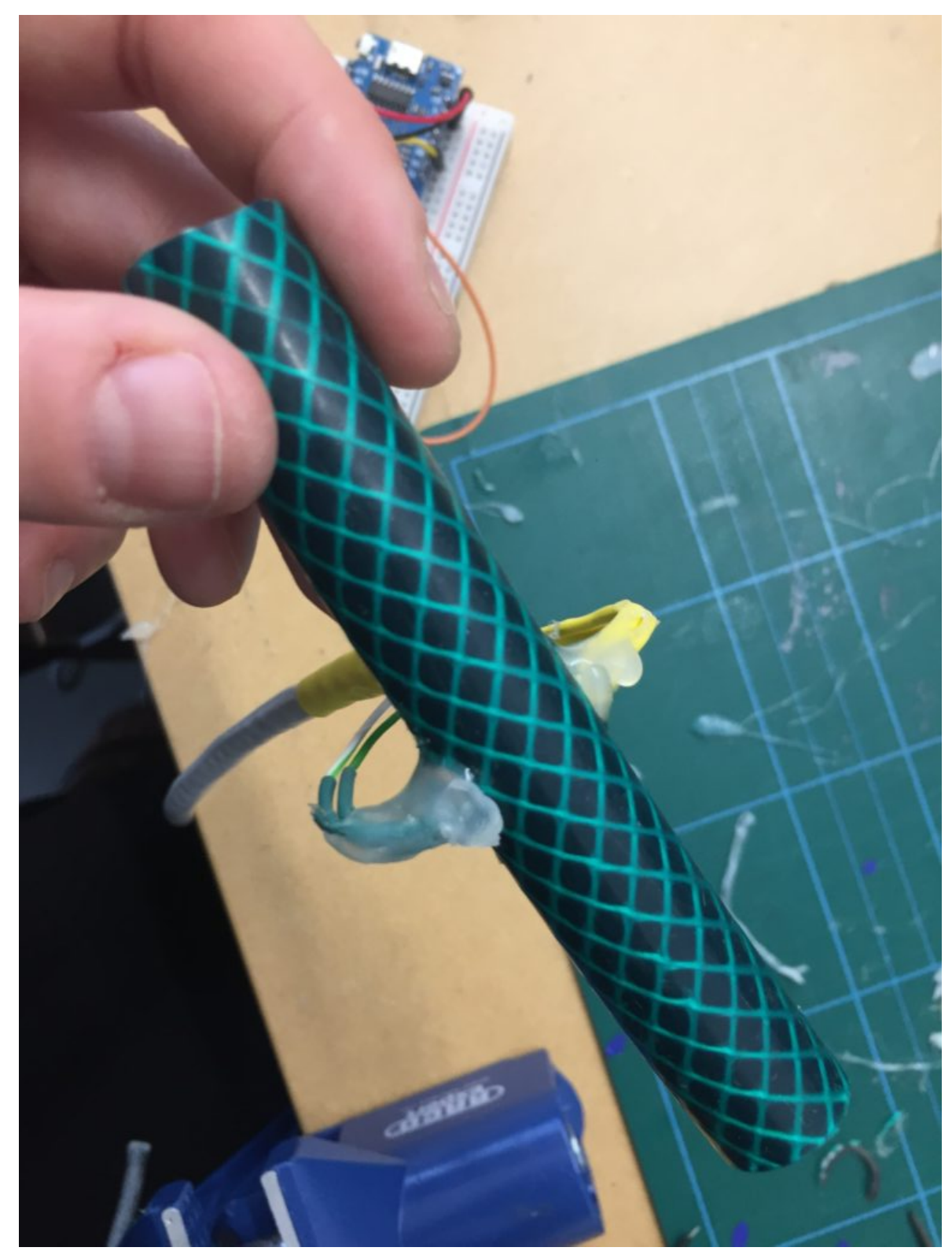

Figure 103. Author. (2018). Detail of the sensor probe with dried hot glue.

Despite the aesthetic shortcomings of this turbidity sensor design, all components seem to be working, and I am ready to compile a version that sends data wirelessly via the MQTT 
network so I can safely test the design in an underwater setting, without needing a laptop attached to any submerged components.

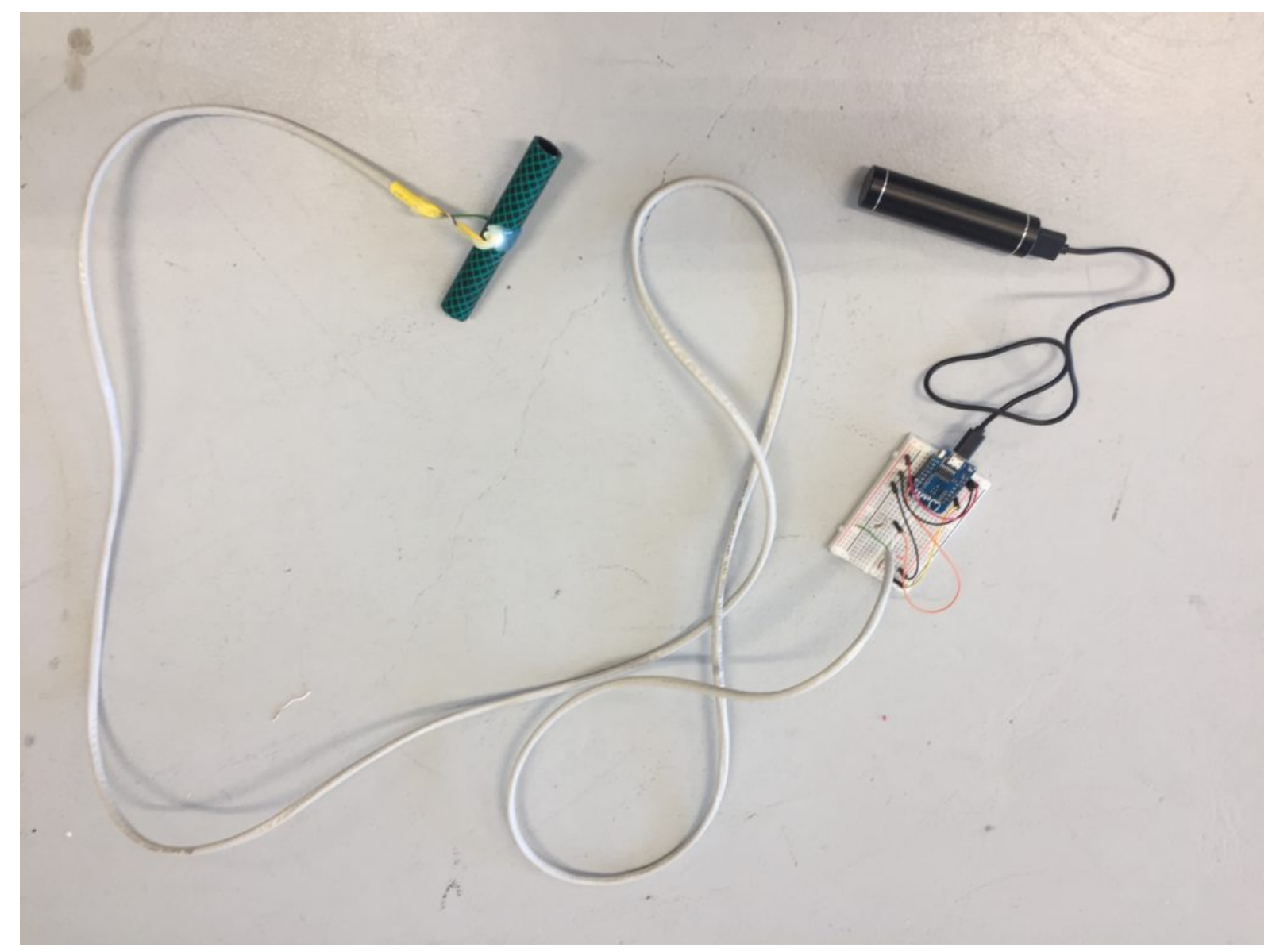

Figure 104. Author. (2018). Test setup of turbidity probe and battery-powered WeMos D1 mini connected to Wi-Fi network.

\section{Continuing the design with a custom LED output}

For this turbidity LED prototype, I wanted to test the use of a transparent material instead of paper. This would make the microcontroller and the battery more visible and provide more literal transparency in the design.

\section{Planning and designing the circuit}

To avoid more plastic usage, I recycled an old bottle from the Moturoa installation for this prototype. As a first step, I removed the Moturoa stickers and cleaned it with methylated spirits. 


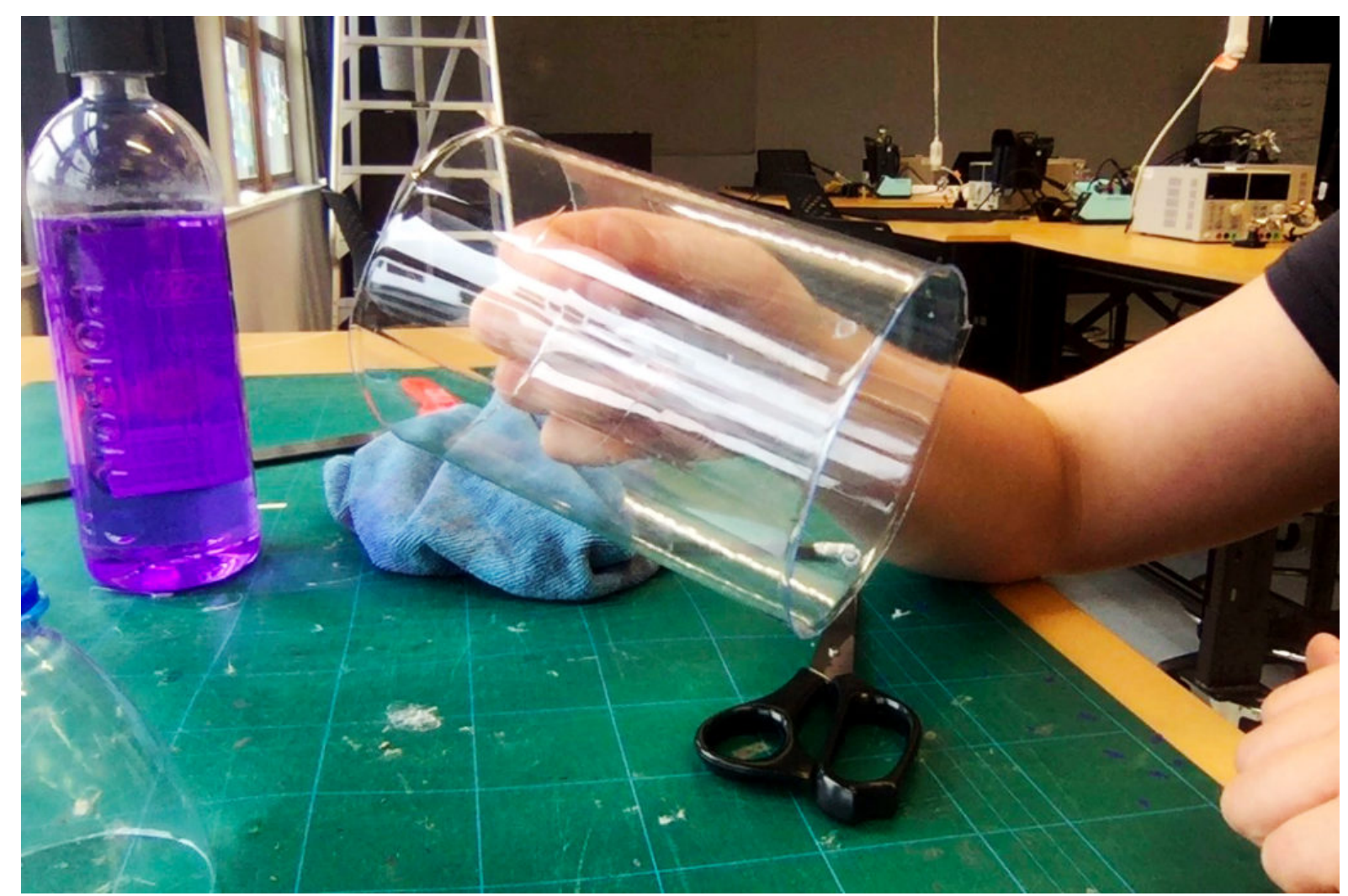

Figure 105. Author. (2018). Cleaning a used bottle casing for repurpose as a new output node.

During my initial research I looked for known scales or visual indicators for turbidity but mostly found visual gradients.

My first idea for visualisation was to link the brightness of an LED to the sensor value. For example, if the LED is at full brightness this means the water is clear; if it is of low brightness the visibility of the water is low. An issue with this approach is that it might be difficult for a viewer to estimate the maximum brightness of a single LED. Hence, a viewer might not be able to extract meaning from the displayed value. LED brightness is generally a hard thing to estimate, especially in bright sunlight.

A solution could be to have a reference LED next to the indicator LED that is always at full brightness as a comparative value. The indicating LED could also be faded on first to a complete maximum brightness and then dimmed to the readout value read by the sensor. This could work, but this method might be too complex and create confusion on how to understand turbidity. 


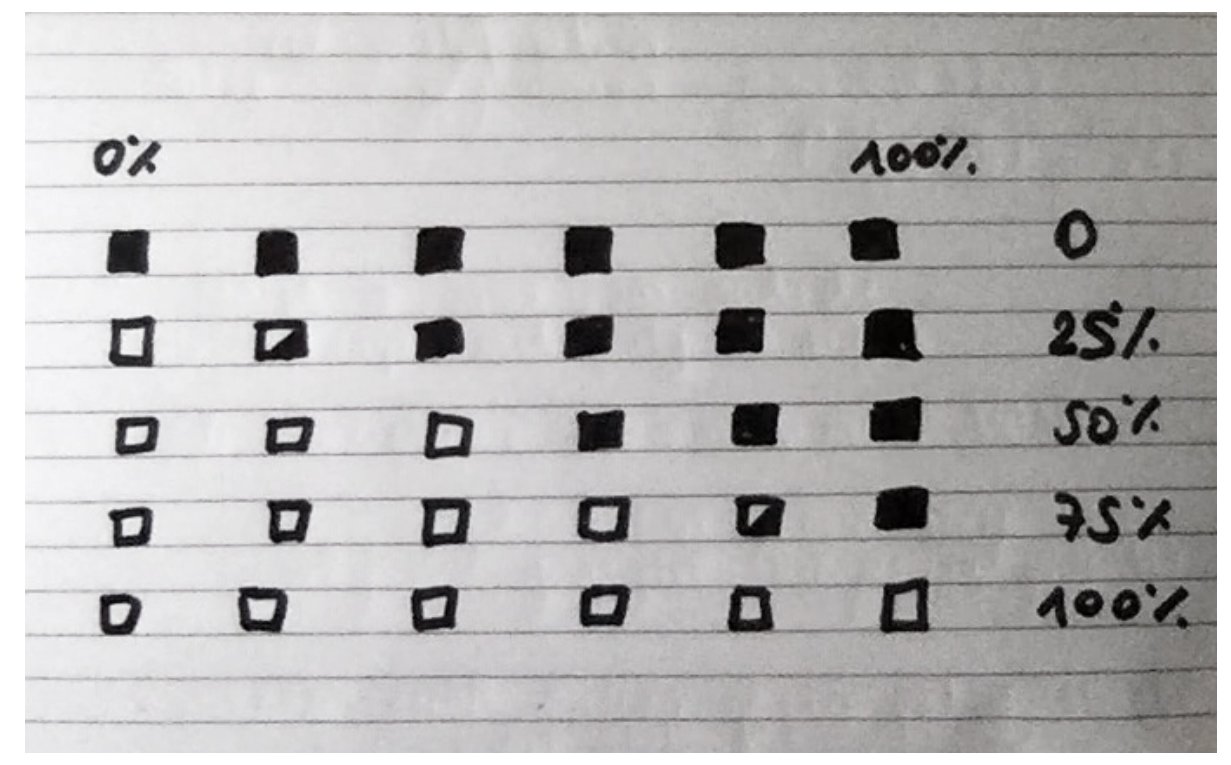

Figure 106. Author. (2018). Draft for turbidity visualisation with six LEDs.

A simpler idea would involve visualising turbidity with an array of LEDs. If water clarity is at 100\%, all LEDs are on. At 0\% all LEDs are off, at 50\% half of the LEDs are on (see sketch above). Using a variable brightness of the rightmost LED could work here to indicate a finer range of turbidity (25\% would be one LED on maximum brightness and the neighbouring LED at 50\%). This idea is simple but does align with the design of previous prototypes. The WeMos D1 mini has nine digital pins in total. I chose to use six LEDs for this prototype because this allows seeing all LEDs from one angle while leaving enough space (around $15 \mathrm{~mm}$ ) in between the $5 \mathrm{~mm}$ copper tape lines. 


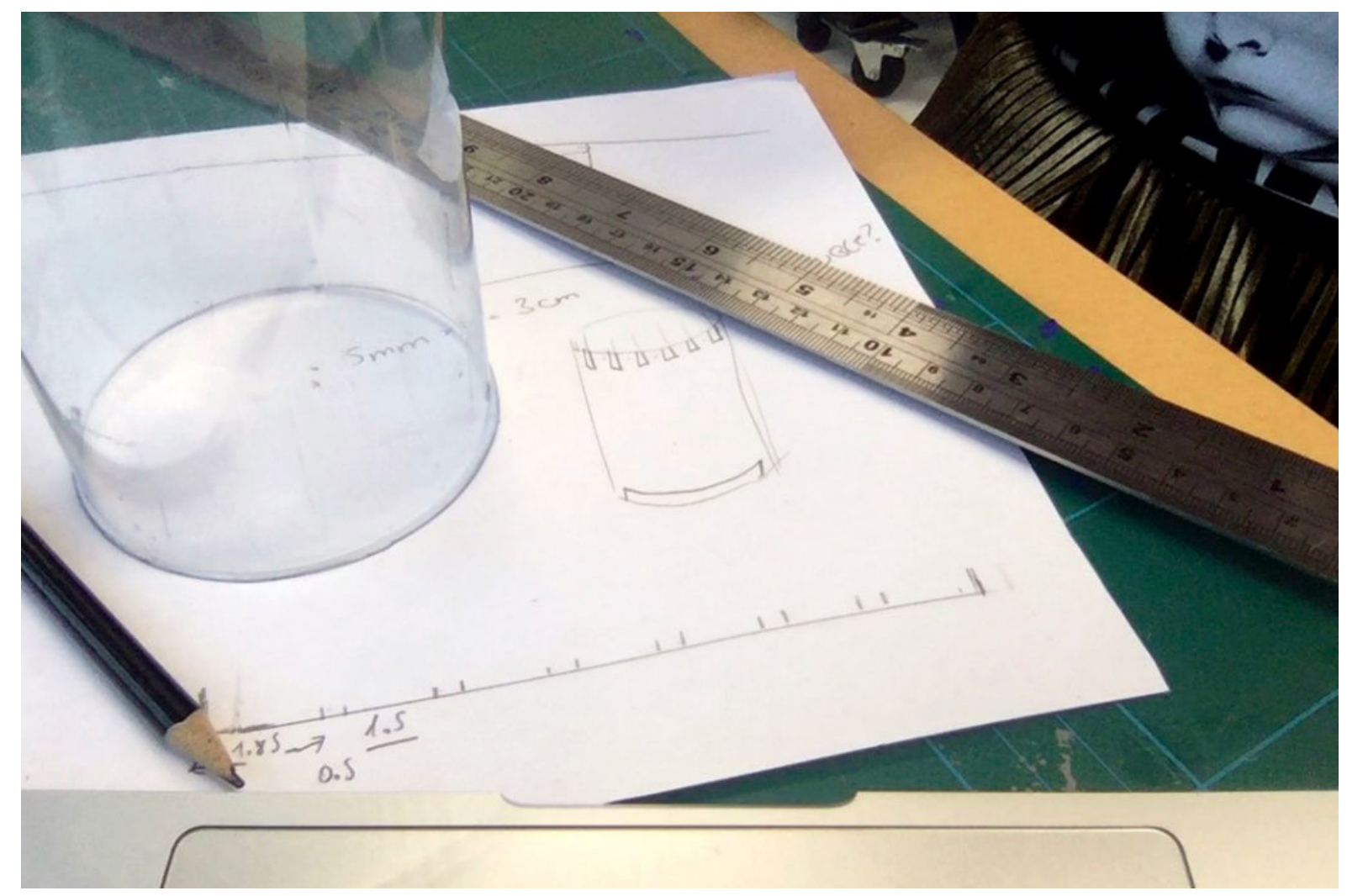

Figure 107. Author. (2018). Measuring out the placement of the copper tape.

\section{Assembly of the copper tape circuit}

The plastic insert should fit inside a glass jar and it should be able to fit into a variety of jar sizes. I placed the six copper trails to the digital pins on the top of the plastic cylinder. I decided to line the bottom of the cylinder with one continuous piece of tape as ground (GND). 


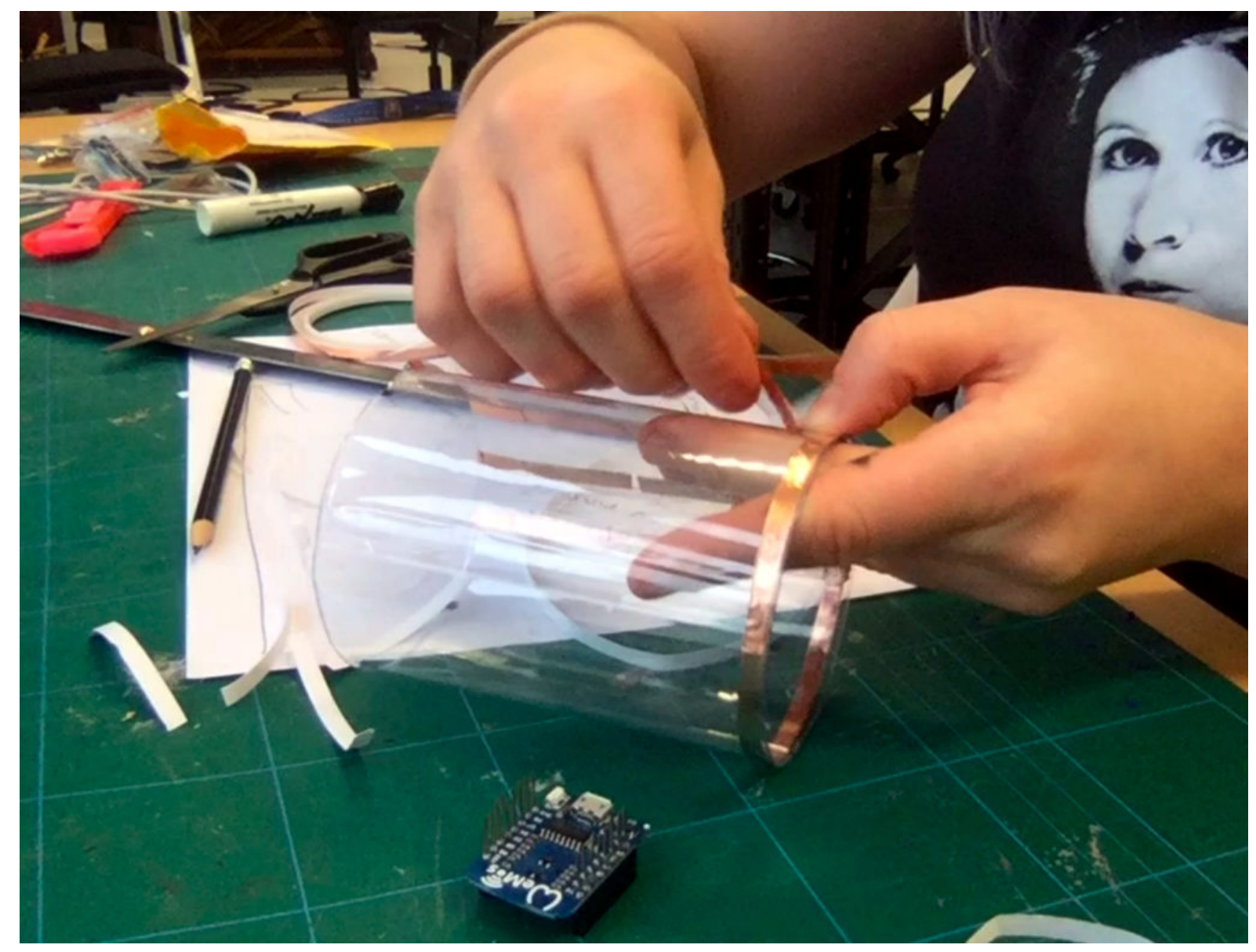

Figure 108. Author. (2018). Attaching the copper tape to the bottle casing.

For this light probe, I chose blue SMD LEDs, mostly because I haven't used this colour in any other probes so far. I apply some solder on the copper tapes first before placing the SMD on the circuit with a pair of tweezers. Then I melt one solder to tentatively put the LED in place before finalising the solder points on both sides. As anticipated, plastic and copper tape are not an ideal combination. Too much heat from the soldering iron melts the tape layer of the copper tape and lifts it off the surface (as with paper). The heat additionally shrinks the plastic and eventually deforms the entire surface. 


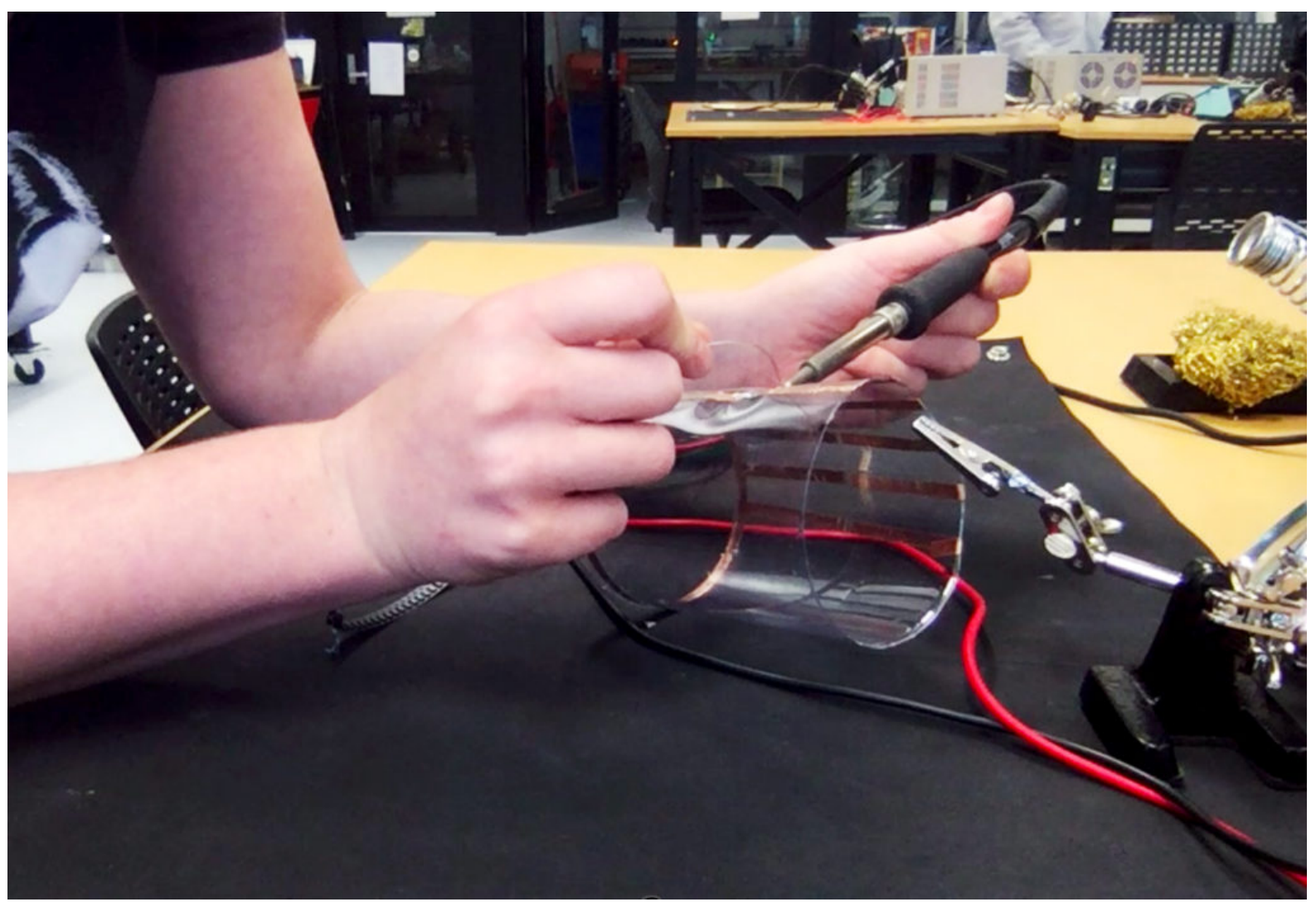

Figure 109. Author. (2018). Soldering the LEDs carefully to not melt the plastic too much.

Before adding another LED, I test every solder connection immediately by connecting a 3V power supply. This involves checking whether the solder point is good enough and if the polarity of the LED is correct. This batch of LEDs was easier to place on the circuit as they feature a clear polarity mark on the top. 


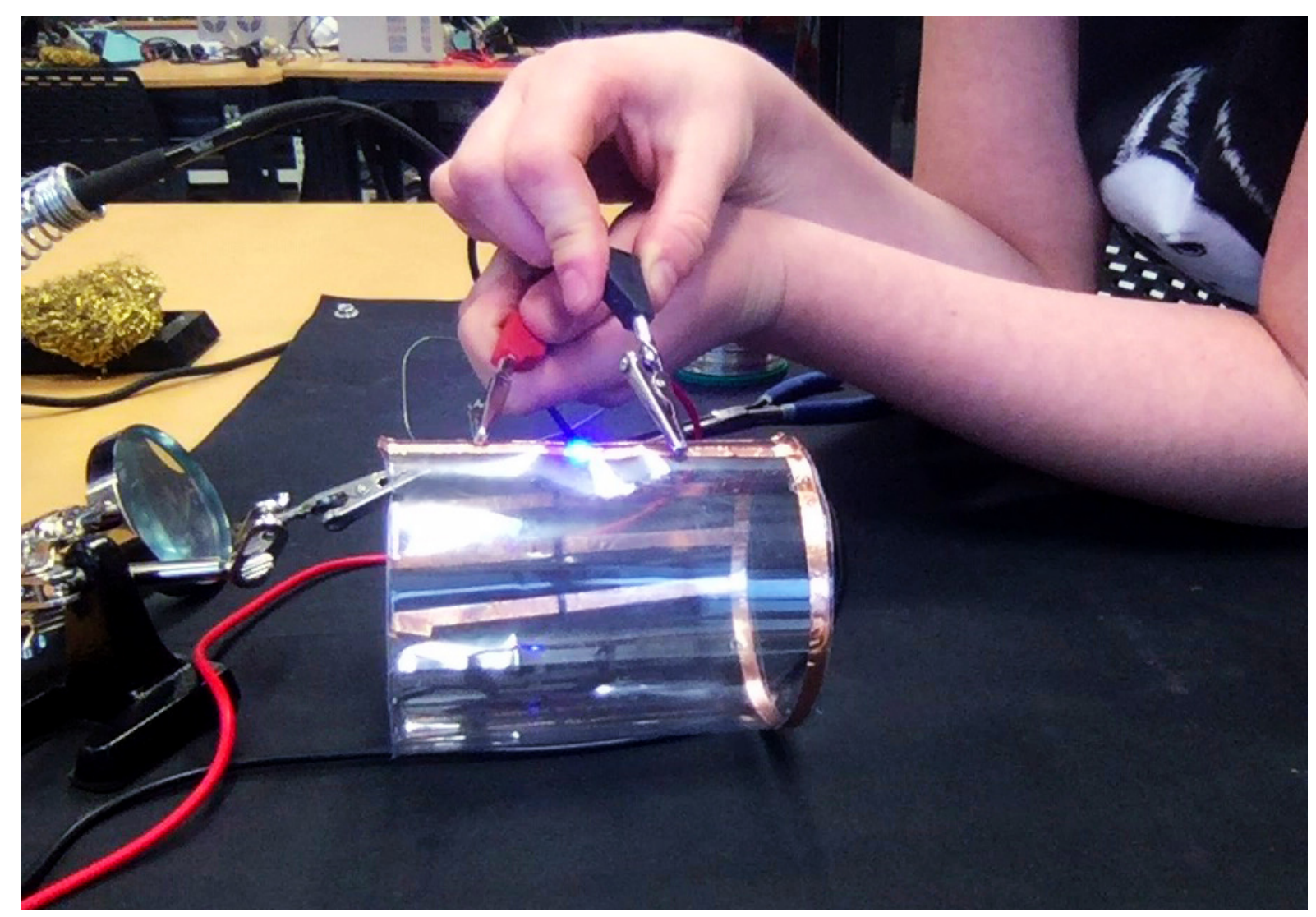

Figure 110. Author. (2018). Testing the solder connection of the LED.

To finalise the circuit, I decided to solder copper tape ends together instead of taping them. Using Sellotape to bridge copper tape ends has turned out to be a weak connection point in previous prototypes. This turned out to be a problem especially once I needed to bend the paper circuit to fit into the jars. The soldered connections are more stable and reliable. Unfortunately, the additionally applied heat and solder gave the GND copper tape a somewhat messy look. 


\section{Connecting the circuit to the microcontroller}

After placing the finished circuit into a glass, aesthetic details of the copper tapes become less obvious. The bright blue LED becoming the visual focus of the prototype.

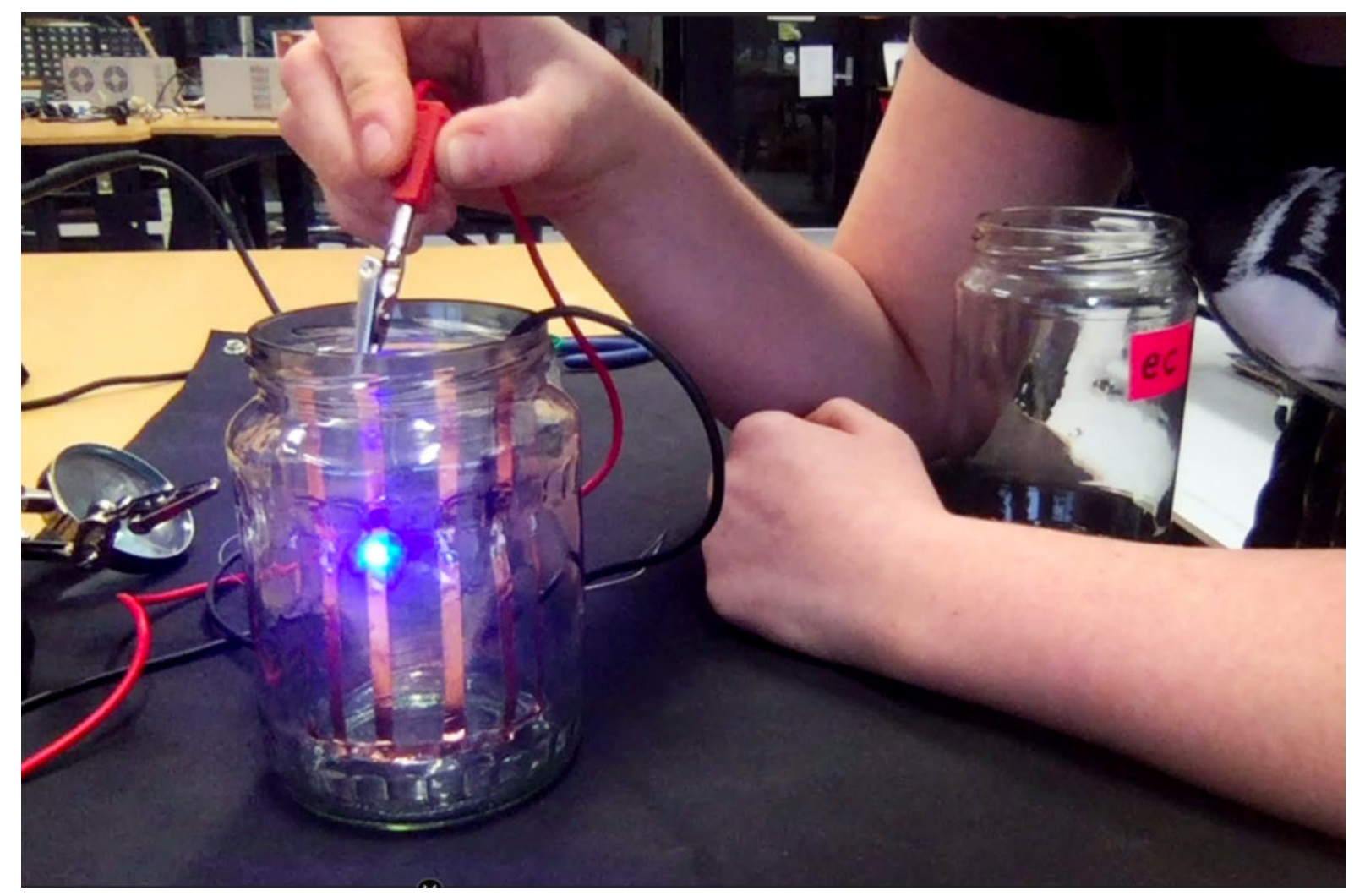

Figure 111. Author. (2018). Testing all six LEDs in planned jar enclosure.

The final soldering job involved attaching wires to the circuit that could connect to the WeMos D1 mini microcontroller.

I chose a piece of CAT5 wire and first soldered seven strands (6 digital pins plus 1 GND) to a row of pin headers to be plugged into the microcontroller. Once completed, I soldered them one by one to the copper tape circuit. 


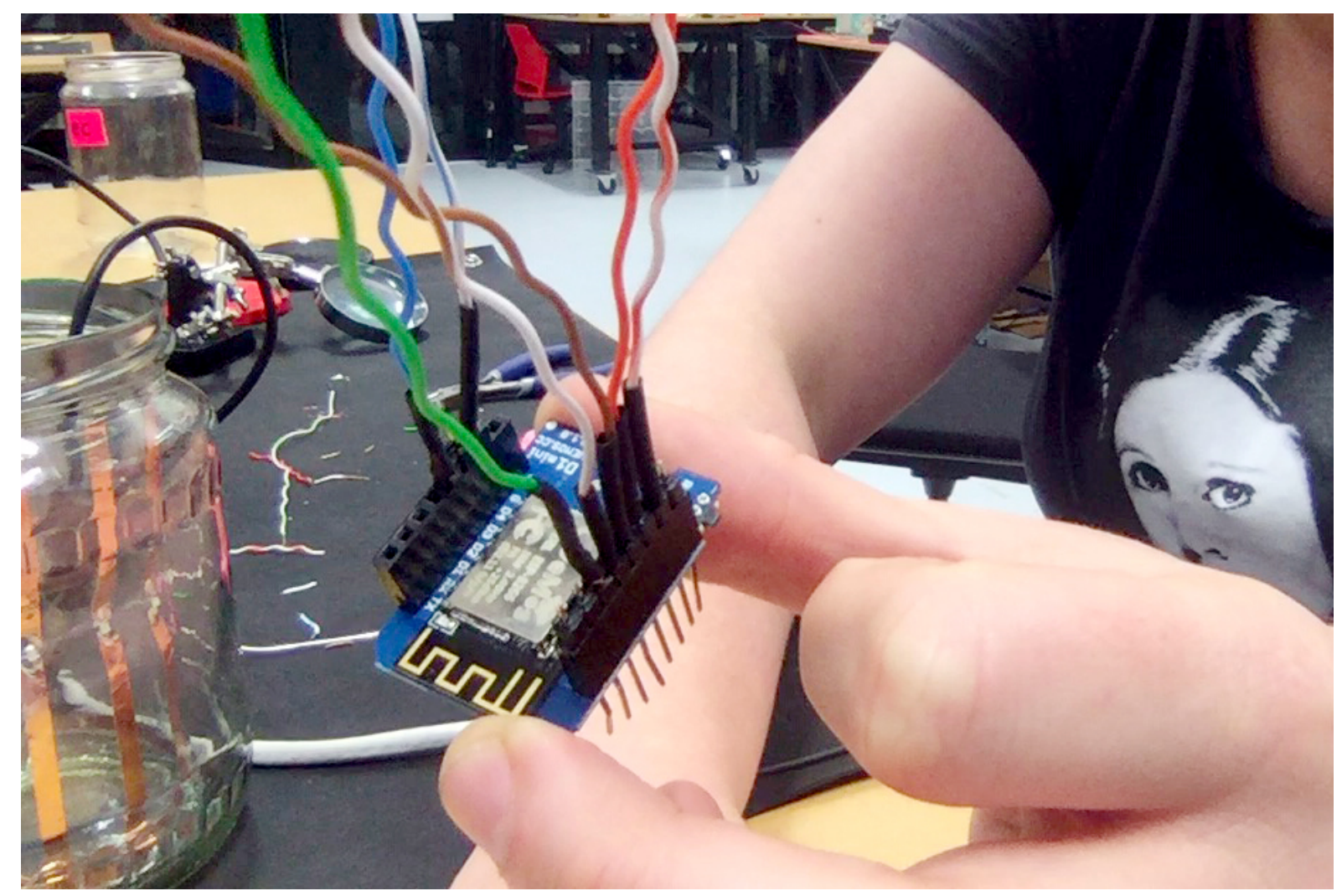

Figure 112. Author. (2018). WeMos D1 mini connected to control the six LEDs.

The assembly of the electronic components of the prototype is completed now. The next step involves writing code to link the LED brightness to the readout of the turbidity sensor.

In the first turbidity node design I re-used leftover plastic of one of the bottle designs as the circuit carrier and copper tape for connections. This proved to be tricky as the heat during soldering would distort the plastic and dissolve the glue of the copper tape, making it lift off the surface and weaken the connections.

In a new attempt to provide a seamful design, this new prototype uses copper coated welding rods and copper wire as conducting elements and at the same time as structural element. This means the circuit would not require a surface, such as paper or plastic, but would only consist of conducting copper elements. For a first test I used a $1.2 \mathrm{~mm}$ rod and experimented with soldering various wires and components to the rod. Soldering wire to the copper rod works well after removing the oxidation layer with sandpaper. The enamelled copper wire also only solders well after sanding, which is time consuming when many components are involved. The advantage of this, however, is that the 3-dimensional 
circuit is less likely to be short-circuited if parts accidentally touch - except for the conductive elements of the LEDs and the solder points.

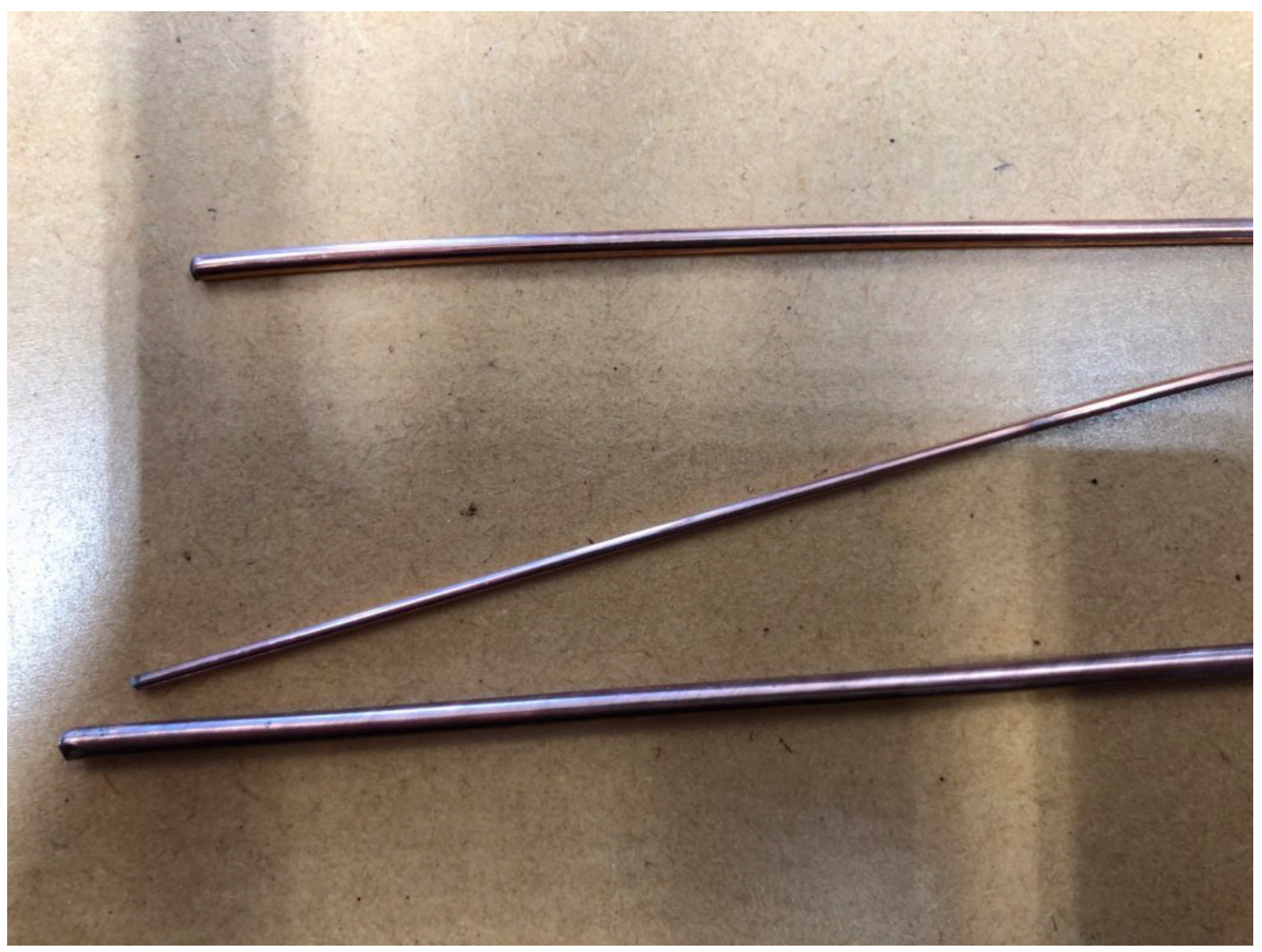

Figure 113. Author. (2018). Copper rods of various diameters. 


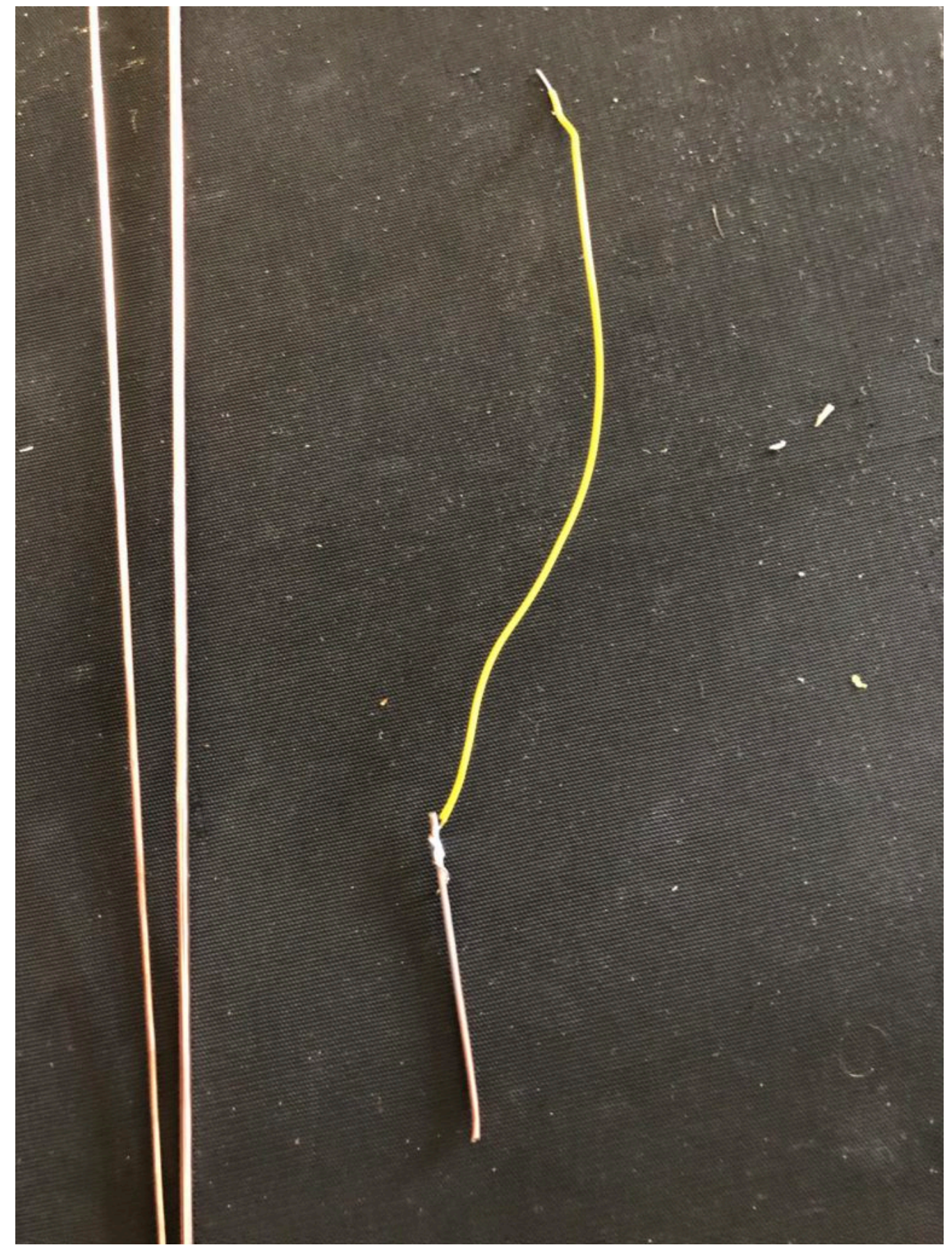

Figure 114. Author. (2018). Tests of soldering cables to the rods. 


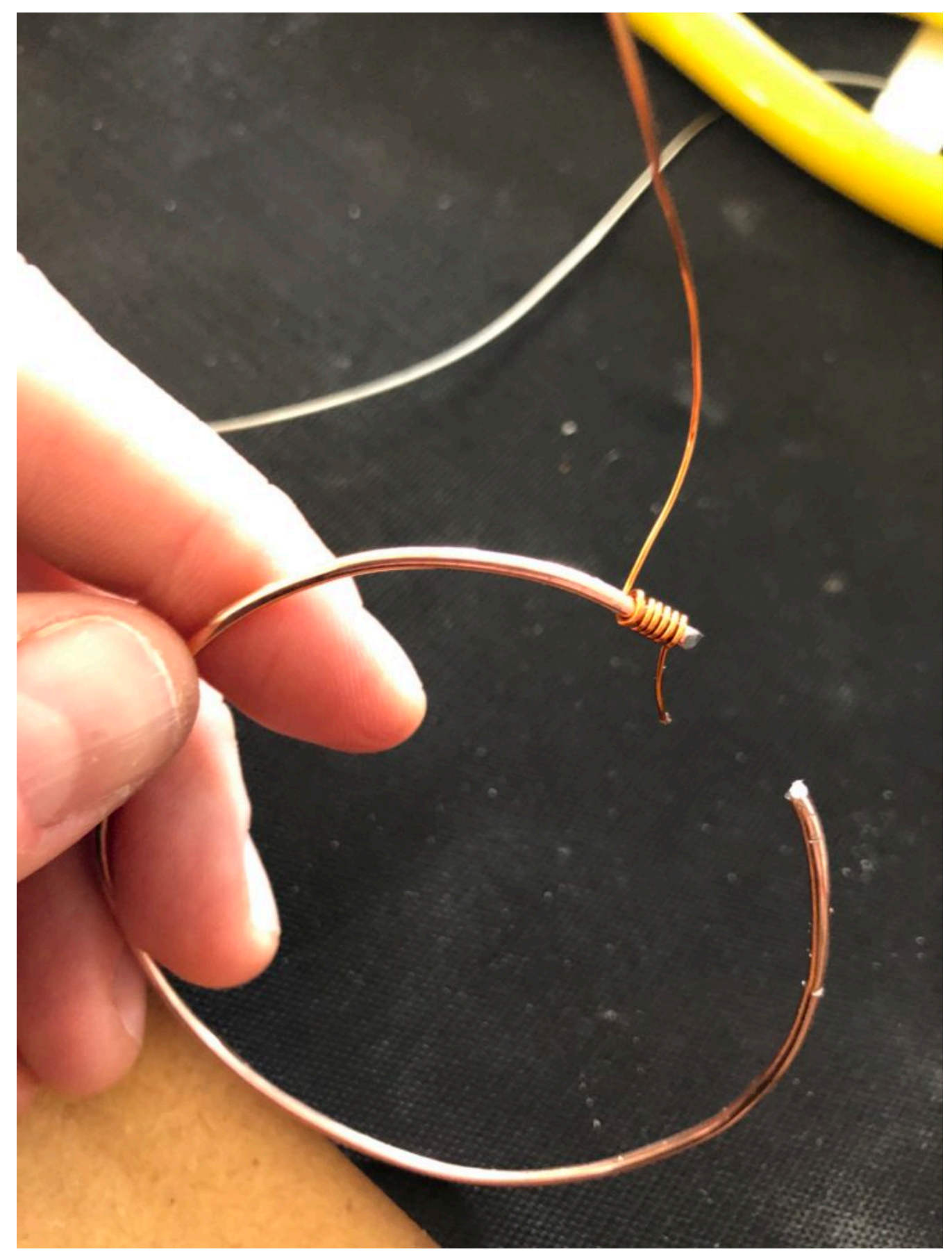

Figure 115. Author. (2018). Shaped copper rod with copper wire before being soldered.

I envisioned the test design to contain a set of 3 addressable LED sets that fit inside a glass jar. I bent the ground wire into a circular shape to act as a base for the circuit which will connect to a set of LEDs to be controlled by a WeMos D1 board. After a hopeless attempt to use SMD LEDs for this circuit I found that 3mm LED diodes are much better suited for this kind of circuit. 


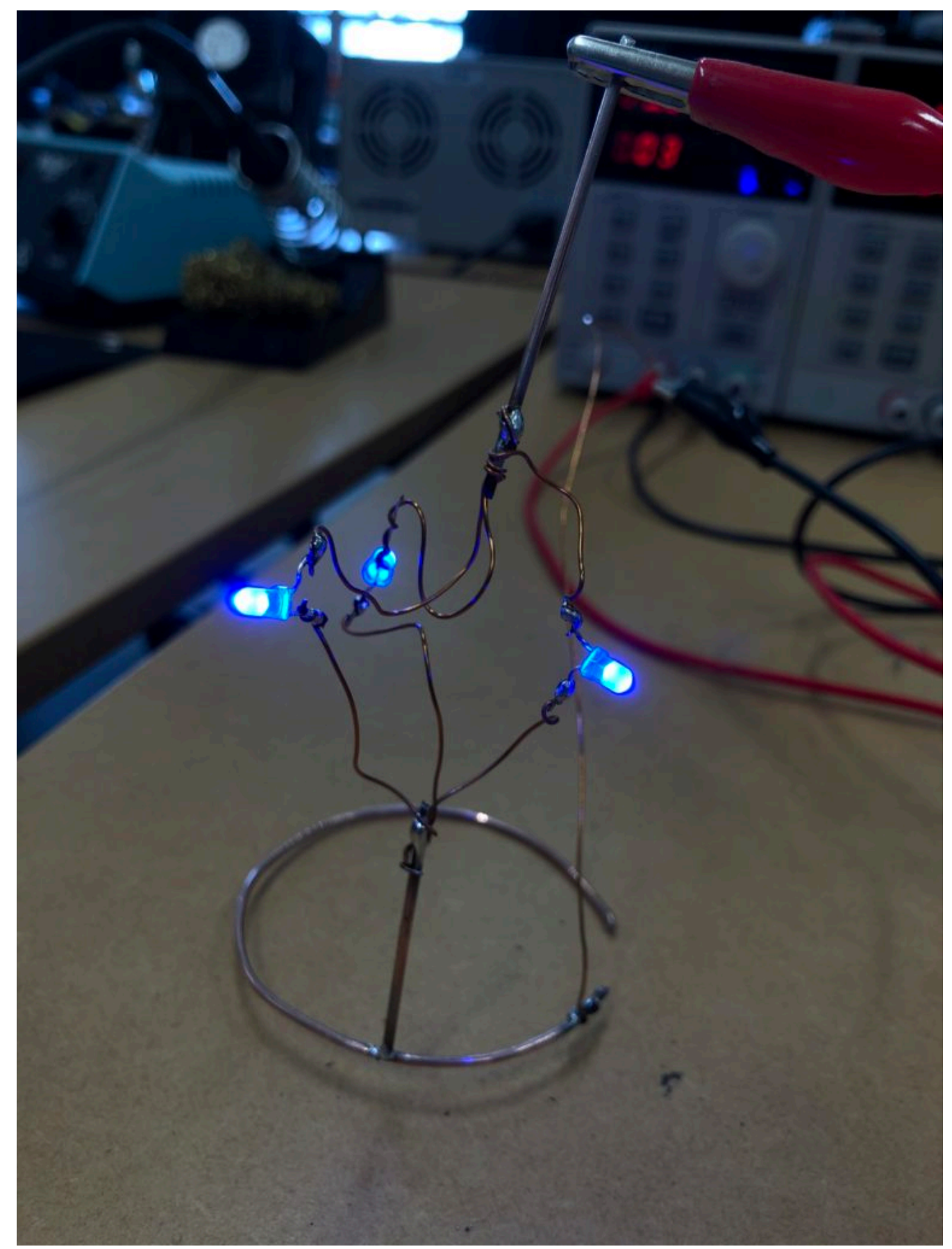

Figure 116. Author. (2018). Testing a group of three LEDs connected to the copper prototype. 


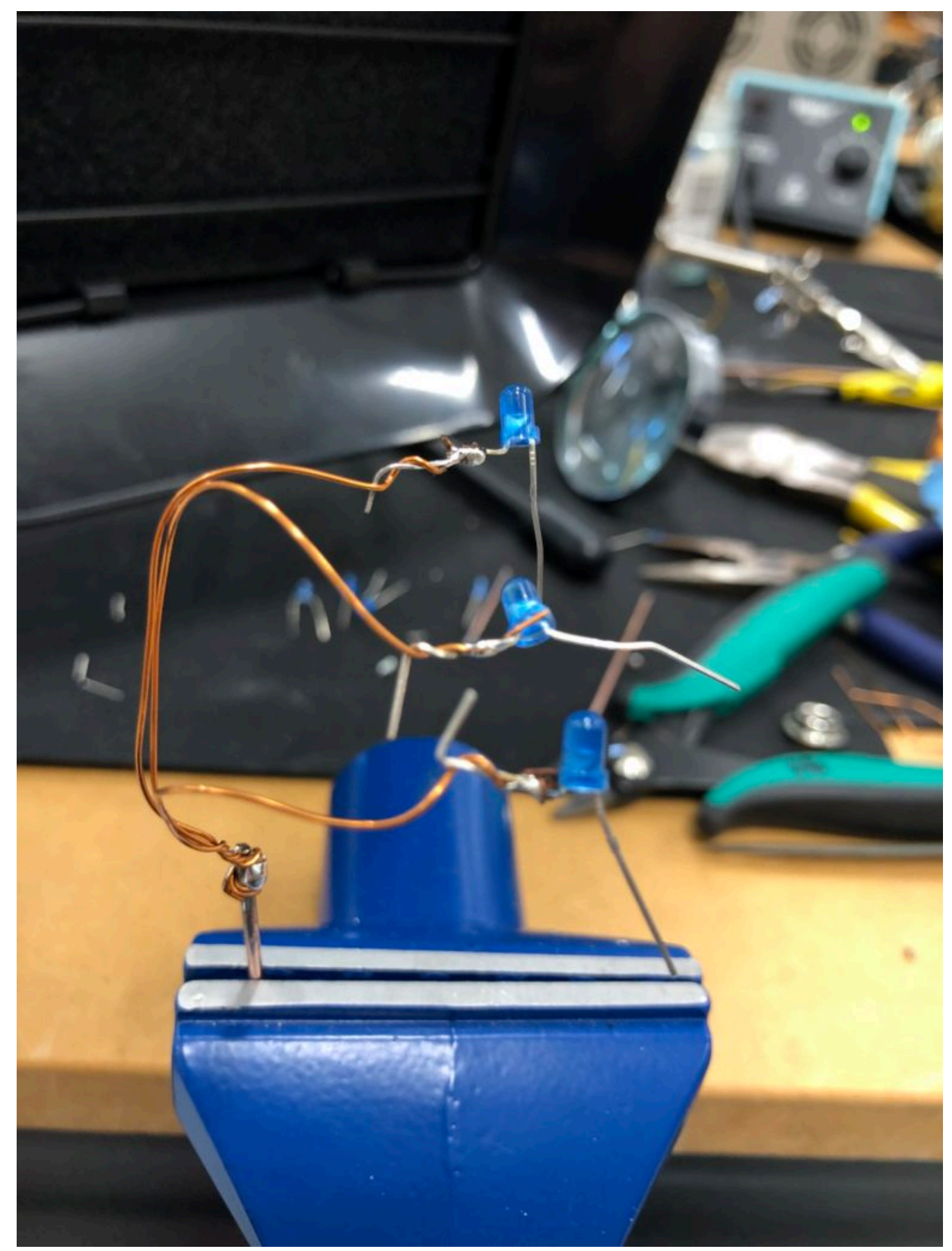

Figure 117. Author. (2018). Detail of assembling grouping of LEDs to copper wire before soldering. 


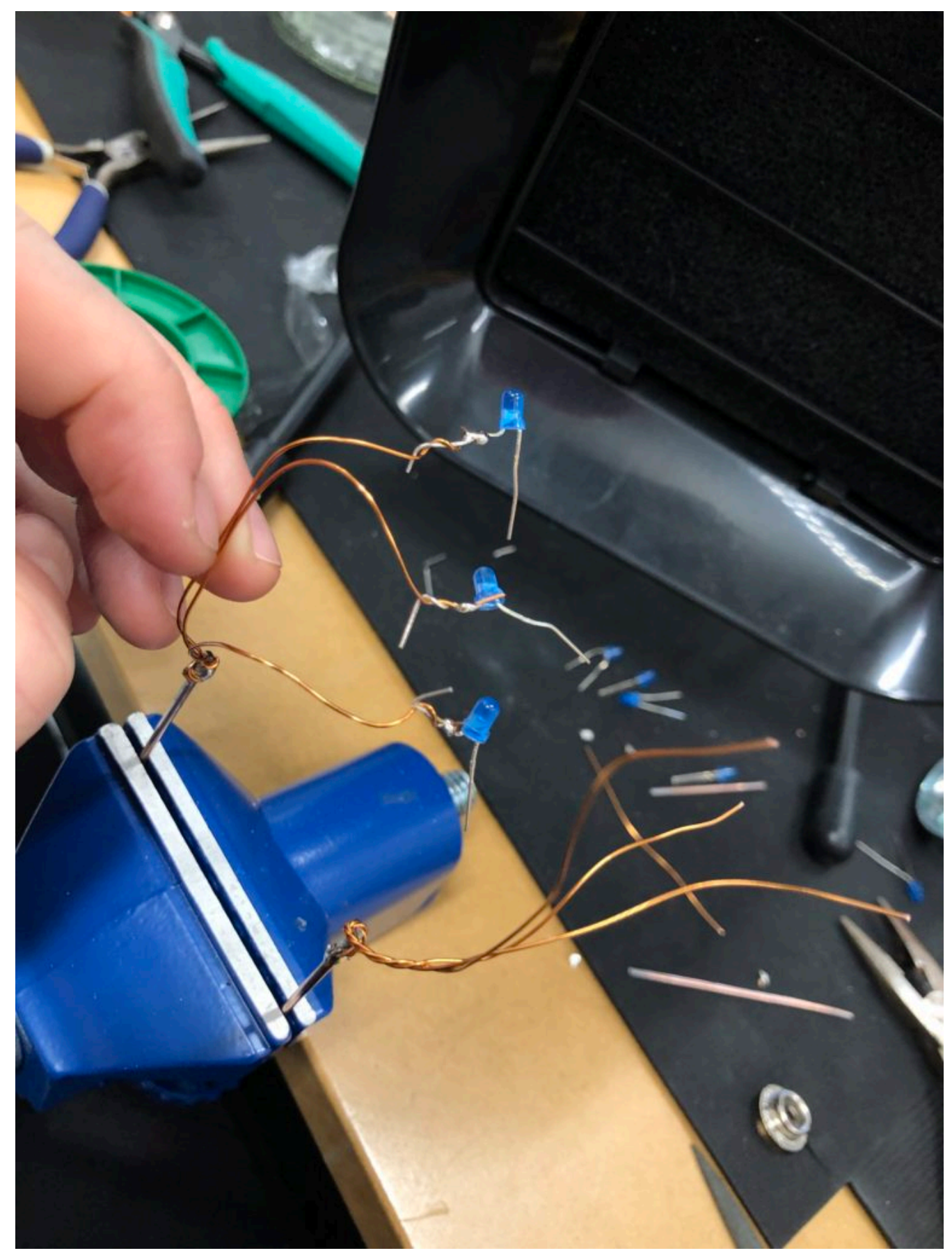

Figure 118. Author. (2018). Detail of copper wire prototype in progress. 


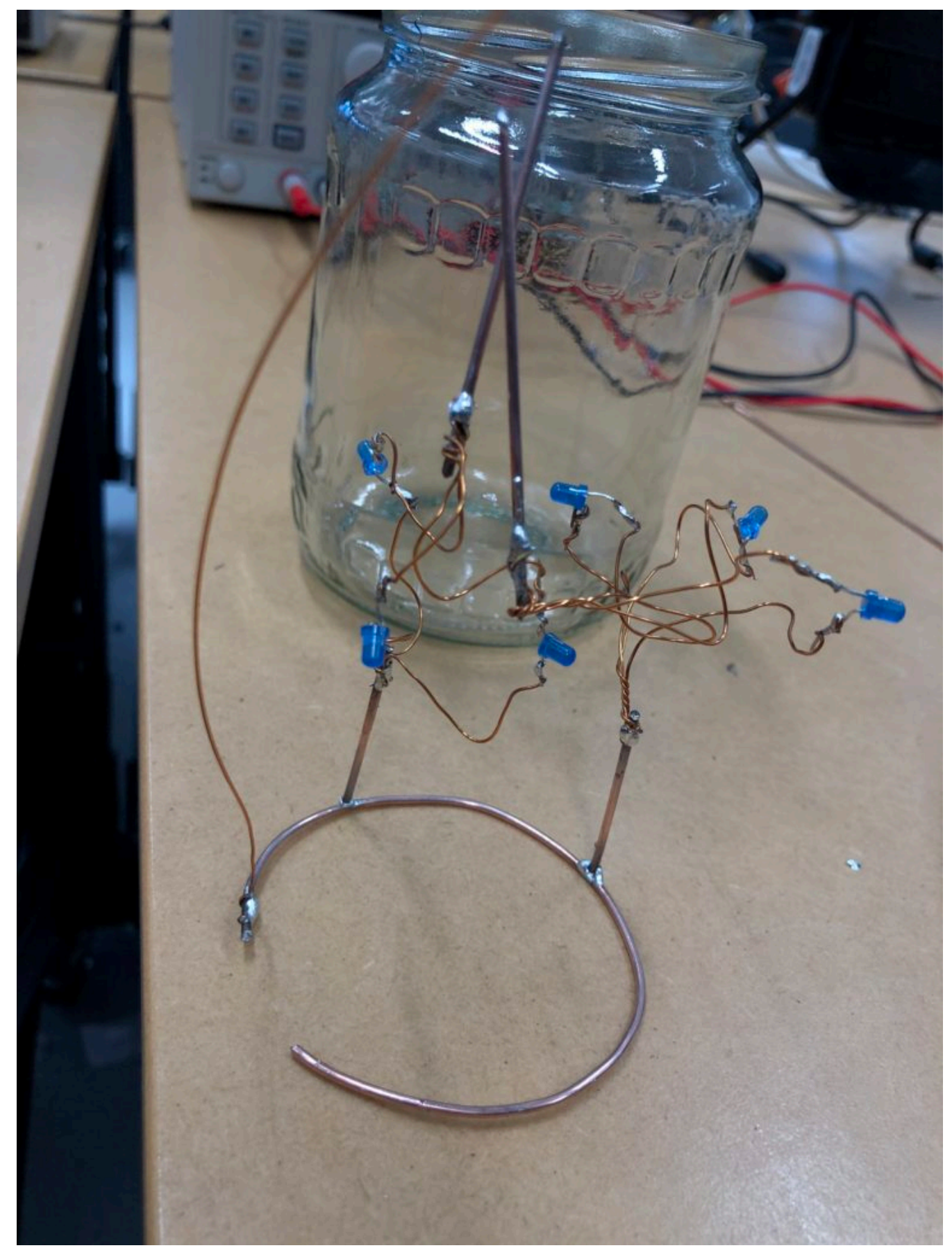

Figure 119. Author. (2018). Prototype with two groupings of three LEDs attached.

In the end, I connected a set of three LEDs to three individually addressable wires. The copper rod needs to be bent carefully with flat pliers while the copper wire bends into shapes very easily. This gives the final circuit a quite messy look and I am unsure the design in this form would be suitable to provide any meaningful visualisation of the turbidity reading. 


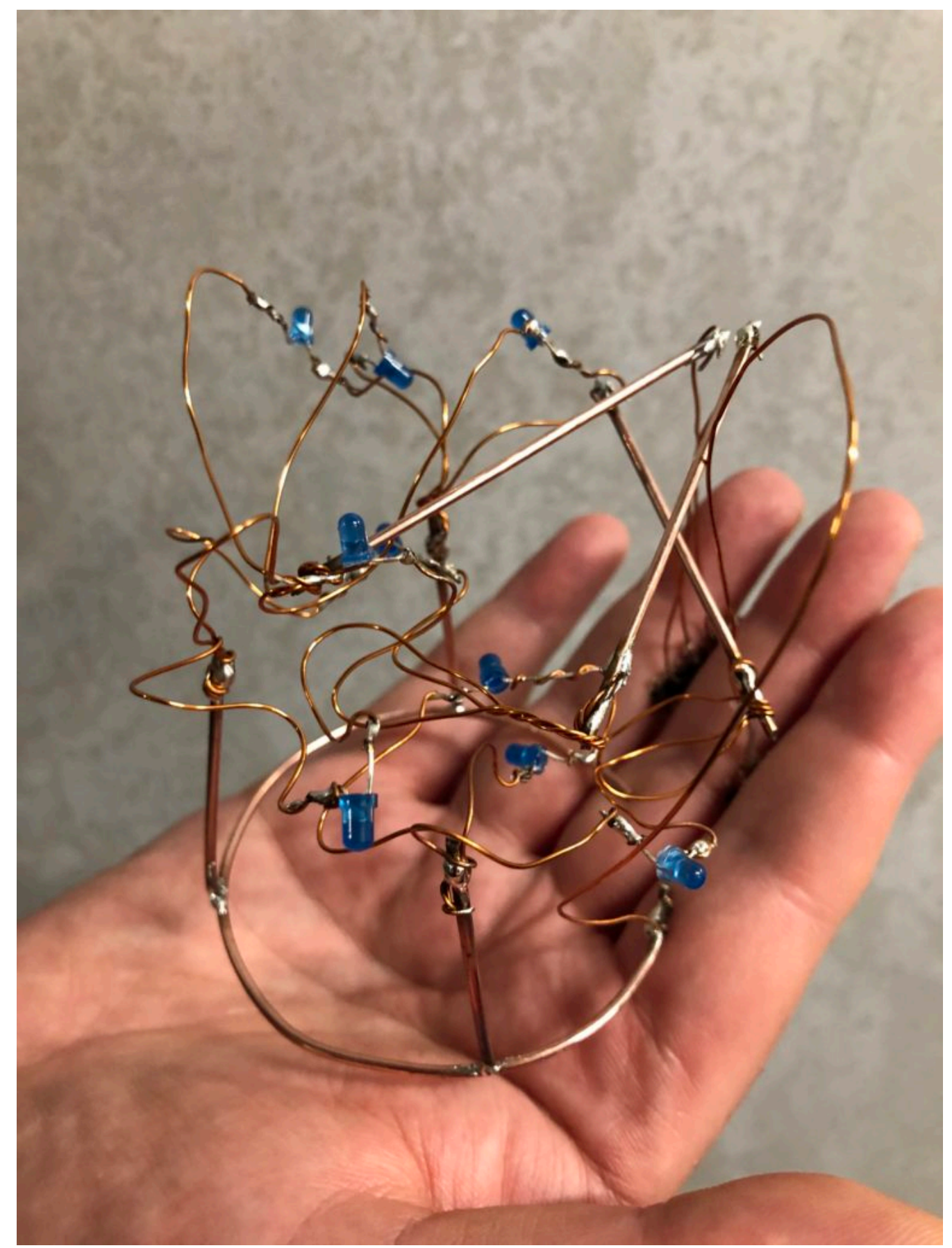

Figure 120. Author. (2018). Final prototype design with three groupings of three LEDs.

The circuit appears quite fragile, the copper wires can be bent and crushed in the hand which gives it quite a unique aesthetic when handheld. Once transferred into a glass jar, the intricacies of the circuit design fade into the background, and the bright blue LEDs, as well as the battery and the small circuit board, distract from the fragile wires. I programmed the board with a simple test sketch that loops through the three LEDs. 


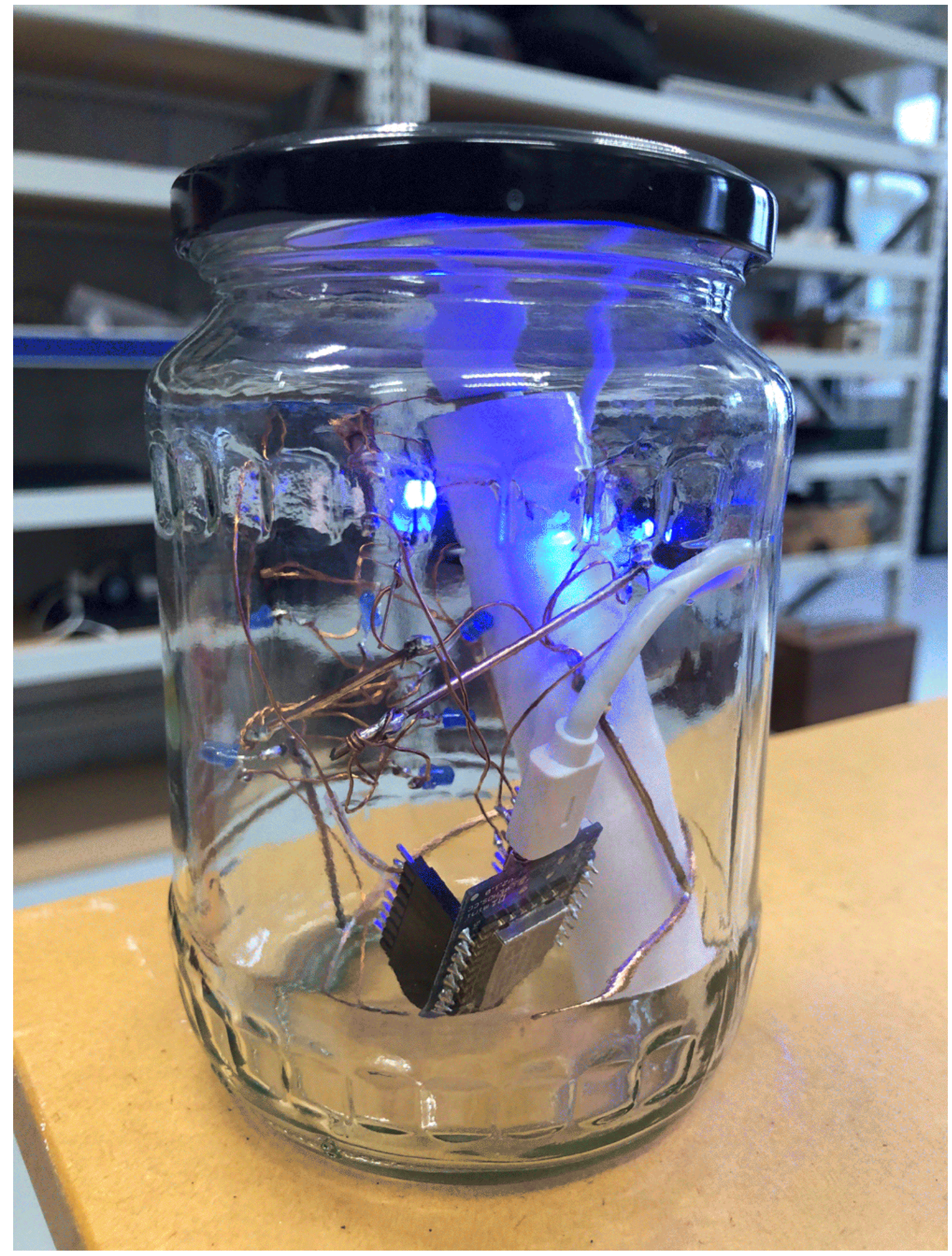

Figure 121. Author. (2018). Detail of assembled prototype with test code.

The next step involves connecting this design to the turbidity sensor through my local MQTT network. I submerge my turbidity sensor into a glass bowl filled with water to get more realistic sensor data readings for this test. Unfortunately, the circuit design appears 
tricky to programme, and only after a while am I able to successfully de-tangle the wires that must have short-circuited somewhere, causing the code to malfunction and print nonsensical glyphs in the serial monitor when I try to debug my code.

Once my LED node is properly connecting to the Wi-Fi network and correctly receiving the sensor data, I map the turbidity to the number of LEDs being switched on. To achieve a murkier fluid for this test I add a teabag to the water. I notice that the value changes are not as extreme as I would have hoped and assume that a different resistor, perhaps a trimmer potentiometer, would help to get more accurate data. Another issue with the sensor data is jumpiness. This could be because the LDR is just not suitable for an accurate measurement, or perhaps the sensor design is not waterproof and hence unreliable. Perhaps the code could be improved by measuring a running average over a couple of milliseconds, instead of measuring the brightness only once and immediately transmitting this data.

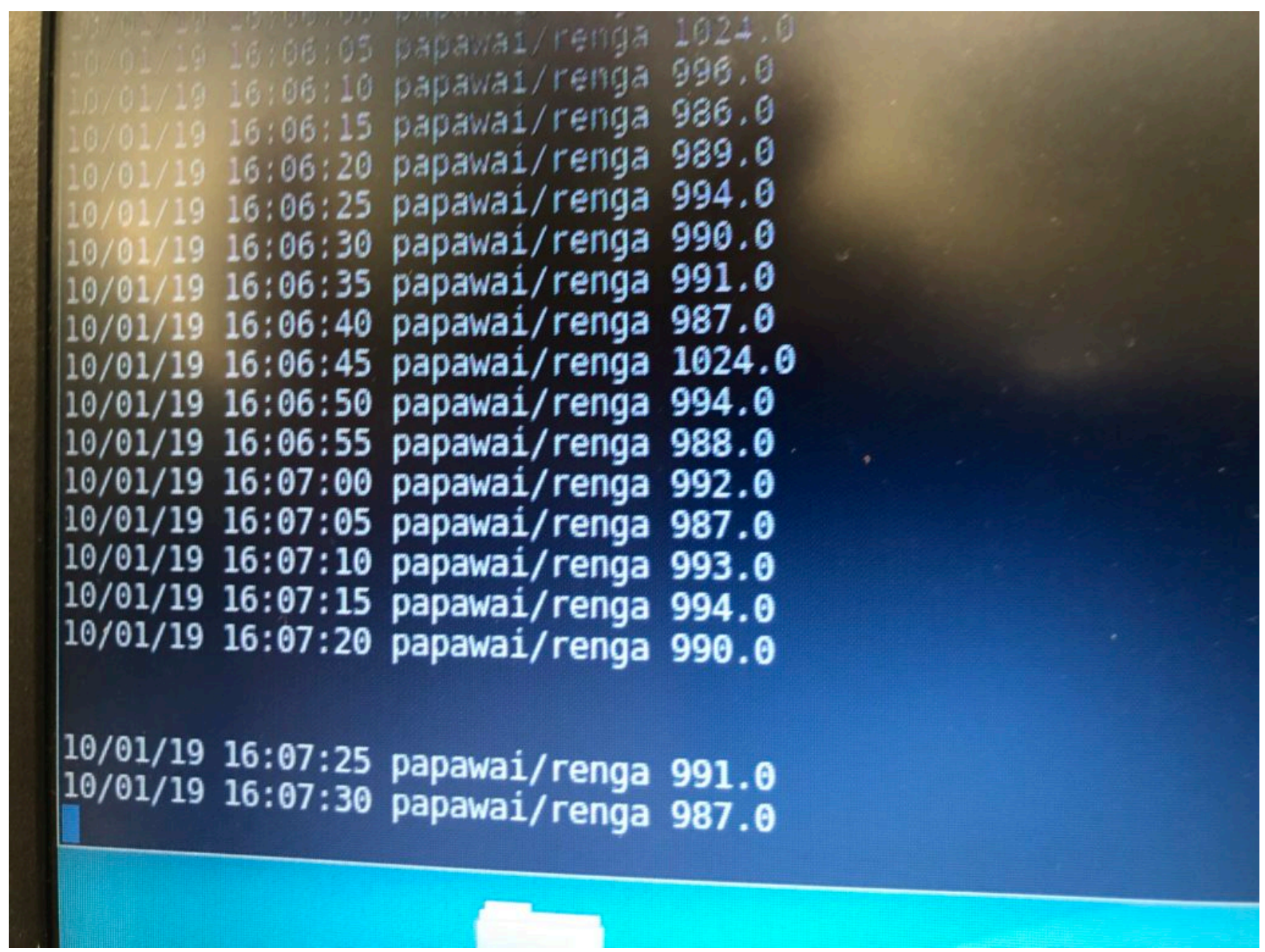

Figure 122. Author. (2018). Terminal output of turbidity data measurements. 


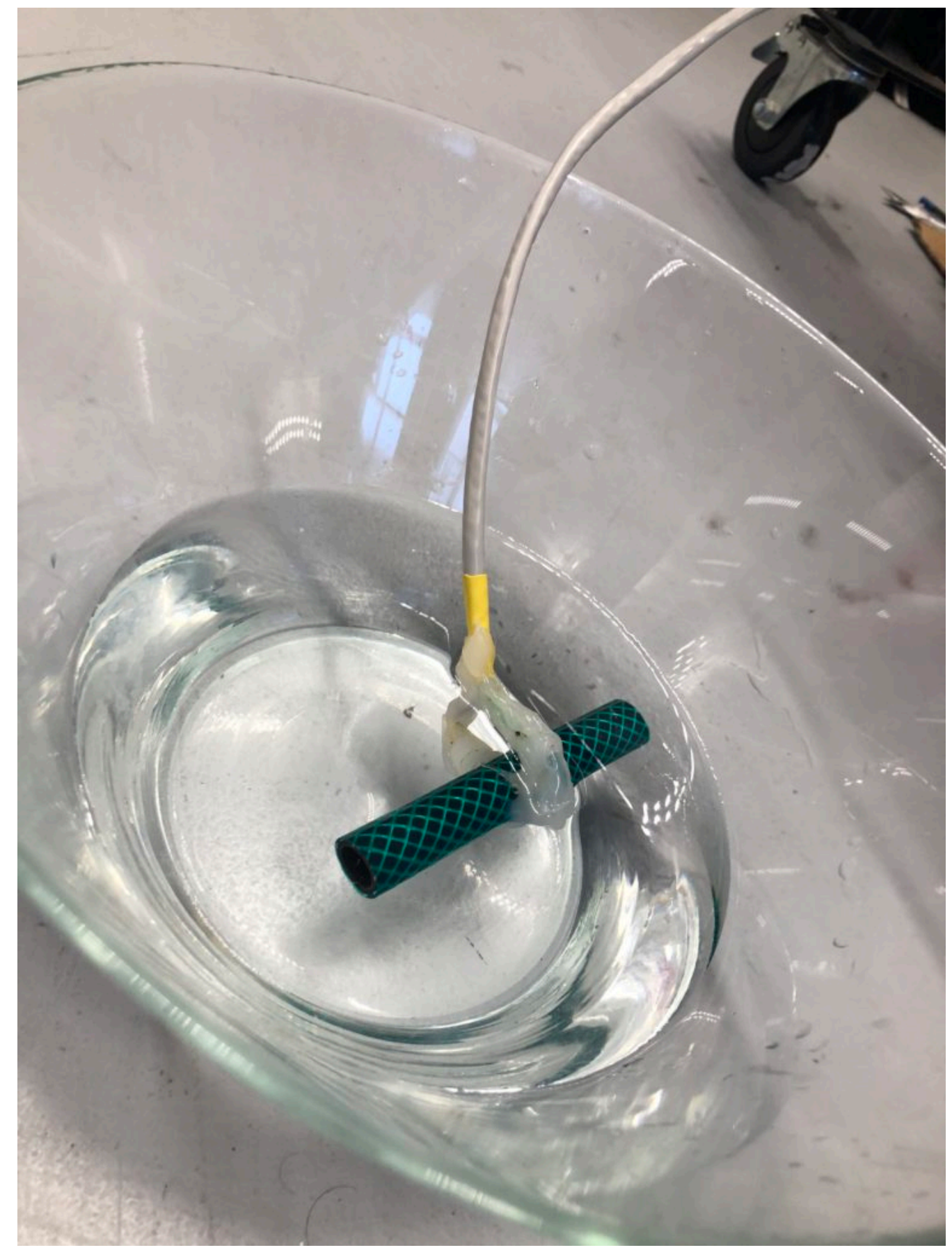

Figure 123. Author. (2018). Turbidity sensor node being tested in a bowl of tap water. 


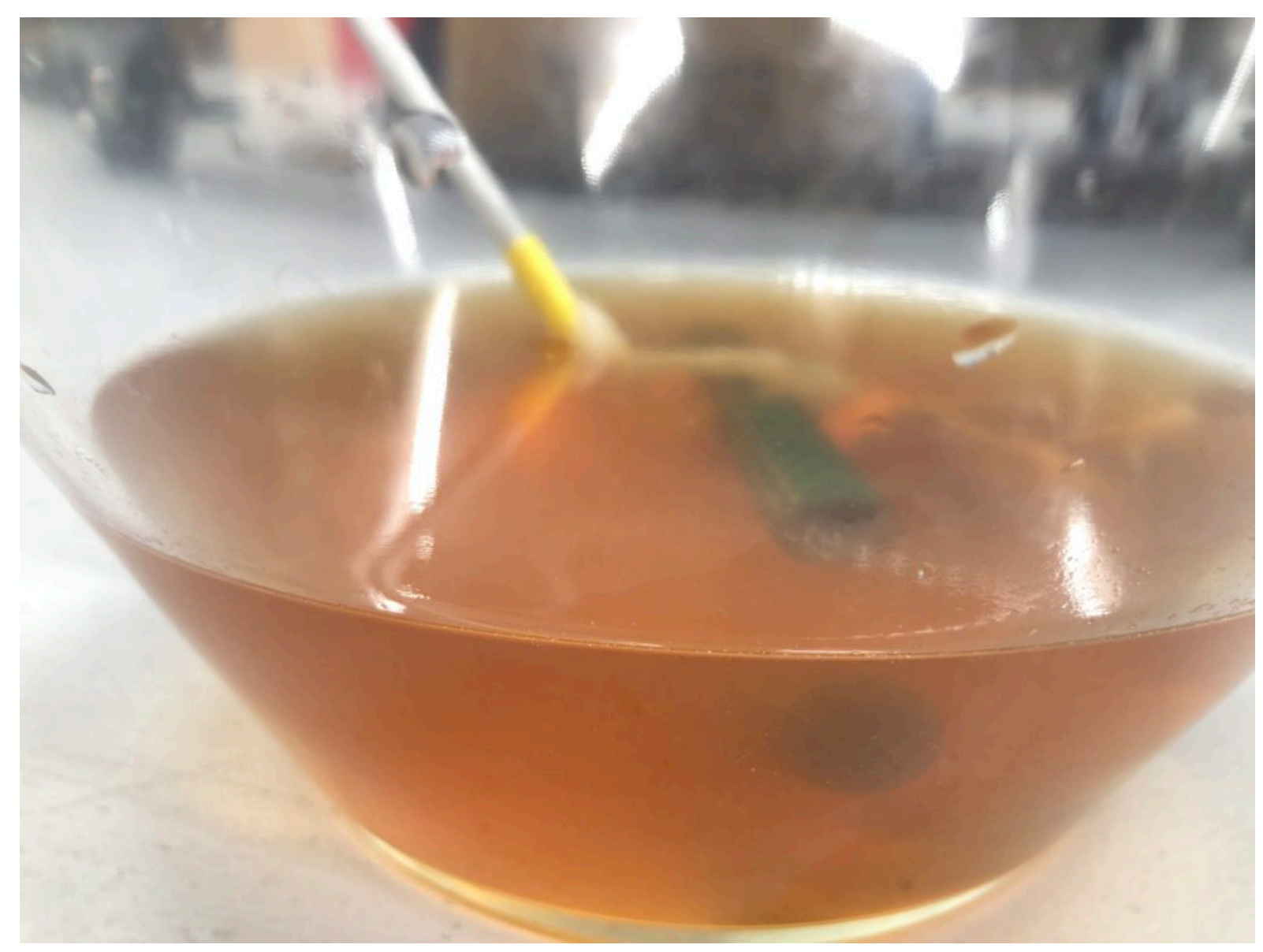

Figure 124. Author. (2018). The turbidity sensor node submerged in a bowl of cold tea. 


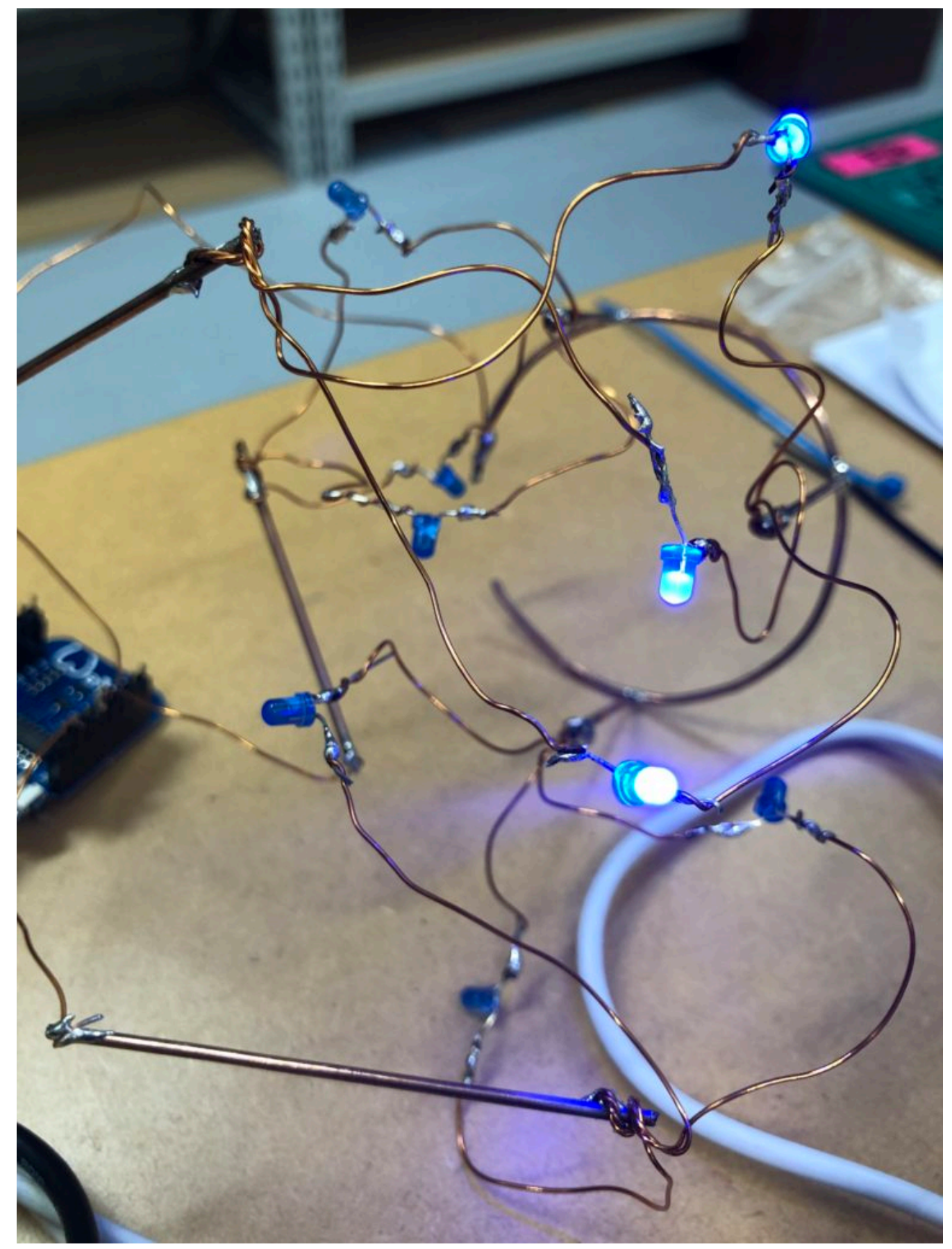

Figure 125. Author. (2018). While the prototype is configurable in space, the visual data readout is not clear.

Despite issues with the quality of sensor data, I learned a lot about the feasibility of this circuit design. While the copper wire gives the circuit a unique, messy look that I generally like, it is unsuitable for providing an easily understandable visualisation of sensor data. Using only copper rods in combination with $3 \mathrm{~mm}$ LEDs could work with a refined sketch on how to accurately map the sensor reading to an array of LEDs. 


\subsection{Giving voice to the stream: Output node iterations}

One of the challenges of building this work was how to make the IoT data that is being collected by the sensor nodes part of the install, without needing to add yet another layer of technology that would possibly disconnect an audience from the site and the install itself. From the onset of the design process, I wanted to avoid the need of a human participant needing to use a device like a smartphone to access the live data being transmitted by the nodes, and as a result, paying attention to their phone rather than allowing themselves to explore the nodes and how they connect to the stream. This section discusses design experiments for what I sum up under output nodes - nodes that have the sole purpose to reveal data to an audience while the work is set up on site.

\section{Organic LED (OLED) screen}

One of the very first attempts to build a node that solely presented data from the network to the human audience featured an OLED shield. I wanted to have this node act as a central display of the network status. The first option was to simply display the messages bounced around on the MQTT network, giving the audience an understanding of the network traffic, but without revealing actual measurements. This idea was based on the intention of making the network itself the central focus of the exhibition, rather than the measured values, which would only be abstract numbers to an audience, and direct the attention away from the install itself. The second, less preferred option, was to roll incoming sensor data over the little $64 \times 48$ screen, giving the audience a live update of the various values recorded and transmitted.

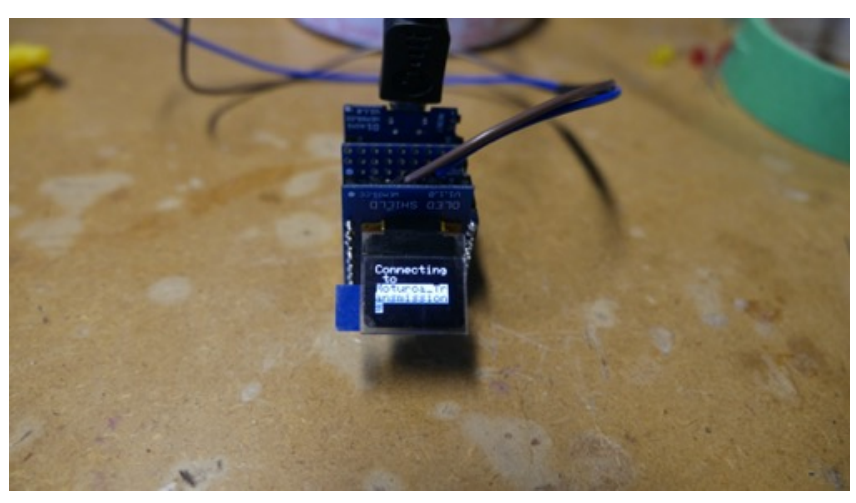

Figure 126: Author. (2017). Early testing of an output node featuring an OLED shield. 
In the end, this node never made it into the final install, as the function of the display would come close to what I tried to avoid by not requiring a smartphone to access the exhibit. With its relatively small size, the screen would also pose accessibility issues, as people would need to come close enough to be able to read the display. Designing this node would take the focus away from the other nodes and might have detracted from exploring the install without having concrete instructions and outputs.

\section{Piezodisk audio node}

Early design sketches featured a piezo speaker on each sensor node that would emit a short beeping tone, every time a message was being sent from that sensor via the MQTT network. While this sound layer might have helped make the activity on the network more comprehensible to the audience, it was not used in Moturoa Transmissions.

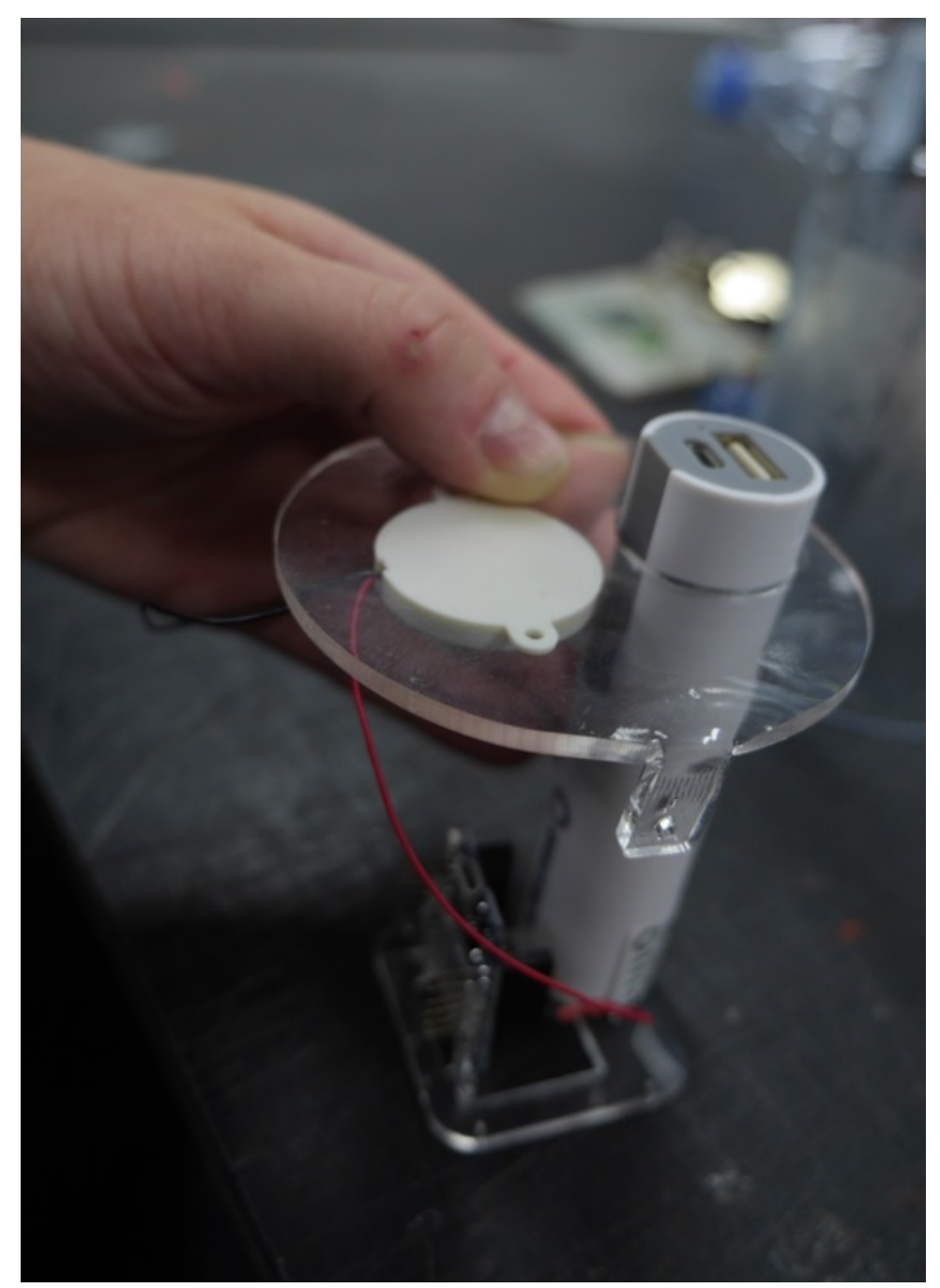

Figure 127. Author. (2017). Early node design featuring a piezo disk for sound output. 
Initially, I wanted to direct the audience's ears to the fact that the stream could be heard but not seen from the site of the install, when paying close attention, and I did not want beeps of the network to disturb this discovery. Eventually, the soundscape was dominated by cicadas during the install days, and the stream was not audible from the install site anymore, so most likely the beeps would have also been drowned in the cicada sounds.

\section{Single multi-color LED}

Following the early experiments with the OLED screen, I considered the WS28128 RGB shield for the WeMos D1 the basis of an alternative visual output of data. The single LED can display any colour from the RGB-spectrum and would give a more abstract visual representation of network traffic.

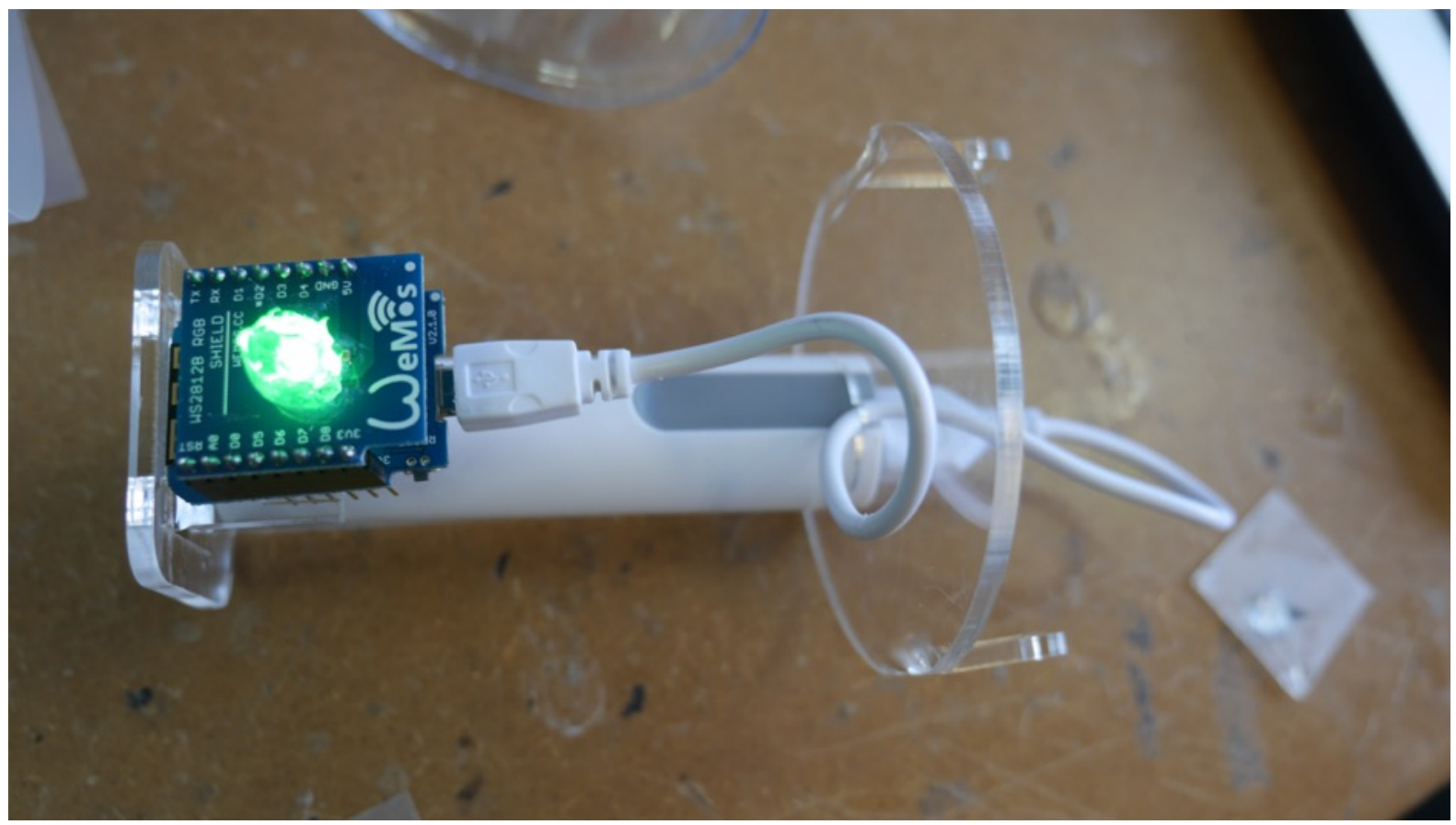

Figure 128: Author. (2017). Moturoa status node in lab without bottle casing.

The code simply prompted the microcontroller to listen to all incoming network traffic and switch the onboard LED on for half a second, when a message of a certain topic was being transmitted. Different colours were used to identify the kind of data being sent through different colours and blinking patterns (see code below). Humidity would just be a single blue blip. If the blue blip was followed by a pink blip, then an air temperature message was being sent. 


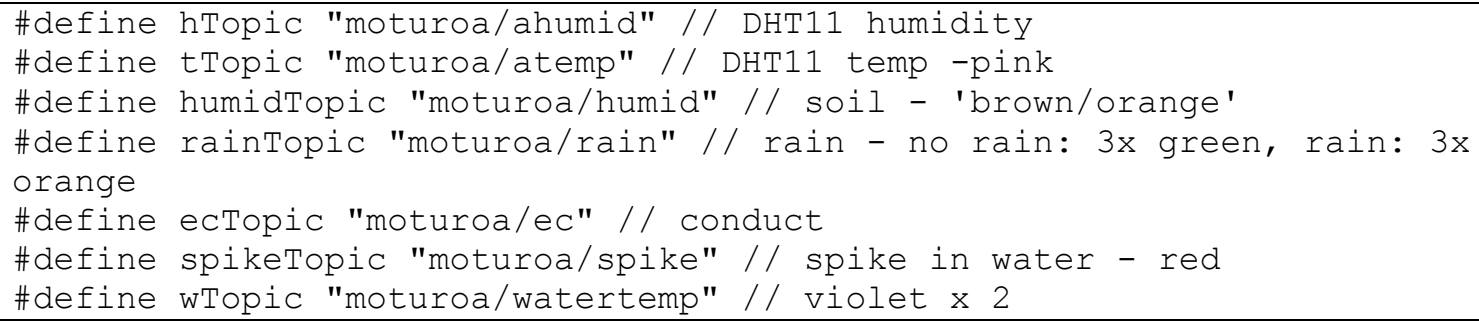

The lights should just visualise the activity of the network at first, with each colour showing a message from a particular sensor node is being transmitted. For simpler sensors, like the rain sensor, that basically measures only two different values, the light would indicate the content of the message itself, e.g., rain would be indicated by the LED blinking orange three times, and no rain would be shown as three green blinks. Such a simple indication of data with just one coloured LED would be too complex to achieve with sensor data like temperature, or electric conductivity.

The code of the EC node, however, already calculated when a spike of conductivity was detected, and transmitted this data separately from the conductivity value, as 'spikeTopic'.

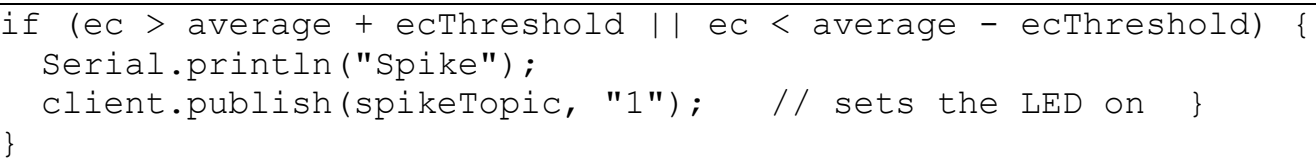

This made it easy to have the LED node only listen to spikeTopic, and then signaling a spike in conductivity with a red colour. 


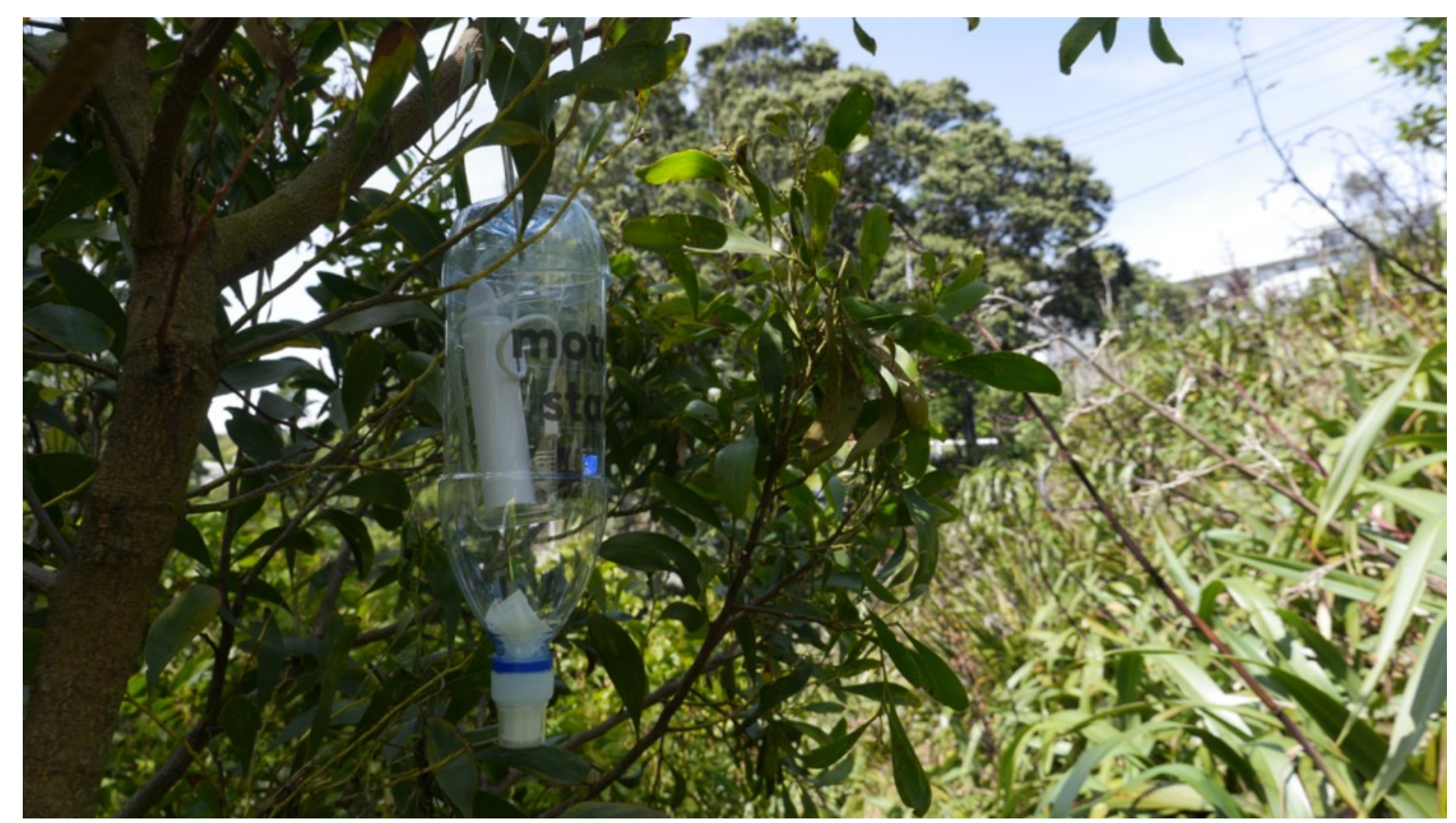

Figure 129. Author. (2017). Moturoa status node showing a blue light, indicating data is being transmitted on the MQTT network.

While I considered the nodes themselves the main component of the work during the first public exhibition, it soon became clear after a day of audience observation, that the public was curious to have access to more tangible data, rather than just getting an abstract sense of communication between the nodes being activated. 


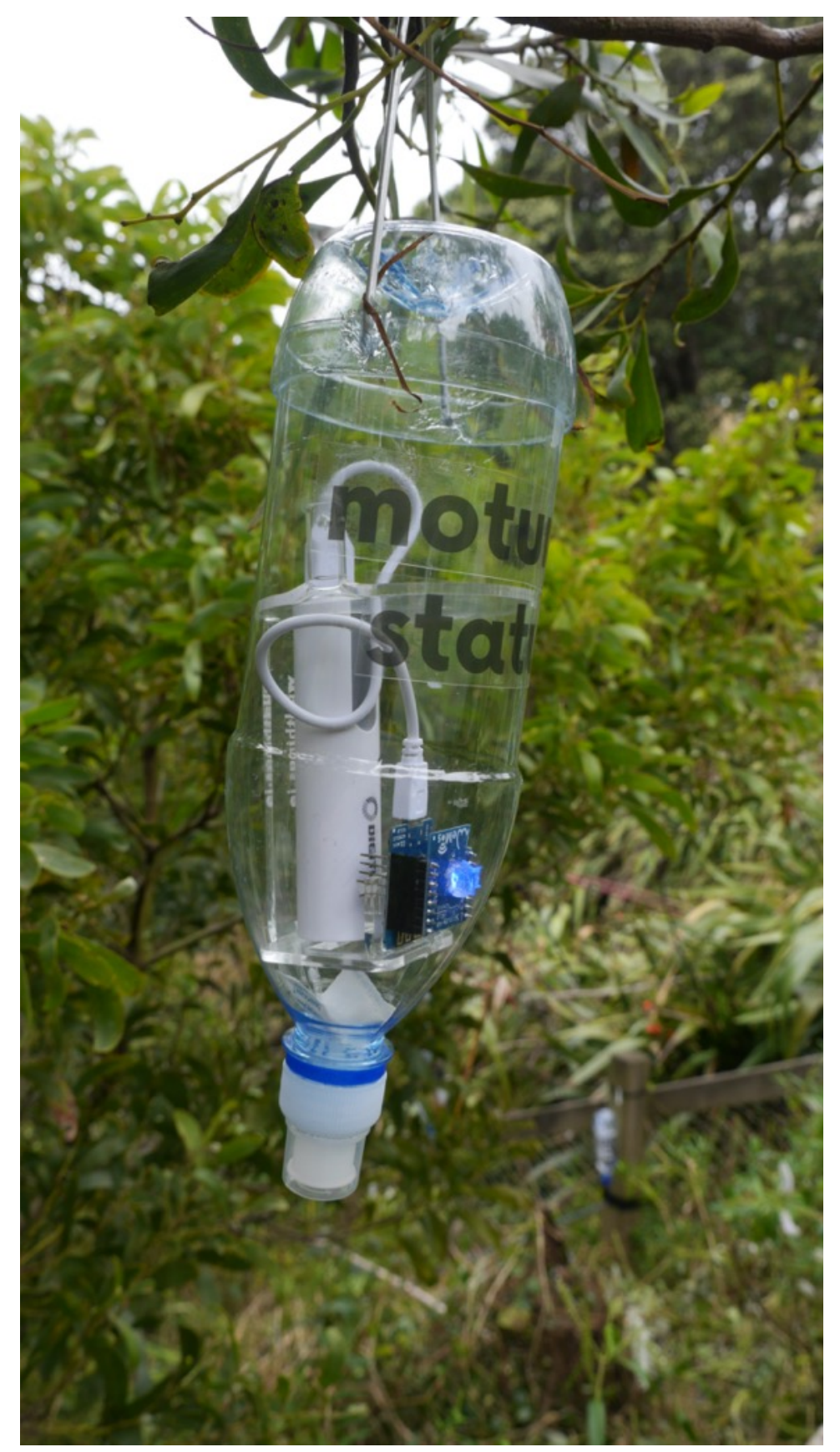

Figure 130. Author. (2017). Moturoa status node showing a blue light, indicating data is being transmitted on the MQTT network.

One major flaw of this system was that the intention to keep the colours and data abstract also made it impossible for an audience to comprehend the colour/pattern key. Adding a key to the exhibition felt like an afterthought and would have again drawn too much attention to a singular node. While the single LED node itself was not successful as a comprehensive visual representation of the sensor data, it sparked the creation of a series of bespoke LED nodes, based on audience feedback wanting to get a better picture of the measured data in real-time. 


\section{Light (LED) nodes}

With the EC sensor already offering a data stream that can be of direct interest to an audience, as the spike value indicates a sudden change in the composition of the stream water, the first bespoke node would be designed for visualising the transmission of the EC data.

The design of this node, using copper tape and paper as basic materials, is inspired by the work of Qi (2012; see also High-Low Tech Group, MIT Media Lab 2012), embracing a lo-fi DIY aesthetic for circuit design.

Please watch the video documentation attached to follow the assembly of this prototype. Featuring five separately addressable white SMD LEDs, the EC_visualisation node either shows that the EC probe is connected to the network and sending data (one LED is fading on and off) or indicates a spike in the EC measurements (all LEDs blink four times at full brightness).

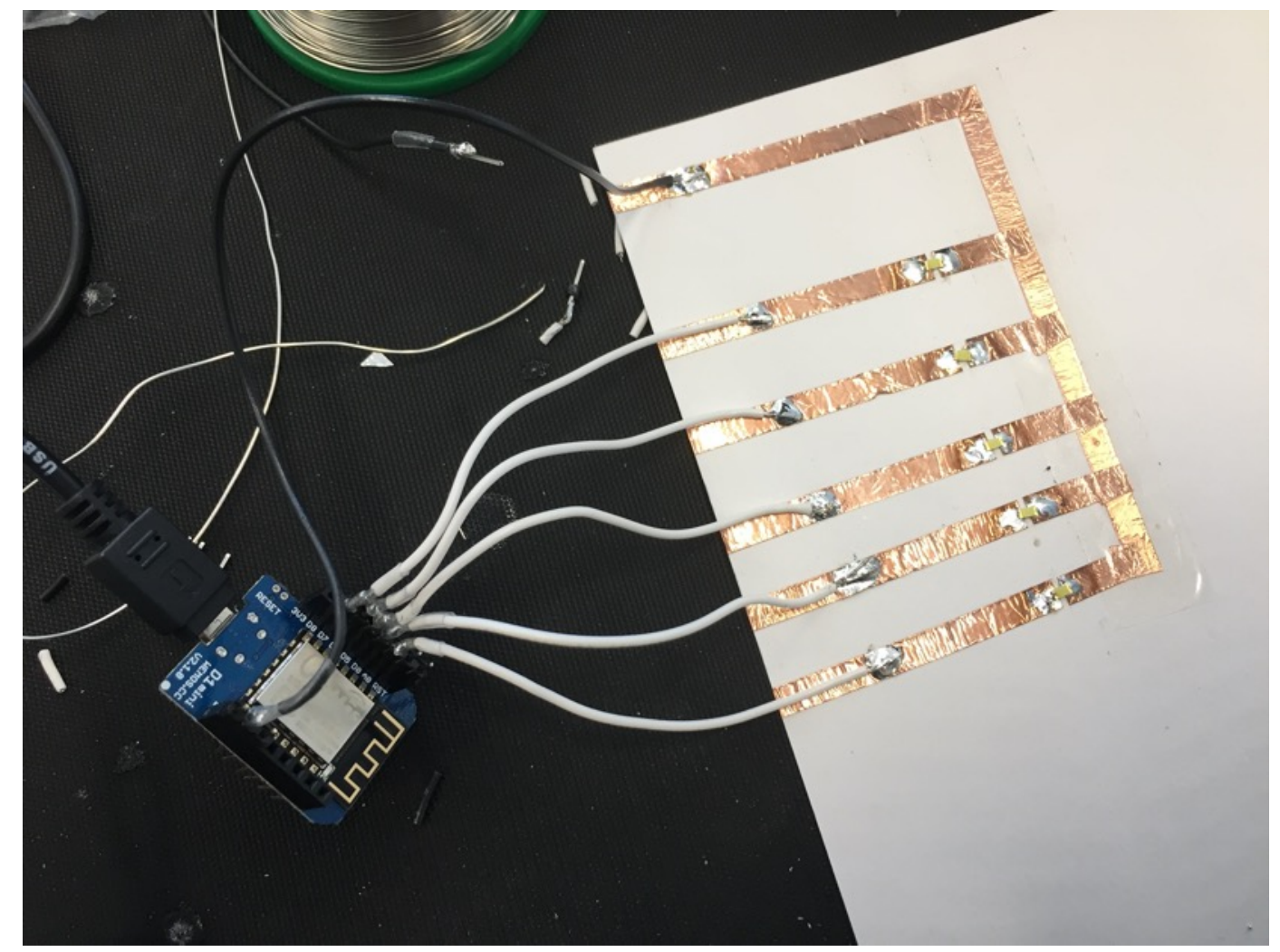

Figure 131: Author. (2019). Final assembled circuit for the first bespoke LED node.

With the paper and copper tape LED design receiving overall positive feedback during lab evaluation interviews, a new visual node was designed for the temperature sensor, followed by another one for the last addition to the DIY nodes, the turbidity sensor. 


\section{Audio node}

In an attempt to move away from the solely visual data representation, I looked at options to use the ESP8266 chip in combination with a simple audio interface. While the WeMos D1 mini does not natively support sound, I opted to test a new setup with a previously purchased microcontroller, the Adafruit Feather Huzzah. This board, also based on the ESP8266 like the WeMos D1 mini, can be expanded with the Music Maker FeatherWing, which adds an audio interface with a headphone jack output to the microcontroller.

Powered by a battery, this board could be used as a standalone sound output node as part of the installation in the field.

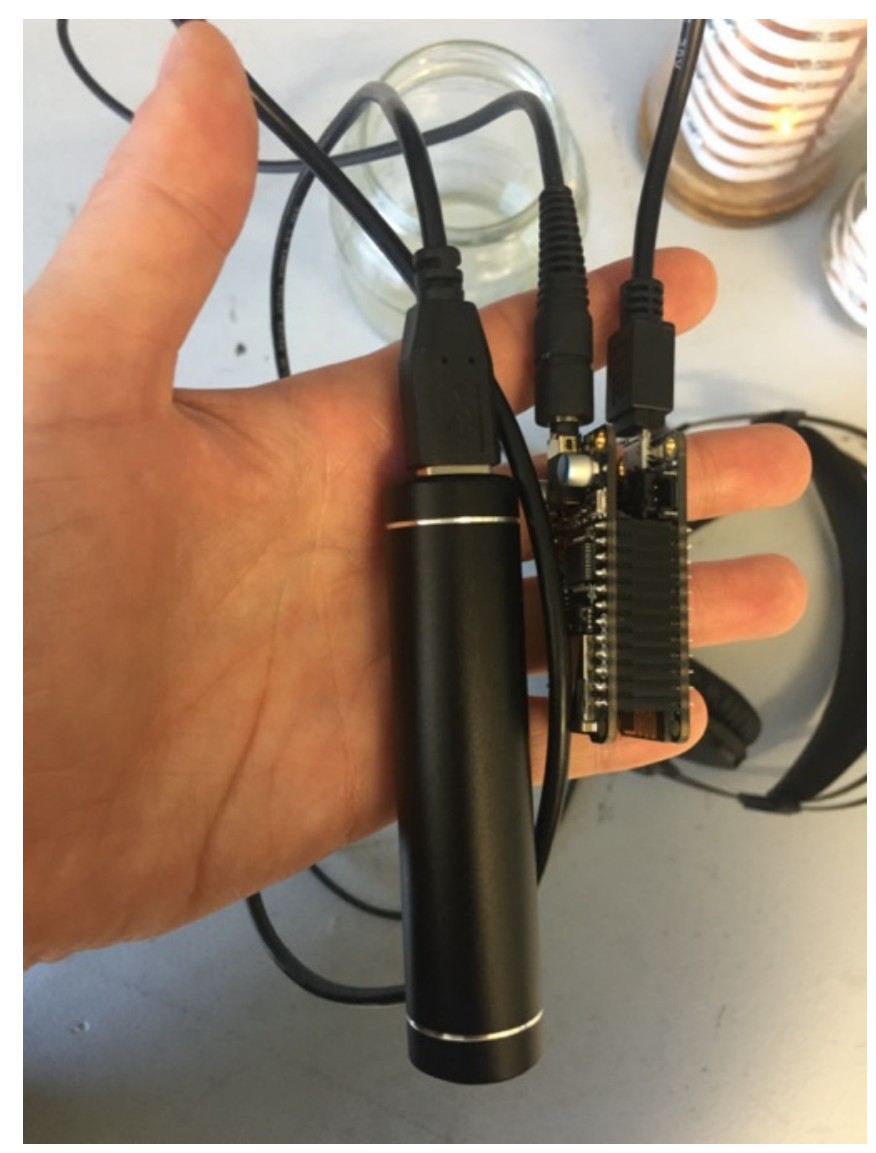

Figure 132. Author. (2018). The components that make up the audio node: The Adafruit Feather Huzzah microcontroller with the Music Maker FeatherWing shield powered by a battery via USB.

As the sound node can either play MIDI sounds or pre-recorded MP3 files, the options to sonify data could range from using MIDI instruments as indicators for network traffic, using for example, pitch or dissonance to indicate the quality of the data, or pre-recording audio tracks to correspond to events on the network. 
Early experiments included mapping a basic MIDI instrument, the 'Ocarina', to the sensor readouts of the EC sensor. While the prototype technically worked, appearing to be a good addition to the sensor family from an accessibility standpoint, the experience of needing headphones to listen to the stream data was less successful, as this node, would again, take the focus of the participant away from the stream and the network, being prompted to focus solely on one particular node. Eventually, the audio node did not make it to the final Papawai_Transmissions installs, but could prompt more research into using audio as a medium to present IoT data.

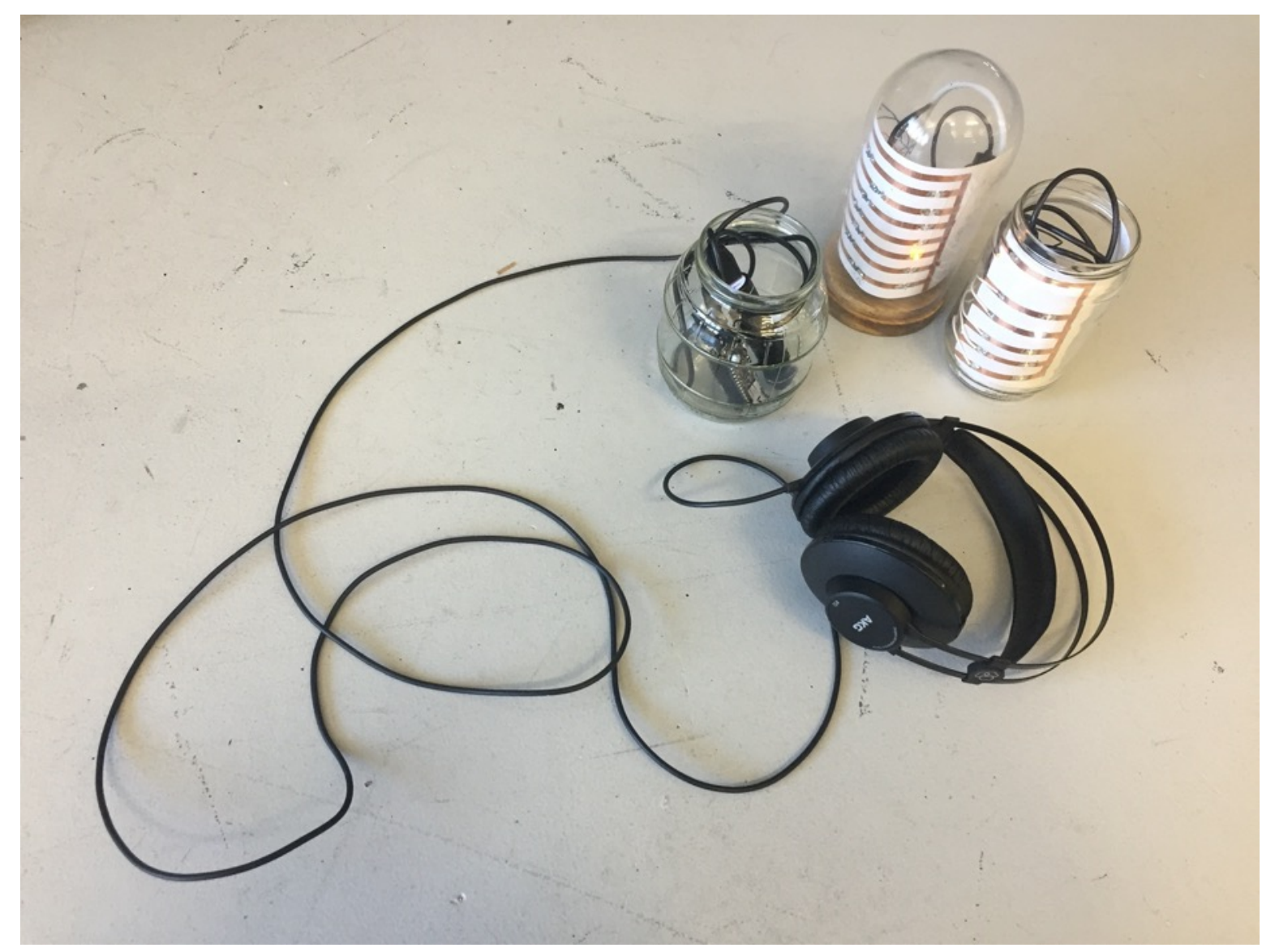

Figure 133. Author (2018). The audio node being tested with a pair of headphones, placed next to the visual nodes for temperature and electric conductivity. 


\section{Concluding Remarks}

The first single RGB LED node was successful because it gave audiences a glimpse into the otherwise obfuscated traffic on the network. The challenge with all visualisation and sonification nodes appeared to be the concern that any representation of data would turn the attention of the audience to one single sense, either vision or hearing, and away from the sounds and sights of the more-than-human world. An audience would be distracted trying to read or interpret data that is continuously being sent over the network. In turn, the network itself, its design, and the efforts to use it as a vehicle to (re-)connect an audience with the stream and its environment would play a secondary role to the experience.

In sum, the iterations of nodes solely dedicated to presenting data to a human audience revealed how seamful design aids the creation of simple design pieces, that evoke curiosity in an audience. Use of basic prototyping materials, such as paper and tape in combination with microcontrollers and electronic components, creates an accessible interface between DIY crafts and citizen science. 


\subsection{Prototyping Log Reflections}

The selected prototyping narratives in this chapter give insight into the hands-on design and development activities that accompanied the field work activities, outlined in Chapter 5 that accumulated in installations, as discussed in Chapter 7, the Stream Lab. Giving step-bystep documentation for one prototyping activity, such as in 6.2 Building a Low-Cost Hydrophone, and discussing development over a few weeks' time, as for 6.5 EC-sensor Iterations, 6.6 Turbidity Probe Iterations and 6.7 Output Node Iterations, shows how the design project evolved from early, loose experiments towards more established prototypes informed by iterative design processes. Giving a glimpse into the lab notebook during the development for a DIY IoT, 6.3 Hello World shows how the project was created using by both familiar and unfamiliar development environments, and 6.4 Wi-Fi in a Bottle illustrates how conceptual and practical considerations pushed the design process of project enclosures further.

The documented narratives give an overview of prototyping development; zooming in on the developments over a few days (6.2), multiple weeks as part of a focused sprint $(6.3,6.5$, 6.6), or show progress of prototyping spanning the length of the project (6.4). Here, I reflect on how these narratives were underpinned by and mobilised the key research themes of slowness, seamfulness, and openness, and the encounters these themes then invited accordingly.

\section{Openness}

Openness was one of the key concepts from the departure of this design research. The preference to solely use open-source or otherwise openly licensed hard- and software ensured all my designs could be easily shared and published online, and to be used and modified by other developers and researchers.

The search for open-source solutions stood at the beginning of each development sprint, assessing open solutions for both hard- and software. Deciding early to work within the Arduino development environment helped establish early criteria for selecting suitable 
hardware and software. Having experience with this environment, having worked with it since 2006, helped me to adopt unfamiliar IoT concepts-such as the Publish/Subscribe protocol-into an environment I am familiar with. The widespread familiarity with Arduino in the maker scene also appeared to be a good way of publishing my code that could be useful for a wider audience.

I published all prototyping work on a github repository irgbit/wildthings.io, starting from GIS experiments to revisions of the sensor code. This allows users who are familiar with the coding environments to get an insight into the workings of my designs, with the opportunity to copy all code, re-use it, modify it and contribute new ideas to the designs. As discussed previously in Chapter 4, this openness is largely limited to the open-source community, as working with multiple coding environments and diverse hardware requires prior knowledge, which might pose a barrier to novice users and non-coders. The open write-ups as published on the blogs, accompanied by photos and video content, aim to lower the entry bar to working with electronics, showing a raw representation of developments in the lab, and providing step-by-step instructions for building sensor nodes. The documentation explicitly features failures (see 6.5 or 6.6), which are important parts of an iterative design approach, as they help illustrate how subsequent design decisions have been made, often as a response to a previous unsuccessful experiment. 


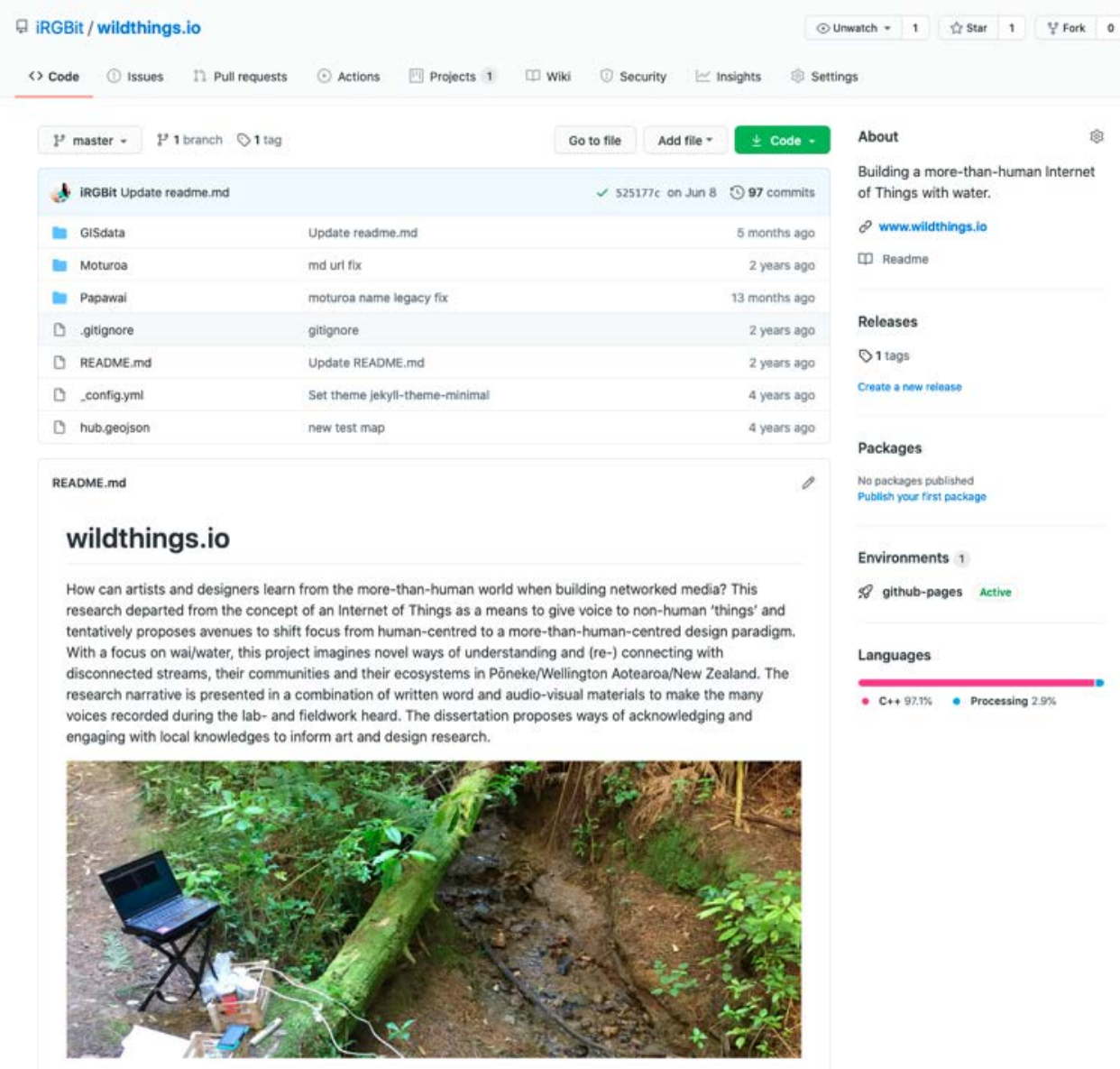

This repository contains code, sketches, prototypes and designs created during the development of artefacts and artistic outputs of this thesis.

Figure 134. Author. (2020). Screenshot of the GitHub repository iRGBit/wildthings.io.

\section{Seamfulness}

Embracing seamfulness as part of the prototyping process involved both acknowledging the seams revealed in the field during exploratory walks and translating those seams into features of the design work, as well as recognising seams inherent in the process of iterative development as productive outputs of a research, that taps into multiple complex territories. Here, seamful design involved drawing together theoretical concerns from more-than-human scholarship, and technical concepts from IoT development, where multiple layers of technology, hardware, and software, are literally patched together to create an IoT network. 
The designs, specifically the enclosures (in 6.4) were linking back to seams revealed during field exploration memories, where rubbish, old bottles, and glass containers, emerged from the stream, disposed of in the past days-or possibly some time during the past decades-was slowly making its way downstream. Thinking with the stream, prototyping also involved thinking through which materials would be the least problematic to use for building devices, aiming to use recycled materials wherever possible. Ideally, prototypes should interfere with the natural flow of the water as little as possible, should not require any alterations of the environment and make use of already present materials and, as much as possible, make use of already present infrastructures, such as tree branches and other vegetation.

My design approach embraced the seams as design features, which involved avoiding any work that would lead to smoothing over rough edges of the design, aiming to make the process that went into creating the work, visible. This was successfully done on a hardware level, prioritising an openly accessible design that reveals all componentry to an audience, instead of hiding away any features. I ensured, as far as practicable, that circuit boards, batteries, LEDs, solder connections, wires, cable ties, hot glue, broken acrylic inlays, cracked bottle enclosures were considered as part of the design. On the level of software development, seamful design had its limitations, as the code that drives the behaviour of the network remained invisible. I did publish the code in the online repository but did not plan on revealing the code in the field. I could have pushed this further, for example by enclosing all code with the designs, e.g., by providing a printed version of the source code next to each node. However, the code of each node is roughly 150-200 lines and would only be accessible to people familiar with Arduino or a related programming language. The discussion of software function was mostly presented to an audience, or to participants through conversation, as presented in the field lab narratives of Chapter 7. 
In order to keep the designs simple, and not to distract from the hardware and designs themselves, I decided to give the nodes short, descriptive names, printed on the outside of the container, e.g. moturoa temp for the water temperature sensor node. This should give participants an opportunity to understand the basic role of each sensor. As the designs evolved, and sensor nodes were equipped with a corresponding output node, the design focussed on visually tying the nodes together. This meant that, for example, in the final install, both the EC sensor and its visual counterpart would be placed the same kind of jar, while the water temperature sensor-whose bottle enclosure was still usable until the last day of the installation-would have a corresponding LED node placed in a bottle enclosure as well. This seamful design allowed nodes to be used interchangeably, switching components as necessary, and re-using parts from retired nodes for new iterations, such as experimenting with old plastic bottle enclosures as surface for copper tape circuits.

Designing the prototypes to be used in a stream environment with the aim of connecting and amplifying the more-than-human voices also revealed the unclear seam inherent in the lab/field relations. While situating prototyping activities in the lab has the advantage of providing access to necessary infrastructure: tools, electricity, components; a fast, reliable internet connection; much of the needed testing could not be done solely in the lab, as complications would only reveal themselves once the nodes were transported to the field. The field was always entangled in the prototyping process, with the learnings from early explorations, walks with and without participants, and later installations and exhibitions of the work in the field, as further elaborated on in Chapter 7.

\section{Slowness}

Slowness, especially as part of the prototyping process, appeared as a hindrance, when 'to do list' items started piling up before installs or presentations were planned. However, appreciating slowness in later stages of the prototyping process allowed working with the aforementioned concepts of seamfulness and openness in a more confident manner. 
During earlier prototyping work, such as 6.3 Hello World, documenting my work required me to pause and note down developments. When working towards an external deadline, keeping a good documentation of process is often hard; when, for example trying different settings, making multiple changes, and reverting some of them back to the original state. As it was often difficult to exactly trace back the steps that it took to arrive at a certain outcome, I would later become more rigorous in taking time to document any developments leading up to the final Papawai Transmissions prototypes.

This materialised through the way I took time to document my work more rigorously, through lab notebook entries, audio recordings, video documentation and photographs. As part of this process, I experienced how, often, pausing in the middle of a development step to take a photo or take notes, helped me to embrace the moment, and gave me time to (re-)consider my prototyping work, assessing it against the design parameters; allowing me to take the time and observe my own process.

I would often plan breaks during days reserved for prototyping activities and dedicate time to a short walk along the stream. I would sometimes visit the accessible part of the stream in the town belt, or I would follow the course of the pipes, which run close to the lab, to take time and reconnect with the kaupapa/purpose of this project. If it was not possible to take a break, I would open my online lab notebook, which features a field recording from the stream as background audio track. I would play this audio track to bring an aspect of the stream into the lab, to immerse myself in the field aurally, not to get too stuck within a small task, such as soldering SMD LEDs for a node, and losing sight of the larger goals of the project, which was to build designs that could connect us the more-than-human world. 


\section{Chapter 7: Field Lab}

\subsection{Introduction}

In this chapter, Field Lab I focus on research activities that sit, at the edges or the seams of this project, somewhere in between my field exploration and prototyping work. This chapter focuses on the moments where development from the electronics lab and explorations from earlier fieldwork meet, in the form of installing, testing, and exhibiting my IoT prototypes at stream sites in Pōneke/Wellington.

This section is presented in three key narratives that outline the process of installing, spending time with, and evaluating DIY sensor networks in the field. In the first section I discuss how early prototyping efforts and field explorations concluded in the first public installation of the networked installation Moturoa Transmissions as part of the Upstream Brooklyn Arts Trail. The second, Papawai Transmissions - Field Evaluations shows the final two public installs of the networked installation as part of the concluding evaluation sessions on site. The third section, Testing DIY ECsensors in Papawai Stream [Lab Notebook, 16 December 2018] highlights the process of installing and testing probes without participants as part of the iterative design development.

The media component of this chapter is a video (fieldlab.mp4) that visually documents the install Moturoa Transmissions. The video could be played to conclude Section 7.2 with moving image documentation, or at any time throughout the chapter, when the reader wants to see an audiovisual documnent of the sensor network being installed in the field.

This chapter attempts to weave together the narratives that emerge across the fields of hard- and software development for IoT design, working within the more-than-human world, and highlighting how these two worlds came to meet as part of this research. The coming together of these two worlds reveals new avenues for creative research, in which the more-than-human world is able to stand central in the development of an Internet of Things and inform the design of prototypes that plug into disconnected and overlooked features of urban freshwater streams. 


\subsection{Moturoa Transmissions - prototyping quick iterations for Brooklyn's Central Park}

This section provides a close look at the install Moturoa Transmissions, specifically the days the work was installed in Brooklyn, Pōneke/Wellington. The preceding prototyping developments that led up this point are outlined in the prototyping log, specifically sections 6.3 and 6.4 discussing early developments of the network and node design, and 6.5 and 6.7 giving some context to the work involved in building particular sensor nodes as part of this install.

The first publicly exhibited iteration of wildthings.io was installed as part of the Upstream Brooklyn Art Trail at Moturoa Stream in Pōneke/Wellington under the title Motuora Transmissions. The installation was centred around one Raspberry Pi single-board computer with an external USB antenna, serving a local Wi-Fi network and acting as an MQTT broker for handling communication between the Wi-Fi various sensor nodes. The nodes were built with WeMos D1 microcontroller boards, supplemented by custom hardware designed to monitor the stream site with a range of environmental sensors: a mix of off-the-shelf shields and DIY sensor solutions based on recycled materials. Located at one of the entrances by the Park, operating during daytime and disassembled for charging overnight, the artwork greeted visitors with the following artist statement:

A networked series of interventions in the surrounding environment of Moturoa Stream that senses and monitors change in a range of variables, such as temperature, humidity and conductivity. Together the stations enter a conversation beyond their mere weather-reflective qualities and given structure of land, water and its human and non-human encounters, to form a visually engaging addition to the ecosystem in which they are situated. 
The artwork was running every day and deinstalled for charging overnight. The inclusion of an early prototypical rendition of the work in a public art exhibition provided an opportunity for audience feedback and allowed quick iterations and updates of the installed work overnight. A few weeks before the install, a lab notebook entry shows the main considerations for the work at this stage:

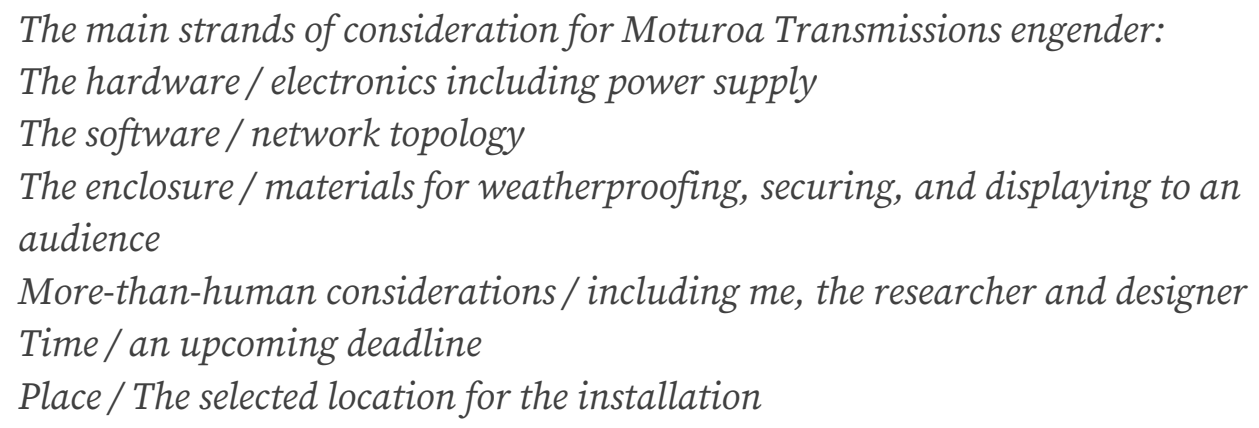
Moturoa Transmissions was installed close to a secondary entrance of Brooklyn's Central Park, where Moturoa Stream is not directly visible but-unbeknownst to many localsemerges from an underground pipe. Hidden from sight behind thick foliage, the stream water cascades from the pipe outlet into a small plunge pool, before making its way down through the park before being piped underground again. The selected location intended to highlight the transition the water went through between the ontological categories of 'stormwater' and 'stream'. However, the site also obfuscated an apparent connection between the exhibited electronic artefacts and the stream, without providing further context to an audience. One of the nodes placed close to the accessible path visualised the local network traffic and the sending and receiving of data through a multi-colour LED. An additional node, recording sensor data to a memory card, was added on day two after feedback from the audience. 


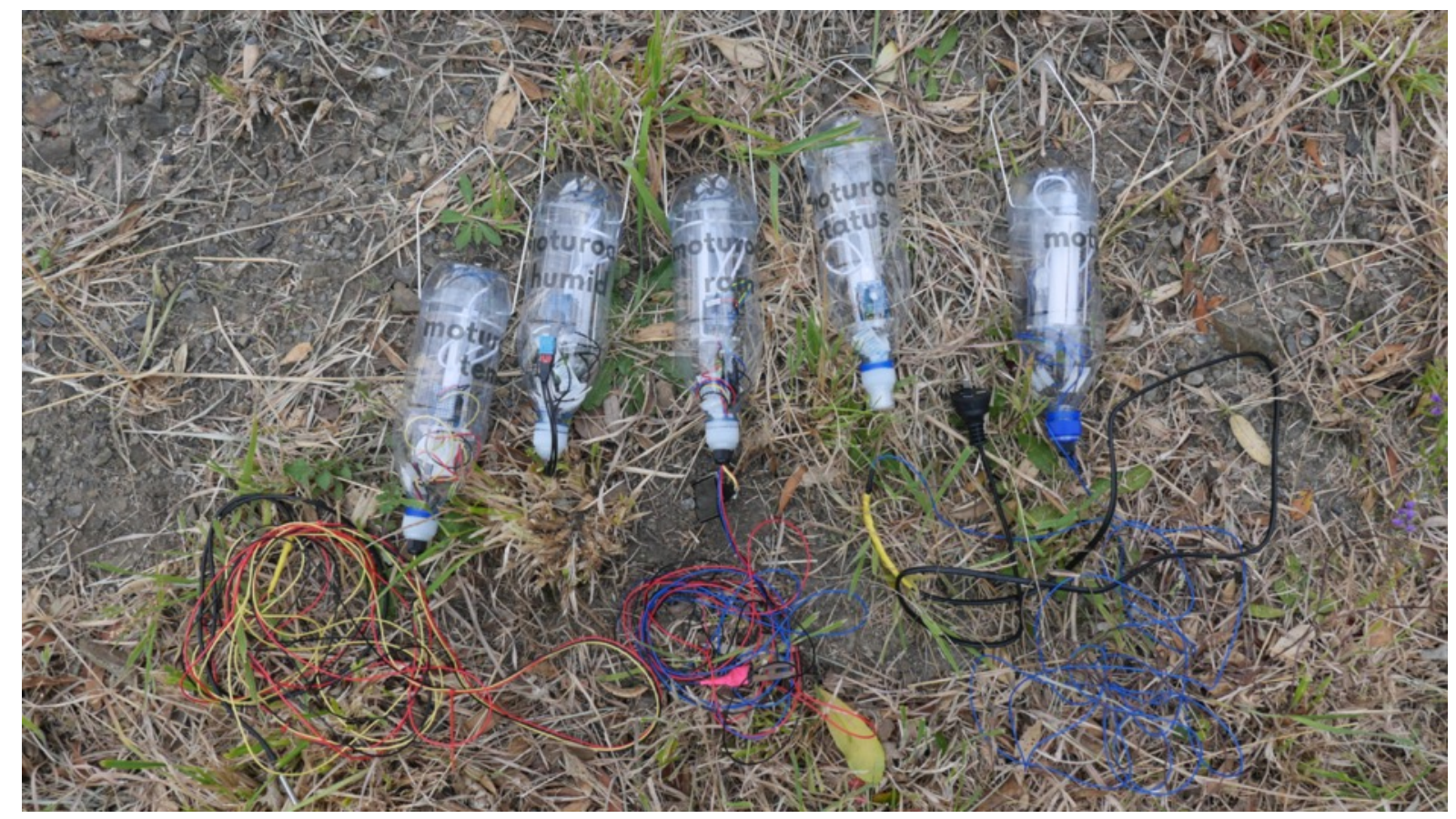

Figure 135. Author. (2017). Moturoa Transmissions nodes (water temperature sensor, air temperature/humidity sensor, rain sensor, status node and EC sensor) gathered by the install location for setup.

Initial challenges for the exhibit included outdoor proofing of the network and providing reliable power to all nodes. The hardware design needed to be suitable for exhibition across multiple days under variable weather conditions, protecting components and circuitry against more-than-human forces such as moisture, wind, and heat. The exhibited design reused water bottles initially used for collecting stream samples and testing DIY nodes in the lab as casing. The transparent casing gives an audience visual access to all componentry and reveals the processes and connections that went into the assembly of the hardware. The piece was installed close to a secondary entrance of Brooklyn's Central Park, where Moturoa Stream is not directly visible and inhabits constructed underground and natural overground environments (see Figure 137).

The Art Trail ran for three consecutive days, with the artwork operating during daytime hours so the batteries could be recharged overnight. This not only allowed some time to do any required repair work, improvement, or cleaning of the nodes, but also provided an opportunity to quickly develop new components from prepared spare parts. 
While the schedule of the public art event pushed the development of the project significantly forward within a few weeks, the compressed timeframe of quick iterations developed overnight-based on feedback from the audience-came with a few drawbacks. Access to the stream had significantly changed between the exhibition and earlier location visits, with flax bushes and other vegetation overgrowing the site, and the chirping of cicadas dominating the soundscape. Having some of the artefacts tested as part of the network in the field for the first time during the exhibition was stressful and demanded onlocation debugging and adjustments. As a result, some of these field updates were not appropriately documented in the online code repository due to the lack of Internet access on location.

Initial challenges for the exhibit included outdoor proofing the installation and providing reliable power to all nodes. The setup needed to be weather-proof and durable, and incorporate recycled materials to protect all equipment, while allow information flow to audiences.

The repurposing of used water bottles as outdoor-proof project enclosures, instead of manufacturing new materials, resonated with a low-cost and low-impact approach to prototyping and significantly extended the usage period of these single-use materials. The bottle enclosure design also linked back to a range of discarded bottles I encountered in the exploratory walks as part of my fieldwork, where I discovered disposed plastic bottles beside the stream banks, and older ones slowly emerging from muddy stream beds after heavy rainfall. A disadvantage of the material, however, was that it slowly degenerated and cracked over time from continuous de-assembling and re-assembling of the nodes for charging and maintenance.

Overall, despite being the result of a quick development cycle, the installation of the prototypes as part of a public art event provided better understandings of the limitations of a networked artwork installed in a stream environment, and yielded material to depart 
from for further iterations of the piece. This cumulated in the work Papawai Transmissions, which I introduce in the next section.

I conclude this section with an overview of visual material showing the piece as installed during the Brooklyn Art Trail, followed by the graphs I generated with help of the software Processing from the data collected during the two days of the install I collected data from. For audiovisual documentation, please have a look at the video fieldlab.mp4, which gives an impression of the environment, and the sonic interferences between the sounds of the stream gurgling, cicadas chirping and traffic passing by the install site.

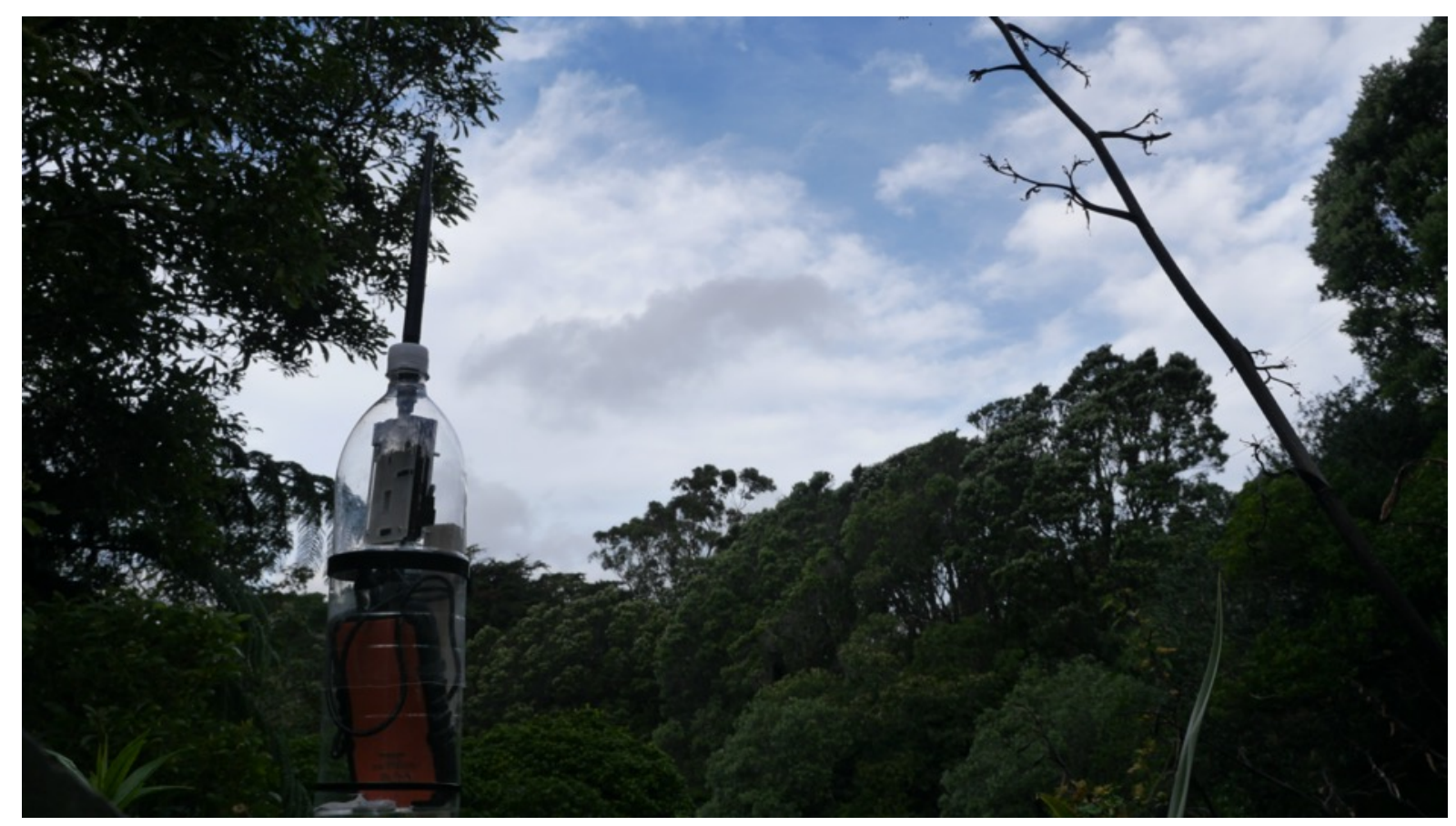

Figure 136. Author. (2016). Moturoa Transmissions base station during install. 


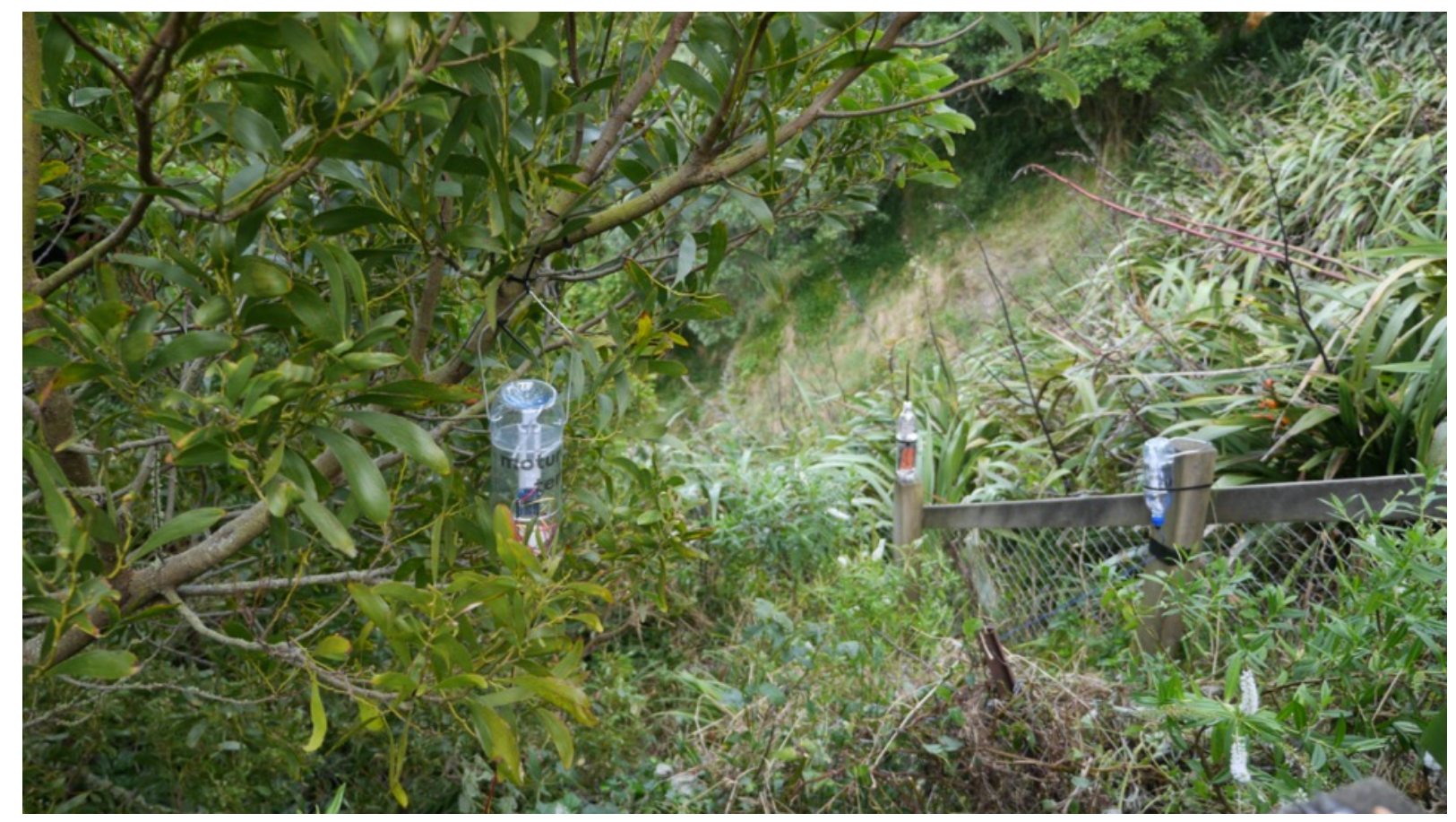

Figure 137. Author. (2017). Installation at Moturoa Stream showing two sensor nodes and one and Wi-Fi Access Point/MQTT Broker.

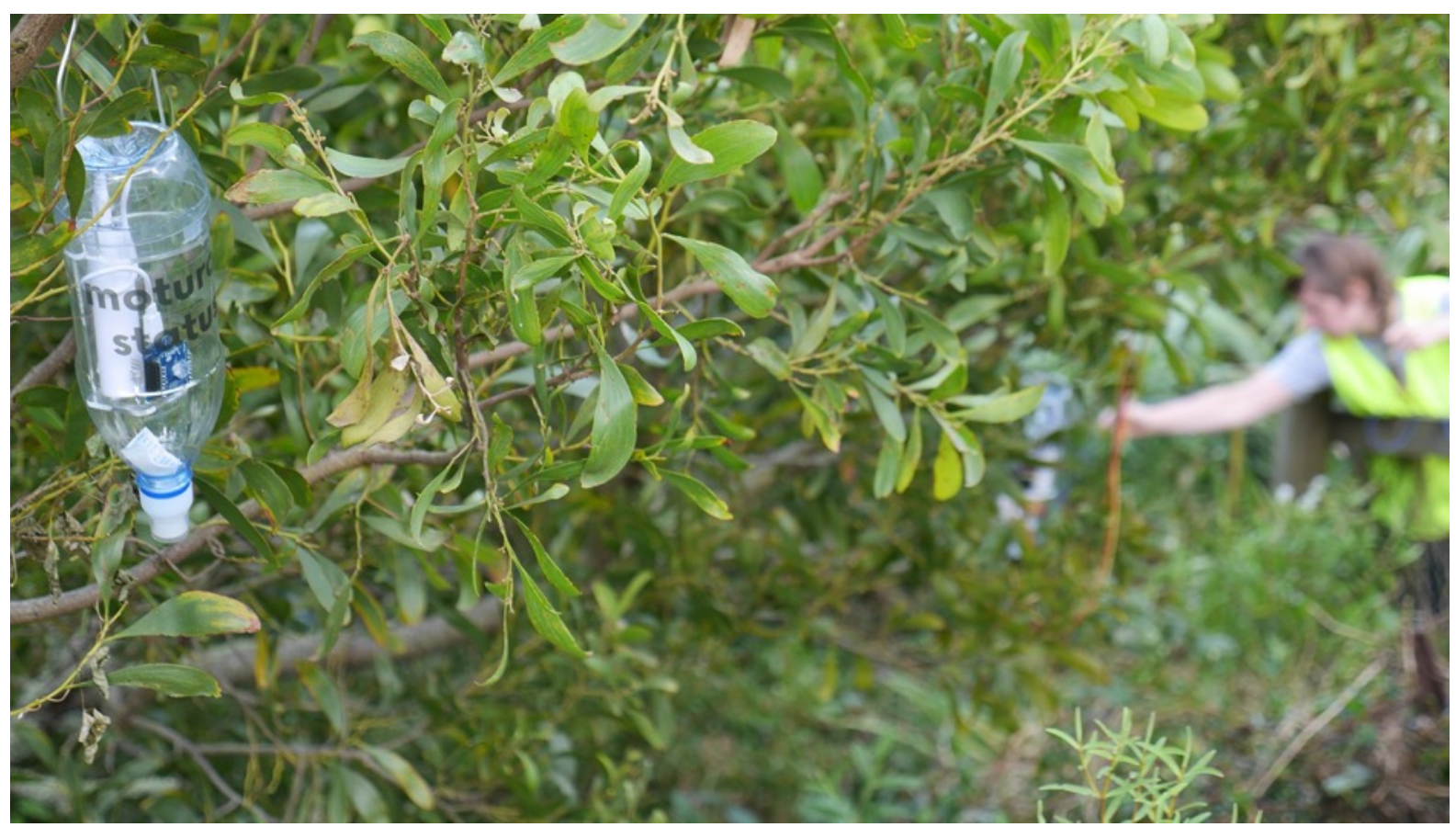

Figure 138. Author. (2017). Adjusting the cables of the temperature sensor node as part of the install. 


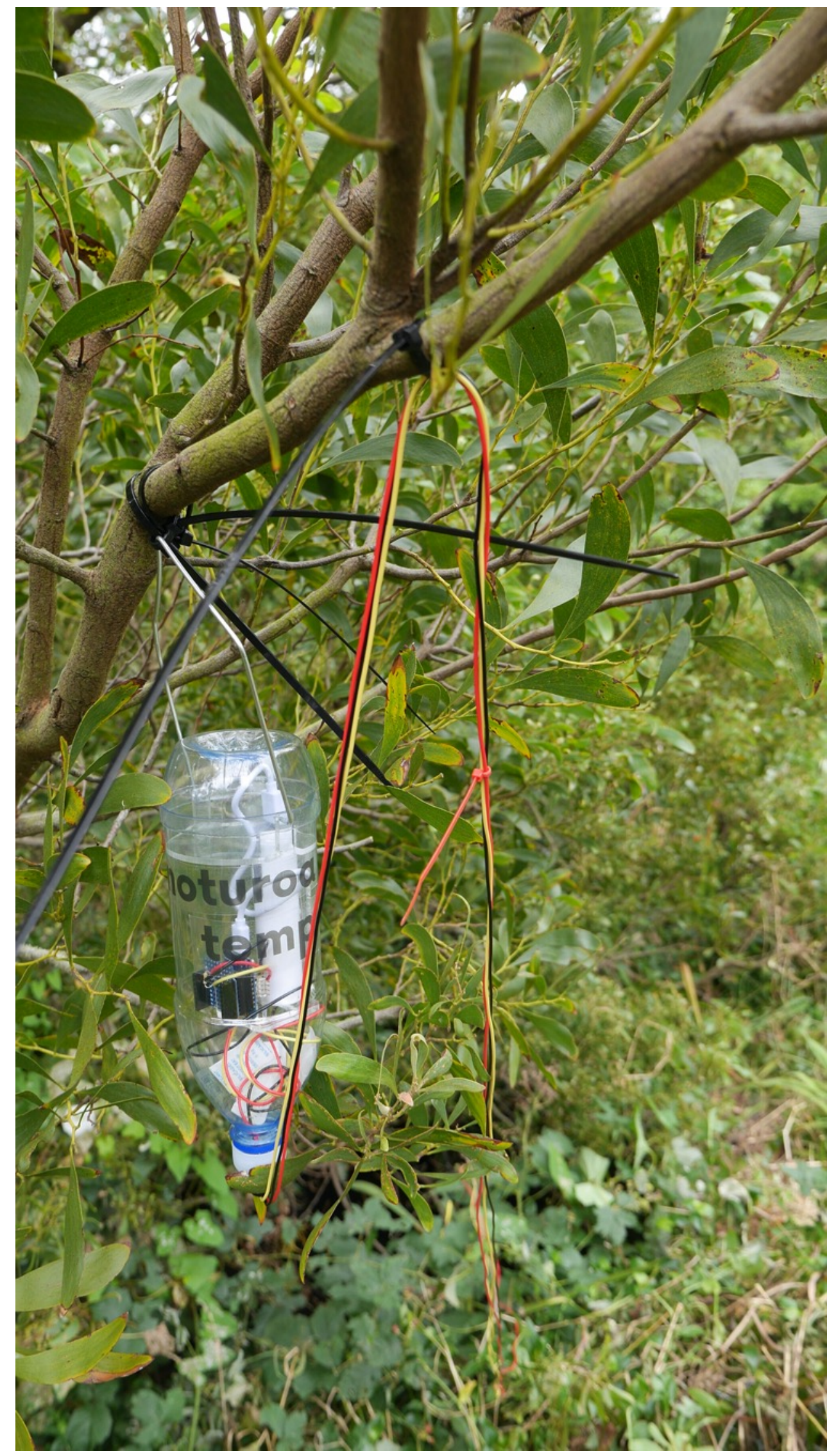

Figure 139: Author. (2017). Moturoa_Temp node suspended from a tree with sensor cables secured to the branch. 


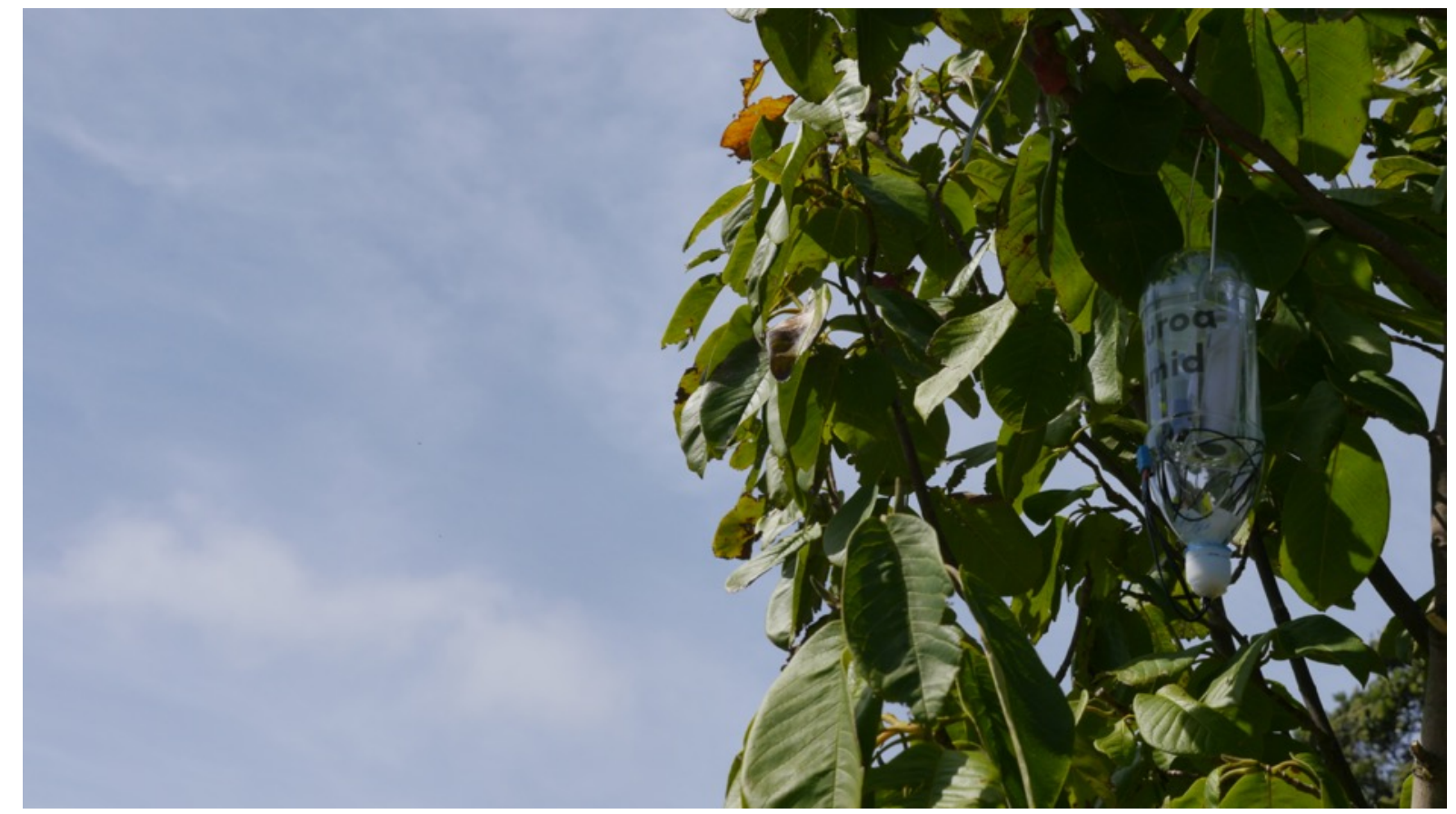

Figure 140: Author (2017). Moturoa humid node suspended in a tree as part of the Upstream Brooklyn Art Trail.

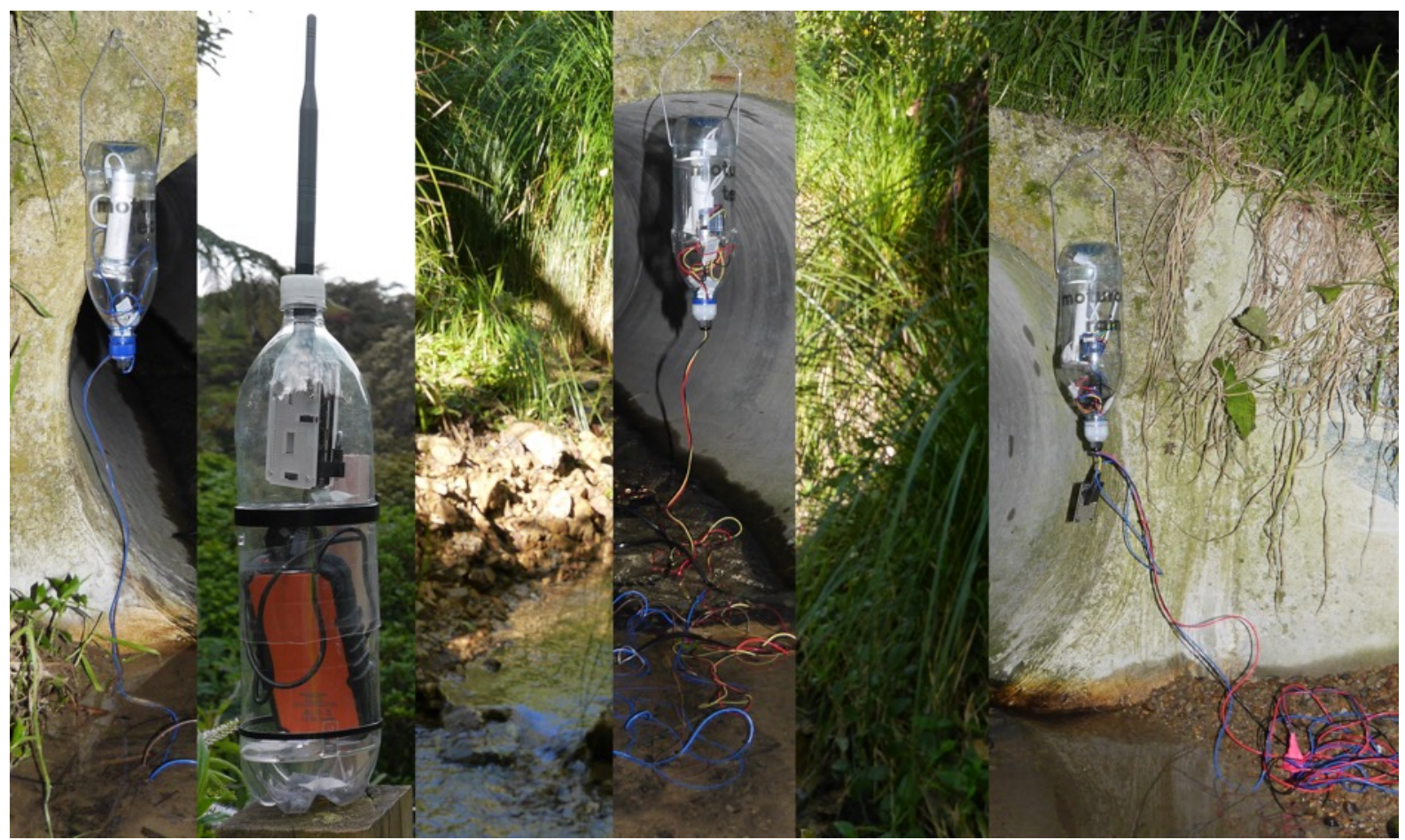

Figure 141: Author. (2017). Test installing Moturoa Transmissions nodes at Papawai Stream. 


\section{Overview of all sensors installed as per documentation:}

\section{Electric conductivity (EC) sensor}

publish: moturoa/ec, moturoa/spike; subscribe: none

\#define node_name "Moutroa-EC"

\#define ectopic "moturoa/ec"

\#define spikeTopic "moturoa/spike"

Measuring interval: 10 seconds.

Example messages:

moturoa/ec 182.0

moturoa/spike 1

\section{Water temperature sensor}

publish: moturoa/watertemp; subscribe: none

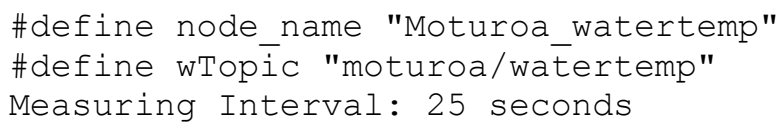

Example messages:

moturoa/watertemp 30.0

\section{DHT11 - Air humidity and air temperature sensor}

publish: none; subscribe: none

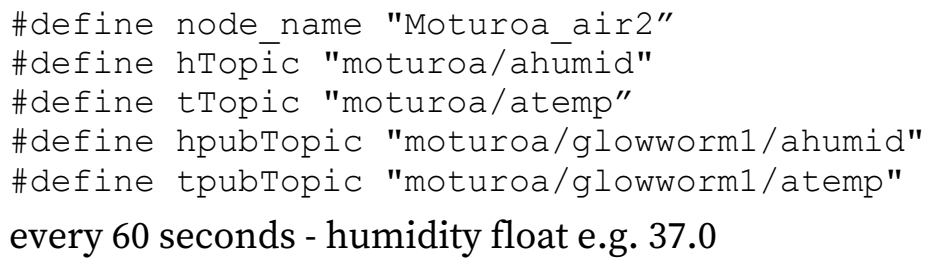

\section{Soil humidity \& rain sensor}

publish: moturoa/rain, moturoa/glowworm1/rain, moturoa/humid,

moturoa/glowworm1/humid; subscribe: none

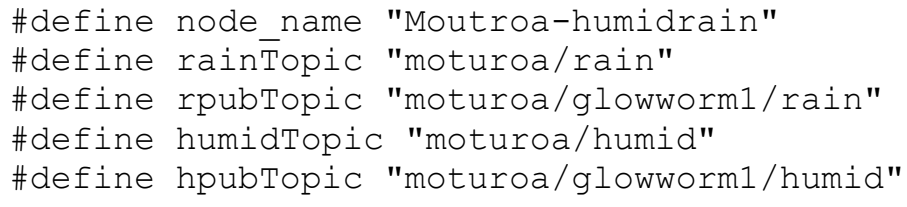

Interval: Rain: Every 30 seconds; Humidity: every 30 seconds

Understanding the values:

$0=$ rain, $1=$ dry;

Humidity range: $0-1024$ (0-300 : dry soil, 300-700 : humid soil, 700-950 : in water) 


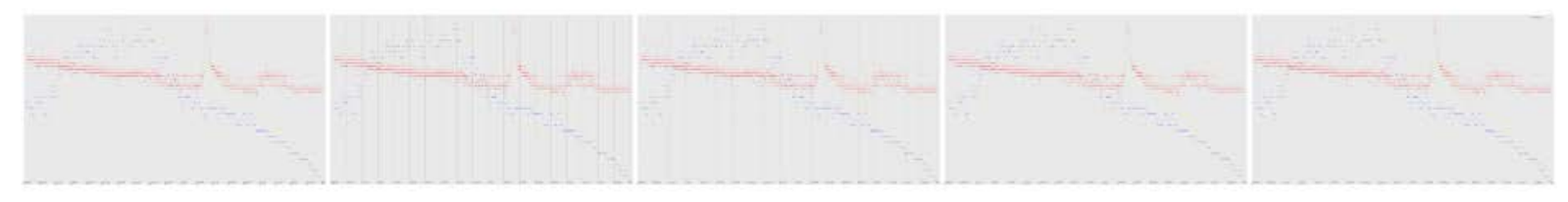

Sunday20-7_11:36:23... Sunday20-7_11:37:55... Sunday20-7_11:38:15... Sunday20-7_11:40:11... Sunday20-7_11:42:28...

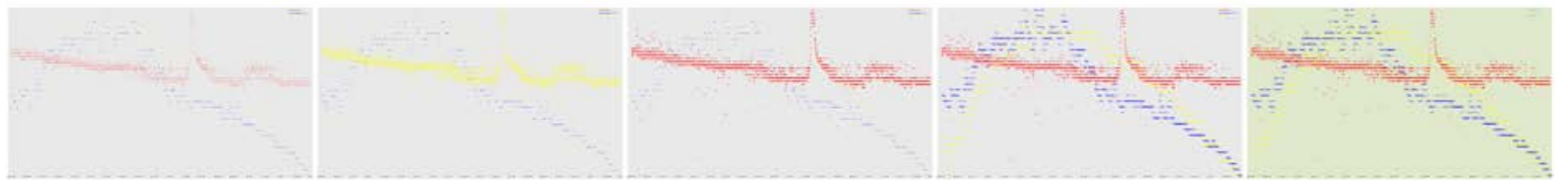

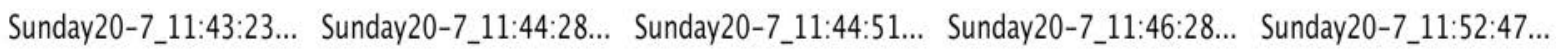

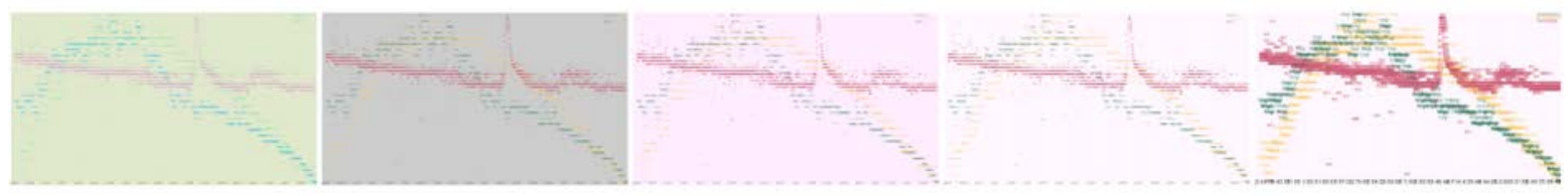

Sunday20-7_11:53:25... Sunday20-7_11:54:50... Sunday20-7_11:55:11... Sunday20-7_11:55:29... Sunday20-7_12:0:53.png

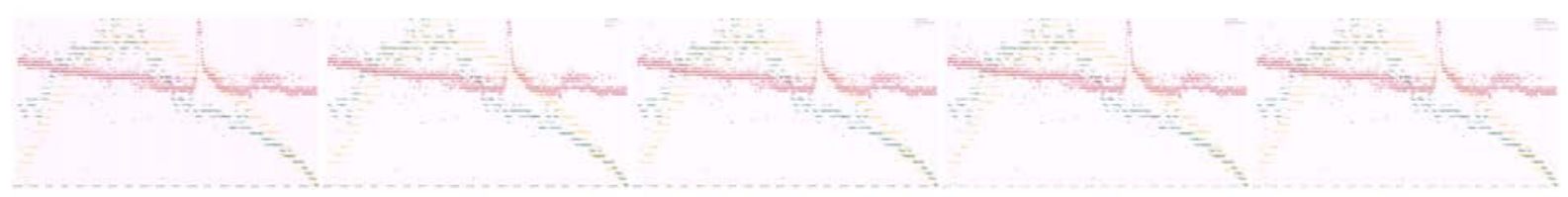

Sunday20-7_12:1:5.pngSunday20-7_12:1:25.pngSunday20-7_12:3:37.pngSunday20-7_12:4:1.pngSunday20-7_12:5:51.png

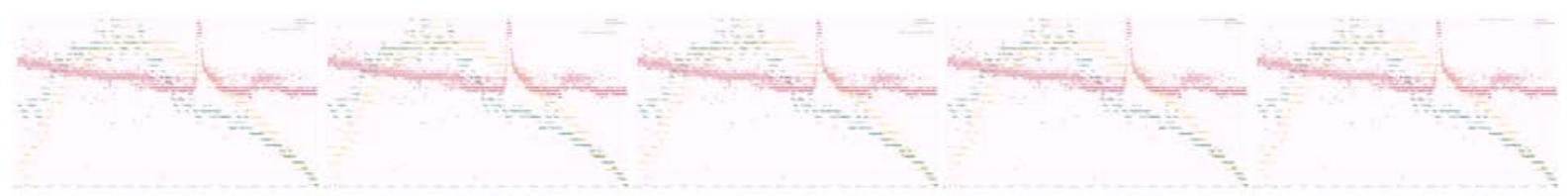

Sunday20-7_12:6:5.pngSunday20-7_12:6:17.pngSunday20-7_12:6:25.pngSunday20-7_12:6:42.pngSunday20-7_12:6:50.png

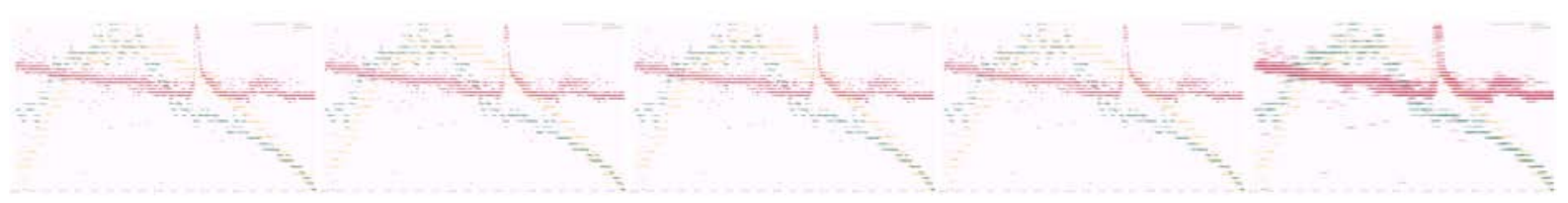

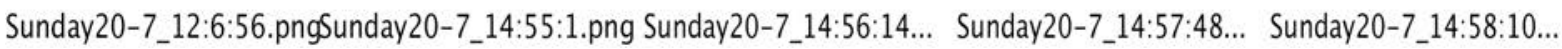
Figure 142: Author. (2017). Contact sheet of iterations of chart design. 


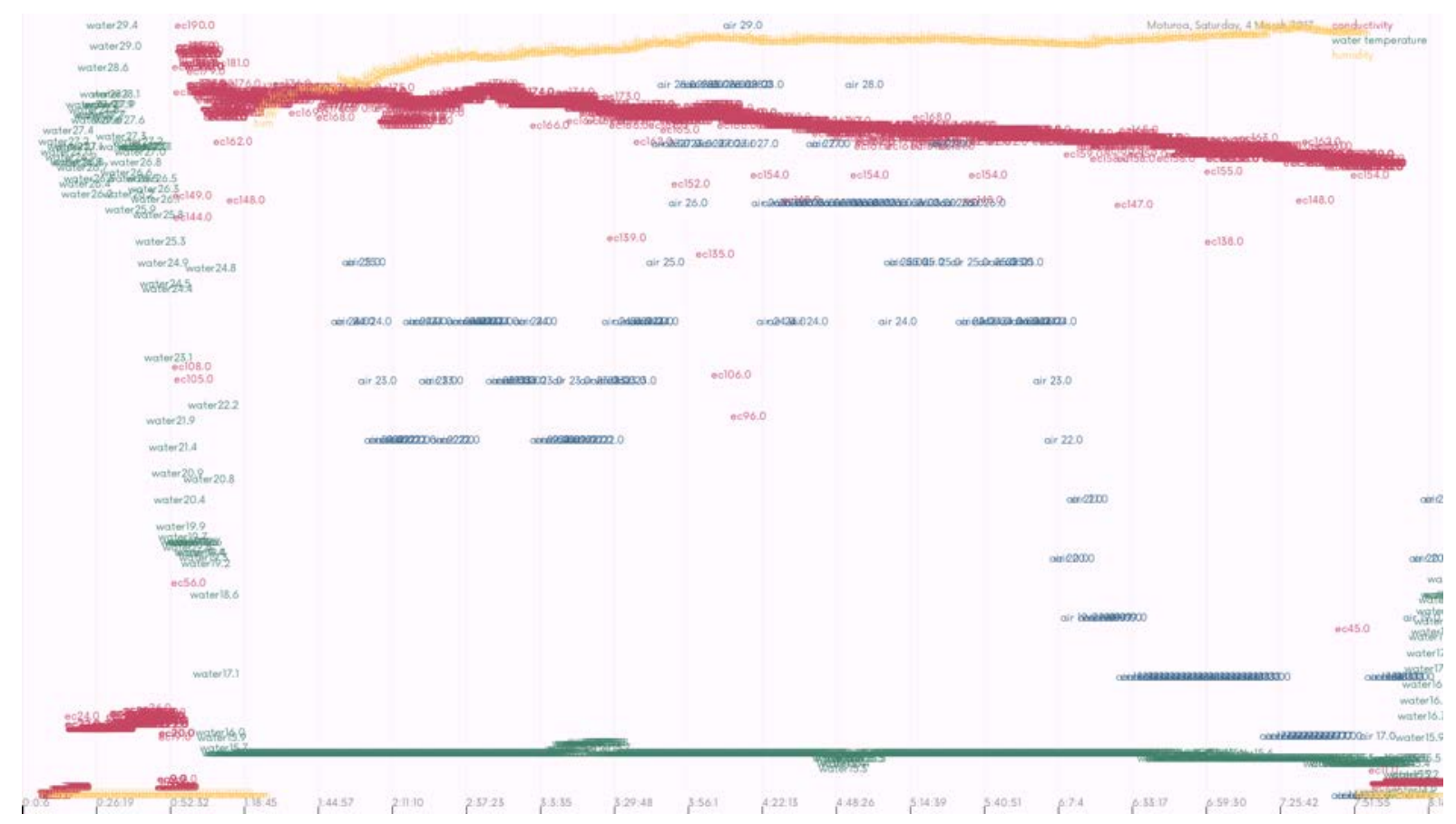

Figure 143: Author. (2017). Moturoa Transmissions chart, Saturday 4 March 2017.

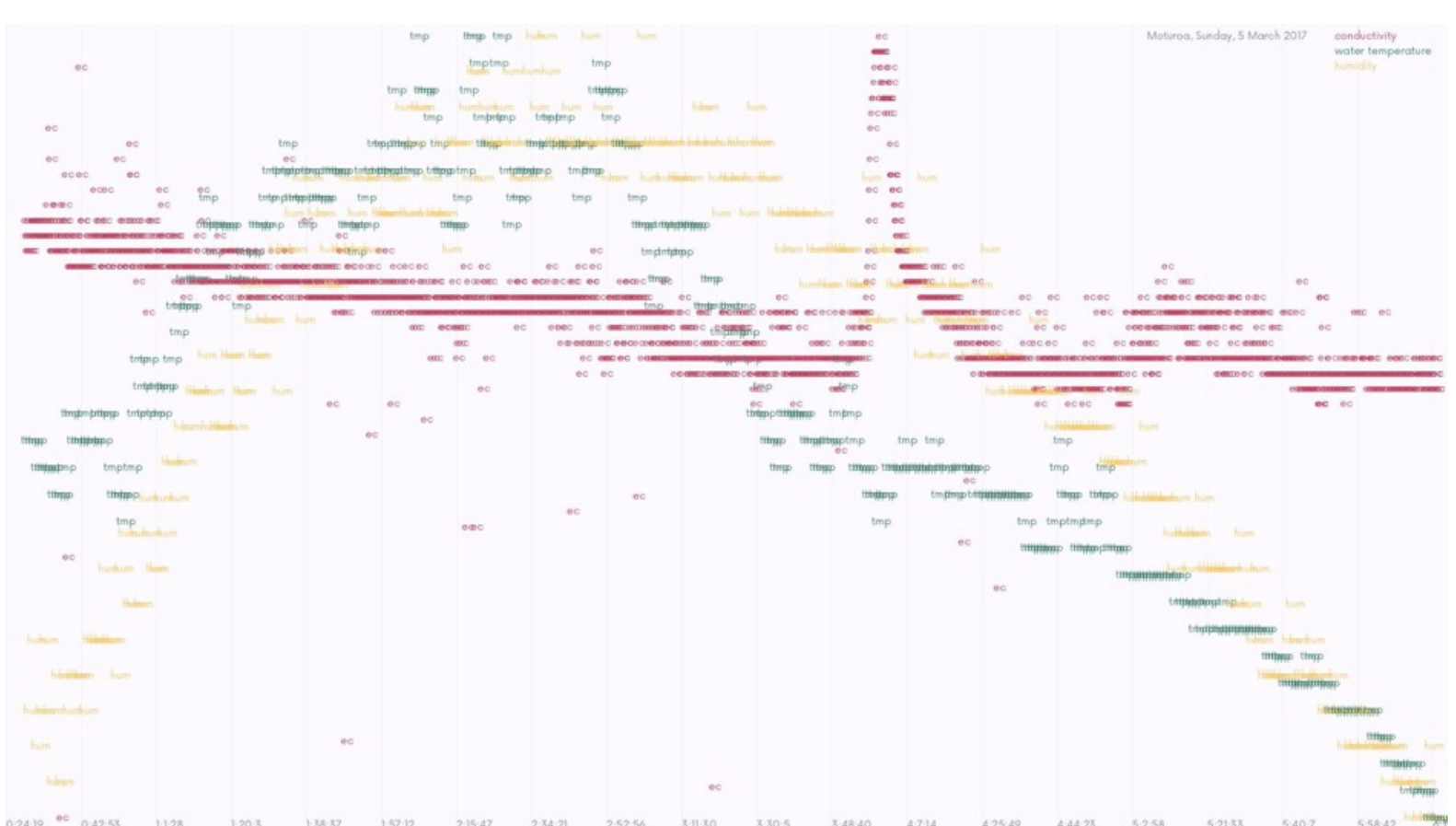

Figure 144. Author. (2017). Moturoa Transmission data visualisation, Sunday 5 March 2017. 


\subsection{Testing DIY EC sensors in Papawai Stream [Lab Notebook, 16 December 2018]}

This narrative outlines the testing of newly built electric conductivity sensors in the field, as part of the iterative sensor design outlined in Section 6.5. Having experimented with different ways of documenting my design work, at this stage of the research I feel comfortable audio-recording myself while I am monologising, to be able to reconstruct the narrative later from a combination of written lab notebook entries and the audio transcripts.

\section{Lab Notebook, 16 December 2018}

It is the first time I install and test the two newly built EC probes at Papawai Stream. I am close to where the wastewater pipe is crossing the stream. I have decided to audio record myself talking along with my fieldwork for better documentation of my process and challenges in the field. The writing in this post is a combination of re-telling of events based on the photos I took with my mobile phone and a transcript of my audio recording, which has been edited to aid clarity and flow of narrative.

I have put together an installation kit that is transportable by one person. The wooden crates make it easy to carry the sensor probes and I imagine they could also come in handy during installation in the field to keep the electronics in place, and be safely stored outside of the water or muddy areas around the stream during the recording of data. 


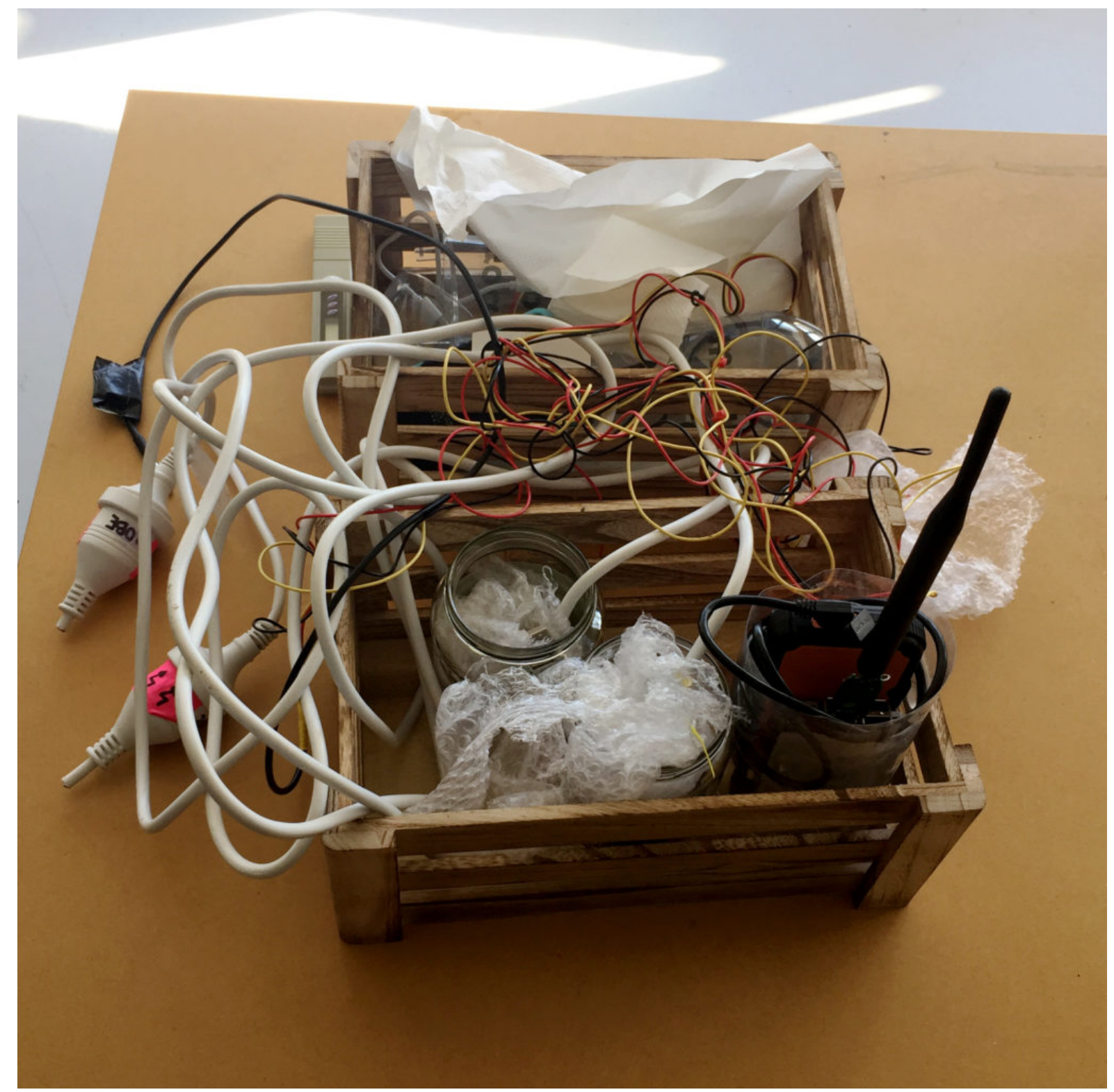

Figure 145. Author. (2018). Two wooden crates containing the Wi-Fi Access point and two EC-sensor probes to be tested in jars. The electronics are temporarily secured in place with bubble wrap. Additional materials in the crates are spare enclosures, cables, and batteries.

Additionally, I am carrying a backpack with my Thinkpad laptop, and a waterproof bag with additional equipment such as the TDS reader, spare batteries and USB cables and other necessities such as extra jars, paper towels, cable ties, pens, and a notebook. I also take a little three-legged camping stool and my smartphone with me. I transport the equipment by car to the Prince of Wales Park and from there carry it from the carpark to the install site. 
After a short hike up the public walkway, I reach the entrance to the site where I plan to install my work, turning into a smaller path just on the left before the bridge. The ground is covered in pine needles, the path lined by grass, bushes, and pine trees.

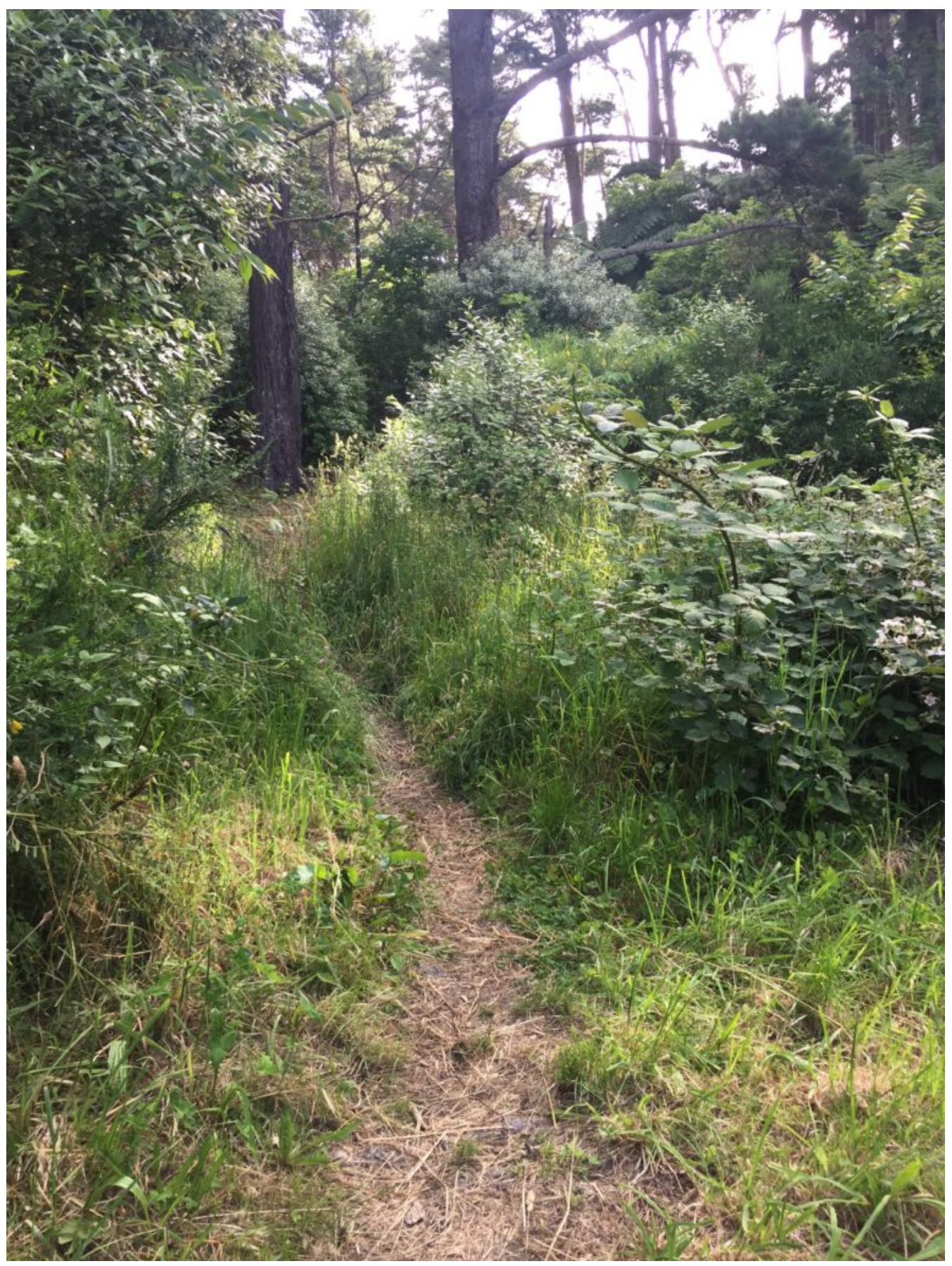

Figure 146. Author. (2018). The pathway to access the installation test site besides Papawai Stream.

If I listen closely, I can hear the stream through the bush, following the pathway just a few steps into the forest. Not much later I can see the stream on the right side flowing about a metre or two below the pathway, merely a shallow trickle today. I pause and take a breath but cannot smell anything out of the ordinary from the water, only the dampness of the 
pine trees. I watch the stream quietly gurgling down the small gully, flowing under the wooden pedestrian bridge, before disappearing from sight further down the bush. I follow the stream further upstream, ducking under fallen pine trees, until I reach the site that I have scouted earlier as a possible install location.

I put the crates on the flattest surface I can find on location. The ground is quite wet, and during install, I need to make sure that my gear is not in the way of any walkers, and safe from dogs, who might use that path. The sensors themselves are only loosely attached into the recycled glass jar enclosures. They are not waterproofed yet-I only temporarily propped the jars up with bubble wrap to protect the electronics from humidity and insects while keeping the electronics easily accessible during the testing.

\section{Setting up}

The Raspberry Pi has been on all the time since I tested it back in the lab, so the Moturoa_Transmissions Wi-Fi network is still up and running, but no nodes are connected to it yet. I start unrolling the sensor cables, which are a couple of metres long, and connect the probes one by one to their batteries. I make sure the crate is in a stable position, so it does not create a hazard once I launch the probes into the stream water. 
As I attempt to submerge the first probe, I notice it does not easily drown. The water is just a couple of centimetres deep. Both prongs need to be touching the water to get accurate readings, and I need a few attempts to position the plugs properly.

Looks weird. Two electrical plugs in the water.

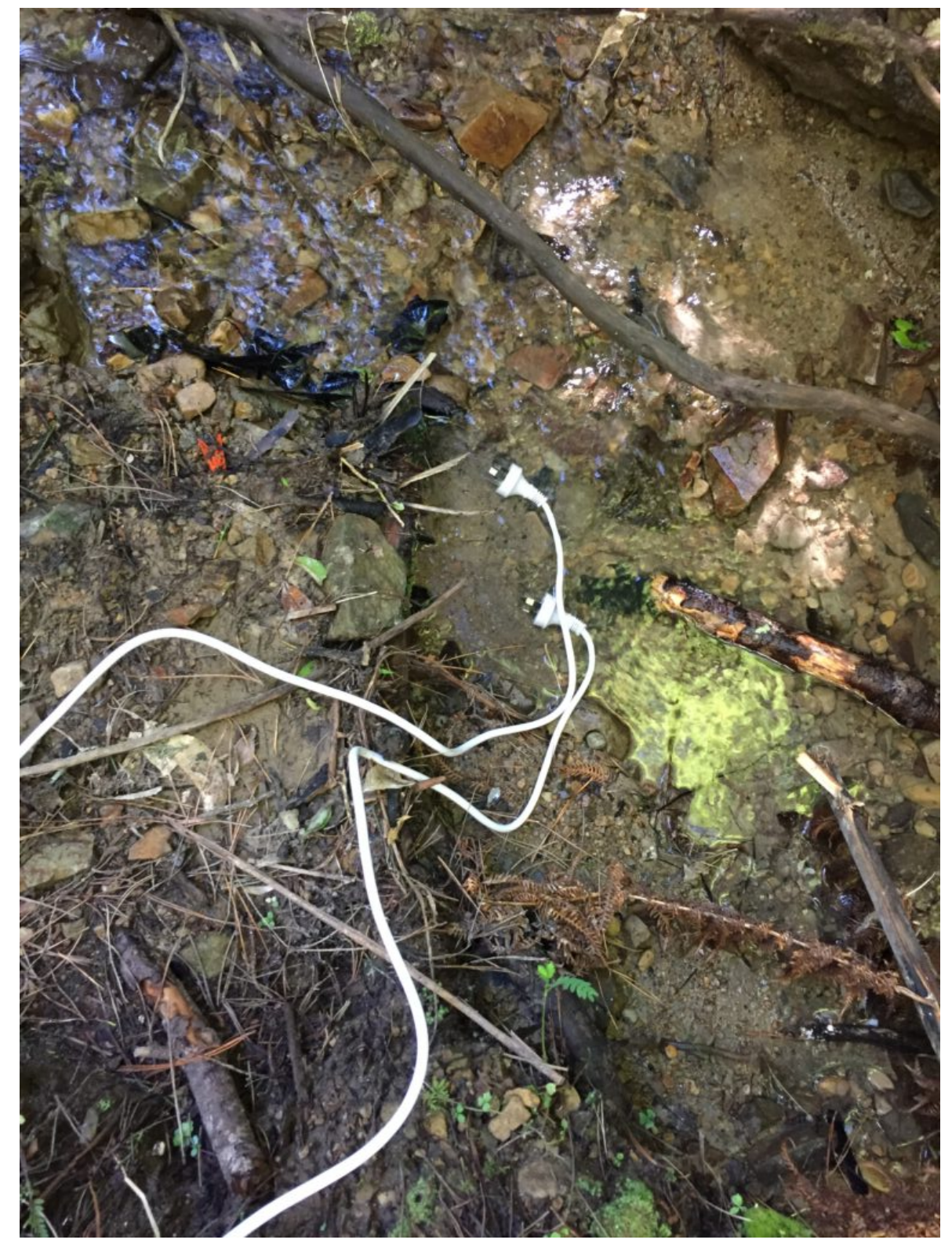

Figure 147. Author. (2018). Two repurposed extension cables measuring electric conductivity in Papawai Stream.

I am also testing to see whether the new code on my SD card node is working. I have updated the code so that a new CSV file is created every time the node is being reset, as opposed to overwriting any old data, which was good enough for earlier experiments, but now I need a more stable way of collecting and keeping data. I chose not to thoroughly test 
the SD card code in the lab because the main focus of this experiment was to evaluate the functionality of EC probes and I needed to get into the field as soon as possible to avoid having to work after dark.

I notice I haven't brought a dedicated battery for the temperature probe, so I need to use the spare one from the equipment bag. The temperature probe is still in the plastic bottle casing from the previous install. It could work if I redesigned the cap to aid with accessibility to charge and change the batteries. The probe itself has a stone gaffer-taped at the bottom of the wire to add weight to it for easier launching into less-reachable parts of water. The previous install location at Moturoa Stream was wildly overgrown, and I made this with what I had at hand in the field. I could add some fluorescent-pink tape over the black to make the submerged probe more visible, as it is difficult to spot under water. The temperature probe has a quite long cable (partly seen in Figure 148 with red, yellow, and black strands) and it already got entangled with a plant, around here even though I have just carefully de-tangled the cable before leaving the lab. I launch the probe into the water close to the already submerged EC probes, carefully, so as not to short-circuit the prongs of the other probes. 


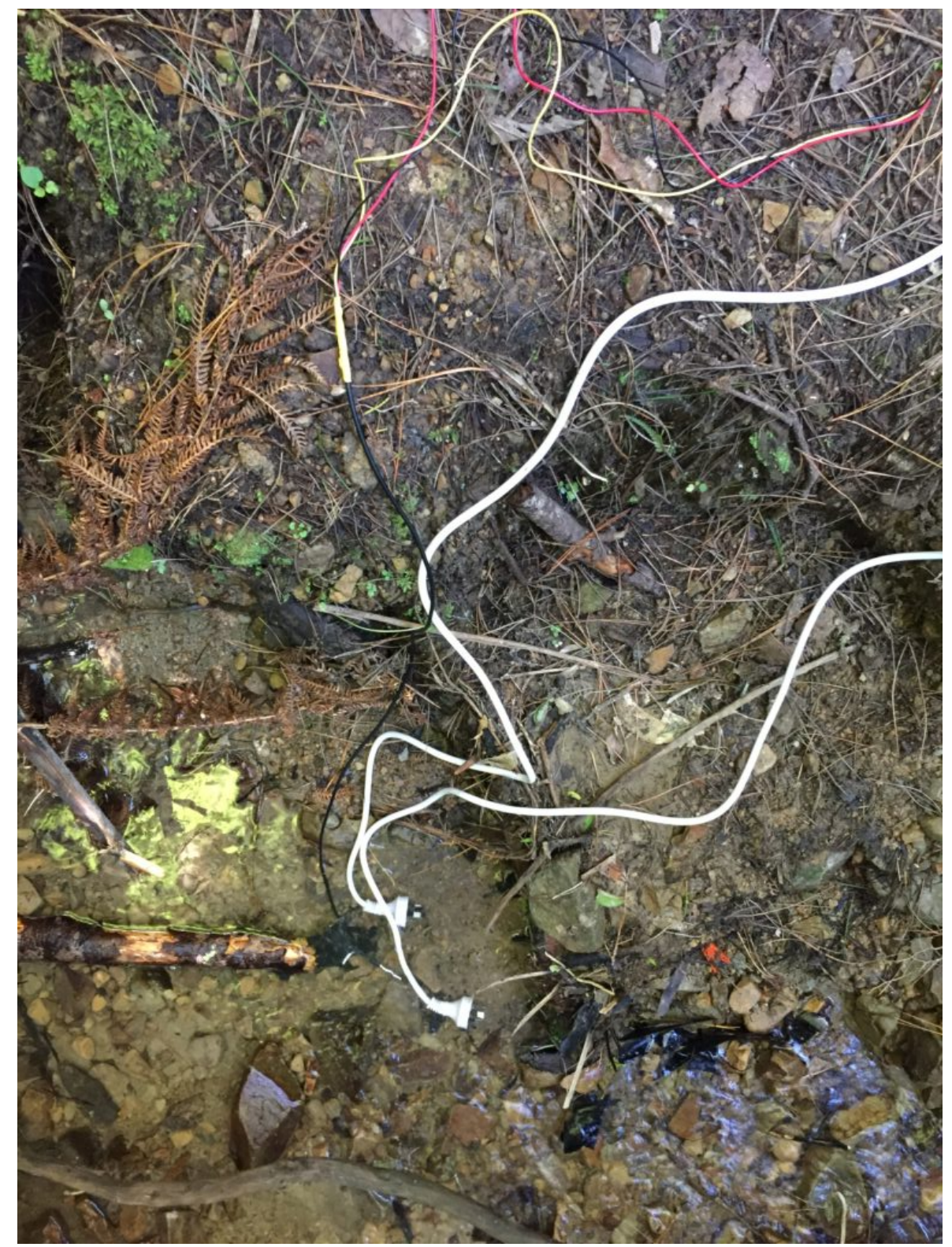

Figure 148. Author. (2018). The temperature node is added to the two EC-probes in the stream.

That looks pretty good. I am also taking a panorama of the setup to capture the relationships of the probes with the stream.

\section{Accessing the sensor data}

So now I have got the probes in the water, I am moving on to the exciting part of seeing the data, and what values the two EC probes return. It's quite sunny and dry today, so I thought it would be fine to take my development laptop with me into the field and use it to live-view the data of my devices.

Plants are already losing some bits and pieces on my keyboard. 
I spend some time failing to connect to my Moturoa_Transmissions Wi-Fi network and need to restart my machine. This takes a while and gives me time to look at the probes again. I rearrange them because the cables are overlapping, but find it difficult to place them the way I like because the tension of the cable dictates their placement on the ground. I also notice that the probes disappear when underwater. I consider adding some elements of colour to set them visually apart from the surroundings. While trying to rearrange cables and probes, I notice that it would have helped to have brought some gloves since I am not entirely sure if it is safe to touch the water. Additionally, some hand disinfectant as used during the SHMAK workshop would have been a good addition to my carry bag. My laptop has rebooted, and I finally see my own Wi-Fi network, but I also notice another wireless network named AI-THINKER, which curiously seems to be another ESP8266-based Internet of Things network.

I open a terminal window on my laptop and use the mosquitto_sub command to subscribe to all topics to see all data floating around in my little PubSub network.

\section{This is exciting.}

I see EC returning a value of 267 and EC_rua on 276, which appears to be consistent with previous readings, where EC_rua returned a slightly higher value than EC. The readings seem to be remaining constant, which is good.

I don't see the temperature in the feed, so maybe the battery I used is not working. It does not have an indicator light, so I do not know whether it is discharging or not. I will swap the power supply to the spare solar battery I brought along. This one is clearly discharging according to the LED, so I should see a temperature value soon.

\section{Oh yeah, watertemp here we go. 14.2}

Now I will cross-check this result (in degrees Celsius) with the temperature my TDS reader returns. I step down towards the probes and submerge the prongs in the water, and it seems to be about 1 degree off. I might jot these values down in my notebook just in case.

EC is 265. EC_rua is 275 . Watertemp sensor returns $14.2^{\circ} \mathrm{C}$, the TDS $15.5^{\circ} \mathrm{C}$.

The TDS reader shows 158 ppm. 


\section{Concluding the experiment}

I would call this a successful experiment; I have collected 15 minutes of data so far. No one has walked past yet. It worked this way, but the gear obstructs the walking path a little, and I need to find a more stable and safer spot for longer installs that can also be left alone for some time. This was easier at the previous install site where most devices were installed behind a fence and the others were safely suspended, with just the probes touching the ground without obstructing anyone's path, sheltered by large harakeke/flax plants. The jars work well, so I should continue working with them as a material and find a suitable solution to make them outdoor- and long-term install-proof as well as provide easy access to the electronics for charging and possible hardware updates. The electrical cords work, but the way they are lying on the ground like this looks quite ugly. The white colour of the cable helps to set them apart from the dark ground, but I should consider some more thoughtful decoration or support for the cables. Suspending the electronics on one of the fallen trees, as previously planned, could work, but needs some careful consideration of the environment. 


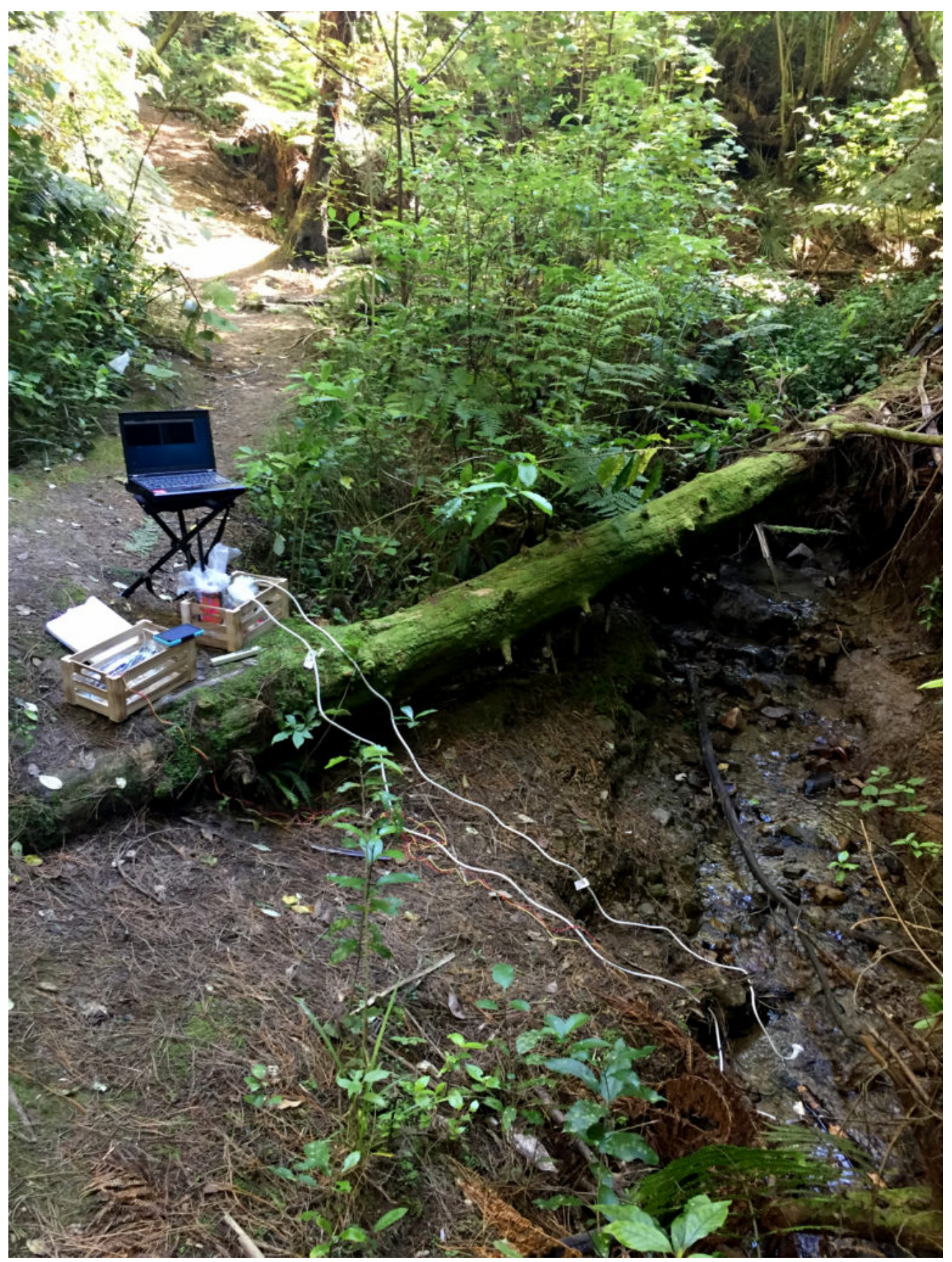

Figure 149. Author. (2018). The test setup beside the stream.

\section{Observations and recommendations for next install:}

\section{Design development and install}

- Draft some designs, so the probes are stable and visible underwater while not disturbing the natural flow of the stream.

- Design versions of outdoor proof but easily accessible lids for glass jars.

- Overall the electrical cords in this setup are visually not appealing. The white colour sets the cables visually apart from the background, which is good for safety but aesthetically unpleasant. 
- The installation of probes should be tested suspended from trees. This needs additional install tools such as hooks, cable ties and a variety of options should be tested in lab and field.

- Replicate the probes and connect them within the network. Build three probe pairs for temperature \& EC and design an output that is accessible from the bridge.

\section{Equipment and electronics}

- Bring all spare batteries just in case.

- Use a lapel microphone attached to an audio recorder for hands-free recording.

\section{Hygiene, health and safety:}

- Bring (plastic) gloves to be able to safely touch the water when adjustment of probes in water is required.

- Add paper tissues and hand sanitiser to the kit if getting in contact with water or dirt.

- Consider bringing gumboots for installing probes in more inaccessible, boggy areas.

- To stabilise the setup, I would need to bring something like pegs to fix the cables to the ground.

- Add some colours to the bottom of the probes or consider using makers to make sensors and cables more visible when submerged in the stream water. Especially the black wire of the temperature probe disappears into the environment.

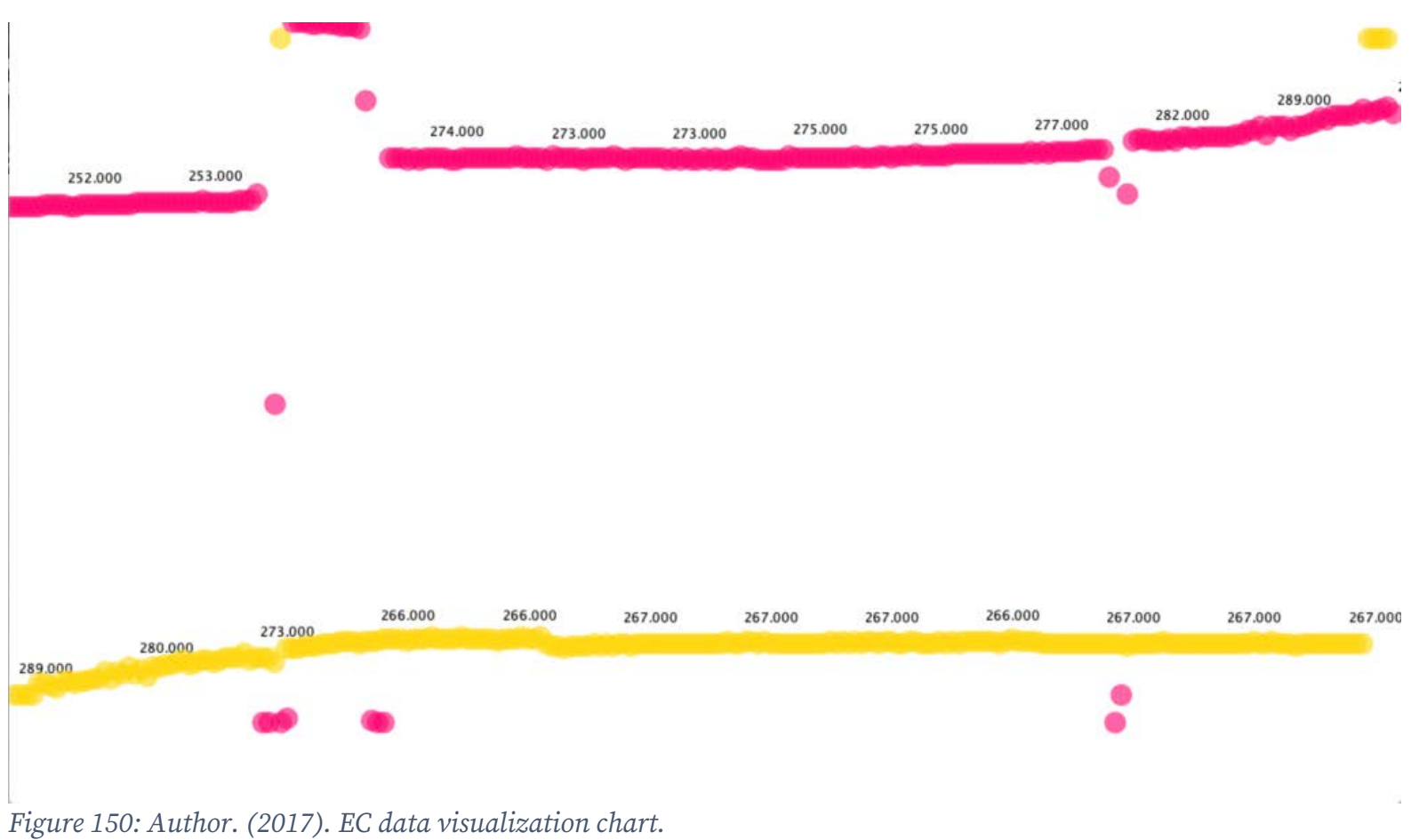




\subsection{Papawai Transmissions - Field Evaluations}

February 2019. The monthly Papawai Gardening meetups have been on summer break and are about to resume on the second Sunday of the month. This weekend would mark the final installation of Papawai Transmissions as part of this thesis research and the last round of evaluation interviews.

\section{Sunday, 10 February 2019.}

I meet the Friends of Papawai Stream briefly during their gardening session and give an update of my project during the morning tea before heading to install. I chose a sunny spot for today's install that is easily reachable from the area the gardeners usually work at during their Sunday sessions. To my surprise, two participants just came to the gardening to see my presentation. I was initially planning to install the work first and then people could come after the gardening session to check it out. But now I had participants with me right from the beginning of setup. This gave me an opportunity to talk them through the installation process step by step and involve them in the installation immediately.

It is sunny, and very hot. I am wearing my preferred outdoor summer research outfit, a boilersuit with a pair of jandals. I have packed all my components in two wooden crates for easy transport and walk with the two participants to the install location, while answering some technical questions about the kind of data I capture and how it is stored. We arrive at the install location and I begin to set up my work while briefly introducing the function of each node to the attending participants.

First, I get the Wi-Fi hub and switch the battery on to launch the Raspberry Pi local network, and carefully prop the components back into the plastic bottle enclosure. "The batteries are the bottleneck of the installation" I explain. We discuss the option of solar power, and that this would work for this particular install location, which is only partly shaded, but not so well for my second installation, further upstream in the pine forest. The hub can stand on its own and I place it at a safe distance away from the water on the packed mud. A similar procedure follows for all the other nodes. I connect the batteries and check 
that the node is powered before placing it in a suitable position. Slowly some of the gardeners arrive on location to have a look at the installation.

The sensor nodes with enclosures recycled from Moturoa Transmissions find a place somewhere suspended from a stable tree branch. Today I make sure that all the nodes are visible to the participants without having to cross the water. The EC-sensor node is placed in a jar, which I made a yarn enclosure for, so it can be hung from a branch. The turbidity sensor is the newest addition to the nodes and its jar enclosure does not feature a hanging mechanism yet, so I place the jar in a safe spot by the Wi-Fi hub. I carefully launch the probes into the stream one by one. Once all probes are in place, I connect the recording node to its battery. It is placed in a small glass jar with a red lid, to be identified by the handwritten letters REC on it. The recording node has no visible in- or output and can be placed anywhere, so I put it on the ground as well.

The temperature output node is in a bottle enclosure, analogous to its sensing counterpart. I hang it in a tree, making sure the node gets a bit of shade. I notice the LEDs are hard to read in the bright sunlight. The EC output node jar has no dedicated mechanism for permanent installation yet, and I hand it to a participant for closer inspection. I find myself demonstrating how the sensor nodes actually work, lifting the EC sensor from the water and demonstrating how the output node visualises the detected change in conductivity by flashing all its white LEDs nervously. The stream temperature appears to be healthy, indicated by the green LED on the output node. I briefly take the temperature sensor out of the stream and place it in the sun, and a participant notices the LED scale changing from green, to yellow to red. 
After about one hour, all participants have left the location and I take some photos before deinstalling the work and heading back to the lab.

I love that you can see the things inside

(Participant).

It's like an intravenous drip hanging in the tree

(Participant).

If you're getting the data, the shape of the device does not really matter. If it works in a plastic bottle or a glass jar, why not?

(Participant).

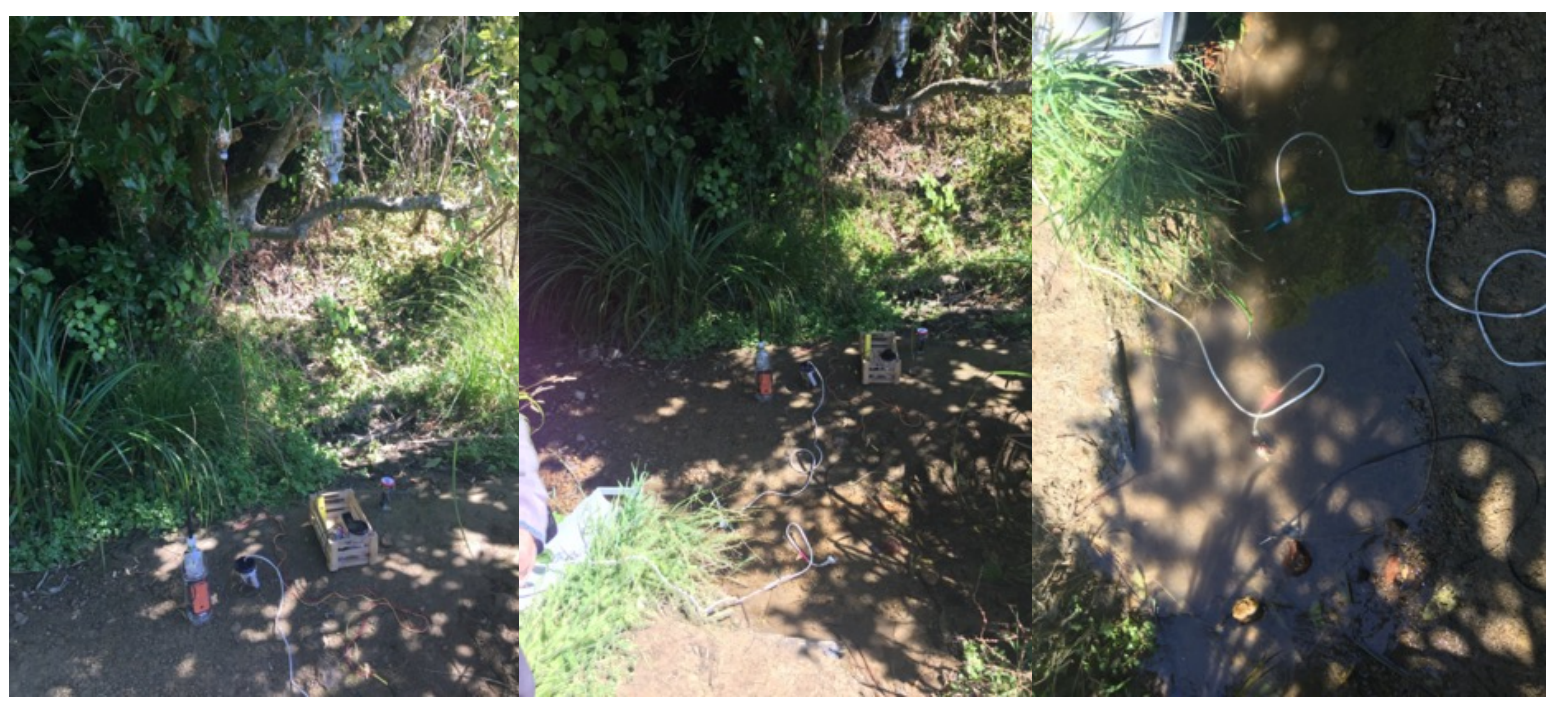

Figure 151. Author. (2019). Papawai Site install beside the sports field. 


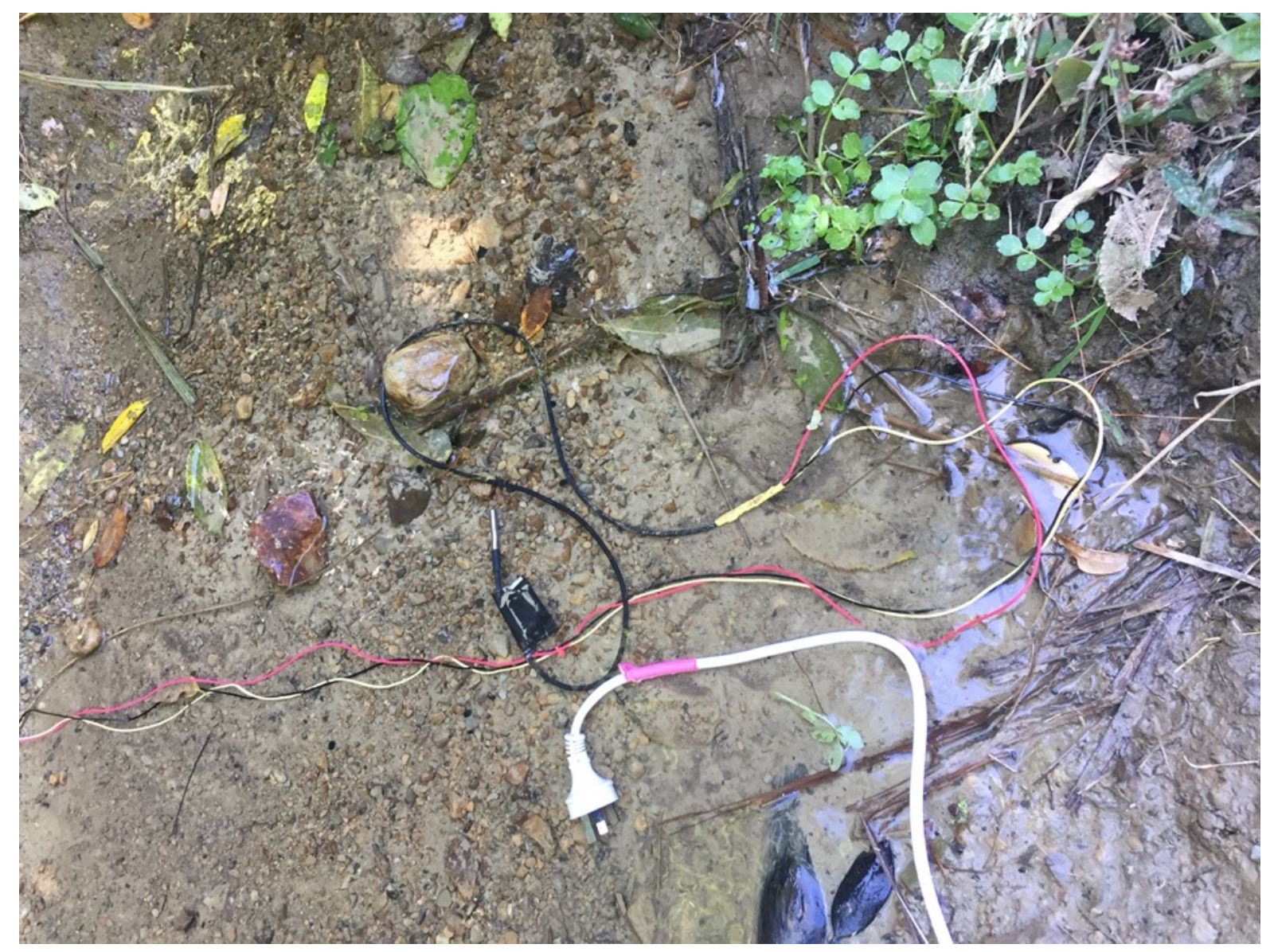

Figure 152. Author. (2019). Temperature and EC-sensor nodes in the stream.

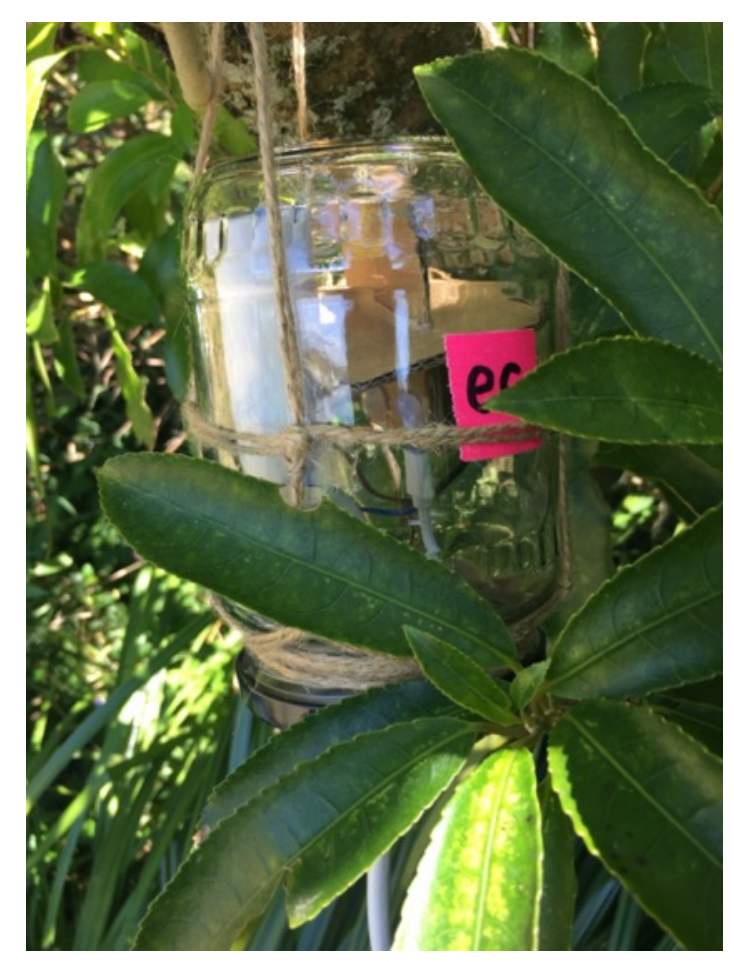

Figure 153. Author. (2019). EC sensor node in a glass jar. 


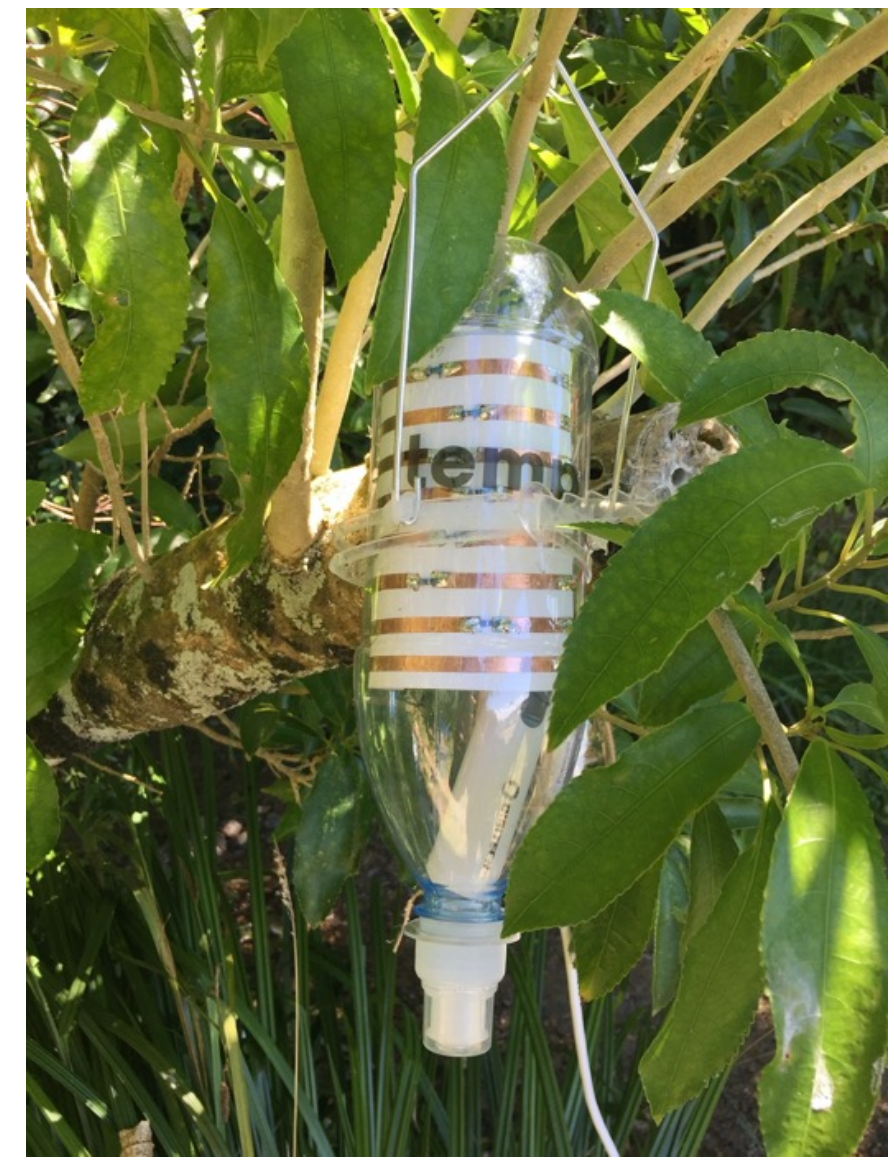

Figure 154. Author. (2019). Temperature LED probe in a bottle repurposed from the Moturoa Transmissions install.

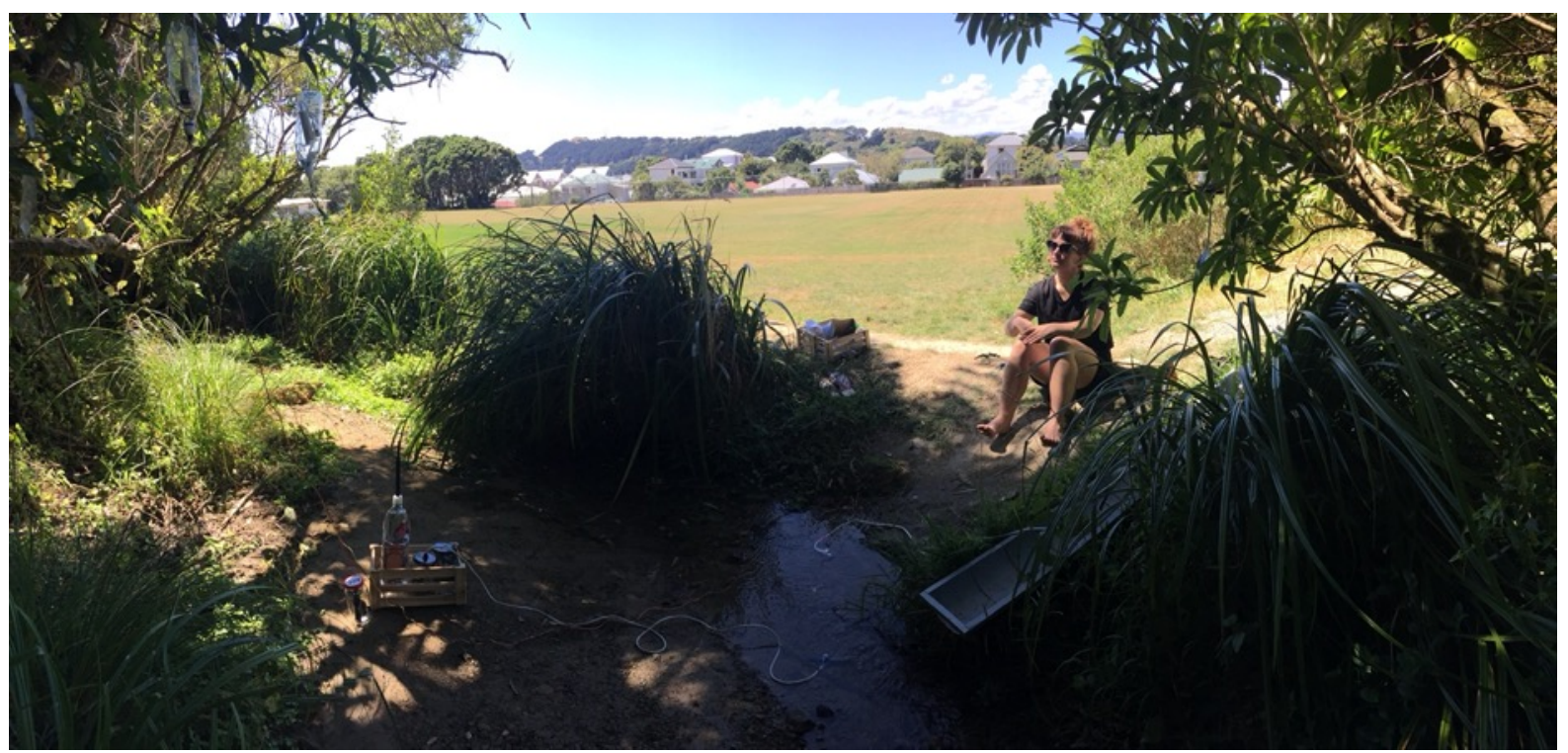

Figure 155. Author. (2019). Overview of the install site by the playing field. 


\section{Monday, 11 February 2019.}

This installation is with two invited participants. We meet in the morning and walk together to the install location, upstream in the pine forest by the stream fork where the wastewater pipe crosses the stream. The participants sit down next to the stream and I install the work, while explaining the context:

That wastewater pipe runs parallel with the stream. You can see it here. There is also this sump, you can access the pipes there through the orange cover. It comes down from Brooklyn somewhere, Connaught Terrace, and when there's a big storm it tends to overflow upstream and slowly creeps down here and further down there's kids playing in the stream...

We have walked up there, it is quite littered, but also a time capsule, with old trash...

I wanted to build a kit where people have the ability to measure the stream themselves, but keep an art angle, installation piece. I have decided to bring earlier versions of my prototypes along so you can see the progress and improvement of the newer ones and the rationale behind the designs.

I mount the two suspended sensing nodes on a branch of a fallen tree that crosses the stream. As the terrain is a bit rough it is harder for me to find stable ground to put the Wi-Fi node, so I leave it inside the wooden crate, along with a bottle of drinking water that I have brought along. I place the LED nodes close to where the participants are sitting, making sure they are in a shaded enough spot for the LEDs to be visible. 


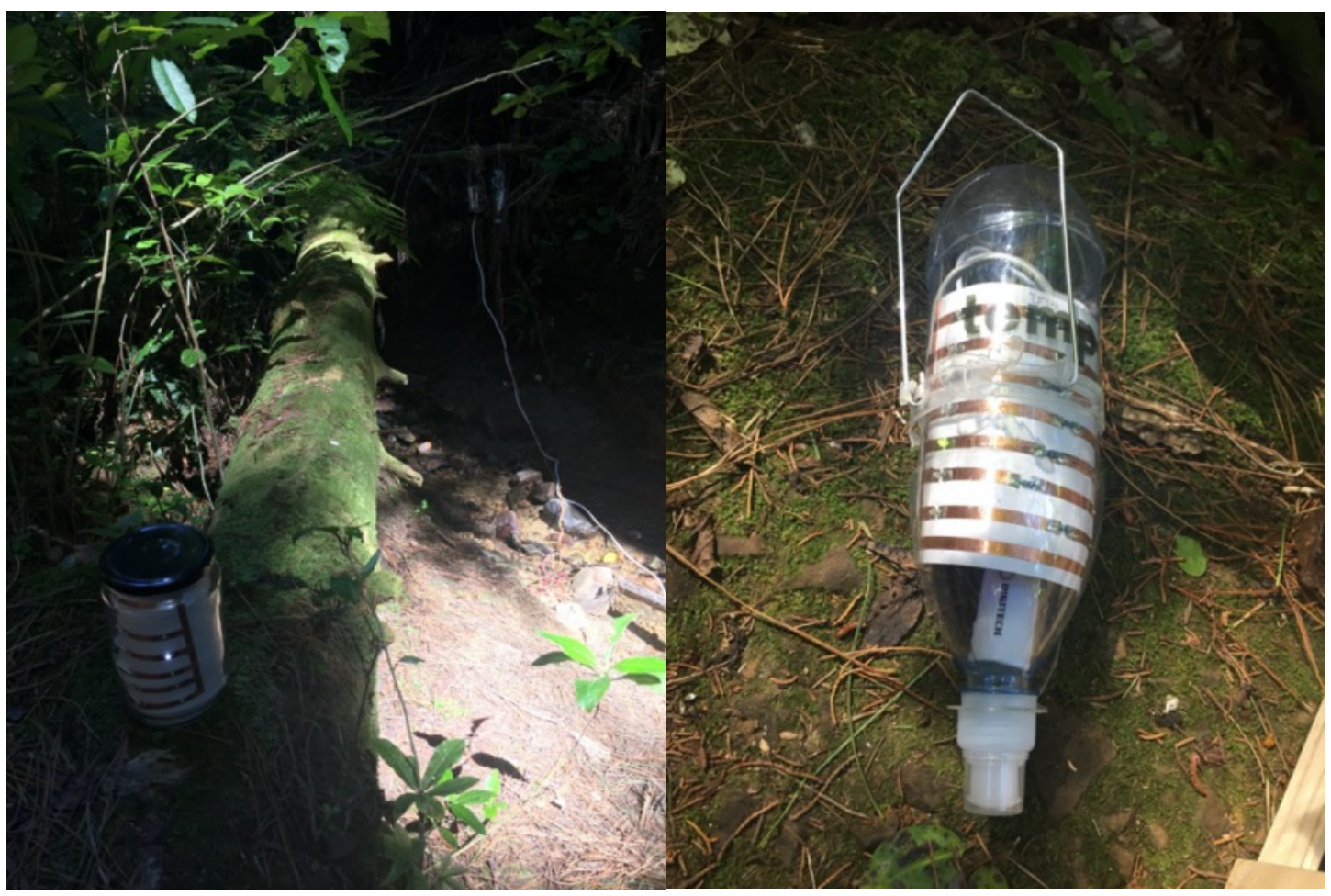

Figure 156. Author. (2019). Two versions of LED probes used for the test install.

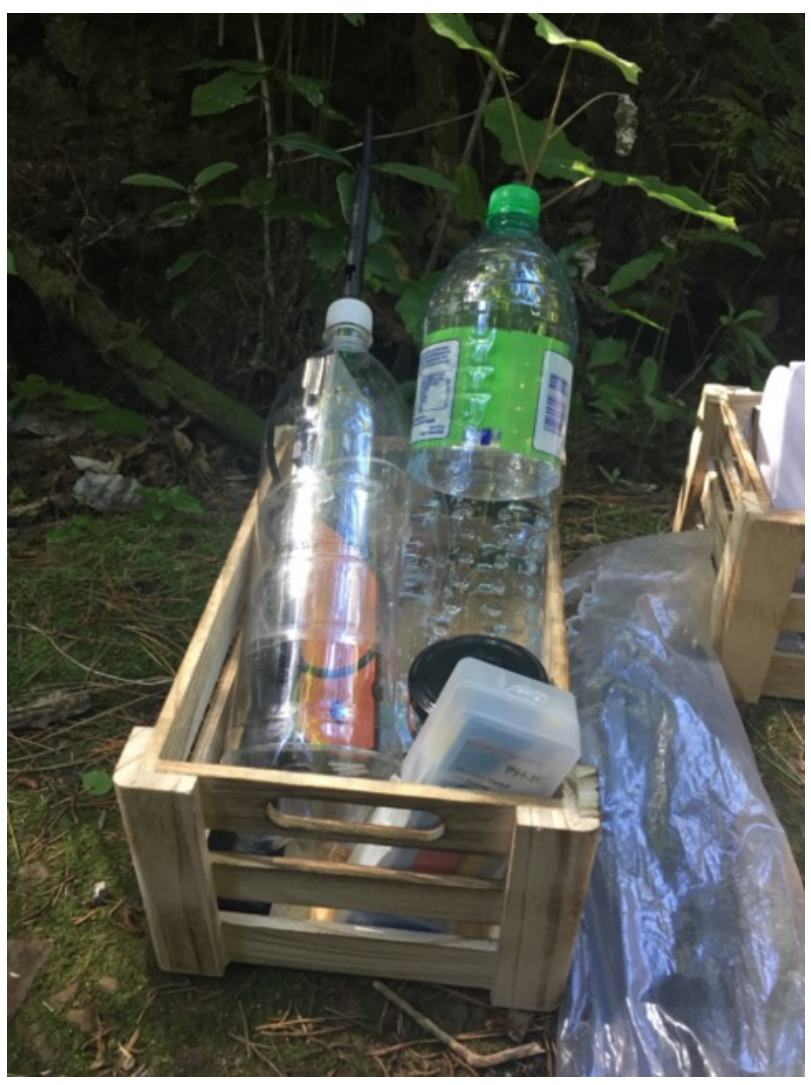

Figure 157. Author. (2019). The Wi-Fi hub bottle rests in the crate with a drinking water bottle. 


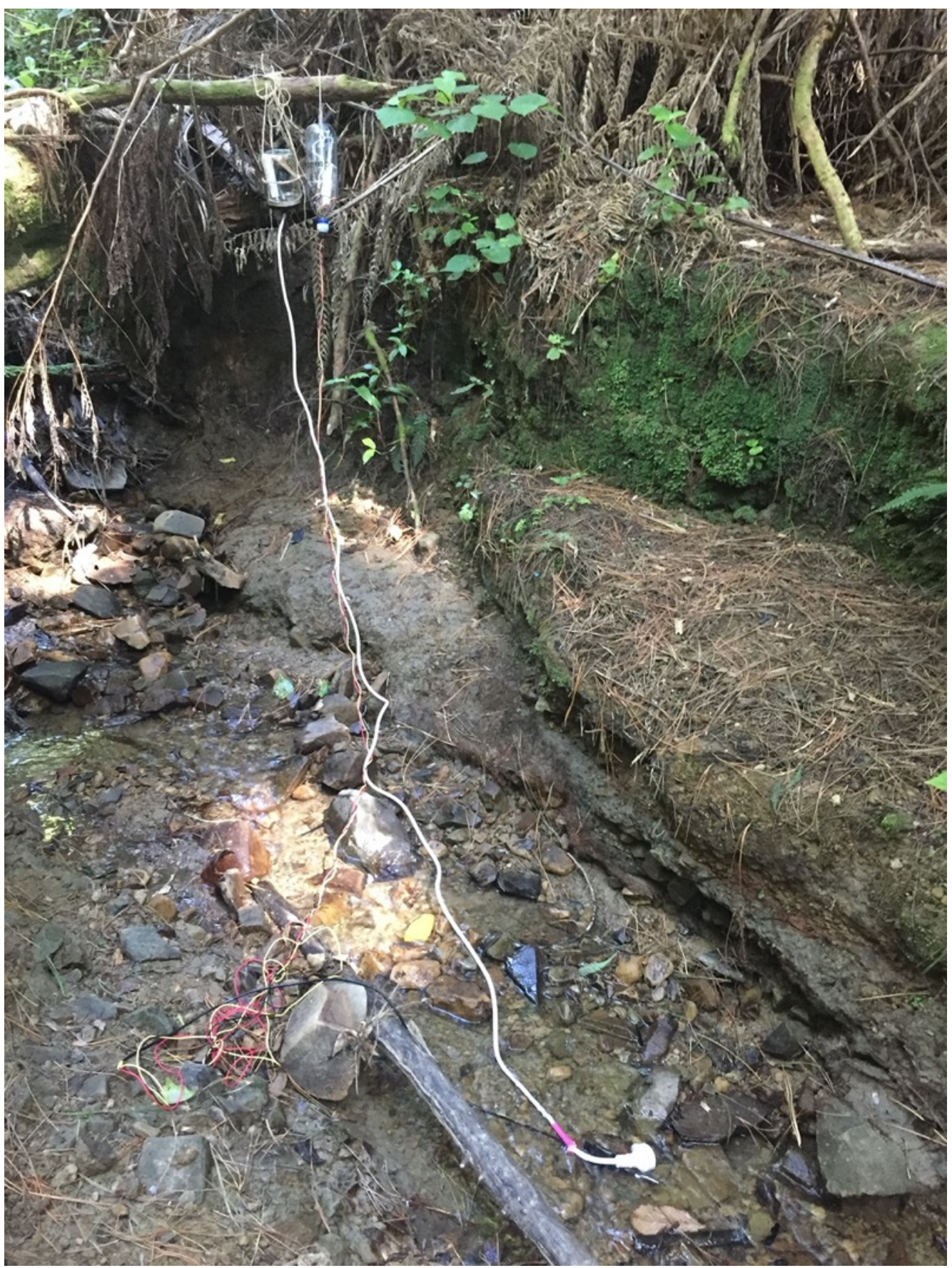

Figure 158. Author (2019). During the install the two sensor probes are suspended from a fallen over tree.

As the installation is set up, I sit down with the participants and we spend some time with the work. We discuss the politics of water and the challenges of doing work at the cross section of art and science. It is sunny and warm, some sunny patches reach the usually shaded stream section through the canopy layer today. We spend about one and a half hours with the work, pausing at times and taking in the more-than-human surroundings, the soft gurgling of the stream, the wind rustling the tall pine trees, bird song. As we pay 
attention to the site, we note the presence of data that the installed sensors cannot capture, such as smells, or how being with the stream makes us feel.

We look at the sensors again, and one participant notes how the nodes expose all this invisible data, making it accessible.

One of the participants discusses one of their own artworks relating to water and they reflect on an anecdote of a person they once observed, "standing in the tourist lookout spot, flying his drone over the lake, totally plugged into his phone”. Referring back to the experience with Papawai Transmissions, they comment

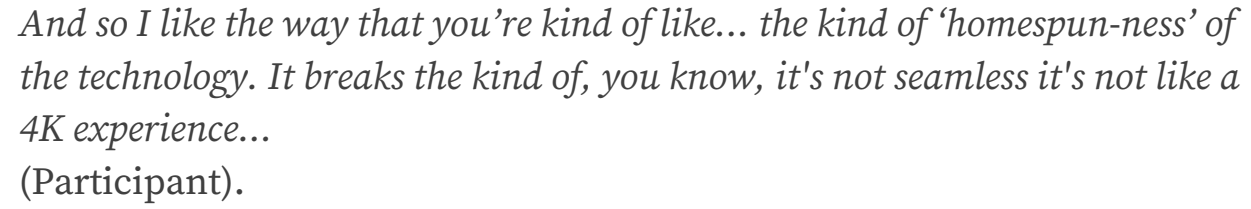

The issue with collecting data and simultaneously displaying it to a human audience to make sense of it in the field remains tricky. A participant mentions that in a previous piece they created, the data visualisation at the end appeared the least interesting part to an audience. We discuss how the installation presents an interface to the stream, and the issues with mediation and representation of the data. We discuss how "getting people to the water" was one of the key things for the making the stream visible, and with that some of the freshwater issues at hand. They note that Papawai Transmission is "a kind of field lab" that could facilitate some of these reconnections between people and water through "logging useful data that you can muck around with later. I like that".

Back in the lab I prepare the logged SCV data for being plotted as a graph with my Processing script sensordata_visualisation.pde. The graphs are inspired from conversations with participants during walks, who imagined the stream data could be visualised as if the stream was painting a picture. The script grabs the values from the table, finds the maximum and minimum value of each sensor reading and then prints each data point on a 1920x1080px canvas, relative to the highest and lowest value recorded. This means the graphs (Figure 159 \& Figure 160) are not directly comparable but paint a relative image of each installation. However, simple narratives can be inferred from the data presented here. 
The Sunday installation site was clearly warmer than the Monday one, but the stream temperature remains relatively stable around $14-15^{\circ} \mathrm{C}$, which is a good to excellent condition, according to the SHMAK manual (NIWA, 2019). The EC values appear relatively stable, but show some spikes in both graphs, which do not necessarily signal any issues with the stream in those particular examples. During the presentation of the work in the field, I explain the network to the participants and often remove the EC-sensor or the temperature sensor from the water to demonstrate how a spike would get visualised by the EC LED jar, or how the temperature visualisation LEDs would respond to a higher temperature, indicating poor stream conditions. 


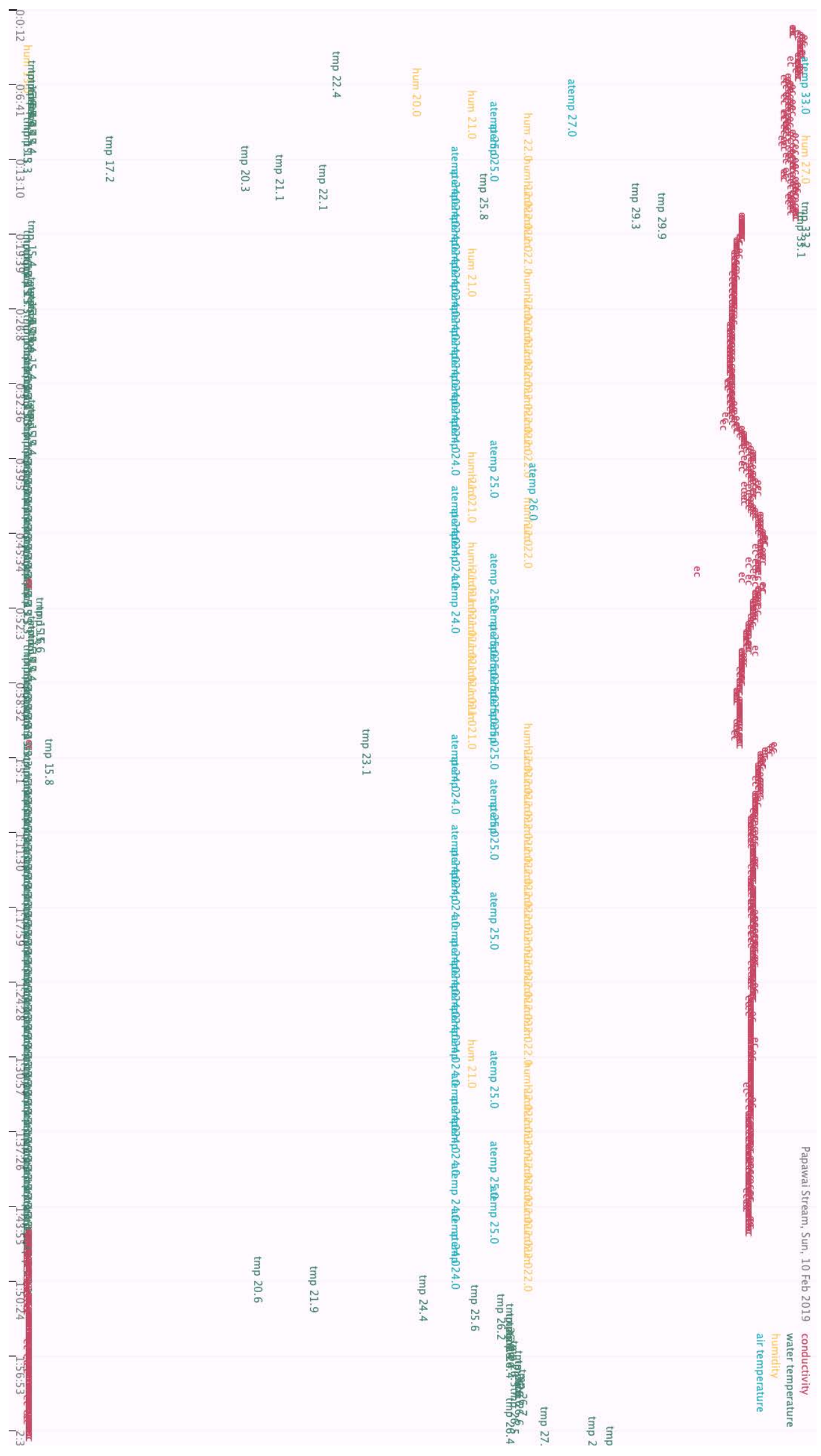

Figure 159. Author. (2019). Sunday install LOG01.CSV data plotted with sensordata_visualisation.pde. 


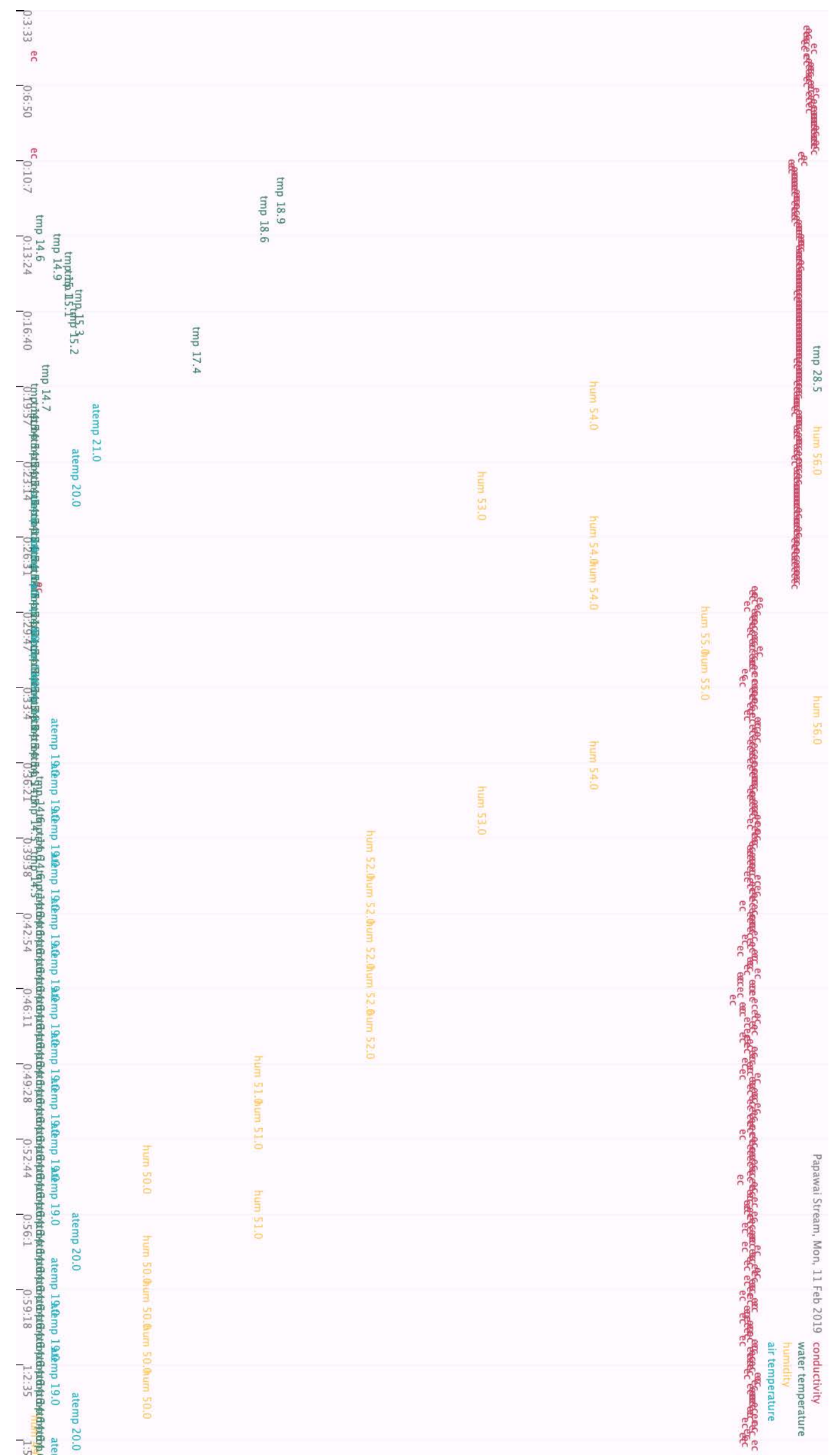

Figure 160. Author. (2019). Monday install LOG02.CSV data as plotted through sensordata_visualisation.pde. 
In summary, the public installaltions felt like a successful conclusion of the field labs, with participant feedback largely positive. While both artists and gardeners in the participant groups generally appreciated the lo-fi approach to the sensor design, and the exploratory aspect of understanding sensor data and the idea of being able to take measurements themselves, the idea of using the data for artistic explorations was more interesting to artists. Gardeners also regarded the install as something that could be more compact and deployed at a larger scale, with one larger device collecting all data at once, instead of having to consider the placement of each sensor separately in the field. This would be generally possible by just leaving sensors in the transport crate. However, this idea could be optimised in the hardware design, by removing the modular quality of the current design and using only one, more versatile microcontroller that handles all sensors, recordings and data visualisations. While this could possibly make the design more usable for a novice, it would not work as well for using the installation for educational purposes, where the modularity and redundancy of multiple microcontrollers helps to visualise the connections between components and their inner workings, and allows a slow, step-by-step install process to introduce each sensor node separately to familiarise participants with each component. 


\subsection{Field Lab Reflections}

The three selected narratives in the Field Lab chapter outline the research activities in which prototyping work and field work get inseparably linked together, while introducing methods of prototyping into the field, and contextualising experiences from previous fieldwork directly into the process of installing, testing, and evaluating my design work by the stream, with and without audiences present.

The narrative of Moturoa Transmissions (7.2) talks particularly about the outputs generated from the first prototyping sprint, while Papawai Transmissions - Field Evaluations (7.4) focusses on the final install of the prototypes in a public context. The testing of the ECsensors as part of the development has been chosen as an example that illustrates my documentation of fieldwork towards the end of the research process, involving audio recordings, field notes and photography, as well as sensor data recordings to tell the story of the work being done when testing IoT nodes in the field. The three narratives of these chapters are a result of fieldwork (Chapter 5) and prototyping activities (Chapter 6), but also in return, informed subsequent fieldwork and prototyping efforts.

This chapter attempts to weave together the narratives that emerged across field of hardware and software development for IoT design and working within the more-thanhuman world and highlighting how these two worlds came to meet as part of this research. In this reflective section I look closely at how slowness, seamfulness and openness informed the field lab, revealing how themes emerging from field explorations and prototyping activities intersect within this chapter.

\section{Openness}

Openness, as discussed in the previous two chapters, permeates this work through an open approach towards inviting the more-than-human world into the research process, the use of open-source technology to build prototypes and openly sharing both process and project outcomes with the local communities involved, as well as the online open-source community. 
The sharing on a larger scale with the development community materialised in the form of an online lab development log in the form of a WordPress page on lab.wildthings.io. This page would provide general information about the research and contain some of the narratives presented in this thesis. The site would also link to a SoundCloud page that contains two of the audio field recordings collected during site visits on Papawai Stream.

The sharing of work with the communities took place in the form of bringing recent work to field explorations and inviting participants to see the work installed in the field during the public exhibitions in March 2017 and February 2019. Openness as part of the research process during public exhibits involved paying attention to the many voices present, giving feedback on the work during installation. While participant observation for Moturoa Transmissions and open evaluation interviews during Papawai Transmissions allowed understanding of what human audiences thought about the installs, an open approach included not prioritising human feedback over the feedback of the more-than-human voices present. This included, for example, paying attention to how the stream reacted to the sensors, if they acted as a barrier to the flow, if they could be installed without impacting the surroundings, not harming any vegetation, using branches and fallen trees to suspend sensors from instead of introducing new structures to the environment. Looking out for any harm that the install could cause to the surroundings also included making sure the nodes are installed safely, and none of the electronics could fall into the water or batteries leak into the ground.

During the first iteration at Moturoa Streams, I carefully looked at where the wind or animals could move nodes around. Whenever I noticed a possibility of a node being interfered with, I secured it with a cable tie, which, being single-use and made from plastic was not a preferred material. However, this provided a secure install, and cable ties were reused where possible. This openness to what could be considered pragmatic solutions involved assessing on the spot what kind of approach is preferable. Where possible, I would use stakes and branches found on the ground to build a pole that would help me install sensors in hard-to-access places. Embracing openness during later installations at Papawai 
Stream let me confidently share prototypes that show signs of wear, have been reused, or re-assembled from previously used parts, and consider them a vital part of the project, as I further elaborate on in the following discussion of seamfulness.

\section{Seamfulness}

The seamful aspects of the project came into the foreground particularly during test installs and public exhibitions of work as part of the field lab activities.

When installing electronic prototypes in the field, seams of connectivity immediately revealed themselves. As the lab notebook entry (7.3) illustrates, the work with prototypes in the field yields many unexpected challenges, such as Wi-Fi networks from the lab lingering in the memory of the machine, instead of displaying the local IoT network for debugging. The absence of flat, dry surfaces and the readily available access to components, electricity, fast internet for troubleshooting, access to fresh water in case components or body parts get muddy and the presence of wind, heat and animals all reveal the complications experienced when travelling with IoT gear in the bush.

However, experiences from the field explorations, as well as from development as part of prototyping, informed the work of the field lab, finding ways to navigate the complexities when doing work with "worlds that were not supposed to meet" (see Chapter 5.5). This experience highlighted my own positionality as a researcher and maker, using my designs to tie together fields and acting as the seam myself, when contextualising the work for a human audience, considering the impact of the work on the more-than-human world, and understanding my own practice through recording my own actions in the field when trying to weave a network between the stream and my sensor devices. This, as discussed in the previous two chapters, links closely together with ideas around slowness, which I elaborate on further below. 


\section{Slowness}

The embracing of slowness as part of the field lab firstly involved slowing down the development process, after experiencing a busy development sprint for the first public exhibition of the work as Moturoa Transmissions. Installing a relatively complex work for a few consecutive days in a row helped push the overall development of the project further but exhausted me as a researcher and did not allow room for prioritising long-term solutions for designs when working towards an external deadline.

The slowly developed prototypes unfolded their qualities after the first install, and this time of reflection provided valuable insights for the further development of Papawai Transmissions. While field explorations revealed that the two streams, Papawai Stream and Moturoa Stream, are geographically close to each other, the narratives present, and the concerns for the more-than-human world were quite different. The communities involved with each of the streams faced different challenges in their caretaking and restoration work, and the streams had different more-than-human inhabitants and connected with different stories. The peculiarities of each stream only revealed themselves after spending time in the field and observing how my design work plugged into the different worlds. Moturoa Stream, for example, is a feature of a public park nestled inside a gully and the main arm is accessible via popular walking and commuter routes. In contrast, Papawai Stream is lesser known, and the connected small reserve is barely known outside its local neighbourhood. The full locality of all its tributaries and forks is not properly recorded and the community feels threatened by a big project for a large water reservoir.

Moturoa Transmissions was aimed to highlight the presence of the stream at a site that is largely disconnected from how the public perceives the stream, on the side of a busy road, above the spot where the pipe directs the stream into the Park. The install provided a good opportunity for audience feedback and learning more about network design in the field. However, spending more time with Papawai Transmissions slowly developed into a project engaging with the multiple complexities faced by Wellington freshwater streams, and understanding that participation and spending time with the work was as important as the 
work itself. While the sensor devices record data such as temperature, conductivity or turbidity, the narratives told by human participants during the install, while paying attention to the more-than-human world surrounding it, supports a slow approach, inviting participants to slow down together with the stream, tuning into its environment, and connecting with the more-than-human world through their own senses, as well as with the sensors of the networked artwork. 


\section{Chapter 8: Conclusions}

Starting from the research question of how artists and designers can learn from the morethan-human world when designing networks, this thesis has demonstrated how networked technology can aid in (re-)connecting with lost or forgotten urban waterways. Specifically, this body of work has looked at Internet of Things technology, and how the idea of connecting 'things' could provide useful avenues for designing IoT prototypes that plug into more-than-human environments, in order to understand narratives that are inaccessible to our human sensorium. The methodological contribution of this thesis has been built upon an analytical lens of slowness, seamfulness and openness as themes that guided the research throughout fieldwork, walks, field recordings, interviews, mapping exercises, network development, IoT prototyping, research blogging, exhibitions, and evaluation sessions.

Ultimately, this thesis has articulated how, through embracing methods of slowness seamfulness and openness, the project wildthings.io responded to the question of how we, as a design community, can learn from the more-than-human world when building networked media. Via the development of experimental prototypes for grassroots, community-run digital networks, and DIY electronic devices as artistic interventions, this research departed from the concept of an Internet of Things as a means to give voice to non-human 'things'. With a focus on wai/water, the design of the networked installations discussed in this thesis specifically engaged with local stream ecologies in Pōneke/Wellington that have largely disappeared from the cityscape as they have been piped underground through urban development. Data collected during fieldwork and labwork has informed the creation of electronic design artefacts to learn how the morethan-human world can inspire the development of networked media, and to imagine novel ways of (re-)connecting with disconnected waters and their more-than-human ecosystems. Doing so calls into focus the role design plays within a growing push for methods that can work with the distributed knowledges, experiences and values of a more-than-human world. 
In my thesis, my engagement with my research question was mapped across six key chapters. In the first, the overview of the conversations around the IoT from its early beginnings to the current day illustrated how the increasing availability of network technology, in combination with business opportunities, has served as the main catalyst for the development of IoT technology. Critics, however, have raised issues inherent in a world where any 'thing' could have access to the internet, indicating a loss of privacy, and issues of agency and control from the user perspective.

As part of the chronological overview of the IoT, in the second chapter, I introduced technological and theoretical milestones and developments from the 1990s until now. Here I also highlighted a number of art and design projects that critique the IoT as a marketdriven concept dominated by anthropocentric interests. The selected creative works explored in this second chapter present an alternative vision for an IoT, established through collaborative co-creation between human and non-humans illustrating how different types of creative experiments across academia, industry and the arts can manifest deep and complex links, as well as clear points of rupture and divergence in IoT research. These are conversations to which this research project aimed to further contribute by specifically seeking to understand how localised more-than-human worlds can be centred during an IoT design process.

By providing narratives from my research progress across field explorations, prototyping activities and field lab work, in the third chapter I discussed how the design work developed as part of the research project wildthings.io has sought to include and amplify voices in the process of developing prototypes that centre the more-than-human world, through embracing methods of openness, seamfulness, and slowness. Here, the project suggests further investigation into how slow development cycles of networked technology can open new avenues for understanding designing within and for the more-than-human world. This thesis showed how learning from the more-than-human world during fieldwork can inspire prototyping processes and creative outputs within an IoT network design. 
Slowness thus formed a crucial research theme for this project. Designing an Internet of Things for wai/water required patience and the acknowledgement that the decentring of the human within a human-led design process needs slow and careful attunement to the more-than-human world. Spending time with streams during exploratory fieldwork first, and later during the testing and installation of prototypes, provided valuable observations on how the work plugs into the existing networks around freshwater. Paying attention to the non-human animals, plants, and rocks that the freshwater stands in a symbiotic relationship with, noticing human interventions in these relationships, in the form of pipes and culverts, or discarded glass and plastic, and as well as taking the time to learn about the wairua/spirit of the place, extending beyond measurable data, provided rich insights to be considered for further iterations of this work. In particular, the research revealed how an IoT, despite initially designed to increase efficiency, could aid modes of slowing down and reconnecting, by revealing layers of our more-than-human world that have been underacknowledged, lost or forgotten.

Investing such time and attention in this environment was also a humbling experience, which taught me to acknowledge the richness and diversity of these worlds beyond what my work could conceivably engage. Understanding how fish commute through the pipes to reach the stream taught me to see the stormwater network as a suburban canvas full of more-than-human stories, that my small network was not able to connect. Further limitations of the work centred on the concern of bringing possibly harmful materials from the microcontrollers and Lithium-ion batteries into the stream environment, potentially adding more harm to an already troubled world.

This understanding of the enormity of the more-than-human world, and my own limitations as a researcher therein, also necessitated a critical self-awareness of my positionality within the context of Aotearoa/New Zealand. As part of the research into the streams and the narratives of land and water, I learned the rich knowledge and stories present in Te Ao Māori that I could not fully grasp as part of this journey. I consider this the most important area in which this research could be developed further in the future. Acknowledging that many concepts currently trending in academia around more-than- 
human thinking have deeper roots in indigenous knowledge is only a first small humble step in a long journey of pushing projects like this closer in the direction of what could be a decolonising design research paradigm. As Oliveira has noted,

\section{A decolonising project dwells on time and moves at a different pace. It rejects the impositions of neoliberal academia and the colonial framework of result-driven, well-defined, problem-solving design (Oliveira in Schultz et al., 2017, p.93).}

Embracing slowness here has taught me to acknowledge the limitations of my own practice, being a European-born and taught researcher in a country that still suffers from colonisation. Such awareness has foregrounded the vital understanding that learning with and about Te Ao Māori should stand central to the development of designers who work in Aotearoa/New Zealand.

However, despite often feeling out of place and underequipped to answer my research question, I want to acknowledge how the generous interactions I had with my research participants, where the rich experiences I collected during fieldwork have shaped me as a researcher. My human participants offered critical but encouraging feedback on my work during times when I had lost my confidence, and when I felt that none of my progress had led me in any way closer towards providing an answer to my research question. My most significant collaborators, however, were the streams. The streams patiently accompanied me when I anxiously tried to make a piece of technology work, whether to take photos, record audio or collect sensor data with my prototypes. It was the stream that taught me how to slow down, and be at ease, and take obstacles along the way as they come.

Integrating the multiple voices present in the more-than-human world of a relatively small stream environment requires attunement to and working with a kind of polyphony that requires paying attention to the various voices present. Emerging at different times and at different volumes, these voices require a careful ear, and the willingness to slow a design process down, in order to include the softest voices, ensuring all are heard. In the particular case of the suburban freshwater, the complexities of the more-than-human world being piped underground were revealed: with fish commuting through stormwater pipes, or the lack of native plantings resulting in freshwater life in general being dependent 
on the shade of invasive species for survival. This research provides a prototypical example of how the complex narratives of the more-than-human world, and its forced adaptations to the interventions of anthropocentrism, can be woven into the design of IoT networks. Future work may expand the inquiry towards including other localised more-than-human communities into the research and grounding the work more rigorously in concepts of Mātauranga Māori.

Alongside slowness, openness was also key to the development, realisation, and dissemination of my research. As part of the explorative work discussed above, openness extended into how I situated and negotiated my own participation within this research. While in the early phases of my research, I needed to be open to the diverse participants of the research, and allow myself to acknowledge the streams, and the more-than-human entanglements, as voices I need to be listening to. Methods of openness contributed new perspectives to my field explorations, through processes of walking with, conversing with and listening to and participating within the communities around the more-than-human worlds of freshwater streams.

From the outset of the research, I considered Participatory Design (PD) as an avenue to involve the more-than-human world into the research process as participants. This required unpacking of what participation means in a more-than-human context, and if and how traditional human-centred participatory design methods can provide new perspectives on designing with and for water and connected ecosystems. While human-centred methods are clearly not directly applicable in a more-than-human context through "add and stir" (cf. Muller, 2009), the sensibilities, especially the embracing of openness towards multiple voices as part of the design process, formed a valuable foundation to this research. This openness also extended into the documentation and dissemination of my research process itself. The published materials of my research, the blueprints for designs of prototypes and all code and documentation are openly available online, developing more educational resources that could help make the project accessible to users who are unfamiliar with coding and hardware prototyping. With my background in open-source development, I needed to carefully leverage my knowledge in FLOSS and DIY methodologies to create work 
that is radically open and accessible, so my research ultimately benefits the more-thanhuman communities it affects. This openness implied open licensing and publishing of hardware, software and my design processes, but was also embraced in the design process itself, through the concept of 'beautiful seams'.

Seamfulness, was also important in the conceptual triad which guided my research in this thesis. Embracing seamfulness as part of the prototyping process involved acknowledging the seams revealed in the field during exploratory work, translating those seams into features of the design work, and recognising seams inherent in the process of iterative development as productive outputs of research, tapping into multiple complex territories. Seamful design here involved drawing together theoretical concerns from more-thanhuman scholarship and technical concepts from IoT development, where multiple layers of technology, hardware and software, are literally patched together to create an IoT network.

The embracing of seamful design required slowing down and taking time to acknowledge rough edges as a feature of a design piece. The first seams as part of the field explorations emerged from my initial efforts to understand how Papawai Stream connected from the mountain to the sea. While this was a literal attempt of understanding the geography, I learned how in Māori philosophy, a ki uta ki tai (mountains to sea) approach encompasses a holistic way of caring for and managing water resources. The difficulty of finding the stream laid apparent how urbanisation has created a rupture within the pre-colonial landscape, removing a clear connection between the freshwater stream and its link to the harbour from the cityscape.

Seamfulness further revealed itself in the practice of the act of walking, documenting the walks and the attempt of piecing various forms of data and media collected together for the write-up. The process of mapmaking, (outlined in 5.3), aided the unearthing of the subterranean canvas of Pōneke/Wellington's forgotten freshwater. The seams between the piped and unpiped freshwater served as places to plug in my design work (as discussed in Chapter 5). Seamfulness is hence reflected in the field explorations in discovering apparent seams present in the field, including piped/unpiped, water/waterproof, and the research 
process, such as generating multiple layers of data and media that underpin the design process as described in Chapters 6 and 7.

The contributions of the thesis can thus be seen in this methodological approach which sought experimental, locally-responsive ways to give voice to more-than-human 'things' within the theoretical paradigm of an Internet of Things. The search for methods involving the decentring of the human helped establish a theoretical grounding for design research that aims to navigate this complex territory, with the aim to introduce new perspectives to the development of an Internet of Things. My research, as situated in Aotearoa/New Zealand, presented further opportunities for engaging in a methodological approach which responds to this call for a diversity of perspectives in design research. As a European-born researcher, having lived in New Zealand for seven years, I need to learn about local, situated knowledges (Haraway, 1988) and perspectives. My context of Aotearoa/New Zealand was very beneficial to such research where the longstanding culture and Māori worldview offers a deep, intricate understanding of networks of 'things'.

From a designer's perspective, new, more malleable and open frameworks for approaching research problems are emerging, among them post-qualitative research (Lather and St. Pierre, 2013) and non-representational approaches (Vannini, 2015). However, given that they are still in their infancies, these new, cross-cultural traditions face many challenges when trying to weave diverse attributes and non-text-focused work into Western academic publishing structures, where they might be described as "messiness" (Law, 2004), or "slowness" (Ulmer, 2017). These factors have become core to my research paradigm where, as I have outlined throughout this thesis, slowness, seamfulness, and openness have been integral to the development of a networked artwork as part of wildthings.io.

Here, I want to briefly highlight the interconnectedness between research and design practice that stood central to this work, by emphasising the importance of being both a reflective practitioner and a reflexive researcher, arguing that reflective design practice also needs to be reflexive. The reflections on my prototyping processes and my design work have changed me as a researcher, allowing me to pay more careful attention to how I do 
research, which continuously shaped and re-shaped the way I conducted my research along the way. Hence, a reflective and reflexive practice tied together emerge as a central part of the iterative process that underpinned my development journey, dependent on and informed by each other.

Ultimately, this research offers a humble exploration of a research trajectory where morethan-human needs are being met with technological developments. While participants from multiple fields and backgrounds generously supported the project, further development will rely on the participation of experts and novices from all worlds, including the open-source community, the stream restoration community, freshwater ecologists, urban engineers, artists, developers, writers, designers, and most importantly people with knowledge of and mana in Te Ao Māori and Mātauranga Māori. I am reminded here again of Linda Tuhiwai Smith's call for research in colonial contexts to be self-aware, self-critical, and attentive to the ongoing lived realities of colonial violence. I thus draw again from the following statement, as a reminder of what is at stake for both myself and future researchers working in this context:

From the vantage point of the colonised, a position from which I write, and choose to privilege, the term 'research' is inextricably linked to European imperialism and colonialism. The word itself 'research', is probably one of the dirtiest words in the indigenous world's vocabulary (Smith, 2012, p.1). 
To conclude, I want to position the value of this thesis as a research artefact itself. As it is presented here, in combination with image and media material, this document is a metatestament to not only how the three themes of slowness, seamfulness and openness informed the research process, but how they materialised in the writing and presentation of this thesis. I set out with the goal of openness, honesty, and transparency in documenting my process; the seams, tensions, and theoretical and ecological sutures that I encountered, and the often frustrating, yet gradual embracing of slowness. This document is not only the story of the creation of electronic design artefacts to learn how the morethan-human world can inspire the development of networked media, but a testament to my own critical reckonings and learnings of myself as a researcher over these years. This research then demonstrates how not only the execution, but also the documentation of design practice, can work to be open and seamful, and sometimes slow. 


\section{References}

Abram, D. (1996). The spell of the sensuous: Perception and language in a more-than-human world (1st ed.). Pantheon Books.

Adafruit. (n.d.). Adafruit Learning System. Internet of Things - IOT.

https://learn.adafruit.com/category/internet-of-things-iot.

Adams, T., Ferguson, G., \& Tobolski, J. F. (2001). An introduction to silent commerce: Creating new sources of value from intelligent objects. Outlook: Accenture's Journal of High-Performance Business.

https://web.archive.org/web/20120108122435/http://www.accenture.com/SiteCollectionDo cuments/PDF/silentcommerceintropovusletter.pdf.

Akama, Y., Light, A., \& Kamihira, T. (2020). Expanding Participation to Design with More-Than-Human Concerns. Proceedings of the 16th Participatory Design Conference 2020 - Participation(s) Otherwise, 1, 1-11. https://doi.org/10.1145/3385010.3385016

Albrecht, K. (2002). Supermarket cards: The tip of the retail surveillance iceberg. Denver University Law Review, 79(4), 534-539 \& 558-565.

Amazon Web Services, Inc. (n.d.-b). AWS IoT and Alexa..

https://aws.amazon.com/iot/solutions/connected-home/iot-and-alexa/.

Amazon Web Services, Inc. (n.d.). AWS IoT. http://aws.amazon.com/iot/.

Apple, Inc. (2014, September 9). Apple announces iOS8. Available September 17 [Press Release]. https://www.apple.com/newsroom/2014/09/09Apple-Announces-iOS-8-AvailableSeptember-17/.

Arduino. (2015). Casa jasmina. http://casajasmina.arduino.cc/about/.

Arnall, T. (2013). Making visible: mediating the material of emerging technology [Doctoral thesis]. Oslo Arkitektur- og designh øgskolen.

Arnall, T. (2014). Immaterials: Satellite lamps / Touch. Nearfield. http://www.nearfield.org/2014/08/satellite-lamps

Ashton, K. (2009). That "internet of things" thing. RFiD Journal, 22(7), 97-114.

Bachler, B. (2016, October 11). [Facebook post]. Wellington Sonic Arts Forum. https://www.facebook.com/groups/571931359535288/permalink/1185181034876981/

Bachler, B. (2019). wildthings. http://www.wildthings.io/

Bannon, L. J., \& Ehn, P. (2012). Design: Design matters in participatory design. In J. Simonsen \& T. Robertson (Eds.), Routledge International Handbook of Participatory Design (pp.37-63). Routledge.

Barad, K. (2007). Meeting the Universe Halfway: Quantum Physics and the Entanglement of Matter and Meaning. Duke University Press.

Barrett, E., \& Bolt, B. (2013). Carnal Knowledge: Towards a "New Materialism" Through the Arts. I.B.Tauris; Bloomsbury.

Barrett, E., \& Bolt, B. (2014). Material Inventions: Applying Creative Arts Research. I.B.Tauris; Bloomsbury.

Bastian, M. (2009). Inventing Nature: Re-writing Time and Agency in a More-than-Human World. Australian Humanities Review, 47, 99-116. 
Bastian, M. (2016). Participatory Research in More-than-Human Worlds (1st ed.). Routledge. https://doi.org/10.4324/9781315661698

Bennett, J. (2010). Vibrant matter: A political ecology of things. Duke University Press.

Berners-Lee, T. (1989). The original proposal of the WWW, HTMLized. https://www.w3.org/History/1989/proposal.html

Blavatsky, H. P. ([1888]1997). The secret doctrine. Theosophical Pub. Society. http://pyramidalfoundational-information.com/file_download/108/SDVolume_I.pdf.

Bleecker, J. (2006). A manifesto for networked objects - cohabiting with pigeons, arphids and aibos in the internet of things [PDF document]. Near Future Laboratory. http://nearfuturelaboratory.com/files/WhyThingsMatter.pdf

Blomberg, J., \& Karasti, H. (2012). Positioning Ethnography within Participatory Design. In Routledge International Handbook of Participatory Design (pp. 86-116). Routledge. https://doi.org/10.4324/9780203108543

Boulton, J. (2014). The Trojan Room Coffee Pot [image]. Digital Archaeology. http://digitalarchaeology.org/wp-content/uploads/2014/02/Trojan_black_3.png

Bowker, G. C., \& Star, S. L. (1999). Sorting things out: Classification and its consequences. MIT Press.

Boyd, M. (2015). Multispecies becomings in the Anthropocene. Antennae, 31(Spring 2015), 5-8.

Braidotti, R. (2013). The posthuman. Polity Press.

Brain, T. (2015, October). Eccentric Wi-Fi: Networks with others - Tega Brain. Presented at the Radical Networks, MAGNET NYU Poly, Brooklyn, NY.

http://ivestream.com/internetsociety/radicalnetworks/videos/102809342

Brewster, T. (2014, March 20). There are real and present dangers around the internet of things. The Guardian. http://www.theguardian.com/technology/2014/mar/20/internet-of-thingssecurity-dangers.

Broll, G., \& Benford, S. (2005). Seamful Design for Location-Based Mobile Games. In F. Kishino, Y. Kitamura, H. Kato, \& N. Nagata (Eds.), Entertainment Computing - ICEC 2005 (pp. 155-166). Springer.

Burnett, M. (2013). Mapping Wellington's Unpiped Streams [PDF document] Wellington: Victoria University of Wellington. https://wellington.govt.nz/ /media/about-wellington/research-andevaluation/natural-environment/2013-mapping-unpiped-streams.pdf.

Butcher, M. (2011, July 21). LogMeln acquires "Internet of Things" Startup Pachube for \$15m In Cash. TechCrunch. https://techcrunch.com/2011/07/20/logmein-acquires-internet-of-thingsstartup-pachube-for-15m-in-cash/

Canonico, T. (2007, November 5). Constraint city, body as a living map. Neural. http://neural.it/2007/11/constraint-city-body-as-a-living-map/.

CASPIAN. (2003, November 14). Position statement on the use of RFID on consumer products. https://w2.eff.org/Privacy/Surveillance/RFID/RFID_Position_Statement.pdf.

Castree, N. \& Nash, C. (2004). Introduction: posthumanism in question. Environment and Planning A, 36(8), 1341-1343. http://doi.org/10.1068/a37127

Chalmers, M, \& MacColl, I. (2003). Seamful and Seamless Design in Ubiquitous Computing. Workshop at the crossroads: The interaction of HCl and systems issues in UbiComp (Vol. 8). 
Chalmers, M. (2003). Seamful design and ubicomp infrastructure. In Proceedings of Ubicomp 2003 Workshop at the Crossroads: The Interaction of $\mathrm{HCl}$ and Systems Issues in Ubicomp. http://citeseerx.ist.psu.edu/viewdoc/download?doi=10.1.1.61.6779\&rep=rep1\&type=pdf

Chalmers, M., Dieberger, A., Höök, K. \& Rudström, Å. (2004). Social navigation and seamful design. Cognitive Studies: Bulletin of the Japanese Cognitive Science Society, 11(3), 171-181.

Christen, K., Merrill, A., \& Wynne, M. (2017). A Community of Relations: Mukurtu Hubs and Spokes. DLib Magazine, 23(5/6). https://doi.org/10.1045/may2017-christen.

Ciortea, A., Boissier, O., Zimmermann, A., \& Florea, A. M. (2014). Open and Interoperable Sociotechnical Networks. 16th International Symposium on Symbolic and Numeric Algorithms for Scientific Computing (SYNASC), 251-257. Los Alamitos, CA: IEEE. http://doi.org/10.1109/SYNASC.2014.41

Clothier, I., Hornblow, A., Priest, J., Soundy, A., Dwyer, M., Visser, A., Wareing, P. \& Ariki, P. (2011). The Park Speaks. Ian Clothier. http://ianclothier.com/theparkspeaks/.

Coole, D. H., \& Frost, S. (2010). Introducing the new materialism. In D. H. Coole \& S. Frost (Eds.), New materialisms: Ontology, agency, and politics (pp.1-43). Duke University Press.

Coulton, P., \& Lindley, J. G. (2019). More-Than Human Centred Design: Considering Other Things. The Design Journal, 22(4), 463-481. https://doi.org/10.1080/14606925.2019.1614320

Countdown NZ Ltd. (2009). Pure Dew Water Ultra Distilled. https://shop.countdown.co.nz/shop/productdetails?stockcode=263889

Crabtree, A., \& Rodden, T. (2008). Hybrid ecologies: Understanding cooperative interaction in emerging physical-digital environments. Personal and Ubiquitous Computing, 12(7), 481-493. https://doi.org/10.1007/s00779-007-0142-7

Crutzen, P.J., \& Stoermer, E. F. (2000). The "Anthropocene." Global Change Newsletter, (41), 17-18.

Da Costa, B. (2006, July). PigeonBlog. Map Your City. https://web.archive.org/web/20120715133001/http://www.pigeonblog.mapyourcity.net/stat ement.php.

Dawson, S. (2012, January 20). Building the "CETOS" directional hydrophone. Whale and Dolphin Trust. http://whaledolphintrust.org.nz/wp-content/uploads/Building- Directional-HPs.pdf.

De Lange, M. (2013). The Smart City You Love to Hate: Exploring the Role of Affect in Hybrid Urbanism. Subtle Revolutions Proceedings of the 2nd International Hybric City Conference, Eds. D. Chartitos, I. Theona, D. Dragona, H. Rizopoulos (Athens: URIAC, 2013), 77-84.

De Ruyter, B. (2003). 365 Days' Ambient Intelligence Research in HomeLab. Philips Research. Neroc Publishers..

Decker, F. C. (2013, June). Underwater sound detection using sea perch through construction and operation of hydrophone. Sea Perch. https://seaperch.byu.edu/wpcontent/uploads/2013/06/Final.pdf.

DeLanda, M. (2006). A New Philosophy of Society: Assemblage Theory and Social Complexity (annotated edition). Bloomsbury.

Deleuze, G. (1992). Postscript on the Societies of Control. October, 59, 3-7. http://www.jstor.org/stable/778828

Dennis, D. (2006). Policing the Convergence of Virtual and Material Worlds. CTheory. www.ctheory.net/articles. aspx?id=567 
Dern, D. P.(1992). INTEROP 92 spring proves a capital idea. ConneXions-The Interoperability Report, 6(7), 15-18.

Deschamps-Sosino, A. (2015, Sep 07). A critical look at Gartner's Internet of things. Huffington Post. http://www.huffingtonpost.co.uk/alexandra-deschampssonsino/internet-ofthings_b_8094266.html.

digifishmusic. (2008, January). How to make a Hydrophone (stereo!). freesound. http://www.freesound.org/forum/production-techniques-music-gear- tips-andtricks/2631/?page=1\#post13253.

DiSalvo, C. \& Lukens, J. (2011). Non-anthropocentrism and the non-human in design: Possibilities for designing new forms of engagement with and through technology. In M. Foth, L. Forlano, C. Satchell and M. Gibbs (Eds.), From social butterfly to engaged citizen: urban informatics, social media, ubiquitous computing, and mobile technology to support citizen engagement (pp. 421437). MIT Press,.

Dodson, S. (2003, Oct 09). The internet of things. The Guardian. http://www.theguardian.com/technology/2003/oct/09/shopping.newmedia

Dolphijn, R., \& Tuin, I. van der. (2012). New materialism: Interviews \& cartographies (1. ed.). Open Humanities Press.

donblair. (2015, August 25). Turbidity 001. Public Lab. publiclab.org/n/12168.

Dougherty, D. (2012). The maker movement. innovations, 7(3), 11-14.

Douglas, E. M. K., New Zealand \& University of Waikato. (1984). Waiora, waimaori, waikino, waimate, waitai: Maori perceptions of water and the environment : proceedings of a seminar. Hamilton, N.Z: The Centre.

Durie, M. (2004, March). Exploring the interface between science and indigenous knowledge. Capturing Value from Science: 5th APEC Research and Development Leaders Forum, Christchurch, NZ.

Eames, A. (2017, January 26). Wireless Remote Sensing with WeMos D1 mini, Arduino IDE, Raspberry Pi and lighttpd web server. Raperry Pi. http://raspi.tv/2017/wireless-remote-sensing-withWeMos-d1-mini-arduino-ide-raspberry-pi-and-lighttpd-web-server.

Easterling, K. (2012). An Internet of things. E-flux journal \#31. http://www.e-flux.com/journal/aninternet-of-things/

Easterly, D., \& Kenyon, M. (n.d.). SWAMP» SPORE 1.1. Swamp. http://www.swamp.nu/projects/spore-1-1/

Eclipse. (n.d.). Paho Python Client. Eclipse. https://eclipse.org/paho/clients/python/

Edson, B. (2015). Creating the Internet of Your Things [PDF document]. Microsoft Corporation. http://download.microsoft.com/download/C/F/7/CF78575B-711E-4E1B-8BAB3ED1657DFA82/Creating_the_Internet_of_Your_Things.pdf

Emoncms/emoncms. ([2012]2020). Emoncms [PHP, git repository]. https://github.com/emoncms/emoncms.

Environmental Health Clinic. (2009). Amphibious Architecture [PDF document]. http://www.environmentalhealthclinic.net/amphibiousarchitecture/wpcontent/uploads/2009/10/aa_pressrelease_4p.pdf.

Ericsson Media Relations Team. (2010, April 13). CEO to shareholders: 50 billion connections 2020 [Press Release]. https://www.ericsson.com/en/press-releases/2010/4/ceo-to-shareholders50-billion-connections-2020. 
Evans, D. (2011, April). The Internet of Things. How the Next Evolution of the Internet Is Changing Everything [White paper]. CISCO.

https://www.cisco.com/c/dam/en_us/about/ac79/docs/innov/loT_IBSG_0411FINAL.pdf

Everhart, C. (1990). Interesting uses of networking. UCSD.

http://cseweb.ucsd.edu/ bsy/coke.history.txt

Facebook. (n.d.). Portal. Smart video calling with Alexa Built-in. Portal from Facebook. https://portal.facebook.com/nz/.

Faludi, R. (2013, April 25). Press. Botanicalls. http://www.botanicalls.com/category/press/

Ferguson, G. T. (2002). Have your objects call my objects. Harvard Business Review. https://hbr.org/2002/06/have-your-objects-call-my-objects.

Fisher, K., \& Parsons, M. (2020). River Co-governance and Co-management in Aotearoa New Zealand: Enabling Indigenous Ways of Knowing and Being. Transnational Environmental Law, 9(3), 455480.

Floridi, L. (2009). Web 2.0 vs. the Semantic Web: A Philosophical Assessment. Episteme, 6(1), 25-37. http://doi.org/10.3366/E174236000800052X.

Fukuyama, F. (2002). Our posthuman future: consequences of the biotechnology revolution. Picador.

Fuller, M., \& Haque, U. (2008). Urban Versioning System 1.0. The Architectural League.

Fure, A. (2016). The Force of Things. Ashley Fure. http://www.ashleyfure.net/ice/.

Gabrys, J. (2016). Re-thingifying the Internet of things. In N. Starosielski \& J. Walker (Eds.), Sustainable Media: Critical Approaches to Media and Environment. Routledge.

Galloway, A. (2006, November 9). Of seams and scars: Tracing technological boundaries and points of attachement. Fleshing Out Seminar, Rotterdam.

http://citeseerx.ist.psu.edu/viewdoc/download;jsessionid=7CA6EA7EBC6F7A7FFE0F2038DB1 A6421?doi=10.1.1.98.7538\&rep=rep1\&type=pdf.

Galloway, A. (2007). Seams and Scars, Or How to Locate Accountability in Collaborative Work. In C. Brickwood, B. Ferran, D. Garcia, \& T. Putnam (Eds.), (Un)common Ground: Creative Encounters across Sectors and Disciplines (pp. 152-159). BIS Publishers.

Ganguly, K. (2004). Temporality and postcolonial critique. In N. Lazarus (Ed.), The Cambridge Companion to Postcolonial Literary Studies (Cambridge Companions to Literature, pp. 162180). Cambridge University Press. doi:10.1017/CCOL0521826942.009

Gasparotto, S. (2019). Open Source, Collaboration, and Access: A Critical Analysis of "Openness" in the Design Field. Design Issues, 35(2), 17-27. https://doi.org/10.1162/desi_a_00532.

Gershenfeld, N. A. (1999). When things start to think (1st ed.). Henry Holt.

Gershenfeld, N., \& Vasseur, J. (2004). As objects go online [PDF document]. MIT. http://www.cba.mit.edu/docs/papers/14.02.FA.pdf.

Gilmore, N. \& Mellish, L. (2004). Cultural Impact Report: Waitangi Park. Wellington Tenths Trust. A report prepared for the Wellington City Council Built and Natural Environment Committee.

Goldberg, K. \& Santarromana, J. (2008). Telegarden Description. Youtube. https://www.youtube.com/watch?v=gbyy5vSg8w8

Google Cloud (n.d.). Google Cloud loT - Fully managed loT services. https://cloud.google.com/solutions/iot/ 
Greenberg, A. (2015, July 21). Hackers remotely kill a jeep on the highway-With me in it. Wired. http://www.wired.com/2015/07/hackers-remotely-kill-jeep-highway/.

Greenfield, A. (2006). Everyware: the dawning age of ubiquitous computing. New Riders.

Greenhough, B. (2014). More-than-human Geographies. In R. Lee, N. Castree, R. Kitchin, V. Lawson, A. Paasi, C. Philo, ... C. W. J. Withers (Eds.), The SAGE handbook of human geography (Vol. 1) (pp. 94-119). SAGE Publications.

Grokhotkov, I., \& Molinari, M. (n.d.). ESP8266 Community Forum. [GitHub Repository]. Retrieved December 9, 2020, from https://github.com/esp8266

Grusin, R. (2015). Introduction. In R. Grusin (Ed.), The nonhuman turn (pp.vii-xxx). University of Minnesota Press. http://www.jstor.org/stable/10.5749/j.ctt13x1mj0.3

Haapoja, T. (2008). Dialogue. Terike Haapoja. http://www.terikehaapoja.net/dialogue/

Hallnäs, L., \& Redström, J. (2001). Slow Technology - Designing for Reflection. Personal and Ubiquitous Computing, 5(3), 201-212. https://doi.org/10.1007/PL00000019

Haque, U. (2007, February 25). Sky Ear. Haque. http://www.haque.co.uk/skyear/information.html.

Haque, U. (2009a). Natural Fuse Research Book [PDF]. https://dl.dropboxusercontent.com/u/253194/NFresearchbook.pdf

Haque, U. (2009b). home / map. Natural Fuse. http://www. naturalfuse.org/index.html.

Haque, U. (2011). Pachube screenshot. Haque. http://www.haque.co.uk/pachube.php.

Haque, U. (n.d.). Design + research. Haque. http://www.haque.co.uk/pachube.php.

Haraway, D. (1988). Situated Knowledges: The Science Question in Feminism and the Privilege of Partial Perspective. Feminist Studies, 14(3), 575. https://doi.org/10.2307/3178066.

Haraway, D. (2015). Anthropocene, capitalocene, plantationocene, chthulucene: Making kin. Environmental Humanities, 6(2015), 159-165.

Harman, G. (2018). Object-Oriented Ontology: A New Theory of Everything (Illustrated edition). Pelican.

Harrison, B. L., Fishkin, K. P., Gujar A., Portnov D. \& Want, R. (1999). Bridging physical and virtual worlds with tagged documents, objects and locations. CHI '99 Extended Abstracts on Human Factors in Computing Systems (CHI EA '99), 29-30. ACM. http://dx.doi.org/10.1145/632716.632738

Hayles, N. K. (1999). How we became posthuman. Virtual bodies in cybernetics, literature and informatics. University of Chicago Press.

Hewlett Packard Enterprise. (2015, November). Internet of things research study. Hewlett Packard Enterprise.

http://h20195.www2.hp.com/V2/GetDocument.aspx?docname=4AA5$4759 E N W \&$ cc $=$ us \& $\mid c=e n$

High-Low Tech Group, MIT Media Lab. (2012, August 21). Paper circuits. http://highlowtech.org/?p=2505.

Hirst, R. (2011, December 14). Mystery Project update 2 "The Time Vampire." Runaway Brainz. https://runawaybrainz.blogspot.com/2011/12/mystery-project-update-3-time-vampire.html 
iMal. (2008). CONTENT. RFID workshop. New Brave World.

https://web.archive.org/web/20120519093117/http://www.imal.org/NewBraveWorld/NBW1 /?page_id=4.

Inman, S., \& Ribes, D. (2019). "Beautiful Seams": Strategic Revelations and Concealments. Proceedings of the 2019 CHI Conference on Human Factors in Computing Systems - CHI '19, 1-14. https://doi.org/10.1145/3290605.3300508.

Intel Corporation. (n.d.). A Guide to the Internet of Things Infographic. Intel. https://www.intel.com/content/www/us/en/internet-of-things/infographics/guide-toiot.html

Internet of Shit [@internetofshit] (n.d.).Twitter. https://twitter.com/internetofshit

Internet of Shit [@internetofshit] (October 8, 2019).when i started this account five years ago, i worried $i$ would run out of material too quickly, but there really is an unlimited supply of shit [Twitter post]. Twitter. https://twitter.com/internetofshit/status/1181204553200472064.

ioBridge. ([2010] 2020). lobridge/thingspeak [Ruby, github repository]. https://github.com/iobridge/thingspeak.

irgbit. (2017, July 18). Wellington-stream-addresses.csv. [csv file]. https://github.com/iRGBit/wildthings.io/blob/master/GISdata/wellington-streamaddresses.csv

Isaac, M. \& Hardy, Q. (2016, January 28). Facebook to shut down Parse, its platform for mobile developers. https://bits.blogs.nytimes.com/2016/01/28/facebook-to-shut-down-parse-itsplatform-for-mobile-developers/

ItKindaWorks. (2016). ESP8266_SimpleMQTT.ino [Arduino, github repository]. https://github.com/ItKindaWorks/ESP8266/blob/master/Home\%20Automation/Part\%201/ES P8266_SimpleMQTT/ESP8266_SimpleMQTT.ino

Jenkins, T. (2015). Designing the "Things" of the IoT. Proceedings of the Ninth International Conference on Tangible, Embedded, and Embodied Interaction - TEI '14, 449-452. https://doi.org/10.1145/2677199.2691608

Jenkins, T. (2018). Cohousing IOT: Designing edge cases in the Internet of things [Doctoral Dissertation, Georgia Institute of Technology]. https://smartech.gatech.edu/bitstream/handle/1853/60758/JENKINS-DISSERTATION2018.pdf

[Joe_Q]. (2016, July 07). Running Mosquitto (MQTT broker) at startup on RPi 2 [Online forum post]. https://www.reddit.com/r/raspberry_pi/comments/4ria15/running_mosquitto_mqtt_broker _at_startup_on_rpi_2/

Jones, A. (2012). Dangerous liaisons: Pakeha, kaupapa Maori, and educational research. New Zealand Journal of Educational Studies, 47(2), 100-112.

Jones, P., \& Evans, J. (2012). The spatial transcript: Analysing mobilities through qualitative GIS. Area, 44(1), 92-99.

Joy, M. (2015). Polluted Inheritance: New Zealand's Freshwater Crisis. Bridget Williams Books.

Kelley, C., Krolick, A., Brunner, L., Buklund, A., Kahn, D., Ball, W., \& Weber-Shirk, M. (2014). An Affordable Open-Source Turbidimeter. Sensors, 14(4), 7142-7155. https://doi.org/10.3390/s140407142

Kellmereit, D., \& Obodovski, D. (2013). The Silent Intelligence: The Internet of Things (1st edition). DND Ventures LLC. 
Kensing, F., \& Greenbaum, J. (2012). Heritage: Having a say. In J. Simonsen \& T. Robertson (Eds.), Heritage (pp. 21-36). Routledge Handbooks Online.

https://doi.org/10.4324/9780203108543.ch2

Khoo, B. (2010). RFID- from Tracking to the Internet of Things: A Review of Developments. 2010 IEEE/ACM Int'l Conference on Green Computing and Communications \& Int'l Conference on Cyber, Physical and Social Computing, 533-538. https://doi.org/10.1109/GreenComCPSCom.2010.22

Kidd, C. D., Orr, R., Abowd, G. D., Atkeson, C. G., Essa, I. A., Maclntyre, B., Mynatt, E., Starner, T. E., \& Newstetter, W. (1999). The Aware Home: A Living Laboratory for Ubiquitous Computing Research. In N. A. Streitz, J. Siegel, V. Hartkopf, \& S. Konomi (Eds.), Cooperative Buildings. Integrating Information, Organizations, and Architecture (pp. 191-198). Springer. https://doi.org/10.1007/10705432_17

Kirsch, M., \& Ängeslevä, J. (2008). URBAN EYES. http://project-urbaneyes.blogspot.co.nz/

Kitchin, R., \& Dodge, M. (2011). Code/space: software and everyday life. MIT Press.

Know Your Meme (n.d.). Internet Coke Machine. http://i2.kymcdn.com/photos/images/newsfeed/000/100/288/409208628_b0d4da75c5.jpg.

Knutsen, J. (2014). Uprooting Products of the Networked City. International Journal of Design, 8(1), 127-142.

Kranenburg, R. (2007). The Internet of Things. A critique of ambient technology and the all-seeing network of RFID. [PDF document] Institute of Network Cultures. http://www.networkcultures.org/_uploads/notebook2_theinternetofthings.pdf

Kranz, M., Holleis, P., \& Schmidt, A. (2010). Embedded Interaction: Interacting with the Internet of Things. IEEE Internet Computing, 14(2), 46-53. https://doi.org/10.1109/MIC.2009.141

Kwan, M.-P., \& Ding, G. (2008). Geo-Narrative: Extending Geographic Information Systems for Narrative Analysis in Qualitative and Mixed-Method Research. The Professional Geographer, 60(4), 443-465. https://doi.org/10.1080/00330120802211752

lady ada. (2013, June 12). Setting up a Raspberry Pi as a WiFi access point. Adafruit Learning System. https://learn.adafruit.com/setting-up-a-raspberry-pi-as-a-wifi-access-point/preparation

Lamming, M., \& Flynn, M. (1994). "Forget-me-not" Intimate Computing in Support of Human Memory. Proceedings of FRIEND21, 11. https://citeseerx.ist.psu.edu/viewdoc/download?doi=10.1.1.421.9872

Lasch, A. (2014). - The brooklyn pigeon project. http://arandalasch.com/works/the-brooklyn-pigeonproject/

Law, J. (2004). After Method: Mess in Social Science Research (1st Edition). Routledge.

Lee, J., \& Ingold, T. (2006). Fieldwork on foot: Perceiving, routing, socializing. Locating the Field. Space, Place and Context in Anthropology, 67-86.

Lewis, J. (2016, February 24). MQTT Tutorial for Raspberry Pi, Arduino, and ESP8266. https://www.baldengineer.com/mqtt-tutorial.html

Li, S., Xu, L. D., \& Zhao, S. (2018). 5G Internet of Things: A survey. Journal of Industrial Information Integration, 10, 1-9. https://doi.org/10.1016/j.jii.2018.01.005

Light, R. (n.d.). MQTT Client Library Encyclopedia - Paho Python. Retrieved February 21, 2017, from https://web.archive.org/web/20170621222846/http://www.hivemq.com/blog/mqtt-clientlibrary-paho-python 
Lindley, J., Coulton, P., \& Cooper, R. (2017). Why the Internet of Things needs Object Orientated Ontology. The Design Journal, 20(sup1), S2846-S2857. https://doi.org/10.1080/14606925.2017.1352796

Lindley, S. E. (2015). Making Time. Proceedings of the 18th ACM Conference on Computer Supported Cooperative Work \& Social Computing - CSCW'15, 1442-1452. https://doi.org/10.1145/2675133.2675157

Liu, S.-Y. (Cyn), Bardzell, J., \& Bardzell, S. (2019). Decomposition as Design: Co-Creating (with) Natureculture. Proceedings of the Thirteenth International Conference on Tangible, Embedded, and Embodied Interaction - TEI '19, 605-614. https://doi.org/10.1145/3294109.3295653

Lloyd, A. (2013, Sep). If this toaster could talk: Narratives in the age of smart objects. The Atlantic. http://www.theatlantic.com/technology/archive/2013/09/if-this-toaster-couldtalk/279276/

Lockwood, A. (1989). A sound map of the Hudson River. Lovely Music.

Lockwood, A. (2008). A sound map of the Danube. Lovely Music.

logMaker360. (2016, November 23). MQTT tutorial on Raspberry pi, Arduino and Python. https://www.youtube.com/watch?v=nAUUdbUkJEI

MacColl, I., Chalmers, M., Rogers, Y., \& Smith, H. (2004, February 22). Seamful ubiquity: Beyond seamless integration. Proc. Ubicomp 2002 Workshop on Models and Concepts for Ubiquitous Computing.

Malamund, C. (2000, Dec 30). Exploring the internet: Round one, San Jose. http://museum.media.org/eti/RoundOne01.html

Manning, R. F. (2009). Place, Power and Pedagogy: A Critical Analysis of the Status of Te Atiawa Histories of Place in Port Nicholson Block Secondary Schools and the Possible Application of Place-Based Education Models [Doctoral Dissertation, Victoria University of Wellington]. http://researcharchive.vuw.ac.nz/handle/10063/1051

Martin, P. (2017, October 3). Using your new Raspberry Pi 3 as a Wi-Fi access point with hostapd. https://web.archive.org/web/20171003143808/https://frillip.com/using-your-raspberry-pi-3as-a-Wi-Fi-access-point-with-hostapd/

[matt_symes]. (2016, January 7). Script not starting up with update-rc.d. Ubuntu Forums. https://ubuntuforums.org/showthread.php?t=2308828

McGuirk, J. (2015). Honeywell, I'm Home! The Internet of Things and the New Domestic Landscape. EFlux Journal, (64).

McNely, B. J., \& Rivers, N. A. (2014). All of the Things: Engaging Complex Assemblages in Communication Design. Proceedings of the 32nd ACM International Conference on The Design of Communication CD-ROM, 1-10. https://doi.org/10.1145/2666216.2666222

Mechen, J., Kilford, A., \& Winter, A. (2017). Wairua, wai-rua. https://www.angelakilford.com/wairuawairua

Microsoft. (n.d.). Internet of things overview | Microsoft. http://www.microsoft.com/en-us/server-cloud/internet-of-things/default.aspx

MIT Project Oxygen: Overview. (2004, June 3). MIT Project Oxygen website. http://oxygen.csail.mit.edu/Overview.html

MIT Senseable City Lab. (2009). Trash | Track. http://senseable.mit.edu/trashtrack/ 
Muller, M. J. (2009). Participatory Design: The Third Space in HCI. In A. Sears \& J. A. Jacko (Eds.), Human-Computer Interaction: Development Process (pp.165-186). CRC Press.

Nelson, T. H. (1987). Computer Lib/Dream Machines. Aperture.

Nest Labs, Inc. (2016). Home. https://nest.com/

NIWA. (2008, December 17). Training notes. https://www.niwa.co.nz/ourscience/freshwater/tools/shmak/manual/15trainingnotes

NIWA. (2019). SHMAK Stream Health Monitoring and Assessment Kit. User Manual. NIWA. https://niwa.co.nz/static/web/SHMAK_Manual.pdf

Node-red/node-red. (2020). [JavaScript]. node-red. https://github.com/node-red/node-red (Original work published 2013)

Noorani, T., \& Brigstocke, J. (2018). More-than-human Participatory Research. In K. Facer \& K. Dunleavy (Eds.), Connected Communities Foundation Series. University of Bristol/AHRC Connected Communities Programme.

O'Rourke, K. (2013). Walking and mapping: Artists as cartographers. MIT press.

O'Leary, N. (2016). pubsubclient: A client library for the Arduino Ethernet Shield that provides support for MQTT. [Arduino, github repository]. https://github.com/knolleary/pubsubclient

Odom, W., Lindley, S., Pschetz, L., Tsaknaki, V., Vallgårda, A., Wiberg, M., \& Yoo, D. (2018). Time, Temporality, and Slowness: Future Directions for Design Research. Proceedings of the 19th International ACM SIGACCESS Conference on Computers and Accessibility - DIS '18, 383-386. https://doi.org/10.1145/3197391.3197392

Open Water Project. (n.d.). Open Water Project. Retrieved January 28, 2019, from https://github.com/OpenWaterProject

OpenHAB. (n.d.). Retrieved December, 12 2016, from https://www.openhab.org/

Oulasvirta, A. (2004b, August 17). SeamfulSystems: What are they and how can we design them? Seamfulness Workshop, Haninge, Sweden.

https://web.archive.org/web/20060209150933/http://www.hiit.fi/u/oulasvir/Haninge/seamf ulness-ws-oulasvirta.pdf

Oulasvirta, O. (2004a, August 18). Notes on Seamful Design. By Antti Oulasvirta, HIIT. https://web.archive.org/web/20050427021945/http://www.hiit.fi/u/oulasvir/Haninge/

Panetta, K. (2018). 5 Trends Emerge in the Gartner Hype Cycle for Emerging Technologies, 2018 [Press Release]. http://www.gartner.com/smarterwithgartner/5-trends-emerge-in-gartner-hypecycle-for-emerging-technologies-2018/

Panetta, K. (2019). 5 Trends Appear on the Gartner Hype Cycle for Emerging Technologies, 2019 [Press Release]. http://www.gartner.com/smarterwithgartner/5-trends-appear-on-the-gartnerhype-cycle-for-emerging-technologies-2019/

Parikka, J. (2010, June 23). What is New Materialism-Opening words from the event. https://jussiparikka.net/2010/06/23/what-is-new-materialism-opening-words-from-theevent/

Parikka, J. (2012). New Materialism as Media Theory: Medianatures and Dirty Matter. Communication and Critical/Cultural Studies, 9(1), 95-100. https://doi.org/10.1080/14791420.2011.626252

Parker, K. (2015). Kumutoto Stream.

Parker, K., Farrant, S., Mahalski, B., McNaught, B., \& Warren, P. (2017). Inanga Love Park. http://commongroundfestival.org.nz/portfolio/inanga-love-park/ 
Parks, L., \& Starosielski, N. (2015). Introduction. In Signal Traffic: Critical Studies of Media Infrastructures (pp.1-28). University of Illinois Press. http://www.jstor.org.helicon.vuw.ac.nz/stable/10.5406/j.ctt155jmd9.4

Parse-community/parse-server. (2020). [JavaScript]. Parse. https://github.com/parsecommunity/parse-server (Original work published 2016)

Parse. (n.d.). Parse | Products \& Platforms. https://parse.com

Parsons, M., Nalau, J., Fisher, K., \& Brown, C. (2019). Disrupting path dependency: Making room for Indigenous knowledge in river management. Global Environmental Change, 56, 95-113. https://doi.org/10.1016/j.gloenvcha.2019.03.008

Patel, P., Pathak, A., Teixeira, T., \& Issarny, V. (2011). Towards application development for the internet of things. Proceedings of the 8th Middleware Doctoral Symposium, 1-6. https://doi.org/10.1145/2093190.2093195

Paulson, W.R. (2001). For a Cosmopolitical Philology: Lessons from Science Studies. SubStance 30(3), 101-119. doi:10.1353/sub.2001.0033.

Pemberton, S. (2006, June). Web 4.0: Start planning now!. Presented at the The Web and Beyond, 10th SIGCHI.NL Conference, Amsterdam, The Netherlands. http://www.w3.org/2006/Talks/12-07-steven-aristote/

Perera, C., Zaslavsky, A., Christen, P., \& Georgakopoulos, D. (2014). Sensing as a service model for smart cities supported by Internet of Things. Transactions on Emerging Telecommunications Technologies, 25(1), 81-93. https://doi.org/10.1002/ett.2704

Pfister, C. (2011). Getting Started with the Internet of Things: Connecting Sensors and Microcontrollers to the Cloud (1st edition). Make Community, LLC.

Platformio (n.d.). http://platformio.org/get-started/ide?dl=platformio-atom-mac.zip

Plumwood, V. (2001). Nature as Agency and the Prospects for a Progressive Naturalism. Capitalism Nature Socialism, 12(4), 3-32. https://doi.org/10.1080/104557501101245225

Porter, M. E., \& Heppelmann, J. E. (2014) How smart, connected products are transforming competition. Harvard Business Review. https://hbr.org/2014/11/how-smart-connectedproducts-are-transforming-competition

Practical Maker. (2011). DIY EC Probe. https://www.youtube.com/watch?v=0NPtmHdbW3k

Price, S. (2015, October 5). Havelock North water supply hit by e-coli-NZ Herald. New Zealand Herald. https://www.nzherald.co.nz/nz/news/article.cfm?c_id=1\&objectid=11523875

Priest, J. (2017). Citizen Water Map Lab. https://julianpriest.org/project/citizen-water-map-lab/

protoman. (2009, July 19). USB casting in transparent resin. Retrieved February 18, 2017, from http://www.instructables.com/id/USB-casting-in-transparent-resin/

Pschetz, L. (2015). Isn't it time to change the way we think about time?. interactions, 22(5), 58-61.

Public Lab contributors. (n.d.). Projects [Projects wiki]. Public Lab. Retrieved November 23, 2020, from https://publiclab.org/n/17013

Qi, J. (2012). The Fine Art of Electronics: Paper-based Circuits for Creative Expression [Master of Science in Media Arts and Sciences, Massachusetts Institute of Technology]. http://web.mit.edu/ jieqi/Public/Jie_Qi_MS_thesis.pdf

Ragan, S. M. (2012a, April 12). The Most Beautiful Homemade Device I've Ever Seen. Make: DIY Projects and Ideas for Makers. http://makezine.com/2012/04/12/the-most-beautifulhomemade-device-ive-ever-seen/ 
Ragan, S.M. (2012b, October 29). 15 Fantastic Project Enclosures. Makezine. http://makezine.com/slideshow/15-fantastic-project-enclosures/.

Raspberry Pi (n.d.). FAQs-Raspberry Pi Documentation. https://www.raspberrypi.org/documentation/faqs/\#pi-power-specs

Raspberry Pi. (n.d.) SSH using Linux or Mac OS. https://www.raspberrypi.org/documentation/remoteaccess/ssh/unix.md.

Ratcliffe, M. (2015, September 4). Three Dollar EC - PPM Meter. Retrieved March 19, 2019, from https://hackaday.io/project/project/7008-fly-wars-a-hackers-solution-to-worldhunger/log/24646-three-dollar-ec-ppm-meter-arduino

Rebaudengo, S. (2012). Addicted products: The story of Brad the Toaster. https://vimeo.com/41363473

Rebaudengo, S., \& Haque Design Research. (n.d.). Addicted Toasters. http://addictedproducts.com

Rekret, P. (2016). A critique of new materialism: Ethics and ontology. Subjectivity, 9(3), 225-245.

Rifkin, M. (2017). Beyond Settler Time: Temporal Sovereignty and Indigenous Self-Determination. Duke University Press Books.

Rivera, J. (2015). Gartner's 2015 Hype Cycle for Emerging Technologies Identifies the Computing Innovations That Organizations Should Monitor [Press Release].

https://www.gartner.com/en/newsroom/press-releases/2015-08-18-gartners-2015-hypecycle-for-emerging-technologies-identifies-the-computing-innovations-that-organizationsshould-monitor

RiverSense. (n.d.). RiverSense I Waikato. Retrieved November 23, 2020, from http://www.riversense.cloud/

RiverWatch. (n.d.). RiverWatch-Making sense of water quality. RiverWatch. Retrieved November 23, 2020, from https://riverwatch.nz

RNZ. (2019, September 21). Climate change protests spread on global day of action. https://www.rnz.co.nz/news/world/399261/climate-change-protests-spread-on-global-dayof-action.

Rogers, A., Castree, N., \& Kitchin, R. (2013). More-than-human. In A Dictionary of Human Geography. Oxford University Press.

https://www.oxfordreference.com/view/10.1093/acref/9780199599868.001.0001/acref9780199599868-e-1216

Rosiek, J. L., Snyder, J., \& Pratt, S. L. (2020). The New Materialisms and Indigenous Theories of NonHuman Agency: Making the Case for Respectful Anti-Colonial Engagement. Qualitative Inquiry, 26(3-4), 331-346. https://doi.org/10.1177/1077800419830135

Russell, S., Frame, B., Lennox, J., \& Manaaki Whenua-Landcare Research New Zealand Ltd. (2011). Old problems new solutions: Integrative research supporting natural resource governance. Manaaki Whenua Press.

Santucci, G. (2009). From internet of data to internet of things. Paper for the International Conference on Future Trends of the Internet (Vol. 28) [PDF version]. Luxembourg.

http://cordis.europa.eu/pub/fp7/ict/docs/enet/20090128-speech-iot-conference-lux_en.pdf.

Sarma, S. Brock, D. L., Ashton, K. (2000). The networked physical world: Proposals for engineering the next generation of computing, commerce \& automatic-identification. MIT. 
Sautner, B. ([2011] 2019). Bsautner/com.nimbits [git repository]. https://github.com/bsautner/com.nimbits.

Savetz, K. (1994). I heard someone hooked a toaster to the Internet?! Really?. Your Internet Consultant: The FAQs of Online Life (11.6). http://www.savetz.com/yic/YIC11FI_6.html.

Savicic, G. (n.d.). Concept. Constraint City. http://www.yugo.at/equilibre/?page_id=8.

Schilit, B. N., \& Theimer, M. M. (1994). Disseminating active map information to mobile hosts. Network, IEEE, 8(5), 22-32.

Schoutsen, P. (2015, October 11). Report the temperature with ESP8266 to MQTT. Home Assistant. https://home-assistant.io/blog/2015/10/11/measure-temperature-with-esp8266-and-reportto-mqtt/.

Schultz, T., Abdulla, D., Ansari, A., Canlı, E., Keshavarz, M., Kiem, M., Martins, L. P. de O. \& Vieira de Oliveira, P.J.S. (2018). What Is at Stake with Decolonizing Design? A Roundtable. Design and Culture, 10(1), 81-101. https://doi.org/10.1080/17547075.2018.1434368.

Schwartz, M.O. (2015, July 28). Home Automation in the Cloud with the ESP8266 \& Adafruit 10. Adafruit Learning System. https://learn.adafruit.com/home-automation-in-the-cloud-withthe-esp8266-and-adafruit-io/introduction.

Science Learning Hub - Pokapū Akoranga Pūtaiao. (2011, May 10). Make and use a hydrophone. https://static.sciencelearn.org.nz/documents/files/000/000/170/original/Make_and_use_a_h ydrophone.docx?1551392251

Seaman, A. (2015, May 20). Dangerous Wellington stream exposed by floods. Stuff. https://www.stuff.co.nz/dominion-post/news/68685403/dangerous-wellington-streamexposed-by-floods.

Shaviro, S. (2014). The Universe of Things: On Speculative Realism. University of Minnesota Press.

Shingleton, D. (2011). Object Geography: The Internet of Things. ISEA 2011(The 17th International Symposium on Electronic Art). Istanbul. https://isea2011.sabanciuniv.edu/paper/object-geography-internet-things.

Smith, L. T. (2012). Decolonizing methodologies: research and indigenous peoples. Zed Books Ltd.

Smith, V., McColgan, M., Hornblow, A. \& Pochin, J.P. (2017). Breathe [Installation]. Nelson Pubic Library, New Zealand.

Speed, C., \& Shingleton, D. (2012). Take Me I'M Yours: Mimicking Object Agency. Proceedings of the 2012 ACM Conference on Ubiquitous Computing, 1167-1170. ACM. http://doi.org/10.1145/2370216.2370465.

Spiess, A. (2016). \#58 ESP8266 Sensor runs 17 days on a coin cell/transmits data to sparkfun.com and ubidots.com. Youtube. https://www.youtube.com/watch?v=IYuYTfO6iOs.

Springgay, S., \& Truman, S. E. (2019). Walking methodologies in a more-than-human world: Walking lab. Routledge.

Stadtgemeinde Knittelfeld. (2004). Umweltbericht der Stadtgemeinde Knittelfeld (No. 52/5; Aus dem Knittelfelder Rathaus). Stadtgemeinde Knittelfeld.

https://www.yumpu.com/de/document/read/10651048/sonderausgabe-der-zeitung-ausdem-knittelfelder-rathaus.

Stafford-Fraser, Q. (2001). On Site: The Life and Times of the First Web Cam. Communications of the ACM, 44(7), 25-26. http://doi.org/10.1145/379300.379327. 
Stanford-Clark, A. (2009). Twitter.com/andy_house.

http://image.slidesharecdn.com/andyscoggcamp-091224085658-phpapp01/95/the-housethat-twitters-26-728.jpg?cb=1261646284.

Star, S. L., \& Griesemer, J. R. (1989). Institutional Ecology, 'Translations' and Boundary Objects: Amateurs and Professionals in Berkeley's Museum of Vertebrate Zoology, 1907-39. Social Studies of Science, 19(3), 387-420. https://doi.org/10.1177/030631289019003001.

Starr, M. (2014, January 19). Fridge caught sending spam emails in botnet attack. CNet. http://www.cnet.com/news/fridge-caught-sending-spam-emails-in-botnet-attack/.

Stead, M. (2017) Spimes and Speculative Design: Sustainable Product Futures Today. Strategic Design Research Journal,10(1), 12-22.

Stead, M., Coulton, P. \& Lindley, J. (2019). Spimes Not Things. Creating A Design Manifesto For A Sustainable Internet of Things. The Design Journal, 22(sup1), 2133-2152. https://doi.org/10.1080/14606925.2019.1594936.

Sterling, B. (2004). Viridian note 00422: The spime. Viridian Design. http://www.viridiandesign. Org/notes/401-450/00422_the_spime.html.

Sterling, B. (2005) Shaping Things. MIT.

Steward, W. (2015). The Internet Toaster. Living Internet. http://www.livinginternet.com/g/toaster_simon2.jpg.

Sullivan, N. (2012). The somatechnics of perception and the matter of the non/human: A critical response to the new materialism. European Journal of Women's Studies, 19(3), 299-313.

Summerson, C. (2019, April 8). Stringify is Shutting Down After Five Years. How-To Geek. https://www.howtogeek.com/410576/stringify-is-shutting-down-after-five-years/.

Swanson, H., Tsing, A., Bubandt, N. \& Gan, E. (2017). Introduction: Bodies tumbled into bodies. In H. Swanson, A. Tsing, N. Bubandt, \& E. Gan (Eds.), Arts of Living on a Damaged Planet (pp. 1-13). University of Minnesota Press. https://www.jstor.org/stable/10.5749/j.ctt1qft070.18.

Taiuru, K. (2015). Impacts and Considerations for Indigenous Populations using Open Source. Open Source/Open Society.

Taylor, J. L., Soro, A., Roe, P., Lee Hong, A. \& Brereton, M. (2017). Situational When: Designing for Time Across Cultures. Proceedings of the 2017 CHI Conference on Human Factors in Computing Systems, 6461-6474. https://doi.org/10.1145/3025453.3025936.

Te Mana Raraunga. (2017). Te Mana Raraunga-Māori Data Sovereignty Network Charter. https://static1.squarespace.com/static/58e9b10f9de4bb8d1fb5ebbc/t/5913020d15cf7dde1d f34482/1494417935052/Te+Mana+Raraunga+Charter+\%28Final+\%26+Approved\%29.pdf.

Te Punga Somerville, A. (2015, June). Culvert: The slipperiness of place. Pukeahu: An Exploratory Anthology. http://pukeahuanthology.org/stories/migrations/culvert/.

The Disappearing Computer Initiative. (2003). The Disappearing Computer. http://www.disappearingcomputer.org/

The Hammersmith Group. (2009). Clicks \& Mortar: Web 4.0, The Internet of Things [PDF version]. The Hammersmith Group.

https://web.archive.org/web/20131126150019/http://thehammersmithgroup.com/images/r eports/web4.pdf.

ThingSpeak. (n.d.). IoT Analytics. https://thingspeak.com/. 
Thomas, P. (2014, January 23). Despite the News, Your Refrigerator is Not Yet Sending Spam. Symantec. http://www.symantec.com/connect/blogs/despite-news-your-refrigerator-not-yetsending-spam.

Thompson, M. (2007). Wi-Fi Dowsing Rod. MOMA. http://www.moma.org/interactives/exhibitions/2011/talktome/objects/145461/.

Tipa, G., Teirney, L. D., New Zealand, \& Ministry for the Environment. (2006). A cultural health index for streams and waterways: A tool for nationwide use. Ministry for the Environment.

Tipa, G., Harmsworth, G.R., Williams, E. \& Kitson, J.C. (2016). Integrating mātauranga Māori into freshwater management, planning and decision making. In P.G. Jellyman, T.J.A. Davie, C.P. Pearson, \& J.S. Harding (Eds.), Advances in New Zealand Freshwater Science. New Zealand Freshwater Sciences Society \& New Zealand Hydrological Society.

Tourism New Zealand. (n.d.). Welcome to New Zealand. Tourism New Zealand. https://www.newzealand.com/nz/.

transmediale. (2015a). The Quantifiable Everything? - STARTS roundtable on the internet of things. http://transmediale.de/content/the-quantifiable-everything-starts-roundtable-on-theinternet-of-things.

transmediale. (2015b). Presentation by Bruce Sterling - The quantifiable everything?. Youtube. https://www.youtube.com/watch?time_continue=13\&v=JUvECBZpefU.

transmediale (2016). Reprogramming the internet of things. http://2016.transmediale.de/content/reprogramming-the-internet-of-things.

Tuters, M., \& Varnelis, K. (2006). Beyond locative media: Giving shape to the internet of things. Leonardo, 39(4), 357-363. doi:10.1162/leon.2006.39.4.357.

Ulmer, J. B. (2017). Writing Slow Ontology. Qualitative Inquiry, 23(3), 201-211. https://doi.org/10.1177/1077800416643994.

United Nations. (n.d.). Sustainable Development Goals: Sustainable Development Knowledge Platform. https://sustainabledevelopment.un.org/?menu=1300.

Valois, A., Davies-Colley, R., Storey, R., Wright-Stow, A., Stott, R., Kin, E. \& van Hunen, S. (2019). Volunteer monitoring as a focus for community engagement in water management in Aotearoa-New Zealand: Review and prospects. Water Supply, 19(3), 671-680. https://doi.org/10.2166/ws.2018.116.

Vertesi, J. (2014). Seamful Spaces: Heterogeneous Infrastructures in Interaction. Science, Technology, \& Human Values, 39(2), 264-284. https://doi.org/10.1177/0162243913516012.

Victoria University of Wellington. (2019a, November 26). DSDN172-Maori Narratives/Storytelling for Design (CRN 30062). https://www.wgtn.ac.nz/courses/dsdn/172/2018/offering?crn=30062.

Victoria University of Wellington. (2019b). CCDN412 - Mātauranga Design (CRN29153). https://www.wgtn.ac.nz/courses/ccdn/412/2019/offering?crn=29153

Waldo, J. (2002). Virtual organizations, pervasive computing, and an infrastructure for networking at the edge. Information Systems Frontiers, 4(1), 9-18.

http://search. proquest.com.helicon.vuw.ac.nz/docview/232037596? accountid=14782.

Walker, S., Eketone, A., \& Gibbs, A. (2006). An exploration of kaupapa Maori research, its principles, processes and applications. International Journal of Social Research Methodology, 9(4), 331344. https://doi.org/10.1080/13645570600916049.

Walkevar, S., \& Agrawai, P. (2016, May 9). Esp8266 ad-hoc network. Youtube. https://www.youtube.com/watch?v=ulbjkl8DsdE. 
Ward, M. (2001, April 6). A small slice of design. BBC.

http://news.bbc.co.uk/2/hi/science/nature/1264205.stm.

Wardrip-Fruin, N. (2003). 21. [Introduction] FromComputer Lib / Dream Machines. In N. Montfort \& N. Wardrip-Fruin (Eds.), The new media reader (pp. 301-302). MIT Press.

Weiser, M. (1991). The computer for the 21st century. Scientific American, 265(3), 94-104.

Weiser, M. (1994). Ubiquitous Computing (Abstract). In Proceedings of the 22nd Annual ACM Computer Science Conference on Scaling Up: Meeting the Challenge of Complexity in Realworld Computing Applications (p.418). ACM. http://doi.org/10.1145/197530.197680.

Weiser, M. (1995). Ubiquitous Computing. UBIQ. http://www.ubiq.com/hypertext/weiser/Usenix95Slides.ps.

Weiser, M., \& Brown, J. S. (1996). Designing calm technology. PowerGrid Journal, 1(1), 75-85.

Weisman, R. (2004, Oct 25). The Internet of Things. The Boston Globe. https://www.boston.com/business/technology/articles/2004/10/25/the_internet_of_things/.

Wellington City Council. (2015, November 3). Wellington City Council Stormwater Pipe. https://koordinates.com/layer/3837-wellington-city-council-stormwater-pipe/.

weputachipinit. (n.d.). We put a chip in it! [Tumblr page]. Tumblr. https://weputachipinit.tumblr.com/?og=1.

Whatmore, S. (2002). Hybrid geographies: natures, cultures, spaces. SAGE.

Whatmore, S. (2004). Mapping posthumanism: an exchange. Environment and Planning A, 36(8), 1360-1363. http://doi.org/10.1068/a37127.

Whatmore, S. (2006). Materialist returns: practising cultural geography in and for a more-than-human world. Cultural Geographies, 13(4), 600-609. http://doi.org/10.1191/1474474006cgj377oa

Wilcox, D. (2012). No Place Like Home, GPS shoes. Dominic Wilcox. http://dominicwilcox.com/portfolio/gpsshoe/.

Wildthings.io. (2018, December 3). Papawai Stream [sound recording].. https://soundcloud.com/wildthings10/papawai-stream-3-december-2018.

Yusoff, K. (2015, August 28). "Future Fossils" Exhibition by Beth Greenhough, Jamie Lorimer and Kathryn Yusoff. Society and Space. https://societyandspace.com/2015/08/28/future-fossils-greenhough-lorimer-yusoff/. 


\title{
Appendix A
}

\author{
Human Ethics Application 0000023322 : Participatory design walkshops for a more-than- \\ human Internet of Things - 20/09/2016
}

\section{Describe the benefits and scholarly value of the project}

Literature reveals that there is currently no stable technological framework for building the a global, market-driven IoT, but the availability of IoT technology allows artists and designers to create alternative visions to the prevailing market-driven scenario. New Materialism and more-than-human theory offer a philosophical context for approaching IoT design from a non-anthropocentric thing-perspective, but they lack established methodological approaches.

\section{Describe the method of data collection. Note that later in this form, in the Documents} section, you will need to upload any relevant documentation such as interview schedule, survey, questionnaires, focus group rules, observation protocols etc. Delays are likely if the interview questions are missing from the Documents section.

Qualitative and quantitative (i.e. sensor) data will be collected during a set of participatory design workshops and evaluated in a follow-up workshop.

Workshop 1 - Generation:

The first workshop consists of three phases and the methods will iteratively change over the course of the workshop series but the general scope of the workshops will remain the same.

The first phase is a general introduction to the research and involves a sensitizing activity (as recommended by Hagen, 2013, p.5). This activity involves exploring participants connections with the stream ecosystem and the IoT through visual methods such as ecosystem mapping, semantic zoom, personas or collages. 
In the second phase participants will explore the stream ecosystem in an immersive 'walkshop', (see for example Korn \& Zander, 2010 ) through a walk along the stream. Data will be collected e.g. through field recordings and sensory probes, aiming to identify IoT related design context in the ecosystem. This involves audiovisual recording of the walk, exploratory use of IoT equipment, logging and tagging of relevant GPS locations and documentation of themes explored and discussed during the walk.

In the third phase of the workshop, participants will reflect on the data and material collected through lo-fi prototyping. In order to provide open access to the workshop data, workshop material, methods and design outputs will be regularly published through an online repository on the website www.wildthings.io

Workshop 2 - Evaluation

The details for this workshop will be amended once the evaluation period is closer.

\section{Describe the objectives of the project}

The objective of this research is to understand how 'things' themselves can be included in the design process of an Internet of Things (IoT). I will first produce and assess a set of IoT prototypes using a variety of design methods, and then propose a set of design methods for future research.

\section{Who will the participants in this project be?}

Adult members of neighbourhood community groups who have an interest in, or an active engagement with, local urban ecosystems, such as community-run initiatives for maintaining native wildlife reserves, or restoring and urban stream ecologies. 
To-date contact has been established with the 'Friends of Papawai Terrace' community group and a first workshop is planned for October 2016. The group representative is also connected to neighbouring community groups and has agreed to help with further participant recruitment. For workshops held as part of the programme of the Hutt Public Art Festival 'Common Ground' in Lower Hutt, which takes place in February 2017, participants will be additionally recruited via festival communications.

\section{How will participants be recruited?}

Local communities will be contacted via their official community group contacts, and via snowball sampling. Additional participants may be reached through social media, email lists and collaboration with relevant arts networks and design events. 


\section{Wildthings.io \\ Materializing a More-than-human Internet of Things}

\section{INFORMATION SHEET FOR PARTICIPANTS}

Thank you for your interest in this project. Please read this information before deciding whether or not to take part. If you decide to participate, thank you. If you decide not to take part, thank you for considering my request.

\section{Who am I?}

My name is Birgit Bachler and I am a Doctoral student in Design at Victoria University of Wellington. This research project is work towards my dissertation.

\section{What is the aim of the project?}

In commercial scenarios the term "Internet of Things (IOT)" describes the idea of a network in which 'things' - such as toasters, fridges, lamps or cars - are connected to the Internet, typically for user convenience. This research looks at how loT technology can instead be used creatively to re-connect people with often invisible urban wildlife.

During a half-day workshop, we will explore local stream ecosystems through walking, and then collaboratively devise and prototype loT technologies that could give a voice to the stream and (re-)connect us and it with the larger environment.

\section{How can you help?}

If you agree to take part in this research, you will participate in a half-day design workshop involving a walk along a local stream and participatory design activities. Location and times will be decided in collaboration with the participants. The workshop will be audio-visually recorded but participants can choose to have their face blurred and/or their voice modified in published materials.

You will also be invited to a second evaluation workshop planned for 2017.

You can stop participating in the workshop at any time, without giving a reason. You can withdraw from the study by contacting me at any point within 14 days after the workshop. If you withdraw, all identifiable data and recordings will be destroyed or returned to you. Due to the nature of the research, it may not be possible to withdraw data that has been collaboratively created with the group up to withdrawal. 


\section{What will happen to the information you give?}

Documentation and outcomes of the workshop will be published, and made publicly accessible, on the website www.wildthings.io. You understand that this research is not confidential or anonymous, but you can choose to keep personal information confidential to the research team. All data not placed in the public repository, e.g. interview transcripts, summaries and any recordings, will be kept securely and destroyed two years after the research ends.

\section{What will the project produce?}

The information from my research will be used in my $\mathrm{PhD}$ dissertation. Design outputs and methods produced during the workshop will be published online (www.wildthings.io). I may also use the results of my research for conference presentations, and academic reports.

\section{If you accept this invitation, what are your rights as a research participant?}

You do not have to accept this invitation if you don't want to. If you do decide to participate, you have the right to:

- $\quad$ choose not to participate in any activity;

- choose whether you want or do not want to be identified in publications

- $\quad$ ask any questions about the study at any time;

- be able to read any reports of this research by emailing the researcher to request a copy.

- $\quad$ withdraw from the study within 14 days of the workshop date

- $\quad$ agree on another name for me to use rather than your real name

If you have any questions or problems, who can you contact?

If you have any questions, either now or in the future, please feel free to contact either:

\section{Student:}

Name: Birgit Bachler

School: School of Design

Phone: 044636203

Email: birgit.bachler@vuw.ac.nz

\section{Supervisor:}

Name: Dr. Anne Galloway

School: School of Design

Phone: 044636230

Email: anne.galloway@vuw.ac.nz

\section{Human Ethics Committee information}

This research has been approved by the Victoria University Human Ethics Committee (\#23322). If you have any concerns about the ethical conduct of the research you may contact the Victoria University HEC Convener: Associate Professor Susan Corbett. Email susan.corbett@vuw.ac.nz or telephone +64-4-463 5480 . 
Wildthings.io

\title{
Materializing a More-Than-Human Internet of Things
}

\author{
CONSENT TO PARTICIPATE
}

Researcher: Birgit Bachler, School of Design, Victoria University of Wellington.

- I have read the Information Sheet and the project has been explained to me. My questions have been answered to my satisfaction. I understand that I can ask further questions at any time.

- I agree to take part in a workshop series that will be audio-visually recorded.

I understand that:

- Identifying information I provide will be kept confidential to the researcher and the supervisor. I understand that the results will be published online (www.wildthings.io) and used for a PhD report, and a summary of the results may be used in academic reports and/or presented at conferences.

- $\quad$ Any recorded information will not be used for any other purpose or released to others without my written consent

- $\quad$ All data not placed in the public repository, e.g. interview transcripts, summaries and any recordings, will be kept securely and destroyed two years after the research ends.

I wish to keep my identity anonymous in published materials:

- through voice modification in audio-visual documentation

- through the use of a pseudonym.

- through blurring of identifying features in video and photographic documentation

$\begin{array}{ll}\text { Yes } \square & \text { No } \square \\ \text { Yes } \square & \text { No } \square \\ \text { Yes } \square & \text { No } \square\end{array}$

I would like to receive a copy of the final report and have added my email address below.

Yes $\square \quad$ No $\square$

Signature of participant:

Name of participant:

Date:

Contact details: 


\section{Wildthings.io \\ Materializing a More-than-human Internet of Things \\ INFORMATION SHEET FOR INTERVIEW PARTICIPANTS}

Thank you for your interest in this project. Please read this information before deciding whether or not to take part. If you decide to participate, thank you. If you decide not to take part, thank you for considering my request.

\section{Who am I?}

My name is Birgit Bachler and I am a Doctoral student in Design at Victoria University of Wellington. This research project is work towards my dissertation.

\section{What is the aim of the project?}

In commercial scenarios the term "Internet of Things (IOT)" describes the idea of a network in which 'things' - such as toasters, fridges, lamps or cars - are connected to the Internet, typically for user convenience. This research looks at how loT technology can instead be used creatively to re-connect people with often invisible urban wildlife, with a focus on local streams.

\section{How can you help?}

I will ask you questions about your perspectives on urban water and design prototypes developed during this study. The interview will take approximately one hour. I will record the interview and write it up later. You can stop the interview at any time, without giving a reason. You can withdraw from the study by contacting me at any point within 14 days after the interview. If you withdraw, the information you provided will be destroyed or returned to you.

\section{What will happen to the information you give?}

This research is not confidential or anonymous, but you can choose to keep personal information confidential to the research team.

Outcomes of the design research will be published, and made publicly accessible, on the website www.wildthings.io. Any data not placed in the public repository, e.g. interview transcripts, summaries and any recordings, will be kept securely and destroyed two years after the research ends. 


\section{What will the project produce?}

The information from my research will be used in my PhD dissertation. Design outputs produced as result of the interview will be published online (www.wildthings.io). I may also use the results of my research for conference presentations, and academic reports.

\section{If you accept this invitation, what are your rights as a research participant?}

You do not have to accept this invitation if you don't want to. If you do decide to participate, you have the right to:

- choose not to answer any question;

- $\quad$ ask for the recorder to be turned off at any time during the interview;

- withdraw from the study within 14 days of the interview

- choose how you want to be identified in publications;

- $\quad$ ask any questions about the study at any time;

- $\quad$ receive a copy of your interview recording;

- $\quad$ read over and comment on a written summary of your interview;

- $\quad$ agree on another name for me to use rather than your real name;

- be able to read any reports of this research by emailing the researcher to request a copy.

If you have any questions or problems, who can you contact?

If you have any questions, either now or in the future, please feel free to contact either:

\section{Student:}

Name: Birgit Bachler

School: School of Design

Phone: 044636203

Email: birgit.bachler@vuw.ac.nz

\section{Supervisor:}

Name: Dr. Anne Galloway

School: School of Design

Phone: 044636230

Email: anne.galloway@vuw.ac.nz

\section{Human Ethics Committee information}

This research has been approved by the Victoria University Human Ethics Committee (\#23322). If you have any concerns about the ethical conduct of the research you may contact the Victoria University HEC Convener: Associate Professor Susan Corbett. Email susan.corbett@vuw.ac.nz or telephone +64-4-463 5480 . 


\section{0: \\ Wildthings.io \\ Materializing a More-Than-Human Internet of Things \\ CONSENT TO INTERVIEW}

Researcher: Birgit Bachler, School of Design, Victoria University of Wellington.

- I have read the Information Sheet and the project has been explained to me. My questions have been answered to my satisfaction. I understand that I can ask further questions at any time.

- I agree to take part in an audio recorded interview.

I understand that:

- Identifying information I provide will be kept confidential to the researcher and the supervisor. I understand that the results will be published online (www.wildthings.io) and used for a PhD report, and a summary of the results may be used in academic reports and/or presented at conferences.

- $\quad$ Any recorded information will not be used for any other purpose or released to others without my written consent

- $\quad$ All data not placed in the public repository, e.g. interview transcripts, summaries and any recordings, will be kept securely and destroyed two years after the research ends.

You can choose how you want to be identified in published materials:

- I wish to be identified by my name

- I wish to be identified through my occupation

Yes $\square \quad$ No $\square$

- I wish to be identified through my organisation/affiliation

Yes $\square \quad$ No $\square$

Yes $\square \quad$ No $\square$

I would like to receive a copy of the final report and have added my email address below.

Yes $\square \quad$ No $\square$

Signature of participant:

Name of participant:

Date:

Contact details: 


\section{Artist Interview Question Samples}

You have been selected as an artist that has a body of work relating to water. What kind of water do you specifically work with?

Can you discuss the methodology you employ in your artistic work, specifically when engaging with water?

What role does media play in this practice? What devices do you use for sensing water? Do you build/create your own devices/media?

What is your perspective on artistic practice and collaboration engaging with a this field? Do you collaborate with scientists, engineers policy makers?

As an artist based in Aotearoa/New Zealand, can you describe how working with water and land here differs from approaches elsewhere?

Is your practice connected to Mātauranga Māori, and if yes, can you describe in what way? 
MEMORANDUM

\begin{tabular}{l|l}
\hline TO & Birgit Bachler \\
\hline COPY TO & Dr Anne Galloway \\
\hline FROM & AProf Susan Corbett, Convener, Human Ethics Committee \\
\hline
\end{tabular}

\begin{tabular}{l|l}
\hline DATE & 20 September 2016 \\
\hline PAGES & 1 \\
\hline
\end{tabular}

SUBJECT $\quad$ Ethics Approval: 23322

Participatory design walkshops for a more-than-human Internet of Things

Thank you for your application for ethical approval, which has now been considered by the Standing Committee of the Human Ethics Committee.

Your application has been approved from the above date and this approval continues until 30 April 2018. If your data collection is not completed by this date you should apply to the Human Ethics Committee for an extension to this approval.

Best wishes with the research.

Kind regards

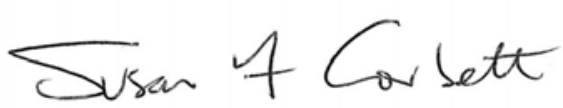

Susan Corbett

Convener, Victoria University Human Ethics Committee 


\section{Appendix B}

\section{Audiovisual media components}

\section{fieldexplorations.mp4}

1280x720, MPEG-4 movie, 24:13, Stereo audio

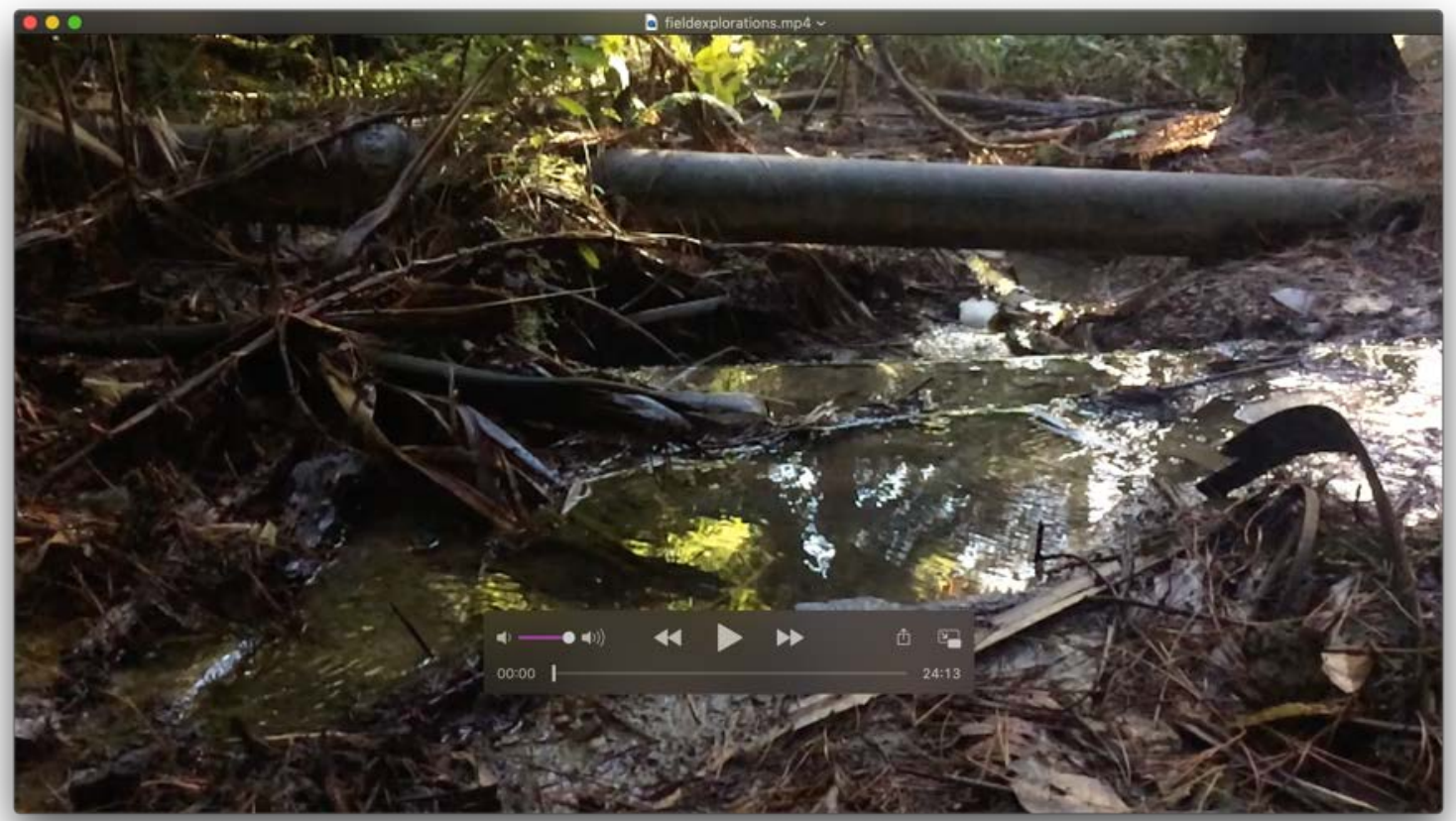

Figure 161: Author. (2020). Screenshot of fieldexplorations.mp4 


\section{prototypinglog.mp4}

1080x720, MPEG-4 movie, 22:53, Stereo audio

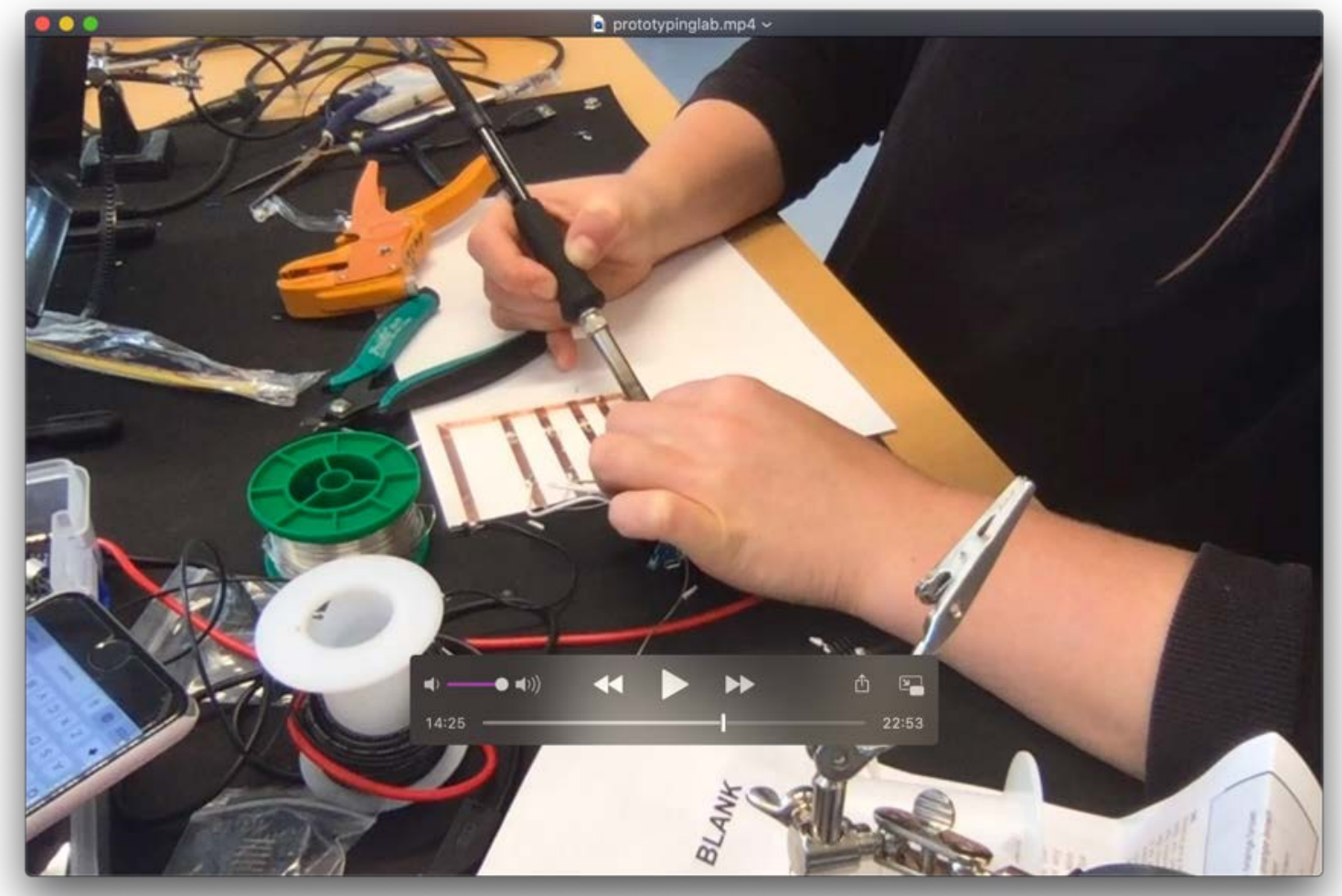

Figure 162: Author. (2020). Screenshot of prototypinglog.mp4 


\section{fieldlab.mp4}

1280x720, MPEG-4 movie, 03:13, Stereo audio

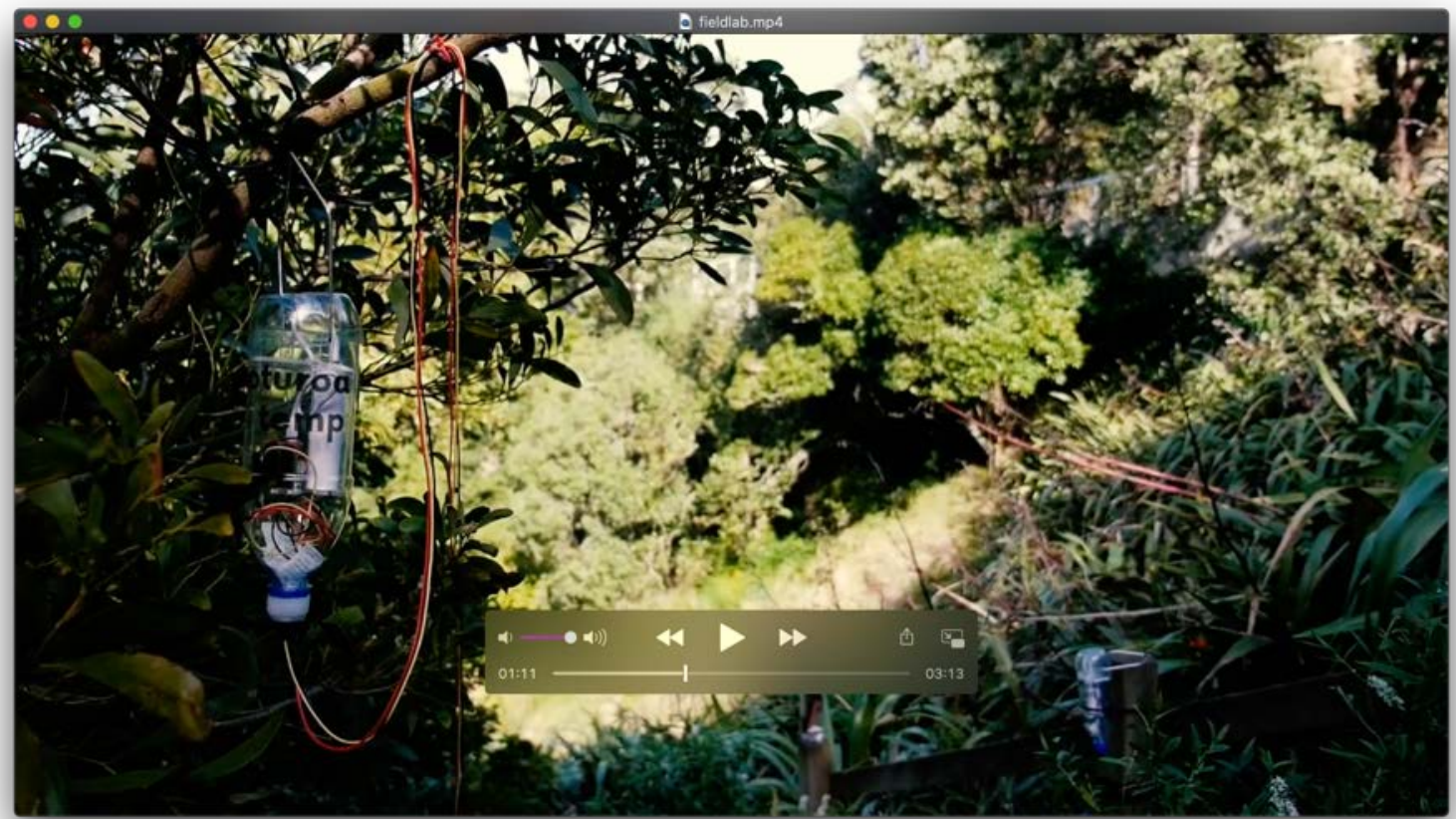

Figure 163. Author. (2020). Screenshot of fieldlab.mp4 